UNIVERSIDAD AUTÓNOMA DE MADRID

PROGRAMA DE DOCTORADO DE BIOCIENCIAS MOLECULARES

\title{
CHARACTERIZATION OF HEMATOPOIETIC STEM CELLS IN THE CIRCULATION
}

I T Z I A R C O S S ÍO C U A R T E R O

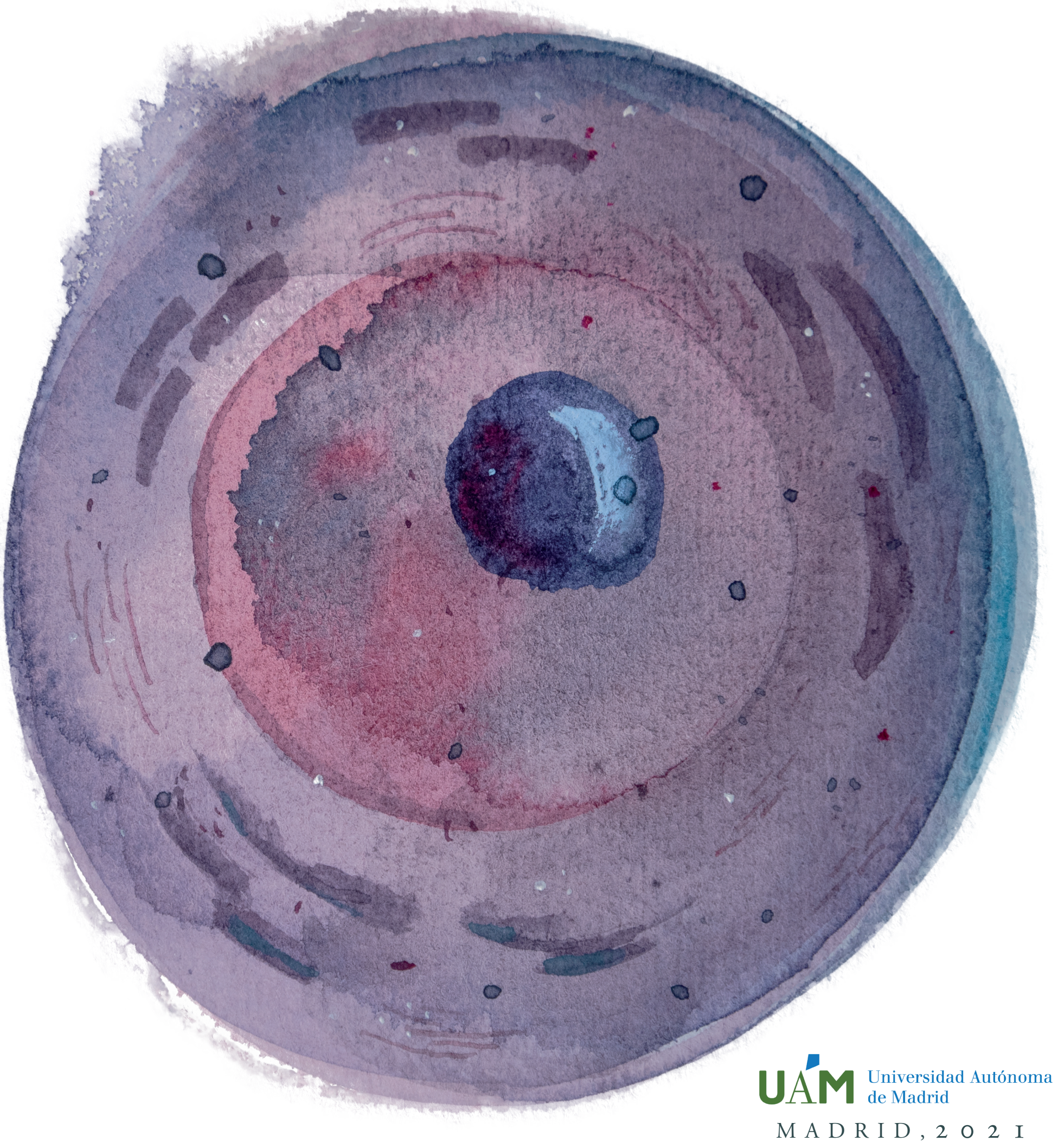


AUTOR/-A: ITZIAR COSSÍO CUARTERO

DIRECTOR/-A(S): ANDRÉS HIDALGO ALONSO

PROGRAMA DE DOCTORADO: BIOCIENCIAS MOLECULARES

TÍTULO: CHARACTERIZATION OF HEMATOPOIETIC STEM CELLS IN THE CIRCULATION

Hago entrega de la citada tesis doctoral en tiempo y forma de acuerdo a la normativa vigente de la Escuela de Doctorado de la Universidad Autónoma de Madrid.

Asimismo, como autor de la citada tesis doctoral,

\section{DECLARO:}

- Que el documento responde al Código de Buenas Prácticas en la Investigación de Universidad Autónoma de Madrid.

- Que es un trabajo original y sin plagios, donde se han seguido los estándares internacionales de citación y normas de publicación.

- Que soy conocedor de que el incumplimiento de las declaraciones anteriores supone la anulación del título de Doctor/-a

Del mismo modo, ASUMO frente a la Universidad cualquier responsabilidad que pudiera derivarse del incumplimiento del compromiso ético de la presente declaración.

Madrid, 12 de febrero de 2021

Fdo: D./D. a Itziar Cossío Cuartero

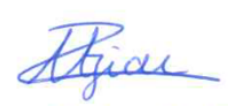


Universidad Autónoma de Madrid (UAM)

\author{
Facultad de Medicina \\ Departamento de Bioquímica
}

Programa de Doctorado en Biociencias Moleculares

\title{
UÁMM
}

\section{"Characterization of Hematopoietic Stem Cells in the Circulation"}

\section{Itziar Cossío Cuartero}

Graduada en Biotecnología por la Universidad de Barcelona

\author{
Dirigida por \\ Dr. Andrés Hidalgo Alonso
}

Realizada en el Centro Nacional de Investigaciones Cardiovasculares (CNIC)

Financiada por el Ministerio de Ciencia Innovación y Universidades (MICINN)

Madrid, 2021 
-2 - 
Dr. Andrés Hidalgo Alonso CERTIFICA:

Que la presente memoria de tesis titulada "Characterization of Hematopoietic Stem Cells in the Circulation", que presenta Da Itziar Cossío Cuartero para obtener el grado de Doctor, ha sido realizada bajo mi dirección, autorizándola para su presentación al Tribunal Calificador.

Madrid, 4 de enero de 2021

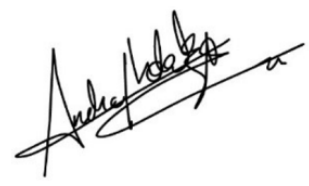

Fdo. Dr Andrés Hidalgo Alonso

Director de la presente Tesis Doctoral

Éste trabajo se ha realizado en el laboratorio del Dr. Andrés Hidalgo Alonso, "Imagen de la Inflamación Cardiovascular y la Respuesta Inmune", englobado en el Área de Biología Celular y del Desarrollo del Centro Nacional de Investigaciones Cardiovasculares (CNIC), en Madrid.

El estudio ha sido financiado por el proyecto SAF2015-65607-R otorgados al Dr. Andrés Hidalgo por el Ministerio de Economía, Industria y Competitividad (MEIC). Por su parte, $D^{a}$ Itziar Cossío Cuartero ha sido beneficiaria de una beca FPI (SAF2015-65607-R). El CNIC recibe financiación del MEIC y la Fundación Pro-CNIC. 
- 4 - 
A mi familia... 
$-6-$ 


\section{Agradecimientos}

Este trabajo os lo dedico a vosotros, familia. No tengo la menor duda de que sin todo vuestro apoyo, hoy no estaría donde estoy. Desde pequeña, me habéis inculcado unos valores magníficos que han hecho que sea la persona que soy a día de hoy. Me habéis enseñado lo que es el sacrificio, el trabajo duro, pero también la humildad y la generosidad. Solo tengo palabras de agradecimiento hacia vosotros. Papi y Mami, gracias por acompañarme en todo mi recorrido académico, por apostar siempre por mí y por haberme ayudado en todo lo que he necesitado sin ningún tipo de reparo. Sin todo eso yo hubiera abandonado este camino seguramente hace mucho tiempo, pero creo que el esfuerzo ha valido la pena. Este camino, llamado tesis, no es, ni mucho menos, un camino de rosas, pero vosotros siempre me habéis dado la fuerza y energía para seguir adelante. $Y$ aunque nos separen muchos kilómetros de distancia, nunca he dejado de notar esas fuerzas positivas y ese cariño que me enviabais.

Feli, que te voy a decir. Ya sabes que lo eres todo para mí, tatico. Has estado todos estos años animándome, viendo siempre el lado positivo de las cosas y haciendo de hermano mayor muchas veces. Sé que estemos donde estemos, vamos a recorrer nuestro camino juntos y eso me da mucha tranquilidad y felicidad. Sin ti, todo esto tampoco hubiera sido posible, así que muchas gracias por traerme hasta aquí. Ya sabéis que los tres lo sois todo para mí, os quiero.

También querría agradecer a todo el resto de mi familia maña y peruana por todos estos años de continuo apoyo y admiración. ¡Tengo mucha suerte de teneros! Sabéis que hay una celebración pendiente :)

No puedo olvidarme de mis niñas catalanas. Izar, Laura y María, mis tres grandes pilares. Sabéis que sois lo mejor que tengo. Sin vosotras esto habría sido un camino muy, muy duro. Gracias por escucharme y apoyarme tantísimo siempre, de verdad. No sé que haría sin vosotras. Sois fundamentales en mi vida, os quiero muchísimo.

Hacer mención también a mis niños catalanes, Isart y Albert, por recorrer este camino juntos.

Dar millones de gracias a mi Gordito Vic. Te ha tocado vivirme en una "mala" etapa y encima durante una pandemia mundial, ique se dice pronto!, así que gracias de verdad por toda esa paciencia que has tenido, y por aguantar mi 
mal humor y mi estrés. Gracias por hacerme reír y sobre todo por darme tu hombro cuando más lo necesito. Eres puro corazón, te quiero.

En el ámbito académico, quería agradecer en primer lugar a Andrés (el Dr. Hidalgo), por brindarme la magnífica oportunidad de formar parte de su equipo. Le estoy y le estaré siempre muy agradecida por apostar por mí en un momento complicado. Me sentí muy arropada por todo el grupo en aquel momento tan difícil de asimilar (empezar una tesis por segunda vez, iquién me lo iba a decir!). Esta experiencia, llamada tesis, no suele ser fácil, pero gracias a Andrés, todo ha sido mucho más llevadero, ya que siempre mantiene la puerta abierta de su despacho dispuesto a echarte una mano, y siempre con ideas brillantes en su mente. ¡Muchas gracias por estos años de enseñanza y aprendizaje continuo! Mención especial también a Linnea, que siempre nos ha recibido en casa con los brazos abiertos y un pastel preparado (jriquísimo, por cierto!).

También querría agradecer uno por uno a los miembros de mi grupo, ya que sin ellos esta tesis no sería una realidad. Primero, a ti Juanchi (JAQ), uno de los pilares fundamentales de mi tesis. Gracias por haberme ayudado tantísimo en todos mis años de tesis (¡salvo el último que te escapaste!). Gracias por apoyarme en todos los malos momentos por los que he pasado durante estos años, siempre has estado ahí para darme los mejores consejos. Has hecho que mis días en el laboratorio fueran mucho más amenos, clarísimamente ¡no me he podido reír más en la poyata! Ha sido genial y al final te quedas con esos pequeños momentos, con las batallitas que nos contábamos, con todas las coñas que hacíamos, apodos que poníamos a la gente... En fin, ha sido genial conocerte, formamos un gran equipo.

Geo, florecilla, muchísimas gracias por ser como eres, sé que puedo contar contigo para todo y has sido uno de los grandes apoyos dentro del laboratorio. Siempre dispuesta a ayudarme cuando me has visto agobiada, triste... Eso siempre lo recordaré, mil gracias. Eres una persona bonita por fuera, pero sobre todo con un corazón gigante por dentro.

Jose, has sido una fuente de sabiduría para mí. ¡Me has enseñado tantas cosas que te debo media tesis! (salvo lo del chorrito de azida). Siempre alegre, contento y con ideas buenísimas jaunque odies las stem, ¡sabes que las acabarás amando!). Ha sido un placer conocerte a ti y a Juanma, por supuesto, que también forma parte de este gran equipo y que tantísimas veces ha hecho que 
llegue un poquito antes a casa. Además, grandes artistas donde los haya ¡Sois lo mejor! ¡Muchísimas gracias por el pedazo de portada!

Iván, ¿cuántas veces te habré ido a dar el coñazo a tu mesa? Mil gracias por ayudarme tanto con tu brillante cabeza y siempre ver el lado bueno de mi proyecto. Sabes que siempre seremos Carmen Sevilla y Parada al piano.

Ángel, el gran gallego. Muchas gracias por todos esos momentos de cordura que me has ofrecido y sobre todo por ayudarme a animar el laboratorio, nos ha costado un poco, ipero creo que lo hemos conseguido! Gracias también por todos estos años de enseñanza.

Sandra, gracias por crear tan buen rollo en el laboratorio, animarme cuando lo necesitaba y siempre estar dispuesta a ayudar en todo.

Andrea, gracias por tanta ayuda y paciencia conmigo, jha sido un placer aprender tanto de ti!

Marianna, puedo llamarte mi primera mentora napolitana. Me has enseñado muchísimas cosas, fue un placer compartir proyecto contigo en nuestros inicios allá en el 2016. Creo que nos complementábamos genial, formamos un buen tándem (sobre todo en los circadianos, jde los que tanto me voy a acordar!, ¡hemos logrado muchas cosas juntas!).

Jackson and Kanako, thank you for sharing with us new ideas, letting us taste food from your lovely countries and introducing us to your cultures, it has been a pleasure to meet you!

Ale, qué te voy a decir que no sepas. Me ha encantado que te cruzases en mi camino y que hayamos pasado por tantos cambios en estos años juntas, ¡te espera un futuro esperanzador!

María, mi compi de cumpleaños, muchas gracias por traer tanta alegría al laboratorio, hemos pasado momentos muy malos y muy buenos juntas. Sois el futuro del laboratorio, y no podía quedarse en mejores manos, junto con los chicos nuevos, Miguel, Albert, Tommaso, Elvira y Jon que habéis traído frescura al laboratorio y buen rollo, sois todos geniales. Tommaso, sabes que eres el sucesor de mis proyectos jasí que cuídalos bien si quieres ganarte el Nature!

No me quiero olvidar de los tres estudiantes que han estado a mi cargo, Edu, Margaux y Marina. Ojalá os haya podido enseñar, aunque sea una milésima parte de mis conocimientos, y que hayáis estado a gusto trabajando conmigo, ha sido un placer haber podido enseñaros.

No me quiero olvidar de dar las gracias a Sara, que ha formado parte de nosotros, y que tanta alegría ha aportado al grupo y a la gente que ha pasado por el laboratorio, Diego, Arturo, Elena, Fran y María. 
Sin duda no podía haber tenido un equipo igual. Estoy y estaré enormemente agradecida de haber estado rodeada de este grupo de profesionales y por encima de todo, me llevo la amistad de todos ellos, igracias Hidalgos!

Asimismo, quiero agradecer a todo el personal del CNIC que ha aportado su granito de arena en esta tesis. A Laura Cabezuela y Eva Santos, por cuidar tan bien de nuestros ratoncillos y por todo el trabajo que les hemos generado a diario, sin vuestra ayuda todo esto no hubiera sido posible. A Elena, Ligos, Raquel y Mariano, de la unidad de citometría, por tanta ayuda técnica y soporte emocional durante las largas horas de sorting. Ha sido un placer aprender de vosotros y haber pasado tan buenos ratos dentro de la unidad. A Vero y Elvira, de la unidad de microscopía por toda su paciencia enseñándome a usar los diferentes miles de microscopios. A las unidades de histología y genómica que tanto me han ayudado siempre que he ido con algún problema. A Cristina Giménez, que tantas veces me ha salvado de saltarme algún plazo o presentar algún papel en la universidad, gracias, Cris, jeres genial!, y a Soriana, por alegrarme con su sonrisa a altas horas de la noche.

Agradecer también a todas las personas colaboradoras externas que han participado en el proyecto: a Elena (CIEMAT), por ayudarme a organizar los experimentos de cuarenta mil animales. A Daniela y Eugenio (Centro San Raffaele), por su tiempo explicándonos los resultados tan complicados.

Por otro lado, querría dar las gracias a todo mi equipo anterior. Maruchi, Carlos, Esme, Vera, Rebeca, lleana y Eleni, creo que lo que nos pasó nos unirá para siempre. Fue genial trabajar con vosotros, además fuisteis las primeras personas que conocí en Madrid y me sentí como en casa nada más llegar.

Maru, Nacho y Carli, mis sevillanos favoritos, muchas gracias por estar ahí siempre que lo he necesitado, espero no perderos nunca.

Dentro del CNIC tengo que agradecer a muchísima gente que ha hecho que estos años de tesis sean mucho más llevaderos y divertidos. A mis compañeros de piso, Javi y Jose, por aguantarme cada día en casa, tanto en los buenos como en los malos momentos. Siempre dispuestos a escucharme cuando lo he necesitado y darme los mejores consejos. Por todas las risas que nos hemos echado, viajes y fiestas que hemos compartido juntos, ha sido genial, ¡muchas gracias, chicos! Y a la reciente incorporación al piso de Diego, Laura, por traer ese positivismo a casa y poder compartir muchas cosas en común. A 
los ex miembros del piso, Miriam y Pablo, por haber hecho mucho más divertido el tiempo en Madrid, jojalá siguierais viviendo aquí!

A Natalia, Mariya, Ana, Macarena, Alberto, Julio, Sergio, Rebeca, Laura, Sara, Carles, Eli, Jesús, Paula, Bonafont, por todas las fiestas, conversaciones en la Yoli, escapadas y viajes que hemos compartido estos años, sin todo eso, estos años hubieran sido mucho más aburridos. A toda la gente de la 3 sur con la que he compartido anécdotas diarias, días largos y limpiezas semanales...

Muchas gracias a todos vosotros, y a los que no he mencionado, por haberme acompañado en estos años de duro trabajo, cada uno de vosotros ha contribuido con su granito de arena a que esta etapa de mi vida sea inolvidable. 
-12 - 
Abstract

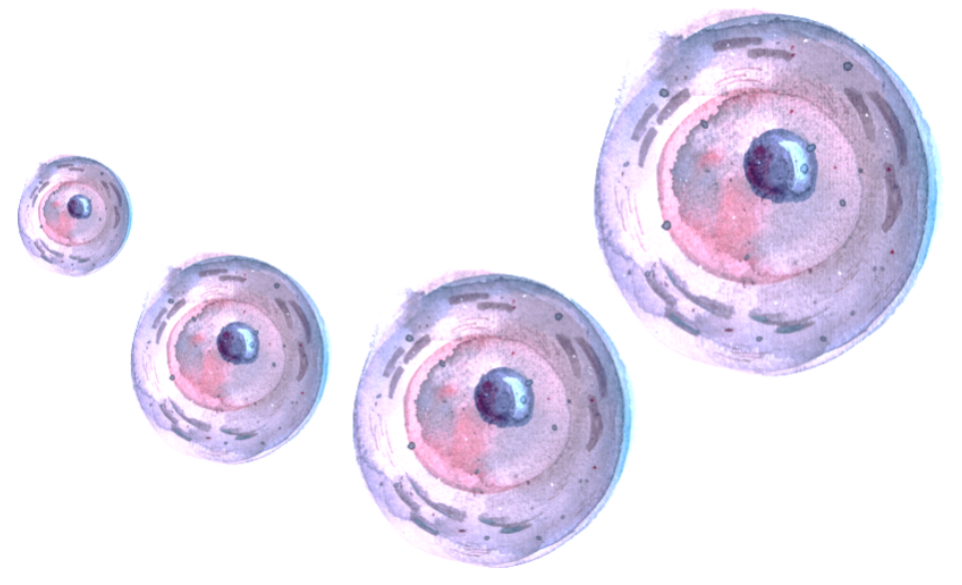

$-13-$ 
- 14 - 


\section{Abstract}

Hematopoietic stem cells (HSCs) have the ability to self-renew and differentiate into multiple cell lineages, giving rise to all blood components and immune cells, during the entire life of an individual. HSCs are localized in the bone marrow inside specialized compartments named "hematopoietic niches". The niche contains stromal cells of mesenchymal origin, as is the case of adipocytes and osteoblasts as well as endothelial cells and cells of hematopoietic origin such as macrophages or megakaryocytes (1). All of these cells produce and deposit elements in the extracellular matrix but also secrete local hematopoietic cytokines that can induce or inhibit the proliferation and differentiation of progenitor cells.

Early studies described that some of these HSCs are found travelling through the circulation of the organism (2). Additionally, the release of HSCs from the BM into peripheral blood follows circadian patterns, i.e. their numbers oscillate between day and night (3).

In the present thesis we have analyzed whether HSCs in the circulation (named here circulating HSCs) have any physiological function and the mechanisms through which cHSCs are released into bloodstream. We have found that circulating HSC have a myeloid bias and are important for the repopulation of damaged niches. In addition, we found that multiple clones of these cHSCs enter the bloodstream and contribute to the regeneration of hematopoiesis in remote niches.

We have found that the chemokine receptor CXCR2 is expressed in HSCs and is important for their homeostatic egress into the circulation. Genetic deficiency of Cxcr2 prevents the release of HSCs and the repopulation of remote damaged niches and gives rise to hematopoietic defects with age. Correspondingly, we have identified a population of perivascular cells inside the BM that express the chemokine ligand CXCL1 and could be key in the signaling of CHSC egress, and ultimately in preserving hematopoietic health through life. 
- 16 - 
Resumen

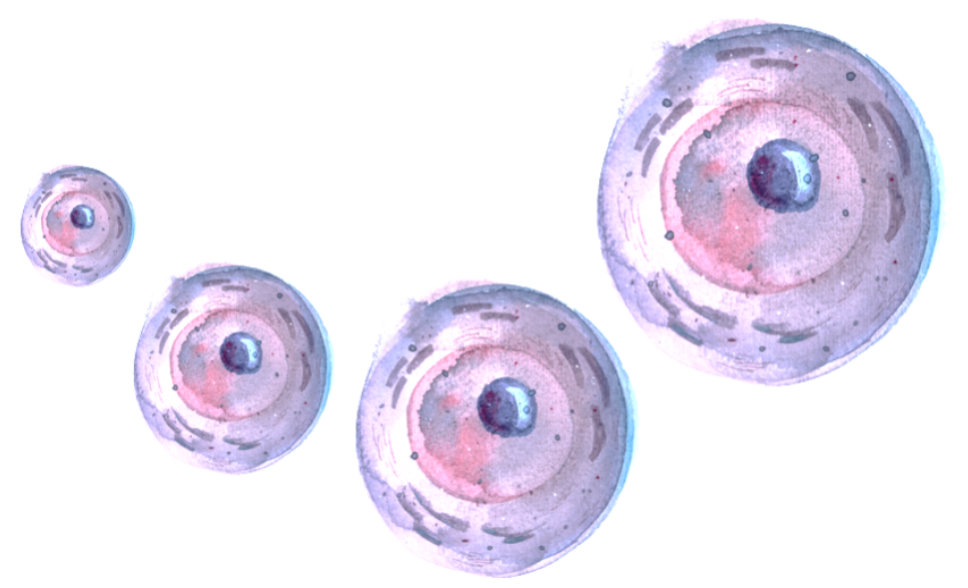

-17 - 
-18 - 


\section{Resumen}

Las células madre hematopoyéticas ( $\mathrm{CMHs}$ ) poseen la capacidad de autorenovarse y diferenciarse a múltiples linajes celulares, dando lugar a todos los componentes de la sangre y el sistema inmune durante la vida del individuo. Las CMHs se ubican en la médula ósea dentro de compartimentos especializados denominados "nichos hematopoyéticos". El nicho hematopoyético contiene células del estroma de origen mesenquimal, como es el caso de los adipocitos y osteoblastos, así como también células endoteliales, o de origen hematopoyético como los macrófagos o los megacariocitos (1). Todas estas células del estroma producen y depositan elementos en la matriz extracelular además de producir citoquinas hematopoyéticas locales que pueden inducir o inhibir la proliferación y diferenciación de células progenitoras.

Se ha descrito previamente que algunas $\mathrm{CMHs}$ se encuentran viajando por el torrente sanguíneo del organismo (2). Además, la salida de $\mathrm{CMHs}$ desde la médula ósea hacia la sangre sigue patrones circadianos, es decir, su número oscila entre día y noche (3).

En la presente tesis hemos analizado si las $\mathrm{CMHs}$ que viajan por la sangre (denominadas aquí como $\mathrm{CMH}$ s circulantes) tienen alguna función fisiológica y los mecanismos mediante los cuales se produce la salida al torrente sanguíneo. Hemos encontrado que estas células con capacidad migratoria y tendencia hacia el linaje mieloide son importantes para la repoblación de nichos dañados. Además, hemos podido observar que múltiples clones de $\mathrm{CMH}$ s entran en la circulación y contribuyen a la regeneración hematopoyética de nichos remotos.

Hemos encontrado que el receptor de quimiocinas CXCR2 se expresa en las $\mathrm{CMHs}$ y es importante para su salida al torrente sanguíneo en condiciones homeostáticas. La deficiencia genética de Cxcr2 impide la salida de CMHs a circulación y por tanto la repoblación de nichos remotos dañados y da lugar a defectos hematopoyéticos durante el envejecimiento. Correspondientemente, hemos identificado una población de células perivasculares dentro de la médula ósea que expresa el ligando de quimiocinas CXCL1. Éste podría ser clave en la señalización de salida de $\mathrm{CMH}$ y en última estancia del mantenimiento de la hematopoyesis durante la vida de un individuo. 
-20 - 


\section{Index}

Agradecimientos ................................................................................................................................................. 7 -

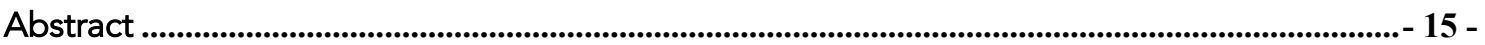

Resumen

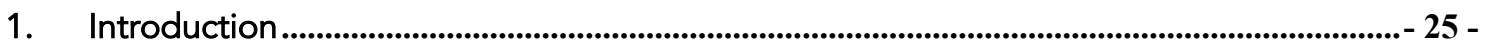

Hematopoietic Stem Cell Biology .......................................................................................... 25 -

Embryonic Origin of Hematopoietic Stem Cells ................................................................ 25 -

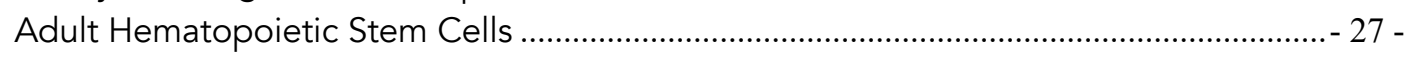

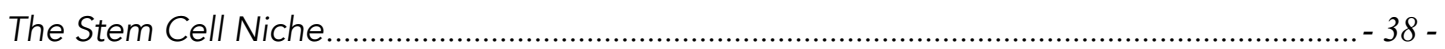

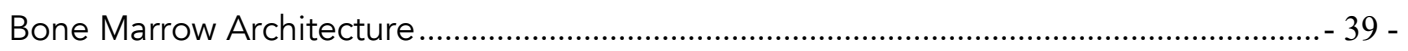

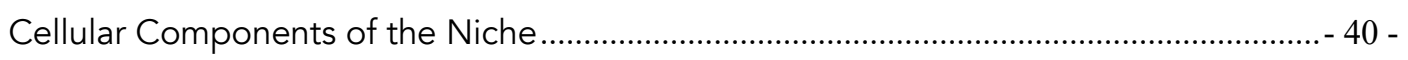

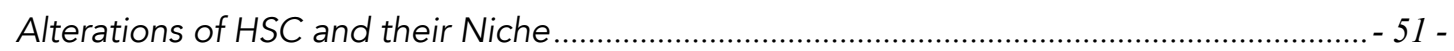

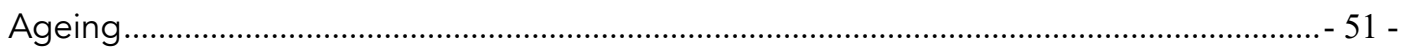

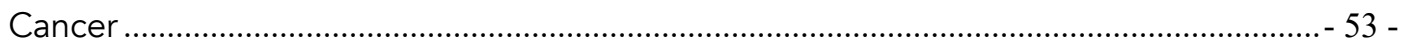

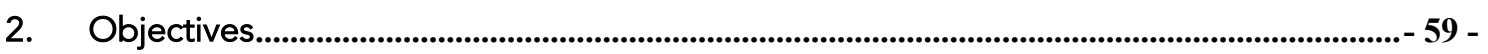

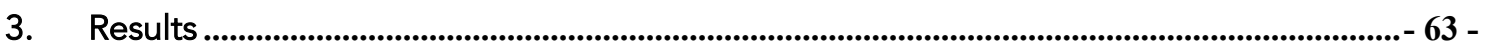

Circulating HSCs repopulate damaged niches ...................................................................... 63 -

Circulating HSCs provide long-term repopulation of damaged bone marrow niches... - 63 -

Repopulation of damaged BM niches is multiclonal .......................................................... 67 -

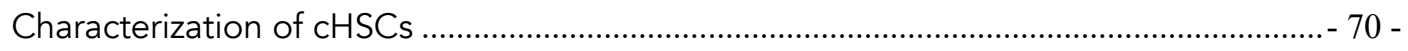

CXCR2 is functional on CHSC and mediates their egress from the BM..............................- 75 -

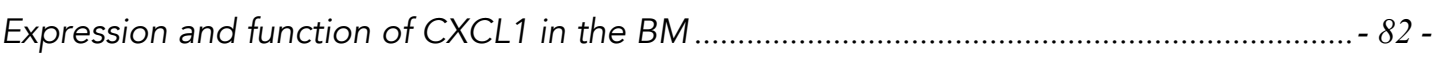

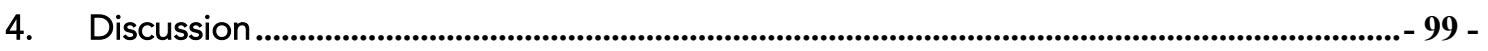

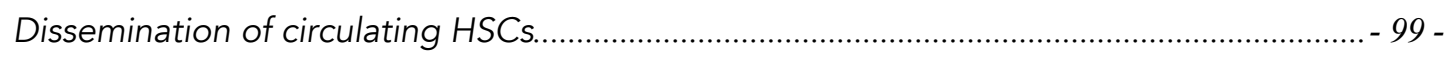

Mechanisms that enable the dissemination of cHSCs ............................................................... 102 -

5. Conclusions ............................................................................................................................ 111 -

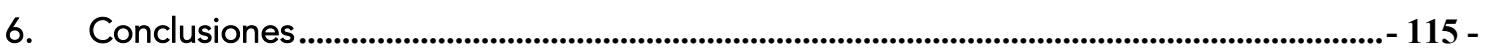

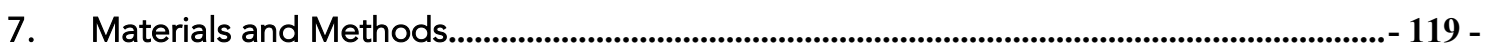

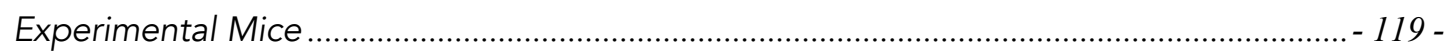

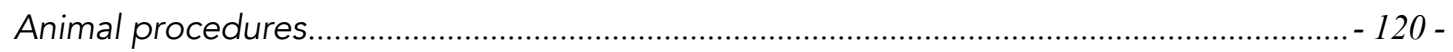

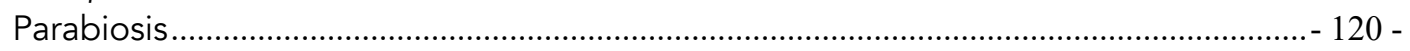

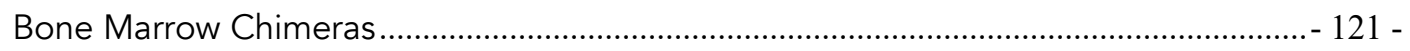

Limiting Dilution Bone Marrow Transplantation Assay ..................................................... 121 -

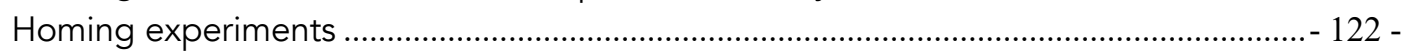

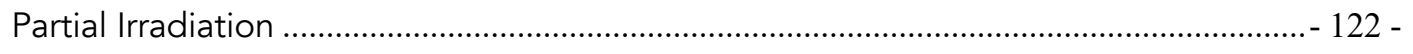

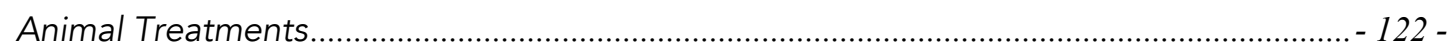


Cell Culture Assays ................................................................................................................. 123 -

Colony Forming Unit Assay (CFU-C) .......................................................................... 123 -

Mesenchymal Stem Cell Culture Assays............................................................................. 124 -

Chemotaxis Assays.................................................................................................. 125 -

Flow Cytometry and Cell Sorting ................................................................................... 125 -

Preparation of single cell suspensions from tissues and staining ................................. 126 -

Molecular and Bioinformatics Analysis ................................................................................... 129 -

RNA Isolation, Reverse Transcription and Real-Time PCR ........................................... 129 -

Clonal analysis of circulating HSC .............................................................................. 130 -

Vector production ...................................................................................................... 130 -

Transduction of hematopoietic progenitors ................................................................... 130 -

Vector copy number (VCN) analysis ........................................................................ 131 -

Retrieval of integration sites (IS) from cell DNA ............................................................. 131 -

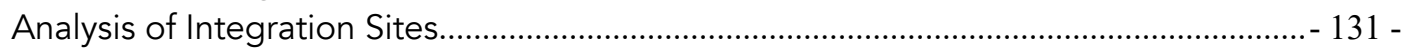

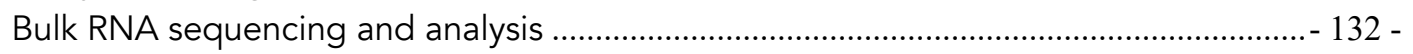

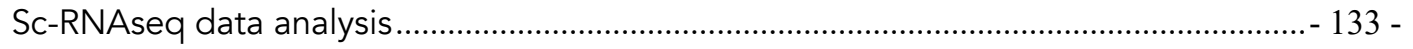

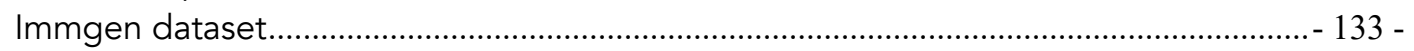

Protein Analysis..................................................................................................... 134 -

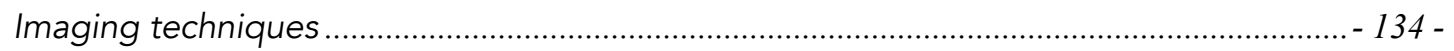

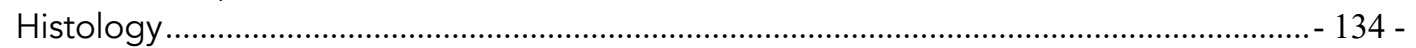

Immunofluorescence staining of BM frozen sections....................................................... 134 -

Whole-mount imaging of BM ....................................................................................... 135 -

Intravital Microscopy of Calvaria Bone Marrow................................................................ 136 -

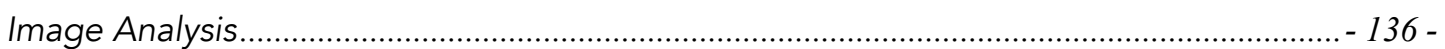

Quantification of perivascular cells by immunofluorescence staining ............................-136 -

Quantification of HSC distances in whole mount ..................................................... 136 -

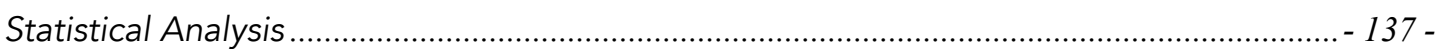

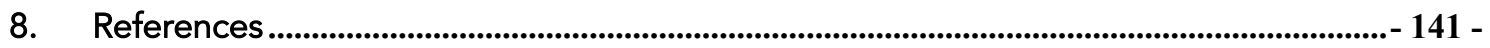

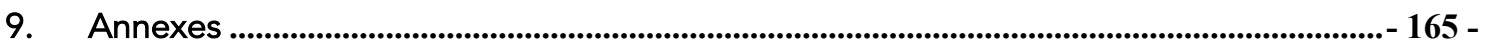

Abbreviations.............................................................................................................. 165 -

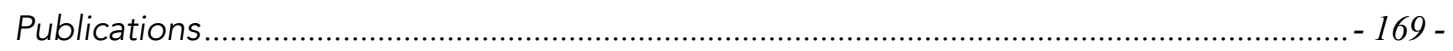




\section{Introduction}

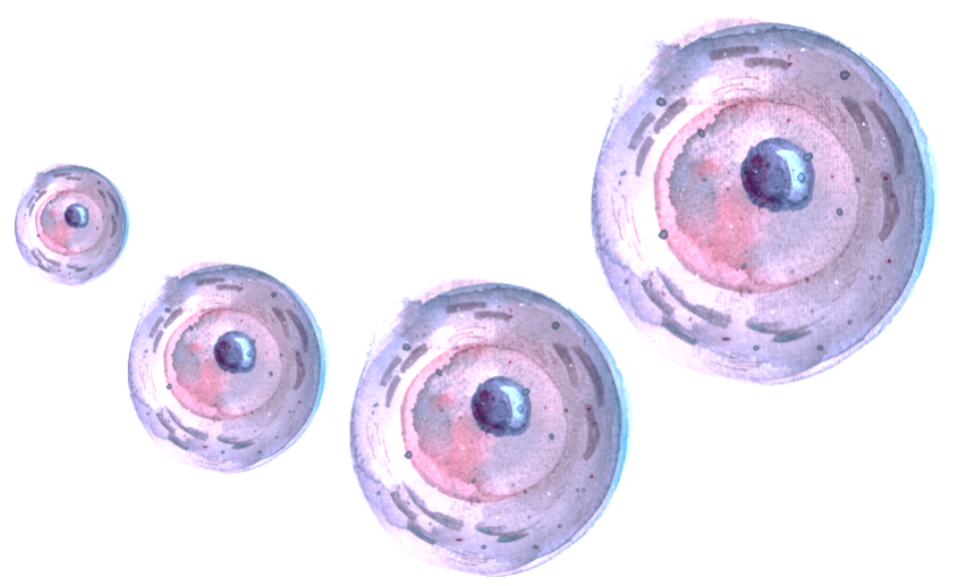


- 24 - 


\section{Introduction}

\section{Hematopoietic Stem Cell Biology}

\section{Embryonic Origin of Hematopoietic Stem Cells}

Hematopoietic stem cells (HSCs) develop during embryogenesis in a rather complex process that involves numerous anatomical sites. In mammals, the sequential sites of hematopoiesis include the yolk sac, an area surrounding the dorsal aorta named the aorta-gonad-mesonephros (AGM) region, the fetal liver and spleen, and finally the bone marrow (BM) that will be the main hematopoietic reservoir in the adult. The placenta has also been recognized as an additional site that participates during the AGM to fetal liver period $(4,5)$. Therefore, constant trafficking appears to be a distinct feature of HSCs already in embryonic stages (6).

During the post-natal period, the HSC pool size is maintained by a tight balance between self-renewal and differentiation, the two main properties of stem cells. This is possible not only due to intrinsic properties of the cell but also to extrinsic signals that derive from a specialized microenvironment, or "niche", that surrounds these cells (7). The challenge of fetal hematopoiesis is to generate differentiated blood cells that are required immediately for embryonic growth and development, and to establish a pool of undifferentiated HSCs even when the BM and its niche has not yet developed. Hematopoiesis shifts from one place to another in the embryo and this provides HSCs with different signals from the numerous niches (Figure 1).

Embryonic hematopoiesis in mice starts after gastrulation, when a subset of specialized mesodermal precursor cells commits to become blood cells. In mammals, hematopoiesis occurs in three different waves $(4,8)$ (Figure 1). In the mouse, the first wave of hematopoiesis occurs in the yolk sac at embryonic day (E) 7 and is referred to as "primitive". The function of these first wave is to produce quick and transient hematopoietic cells to meet the immediate needs of the embryo, including the generation of primitive erythroid progenitors, necessary for oxygen production; embryonic macrophages, required for tissue remodeling and defense; and primitive megakaryocytes (MK) which have a role 
in vascular maintenance (9). This first wave, however, does not produce lymphoid progenitors or HSCs. The initial "primitive" wave is soon replaced by an adulttype "transient-definitive" hematopoiesis, which is referred to as second hematopoietic wave. This second wave occurs in the yolk sac around E8.25 and is marked by the emergence of erythro-myeloid progenitors (EMPs) together with lymphoid progenitors; two progenitor types that seem to complement each other with their differentiation potential $(10,11)$ (Figure 1). Is in the third wave (starting at E10.5), when adult-type HSCs emerge from the AGM region of the embryo (12-14). The AGM is an embryonic tissue derived from the mesodermal germ layer that contains the dorsal aorta, the genital ridge (origin of the gonads) and the mesonephros (origin of the kidneys). HSCs arise from the hemogenic endothelium within the AGM in a process termed endothelial-to-hematopoietic transition (EHT). Distinct AGM sub-regions seem to exhibit different HSC repopulating activity, but HSCs distribute almost exclusively to the ventral wall of the dorsal aorta. Afterwards, AGM-derived HSCs are released into circulation and migrate into the mouse fetal liver at E11, where they can give rise to all the cell types of the hematopoietic hierarchy. The fetal liver is mainly composed by stromal cells that support the highly proliferative capacity of HSCs in this organ. Following this great expansion, HSCs migrate to the spleen and thymus (around E15.5) and later to the BM (E17.5) which will be the main hematopoietic niche site during the early postnatal period (Figure 1). HSC activity in the spleen is detectable until a few weeks after birth, but later, commitment in adult mice is considered to be limited to erythropoiesis under steady-state conditions. In the neonatal BM, HSCs proliferate markedly during the first 3 weeks and then become quiescent (that is, HSCs exit the G1 phase and subsequent division cell cycle phases and enter into G0 phase) (8). 


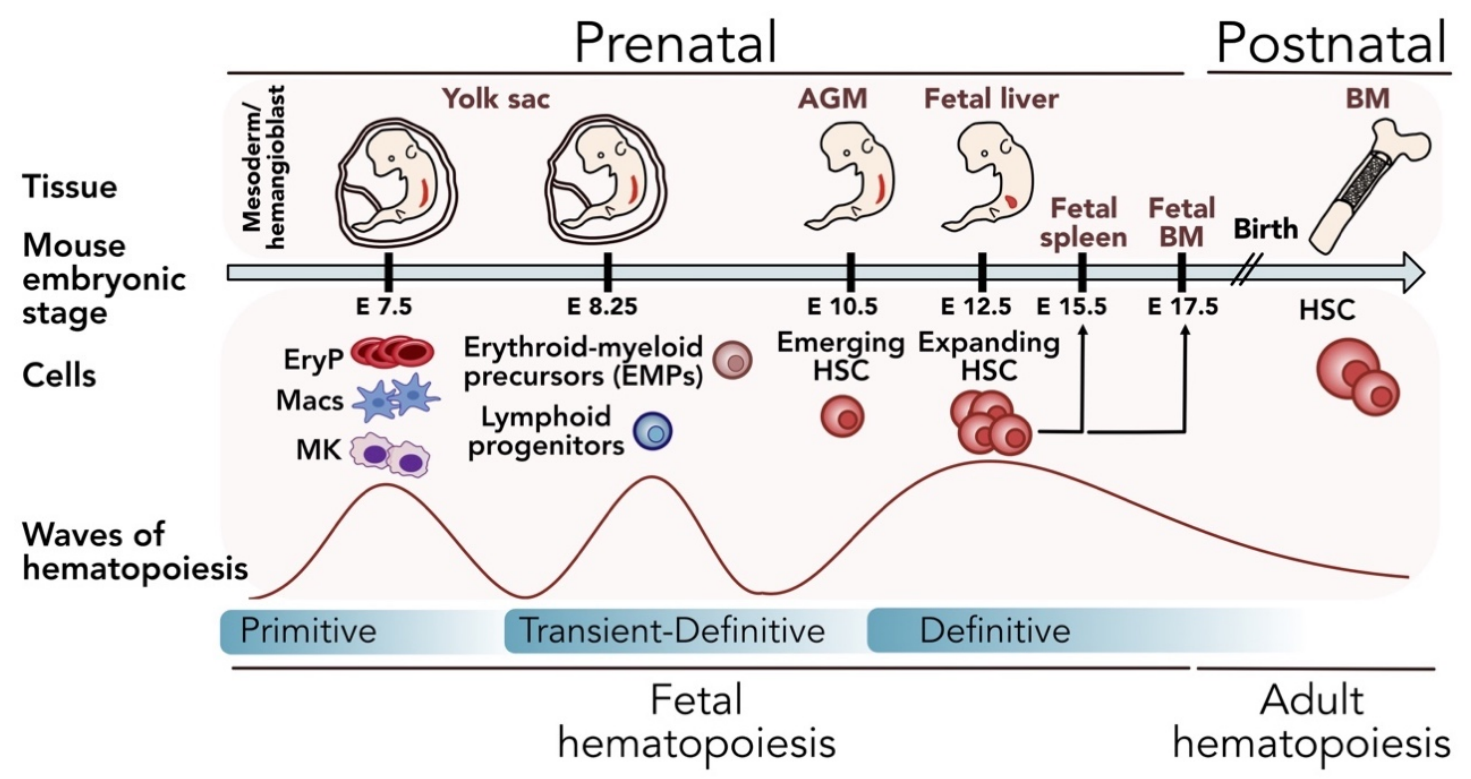

Figure 1. Hematopoiesis in the developmental stages of the embryo. Fetal hematopoiesis in the prenatal stage takes place in three different waves and through different anatomical sites such as the yolk sac, AGM, fetal liver, spleen and BM. Each of these waves produces a different subset of hematopoietic cells although "true" HSCs don't arise until E10.5. After birth, in postnatal stages, the BM becomes the main site of hematopoiesis throughout the entire adulthood and the spleen remains as a secondary hematopoietic organ.

\section{Adult Hematopoietic Stem Cells}

HSCs are characterized by their unique ability to self-renew and give rise to the entire blood and immune system throughout the lifetime of an individual (15, 16). HSCs represent a rare population inside the adult BM constituting only about 0,005-0,01\% of BM nucleated cells (17). Murine HSCs were first identified by the ability of forming myeloid and erythroid colonies in the spleen of lethally irradiated mice, following BM transfer $(18,19)$. The number of colonies found in the irradiated spleen, or CFU-S, was proportional to the BM cells that were injected, indicating that a particular population of HSCs inside the BM was able to reconstitute hematopoiesis in vivo $(18,20)$. The first definition of hematopoietic stem cell was thus the ability of a cell to repopulate an irradiated marrow, and still today the most common functional assay to measure stem cell activity is serial BM transplantation, which requires that HSC-containing donor BM cells can be re-transplanted into secondary and even tertiary recipients while retaining both the ability of self-renewal and multilineage differentiation (21). This functional assay permitted to identify cell-surface markers of mouse HSCs, 
thereby allowing their first isolation in 1988 by fluorescence-activated cell sorting (FACS) (22).

All functional HSCs are found within the population of BM cells that are negative for the cell-surface markers normally present on lineage (Lin)-committed hematopoietic cells, but express high levels of stem-cell antigen 1 (Sca-1) and the transmembrane receptor for the stem cell factor (c-Kit). This HSC-containing subset of BM cells is therefore known as LSK (Lin-Sca- $\left.1^{+} \mathrm{c}-\mathrm{Kit}{ }^{+}\right)$, a term that will be repeatedly used throughout this thesis. However, only 1 out of 10 LSK-cells has repopulating capacity, suggesting functional heterogeneity among these cells (17). A major advance in this field was the discovery that the expression of the SLAM receptor, CD150, together with the absence of CD48, and the expression of CD34, highly enriched for murine stem cells with repopulating activity $(17,23)$. Approximately, half of this population was able to reconstitute lethally irradiated mice when competitively transplanted with limiting numbers of cells (17). Taking advantage of the differential surface expression of these three markers, LSK HSCs were further subdivided into three different subpopulations: long-term (LT)-HSCs which are CD48-CD150+ and contain all longterm repopulating (LTR) activity, short-term (ST)-HSCs which are CD48CD150and have only limited self-renewal activity; and multipotent progenitors (MPP), which are $C D 48{ }^{+} \mathrm{CD} 150$ and give rise to more committed myeloid and lymphoid progenitors (CMP and CLP, respectively) (Figure 2). At the same time, these three subpopulations have been divided into three subsets on the basis of CD34 and CD135 expression (24) (Figure 2). The most primitive HSC population (CD34CD48-CD150+CD135-) gives rise to a MPP population equivalent to ST-HSCs, named multipotent progenitor type 1, MPP1 (CD34+CD48-CD150+CD135) which has acquired CD34 cell surface expression. The second step in initiating the differentiation of HSCs is likely to be the appearance of CD48 (MPP2) followed by the loss of CD150 (MPP3) and gain of CD135 (MPP4) which has been functionally associated with differentiation towards a lymphoid-primed multilineage progenitor (24) (Figure 2). The denomination of LT- and ST-HSC, as well as MPP1-4 will be also used through this work. Besides all these markers, more recent genetic tools have sought to identify genes uniquely expressed in HSCs within the hematopoietic system in order to develop HSC-specific reporter lines. A very recent work, for instance, has identified that the expression of the myelodysplastic syndrome 1 (Mds1) gene is highly enriched in LT-HSCs and this has been useful to even visualize these cells with intravital imaging approaches (25). 
Several groups have studied the cell cycle state of these cells and have identified a highly dormant population of functional HSCs within the LSK-CD150+CD48CD34- population (26-28). Dormant HSCs are found in a prolonged quiescence state and are tracked as label-retaining cells ( $L R C$, according to the capacity of these long-lived dormant cells to retain BrdU, a compound that labels DNA in vivo) $(26,29)$. The DNA replication machinery is down regulated in these cells and only divide about five times during the remaining lifespan of the organism, probably to prevent stem cell exhaustion; even though they can be efficiently activated upon a hematopoietic insult and restored back to dormancy after such stress. Interestingly, the acquisition of CD34 expression on the cell surface is one of the firsts events during the activation of dormant HSCs. A new paradigm in the field, thus, considers that under basal conditions, the multipotent progenitor populations (MPP1-4), instead of a quiescent HSC population, are responsible for the massive production of daily immune cells (30). Moreover, it has been shown that MPPs have a lineage-restricted fate, such that MPP2 and 3 have a myeloid bias while MPP4 have a lymphoid bias $(30,31)$ (Figure 2).

Decades ago, classical studies proposed a hematopoietic hierarchy model that worked in a discrete stepwise hierarchy, with a series of branching steps, and where self-renewal hematopoietic stem and multipotent progenitor cells were at the very top; more committed, lineage-restricted progenitors in the middle and mature functional blood cells at the bottom (Figure 2). More recently, however, the new generation of experimental tools such as fate mapping, barcoding tools or single-cell RNA sequencing are challenging this classical view of the hematopoietic hierarchy. The hematopoietic system is now envisioned as a continuum of hematopoietic progenitors that are not uniform and contain a variety of gene expression states that can dynamically change in response to different environmental situations. Much of these advances have been possible because hematopoiesis has been studied in its native, unperturbed state, without the need to perform transplantation assays, which substantially modifies the basal properties of HSCs. The complexity of this tree starts at the very top, where HSCs have been shown to be themselves heterogenous and not an absolute and fix population of cells. In addition, this model was supported by the subdivision of multipotent progenitors (MPP1-4) that could be primed to certain lineages $(30,31)$. The lymphoid and myeloid lineage decision step appears to occur in later phases and not in two early, separate blocks as previously thought (common lymphoid progenitors and common myeloid 
progenitors, CLP and CMP, respectively). In turn, megakaryocyte-erythroid progenitors (MEPs) may derive directly from HSCs, thereby bypassing the multipotent progenitors step and appearing earlier than the other lineages (3234). Moreover, in contrast to the classical transplantation approaches, analyses of unperturbed hematopoiesis have suggested that the subset of cells that function as LT-HSCs during transplantation does not play a significant role in the steady-state. Instead, native hematopoiesis is driven by cells within the ST or MPP compartment, which have been shown to have higher self-renewal potential than previously thought (35). However, the dormant fraction of HSCs appears to be the one contributing to LT engraftment in transplantation settings, and can be activated upon stress conditions (26). In addition, barcoding experiments suggested that MPPs contribute predominantly to the myeloid and megakaryocytic lineage under homeostatic conditions $(36,37)$.

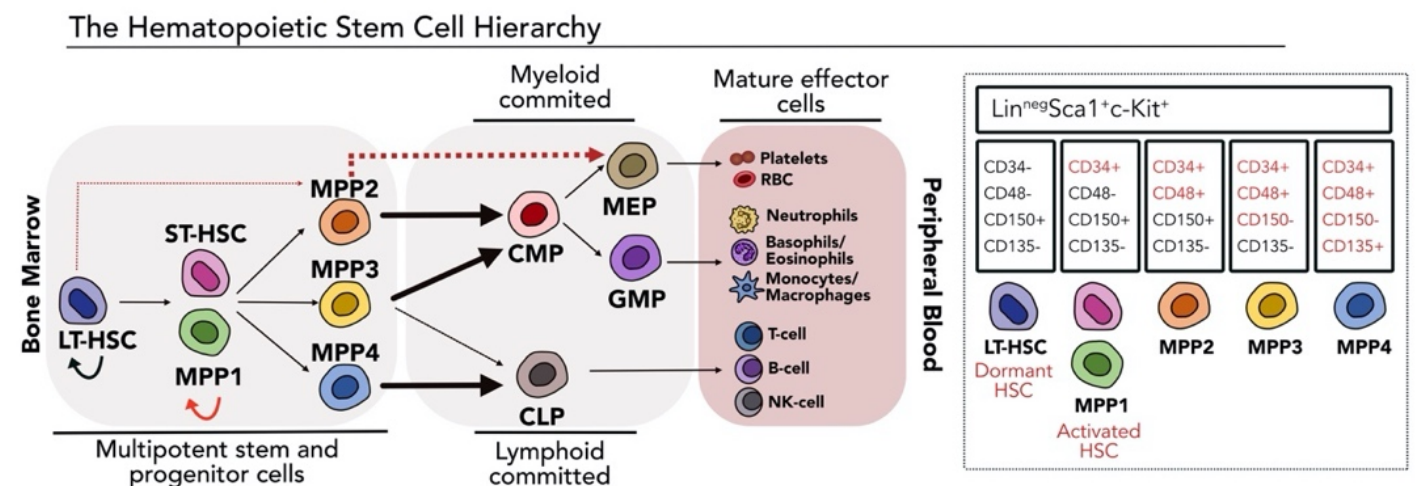

Flow Cytometry Gating Plots
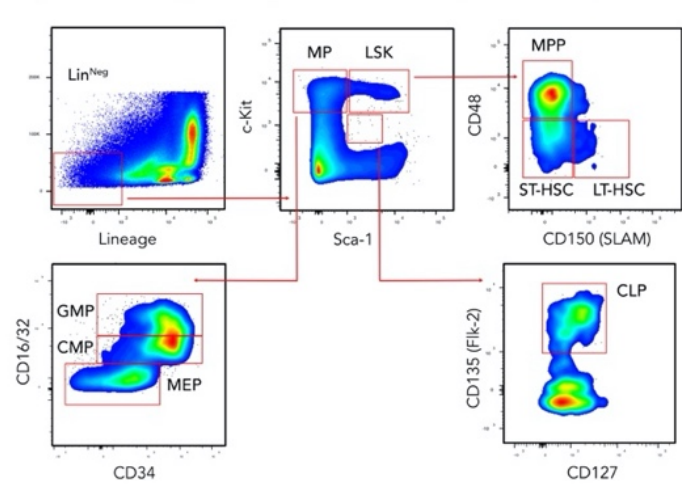

CD127

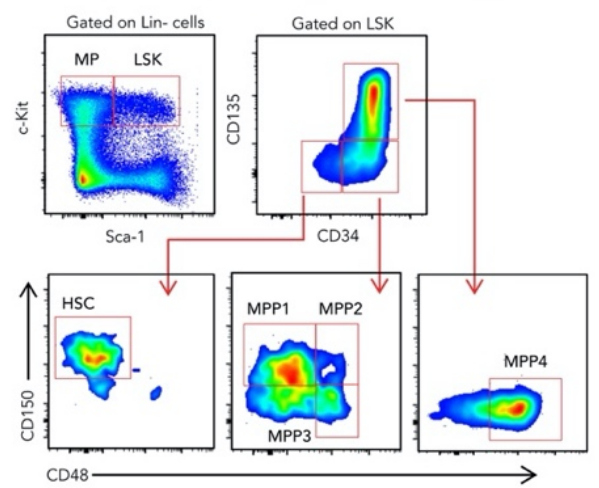

Figure 2. The Hematopoietic Stem and Progenitor Hierarchy. The upper panel shows a scheme representing the classical (black) and the new concepts (red) of the hematopoietic stem cell hierarchy. The table at the right shows the phenotypic cell surface markers of each stem and progenitor subset of cells (the acquisition of each surface marker in each cell type is indicated in red). The bottom panels show the flow cytometry gating plots performed in the lab based on the above-mentioned cell surface markers. Such gating strategies will be used along the work. 


\section{Hematopoietic Stem Cell Trafficking}

The mammalian immune system has evolved to respond and to eliminate a variety of infectious agents. This task requires continuous movement of motile immune cells that follow pre-established routes between one tissue to another using blood and lymphatic vessels for quick accessibility. These migratory paths specific for different immune cells are an integral part of their function and are imprinted during the process of cell differentiation and activation, as the cells acquire the expression of a repertoire of cell surface molecules that enable their migration to defined tissues and microenvironments. Remarkably, not only mature immune cells migrate, but also hematopoietic stem and their immediate descendant's progenitor cells (HSPCs) are motile and actively recirculate throughout the body $(2,38)$.

\section{The role of CXCL12/CXCR4 Axis in HSC Homing}

Although the main reservoir of HSCs is the BM, it has been known from decades that a small fraction of progenitors leaves the BM to enter the circulation (39). It is remarkable that this phenomenon occurs every day (i.e., circadianally), as part of their steady-state homeostasis $(2,3,38)$, and in an accelerated way during stress hematopoietic situations, such as acute or chronic inflammation $(40,41)$. Migration of circulating HSCs across the endothelium back to the BM niche requires active navigation, a process referred to as homing. The ability of stem cells to home to the BM is the first essential step for successful BM transplantation, in which the transplanted HSCs need to enter the donor irradiated marrow for colonization.

Homing of HSCs consists in a multistep adhesion-cascade that starts with the anchoring of HSCs to the BM endothelium, which interestingly exhibits constitutive expression of adhesion molecules that enable cell trafficking, such as P-and E- selectin and VCAM1 (42). The homing process is initiated by the stromal cell-derived factor 1 (SDF-1) also known as CXCL12. Binding of CXCL12 to its receptor, CXCR4, allows rapid activation, firm adhesion and docking to the $\mathrm{BM}$ sinusoidal wall by inducing cytoskeletal rearrangements and activation of integrins and metalloproteinases (43). CXCR4-expressing HSCs rapidly adhere, extravasate and navigate towards special stromal niches that express CXCL12, 
which further provide essential signals for proliferation, motility and differentiation.

The CXCL12/CXCR4 axis has been implicated in numerous processes such as retention, migration, homing and mobilization of HSCs during steady state but also upon stress (44-47). Murine BM stromal cells, including endothelial cells, endosteal bone-lining osteoblasts and perivascular cells secrete high levels of CXCL12, and are found adjacent to HSPCs. The expression of CXCR4 in circulating HSCs allows the chemoattraction towards regions of high levels of CXCL12 in the BM cavity thus facilitating homing and definitive settlement of progenitors inside the BM. Consistent with this, HSCs lacking CXCR4 accumulate in the circulation and fail to undergo normal lympho/myelopoiesis, as they are not able to receive maturation signals (48). In addition, depletion of CXCL12producing stromal cells result in defective hematopoiesis and reduced homing of transplanted HSCs to the BM (49). These studies demonstrated that disturbances on either the stromal or hematopoietic side modify stem cell migration in vivo, and is nowadays being exploited in clinical transplantation settings (50).

The CXCL12/CXCR4 axis is also essential in the retention of HSCs in the BM and the maturation of B-cell progenitor cells. Indeed, disruption of the axis leads to the massive release of HSCs into circulation and impairment of B-cell function $(51,52)$. In fetal development, deficiency in either CXCR4 or CXCL12 results in multiple defects, such as defective B-cell lymphopoiesis, severely impaired myelopoiesis in the BM, despite normal myeloid development in the fetal liver, and reduced pre-B and pro-B cells (51-53).

Many other factors contribute to HSC homing and retention in the marrow. Among these, the cytokine stem cell factor (SCF, also named c-Kit ligand) plays a prominent role. Stimulating HSCs with SCF improves in vivo homing capacity through increased migration and adhesion via very late antigen (VLA) 4 and 5 integrins (54).

\section{Hematopoietic Stem Cell Mobilization}

The small amount of circulating HSCs that are present in the blood were firstly discovered both phenotypically and functionally in animals that were surgically joined to share a common blood circulation, a technique called parabiosis (2, 
$38,55)$ that will be used thoroughly during this work. These studies have shown continuous exchange of HSCs (and their descendants, HSPCs) between the BM and the blood under steady-state conditions. It has been estimated that around 400 HSPCs can be found in murine circulation at any given time $(2,38,39)$, suggesting that circulation in blood is a normal physiological activity of HSCs. Massberg et al. discovered that BM-derived HSCs travel from the blood into multiple peripheral tissues, from the tissues into the lymph, and from the lymph via the thoracic duct back into the blood, where they may return to the marrow or enter another cycle of recirculation (38). Although the physiological significance of circulating HSCs is still unknown, one proposed explanation for this behavior is that the constitutive circulation of HSCs through the peripheral tissues provides a source of progenitors for rapid supply and local production of immune and inflammatory effector cells at sites of injury or infection $(38,56,57)$. Notably, however, the physiological significance of circulating HSC -if anyremains to this day unknown.

Stresses such as bleeding, inflammation or injury can increase stem cell proliferation and accelerate HSC egress, a process called mobilization. This leads to a rapid increase in the number of leukocytes circulating into the bloodstream to the inflammatory site (58). Mobilization can be induced clinically or experimentally in animal models by a wide variety of factors that include cytokines, chemokines, antagonists of adhesion and chemotactic receptors, cytotoxic drugs and chemotherapeutic agents (59-61). These molecules differ in their mechanism and time frame to achieve mobilization, the type of cells mobilized, and their efficiency (62). A common protocol currently used in the clinics is the administration of a CXCR4 antagonist, such as AMD3100 (also called plerixafor), together with the cytokine granulocytic colony stimulating factor (GCSF). This procedure is widely used to facilitate the collection of repopulating stem cells from the circulation of the donors or patients before BM transplantation $(63,64)$.

HSC mobilization involves motility mechanisms, activities of various cytokines, chemoattractants, proteolytic enzymes and other extrinsic factors that ultimately detach HSCs from the BM stroma. Sinusoidal vessels are believed to be the site of HSPC exchange between the BM and the circulation (65). Under stress conditions, such as irradiation or inflammation, the permeability of the BM endothelium is increased, and enhances HSC migration in both directions (66). 
Mobilization by repeated doses of G-CSF provokes "awakening" of quiescent HSCs, generation of guiding signals, repression of the inhibitory attachment machinery and gain of stem cell motility. Importantly, the basal expression of CXCL12 in the BM, which retains HSCs in this organ, is reduced. Similarly, its receptor CXCR4 on BM HSCs is cleaved by proteases, favoring HSC mobilization into the blood $(67,68)$. HSC mobilization also involves upregulation of proteolytic enzymes such as metalloproteinases (MMP-2 and MMP-9) that are necessary for the degradation of the extracellular matrix and for transendothelial migration (69). There is also an increased release of elastase and cathepsin $G$, from BM neutrophils that favors HSC egress 4-5 days after G-CSF treatment (70). These enzymes cleave the unions between VCAM1 expressed in stromal cells and VLA-4 expressed on the surface of progenitor cells, loosening their contact, but also inactivate and degrade CXCL12 in the BM, altogether promoting mobilization (50) (Figure 3).

Signals from the sympathetic nervous system (SNS) are also implicated in controlling HSC traffic. This type of neural regulation can be direct by the secretion of neurotransmitters or myeloid cytokines, or indirect by controlling bone remodeling processes such as bone formation by osteoblasts, bone resorption by osteoclasts, or other changes in the stromal niche $(71,72)$. The BM is extensively innervated by autonomic nerve fibers, including sympathetic nerves which have been shown to be responsible for cytokine-elicited mobilization of HSCs (73). G-CSF promotes the release of noradrenaline (NA) by autonomic neurons localized in the marrow. Released NA mediates the suppression of osteoblasts in the endosteal marrow and reduces CXCL12 levels thus causing HSC mobilization (73) (Figure 3). Additionally, sympathetic nerves regulate a certain type of perivascular cells by acting on $\beta 3$ adrenergic receptors (49). This neural-mesenchymal axis is responsible for the circadian expression of CXCL12 by BM stromal cells, and control the homeostatic release of HSCs into circulation in a circadian fashion (3). Importantly, this neural regulation of HSC mobilization has been causally related to susceptibility to inflammatory disease in the context of myocardial infarction or stroke (74).

\section{Circadian Regulation of HSC Trafficking}

The hematopoietic system, like most mammalian systems, is under circadian regulation. Circadian rhythms make reference to the endogenous oscillations of 
the organism associated with the daily rotation of the earth around the sun (75). The appearance of circadian rhythms in aerobic organisms is believed to be a beneficial adaptation to the environment, as it allows to anticipate periodic changes, such as oxygen levels (76). In mammals, indeed, around $10 \%$ of the genome is under circadian control (77).

Circadian rhythms present an approximate duration of 24h. For a rhythm to be circadian, it needs to oscillate in a specific environment independently of external factors, such as light or food. The German term, Zeitgeber (ZT), refers to these external factors that an organism receives and that enable the synchronization of the internal clocks with the rotational cycles of the earth. Light represents the principal stimuli or zeitgeber to align the internal rhythms of the organism to those of the environment that surrounds it (78).

The SNS is an important regulator of circadian rhythms in the hematopoietic system, as mentioned above. The levels of the neurotransmitter NA are elevated at the beginning of the behavioral active phase in mice (ZT13, or 13 hours after the onset of light) and are delivered locally to the BM (79). In addition, a functional interaction exists between the noradrenergic nerves and the $\beta 3$ receptors in stromal cells that promote the decline in the expression of certain genes implicated in the maintenance of HSCs such as $\mathrm{Cxcl12}$ (49) (Figure 3).

The first observation of the circadian regulation of the HSC niche was in 2008 when Frenette and colleagues showed that the number of HSCs in blood presented circadian oscillations governed by rhythmic changes in the levels of CXCL12 in the BM (3). HSC egress takes place in the resting phase of the mice and exhibit the highest levels at ZT5 (around noon) coinciding with the lowest levels of CXCL12 in the marrow; minimum levels are found at night (ZT13) (the behavioral active phase of the mice) in coincidence with the highest levels of CXCL12 in the marrow (3) (Figure 3). In contrast to mice, humans are diurnal and display inverted circadian oscillations with maximum levels of progenitors in blood in the evening (80). The rhythmic adrenergic signaling promotes the degradation of the transcription factor SP1 and the consequent decrease in the CXCL12 levels in the BM (3).

Adrenergic nerves also control the expression of endothelial-adhesion receptors in the medullary vasculature, as these adhesion molecules are necessary for HSC 
homing back to the marrow (81). It is also likely that a crosstalk exists between the levels of CXCL12 and endothelial adhesion molecules, such as P- and Eselectin, VCAM1 or ICAM1, as higher levels of CXCL12 at night correlate with higher retention of HSCs in the marrow (81).

Interestingly, mature leukocytes also infiltrate the BM in a circadian manner (81) and the highest peak of infiltration in the marrow and other tissues occurs at ZT13. This could be beneficial to provide a rapidly available immune response in the active phase of mice, where their probability of injury or encountering pathogens is highest when the animal is active. The circadian cycles of leukocyte infiltration may also exert control on BM niches. For instance, myeloid subsets regulate circadian oscillations of HSCs through TNF $\alpha$ production. Indeed, this cytokine has been shown to be involved in migration, proliferation and differentiation through modulation of reactive oxygen species (ROS) and melatonin signaling in HSCs $(82,83)$. These studies suggested that diurnal or nocturnal HSCs found inside the BM cavity differ in phenotype and function: the diurnal population of stem cells are more active, with increased rates of differentiation towards myeloid production, compared to the nocturnal subpopulation, which instead appear to have a higher repopulation capacity (82).

Neutrophils are the most abundant myeloid population found in the BM. Due to its short lifespan, vast amounts of these cells need to be released every day to maintain homeostatic levels in blood while at the same time a large number must be eliminated daily (84). Neutrophils follow circadian oscillations throughout the day and are completely cleared from the circulation at ZT13. Neutrophils that are cleared from circulation are ultimately engulfed and eliminated by tissue macrophages inside the different tissues (85). The BM is one the tissues where neutrophils are cleared in large numbers. Clearance in this organ not only serves to control neutrophil numbers but also generates homeostatic cues that modulate the bone marrow niche (86) (Figure 3). When aged neutrophils infiltrate the BM, these are engulfed by tissue-resident macrophages. This efferocytic process generates LXR-dependent signals that downregulate the number of niche cells and consequently the levels of CXCL12 in the marrow, thereby promoting HSC egress (86). Because neutrophil infiltration in the BM occurs with circadian frequency, this process entrains the rhythmic release of HSC into blood. Consequently, depletion of neutrophils or macrophages 
completely blunts the diurnal oscillations of HSCs in circulation, overall revealing an immune-driven mechanism for circadian mobilization of HSC under homeostatic conditions (86).

Altogether, neural, humoral and immune inputs in the BM provide integrated cues that regulate the circadian oscillations of HSCs and leukocytes in the circulation. The redundancy and sophistication of mechanisms controlling HSC egress into blood are an indirect proof of the importance of these circulating cells for organismal physiology, which represents the central query of this thesis.

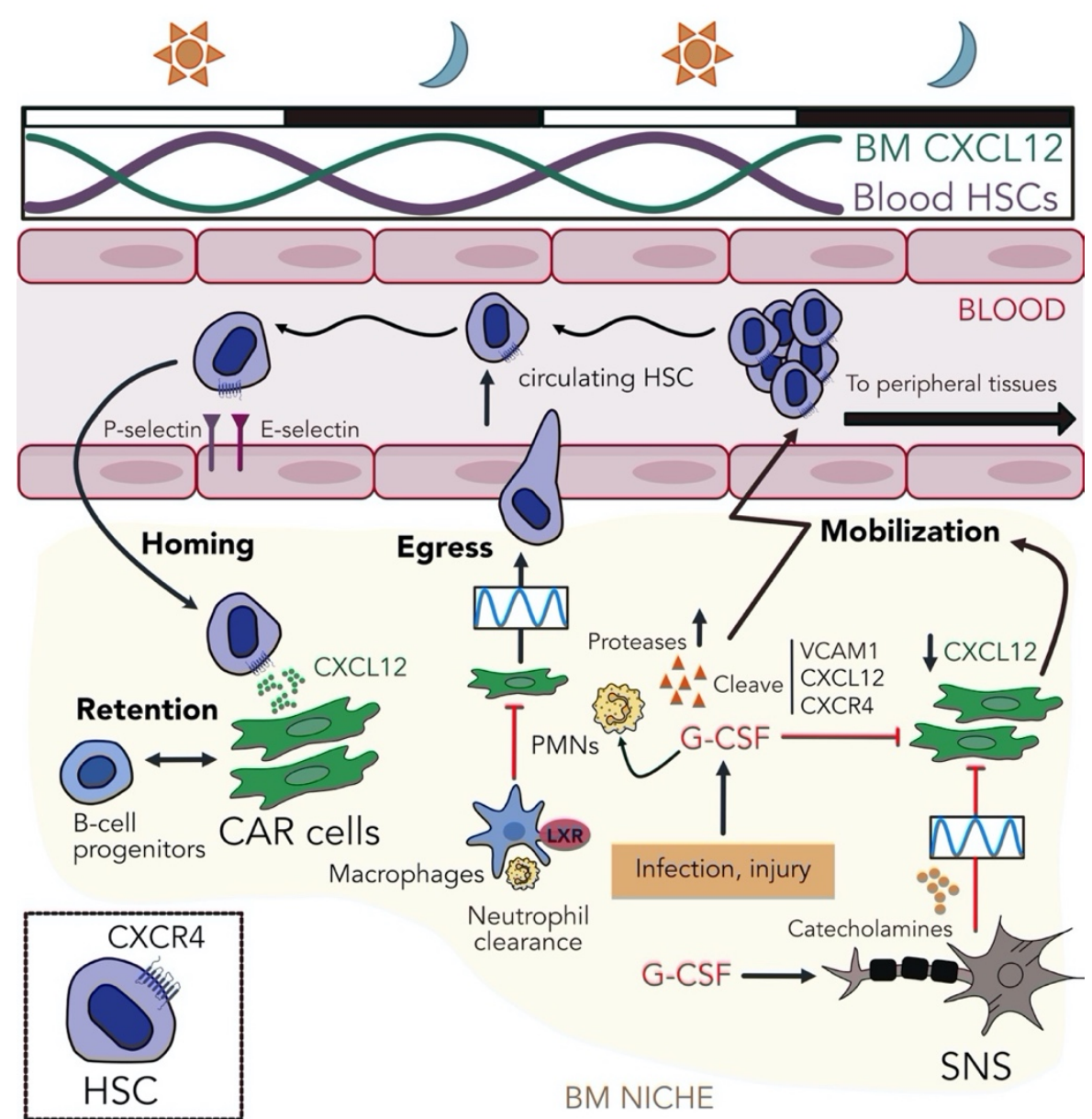

Figure 3. Hematopoietic Stem Cell Trafficking. HSCs dynamically recirculate between peripheral tissues and the BM through the blood stream. Under homeostasis, HSCs are circadianally released into the blood with a peak in the morning (upper panel, purple curve and white box indicates ZT5). This homeostatic process is controlled by the SNS which dictates CXCL12 levels in the BM (upper panel, green curve), and by homeostatic signaling derived from the clearance of neutrophils. Infection or injury induce expression of G-CSF, causing niche remodeling, downregulation of CXCL12 in the BM and HSC mobilization in consequence (indicated with red arrows). Once in the bloodstream, HSCs travel through blood and lymph vessels into different tissues and home back to 
the BM. Homing of HSCs is an active process driven by the CXCR4 receptor (explained in more detail in the upper text, section "The role of CXCL12/CXCR4 axis in HSC homing").

\section{The Stem Cell Niche}

HSC activity is regulated by multiple cell-intrinsic factors, such as transcriptional or epigenetic regulators and metabolic pathways that are involved in its normal function. However, cell-extrinsic factors also control HSC activity; these include humoral and neural signals derived from the BM microenvironment that surround HSCs, which is commonly referred to as the "stem cell niche". The HSC niche is critically responsible for controlling proliferation, differentiation and migration of HSCs during basal and stress conditions, as hinted in the previous chapter.

The initial concept of a niche emerged in 1978, when Ray Schofield referred to a regulatory unit that maintained and directed HSC self-renewal and differentiation (87). Postnatal hematopoiesis takes place in the cavities of long bones and HSCs are proposed to be strategically positioned within unique BM microenvironments with defined anatomical and functional features, in which they receive and integrate regulatory cues from neighboring cells, the extracellular matrix, and/or soluble or membrane-bound factors (5). The precise location of these niches has been hampered for many years due to the technical difficulties encountered when imaging bones, and the lack of HSC and niche specific markers. Thanks to recent genetic tools, advances in imaging methods and discovery of more specific markers, we have now a better understanding of the complex and heterogenous cellular network that surrounds and nurtures HSCs. Although many distinct BM niche constituents have been found, the exact roles and complex relationships among all these cells remains largely unknown. This scenario becomes even more complicated by the fact that the HSC compartment itself is functionally and molecularly heterogenous, raising the possibility that distinct, specialized niches exist for different HSC subpopulations and that each niche may be composed by multiple cell types that contribute to HSC maintenance in unique, as well as redundant ways $(1,7,88)$. 


\section{Bone Marrow Architecture}

In postnatal stages, embryonic-derived HSCs lodge in the BM, where they will remain through the whole life of the organism. Evolutionary studies have suggested that during the transition of aquatic to terrestrial environments, HSPCs relocated into the BM, that is protected from ultraviolet (UV) light by the cortical bone around the marrow (89). In some species, melanocytes (melaninproducing cells) above the hematopoietic niche protect HSPCs from UV light, which suggests that UV light was an evolutionary pressure affecting the location of the hematopoietic niche (89). This may be the reason why hematopoiesis takes place inside the central cavities of long bones, such as the femur and tibia but also in other bones such as the sternum, cranium or limb bones. Long bones are composed of a hollow shaft, or diaphysis; flared, cone-shaped metaphysis below the growth plates, and rounded epiphysis above the growth plates. The diaphysis is composed of dense cortical bone, whereas the metaphysis and epiphysis are composed of a trabecular meshwork of bone surrounded by a relatively thin layer of dense cortical bone. Cortical bone has an outer periosteal surface and inner endosteal surface. The periosteum is the outer layer of tissue that covers the surface of the bone and consists in two layers: a fibrous exterior layer that contains fibroblasts, and collagen fibers and an inner layer that consists in osteoblasts and skeletal stem cells. This layer is followed by the bone and the endosteum which is a thin vascular membrane of connective tissue that lines the inner surface of the bone tissue and forms the medullary cavity of long bones. The periosteum is a highly vascularized and innervated tissue, thus enabling the entrance and exit of blood vessels and of nerve fibers to the bone and marrow cavity. The endosteum is in contact with the BM space, trabecular bone and blood vessels. Within the BM cavity, the mesenchymal compartment forms a dense network of perivascular cell bodies interconnected through large cytoplasmic projections that invades the entire BM tissue. These fibroblast-like reticular cells are responsible for the secretion of regulatory factors such as IL-7, SCF and CXCL12 that influence HSC activity (90-93). Vascular networks in turn are essential for nutrient delivery, waste removal and cellular trafficking, and we now know that they play important roles in hematopoiesis (65). 3D imaging has provided an overview of the microarchitecture of the microvascular system which is composed of arterial and sinusoidal vessels interconnected through a recently defined intermediate vascular type (94-96). These small channels have gained more importance for the trafficking of leukocytes outside the BM (97). However, 
the vast majority of the BM cavity is occupied by a vast sinusoidal network and thus many BM cells lie proximal to sinusoidal surfaces $(65,95,98,99)$.

Even though the BM is the primary site of hematopoiesis, extramedullary hematopoiesis can occur under physiological or pathological conditions, in other organs such as spleen, liver and lung (100-103).

\section{Cellular Components of the Niche}

HSCs and their downstream progeny are tightly regulated by a plethora of cellular components, which regulate their activity by direct contact or by supplying growth factors and/or retention factors. As discussed below, the niche is complex in terms of cellular composition, with several stromal and hematopoietic cells contributing to its activity. Although many components of the niche have been described, is still unknown whether some specific niche populations exist to regulate HSC behavioral processes such as, for instance, the homeostatic release that occurs daily.

\section{Non-hematopoietic cells}

\section{Osteolineage cells}

The BM comprises a number of osteolineage-related cells, derived from the most primitive mesenchymal stem cells (MSCs), which give rise to osteoprogenitors, mature osteoblasts and finally mature osteocytes, which are osteoblasts that have been embedded in the bone matrix (Figure 4). Osteolineage cells were the first cellular component in the niche described to regulate HSC levels in the BM $(104,105)$. They have the ability to support expansion of hematopoietic progenitors cells in vitro $(106,107)$. However, more recent in vivo studies have questioned the role of osteoblasts in $\mathrm{HSC}$ regulation. The conditional deletion of CXCL12 and SCF from these cells did not affect the cellularity, composition or ability of HSCs to reconstitute lethally irradiated mice, implying lack of direct regulation over HSCs (90-92).

Osteoblasts can produce other molecules implicated in the maintenance of HSCs, such as osteopontin (OPN), a matrix glycoprotein that acts as a negative regulator of the HSC pool size $(108,109)$. Indeed, mice deficient in OPN had an 
increased stem cell pool size in vivo with an increased expression of two ligands known to modify stem cell function: the Notch1 ligand, Jagged 1, and the Tie-2 ligand, Angiopoietin-1 (Angpt-1) (109). When osteoblasts were stimulated with the parathyroid hormone, the ability of OPN to restrict stem cell numbers was enhanced, therefore providing OPN with a constraining function on stem cell numbers in the HSC niche (109). Moreover, thrombopoietin (THPO)/MPL signaling contributes to the regulation of HSC quiescence in the osteoblastic niche $(110,111)$. Furthermore, Angpt-1 which binds the HSC receptor tyrosine kinase Tie2, enhances the ability of HSCs to become quiescent resulting in the protection of HSCs from myeloablation (112).

Overall, controversy remains, as secretion of these factors is not osteoblastspecific and thus the contribution of mature osteoblasts to HSC regulation remains elusive. However, osteolineage cells seem to support the maintenance of more committed common lymphoid progenitors (CLP). Indeed, selective elimination of osteoblasts in Col2.34-TK transgenic mice (mice expressing a truncated version of the herpes virus thymidine kinase under a $2.3 \mathrm{~kb}$ fragment of the Collagen $\alpha 1$ promoter, which causes depletion of osteoblasts) severely depleted pre-pro-B and pro-B cells from the BM (113). Furthermore, depletion of CXCL12 in osteoblasts lead to a decrease in CLPs thus suggesting a role of the endosteal niche for the maintenance of lymphoid progenitors and B-cell maturation processes $(91,92)$ (Figure 4).

\section{Endothelial and Perivascular cells}

\section{CXCL12-Abundant Reticular Cells}

The essential role of CXCL12 and its receptor, CXCR4, in the niche was first introduced by studies in $\mathrm{Cxc} / 12^{-/-}$mice. These mice only survived until approximately E18.5 and the BM of these mice was hypocellular and presented a reduced number of myeloid progenitors in the $\mathrm{BM}$, together with a decrease in $B$ lymphoid progenitors in fetal liver and $B M(51,52)$.

HSCs and B cell precursors are scattered around the BM in close contact with a small population of reticular cells expressing high amounts of CXCL12, named CXCL12-abundant reticular (CAR) cells. These cells were surrounding sinusoidal endothelial vessels and also localized near the endosteum, suggesting that CAR 
cells are a key component of both, HSC and B cell niches (114-116). Conditional deletion of CXCL12-expressing cells by diphtheria toxin (DT) administration in CXCl12DTR mice reduced the numbers of lymphoid, erythroid and myeloid progenitors in the BM, indicating that CAR cells support the survival and proliferation of $B$ cell progenitors and proliferation of erythroid progenitors (117). In the absence of CAR cells, LT-HSCs and its repopulating activity were reduced but HSC quiescence was increased (117). Interestingly, these studies also demonstrated that CAR cells contain MSC activity measured by the ability to form fibroblastic colonies (colony-forming unit fibroblasts, CFU-F). MSCs can self-renew and differentiate into bone, fat, cartilage and stromal cells. Finally, CAR cells are the major producers of SCF and CXCL12 (117), while endothelial cells and mature osteoblasts are only minor contributors of $\operatorname{CXCL12}(91,92)$. Given that these cells are defined functionally (production of CXCL12), the population overlaps with other stromal cell subsets described below.

\section{$\underline{\operatorname{Prx}^{+} \text {cells }}$}

The paired-related homeobox gene-1 (Prx1) is expressed in the early limb bud mesenchyme in mouse during development and in adult BM mesenchymal progenitor cells (118). Prx $1^{+}$cells have osteogenic and adipogenic differentiation potential in vitro, consistent with a MSC phenotype and have the capacity to form CFU-F. Importantly, deletion of Cxcl12 in Prx $1^{+}$cells led to loss of HSC LTR activity, HSC quiescence and CLPs, indicating an important role of $\mathrm{Prx}^{+}$ perivascular cells in the maintenance of $\operatorname{HSCs}(91,92)$.

\section{Leptin Receptor ${ }^{+}$cells}

Cells expressing the leptin receptor (referred to as LepR ${ }^{+}$cells) arise postnatally in the BM and make little or no contribution to bone or cartilage during development (119). In the adult mouse, however, they contain high MSC activity and are one of the main sources of osteolineage cells and adipocytes. Indeed, they are highly enriched for MSC markers such as Prx1, PDGFR $\alpha$ and CD51. They are found surrounding both sinusoids and arterioles in the BM (119). They also account for the production of growth factors that contribute to the maintenance of HSCs as they have been shown to be one of the major sources of SCF and CXCL12 in the BM $(90,92)$. Importantly, conditional deletion of Scffrom LepR- 
perivascular stromal cells depleted quiescent HSCs, while deletion of Cxc/12 led to HSC egress from the marrow $(90,92)$.

\section{$\underline{\text { Nestin+ cells }}$}

Perivascular MSCs expressing the intermediate filament nestin were identified using a transgenic mice in which the expression of the green fluorescent protein (GFP) is driven by the nestin (Nes) promoter (hereafter referred to as Nes-GFP+ cells) (120). Nes-GFP+ cells were found to be spatially associated with adrenergic nerve fibers and HSCs, and contained all MSC activity, suggesting an important HSC niche component (49). Nes-GFP+ perivascular cells express high levels of SCF and CXCL12, as well as other regulatory elements such as ANGPT1, OPN, IL-7 and VCAM1 (49). However, although Nes-GFP+ perivascular MSCs strongly overlap with other perivascular cells described later, deletion of Scf or Cxcl12 from Nestin ${ }^{+}$cells did not alter HSC numbers $(90,92)$. Whole-mount 3D imaging

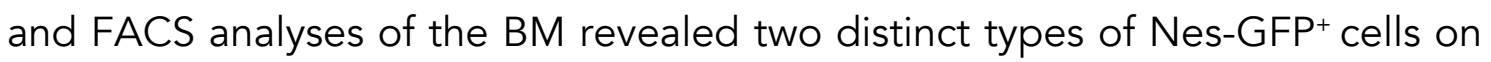
the basis of their GFP expression levels and cellular morphology. Nes-GFPbright cells are much rarer ( $0.002 \%$ of BM cells) than Nes-GFPdim cells and are found exclusively along arterioles. In contrast, Nes-GFPdim cells have reticular shape and are ubiquitously distributed among sinusoids (96) (Figure 4). Although both Nestin $^{+}$cell subsets account for MSC activity in the BM, most CFU-F activity is contained in Nes-GFPbright cells. These cells are additionally, positive for the pericyte markers NG2 and $\alpha$-smooth muscle actin ( $\alpha$-SMA) and are associated with nerves and a subset of quiescent HSCs, whereas Nes-GFPlow cells overlap with Leptin Receptor ${ }^{+}$cells and CAR cells (96).

\section{$\underline{\mathrm{NG} 2{ }^{+} \text {cells }}$}

Stromal cells that express the nerve/glial antigen 2 (NG2) are mainly perivascular cells that surround arterioles in the BM and are important for the maintenance of quiescent HSCs (96) (Figure 4). Depletion of $\mathrm{NG}^{+}$cells induced HSC cycling and, consistently, depletion of $\mathrm{Cxc} 12$ from arteriolar $\mathrm{NG}^{+}$cells lead to HSC reduction in the $\mathrm{BM}$, and altered localization away from arterioles, an effect that was accompanied by massive HSC mobilization into spleen and blood (96). 


\section{Endothelial cells}

The BM is a highly vascularized tissue that enables several blood cells, including HSCs, to enter or leave the bloodstream. BM-endothelial cells that line the interior of vessels secrete various factors such as Notch ligands, CXCL12, SCF and pleiotrophin, that promote HSC activity in steady-state $(90,91,121-124)$, and regeneration after BM damage $(122,123,125-127)$. In vivo studies have shown that deletion of $\operatorname{Scf}(90)$ or $\operatorname{Cxc} 12(91,92)$ using endothelial-specific Cre lines (Tie2-Cre or Cadherin 5 (Cdh5)-Cre) impairs HSC maintenance at steady state, suggesting an important role for endothelial cells as niche components and HSC regulators.

Conditional deletion of Scf from perivascular stromal cells (LepR-Cre) or endothelial cells (Tie2-Cre) reduced HSCs from the BM, but did not have an effect on HSCs when the depletion was done in Nestin ${ }^{+}$perivascular stromal cells, osteoblasts or hematopoietic cells (90). Most studies of perivascular niches in the BM have focused on sinusoids because they are the most abundant blood vessels in the BM, and because most HSC, MSC, SCF-expressing cells and CXCL12-expressing cells are in close proximity to sinusoidal vessels. However, SCF deletion in arteriolar endothelial cells (AECs) but not sinusoidal endothelial cells (SECS) reduced dramatically the number of HSCs in the BM. In addition, AECs-derived SCF contributes to the regeneration of HSCs after myeloablative conditions (128).

Arteriolar and sinusoidal endothelial cells can be phenotypically distinguished by their differential expression of Sca-1 and podoplanin (PDPN); AECs are CD45TER119-Sca-1 high PDPN $^{-}$whereas SECs are CD45-TER119-Sca-1+PDPN ${ }^{+}$(128). Arterioles are structurally distinct from sinusoids as they are surrounded by sympathetic nerves and layers of smooth muscle cells. The permeability of these two types of blood vessels affects the levels of reactive oxygen species (ROS) in the vicinity and therefore HSCs that reside near them. HSCs that reside close to the less-permeable arterioles contain low levels of ROS and are thus quiescent, whereas those close to more permeable, sinusoidal cells have increased ROS levels leading to their activation and increasing its differentiation and migration (65). This was confirmed by another study showing that quiescent HSCs localized in proximity to $\mathrm{NG2}^{+}$-Nestin-GFPbright periarteriolar niches, and that depletion of these cells altered HSC localization and quiescence status (96). 
The BM vasculature has an essential role in the regeneration of HSCs after a myeloablative injury such as chemotherapy or radiotherapy. These procedures are commonly used before BM transplantation to eradicate malignant host cells. Unfortunately, these treatments cause a complete destruction of the vascular niche in the BM. More specifically, sinusoidal vasculature and associated perivascular cells are ablated while arteries and arterioles remain almost intact (126, 127, 129-131). While transplanted HSCs can shortly engraft close to an arteriolar and megakaryocytic niche $(132,133)$, long-term restoration of normal hematopoiesis demands a healthy sinusoidal network. This restoration is enabled by factors that the sinusoidal network produces in response to injury, such as Notch ligands and pleiotrophin $(122,123)$.

Overall, HSCs interact with different types of stromal cells that express important regulatory molecules including SCF, CXCL12 and TGF- $\beta$. Although early mouse studies implicated mature bone-forming osteoblasts as key HSC niche cells, recent work has refined the identity of HSC-supportive cells to several populations of MSCs and their early osteoblastic lineage cell derivatives. Perivascular MSC-like cells expressing either nestin (49), CXCL12 (117) or leptin receptor (90) have been shown to be more important for HSC maintenance. Endothelial cells also have important HSC-supporting roles both in steady state and during regeneration (134). The emerging picture of the HSC BM niche is a complex array of regulatory cell types with a predominant role for MSCs and their early OBC derivatives in forming both perivascular and endosteal BM niches that maintain HSCs and regulate blood production (Figure 4).

\section{Neural regulation}

Besides all the aforementioned cellular types, the nervous system also regulates the hematopoietic niche and HSC properties $(3,73,135)$. The SNS innervates both the bone and the marrow through sympathetic and sensory nerves. Sympathetic fibers release NA which signals through adrenergic receptors. Importantly, sympathetic nerves that align with medullary arterioles regulate the expression of stromal CXCL12 and therefore the traffic of HSCs between the BM and peripheral blood, both under homeostasis and stress $(3,49,73,136)$. Specifically, release of NA by the SNS targets stromal cells through the $\beta_{3^{-}}$ adrenergic receptor (ADR 33 ) leading to rapid downregulation of CXCL12 in the 
BM, and rapid HSC egress into blood. Interestingly, and related to the aforementioned diurnal patterns of HSC in blood, the release of NA from the SNS follows circadian patterns controlled by the core clock genes, thereby explaining the diurnal release of HSCs into blood (3).

Another level of modulation is afforded by non-myelinating Schwann cells, which are glial cells expressing the glial fibrillary acidic protein (GFAP). These cells insulate sympathetic and sensory nerves along arteries, and can promote HSC quiescence through integrin-dependent activation of transforming growth factor- $\beta$ (TGF- $\beta$ )/SMAD signaling (137).

Finally, SNS signals also control G-CSF-dependent HSPC mobilization into the circulation. In this case, adrenergic signals cause osteoblast and stromal suppression, and thus a reduction in CXCL12. Consistently, pharmacological or genetic ablation of adrenergic neurotransmitters suppresses HSC egress after G-CSF treatment (73).

\section{Adipocytes}

In comparison with other niche cells, adipocytes have been associated with a negative regulation of HSCs (138). The content of fatty tissue in the BM negatively correlates with the engraftment and maintenance of HSCs, and BM recovers more quickly after transplantation or chemotherapy when mice are treated with an adipocyte inhibitor $(138,139)$. This is apparent also during ageing, when hematopoietic sites are replaced by fatty tissue and this coincides with a decline in HSC function (138, 140).

\section{Hematopoietic descendants}

In addition to the diverse stromal niche components, the hematopoietic descendants can also regulate HSC activity in a feedback loop-type of process. Among these, megakaryocytes (MKs), macrophages, neutrophils and regulatory $\mathrm{T}$ cells (Tregs) have been best described to regulate the hematopoietic niche, both in homeostatic and stress conditions.

\section{Myeloid cells (macrophages and neutrophils)}


BM-resident macrophages were the first among hematopoietic cells shown to regulate HSCs. BM macrophages promote the retention of HSCs by enhancing the function of Nestin ${ }^{+}$cells and osteoblasts $(141,142)$. Consequently, depletion of $\mathrm{CD} 169^{+}$macrophages was sufficient to induce HSC egress into the bloodstream (141). BM macrophages also regulate BM recovery under stress conditions. In a transplantation setting were radiation eliminates the vast majority of leukocytes, a population of CD169+ radiation-resistant macrophages is needed to repopulate the spleen and BM via cell-autonomous expansion, and are essential for optimal donor-derived HSC repopulation (143). Finally, a rare population of $\alpha$-SMA-positive macrophages localizes adjacent to HSCs in the $\mathrm{BM}$ and prevents HSC exhaustion by diminishing the levels of ROS under stress conditions (144). Whether the two populations of macrophages are the same remains unknown.

Neutrophils are the most abundant myeloid cells inside the BM. Their short lifespan (around $12 \mathrm{~h}$ in mice) demands not only high production rates, but also a quick and efficient way to eliminate them, as high amounts of these cells could lead to toxic, undesired side-effects in tissues. Neutrophils undergo daily circadian oscillations in number and phenotype, whereby they generate a socalled population of "aged" neutrophils that are more abundant at ZT5 (86). This aged population of $\mathrm{CXCR} 4^{\text {high }} \mathrm{CD} 62^{\text {low }}$ neutrophils migrates into tissues at night (86). In the BM, these cleared aged neutrophils are phagocytosed by macrophages resulting in activation of the transcription factor liver $X$ receptor $(L X R)$. Activation of $L X R$ ultimately results in niche-suppressive signals that blunt CXCL12 levels in the marrow and promote the circadian egress of HSCs into circulation (86) (Figure 4).

In addition to this mechanism of steady-state release, G-CSF activation of the SNS was shown to stimulate the production of prostaglandin E2 ( $\left.\mathrm{PGE}_{2}\right)$ by neutrophils. $P G E_{2}$ in turn targeted osteolineage cells to promote HSC retention (145). Finally, a subset of $\mathrm{Gr} 1{ }^{+} \mathrm{CD} 115^{-}$neutrophils produces TNF $\alpha$ upon irradiation and injury and promotes sinusoidal vascular regeneration in the host, thus facilitating HSC engraftment and medullary regeneration (83).

In addition to macrophages and neutrophils, a myeloid population expressing the histidine decarboxylase $\left(\mathrm{Hdc}\right.$ ) forms spatial clusters with a $\mathrm{Hdc}^{+}$myeloid- 
biased (MB) HSC population. These myeloid cells secrete histamine which enforces Hdc $\mathrm{MB}-\mathrm{HSC}$ quiescence through the histamine receptor 2 (146). This negative feedback histaminergic circuit elicited by granulocytes and possibly other myeloid cells, is important for HSPC maintenance because ablation of histamine-producing cells causes HSCs to exit dormancy and induces loss of serial transplantation capacity (146).

Thus, granulocytes and myeloid cells appear to be important to maintain HSC homeostasis and to enhance BM recovery in transplantation settings $(83,146)$.

\section{Megakaryocytes}

Megakaryocytes (MK) are responsible for the production of thrombocytes (platelets). They localize in the BM around sinusoids, where they extend their protrusions to release pro-platelets into the bloodstream. The megakaryocytic lineage has been shown to bypass multipotent progenitors (Figure 2) and to directly differentiate from lineage-biased HSCs (32). Depletion of MKs leads to HSC proliferation indicating a direct role in maintaining HSC quiescence (132, $133,147,148)$, mediated in part by the release of CXCL4 (also known as PF4) (133), TGF $\beta(132)$ and THPO $(147,148)$. More recently, MKs have been shown to control the quiescence of a platelet- and myeloid-biased subset of HSCs expressing von Willebrand factor ( $\mathrm{VWF}^{+}-\mathrm{HSCs}$ ). MK form a niche that differs functionally and spatially from the $\mathrm{NG}^{+}$arteriolar niche. This last one seem to control a lymphoid-biased, vWF- HSC subset (vWF--HSCs) (149). Thus, at least two separated HSC niches may co-exist, a sinusoidal megakaryocytic niche and an arteriolar- $\mathrm{NG}_{2}{ }^{+}$niche, which seem to regulate distinct HSC subsets. In contrast to this, in irradiation settings, MKs promote HSC niche remodeling and HSC recovery through osteolineage cell expansion $(150,151)$ and the secretion of fibroblast growth factor 1 (FGF-1) (132).

\section{$\underline{\text { Regulatory T cells }}$}

The $\mathrm{BM}$ is a reservoir of $\mathrm{CD} 4^{+} \mathrm{CD} 25^{+} \mathrm{T}$ lymphocytes with immune-modulatory functions (152). In vivo intravital microscopy imaging revealed that allogenictransplanted HSCs colocalized proximal to a subset of FOXP3 $3^{+}$regulatory $T\left(T_{\text {regs }}\right)$ cells in the endosteal surface after transplantation (153). These $T_{\text {reg }}$ cells promote 
the survival of "allo-HSC" by secreting the immunoregulatory cytokine IL-10, which provides the HSC niche with immune privilege properties (allowing transplanted HSCs to escape from allogenic rejection) (153). More recently, Hirata and colleagues described a subset of $\mathrm{T}_{\text {regs }}$ that express the HSC marker, $\mathrm{CD} 150$. This population of $\mathrm{CD} 150^{+} \mathrm{T}_{\text {regs }}$ is also important to promote allo-HSC engraftment but also to maintain HSC quiescence inside the BM (154) (Figure 4).

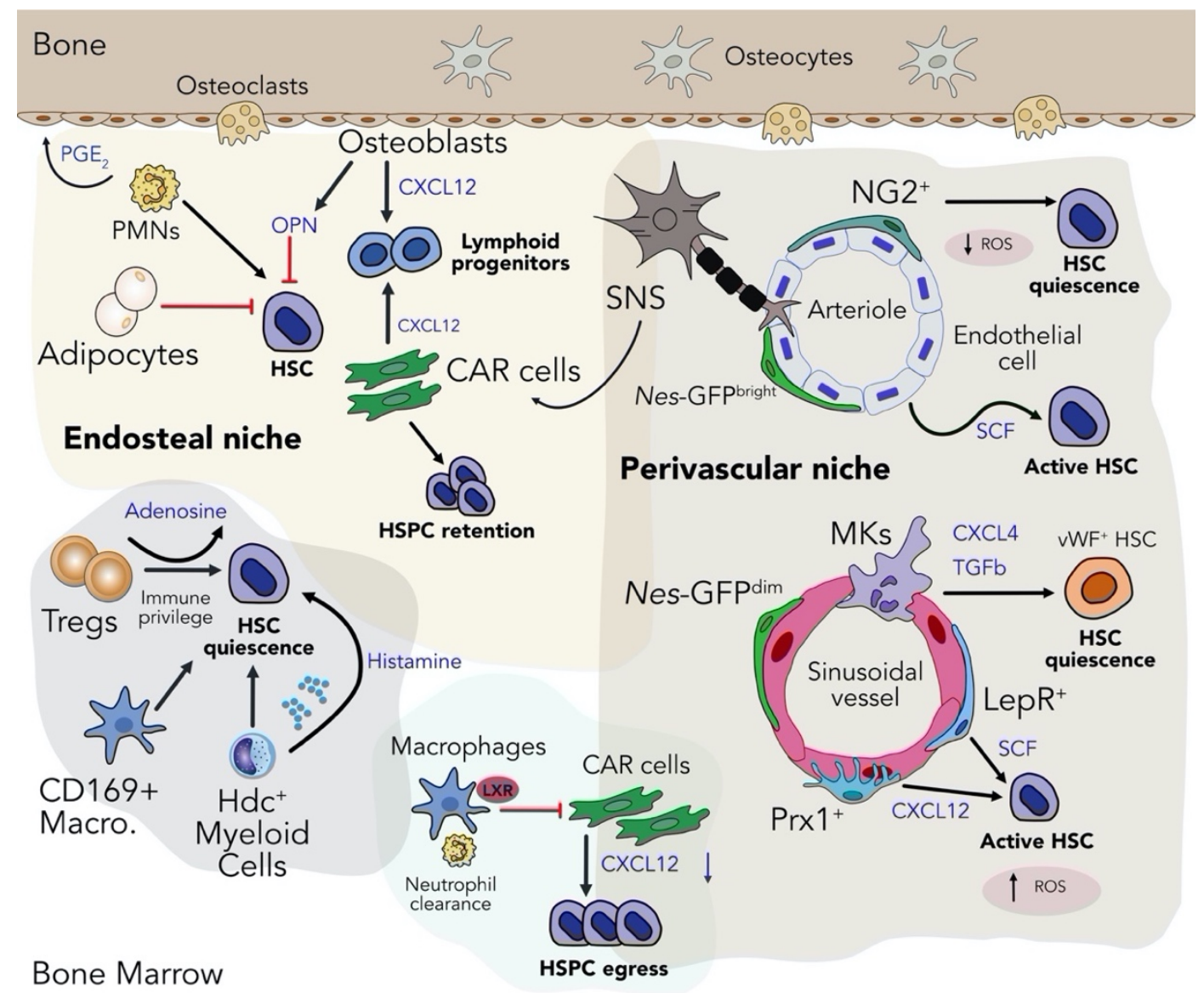

Figure 4. The Hematopoietic Stem Cell Niche. Scheme representing the main cellular components of the BM niche mentioned above and the principal regulatory pathways by which they regulate HSCs.

\section{Localization of HSCs within the Niche}

HSCs inside the BM niche have to compete for the space with mature immune cells and many other stromal components. In a non-deterministic model, HSCs could localize randomly throughout the entire BM space, or instead strategically near specific cellular and molecular compartments. Defining the exact HSC location relative to other components of the BM has been challenging, in large part due to the difficulty of imaging the marrow encapsulated by a fully calcified 
bone. Another hurdle has been the lack of specific reporters for HSC and their immediate descendants. Recently, myelopoiesis has been mapped in situ in the BM by co-localization of numerous cell surface markers. It was shown that myeloid progenitors, which are proximal descendants of HSCs, abandon the HSC niche soon upon differentiation (155). Myelopoiesis appears to occur near sinusoids although different sinusoids produce unique signals to regulate specific subsets of myeloid cells. This matches the idea that individual granulocyte-macrophage progenitors (GMPs) are scattered throughout the BM under steady state conditions and form clusters that locally produce granulocytes, under regeneration conditions (156).

Early observations claimed that progenitor cells with hematopoietic colonyforming capacity were enriched in the proximity of endosteal zones (157-159). However, studies using the phenotypically stem cell marker CD150 and 3Dimaging have shown that HSCs are broadly distributed but typically in close contact with endothelial cells and Nes-GFP+ perivascular cells $(17,49)$. The use of transgenic mice for labelling adult BM HSCs and the optical clearance of bones has enabled deeper knowledge of HSC distribution. Many of these studies suggest that HSCs are largely distributed in perisinusoidal niches and in contact with SCF-producing, LepR+ CAR cells (93-95), as discussed above. More recently, this traditional way of characterizing the HSC niche as endosteal or perivascular has been questioned. In vivo experiments in the BM calvarium deciphered that bone remodeling contributed to an additionally degree of heterogeneity in the niche (25). Cavities that contain a mix of bone deposition and bone resorption seem to favor HSC expansion, supporting the idea that HSCs expand clonally in restricted physical domains, as also mentioned below (25).

Distinct niches have been described for different subsets of quiescent HSCs. Studies showed that quiescent HSCs are proximal to arterioles, while activated or proliferating HSCs move away from these vessels (96). Whole-mount 3Dimaging revealed that quiescent HSCs are also adjacent to MKs forming a niche that seems different from the arteriolar one (133). Different microenvironments exist that either support clonal expansion of HSCs or promote their quiescence. It is thus plausible that HSC distribution changes according to their activity or cell cycle status. Additionally, lineage-committed HSCs also associate with distinct niches. As mentioned above, platelet and myeloid-biased HSCs 
associate with sinusoidal-MK, whereas $\mathrm{NG}^{+}$arteriolar niches selectively regulate lymphoid-biased HSCs (149).

In addition to HSCs, their downstream progenitors may have specific niches separated away from HSCs. Lymphoid progenitors for instance are supported by the endosteal niche and both IL-7 and CXCL12 derived from osteoprogenitors are essential for B cell maturation and maintenance (91-93). In the same line, a macrophage niche is essential for erythroid progenitors to form the so-called erythroblastic islands, where both cells interact, and macrophages instruct and facilitate erythroblast proliferation and differentiation $(141,160)$.

While the exact localization of HSCs is still unclear, it seems feasible that within the entire BM cavity multiple specialized, micro-niches coexist in the same space. Evidence suggests that different hematopoietic progenitors and mature immune blood cells have distinct niches, each of them with distinct signals, but tightly coordinated within the rest (155). In summary, the hematopoietic niche is now envisioned as a changing, dynamic tissue that receives and integrates signals from its environment to maintain and instruct HSCs.

\section{Alterations of HSC and their Niche}

\section{Ageing}

Many physiological processes, including those affecting HSCs, change with age. One of the most remarkable age-related changes in hematopoiesis is a progressive decline that makes the organism vulnerable to infections, autoimmune disease, anemia or cancer (161). The reduced immune function associated with age affects both the myeloid and lymphoid compartment and is in part due to a functional decline of aged HSCs. HSC ageing is caused by both cell-intrinsic mechanisms, such as epigenetic and metabolic alterations or DNA damage, as well as cell-extrinsic mechanisms such as alterations in niche composition.

To compensate for the decrease of HSC function with age, the size of the HSC pool increases as shown by the expansion of phenotypically HSCs in aged mice albeit their low regenerative potential $(162,163)$. Furthermore, aged HSCs show a myeloid-biased differentiation upon transplantation, accompanied by a 
reduction in lymphopoiesis, and a decrease in self-renewal when compared to HSCs from young donors $(163,164)$. Aged mouse HSCs also present decreased homing and engraftment potential, and increased HSC mobilization into peripheral blood $(165,166)$.

CD41 marks a population of myeloid/megakaryocytic-biased LT-HSCs that accumulates with age (167). This phenotypic change is accompanied by an increase in the number of platelets in the peripheral blood of aged mice and MK progenitors in the BM $(167,168)$. Indeed, CD41 loss results in decreased survival and quiescence of HSCs (167). Aged HSCs also present more DNA strand breaks and this increases mutational load that contributes to increased initiation and progression of hematological cancers $(169,170)$. Finally, aged HSCs suffer a loss of polarity in Cdc42, tubulin and AcH4K16 (171).

Interestingly, young HSCs transplanted into aged mice engrafted with lower efficiency, indicating that age-related changes in the BM niche could also impact HSC function. This process is related to the changes in chemokines such as CCL5 and matrix proteins, which partly accounts for the myeloid-bias in HSCs (172), or osteopontin, which attenuates the ageing effects of HSCs (173). Additionally, in vivo multiphoton intravital imaging revealed that old HSCs reside further away from the endosteal zone (which normally favors lymphoid niches) compared to young HSCs (174), and that aged HSCs localize away from arteriolar and megakaryocytic niches and closer to the perisinusoidal Nes-GFPlow niche (175). Thus, altered HSC distribution within the niche is a hallmark of ageing (176).

Age-related bone loss has also been associated with vascular changes in the BM, such as reduction of transitional vessels and arterioles. The BM vasculature also exhibits increased leakiness, elevated ROS levels and decreased expression of CXCL12, SCF and Jagged1 with age (177). Likewise, arteriolar segments suffer shortening and loss of sympathetic innervation (176). Indeed, surgical denervation in young mice recapitulates all major age-related anomalies in HSC, while administration of a $\beta 3$-adrenergic receptor (ADR 33 )-selective agonist in old mice partially rejuvenated aged HSCs by acting on BM Nestin ${ }^{+}$stromal cells (176). However, more recent data suggests that there is a functional change of neurotransmitters, $\beta 2$ over $\beta 3$ - signaling, instead of a general decline in the sympathetic tone during physiological ageing. $\beta 2$-signaling promotes myelopoiesis and megakaryopoiesis through stromal-derived IL-6, whereas $\beta 3$ - 
signaling inhibits myelopoiesis in aged mice (178). MSCs also suffer an expansion during ageing but with reduced clonogenic capacity, and display skewed differentiation towards adipogenesis, accompanied by a reduction in bone formation, with an overall negative impact on hematopoiesis $(138,140)$.

In summary, the BM microenvironment is critical for hematopoiesis during all the stages of the organism, from embryonic until the old age of the organism, and, importantly, can be modulated for therapeutic purposes.

\section{Cancer}

Hematological malignancies have common alterations in hematopoietic function, and affect the blood, BM, lymph nodes and lymphatic system.

As mentioned above, the BM niche supports healthy HSCs for correct functioning. In a similar fashion, altered niches can support malignant hematopoiesis, such that a perturbed BM niche can remodel into a selfreinforcing leukemic niche that impairs normal hematopoiesis and favors leukemic stem cell (LSC) growth, leading to neoplasia (179). Intriguingly, there is substantial evidence for niche-driven malignancies in which deletion of certain genes in different niche compartments, or activation of cancer-related pathways (e.g., RAS signaling or NFK $\beta$ ), favor the proliferation of malignant cells and lead to the development of leukemias. Studies have shown, for example, that genetic ablation of the retinoblastoma gene in BM stromal cells promotes myeloproliferative neoplasia (180).

Just as the microenvironment can contribute to disordered hematopoiesis, malignant cells can disrupt and remodel normal niches creating a cancersupportive environment. LSCs do not die off in a normal cycle and instead they keep dividing and eventually push out other healthy HSCs that compete for nutrients and metabolites in the same space. For instance, LSCs in acute myeloid leukemia (AML) inhibit adipogenesis and promote the differentiation of MSCs into altered osteolineage cells that promote their own growth $(181,182)$. BM vascular abnormalities are also found in hematological malignancies. Proangiogenic cytokines such as VEGF are increased; these stimulate new angiogenic processes that support and nurture LSCs (183). In AML the BM vasculature presented increased permeability that lead to overproduction of 
ROS and nitric oxide (NO) which increased HSC motility (184). LSCs also upregulate cell adhesion molecules such as CXCR4, VLA-4 or CD44. These molecules mediate leukemia cell adhesion and survival that confer chemoresistance, and this correlates with a worst outcome of the disease (185189).

More recent studies have analysed the BM stroma in detail by using single-cell RNA sequencing to define the cellular taxonomy under basal conditions and its perturbation by malignancies (190). This study identified 17 stromal subsets that express different hematopoietic regulatory genes and described changes caused by $A M L$ in mice. Leukaemia impaired mesenchymal development blunting adipogenic and osteogenic differentiation, and significantly changed the proportions of key subsets of stromal cells in favour of malignant cells. This was further accompanied by a loss of HSC niche factor production by multiple cell types, overall indicating that the BM stroma responds and favours malignant cells. Another study used single-cell mass cytometry to measure protein levels to define 28 subsets of BM stromal cells and observed that after radiation conditioning of the $\mathrm{BM}, \mathrm{LepR}^{+}$and Nestin ${ }^{+}$putative niche cells are lost while a set of $\mathrm{CD} 3^{+} \mathrm{BM}$ stromal cells remained resistant, and contributed to HSPC engraftment and acute hematopoietic recovery (191).

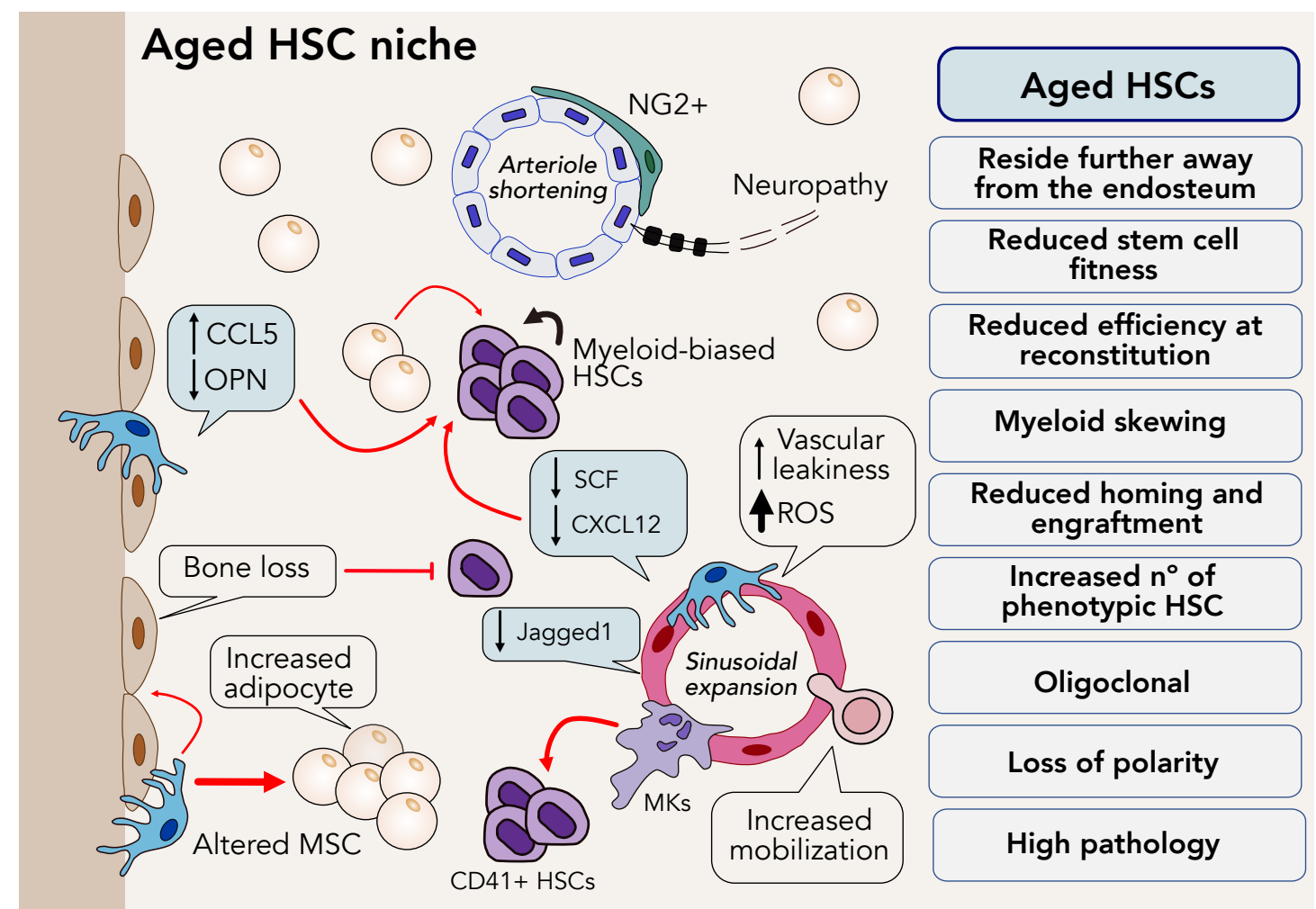




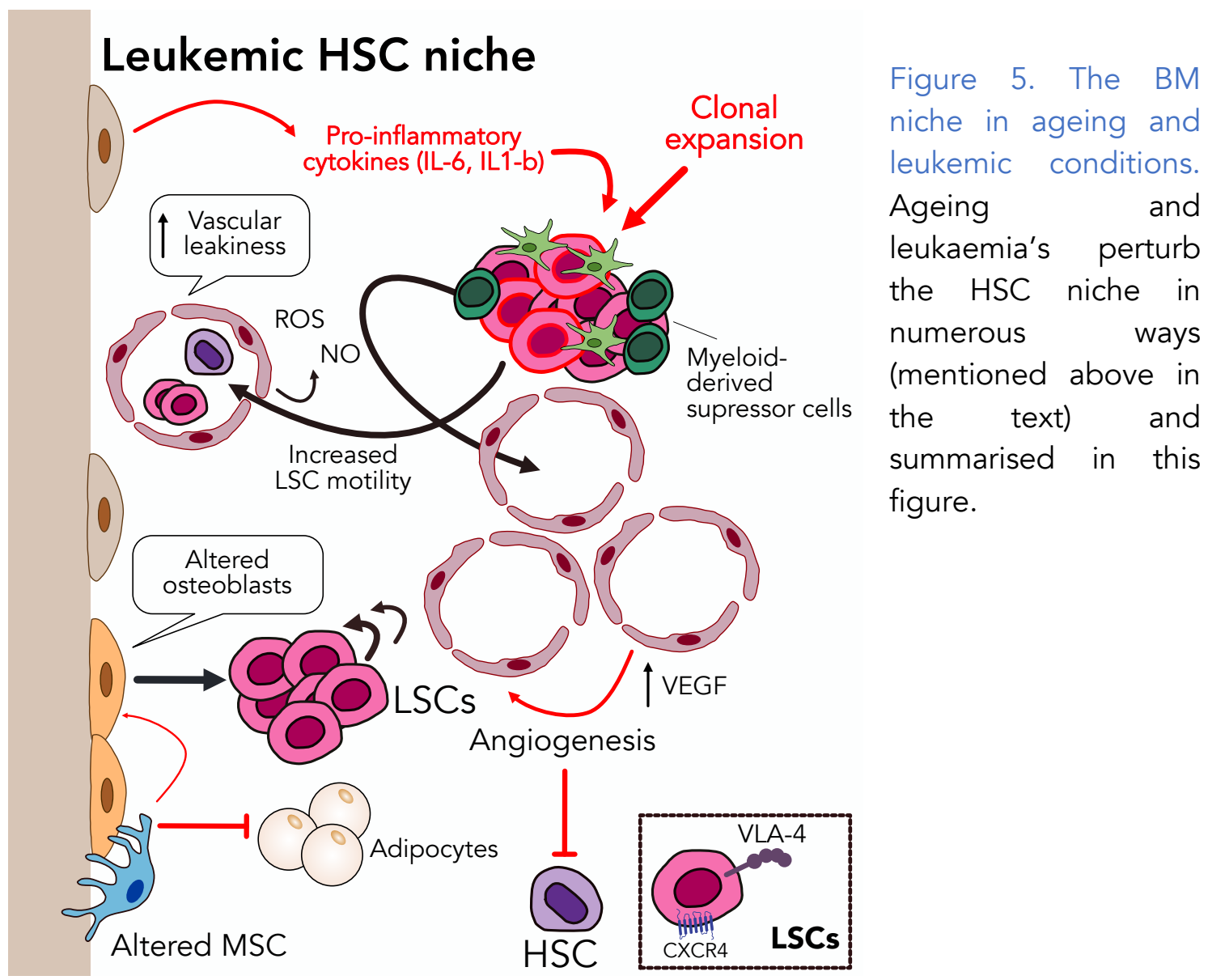

Altogether, the BM niche is no longer seen as a static structure that hosts HSCs, but rather as a dynamic tissue that continuously remodels and adapts to specific situations and demands. This is the case not only in diseases such as myeloid proliferative cancers, but also in physiological events, such as ageing, where functional and phenotypic changes, provoke changes in stem cell behavior. Whether these processes are the consequence or the cause of hematological disfunctions still needs to be clarified. Likewise, whether other components in the niche exist or acquire new roles on HSC function remains elusive. Understanding how the niche contributes to malignant transformation or ageing processes will be extremely useful, for example in the design of therapeutic approaches to prevent or treat hematological malignancies. 


\section{Objectives}

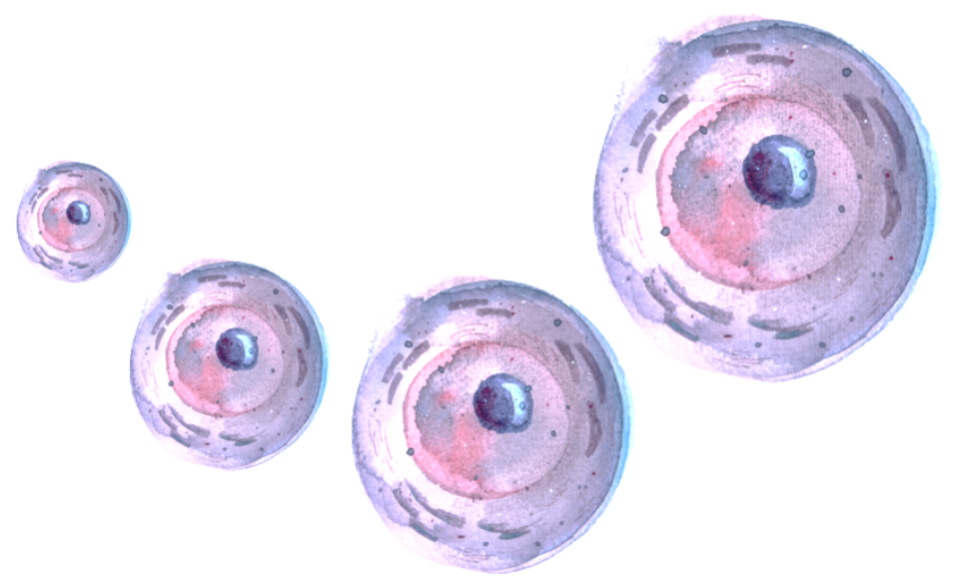


$-58$ 


\section{Objectives}

The main objective of this doctoral thesis is to characterize the physiological roles of circulating hematopoietic stem cells in the organism. The specific objectives of the study are the following:

1. Determine the phenotype and function of circulating hematopoietic stem cells (HSC).

2. Define the molecular pathways that drive the release of circulating HSC into blood.

3. Analyse the relevance of circulating HSC in ageing. 
- 60 - 
Results

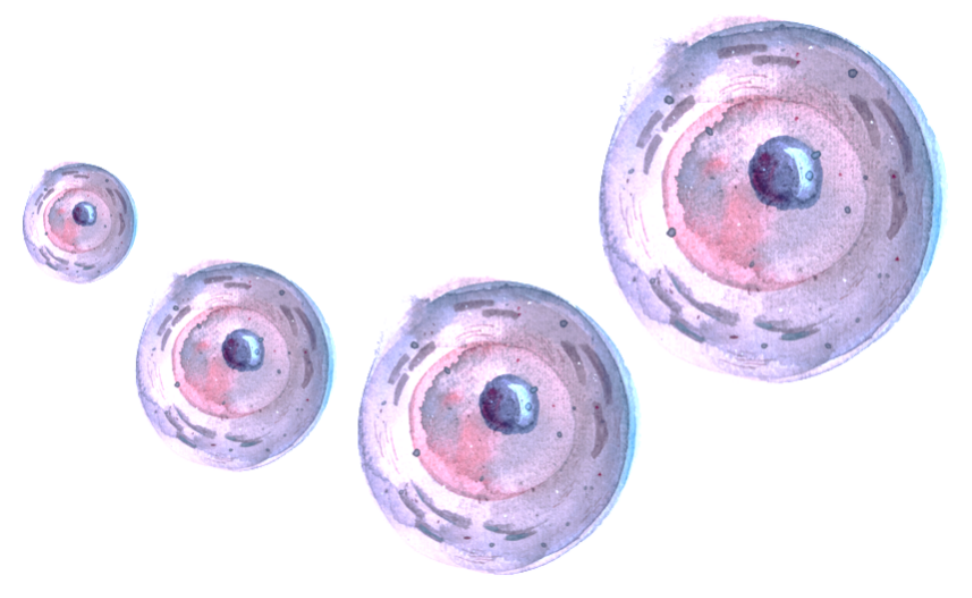


$-62-$ 


\section{Results}

\section{Circulating HSCs repopulate damaged niches}

It has been known for decades that a small population of circulating hematopoietic stem cells (cHSCs) is present in the circulatory system of mice even under steady-state conditions $(2,39)$. However, the biological and functional properties of these cells are still unknown. In order to clarify these uncertainties, we first analysed their properties.

Circulating HSCs provide long-term repopulation of damaged bone marrow niches

We first tested the capacity of a hematopoietic tissue to regenerate in the absence of an external HSPC source (as occurs in BM transplantation). For this purpose, we applied high-dose radiation, 10 grays (Gy), only to the lower limbs of WT mice (femur and tibiae) while the rest of the body was protected by a leaded shield (Figure 1a). We analysed BM recovery in the irradiated bones every week during one month by histology and flow cytometry. We observed a complete depletion of the marrow cellularity at day 6 after irradiation (Figure $1 \mathrm{~b}$ e). More importantly, we observed a progressive and fast regeneration of the marrow, such that by four weeks, the initially depleted marrow was histologically normal (Figure 1b), correlating with normal BM cellularity (Figure 1e). Moreover, the numbers of phenotypical and functional stem and progenitor cells were progressively restored, even though, after one month, did not reach to initial levels (Figure 1c-d). We also measured the levels of colony-forming units (CFUC) as a functional proxy of progenitors in blood and BM (LSK and MP). In BM we observed a small recovery at day 7 after irradiation with a progressive increase up to day 21 that failed however to reach baseline levels (Figure 1f). In blood, however, the level of progenitors remained low at all time points, suggesting that all progenitors might localized at the injured site (Figure $1 \mathrm{~g}$ ). The recovery of fully ablated marrows suggested that migration of HSCs from healthy niches enables niche regeneration upon local hematopoietic ablation. This approach, however, did not rule out the possibility that reconstitution could be due to residual HSCs that survive locally to the irradiation. 

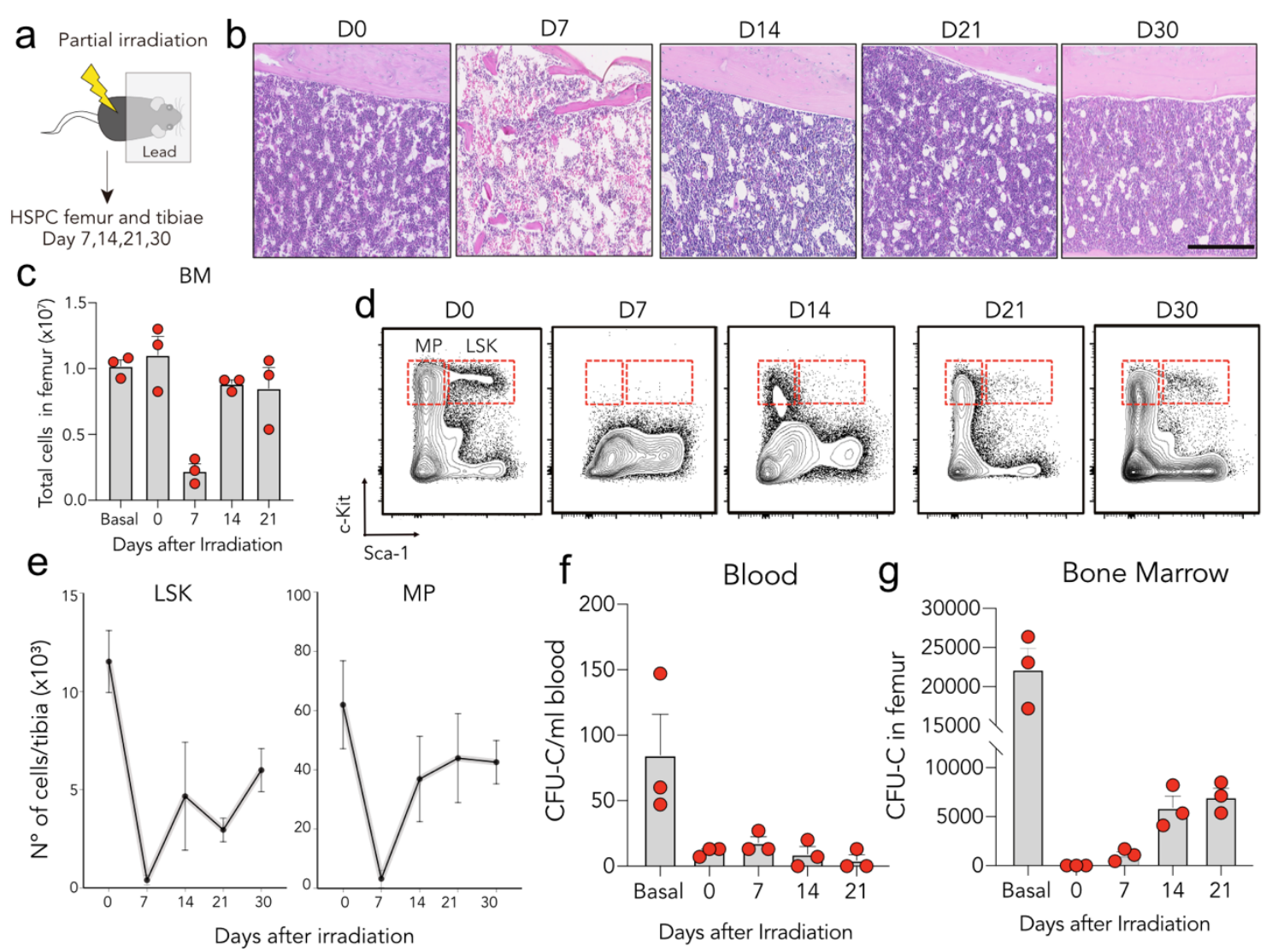

Figure 1. Healthy cHSC restore haematopoiesis in damaged niches. (a) Scheme of partial irradiation using a lead shield to expose only the lower limbs to 10Gy irradiation. (b) Representative H/E stainings of tibial marrow from WT mice at different times after irradiation. Scale bar, $250 \mu \mathrm{m}$. (c) Flow cytometry plots of BM progenitors at the indicated times after irradiation. LSK ( inn $\left.^{\mathrm{NEG}} \mathrm{Sca}-1^{+} \mathrm{c}^{-} \mathrm{Kit}^{+}\right), \mathrm{MP}$, myeloid progenitors (Lin ${ }^{\mathrm{NEG}} \mathrm{Sca}-1^{\mathrm{NEG}} \mathrm{c}-\mathrm{Kit}^{+}$). (d) Plot showing the total numbers of progenitors per tibia at the indicated times; $\mathrm{n}=3-8$ mice per time. (e) Total BM cellularity in femurs at the indicated days after irradiation; $n=3$ mice per time point. ( $f$ ) Total number of CFU-C in femur at the indicated time points after irradiation; $n=3$ mice per time point. (g) Total number of CFU-C in peripheral blood at the indicated time points after irradiation; $n=3$ mice per time point.

To determine whether cHSCs, rather than residual HSCs, were responsible for the marrow recovery, we used a model of parabiosis, in which two mice are surgically conjoined, and start to share the circulation by the formation of new vessels (see Materials and Methods, section 6.2 for a detailed explanation). Here, we joined GFP-expressing, non-irradiated mice together with non-fluorescent mice that had been previously subjected to lethal irradiation (8Gy) (Figure 2a). This model allowed us to discriminate between locally surviving HSCs (nonfluorescent cells) and those derived from cHSCs that arrived from the partner through circulation and can be identified by GFP+ expression (Figure 2b). After 
3 weeks of shared circulation, the parabiotic partner mice were surgically separated and monitored for the presence of partner derived GFP+ leukocytes in the blood of the non-fluorescent irradiated "recipient" partner for 16 weeks, and in the BM at the endpoint only (Figure 2c-d). We found long-term (LT) reconstitution of all hematopoietic lineages, which were exclusively derived from the GFP+ ${ }^{+}$partner, indicating that cHSCs that had crossed between partners were endowed with LT-reconstituting potential, and were indeed capable of regenerating damaged haematopoiesis (Figure $2 c$ and $d$, grey lines and bars).

We next examined if marrow regeneration depended on the elimination of preexisting HSCs, rather than on a competitive advantage of cHSCs over BMresident HSCs. For this purpose, we repeated the parabiosis experiments but this time we subjected the "recipient" partners to decreasing doses of irradiation $(8,4,2$ and 0 Gy, Figure 2a-d). Partner-derived haematopoiesis (measured in blood) by the non-irradiated mice was proportional to the irradiation dose, in the case of myeloid cells, with progressively decreasing contribution at lower doses, and only residual reconstitution in the absence of irradiation (Figure 2c). Analysis of the BM recipient parabiotic mice at the end of the experiment (16 weeks) showed contributions by cHSCs to all hematopoietic progenitor subsets, including the most primitive phenotypic LT-HSCs, and were proportional in frequency to partner-derived leukocytes in blood (Figure 2d). Finally, we sought for cHSCs in other organs that have been described to support extramedullary haematopoiesis, such as spleen, liver or lung $(101,192)$. Parabiotic experiments, in non-irradiated conditions revealed that cHSCs only seeded the main hematopoietic tissues, i.e., the BM and spleen, but we did not find evidence of these cells in liver or lung (Figure 2e).

Overall, these data indicated that cHSCs efficiently repopulate and reconstitute damaged haematopoietic niches with long-lasting and multilineage potential and have the capacity to regenerate the full hematopoietic tissue of a mouse only when the endogenous haematopoiesis has been ablated. 

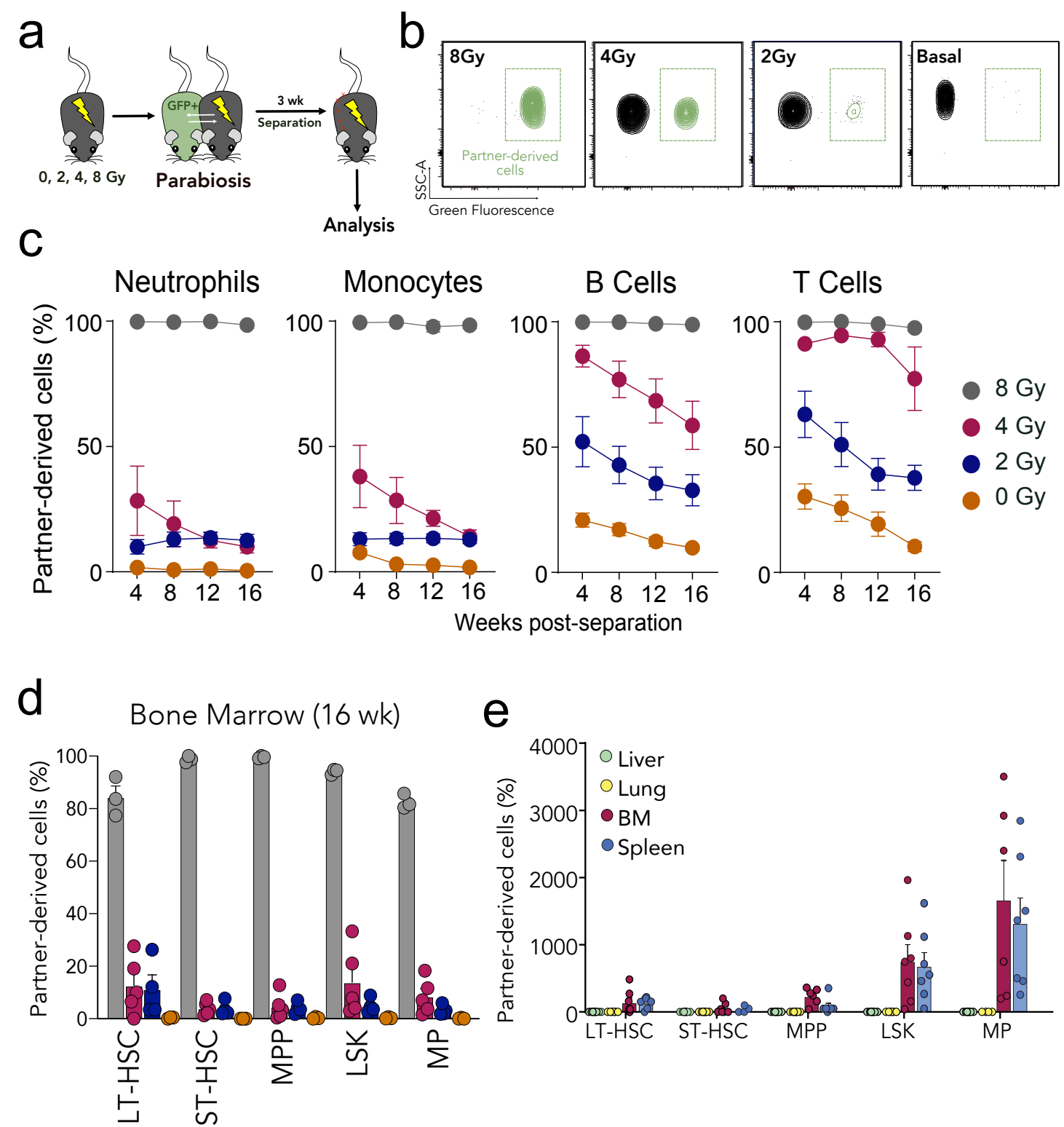

Figure 2. cHSCs long-term reconstitute damaged niches. (a) Experimental design to assess the repopulation of haematopoiesis in parabionts after surgical separation. (b) Gating strategy used to identify GFP+ partner-derived leukocytes in peripheral blood and HSPCs in the BM by flow cytometry. (c) Percentages of partner-derived blood leukocytes in the irradiated partner over time (weeks) after separation, in the different irradiation dose groups; $n=3-10$ parabionts per dose. (d) Percentage of partner-derived hematopoietic progenitors in the BM of the irradiated recipient partner at 16 weeks after parabiont separation; $n=3-10$ parabionts per dose. (e) Total number of partnerderived hematopoietic stem and progenitor cells in the liver, lung, BM and spleen of parabiotic partner; $\mathrm{n}=7-8$ mice. Data shown as mean $\pm \mathrm{SEM}$.

It is well established that the release of cHSCs into circulation follows circadian patterns (3), although their numbers in blood at any given time are low. Further, it is unclear whether the HSCs that enter the circulation are different from the 
bulk of HSCs found in the BM, or whether all the cHSCs have the choice and potential to enter the circulation and patrol tissues. To discriminate between both possibilities, we established parabiosis between DsRed reporter mice and WT non-fluorescent mice (Figure 3a). After one month in parabiosis, we separated the mice and examined whether the few circulating progenitors (CFU$\mathrm{C}$ assay as a proxy for $\mathrm{cHSCs}$ ) from the partner (circulating progenitors will be differentiated based on the fluorescent reporter) persisted in circulation over time (Figure 3b). In our approach, persistent presence of partner CFU-C would indicate that they represented a population distinct from the medullary pool. We found, however, that partner-derived CFU-C disappeared soon after separation of the parabionts, suggesting that cHSC are part of a common medullary pool.
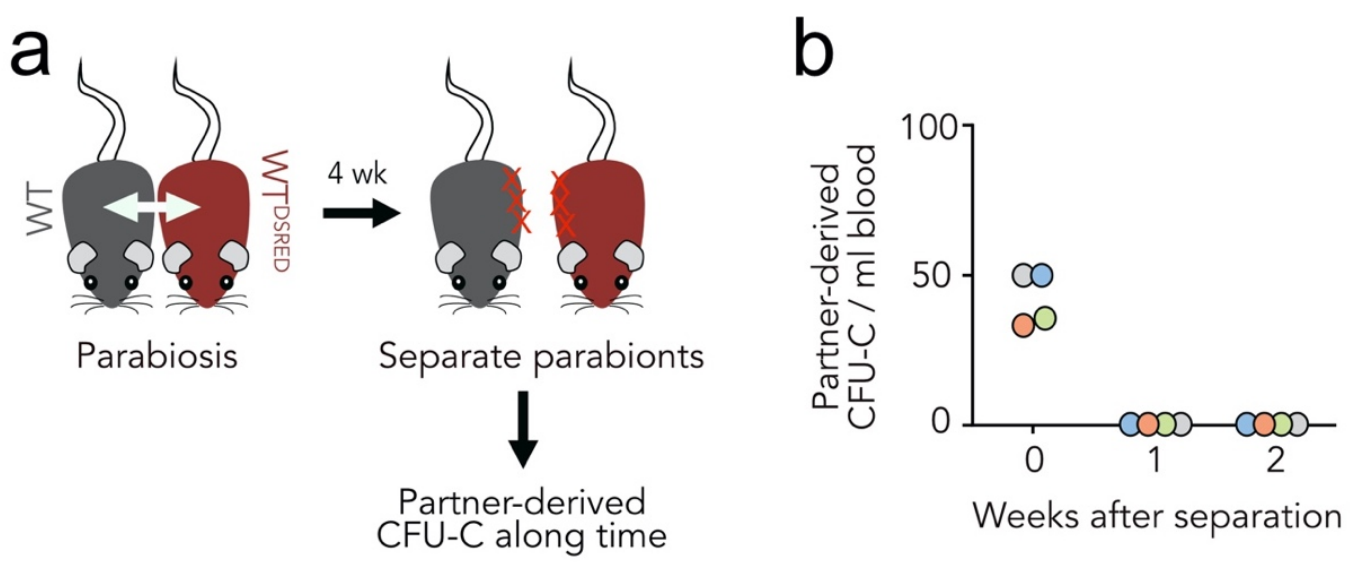

Figure 3. cHSCs are part of the medullary pool of HSCs. (a) Experimental scheme of parabiosis to analyse the time of partner-derived hematopoietic progenitors in circulation. After 4 weeks of sharing circulation parabionts were surgically separated and blood was collected at the moment of separation (time 0), as well as 1 and 2 weeks after separation, to estimate the number of DsRed+ partner-derived CFU-C (b) Number of partner-derived CFU-C in blood. Each dot colour represents a different mouse analysed at different times; $n=4$ mice.

\section{Repopulation of damaged BM niches is multiclonal}

To estimate the frequency of cHSC that enter the circulation and engraft the irradiated BM, we next used lentiviral-driven marking of clones at unique integration sites (193). We setup an experiment in which WT mice were first lethally irradiated (12 Gy) and reconstituted with lentiviral-transduced Lineage ${ }^{\text {NEG }}$ $\mathrm{GFP}^{+} \mathrm{BM}$ cells (Figure 4a). This system permitted us to trace every single clone derived from a stem cell (193). 6 weeks after BM transplantation (BMT) these 
mice were used as "donor" mice in parabiosis. The "recipient" mice in these parabionts were previously subjected to sublethal irradiation (6 Gy) to facilitate cHSC engraftment, and then surgically joined in parabiosis to the donor mice (Figure 4a). After 4 weeks of sharing circulation, mice were separated, and the dynamics and identity of single HSC clones that had crossed to the "recipient" mice were analysed for several months (Figure 4a). The "donor" BM and spleen were taken as reference for the source clones. To track HSC clones, we isolated from peripheral blood both myeloid cells (CD11b+ cells) and lymphoid cells (CD19+ cells) that were GFP+ (i.e., lentiviral transduced) every 4 weeks for 16 weeks (Figure 4b-c). At the end of the experiment, we also isolated the spleen and bones (femur, tibia, sternum, and arms) of the recipient mice for clonal analysis. In the analysis, however, we focused on $\mathrm{CD} 11 \mathrm{~b}^{+}$myeloid cells as they better reflect $\mathrm{HSC}$ activity, rather than long-lived $\mathrm{CD} 19^{+}$lymphoid cells that cross between partners and mirror the composition of the spleen. We additionally controlled for the engraftment levels and transduction efficiencies in all parabiotic pairs (vector copy number, Figure 4c-d). Unexpectedly, we found that many myeloid clones from the donor mouse were found in the recipient partner (Figure 4e). Moreover, estimation of HSC numbers based on the capturerecapture method (194) revealed high numbers of active partner-derived cHSCs that were present in the recipient mice (Figure $4 f$ ). This mathematical model used for HSC estimation, is able to estimate the overall population size, by exploiting repeated sampling of marked elements over time, in this case short-lived myeloid cells (as a reflection of true HSCs), and accounting for the number of shared elements among samplings (195).

Figure 4. Multiple clones of cHSC repopulate remote hematopoietic niches (next page). (a) Experimental design to study clonal dissemination of HSC in parabionts. Lin ${ }^{\mathrm{NEG} G F P+}$ cells transduced with the lentiviral vector were transplanted into lethally irradiated mice, which were then set in parabiosis with partially irradiated (6Gy) mice. Parabionts were separated after 4 weeks, and insertion sites determined in myeloid and lymphoid cells to estimate the number of hematopoietic clones circulating between the parabionts. (b) Sorting strategy used for the isolation of partner derived $\mathrm{GFP}^{+}$myeloid and lymphoid cells. c) Quantification of the percentage of partner-derived cells within the myeloid and lymphoid lineage along time. (d) Table indicating transduction efficiencies (given as vector copy number or VCN) for each parabiotic donor mouse in BM and spleen (e) Representative heatmap showing the abundance of shared integration sites (IS; a measure of clonal diversity) over time in both CD11b myeloid and CD19 B-lymphoid lineages, compared with the donor BM in a parabiotic pair. ( $f$ ) Number of active HSC in each parabiotic pair, estimated by capture-recapture modelling. 

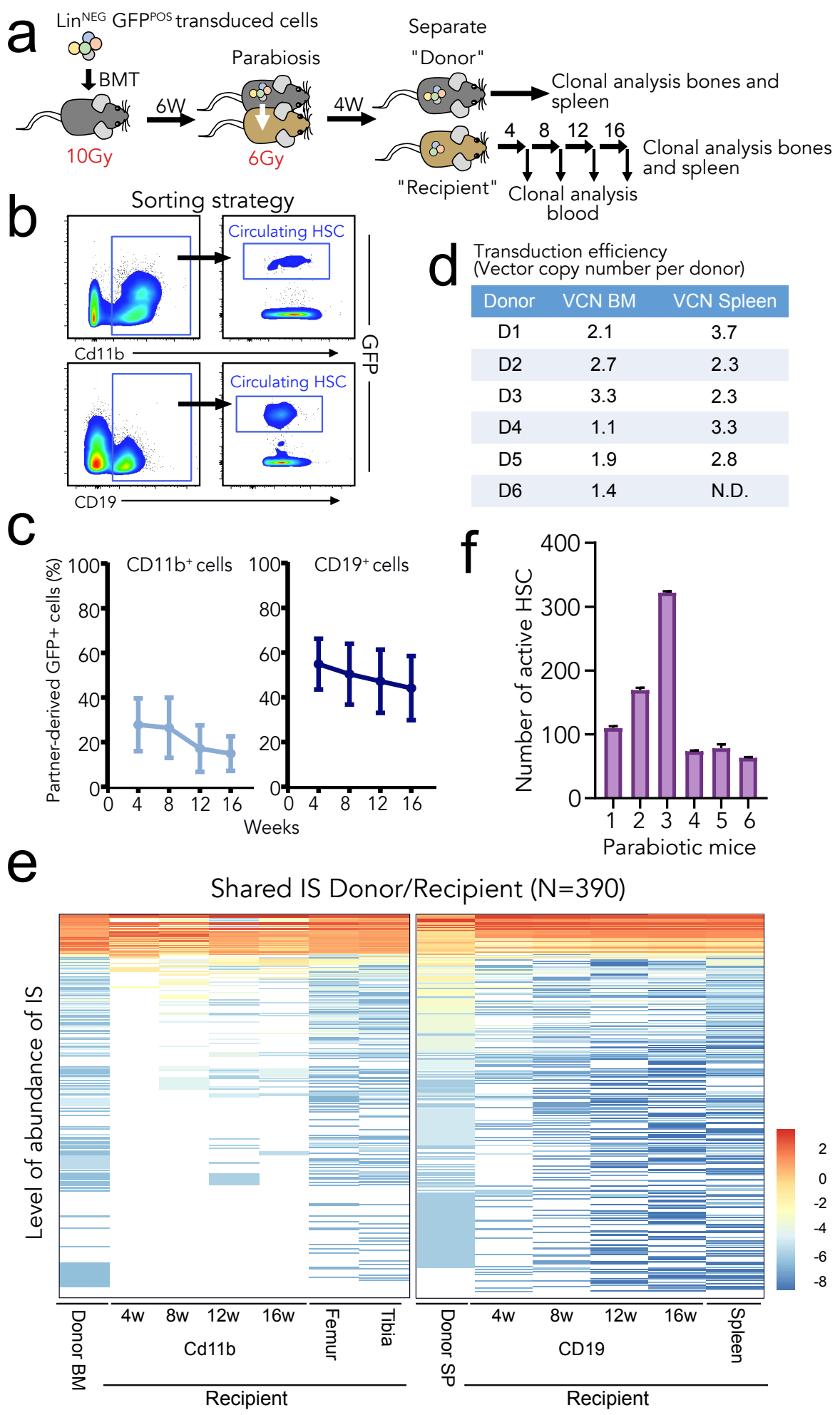

Using the Shannon diversity index (h-index), which considers both the absolute number and the relative abundance of each integration site (IS) in defined cell subsets (195), we confirmed that the clonal complexity in recipient mice was comparable to that found in the donor mice, and was only slightly lower than that of the long-lived B lymphoid compartment (Figure 5a,b). This finding 
suggested that cHSCs crossing between parabionts reflected the clonal composition of the donor HSC pool. Finally, streamgraph analyses revealed that the cHSC clones that reconstituted the partner mouse were long-lived, as they were detectable for at least 16 weeks (Figure $5 c$ ). These data thus suggested that a large fraction of the HSC pool is normally released into the bloodstream to surveil for, and repopulate, damaged niches.

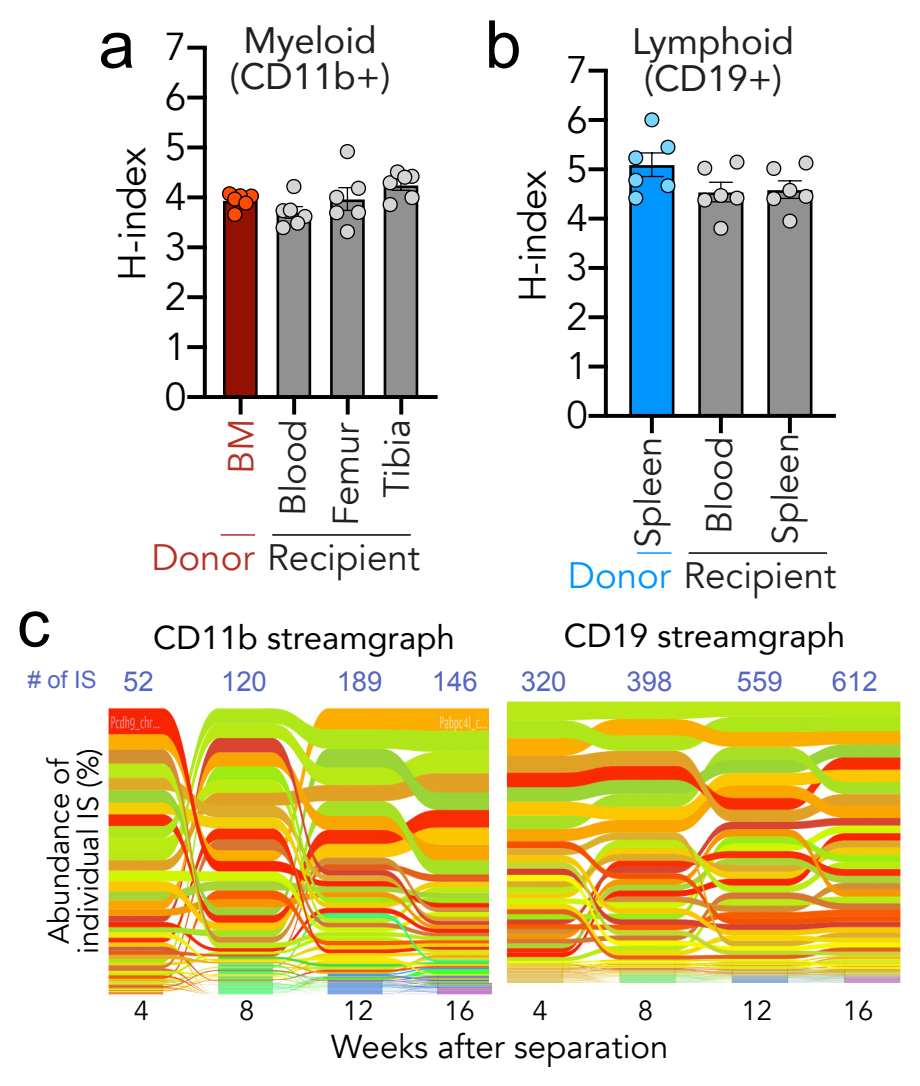

Figure 5. cHSCs reflect the clonal composition of the donor HSCs. (a) H-indexes reflecting the clonal complexity of the donor BM, myeloid and lymphoid (b) compartments, as well as in blood, the indicated bones and the spleen of the recipient partners; $n=6$ parabiotic pairs. (c) Representative streamgraphs showing the abundance of each individual IS within the myeloid (left panel) and lymphoid lineage (right panel) of a representative recipient parabiont, at the indicated time points after parabiosis separation. Numbers on the top indicate the total number of unique IS found at each time point.

\section{Characterization of cHSCs}

Given the long-lived and efficient replacement of damaged haematopoiesis by cHSCs found in our experiments, we asked whether cHSCs had distinctive features compared with the bulk of medullary-resident HSCs. For this purpose, 
we established parabiotic pairs of CD45.1 with sublethally irradiated (6Gy) CD45.2 mice and performed transcriptomics analyses of Lineage ${ }^{\mathrm{NEG}} \mathrm{Sca}-1^{+} \mathrm{cKit}^{+}$ (LSK) cells (that contain all the immature hematopoietic fraction). We isolated three populations of LSK cells: firstly, CD45.1 ${ }^{+}$cells from the donor marrow that we referred to as "endogenous", secondly, CD45.2+ that have survive the irradiation referred to as "irradiated" and finally, CD45.1+ partner-derived cells that have travelled to the irradiated marrow, referred to as "circulating" LSK, isolated from the recipient's BM (Figure 6a). It is important to highlight that we isolated circulating HSCs that engrafted the irradiated marrow, an approach that we took due to the low abundance of circulating HSCs found in peripheral blood at any time. Upon the 14461 genes identified, 375 genes were differentially expressed among the three groups (Figure 6b). Comparison of these genes among groups identified a group of genes that were specifically upregulated in circulating HSCs (Figure 6b, cluster 2 in green). Interestingly, this set of genes revealed a prominent myeloid signature, including granulocytic genes such as Mpo, Elane or Ctsg and monocytic genes such as Ly6C and Ccr2 (Figure 6b). The gene signature profile also showed that cHSCs enter cell cycle, although this feature was shared with the "irradiated" population (Figure 6b, pink), suggesting that proliferation is driven by the recipient irradiated BM microenvironment (Figure 6b, cluster 1). Both signatures were confirmed by flow cytometry analyses in the same experimental settings (Figure 6c). The transcriptomic analyses also showed loss of hematopoietic potential (Figure 6b, cluster 5), suggesting that cHSCs might lose stemness as they leave their native medullary niches. 


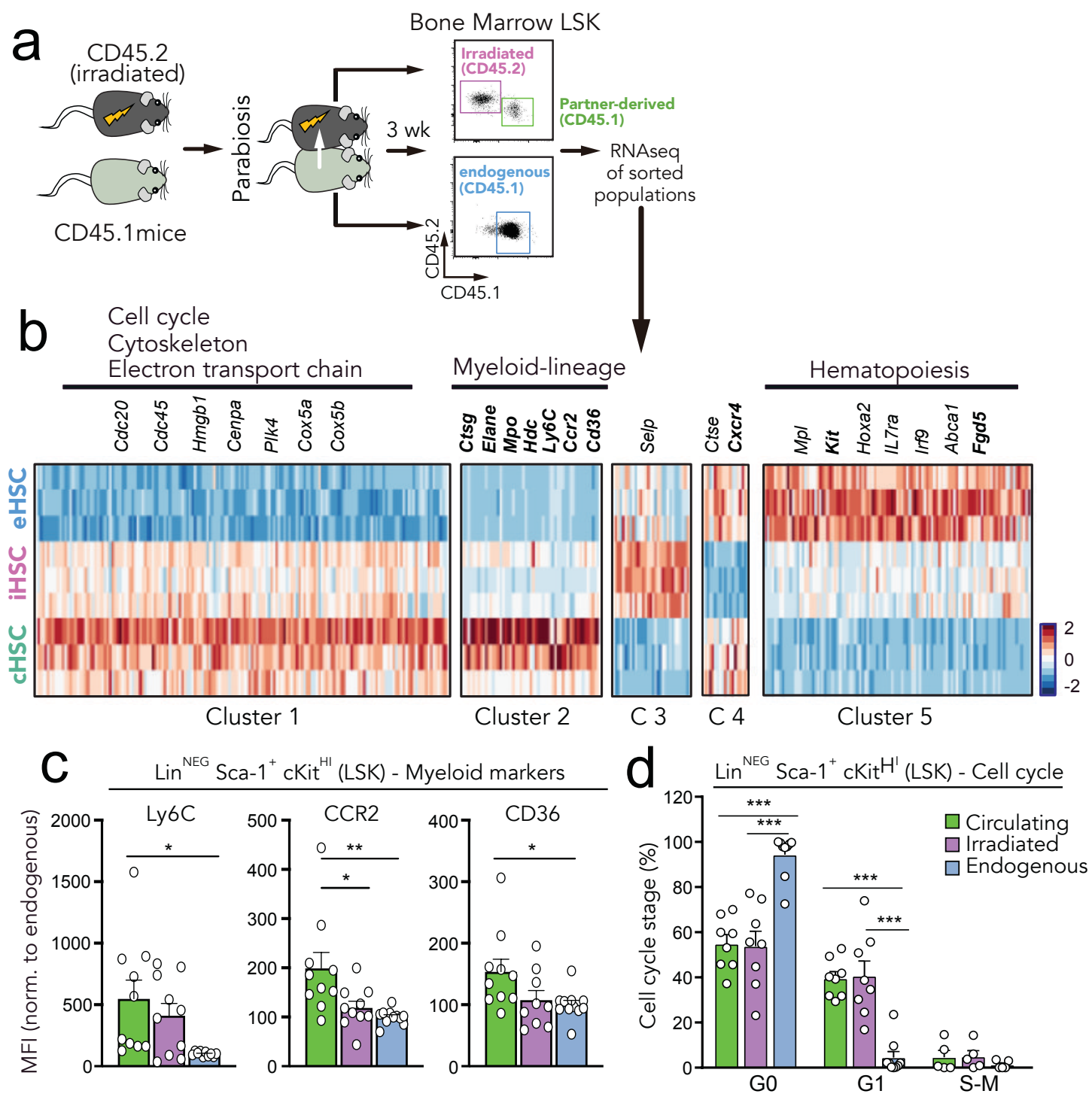

Figure 6. Transcriptomic profiling of cHSC. (a) Experimental design to isolate circulating HSC from the BM of parabiotic mice, in which circulating HSC were CD45.2 (green), residual HSC from the irradiated host were CD45.1 (pink) and endogenous HSC were also CD45.1 but extracted from the "donor" marrow (blue). Bulk RNA-seq was performed on these three populations. (b) Heatmap of the differentially expressed genes (DEGs) between the three different groups: circulating HSC (cHSC, green), irradiated HSC (iHSC, pink) and endogenous HSC (eHSC, blue). DEGs were grouped in 5 different clusters. (c) Expression profile (mean fluorescence intensity, MFI) of Ly6C, CCR2 and CD36 in the indicated LSK cells normalized to endogenous LSK (MFI = 100), as measured by flow cytometry; $n=9-11$ mice. (d) Cell cycle analysis of circulating, irradiated and endogenous LSK cells from mice that have been in parabiosis for 3 weeks; $\mathrm{n}=9$ mice. Data shown as mean \pm SEM. * $\mathrm{p}<0.05 ; \mathrm{p}^{\star \star}<0.001 ; \mathrm{p}^{\star \star \star}<0.0001$, as determined by one-way ANOVA comparing all groups (c), or 2-way ANOVA with Bonferroni post-test comparing all groups to endogenous control group. 
To functionally validate if this was the case, i.e. that cHSC lose stem properties, we used a competitive setting in which BM cells collected from our parabiotic system (without irradiation) were serially transplanted into lethally irradiated recipients (Figure 7a). Under non-myeloablative conditions, the BM of parabionts contain around $5 \%$ of partner-derived progenitors. Deviations from this frequency in recipient mice allowed us to compare the reconstituting potential of cHSC vs. marrow-resident HSC. We found a consistent reduction in all blood lineages and BM progenitors derived from the partner in primary recipient mice and complete depletion in secondary recipient mice (Figure 7a).

To control for the low starting amount of donor BM cells, we performed a control BM transplant in which progenitors from BM donors were mixed at similar ratios to those in the parabiosis system (5-95\%) (Figure 7b). In this system, we observed that the frequency and multilineage reconstitution was preserved over time both in primary and secondary recipients (Figure 7b) suggesting that the disappearance of cHSC in BMT is not due to the low starting numbers but to the quality of the cells. Thus, cHSC are unique in their capacity to traffic to distant hematopoietic niches and to rescue long-term haematopoiesis, but preferentially commit to the myeloid lineage and are outcompeted by marrowresident HSC.

Figure 7. cHSC are outcompeted by medullary HSC (next page). (a) Top, experimental scheme used for primary transplantation. Donor BM cells from parabiotic mice containing approximately 5\% of circulating HSPC (green in graph at the top) and 95\% of endogenous HSPC (grey) were transplanted into primary lethally irradiated recipient mice $(n=16)$ and engraftment was measured for 16 weeks. Note that the contribution from partner-derived HSC decreased over time and disappears fully upon secondary transplantation (lower panel), n=14 mice. (b) To control for the low fraction of partnerderived HSC in the experiment in (a), we transplanted a mix of BM cells from nonparabiotic mice at a 5:95 ratio into lethally irradiated primary and secondary recipient mice; $n=9$ mice. Note that in this case, engraftment from the $5 \%$ donor (red) was sustained even in the secondary recipients; $\mathrm{n}=10$ mice. 
a

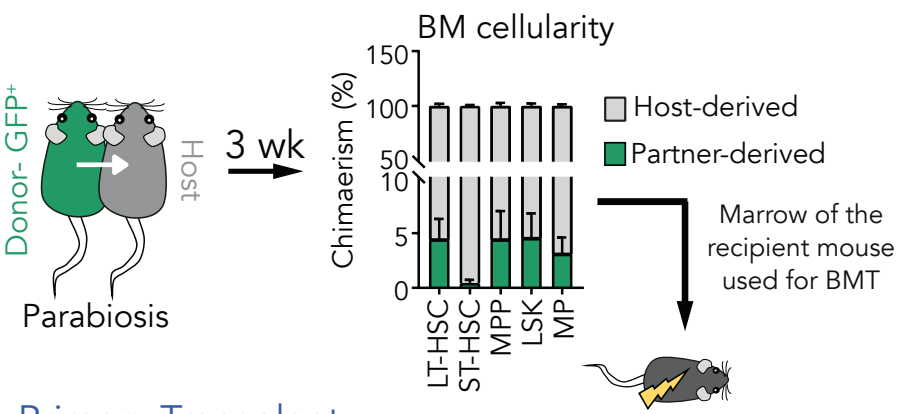

Primary Transplant
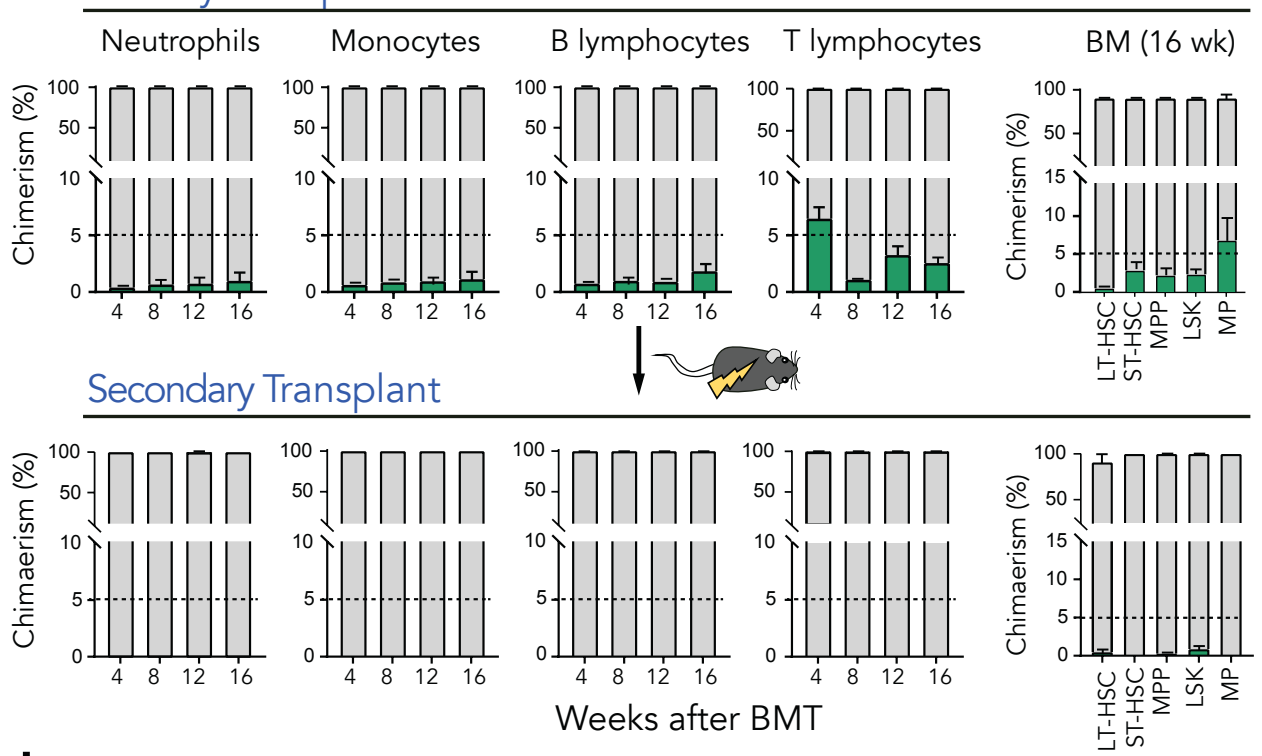

b Control group (no parabiosis)

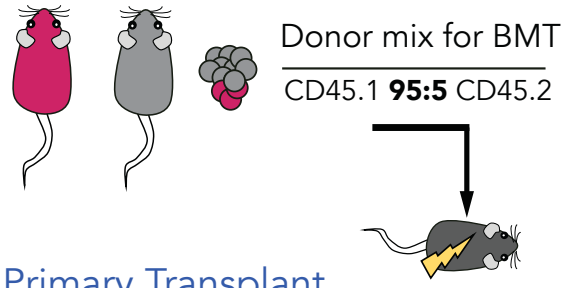

Primary Transplant
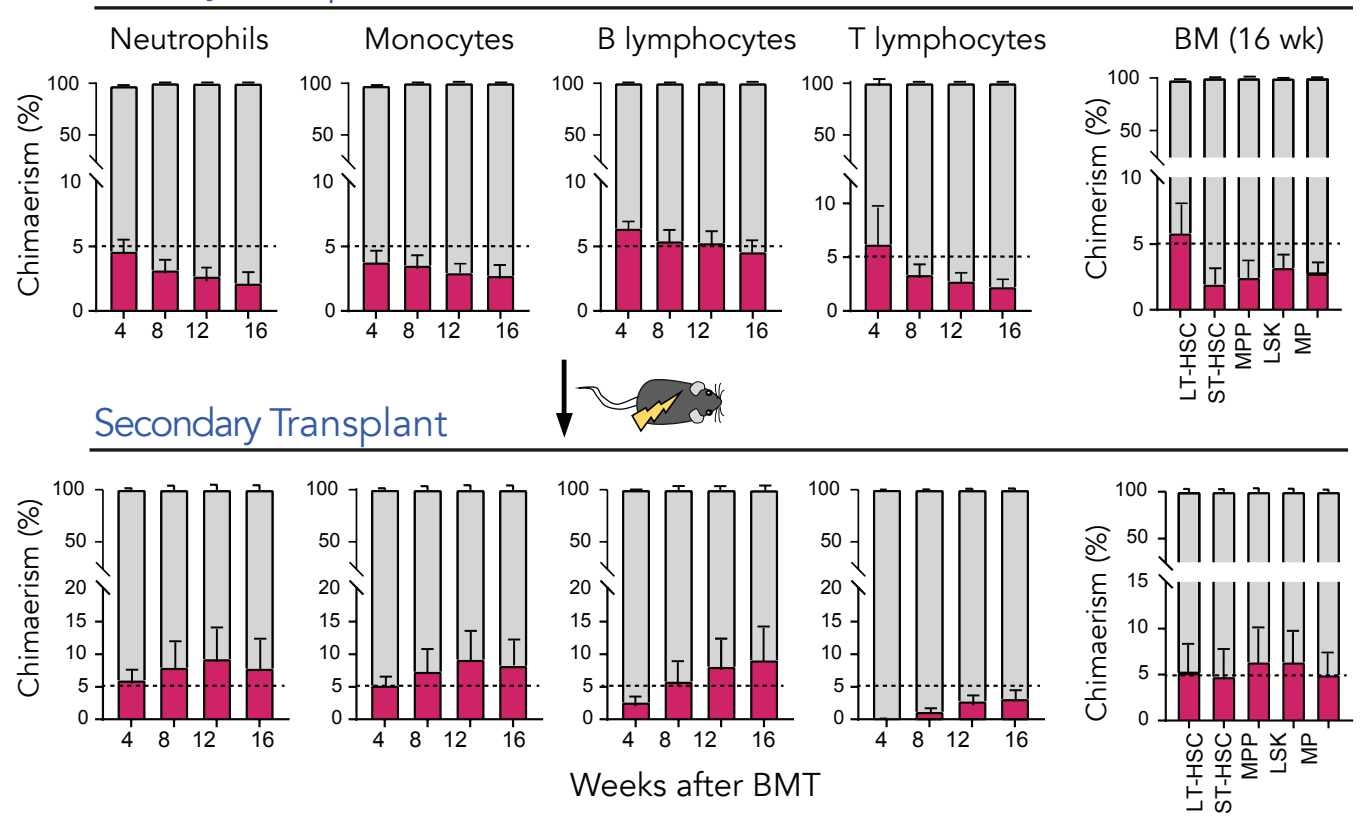


\section{CXCR2 is functional on $\mathrm{CHSC}$ and mediates their egress from the $\mathrm{BM}$}

We next sought to identify the relevant receptor in HSC that could be mediating the egress of cHSC in the steady state. As leukocyte trafficking is mediated through chemokine receptors, we first carried out an unbiased search in public databases (http://www.immgen.org/) for chemokine receptor(s) whose expression was specifically high in HSCs (Figure 8a). We found that some receptors had increased expression as HSCs differentiated along the myeloid lineage (Ccr2, $\mathrm{Cxcr} 3$, or $\mathrm{Cxc} 3 \mathrm{cr} 1)$ (Figure 8b), other were expressed at low levels in all stages (Ccr1, Ccr8 or $\mathrm{Cxcr5})$, and only one receptor was always expressed at high levels (Cxcr4) (Figure 8b). Interestingly, Cxcr2 was the only receptor preferentially expressed in the most primitive compartment (Figure 8a-b). Given this pattern of expression and the fact that CXCR2 mediates the egress of some leukocytes from the marrow $(196,197)$, we hypothesized that CXCR2 could be a key regulator of HSC trafficking.

In our own experiments, using cytometric analyses we found low, but detectable, surface levels of the receptor on all hematopoietic progenitors tested, from LTHSCs to myeloid progenitors (MP) in WT mice (Figure 8c). We took advantage of the germline CXCR2 deficient $\left(\mathrm{CXCr}^{-/-}\right)$mice (198) available in our laboratory

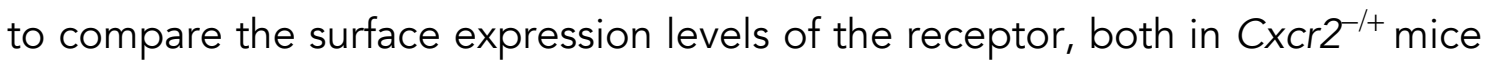
(contains one copy of the receptor, herein referred to as CXCR2 ${ }^{\text {HET }}$ mice) and $\mathrm{CxCr}^{-/-}$mice (referred to as CXCR2 ${ }^{\mathrm{KO}}$ mice, which lack both copies of the gene) to WT levels. Cytometric analyses indicated very low levels of CXCR2 in CXCR2 ${ }^{\mathrm{HET}}$ mice and an absence in CXCR2 ${ }^{\mathrm{KO}}$ mice in HSPCs (Figure 9a) and $\mathrm{Lin}^{+}$cells, which contain in great majority neutrophils (Figure 9b).

Figure 8. CXCR2 expression in HSPC and myeloid populations (next page). (a) Heatmap showing scaled expression of chemokine receptors in hematopoietic progenitors. (b) Normalized gene expression of chemokine receptors in the indicated populations. $\mathrm{Cxcr} 2$ expression, highlighted in red, is highest in primitive HSCs and $\mathrm{Cxcr} 4$, highlighted in orange, is higher in all HSPC populations. (c) CXCR2 protein levels (MFI) measured by flow cytometry in progenitor subsets (left) and in lineage+ cells (enriched in neutrophils; right) in WT mice; $n=11$ mice. Data shown as box and whiskers (c). 

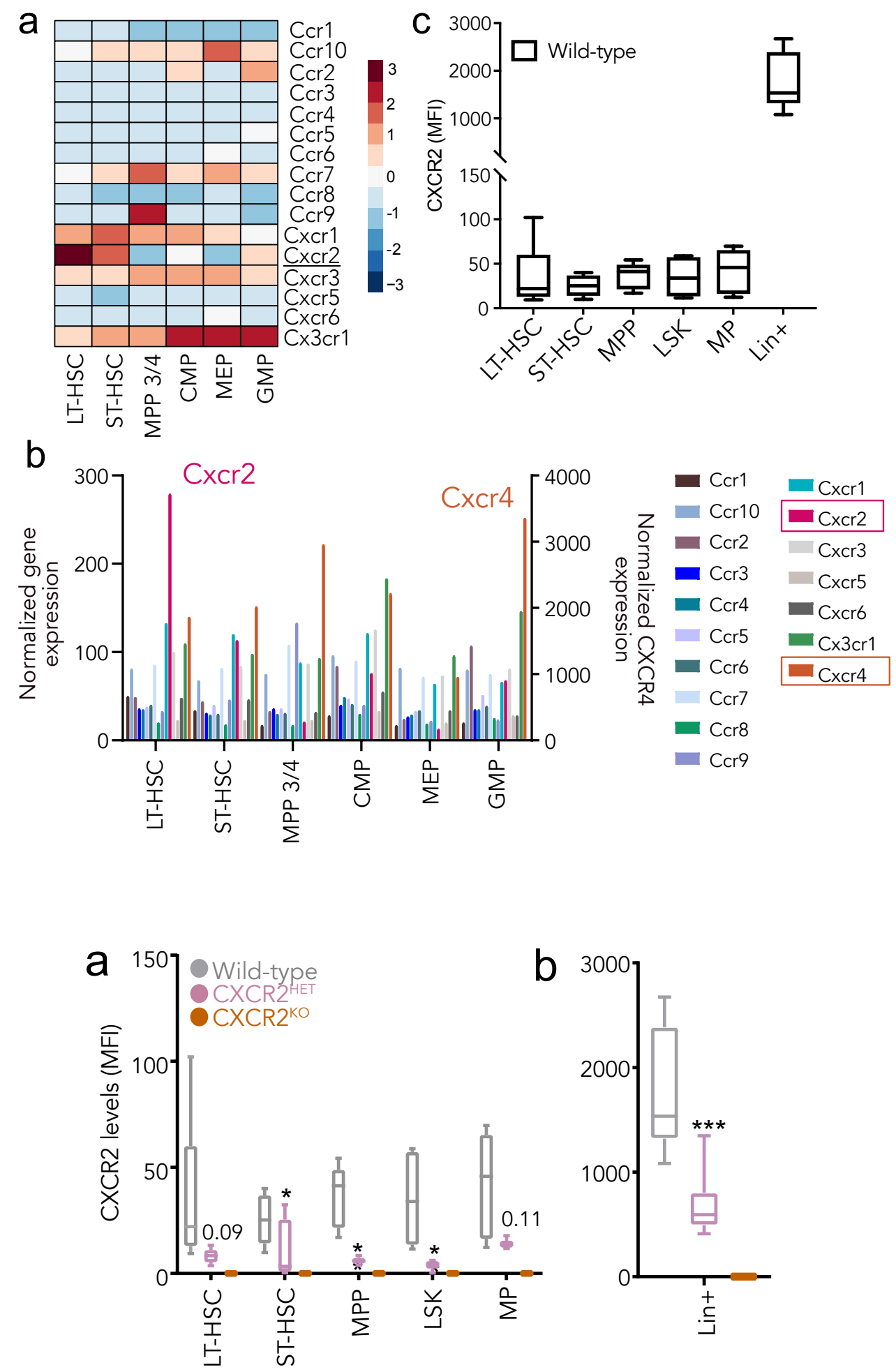

Figure 9. CXCR2 surface expression in HSPCs and mature cells. (a) CXCR2 protein levels (MFI) measured by flow cytometry in the surface of WT, CXCR2 ${ }^{\mathrm{HET}}$ and CXCR2 ${ }^{\mathrm{KO}} \mathrm{HSPC}$ subsets and (b) mature lineage ${ }^{+}$cells (enriched in neutrophils). The MFI values of CXCR2 ${ }^{\mathrm{KO}}$ were subtracted from WT and CXCR2 ${ }^{\mathrm{HET}}$ cells; $\mathrm{n}=3-13$ mice per group. Data shown as box and whiskers. ${ }^{\star} p<0.05 ;{ }^{\star \star} p<0.01 ;{ }^{\star \star *} p<0.001$ as determined by one-way ANOVA with Bonferroni post-test. 
To test if CXCR2 was functional on HSPCs, we first performed ex vivo transwell migration assays. BM mononuclear cells from either WT, CXCR2 ${ }^{\text {HET }}$ or CXCR2 ${ }^{\mathrm{KO}}$ mice were allowed to migrate through $5 \mu \mathrm{m}$-pore transwells towards CXCL1, or towards CXCL12 as a positive control, and RPMI medium as a negative control (Figure 10a). Transmigrated cells were collected after $2 \mathrm{~h}$ and either, plated for clonogenic cultures in a CFU-C assay, analysed by flow cytometry to count migrated neutrophils for reference, or transplanted into irradiated recipient mice to test the ability of migrated HSPC to engraft irradiated recipients (Figure 10a). BM cells in WT mice were capable of migrating towards CXCL1 and had the ability to form clonogenic colonies in culture (CFU-C), indicating that progenitors were migrating (Figure 10b, left panel, grey column). This migration depended on CXCR2, as CXCR2 ${ }^{\mathrm{HET}}$ and $\mathrm{CXCR} 2^{\mathrm{KO}}$ mice presented impaired migration towards CXCL1 and thus impaired formation of colonies, whereas migration to CXCL12 remained unaffected (Figure 10a-b). We also analysed neutrophil migration towards CXCL1 as a reference and observed that migration was completely blunted in CXCR2 ${ }^{\mathrm{KO}}$ mice compared to WT, although it remained almost normal in CXCR2 ${ }^{\text {HET }}$ mice (Figure 10c). More importantly, transmigrated cells were able to reconstitute, at low but detectable levels, hematopoiesis in lethally irradiated mice for 16 weeks, indicating that functional LT-HSCs were migrating to $\mathrm{CXCL1}$. In CXCR2 ${ }^{\mathrm{KO}}$ mice $\mathrm{BM}$ reconstitution was lost indicating again specificity of the receptor (Figure 10d). Altogether, these results suggested us that CXCR2 could be the receptor guiding the homeostatic egress of HSC, and we predicted that its deficiency would cause severe reductions in cHSCs. 

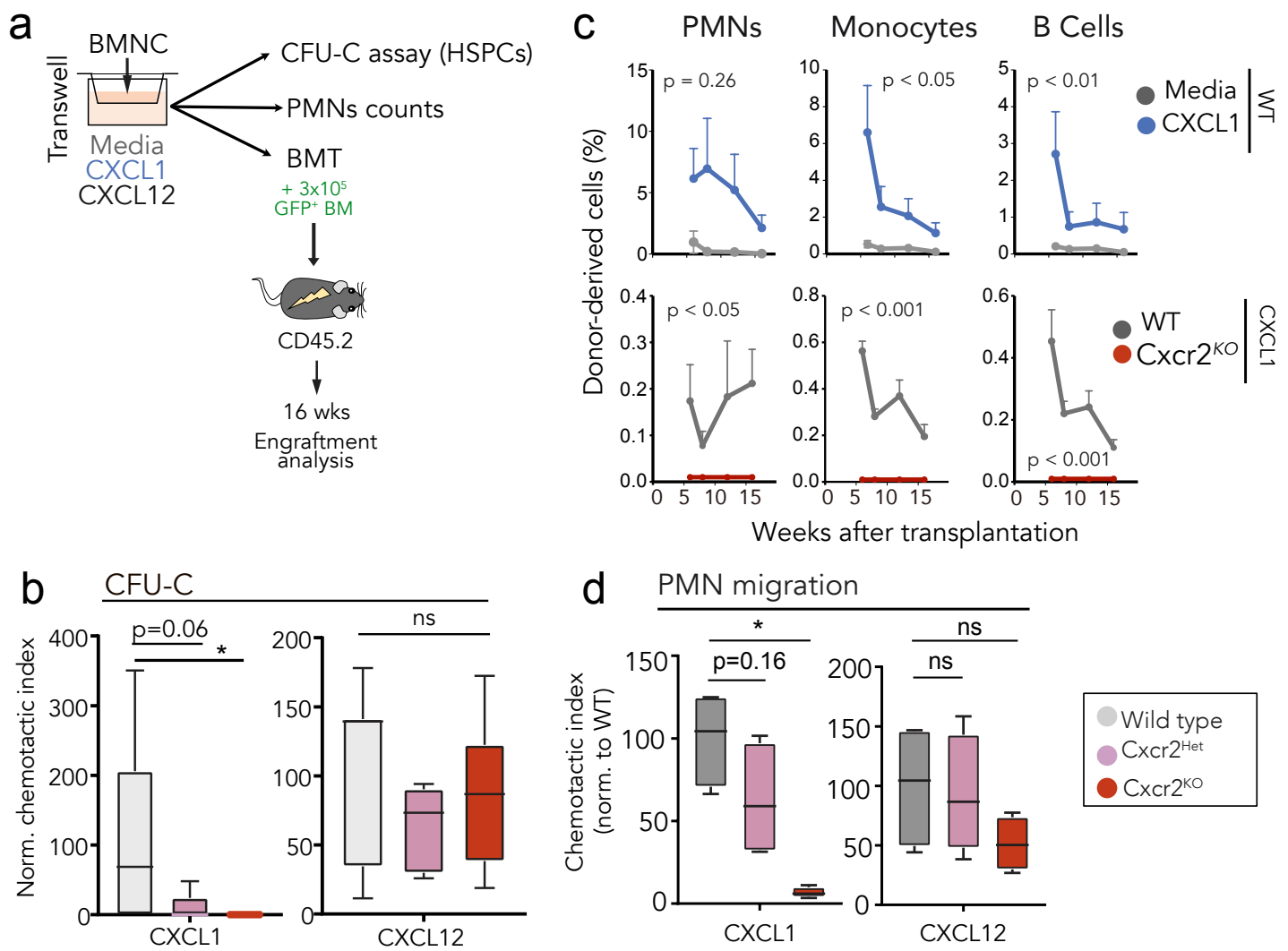

Figure 10. CXCR2 mediates HSPC migration. (a) Experimental setup to test the function of BM cells that have migrated to CXCL1 in vitro. Transmigrated BM cells from WT, Cxcr2 ${ }^{\mathrm{HET}}$ or $\mathrm{Cxcr} 2^{\mathrm{KO}}$ mice were collected for CFU-C assay, PMN counts or BM transplantation (together with $3 \times 10^{5}$ helper $\mathrm{GFP}^{+} \mathrm{BM}$ cells) into lethally irradiated CD45.2 mice, and long-term engraftment was analysed for 16 weeks. (b) In vitro chemotaxis of BM progenitors from WT, Cxcr2 ${ }^{\mathrm{HET}}$ or $\mathrm{Cxcr} 2^{\mathrm{KO}}$ mice, measured as CFU-C, towards CXCL1 (left) or CXCL12 (right). Values are normalized to WT cells; $n=6-9$ mice. (c) Migration efficiency of WT, $\mathrm{Cxcr}^{+/-}$and $\mathrm{CxCr}^{-/-}$mice neutrophils towards $\mathrm{CXCL} 1$ (left) or CXCL12 (right) in in vitro assays (normalized to WT cells); $n=4$ mice per group and per condition. (d) Long-term chimerism in myeloid (neutrophils and monocytes) and lymphoid (B lymphocytes) cells. Although low, reconstitution was specific because WT cells migrating to media alone, or $\mathrm{CxCr}^{\mathrm{KO}}$ cells migrating to $\mathrm{CXCL} 1$, yielded no engraftment; $n=3-4$ mice per group. Data are shown as mean \pm SEM (d) or as box and whiskers (b and $c)$. ${ }^{\star} p<0.05 ;{ }^{*} p<0.01 ;{ }^{\star \star *} p<0.001$; ns, not significant, as determined by one-way ANOVA (b and c) or two-way ANOVA (d).

We then analysed CFU-Cs in CXCR2 ${ }^{\mathrm{KO}}$ mice. We hypothesized that if CXCR2 was necessary for the release of HSCs into periphery, CXCR2 ${ }^{\mathrm{KO}}$ mice would present reduced levels of HSCs in peripheral tissues. Surprisingly, however, CXCR2 ${ }^{\mathrm{KO}}$ mice presented an increase rather than a decrease in the number of CFU-C in blood, spleen, liver and BM compared with WT cells (Figure 11a). Unexpectedly, CXCR2 ${ }^{\mathrm{HET}}$ mice presented low levels of progenitors in periphery; blood, spleen 
and liver, whereas the BM remained normal (Figure 11a). The phenotype observed in the CXCR2 ${ }^{\mathrm{KO}}$ mice has been typically seen in mice with defective neutrophil migration (199). This provokes an increase in the levels of G-CSF in plasma that causes increased mobilization of HSCs and increased myelopoiesis $(196,199)$. Consistent with this possibility, CXCR2 ${ }^{\mathrm{KO}}$ mice presented higher levels of G-CSF in plasma (Figure 11b). To confirm that the increase in the number of progenitors was indeed due to defective neutrophil migration, we generated and analysed progenitors in $\mathrm{CxCr}^{\mathrm{ff} / \mathrm{fl}}$; Mrp8 ${ }^{\mathrm{CRE}}$ mice which specifically lack $\mathrm{Cxcr2}$ in neutrophils. As predicted, we found similar elevations of CFU-C progenitors in blood and spleen (Figure 11c), implying that the unexpected increase of progenitors in CXCR2 ${ }^{\mathrm{KO}}$ mice was indirectly caused by trafficking impairment of neutrophils.
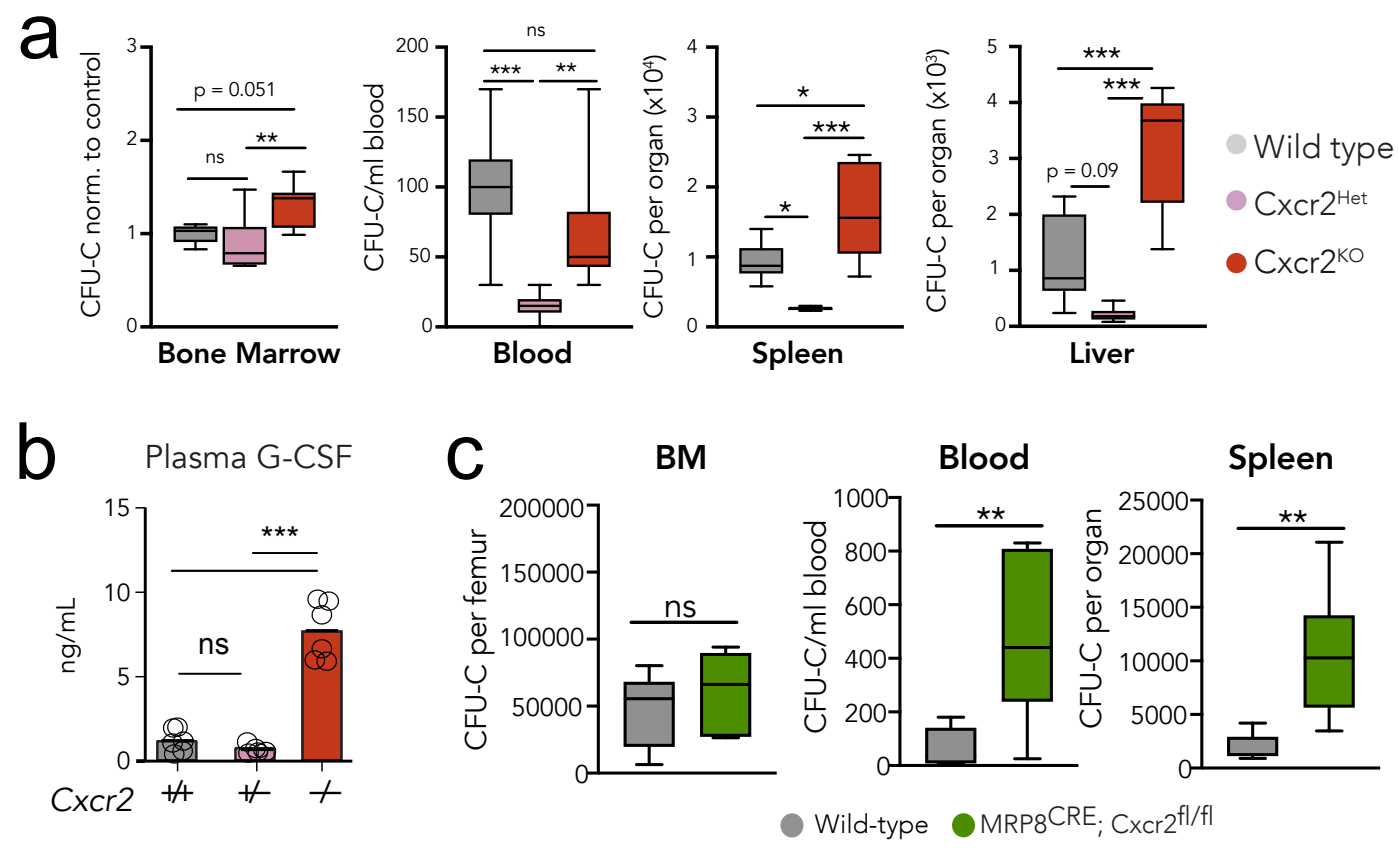

Figure 11. CXCR2 ${ }^{\mathrm{KO}}$ mice create inflammatory signals due to impaired neutrophil migration. (a) Number of CFU-C in BM, peripheral blood, spleen and liver of WT, $\mathrm{CxCr}^{+/-}$and $\mathrm{CxCr2} 2^{--}$mice (values are normalized to WT group in BM); $\mathrm{n}=6-7$ mice per organ. (b) Levels of G-CSF in plasma of WT, $\mathrm{CxCr}^{+/-}$and $\mathrm{Cxcr}^{-/-}$mice; $\mathrm{n}=6$ per genotype. (c) Number of CFU-C in BM, peripheral blood and spleen of WT and MRP8CRE; $\mathrm{Cxcr2}^{\mathrm{f} / \mathrm{f}}$ mice; $\mathrm{n}=6-8$ mice per organ. Data are shown as mean $\pm \mathrm{SEM}(\mathrm{b})$ or as box and whiskers $(a, c) .{ }^{*} p<0.05 ;{ }^{\star \star} p<0.01 ;{ }^{* \star} p<0.001$; ns, not significant, as determined by one-way ANOVA ( $a, b$ and $c)$.

Using CXCR2 ${ }^{\mathrm{HET}}$ mice we have thus discovered that CXCR2 signalling in hematopoietic progenitors mediates the homeostatic egress of cHSCs into the 
periphery (blood and other peripheral organs). We thus reasoned that CXCR2 ${ }^{\mathrm{HET}}$ mice might provide a suitable model to study the biology of $\mathrm{cHSC}$ without altering neither the trafficking of mature leukocytes nor the haematological parameters in blood or BM (Figure 12a-b).

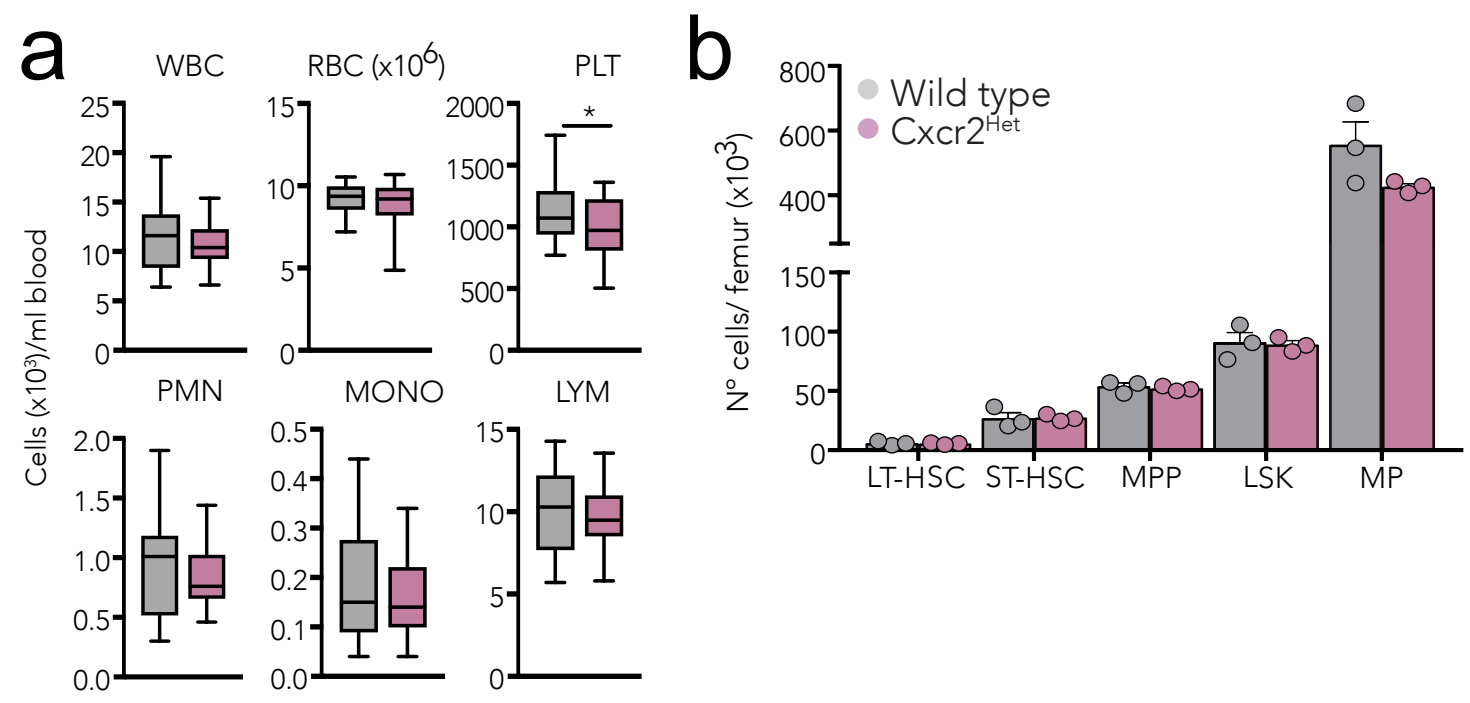

Figure 12. CXCR2 ${ }^{\mathrm{HET}}$ mice present normal haematopoiesis. (a) Peripheral blood counts in WT and CXCR2 ${ }^{\text {HET }}$ mice; $n=6-7$ mice. (b) Total number of the indicated progenitors in the BM of WT and CXCR2 ${ }^{\text {HET }}$ mice; $n=3$ mice. Data are shown as mean \pm SEM (b) or as box and whiskers (a). ${ }^{\star} p<0.05 ;{ }^{* *} p<0.01 ;{ }^{* \star *} p<0.001$; ns, not significant, as determined by unpaired t-test.

To further determine if the reduction in progenitors found in circulation of CXCR2 ${ }^{\text {HET }}$ mice translated in reductions in functional $\mathrm{CHSC}$, we first performed parabiosis experiments with WT and CXCR2 ${ }^{\text {HET }}$ mice. We followed our previous strategy, in which partner mice received different doses of irradiation to create different degrees of hematopoietic damage and HSC ablation. Partner-derived haematopoiesis derived from either WT or CXCR2 ${ }^{\mathrm{HET}}$ mice in the irradiated mice was analysed in peripheral blood and in BM at the end of the experiment (16 weeks). Remarkably, in partially irradiated partners (4Gy) we found major reductions in all leukocyte lineages derived from CXCR2 ${ }^{\mathrm{HET}}$ partners compared with WT controls (Figure 13a-b). In contrast, lethal irradiation (8Gy) or no irradiation (OGy) resulted in similar contributions of CXCR2 ${ }^{\text {HET }}$ mice to the partner's haematopoiesis (Figure 13b). Analysis of the BM at 16 weeks after separation of the parabionts confirmed the chimerism in blood in both WT and CXCR2 ${ }^{\text {HET }}$ (Figure 13c). Thus, cHSCs driven by CXCR2 are important for the repopulation of remote damaged niches. To confirm that the defective 
reconstitution of $\mathrm{CXCR} 2{ }^{\mathrm{HET}}$ progenitors in the parabiotic partners was due to the impaired HSC egress from the BM and not a defect in homing into the irradiated marrow, we performed homing experiments in which BM cells from WT or CXCR2 ${ }^{\text {HET }}$ mice were i.v. injected into WT mice. We found the same amount of WT and CXCR2 ${ }^{\text {HET }}$ cells $16 \mathrm{~h}$ after injection, indicating that Cxcr2 haploinsufficiency did not affect the entrance into the BM (Figure 13d).

Altogether, these results indicate that CXCR2 is required for the homeostatic egress of cHSCs into blood, without affecting homing back to the marrow, and that the few cHSCs in the blood of $\mathrm{CxCr}^{+/-}$mice are sufficient to regenerate haematopoiesis, only in the absence of competing HSC, indicating that "vacant" niches are needed for engraftment by cHSCs.
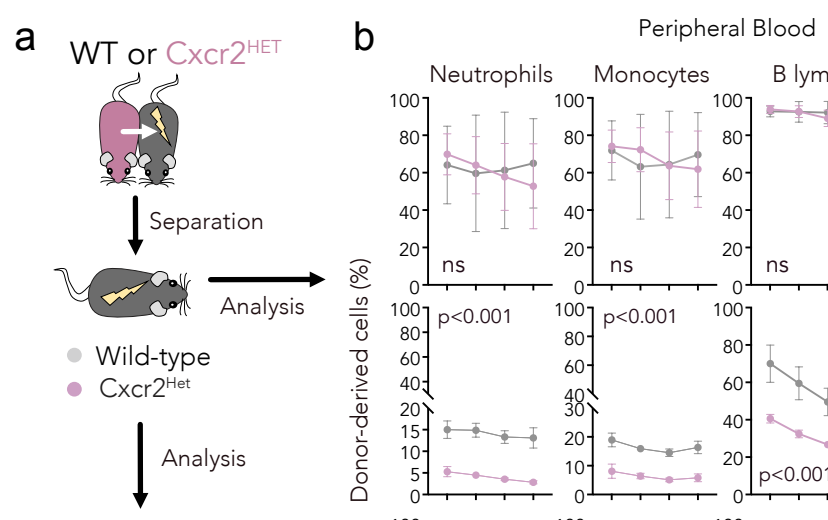

C Bone Marrow (16 wks)
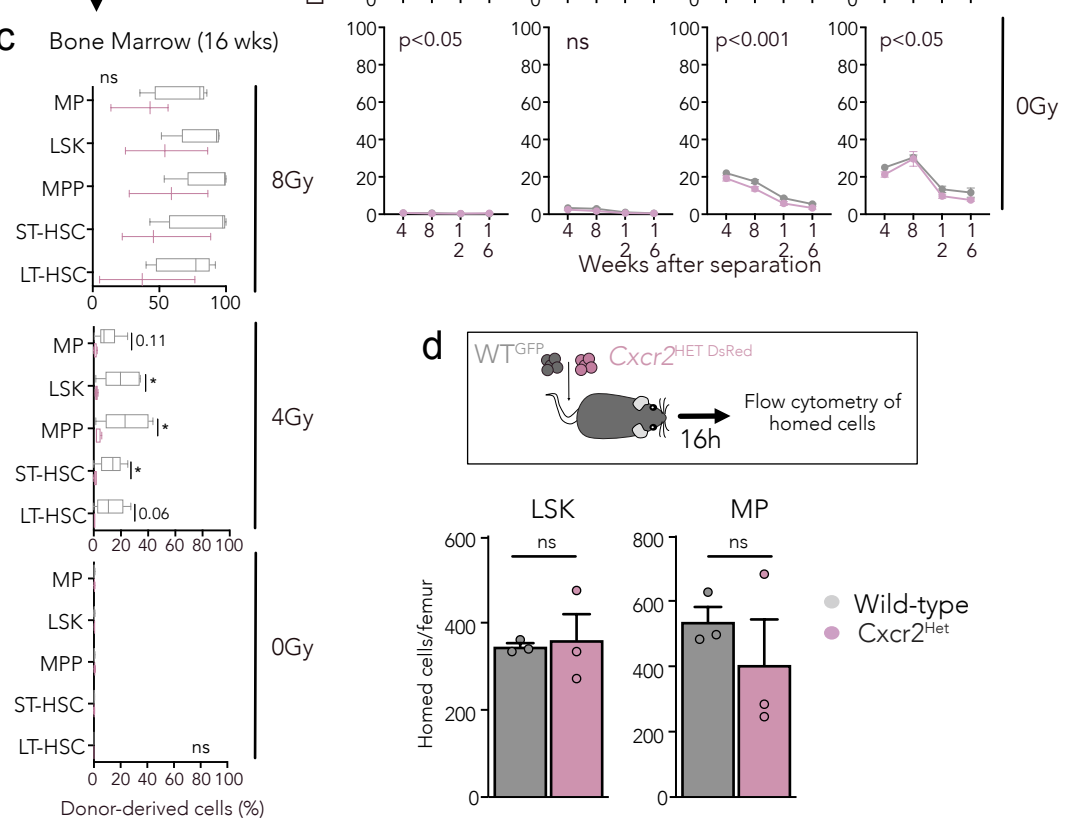

Figure 13. CXCR2 mediates HSC egress. (a) Experimental scheme to assess the reconstitution of irradiated partner mice after parabiosis for 4 weeks with either WT or CXCR2 ${ }^{\mathrm{HET}}$ mice. Mice were separated and blood from the irradiated partners was 
analysed for 16 weeks together with the BM. (b) Percentage of donor-derived leukocytes in the irradiated mice, which had been treated with the indicated doses of irradiation (8, 4, 0Gy); n=3-6 parabiotic pairs per dose. (c) Percentage of donor-derived progenitor cells in the BM of the irradiated partner at 16wk after separation of the parabionts; $n=3-6$ mice. (d) Experimental scheme of competitive homing experiments between WT and CXCR2 ${ }^{\mathrm{HET}}$ BM cells to the BM of WT recipient mice. The number of homed LSK and MP cells in the BM was estimated $16 \mathrm{~h}$ after injection, as shown in the bar graph; $n=3$ mice per group. Data are shown as mean \pm SEM (b and $d$ ) or as box and whiskers (c). ${ }^{\star} p<0.05 ;{ }^{\star \star} p<0.01 ;{ }^{\star \star \star} p<0.001$; ns, not significant, as determined by unpaired t-test (c-d) or two-way ANOVA (b).

\section{Expression and function of CXCL1 in the BM}

Our data show that cHSCs fail to reconstitute "closed", non-damaged niches and are outcompeted by medullary HSCs when these are present, but can efficiently long-term reconstitute distant, open-damaged niches. We also discovered that the chemokine receptor CXCR2 is important in homeostatic HSC egress. Therefore, to better understand the biology of cHSCs we next investigated the cues driving the homeostatic egress of HSCs from the BM, which are currently unknown. We reasoned that differential expression of chemokines, a family of small cytokines specialized in guiding cell migration, by cells strategically localized in the BM could drive HSCs out of the marrow, in a manner similar (but reversed) to how CXCL12 drives HSC homing inside the BM by binding to CXCR4 (43). Due to the importance of CXCR2 in HSC egress, we hypothesized that either CXCL1 or CXCL2, the main ligands of CXCR2, could be promoting steady-state HSC egress.

We therefore examined the expression of these chemokines in the native BM. We took advantage on the recently published single-cell dataset of the whole BM stroma transcriptome (190) (Figure 14a). Among all the niche components, we specifically focused on pericytes, as these localize around blood vessels and have been shown to regulate HSCs and leukocyte trafficking $(96,200,201)$. We found out that among all chemokines detected at the transcriptional level, only Cxcl1 and Cxcl12 were expressed at relatively high levels in pericytes (Figure 14b-d). Because CXCL12 mediates HSC retention $(91,92,114)$ rather than egress, we turned our attention to CXCL1. We observed that CxCl1 transcripts were present in multiple stromal populations such as MSCs, fibroblasts or ECs, although it was most prominent in the pericyte cluster (Cluster 12 in Figure 14a) compared to other chemokines, such as $\mathrm{Cxcl} 12$ or Ccl2 (Figure 14c), which were 
preferentially expressed by MSCs (Cluster 1 in Figure 14a). Incidentally, CXCL1 is known to induce the migration of neutrophils (202-204), a finding that could be possibly related to the strong granulocytic transcriptional profile of $\mathrm{cHSC}$ that we observed in our previously mentioned bulk RNA-seq (Figure 6b).
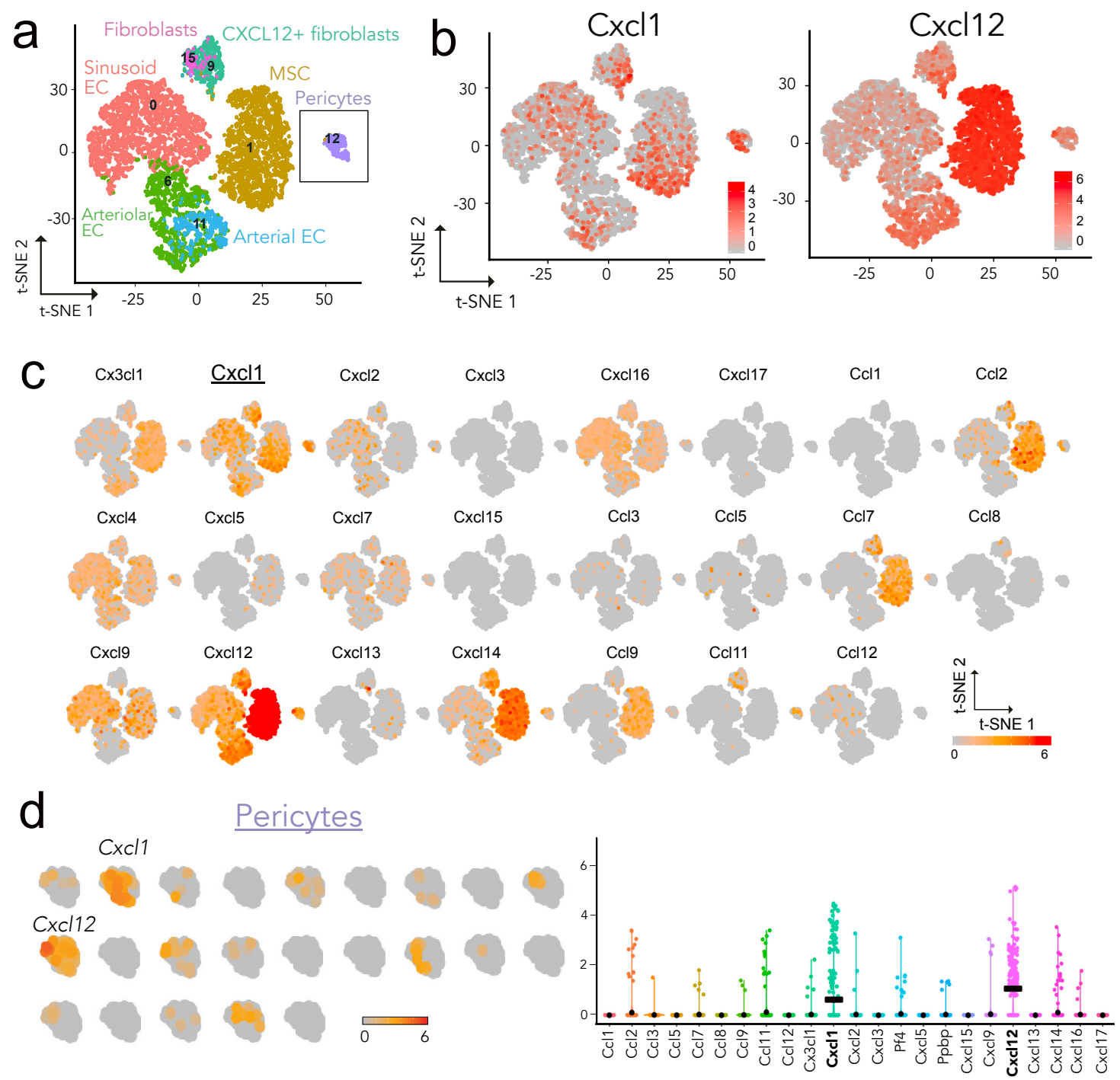

Figure 14. CXCL1 defines a population of perivascular mesenchymal cells. (a) t-SNE plot of the single-cell transcriptomics dataset of the BM stroma, from the previous published dataset (190). The pericyte cluster (box) was analysed for the expression of 23 different chemokines (c-d). MSC, mesenchymal stem cell; EC, endothelial cells. (b) t-SNE plots showing the expression levels of $\mathrm{Cxc} / 1$ and $\mathrm{Cxc} / 12$ in all clusters. (c) Transcriptional expression levels of different chemokines in the different clusters. Only Cxcl1 and Cxcl12 were expressed at relatively high levels in the pericyte cluster (Cluster 12). (d) Transcriptional expression levels of different chemokines in the pericyte cluster (upper panel) and violin plot showing the level of expression of each chemokine inside the pericyte cluster (Cluster 12) (bottom panel). 
To map the actual distribution of CXCL1-producing cells in the BM we generated a mouse encoding the Cre recombinase in exon 1 of the $\mathrm{CxCl} 1$ gene ( $\mathrm{CxC} / 1^{\mathrm{CRE}}$ ) and crossed these mice with the Rosa26 ${ }^{\text {TdTomato }}$ line to generate a CXCL1-reporter mouse, which we refer to as CXCL1 $1^{\text {TdTom }}$ mice (Figure 15a). To visualize CXCL1producing cells we first performed whole organ imaging of marrows from CXCL1 1dTom mice. Interestingly, CXCL1-producing cells were widely distributed across the whole medullary space (Figure 15b). We then examined histological thick BM sections stained with an anti-endomucin antibody, which stains for all types of blood vessels in the marrow, by high resolution imaging. We observed that CXCL1-TdTom ${ }^{+}$cells were mainly found around blood vessels (Figure 15c). Apart from the perivascular distribution, some of the Tomato ${ }^{+}$cells had an interstitial distribution with stroma-like morphologies (Figure 15d).
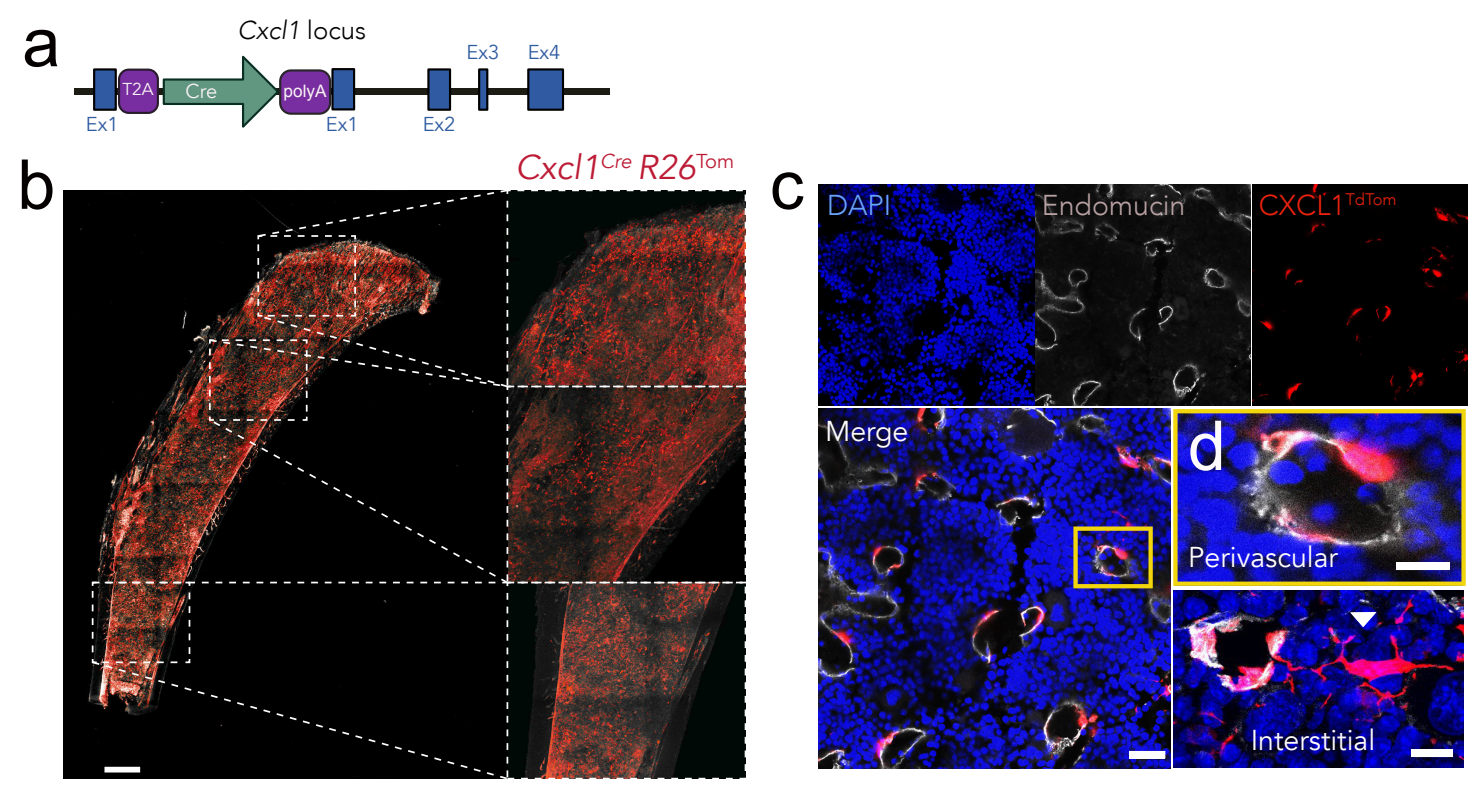

Figure 15. Generation and characterization of CXCL1 ${ }^{\text {TdTom }}$ mice. (a) Targeting strategy to introduce the Cre recombinase in exon 1 of the Cxcl1 locus. Closed boxes (blue) represent exons. (b) Whole-mount staining of a femur from a CXCL1 ${ }^{\text {TdTom }}$ mice $(\mathrm{Cxc} 11+$ cells in red). Images at right show enlarged areas of the proximal epiphysis, diaphysis and metaphysis with numerous Cxcl1+ cells. Scale bar, $500 \mu \mathrm{m}$. (c) Representative image of a femur from CXCL1 $1^{\text {TdTom }}$ mice, with CXCL1+ perivascular cells in red, vessels in grey (stained for endomucin) and nuclei in blue (DAPI). (d) Right lower panels show in more detail a perivascular (upper) and interstitial distribution (lower). Scale bars, $30 \mu \mathrm{m}$ and $10 \mu \mathrm{m}$ in lower panels.

We next compared the distribution of CXCL1 ${ }^{\text {TdTom }}$ perivascular cells with that of well-known niche cells labelled in reporter mice as Prx1-, Leptin-receptor (LepR), NG2- or CXCL12-expressing cells. We used quantification of the percentage of 
perivascular cells in immunofluorescence images (Figure 16a) and used Cadh5 ${ }^{\text {CREERT2 }}$ expressing endothelial cells as control. Importantly, by quantifying perivascular cells (cells in contact with a vessel), we found that CXCL1 ${ }^{\text {TdTom }}$ cells were more restricted to the perivascular distribution than the other known cellular types that form the hematopoietic niche (Figure 16b).
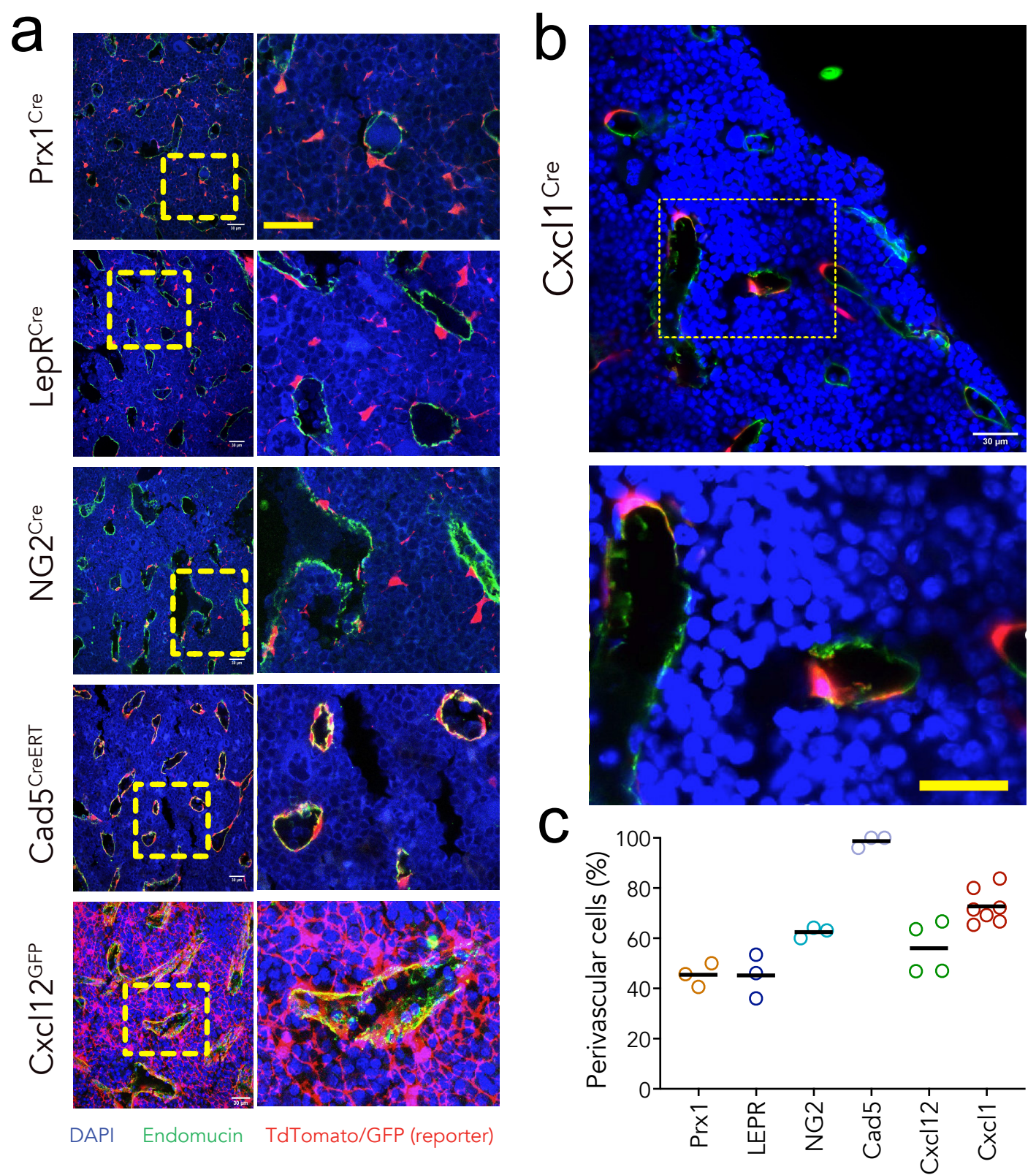

Figure 16. CXCL1 labels a subset of perivascular cells. (a) Representative immunofluorescence images of femoral BM from the indicated niche and vascular reporter lines. Cells labelled by the reporter protein (TdTomato, or GFP in the case of CXCL12) are visualized in red, and vessels (labelled for endomucin) are in green. All scale bars, $30 \mu \mathrm{m}$. Lower images show the dotted areas in upper images. (b) Percentages of each cell lineage found in direct contact with vessels (perivascular), estimated from images as in (a); $n=3-7$ mice. 
To further characterize these cells, we performed flow cytometric analysis of the BM of CXCL1 $1^{\text {TdTom }}$ mice. We observed that the majority of CXCL1 ${ }^{\text {TdTom }}$ cells were

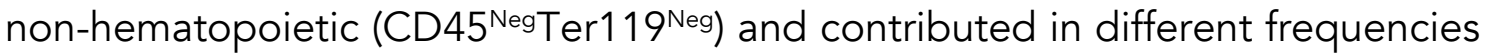
to various stromal populations (Figure 17a). We observed that the majority of Tomato+ cells within this fraction were endothelial cells (CD45-Ter119CD31+Sca1 hi) (Figure 17b). We hypothesized that this could be due to inefficient enzymatic separation (as previously reported (205)) of the endothelium and the perivascular cells that surround the vessels, in agreement with the immunofluorescence analyses (Figure 17c). Further analyses of CXCL1producing cells with other stromal and endothelial cell-surface markers (Figure 17c) revealed that Tomato ${ }^{+}$cells were not exclusively marked by any of the markers tested. We found, however, that they were highly enriched for CD34 and ICAM, which are endothelial cell markers. Thus, our imaging and flow cytometry analyses indicated that $\mathrm{Cxcl1}{ }^{\text {TdTom }}$ cells are perivascular cells that surround sinusoidal vessels and, to a lesser extent, stromal cells in the medullary interstitium.

Figure 17. Flow cytometric characterization of

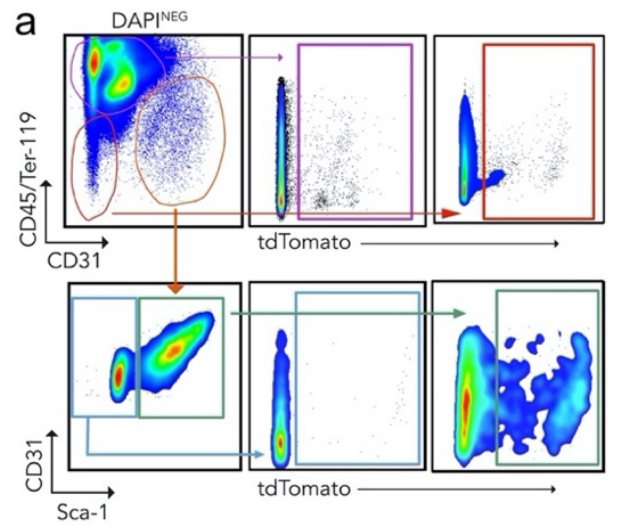

b
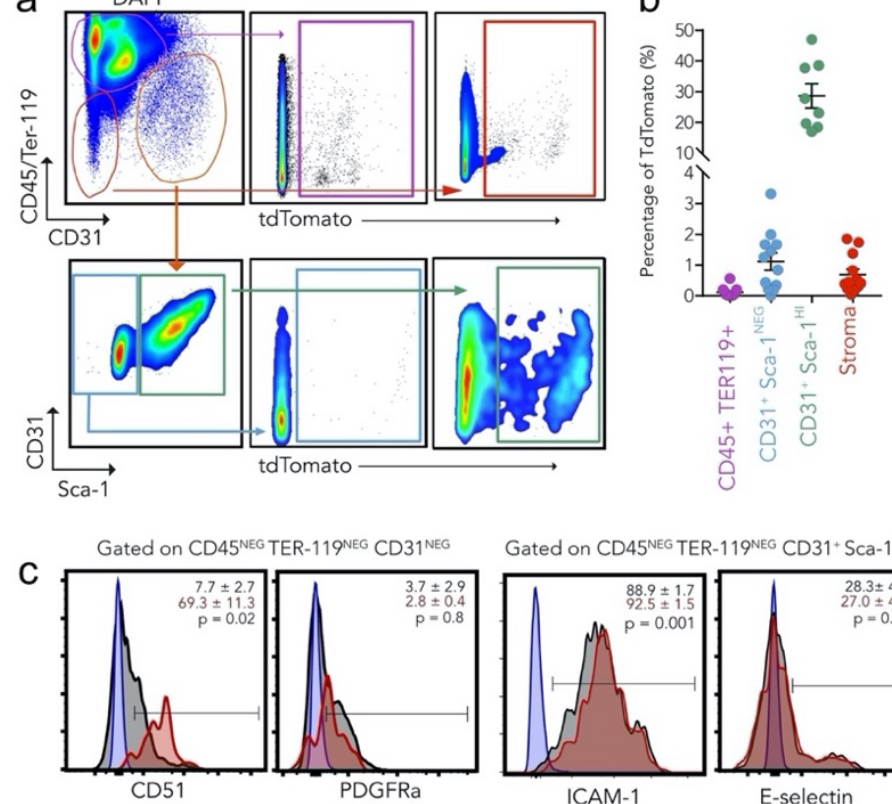

Gated on CD45 ${ }^{\text {NEG }}$ TER-119NEG CD $31+$ Sca-1 ${ }^{\text {hi }}$
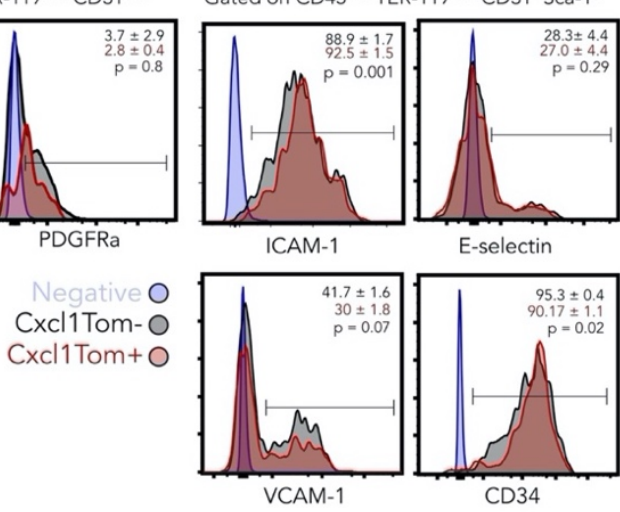

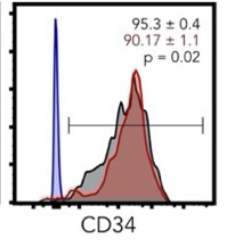

Cxcl1'TdTom mice. (a) Flow cytometry gating strategy for the analysis of BM stromal components. Color boxes indicate Tomato positive gating. (b) Quantification of the percentage of Tomato+ cells inside each of the indicated populations; $\mathrm{n}=$ 8 mice (c) Flow cytometric analysis of stromal and endothelial surface markers. Shown are negative staining (blue),

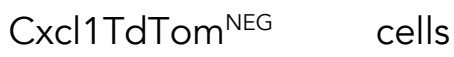
(black) and Cxcl1TdTom ${ }^{+}$ cells (red). Numbers in plots show the mean percentage of positive cells \pm SEM and p-values indicate paired t test comparison between $\mathrm{Cxcl1TdTom}{ }^{\mathrm{NEG}}$ cells and $\mathrm{Cxcl1TdTom}{ }^{+}$cells; $\mathrm{n}=3-8$ mice. 
Perivascular and stromal cells have been reported to have mesenchymal stem cell activity when cultured in vitro. Specifically, MSCs are able to form fibroblastic colonies (CFU-F) and give rise to differentiated mesenchymal populations such as adipocytes, osteoblasts and chondrocytes $(49,206,207)$. We tested whether CXCL1 $1^{\text {Tom+ }}$ cells in CXCL1 $1^{\text {TdTom }}$ mice were endowed with this capacity. For this purpose, we obtained BM single cell suspensions by enzymatic digestion and cultured them in RPMI medium in vitro. We first observed that Tomato+ cells were able to form CFU-F colonies in vitro, and thus performed cell dilution assays to calculate the frequency of MSCs in the Tomato+ population (Figure 18a). We observed that Tomato-positive cells contained almost half the CFU-F activity in the whole marrow. Additionally, $\mathrm{Cxcl} 1^{\text {TdTom }}$ cells were able to generate adipocytes and osteoblasts in vitro, when cultured in specialized differentiation media (Figure 18b). Altogether, these data suggested that a population of perivascular cells with mesenchymal stem activity expresses $\mathrm{Cxcl} 1$ in the steady state in the BM.
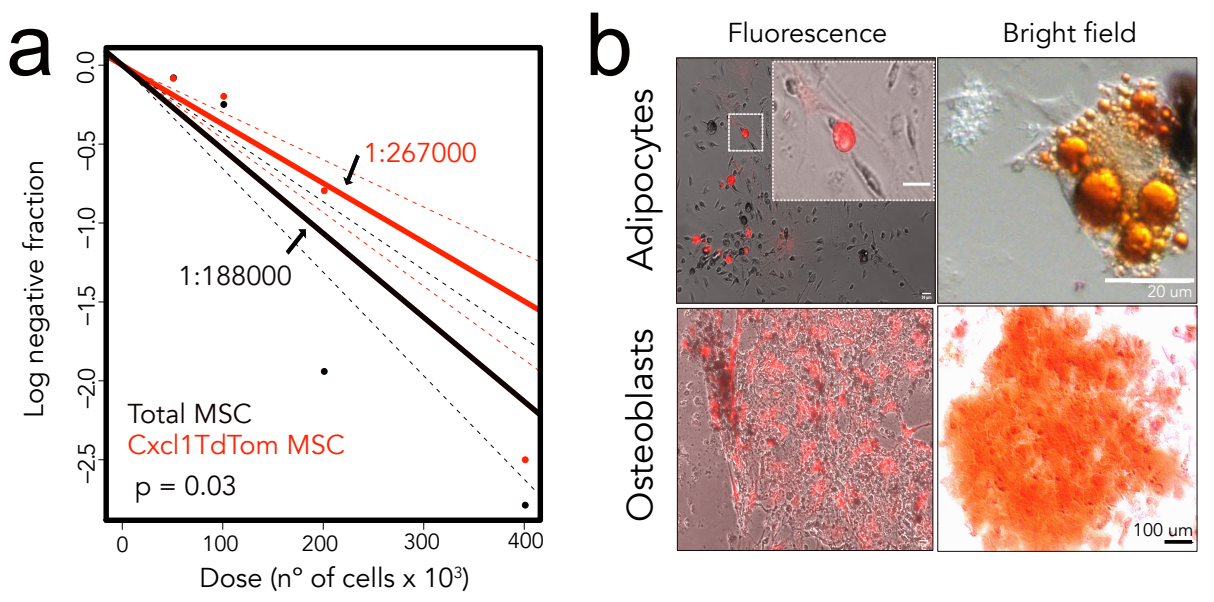

Figure 18. Cxcl1TdTom cells have MSC activity. (a) Limiting dilution analysis to determine the frequency of mesenchymal activity among CXCL1-tdTom + cells and total $\mathrm{BM}$ in $\mathrm{Cxcl} 1^{\text {TdTom }}$ mice; $\mathrm{n}=16$ wells for every dilution (50, 100, 200 and $400 \mathrm{~K}$ cells) from 2 mice. Indicated are the frequencies of MSCs and p value using Poisson statistics. (b) Representative images showing adipocytes (Oil Red $O$ staining) and osteoblasts (Alizarin Red staining) derived from Cxcl1-tdTom+ cells. Scale bars, $20 \mu \mathrm{m}$ (top) and 100 $\mu \mathrm{m}$ (bottom).

Because perivascular cells have an essential role in leukocyte and HSC trafficking and CXCL1 drives the egress of certain leukocytes subsets from the BM (203, 204), we hypothesized that CXCL1 mediated the release of cHSCs. To assess this, we first analysed the location of phenotypical CD150+ $\mathrm{HSC}$ s relative to 
$\mathrm{CXCL}^{+}$perivascular cells, to see whether HSCs localized proximal to these cells, and could thus have a functional relationship. We performed whole mount imaging of cleared femurs from CXCL1 $1^{\text {TdTom }}$ mice that were stained with the lineage antibody cocktail, CD41 and the CD150 SLAM marker (Figure 19a). We observed that Lineage ${ }^{\text {NEG }}$ CD41 ${ }^{\text {NEG }}$ CD $150^{+}$HSCs cells localized proximal to CXCL1+ perivascular cells when compared with randomly distributed points generated in the same frequency as HSCs (Figure 19b). Image quantification revealed that most HSCs were located $20 \mu \mathrm{m}$ or less from the nearest TdTom+ cell (Figure 19b). To confirm this observation, we performed in vivo imaging of the calvaria of CXCL1 ${ }^{\text {TdTom }}$ mice in which we transferred sorted Lineage ${ }^{\mathrm{NEG}} \mathrm{CKit}^{+}$ progenitor cells labelled with a cell tracker (Figure 19c). During the period of observation ( 2 hours) we found that, although the transferred cells remained sessile, they positioned proximal to CXCL1+ perivascular cells compared again to random points generated in the same frequency as the c-Kit+ cells (Figure 19c-d).
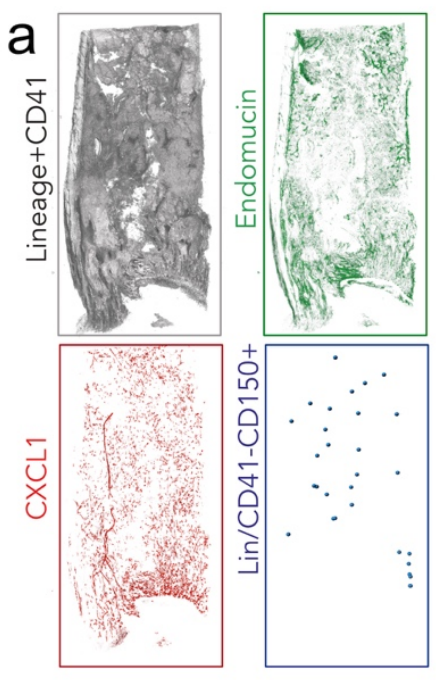

d

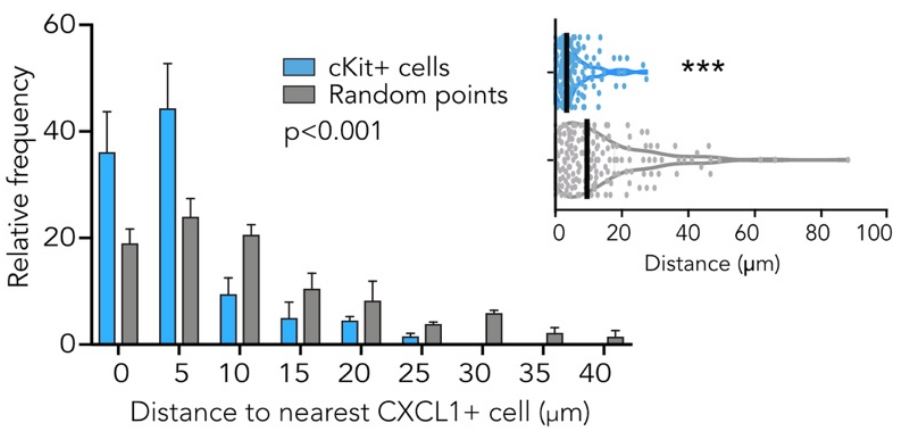

b
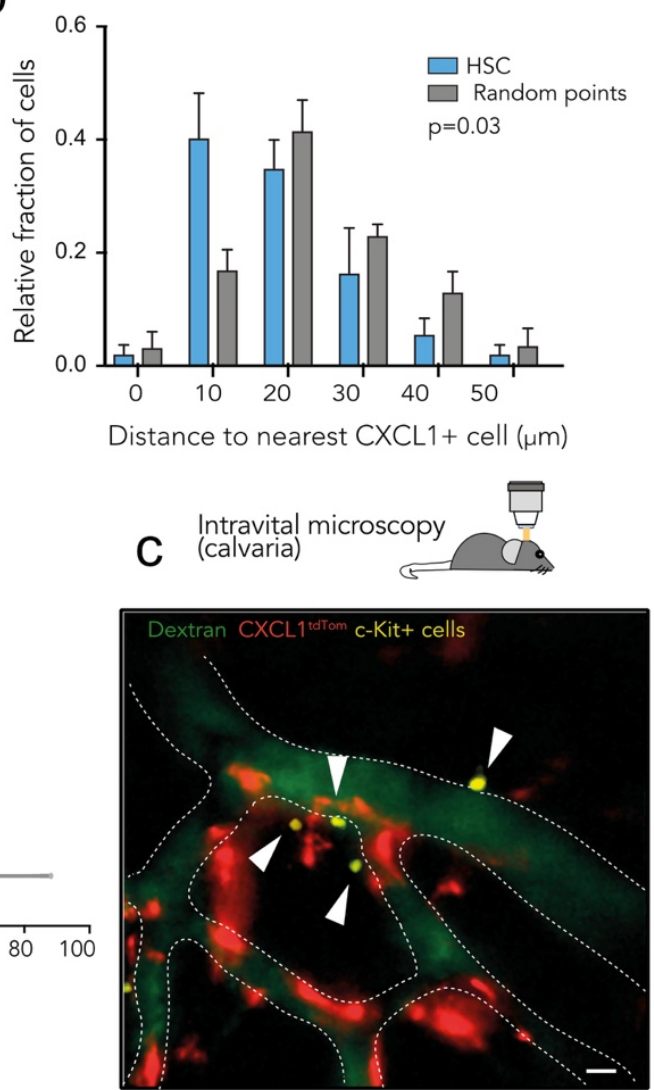
Figure 19. Phenotypic HSPCs localize close to CXCL1-producing cells. (a) Representative whole-mount staining of femora from Cxcl1TdTom mice, showing Lineage/CD41+ cells (grey), Lineage/CD41 NEG CD150+ HSCs (large blue dots for visualization), vessels (endomucin, green) and CXCL1TdTom cells (red). Scale bar, 200 $\mu \mathrm{m}$. (b) Distribution of HSC or random points by distance to CXCL1TdTom cells as determined from the whole-mount images as in (a); $n=48 \mathrm{HSCs}$ and $n=50$ random points from 3 different mice. (c) Intravital imaging of cell tracking red dye-labelled cKit+ cells (yellow, marked by arrowhead) in the BM calvaria of Cxcl1TdTom mice to visualize CXCL1-producing cells (red). Dashed lines outline vessels identified by fluorescent dextran (green). Scale bar, $20 \mu \mathrm{m}$. (d) Distances of the transferred progenitor cells shown as distance distribution (lower panel) and average distances compared with random points (inset). Data are shown as mean \pm SEM. * ${ }^{*} p<0.05$; ${ }^{*}, p<0.01$; ***, $p<0.001$; ns, not significant, as determined by 2-way ANOVA with Sidak multiple comparison post-test $(b, d)$.

To determine whether CXCL1 was required for the egress of hematopoietic progenitors from the BM, we generated CXCl1 CRE/CRE homozygous mice in which the expression of the chemokine is fully disrupted (Figure 20a). We analysed blood counts and progenitors in the BM of Cxcl1-deficient mice ( $\mathrm{Cxcl} 1^{-/-}$mice) to test whether these mice presented any haematological defect. We found normal leukocyte counts and progenitors in the BM compared with WT mice (Figure 20b-c). However, when we analysed the distribution of CFU-C progenitors, we observed that $\mathrm{CxCl}^{-1-}$ mice presented lower number of progenitors in blood and spleen whereas the numbers in BM remained unchanged compared with WT mice (Figure 20d). This effect mirrored that seen in CXCR2 ${ }^{\text {HET }}$ mice (Figure 11a), suggesting that CXCL1 could be guiding homeostatic release of HSC from the BM. 

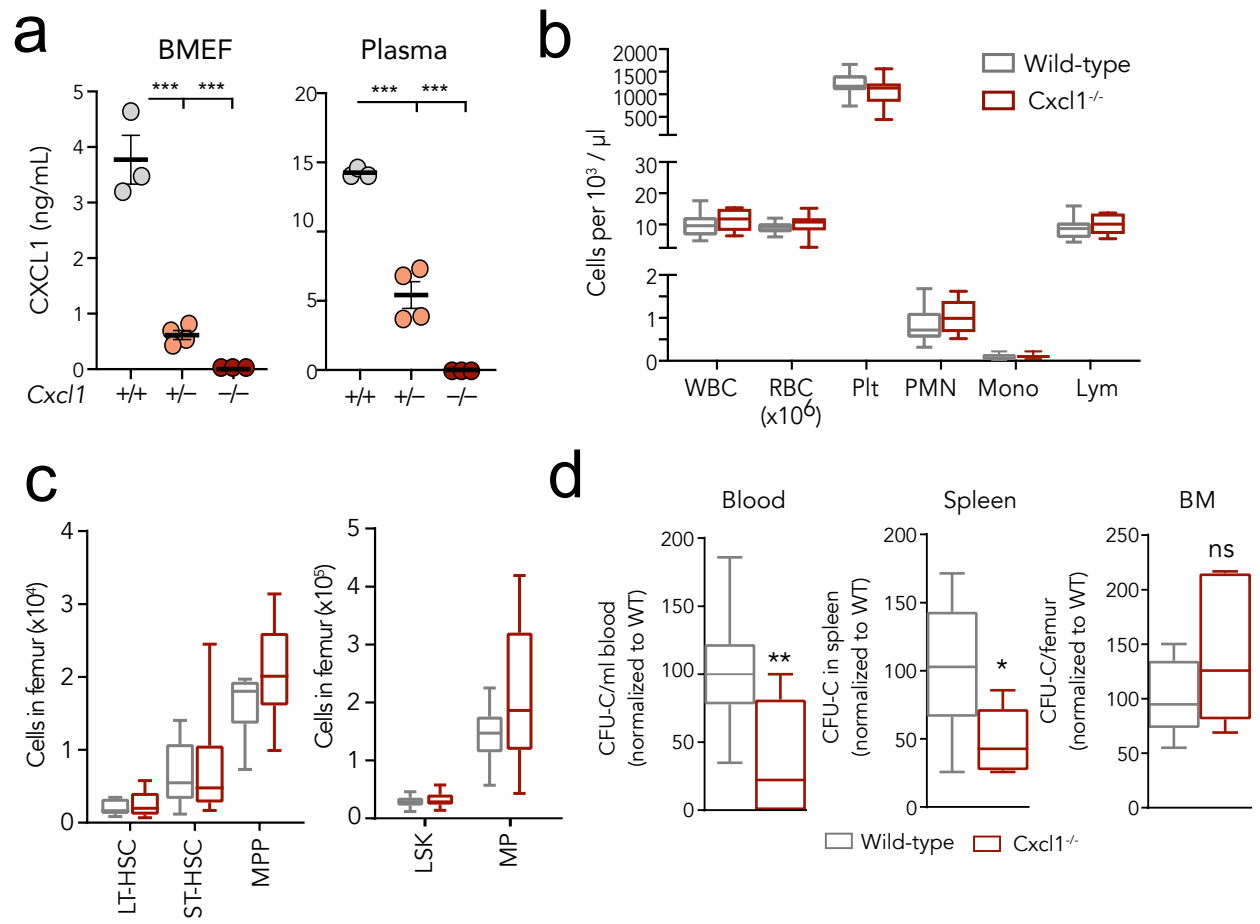

Figure 20. Characterization of $\mathrm{CxCl}^{-/-}$mice. (a) Protein levels of $\mathrm{CXCL} 1$ in $\mathrm{BM}$ and plasma of WT ( $n=3), \mathrm{CxCl}^{+/-}(\mathrm{n}=4)$ and $\mathrm{CxCl}^{-/-}$mice $(\mathrm{n}=3), 24 \mathrm{~h}$ after LPS treatment. (b) Peripheral blood counts in WT $(n=13)$ and $C x c / 1^{-1-}$ mice $(n=10)$. (c) Total number of progenitors in the BM of WT $(n=9)$ and $\mathrm{CxCl}^{-1-}$ mice $(n=9)$. (d) Number of CFU-C in blood of WT and $\mathrm{CxCl1}^{-/-}$mice ( $\mathrm{n}=11-15$ mice), in spleen $(\mathrm{n}=5-11)$ and BM ( $n=5-9$ mice). Data are normalized to WT values. Data are shown as box and whiskers plots. ${ }^{*} \mathrm{p}<0.05$; ${ }^{\star *} p<0.01 ;{ }^{* \star *} p<0.001$; ns, not significant, as determined by unpaired t-test $(b, c, d)$ and one-way ANOVA followed by Bonferroni's post-test comparing mutant mice with WT group (a).

To functionally validate the role of CXCL1-expressing pericytes in HSC egress, we performed the same parabiotic experiments as before in which the "recipient" mouse was sub-lethally irradiated (4Gy) and the "donor" mice were either WT or $\mathrm{CxCl1}^{-/-}$(Figure 21a). We assessed partner-derived cells in the peripheral blood and BM of the irradiated parabiotic mice for 16 weeks and found no differences in the reconstitution between WT and $\mathrm{CxCl}^{-/-}$mice (Figure $21 \mathrm{~b}-\mathrm{c})$. This result suggests that the release of circulating, marrow-reconstituting HSCs was independent of CXCL1, or that alternatively they used other compensatory cues, such as CXCL2.

Because the alterations in CFU-C levels in blood suggested possible roles in progenitor mobilization, we also evaluated whether CXCL1 affected the migration back into the BM. For these homing experiments we transferred $3 \times 10^{6}$ BM progenitors into WT and $\mathrm{CxCl}^{-/-}$mice and analysed the recipient BM $16 \mathrm{~h}$ 
later (Figure 21d). We found similar or even higher migration in $\mathrm{Cxcl} 1^{-1-}$ mice, indicating that this chemokine is not involved in progenitor homing to the BM (Figure 21d).

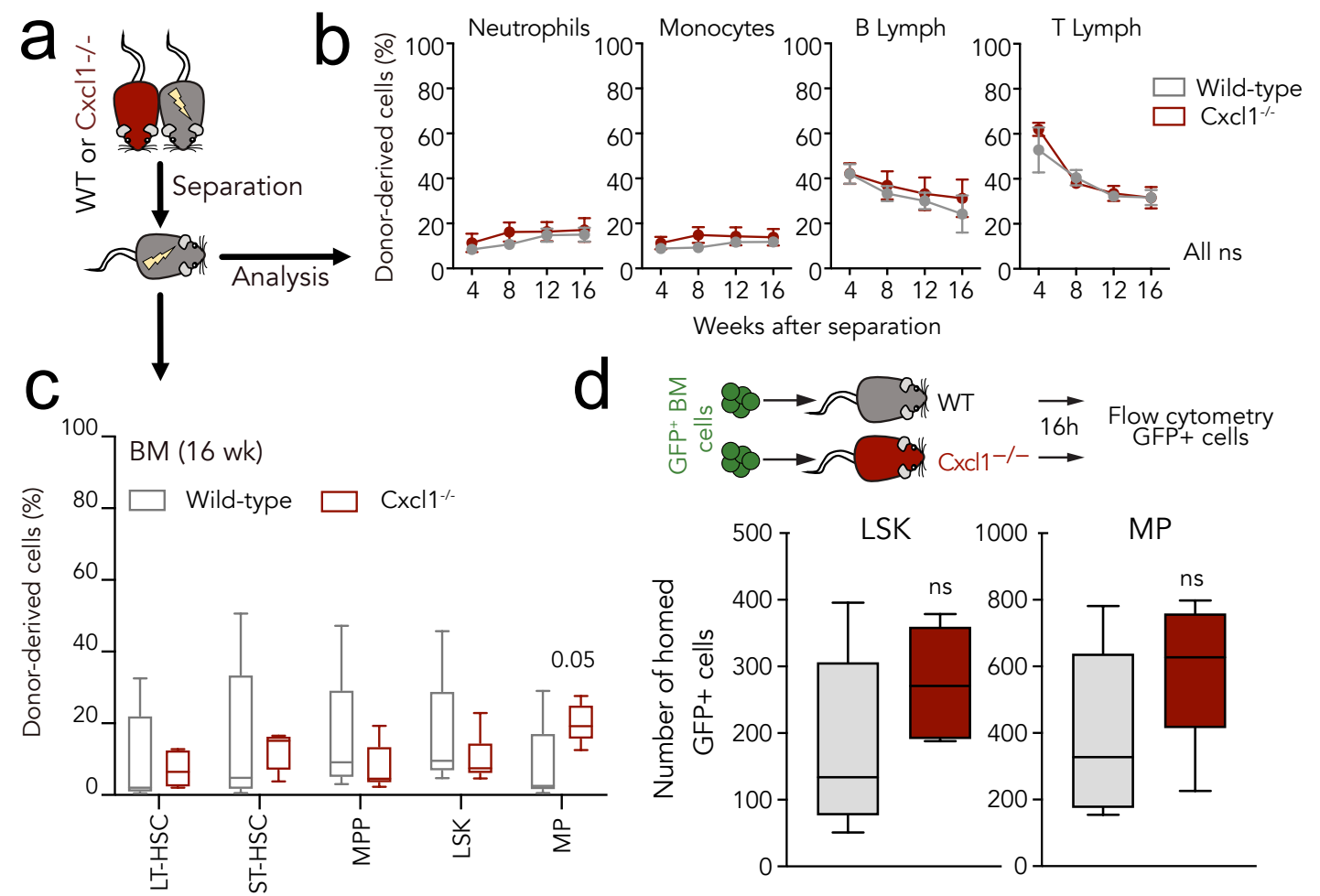

Figure 21. Role of $\mathrm{Cxcl} 1$ in HSPC homing and egress. (a) Experimental scheme to assess the reconstitution of irradiated partner mice after parabiosis for 4 weeks with either WT or $\mathrm{CxCl}^{--}$mice. (b) Percentage of donor-derived leukocytes in the irradiated mice, which had been treated with irradiation (4Gy) ( $n=6$ parabiotic pairs). (c) Percentage of donor-derived progenitor cells in the BM of the irradiated partner at 16wk after separation of the parabionts; ( $n=6$ mice/group). (d) Experimental scheme of the homing experiments of WT-GFP BM cells into WT or $\mathrm{Cxcl} 1^{-1-}$ recipient mice ( $\mathrm{n}=5$ mice/group). Bottom, analysis of the total number of GFP+ LSK and MP cells found $16 \mathrm{~h}$ after injection in the recipient BM. Data are shown as box and whiskers plots (c-d). ${ }^{*} p<0.05$; ${ }^{\star \star} p<0.01$; $\star \star \star x<0.001$; ns, not significant, as determined by unpaired t-test $(c, d)$ and one-way ANOVA (b).

The single cell datasets revealed that CXCL1+ perivascular cells also expressed CXCL12 (Figure 14b). We confirmed that TdTom+ cells sorted from Cxcl1 ${ }^{\text {TdTom }}$ mice expressed Cxcl1, as expected, as well as stem cell factor (Kitlg) and Cxcl12, relative to the TdTom-negative stroma (Figure 22a). We validated these findings by cytometric and imaging analyses of marrows from $\mathrm{Cxcl1}{ }^{\text {TdTom; }}$ Cxcl12 ${ }^{\text {GFP }}$ double reporter mice, in which we found $10-20 \%$ co-expression of both reporters in vascular and mesenchymal cells (including osteoblasts and CAR cells) (Figure 
22b). Imaging analyses of the double reporter mice confirmed the preferential perivascular distribution of CXCL1-TdTom + mice compared with CXCL12-GFP+ reticular cells (Figure 22c).

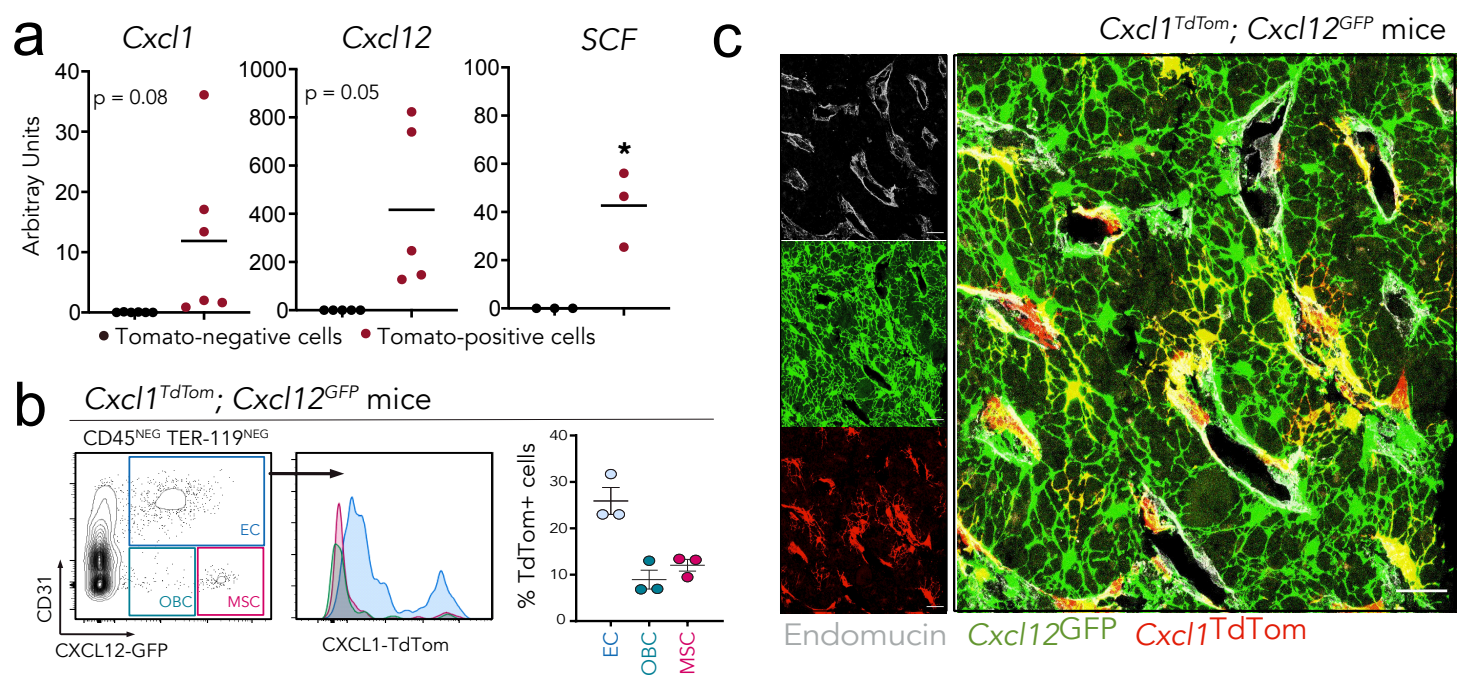

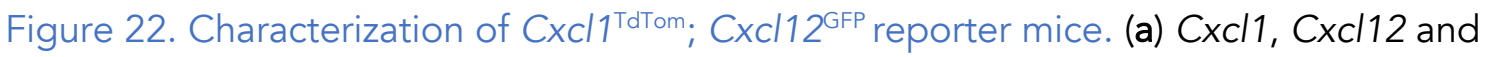
SCF transcripts in sorted TdTom ${ }^{\mathrm{NEG}}$ and TdTom ${ }^{\mathrm{POS}}$ cells from $\mathrm{Cxcl} 1^{\mathrm{TdTom}}$ mice $(\mathrm{n}=3-6$ mice). (b) Flow cytometric gating in the BM of $\mathrm{Cxc} / 1^{\text {TdTom; }}$ Cxcl12 $2^{\text {GFP }}$ reporter mice showing the overlap of cells expressing each reporter, as quantified in the right graph ( $n=3$ mice). (c) Representative immunofluorescence of BM from Cxcl1 ${ }^{\text {TdTom; }}$ Cxcl12 ${ }^{\text {GFP }}$ reporter mice. Scale bar, $30 \mu \mathrm{m}$. Data in a) is shown as mean and b) mean \pm SEM. ${ }^{\star} \mathrm{p}<0.05 ;{ }^{* \star} \mathrm{p}<0.01 ;{ }^{\star \star *} \mathrm{p}<0.001 ; \mathrm{ns}$, not significant, as determined by paired t-test (a).

Because CXCL12 is a key regulator of HSC trafficking, we next examined whether CXCL12 produced by this population of CXCL1+ perivascular cells had an effect on HSPC. For this purpose, we generated $\mathrm{CxCl} 1{ }^{\mathrm{CRE} /+} ; \mathrm{CxCl} 12^{\mathrm{fl} / \mathrm{ll}}$ mice and confirmed by $\mathrm{qPCR}$ analysis that $\mathrm{Cxc} / 12$ was efficiently depleted in sorted

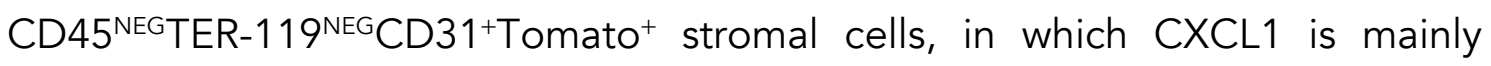
expressed (Figure 23a). Analysis of progenitors in these mice revealed mild reductions in circulating progenitors (measured as CFU-C), but this did not reach significance (Figure 23b). Moreover, these mice presented a normal distribution of stem and progenitor cells in the BM (Figure 23c) and unaltered leukocyte counts in blood (Figure 23d), indicating that CXCL12 produced by CXCL1+ perivascular cells does not control the egress or retention of HSC and their descendants. 

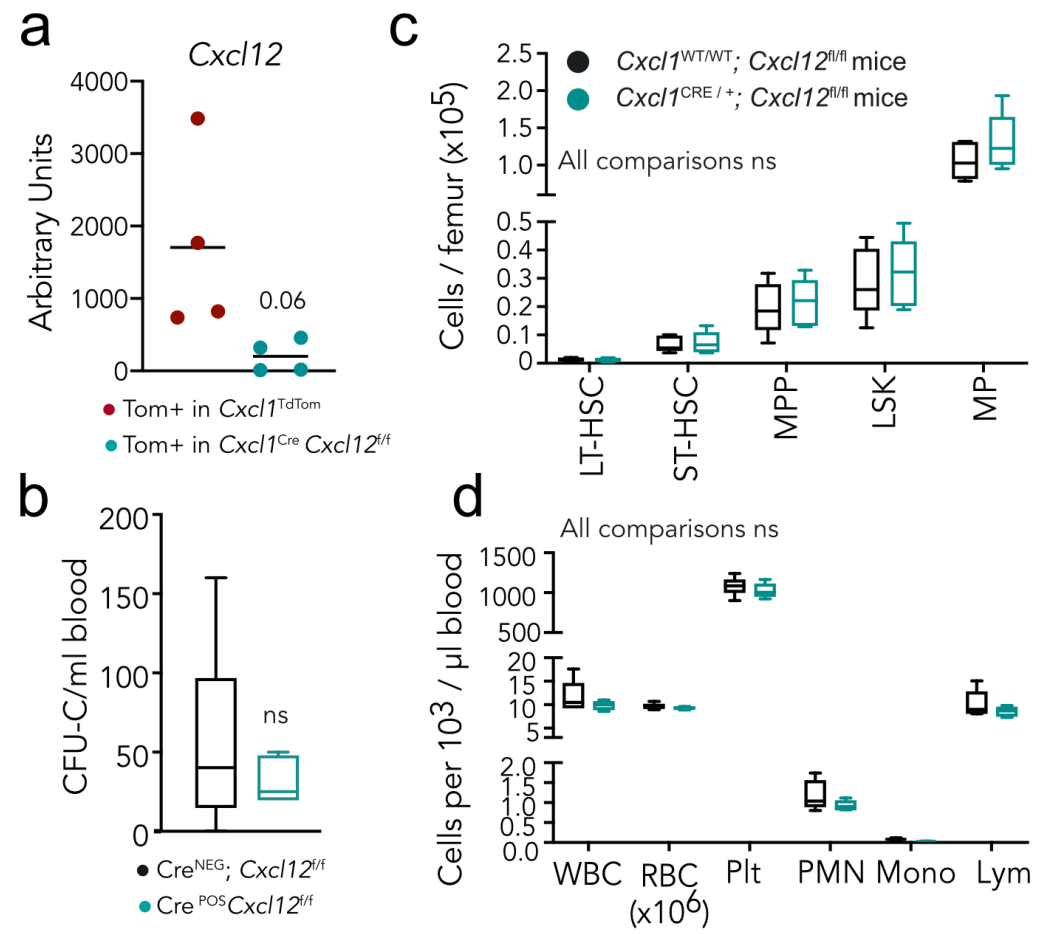

Figure 23. CXCL12 in CXCL1-perivascular cells does not alter HSC distribution. (a) Cxcl12 expression in sorted CD45 ${ }^{\mathrm{NEG}}$ TER $119^{\mathrm{NEG}} \mathrm{CD} 31^{+}$Tomato $^{+}$cells in either Cxcl1 $1^{\text {TdTom }}$ mice or $\mathrm{Cxc} / 1^{\mathrm{Cre}} \mathrm{Cxc} / 12^{\mathrm{fff}}$ mice, $\mathrm{n}=4$ mice. (b) Number of CFU-C in blood of $\mathrm{Cxc} / 1^{\mathrm{WT}}$; $\mathrm{CxCl} 12^{\mathrm{f} / \mathrm{f}}$ and $\mathrm{CxCl} 1^{\mathrm{Cre}} ; \mathrm{Cxcl} 12^{\mathrm{fff}}$ mice; $\mathrm{n}=4-5$ mice per group. (c) Total progenitor numbers

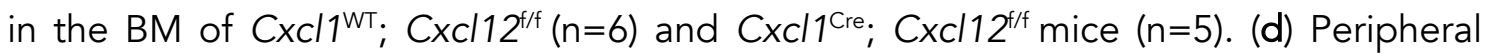

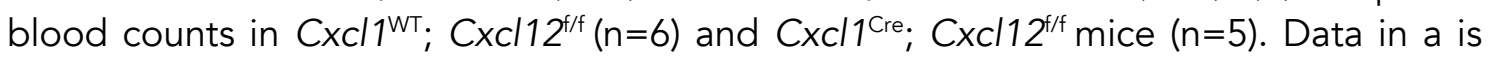
shown as mean and b-d are shown as box and whiskers plots. ${ }^{*} p<0.05$; ${ }^{* *} p<0.01$; ${ }^{\star \star \star} \mathrm{p}<0.001$; ns, not significant, as determined by unpaired t test (a-d). 


\section{cHSC support hematopoietic fitness}

The fact that cHSCs have the ability to regenerate myeloablated niches in distant bones suggested that a physiological role for CXCR2-mediated release of HSC might be to preserve hematopoietic quality throughout the organism by replacing faulty HSC, implying that the absence of CXCR2 could result in defective hematopoiesis. To test this possibility, we first analysed the functional activity of HSC from $\mathrm{Cxcr} 2^{\text {HET }}$ mice using limiting dilution assays, which allowed us to determine the actual number of functional, reconstituting HSC (208) (Figure 24a). We found that, despite comparable number of phenotypic HSC in the marrow of $\mathrm{Cxcr} 2^{\text {HET }}$ mice (Figure 12b), Cxcr2 ${ }^{\text {HET }}$ mice had an almost 7 -fold reduction in the frequency of LTR-HSC (Figure 24b, left panel). Interestingly, despite the natural expansion of HSC with age $(161,209)$, these differences were maintained in old mice (60-80 weeks of age) (Figure 24b, right panel).



Figure 24. Reduced LT-HSCs in Cxcr2 ${ }^{\text {HET }}$ mice. (a) Experimental scheme for the BM dilution assay with either young or old WT and $\mathrm{Cxcr} 2^{\mathrm{HET}}$ donor mice. 10 and $25 \mathrm{k}$ cells from donor mice were transplanted into lethally irradiated recipient mice together with $3.5 \times 10^{6}$ helper GFP BM cells. Chimerism in recipient mice was analysed 16 weeks after transplantation in peripheral blood. (b) Limiting dilution analysis to determine the frequency of HSC reconstituting activity in the BM of WT and CXCr2 ${ }^{\text {HET }}$ mice at young and old ages ( 8 and 60-80 weeks of age, respectively); $n=6-10$ mice per group and dose. Shown are the estimated frequencies and $p$ values using Poisson statistics. 
Ageing affects the function and composition of the mature blood cell compartments $(161,210)$. To examine age-related alterations, we analyse the distribution of leukocytes in blood. We observed that $\mathrm{Cxcr}{ }^{\text {HET }}$ mice underwent a greater expansion with age of their myeloid compartment in blood (particularly monocytes and platelets) (Figure 25a) and presented higher levels of CD41 in the HSC compartment (Figure 25b), which are two hallmarks of ageing $(167,168$, 209). Consistent with these alterations, we found reduced competitive repopulating capacity of $\mathrm{Cxcr} 2^{\mathrm{HET}}$ derived haematopoiesis when confronted with WT competitors and this, too, became more pronounced with age (Figure 25c and d). Altogether, these findings revealed cumulative loss of hematopoietic fitness in mice harbouring fewer CHSC, suggesting a role for these cells in surveilling and replenishing faulty hematopoietic sites that may appear during the lifespan of an organism.
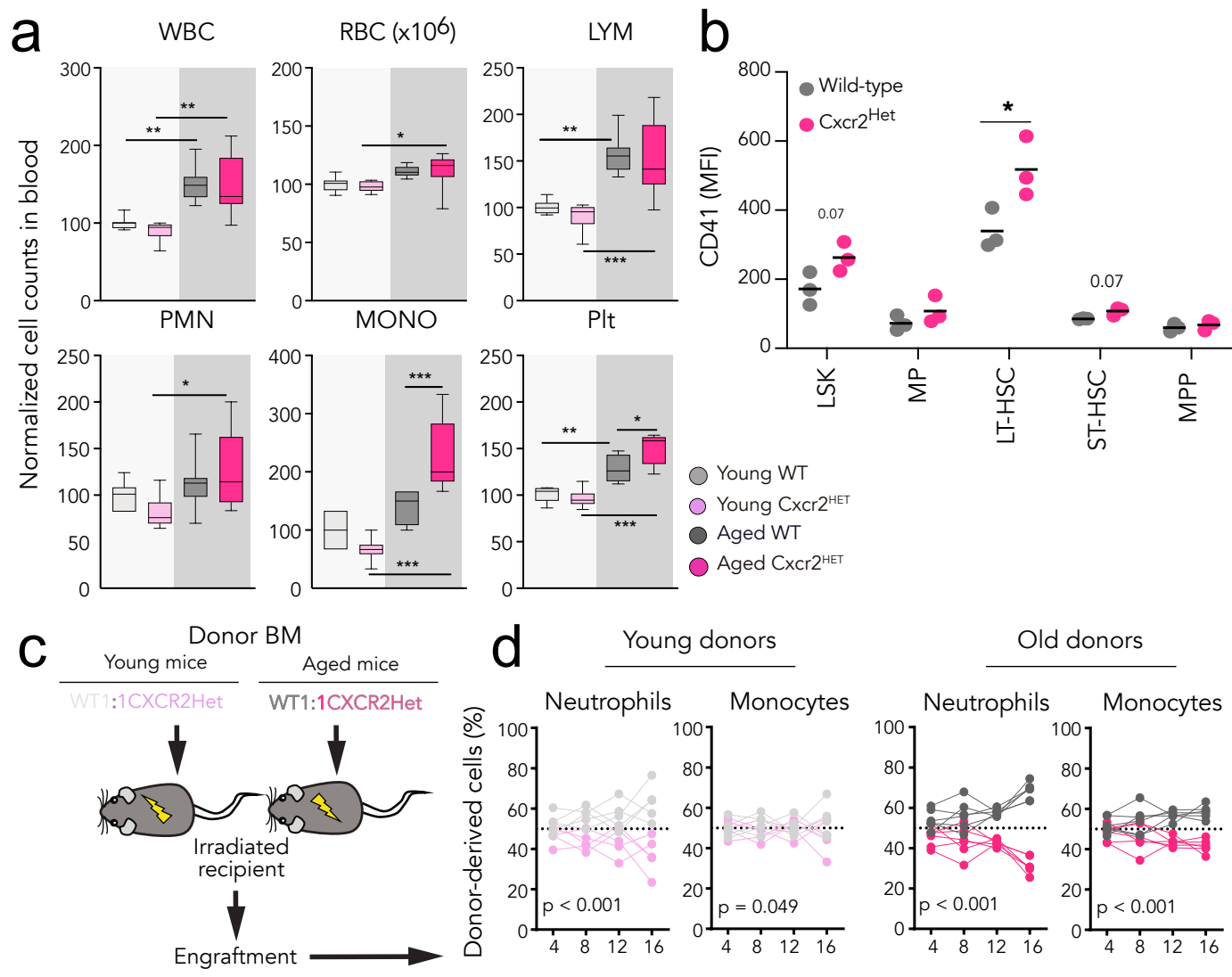

Young WT

Young Cxcr2 ${ }^{\text {HET }}$

- Aged WT

Aged Cxcr2 ${ }^{\text {HET }}$

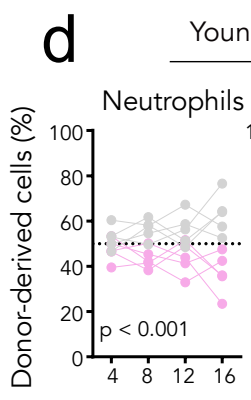

donors

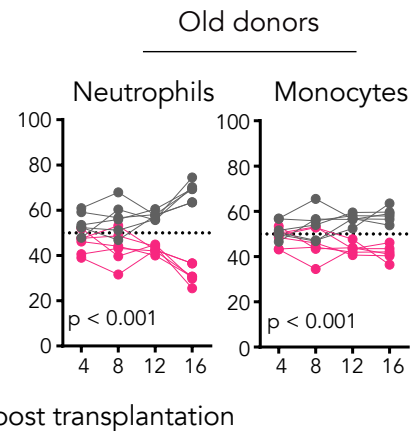

Figure 25. CXCR2 supports hematopoietic fitness. (a) Peripheral blood counts (normalized to young WT group) in young and aged WT and $\mathrm{CxCr}^{\mathrm{HET}}{ }^{\text {mice; }} \mathrm{n}=6-7$ for young, $n=8-9$ for aged. (b) CD41 expression levels (MFI) measured by flow cytometry in the hematopoietic compartment in the BM of aged WT and $\mathrm{Cxcr} 2^{\mathrm{HET}}$ mice; $\mathrm{n}=3$ mice. (c) Experimental scheme for the competitive BM transplantation setup. Mixed BM chimeras were generated by mixing equal number of BM cells from either young or old 
WT or $\mathrm{CxCr}^{\mathrm{HET}}$ mice and transplanted into lethally irradiated mice. (d) Analysis of longterm myeloid engraftment in the peripheral blood of chimeric mice; $n=5$ young chimeras, $n=6$ old chimeras. Data are shown as box and whiskers (a) or mean (b). ${ }^{*} p<0.05 ;{ }^{* \star} p<0.01 ;{ }^{* \star} p<0.001$ as determined by one-way ANOVA (a), 2-way ANOVA with Bonferroni post-test for multiple comparison (d) and unpaired t test (b). 


\section{Discussion}

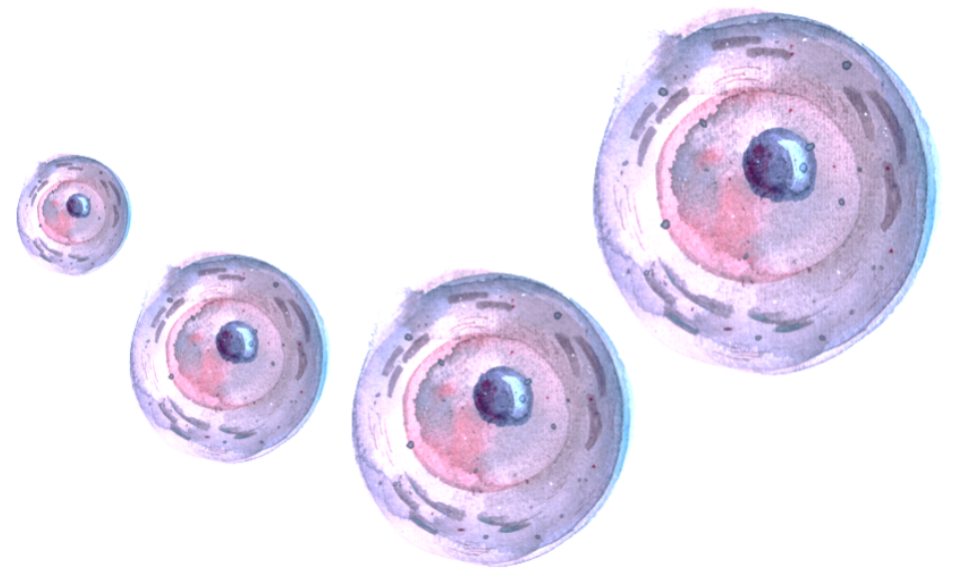

- 97 - 
$-98-$ 


\section{Discussion}

\section{Dissemination of circulating HSCs}

In the present thesis I have defined several aspects of the function of circulating hematopoietic stem cells (cHSCs) that were previously unknown. The fundamental finding of my work is the discovery of a process whereby cHSCs serve to repopulate remote damaged BM niches, to the extent that they can rescue the whole hematopoietic tissue of an organism, and thus maintain hematopoietic quality.

In the first part of this work, I have focused on deciphering the biological functions of cHSCs. Using different approaches, such as parabiosis or partial irradiation experiments, we have shown that these cells have the capacity to enter and replenish empty BM niches and give rise to new progeny. Our data is in alignment with previous results shown in 2001 by Weissman and colleagues demonstrating that circulating $\mathrm{HSCs} /$ progenitors migrate rapidly and constitutively through the blood (2). We extend these findings by showing that HSC migration is important to maintain hematopoietic homeostasis by ensuring that HSC niches are not left unoccupied after HSC dysfunction, i.e., a genetic mutation that blocks HSC differentiation could be substituted by a healthy circulating HSC that comes from a different niche.

As mentioned in the Introduction, migratory HSCs also function as an immediately available pool that can be rapidly recruited for extramedullary haematopoiesis after conditions of infection or inflammatory diseases (101). Consistent with this idea, we find that cHSCs that have engrafted the BM of an irradiated mice have a strong and unique myeloid-biased signature (Figure 6b, c). This could indicate that, as in blood regeneration processes after stress, cHSCs could be transiently induced to a myeloid differentiation program, ensuring the production of myeloid cells (mostly granulocytes and monocytes) and platelets, which are the most needed blood elements after myeloablation, to protect from pathogens and preserve homeostasis, respectively (211). Additionally, in cancer patients with solid tumours it was observed that the composition of circulating HSPCs was altered, with an increased myeloid-bias 
and a decreased lymphoid potential (212). This is important as tumorigenic processes are associated with perturbations in myelopoiesis. Solid tumours recruit myeloid-derived suppressor cells (MDSCs) to suppress anti-tumour immunity and thus promote tumour growth and metastasis (213).

I propose here that cHSCs patrol the organism and serve as a rapidly available pool of HSPCs with stem cell properties that migrate and infiltrate damaged sites to restore haematopoiesis or improve the HSC pool. My work suggests that a deficiency in the dissemination of circulating HSPCs could lead to an inefficient restoration of normal haematopoiesis with serious consequences for the organism.

Previous literature has reported that donor HSCs can engraft and expand even in the absence of myelosuppressive conditioning, indicating that numerous vacant niches exist distant from the niches filled in by endogenous HSCs (214). However, our data with parabiosis experiments, indicates that for this process to succeed, it is necessary that vacant niches are available for external cHSCs to engraft and proliferate, as HSC engraftment between parabionts in basal conditions is minimal (around 2\%) (Figure 2b, c). In this situation we hypothesized that either most of the cHSCs that enter the BM do not have a physical space to engraft into and expand, or that cHSCs are outcompeted by endogenous medullary HSCs. It is likely that endogenous medullary HSCs outcompete cHSCs, as supported by the experiments where these cells are transplanted together (Figure 7a). Consistent with this idea, previous literature has described that competition among HSC with different levels of p53 allows elimination of damaged progenitors and prevents clonal expansion of leukemic cells (215). Also, in line with this idea, when HSCs remain functional and no irradiation is applied, cHSCs remain in competitive disadvantage and thus are lost. The opposite occurs when the BM is subjected to high doses of myeloablative conditioning. This irreversibly damages endogenous HSPCs and the BM becomes vacant for new donor HSCs to enter and successfully engraft the marrow, as we observed when we apply an 8Gy irradiation dose (Figure 2b-c). We thus believe that a mild irradiation dose creates a competitive scenario for cHSCs in the receiving niche (Figure $2 b-c$ ). However, we have to be cautious as our model is a rather artificial approach. We now aim to study the role of cHSCs in a system where we do not need pre-conditioning of the niche by irradiation, but instead HSPCs are intrinsically (e.g., by endogenous mutations) weak or 
defective. For this purpose, important future experiments include parabiosis of wild-type mice with $\mathrm{Kit}^{\mathrm{w}-41 J}$ mutant mice. These mice have a partial loss of function of Kit that leads to increased proliferation of HSPCs in the steady state (216). Critically, HSCs in these mice are functional in an isolated system but are replaced when competing with healthy HSCs $(216,217)$. For this reason, this scenario should allow us to test the capacity of cHSCs to repair intact niches that contain genetically suboptimal HSCs.

From an evolutionary point of view, the fact that a small pool of HSCs leaves the secure BM site and exposes to stress-induced environments has several benefits. First, it protects the whole set of medullary HSCs to be exposed to external inflammatory or stress signals that could compromise the entire hematopoietic system. Second, the patrolling by cHSCs of different origin enables a rapid, onsite production of mature immune leukocytes in response to infection, as previously suggested (38). Finally, cHSCs facilitate the replenishment of damaged niches outside the BM that may be needed to respond to BM failure or stress, as seen in the context of infections or chronic hematopoietic demands $(57,218)$.

In this work, we also asked whether migratory HSCs that enter the circulation are different from the bulk of HSCs found in the BM, and whether all HSCs have the "choice" and potential to enter the circulation. We found that cHSCs form part of the common medullary HSCs, since in parabionts that were separated, partner-derived cHSCs quickly disappeared from the circulation suggesting that they soon integrated into the main HSC pool away from the circulation (Figure $3 a, b)$. These data suggest that all medullary HSCs have the ability to circulate, although we cannot rule out the possibility that a subset of medullary HSCs have special migrating properties, as occurs in HSCs mobilized with AMD3100 in combination with CXCL2, which have higher engraftable activity when compared with mobilized HSCs in G-CSF treatment (219). This suggests that inside the BM different subsets of HSCs with distinct properties co-exist.

Consistent with this notion of a common HSC pool, we identified in clonal analyses with lentiviral barcoding of parabiont mice that the dissemination of cHSCs consisted in multiple clones, as we found that many myeloid clones from the donor mouse reconstituted the recipient partner (Figure 4e). Capturerecapture analyses revealed high numbers of active partner-derived cHSC 
(Figure 4f). The clonal complexity in the recipient mice was comparable to that found in the marrow of the donor partner suggesting that cHSCs crossing between parabionts "capture" the clonal composition of the donor HSC pool (Figure 5a). Although we acknowledge that this system is not optimal to study native haematopoiesis, as it involves transplantation settings, previous studies performed in basal conditions have indicated similar polyclonal processes (220). Moreover, streamgraph analyses revealed that the cHSC clones that reconstituted the partner mouse were stable and long-lived, as they were detectable for at least 16 weeks (Figure $5 c$ ). We also observed the presence of some dominant clones along time, suggesting that even though the repopulation of damaged BM by cHSCs is polyclonal, some of the "fittest" clones might be dominating over others. An interesting observation was that the spleen reflected the clones found in the lymphoid lineage, while the BM reflected the clones found in the myeloid lineage (Figure $4 \mathrm{e}$ and $5 \mathrm{~b}$ ). Additional experiments revealed that cHSCs seeded hematopoietic organs such as the BM and spleen but no other organs such as liver or lung (Figure 2e). Our results argue for a model where multiple clones of HSCs are released into the bloodstream and stably engraft the BM that has been ablated but spares other organs. The reason for this is currently unknown.

\section{Mechanisms that enable the dissemination of cHSCs}

In this thesis work I have also identified the molecular mechanisms by which HSCs are released from the BM into circulation under homeostasis. We found that HSPCs in the BM express low levels of the chemokine receptor CXCR2 on its surface (Figure $8 c$ ) in accordance with the available gene expression databases (Figure 8a-b). Previous work indicated that CXCR2 participates in the maintenance of normal HSPC, including self-renewal and survival (221), although others have questioned the expression of this receptor on HSPCs (219). Here, using CXCR2 ${ }^{\mathrm{HET}}$ and $\mathrm{CXCR} 2^{\mathrm{KO}}$ mice, we demonstrate that hematopoietic progenitors migrate ex vivo to CXCL1 in a CXCR2-dependent manner (Figure 10a-c), and that the cells that migrate have long-term repopulating capacity (Figure 10d). This implies that cells that migrate in a CXCR2-dependent way towards CXCL1 are bona fide HSCs. We have shown that CXCR2 is essential for the egress of HSPCs into blood and other peripheral tissues in vivo (Figure 11a). However, we have also observed that the forced mobilization egress of HSCs 
into peripheral blood is not dependent on CXCR2, as mice deficient in CXCR2 mobilized HSCs to the same extent as WT mice (data not shown). Importantly, we have demonstrated that genetic disruption of the receptor causes a reduction in cHSCs and this leads to an impairment of the repopulation of remote hematopoietic sites (Figure $13 \mathrm{c}$-d). These data suggested that the absence of cHSCs could derive in long-term functional hematopoietic decline. Indeed, analysis of haematopoiesis in aged mice revealed that aged CXCR2 ${ }^{\mathrm{HET}}$ mice contained less LT-repopulating HSCs as measured by BM dilution assays, even if young CXCR2 ${ }^{\mathrm{HET}}$ mice presented already lower frequency of LTR-HSCs (Figure $24 \mathrm{a}-\mathrm{b})$. This finding also suggests that the reduced repopulation of the partner mouse observed in parabiosis with CXCR2 ${ }^{\mathrm{HET}}$ mice (Figure $13 \mathrm{a}-\mathrm{C}$ ) could be due to the reduced availability of HSCs found in the donor mouse and not due to reduced egress. Thus, in order to distinguish between both scenarios, future experiments will consist in treating WT mice with a CXCR2 antagonist (SB225002) to confirm whether short-term inhibition of CXCR2 alters the number of repopulating HSCs in the BM, and its effect in HSC egress.

We have observed an expansion of the myeloid compartment in peripheral blood (especially platelets and monocytes) in CXCR2 ${ }^{\mathrm{HET}}$ aged mice (Figure 25a) accompanied by an increase in phenotypical HSPCs in the BM (data not shown), suggesting an exaggerated aged phenotype in these mice. This phenomenon was accompanied by an increase in the levels of CD41 in phenotypic HSPCs, in agreement with the previously reported megakaryocytic bias of HSCs in aged mice (Figure 25b) $(167,168)$. Altogether, these results suggest that cHSCs are necessary for the correct maintenance of haematopoiesis and a deficiency leads to early deterioration of HSCs.

An interesting implication of the finding that HSCs disseminate through CXCR2signalling is that it could be similarly important for the dissemination of other stem-type cells, such as circulating tumour cells (CTCs). Previous literature has shown the implications of CXCR2 and its chemokine ligands in the progression of different types of cancer such as lung, breast or ovarian cancers (222). Expression of CXCR2 in the tumours have correlated with a poor prognosis of the disease as it controls angiogenesis, cell migration and proliferation. Pharmacological inhibition of CXCR2 has an overall positive impact on the cancerous lesion (222). Likewise, it is conceivable that similar mechanisms 
underlie the dissemination of leukemic stem cells, an area of important clinical relevance that could benefit from our studies.

In line with this idea, an important area of future study will be to understand the mechanisms of dissemination of pre-leukemic cells. For this purpose, we will study the dissemination of cHSC in a context of clonal haematopoiesis $(\mathrm{CH})$. Clonal haematopoiesis of indeterminate potential (CHIP) describes the expansion of a clonal population of blood cells bearing one or more somatic mutations. This phenomenon is associated with a higher risk of suffering haematological malignancies, cardiovascular disease and increased mortality from non-haematological cancers (223-226). Even though HSCs divide slowly, with time they may acquire mutations that are passed on to the next generation of cells, affecting both the HSC compartment and daughter cells (227). The mutations that promote increased self-renewal or increased proliferation have the capacity to expand the HSC clone at a disproportionate rate compared to other clones and this will yield a proliferative advantage over non-mutant cells. $\mathrm{CH}$-mutations in genes such as Dnmt3a, Tet2, Jak2 or Flt3 have been associated with increased incidence of haematological cancers (220). A more recent finding is that these mutations also course with high prevalence of cardiovascular complications, including those associated with atherosclerosis, such as coronary heart disease or stroke, and overall increased frequency of cardiovascular disease (CVD)-related deaths $(223,228)$. Importantly, $\mathrm{CH}$-related mutations are very prevalent in older individuals suggesting that this is a common derailment of haematopoiesis $(227,229)$. With this background, we plan to generate a mouse model deficient for TET2 and CXCR2 (Tet2 ${ }^{-1-}$; Cxcr2 ${ }^{+-}$mice) that will help us to understand if the dissemination of HSPCs bearing somatic mutations in Tet2 is under the control of the CXCR2 axis, and how this could impact atherosclerosis and other associated pathologies. In accordance with this idea, a previous study has described an important role for a subset of circulating HSPCs positive for CCR2 in the healing process after myocardial infarction (74). Overall, these studies suggest that cHSCs could have a role also in pathological conditions.

In this study we provide insights into the chemokine ligands that could be involved in signalling the egress of HSCs. Interestingly, we have found a subset of cells inside the BM niche that expresses CXCL1. By using the reporter mice CXCL1 ${ }^{\text {TdTomato }}$ we have observed that these cells are mainly perivascular and, in 
less frequency, stroma-like cells in the interstitium (Figure 15c-d). Previous literature has focused mainly on perivascular stromal cells that express CXCL12 and drive the homing or retention of HSCs inside the BM. These include the LepR, Prx1-expressing perivascular cells or endothelial cells (90). Here, we describe for the first time, a subset of niche cells that rather than retaining HSCs, drive their release out of the BM. CXCL1-deficient mice present reduced levels of HSCs in circulation mirroring those in CXCR2 ${ }^{\text {HET }}$ mice (Figure 20d). In contrast, these mice presented normal repopulation of remote hematopoietic sites in parabiosis experiments (Figure $21 \mathrm{a}-\mathrm{c}$ ). We speculate that in this situation, other ligands for CXCR2, such as CXCL2 or others (230), could compensate for the absence of CXCL1. Further investigation will be required to analyse the exact ligand(s) for CXCR2 mediating HSC egress.

Based on the observation that cultured BM CXCL1-producing cells in vitro fulfilled all mesenchymal stem cell (MSC) properties, i.e., had fibroblast-like morphology, form colonies in vitro and differentiate into bone and fat cells, we hypothesized that these cells could be of mesodermal origin, although further studies need to be done. It has been documented the suppressor effects of BM MSCs on the immune system of tumour-bearing hosts, facilitating tumour growth and proliferation $(231,232)$. An open question is to determine whether CXCL1perivascular cells outside the BM are affected by pathological conditions or whether they are also involved in tumorigenesis.

RNA-seq data from isolated CXCL1-expressing cells showed an enrichment in several genes belonging to the NF-kB pathway, such as JunB or Fosb (data not shown). This pathway plays an important role in the activation of genes involved in inflammation, cell proliferation and survival (233). It has also an important role in leukocyte migration and it is therefore conceivable that this pathway is involved in the release of cHSCs. Deeper research into the regulation of the CXCR2-CXCL1 axis will be key to understand the functions and dynamics of dissemination of cHSCs. At present, however, we cannot discriminate whether this perivascular CXCL1-producing subset of cells is functionally relevant for HSC egress; thus, an important future task will be to identify if this population of CXCL1-producing cells is responsible for enabling the release of HSCs into periphery. For that purpose, we aim to use a DTR-based system to induce death of this population and evaluate the effects on circulating HSC. 
Further regulation of the secretion of factors and chemokines by perivascular cells inside the BM is by the sympathetic nervous system (234). Our results show that the levels of systemic CXCL1 in plasma follow circadian oscillations and the levels were higher in the night (with a peak at ZT17, 23 pm). This elevation in CXCL1 in plasma could be preceding the morning egress of CHSCs (peak at ZT5, $12 \mathrm{am}$ ) into circulation (data not shown). This oscillatory pattern suggests that local innervation of the BM by the SNS could be also regulating the production of CXCL1 by specialized perivascular cells, and this will be an important aspect of future studies.

In summary, this thesis work identifies previously unknown physiological roles for cHSC in the repopulation of damaged niches or with defective HSCs, maintenance of hematopoietic quality, and unveils the mechanisms by which HSC exit the BM under homeostasis (Figure 26). The CXCR2 axis is implicated in the release of cHSCs, and future studies will be required to confirm the role of CXCL1 or other ligands in this process. The identification of signalling pathways mediating the release of cHSCs will be key for a better understanding of the role of these cells in homeostatic and pathological conditions, and in defining potential therapeutical targets for this population of somatic stem cells. 


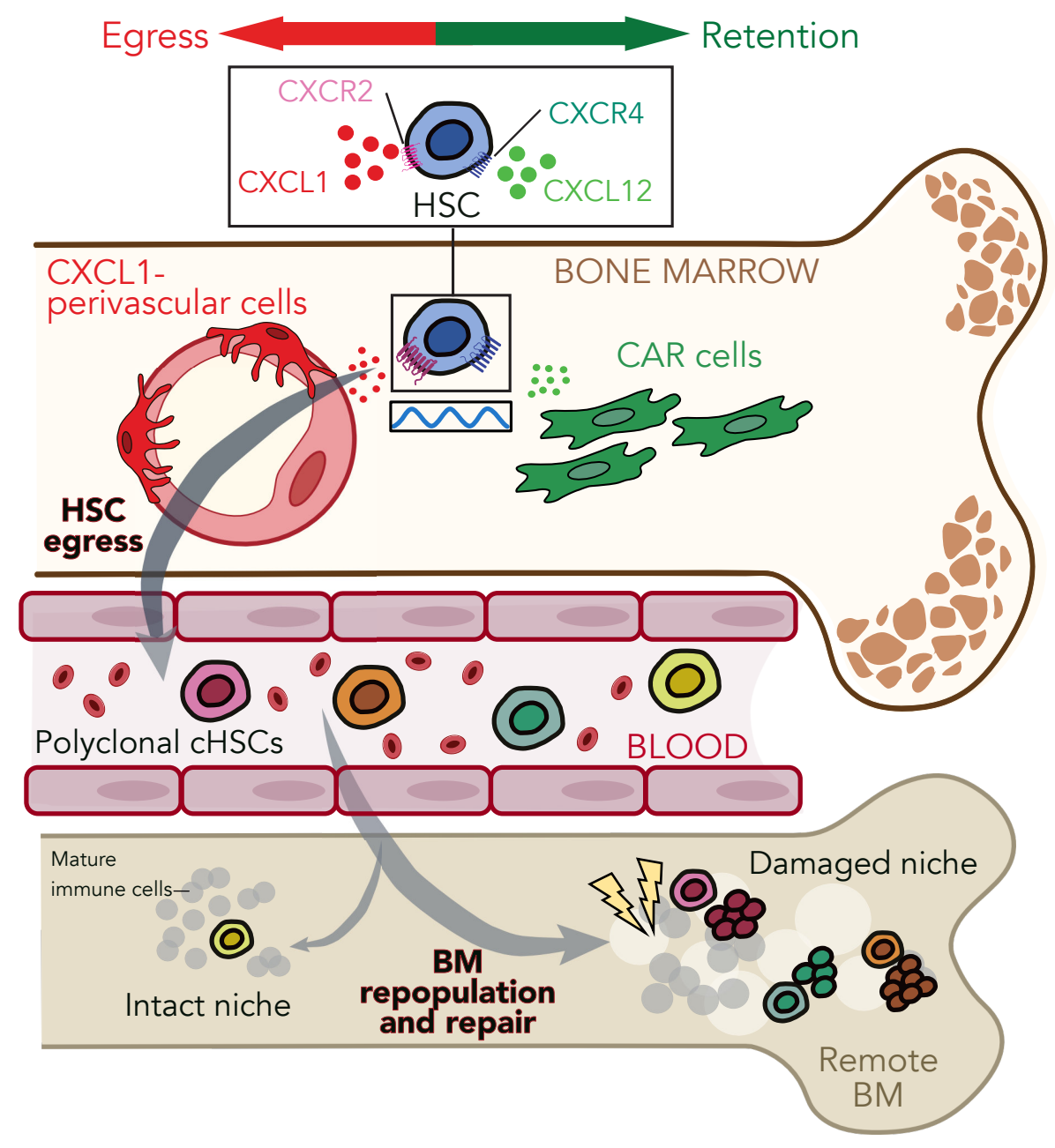

Figure 26. Proposed model of dissemination and function of cHSC. Model of the mechanism reported in this thesis. HSCs that reside inside the BM niche are retained through the CXCR4-CXCL12 axis. They are released daily into circulation following circadian rhythms. The egress is driven by CXCR2 expression in HSCs and presumably by CXCL1 expressed by perivascular cells inside the marrow. Multiple clones of HSCs are released into the periphery and are found in remote damaged niches. Intact niches are not replaced by cHSCs. 
- 108 - 


\section{Conclusions}

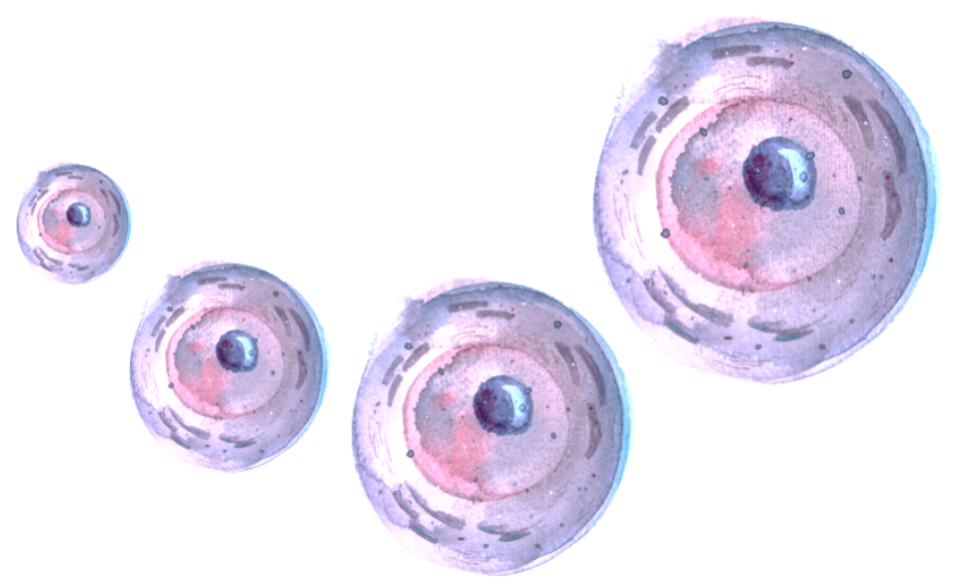


-110 - 


\section{Conclusions}

The main conclusions extracted from this doctoral thesis are listed below:

1. Circulating HSCs are able to reconstitute damaged BM niches and give rise to long-term cells with multilineage differentiation potential.

2. Repopulation of damaged BM niches by cHSCs involves multiple clones of HSCs.

3. Circulating HSCs that engraft in damaged BM niches have a strong myeloid-bias and a high proliferative rate.

4. The chemokine receptor CXCR2 is expressed on BM HSPCs and is functional, as cells that migrate ex vivo to CXCL1 are able to reconstitute multi-lineage parameters in irradiated mice, in a CXCR2-dependent manner.

5. CXCR2 is important for the egress of HSPCs from the BM into peripheral blood and tissues in vivo.

6. A subset of perivascular cells inside the hematopoietic niche expresses CXCL1.

7. CXCL1 deficiency leads to a reduction in the number of circulating HSCs in vivo.

8. Reductions in cHSCs lead to impaired repopulation of damaged niches, and an aged-like premature hematopoietic phenotype. 
-112 - 


\section{Conclusiones}

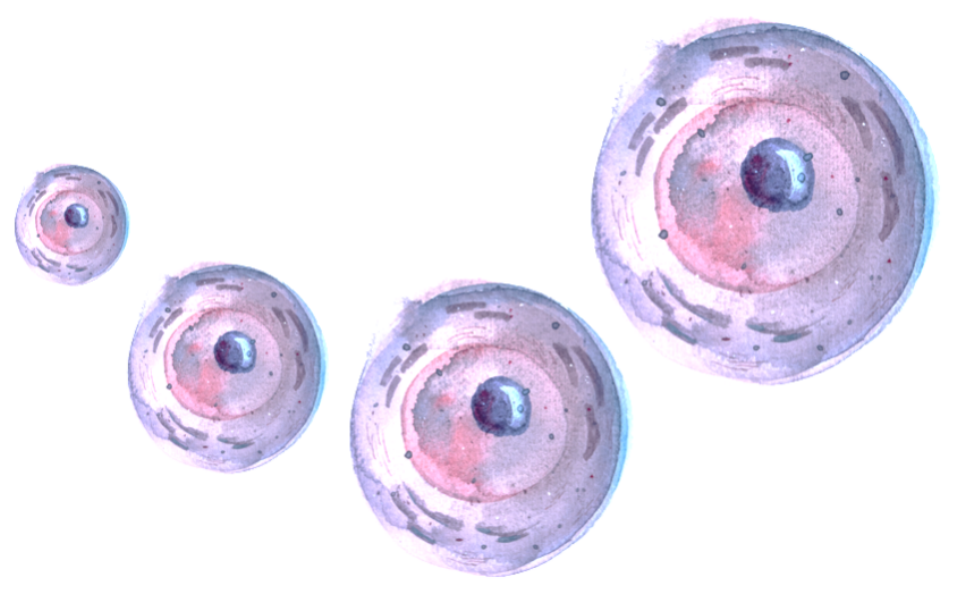


$-114-$

$14-$ 


\section{Conclusiones}

Los resultados presentados en este trabajo permiten extraer las siguientes conclusiones:

1. Las células madre circulantes son capaces de reconstituir nichos dañados y dar lugar a células con potencial de diferenciación hacia los diferentes linajes.

2. La repoblación de nichos dañados mediante células madre circulantes es un proceso que involucra a varios clones de estas células (proceso policlonal).

3. Las células madre circulantes que anidan en los nichos dañados tienen una parcialidad hacia el linaje mieloide y tienen una tasa de proliferación alta.

4. El receptor de quimiocinas CXCR2 se expresa en la superficie de las células madre hematopoyéticas dentro de la médula ósea. Además, es funcional, ya que las células que migran hacia CXCL1 ex vivo son capaces de reconstituir todas las células de la sangre en ratones irradiados, de manera dependiente de CXCR2.

5. CXCR2 es importante para la salida de células madre hematopoyéticas desde la médula ósea hacia la sangre y otros tejidos periféricos in vivo.

6. Un grupo de células perivasculares dentro del nicho hematopoyético expresa el ligando CXCL1.

7. La deficiencia de CXCL1 da lugar a una reducción en el número de células madre circulantes in vivo.

8. La reducción en el número de células madre circulantes da lugar a un defecto en la repoblación de nichos dañados y acelera el envejecimiento de las células madre hematopoyéticas. 
$-116-$ 
Materials and Methods

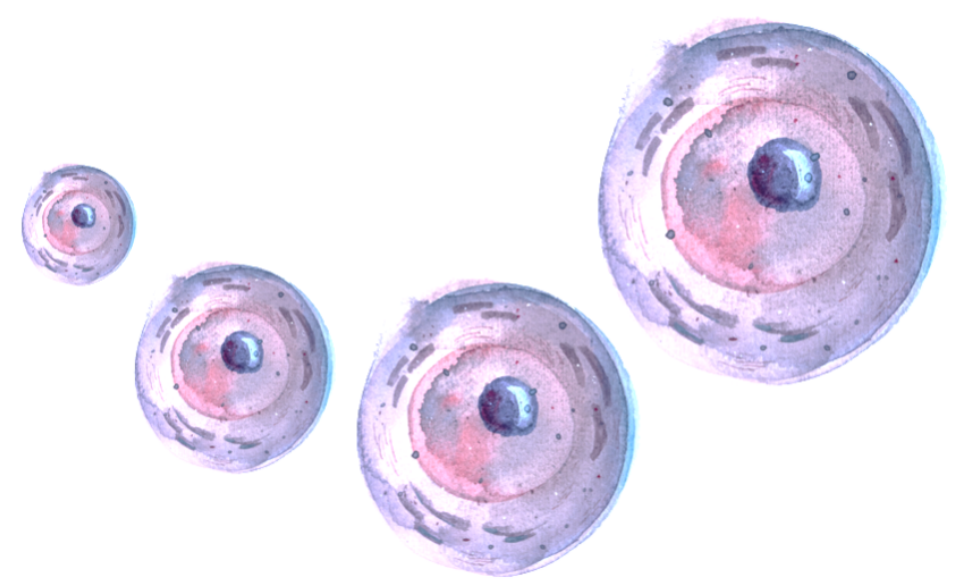


$-118-$ 


\section{Materials and Methods}

\section{Experimental Mice}

All experiments were performed in 7- to 20- week-old male mice in a C57BL/6 genetic background, except where indicated (ageing experiments). Mice were housed in a specific pathogen-free facility at Fundación Centro Nacional de Investigaciones Cardiovasculares (CNIC). Chow and water were available ad libitum. All mice were maintained in a $12 \mathrm{~h}$ light $/ 12 \mathrm{~h}$ darkness schedule. Experimental procedures were approved by the Animal Care and Ethics Committee of the CNIC and local authorities.

In this work, the following experimental mouse models were used:

Table 1. Mouse models used in this work

\begin{tabular}{|c|c|c|c|}
\hline Name & Strain & Phenotype & Reference \\
\hline Cdh5 CREERT2 & Tg (Cdh5-cre/ERT2)1Rha & $\begin{array}{l}\text { Cre recombinase inducible } \\
\text { by tamoxifen expressed } \\
\text { under } \mathrm{Cdh} 5 \text { promotor }\end{array}$ & (235) \\
\hline $\mathrm{CXCL}^{-/-}$ & & & This work \\
\hline CXCL12 $2^{f / f f}$ & B6(FVB)-Cxcl12 $2^{\text {tm1.1Link }}$ & $\begin{array}{l}\text { Cxcl12 gene flanked by flox } \\
\text { sequences }\end{array}$ & (91) \\
\hline CXCL12 ${ }^{\text {GFP }}$ & B6.129P-Cxcl12 $2^{\mathrm{tm} 2 \operatorname{Tng}}$ & $\begin{array}{c}\text { GFP expression in cells that } \\
\text { express CXCL12 }\end{array}$ & $(114)$ \\
\hline $\mathrm{CXCR}^{-/-}$ & B6.129(C)- $118 r^{t m} 1 \mathrm{Mwm}$ & CXCR2 full KO mice & (198) \\
\hline $\mathrm{Cxcr} 2^{\mathrm{fl} / f 1}$ & C57BL/6-Cxcr2 ${ }^{\text {tm1Rmra }}$ & $\begin{array}{l}\text { Cxcr2 gen flanked by flox } \\
\text { sequences }\end{array}$ & (236) \\
\hline $\mathrm{CXCR}^{\Delta \mathrm{N}}$ & Mrp8 $^{\text {CRE; }}$ CXCR2 $2^{f / f l}$ & $\begin{array}{l}\text { Neutrophil deficiency in } \\
\text { Cxcr2 }\end{array}$ & \\
\hline DOCK2-GFP & & $\begin{array}{l}\text { GFP expression under Rac } \\
\text { GTPase promoter control }\end{array}$ & $(237)$ \\
\hline DsRed & $\begin{array}{l}\text { B6. Cg-Tg (CAG-Ds- } \\
\text { Red*MST)1Nagy }\end{array}$ & $\begin{array}{l}\text { DsRed expression under } \\
\text { beta-actin promoter control }\end{array}$ & (238) \\
\hline LepR ${ }^{\text {CRE }}$ & Lepr $^{\text {tm2(Cre)Rck }}$ & $\begin{array}{l}\text { Cre recombinase under } \\
\text { Lep } R \text { promoter }\end{array}$ & $\begin{array}{l}\text { Jackson } \\
\text { Laboratory }\end{array}$ \\
\hline
\end{tabular}




\begin{tabular}{|c|c|c|c|}
\hline Mrp8CRE & $\begin{array}{c}\text { B6. Cg-Tg (-S100A8-cre, - } \\
\text { EGFP)1llw }\end{array}$ & $\begin{array}{l}\text { Cre recombinase under } \\
\text { Mrp8 promoter }\end{array}$ & (239) \\
\hline Rosa26 TdTom & $\begin{array}{l}\text { B6. Cg-Gt (ROSA)- } \\
\text { 26Sor }{ }^{\text {tm14(CAG-tdTomato) Hze }}\end{array}$ & $\begin{array}{l}\text { STOP codon in tdTomato } \\
\text { sequence flanked by loxP } \\
\text { sequences }\end{array}$ & (240) \\
\hline PrX1CRE & Tg (Prrx1-cre)1Cjt & $\begin{array}{l}\text { Cre recombinase under } \\
\operatorname{Prx} 1 \text { promoter }\end{array}$ & $\begin{array}{l}\text { Jackson } \\
\text { Laboratory }\end{array}$ \\
\hline$N G 2^{\mathrm{CRE}}$ & Tg (Cspg4-cre)1rkl & $\begin{array}{l}\text { Cre recombinase under } \\
\text { NG2 promoter }\end{array}$ & $\begin{array}{l}\text { Jackson } \\
\text { Laboratory }\end{array}$ \\
\hline WT & $\mathrm{C} 57 \mathrm{BL} / 6$ & Wild type mice & $\begin{array}{l}\text { Charles } \\
\text { Rivers }\end{array}$ \\
\hline WT & CD45.1 or BL/6. SJLC 57 & $\begin{array}{l}\text { Wild type mice (with a } \\
\text { different isoform of Ptprc, } \\
\text { which encodes for CD45) }\end{array}$ & $\begin{array}{l}\text { Jackson } \\
\text { Laboratory }\end{array}$ \\
\hline
\end{tabular}

\section{Animal procedures}

\section{Parabiosis}

The generation of parabiotic mice permits us to analyze the trafficking of HSPC between two different mice. Parabiosis is a surgical procedure that allows the union (anastomosis) of circulatory systems of two animals. This process creates new angiogenic responses between the tissues of the two parabiotic members after the physical union of the dermis. The procedure was done according to previously described published protocols (2). Animals were anesthetized with a mixture of 7.5\% ketamine (Imalgene, Merial Laboratories, Madrid) and 5\% xylazine (Rompum, Bayer, Leverkusen, Germany), injected intraperitoneally $10 \mu \mathrm{l} / \mathrm{g}$. Then animals were shaved at the corresponding lateral aspects and matching skin incisions were made from the olecranon to the knee joint of each mouse and the subcutaneous fascia was bluntly dissected to create about $0.5 \mathrm{~cm}$ of free skin. The olecranon and knee joints were attached by a single 5-0 polypropylene suture (Lorca Marín, Murcia) and tie, and the dorsal and ventral skins were approximated by continuous suture. After the surgery, parabionts received $0,1 \mathrm{mg} / \mathrm{kg}$ buprenorphine (Buprex, Berkshire, United Kingdom) subcutaneously and let them recover in the recovery chambers where temperature and oxygen pressure were 
controlled. We waited at least 3 weeks after the surgery to take blood and other organs from each parabiotic mice. This system used along the whole study permits us to distinguish between cells that are being produced locally and cells that come from the partner exclusively by the circulation. When parabiosis was performed with irradiation, one of the mice was irradiated immediately before the surgery.

\section{Bone Marrow Chimeras}

To analyze mutant HSPC in the same physiological context as WT HSPC we generated BM chimeras. To do so, femur from both WT and mutant mice were collected and the head of the femur was cut. The BM was perfused with $1 \mathrm{ml} \mathrm{PBS1x}$ in sterile conditions and homogenized gently by pipetting. The cell suspension was centrifuged 5 minutes at $1500 \mathrm{rpm} 4^{\circ} \mathrm{C}$ and the cell pellet was resuspended in $0.5 \mathrm{ml}$ red blood cell (RBC) Lysis Buffer $1 \mathrm{x}$ and incubated for 5 minutes at room temperature (RT). Cells were washed with PBS1 $x$ and bone marrow nucleated cells (BMNC) were counted with the Neubauer Chamber.

To destroy HSPC activity, recipient WT mice were lethally irradiated with two irradiation doses of 6Gy separated by 4 hours. Irradiated recipient mice were intravenously (i.v.) injected with a determined number of cells (indicated in each different experiment) containing an equal mix of WT and mutant cells. BM reconstitution was analyzed 4 weeks after transplantation during 4 months by analyzing the percentage of WT and mutant leukocytes in peripheral blood. At 4 months animals were sacrificed and the BM of transplanted mice was analyzed for HSPC chimerism.

\section{Limiting Dilution Bone Marrow Transplantation Assay}

A limiting dilution assay is an experimental technique for quantifying the proportion of biologically active cells in a larger population. It is a type of doseresponse experiment in which each individual culture allows a negative or positive response. The process of dilution of the dose is typically continued to extinction of the response, or close to it. The rate of positive and negative responses at each dose allows frequency of biologically active cells to be inferred. In stem cell research, this assay consists in determining the frequency of LT-repopulating stem cells in a given BM population. The assay consists in transplanting different doses 
of BM cells into irradiated mice and analyze for LT multilineage reconstitution at 16 weeks after transplantation. Each recipient mouse is scored as positive or negative depending on the percentage of donor reconstitution and Poisson statistics is applied to estimate HSC frequency. Briefly, $10 \mathrm{~K}$ and $25 \mathrm{~K}$ WT or CXCR2 ${ }^{\text {Het }}$ BMNC were i.v. injected together with $3 \times 10^{5}$ supportive WT ${ }^{\text {GFP }}$ cells from Dock2-GFP mice for the survival of the mice, into 6-10 lethally irradiated (two doses of 6Gy, 4h apart) CD45.1 WT mice per dose. Engraftment was determined in the blood 16 weeks after transplantation and mice were scored positive when donor contribution was $>1 \%$. The frequency of repopulating cells was calculated using the ELDA software (http://bioinf.wehi.edu.au/software/elda/).

\section{Homing experiments}

The early migration of HSCs to the BM is commonly defined as homing. To study whether WT or CXCR2 ${ }^{\mathrm{HET}}$ mutant cells had any defect in this process we i.v. inject the same number of WT ${ }^{\text {DSRED }}$ and CXCR2 ${ }^{\text {HET }}$ mutant BM cells into WT recipient mice. The BM of the recipient mice was analyzed for LSK and MP populations by flow cytometry $16 \mathrm{~h}$ after the injection. Furthermore, to study if the homing within the $\mathrm{Cxcl} 1^{\mathrm{KO}}$ niche was impaired, $3 \times 10^{6} \mathrm{WT}^{\mathrm{GFP}}$ cells were i.v. injected and analyzed by flow cytometry $16 \mathrm{~h}$ after injection. The same amount of WTGFP cells were i.v. injected also in WT recipient mice as a control.

\section{Partial Irradiation}

Mice lower limb (femur and tibia) were exposed to 1000 rad X-ray irradiation (MG324, 300 kV, 12.8 mA, Philips, Hamburg, Germany) covering the rest of the body with a lead box. Femur and tibia were analysed at different time points after irradiation by flow cytometry and histology and arms were analysed as a control of non-irradiation. Day 0 indicates before irradiation. These experiments were carried out at Centro de Investigaciones Energéticas, Medioambientales y Tecnológicas (CIEMAT).

\section{Animal Treatments}

\section{Induction of CreERT2 Recombinase with Tamoxifen}


Fluorescent protein expression in ECs was induced in Cdh5 $5^{\text {CreeRT2 }}$ Rosa26 $6^{\text {TdTom }}$ by oral gavage treatment with Tamoxifen ( $5 \mathrm{mg} / \mathrm{mice}$, Sigma) diluted in Corn Oil (Sigma) during 3 consecutive days, at day 7 before analysis.

\section{Cell Culture Assays}

\section{Colony Forming Unit Assay (CFU-C)}

The colony-forming unit cell (CFU-C) assay measures committed myeloid progenitor cell content as a proxy of HSC content directly via an in vitro assay for colonies with methylcellulose media that is supplemented with specific growth factors in sterile conditions. The semi-solid methylcellulose media used to plate the cells consists in all cases in the following: 1,25\% methylcellulose, 30\% FBS, 1\% BSA deionized, 10-4M 2-mercaptoethanol and conditioned media to a final concentration of $12,7 \% \mathrm{v} / \mathrm{v}$ from cell lines WEHI, HM-5 and BHK/MKL. This conditioned media consists in a source of cytokines containing, IL-3, GM-CSF and SCF, necessary for the growth of hematopoietic progenitors. In all cases, cells were plated in duplicates in $35 \mathrm{~mm}$ culture dish (NUNC A/S; Roskilde, Denmark) plates and incubated for 7 days at $37^{\circ} \mathrm{C}$ in $5 \% \mathrm{CO} 2$. Colonies were counted using a low magnification inverted microscope. To visualize partner-derived CFU-C we scored for total and DsRed-positive colonies.

\section{- CFU-C in peripheral blood}

For the purification of hematopoietic progenitors, 100 or $200 \mu \mathrm{l}$ of blood was separated using a density gradient in Lympholyte (Cedarlane Labs). First, blood was resuspended in $2 \mathrm{ml} \mathrm{RPMI}$ medium with $2 \mathrm{mM}$ EDTA and 1\% P/S and carefully transferred to $2 \mathrm{ml}$ of Lympholyte, so the two phases did not mix. Then, samples were centrifuged for 25 minutes at $1200 \mathrm{rpm}$, RT without break and acceleration. The mononuclear fraction in the middle phase was washed once with RPMI and seeded in semi-solid methylcellulose medium. The number of CFU-C was represented as CFU-C by volume (ml) of blood.

\section{- CFU-C in BM}

The BM of one femur was extracted by perfusion with $1 \mathrm{ml}$ of RPMI with $1 \% \mathrm{P} / \mathrm{S}$. BM cells were RBC lysed and counted with the Neubauer Chamber. $1 \times 10^{4}$ cells 
were plated on the methylcellulose. The number of CFU-C was represented as total CFU-C per femur.

\section{- CFU-C in Spleen}

For spleen, the whole tissue was smashed in a 100- $\mu$ m nylon mesh and washed with RPMI 1\% P/S. Cells were centrifuged for 5 minutes at $1500 \mathrm{rpm}$ and RBC were lysed for 10 minutes with 1x RBC lysis buffer. Cells were then washed with RPMI and $1 \%$ was plated to avoid saturation of the culture. The total number of CFU-C was represented in relation to the total present in the whole spleen.

\section{- CFU-C in Liver}

Livers were processed in the same way as described for the spleen. A $5 \%$ of the total liver was transferred to a Percoll gradient prepared at $36 \%$ for the total elimination of hepatocytes. The cell fraction obtained from the purification was washed with RPMI 1\% P/S and plated in the methylcellulose. The total number of CFU-C was represented in relation to the total present in the whole liver.

\section{Mesenchymal Stem Cell Culture Assays}

For the isolation of primary BM cells for the culture of MSCs, femora and tibiae were crushed and digested in HBSS with Liberase $(1 \mathrm{U} / \mathrm{ml}$, Roche) and DNase I $\left(1 \mathrm{mU} / \mathrm{ml}\right.$, Sigma) for 40 minutes at $37^{\circ} \mathrm{C}$. Then, BM suspensions were filtered, and RBC lysed for 5 minutes at RT.

For the differentiation assays and MSC limiting dilution assays, cells were seeded in serial dilutions (400-50K) in 48-well culture plates with DMEM media (Gibco) supplemented with $10 \%$ FBS (Sigma) and 1\% penicillin-streptomycin (Gibco). Cultures were kept at $37^{\circ} \mathrm{C}$ with $5 \% \mathrm{CO}_{2}$ in a water-jacketed incubator and left untouched for 1 week to allow cell attachment. One-half medium changes were performed every 2 days for one more week. Thereafter, wells were scored for total or Tomato positive colonies (for Poisson statistics) using the Nikon Time-Lapse microscope and at that point DMEM medium was switched to differentiation medium to induce production of adipocytes (StemPro ${ }^{\mathrm{TM}}$ Adipogenesis Differentiation Kit, Thermo Fisher) or osteoblasts (StemPro ${ }^{\text {TM }}$ Osteogenesis Differentiation Kit, Thermo Fisher). 


\section{- Adipocytes}

After 1 week under differentiating conditions adipocytes were stained with Oil Red O (ORO; Sigma) as follows: cells were washed with PBS once and fixed with $10 \%$ Formaldehyde for 10 minutes at RT. ORO working solution was prepared as a $3: 2$ dilution in bidistilled water of a $5 \mathrm{mg} / \mathrm{ml}$ ORO solution in isopropanol (Sigma) and filtered $10 \mathrm{~min}$ later. Cells were incubated for $10 \mathrm{~min}$ with ORO working solution and rinsed four times with water.

\section{- Osteoblasts}

After 21 days under differentiating conditions, osteoblasts were stained with Alizarin Red (Sigma) as follows: cells were washed with PBS once and fixed with $10 \%$ Formaldehyde for 10 minutes at RT. Cells were incubated with a $2 \%$ Alizarin

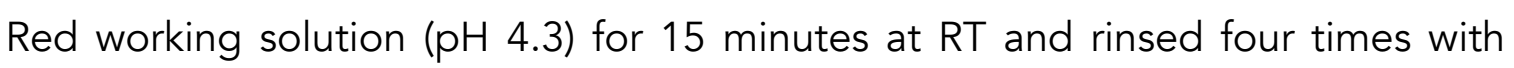
water. Plates were maintained in water at $4^{\circ} \mathrm{C}$ and imaging of each positive well was performed using the Olympus PALM microscope.

\section{Chemotaxis Assays}

For in vitro chemotaxis assays, total BM cells were harvested as previously described and RBC were lysed. $1 \times 10^{6}$ BMNC cells were plated in $6.5 \mathrm{~mm}$ polycarbonate transwells with $5 \mu \mathrm{m}$ pores (Corning, Corning, USA) in RPMI containing $0.5 \%$ BSA. In the bottom well, a single chemokine was added to allow chemotactic migration: $25 \mathrm{ng} / \mathrm{ml} \mathrm{CXCL12} \mathrm{(R \& D),} 80 \mathrm{ng} / \mathrm{ml} \mathrm{CXCL1} \mathrm{(R \& D)} \mathrm{or} \mathrm{RPMI}$ media as negative control. Transwells were incubated $2 \mathrm{~h}$ at $37^{\circ} \mathrm{C}$ and transmigrated cells were harvested from the bottom well and stained for flow cytometry analyses, plated for CFU-C or used as donor cells for BM transplantation. In the case where WT and CXCR2 ${ }^{\mathrm{KO}} \mathrm{BM}$ cells were co-culture together for migration, $5 \times 10^{5} \mathrm{BMNC}$ cells from each group were mixed and plated in the transwells. For BM transplantation after chemotaxis, transmigrated cells were collected and $3 \times 10^{5}$ helper GFP reporter cells were added.

\section{Flow Cytometry and Cell Sorting}

All cytometric analyses were done in a LSRII Fortessa (BD Biosciences) or Canto HTS 3L (BD) equipped with DIVA software (BD). Sorting experiments were performed in a FACS Aria (BD). All the analyses were performed at the Celomic 
Unit at Centro Nacional de Investigaciones Cardiovasculares (CNIC). The FlowJo software (FlowJo LLC, Ashland, OR) was used to analyze the data. All the antibodies used are listed in Table 2. To determine the purity of the cells obtained or quantify the populations of interest, cells were incubated with specific cell surface antibodies (Table 2) during 15-30 minutes at $4^{\circ} \mathrm{C}$ and protected from light. The excess of antibody was removed by washing the cells with $1 \mathrm{ml}$ of a buffer containing EDTA 0.5M, FBS 0.5\% (PEB) and centrifuging for 5 minutes at 1500 rpm $4^{\circ} \mathrm{C}$. In the staining's were biotinylated antibodies were used, after washing the primary antibody the cells were incubated with streptavidin antibody conjugated to fluorochromes (Table 3 ) for 15 minutes at $4^{\circ} \mathrm{C}$ protected from light. After the second washing, cells were resuspended in a PEB solution with DAPI (1/10000) (Life Technologies). When total number of cells were going to be analyzed cells were resuspended in a PEB solution with DAPI and True Count Beads (BD).

\section{Preparation of single cell suspensions from tissues and staining}

\section{- Hematopoietic Stem and Progenitor Cells in the BM}

For the analysis of HSPC populations in the BM, both femur and tibia were flushed with $1 \mathrm{ml}$ PEB into an Eppendorf tube. The cell suspension was centrifuged for 5 minutes, $1500 \mathrm{rpm}, 4^{\circ} \mathrm{C}$ and the pellet was resuspended in $0.5 \mathrm{ml} 1 \times R B C$ Lysis Buffer for 5 minutes at RT. Cells were washed with PEB and a fraction of cells was taken for antibody staining. For the identification of HSPC cells were stained with antibodies that recognize antigens from the hematopoietic lineage such as antiCD3, B220, Ter119, Mac-1 and Gr-1 (Lineage markers or LIN) together with Sca$1(1: 100)$ and $c-K i t$ (1:100). The population enriched for HSC was identified as

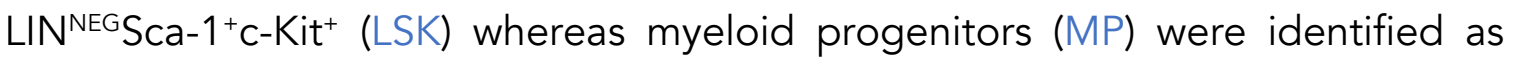
LIN ${ }^{N E G S C a-}{ }^{N E G} \mathrm{C}-\mathrm{Kit}^{+}$. For the identification of more primitive HSCs, we stained for CD48 (1:200) and CD150 (1:50) together with the previous markers. Within the LSK population, the most primitive progenitors or "long-term" (LT-HSC) were

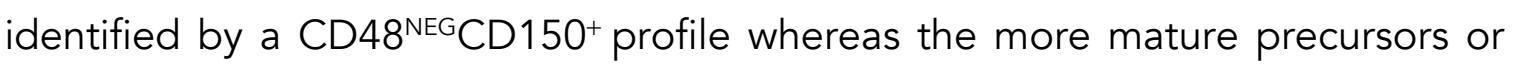

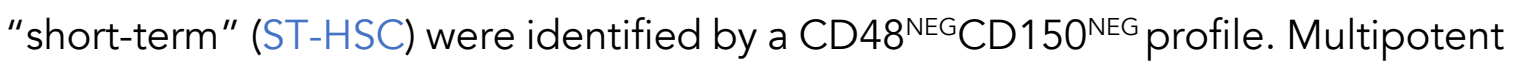
progenitors (MPP) were identified in the same panel as CD48+CD150 NEG (Figure 2 in Introduction). For the identification of more specific myeloid progenitors in the BM we stained cells with the previous antibodies mentioned, LIN, Sca-1 and c-Kit 
together with CD16/32 (1:200) and CD34 (1:50). The granulocyte/macrophage

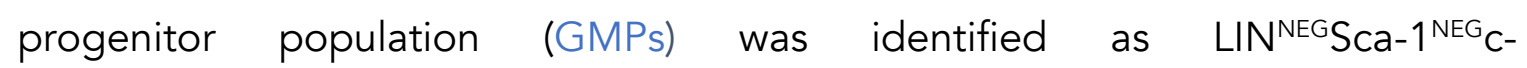
$\mathrm{Kit}^{+} \mathrm{CD} 16 / 32^{+} \mathrm{CD} 34^{+}$, common myeloid progenitors (CMPs) were identified as

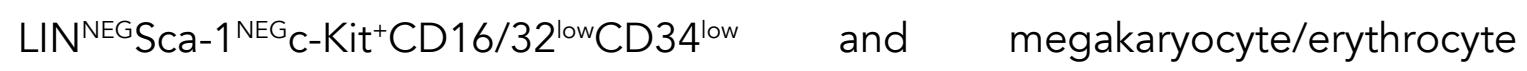

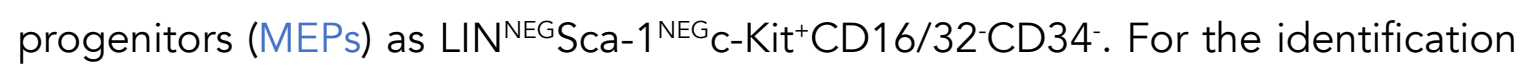
of common lymphoid progenitors (CLPS) we stained for LIN, Sca-1 and c-Kit together with CD135 (Flt3) (1:50) and CD127 (IL-7R) (1:50) and were identified as LIN $^{\text {NEGSCa- }}{ }^{\text {low }_{\text {C-Kit }}}{ }^{\text {low } C D 127+}$ CD $135^{+}$(Figure 2 in Introduction).

\section{- Endothelial and Stromal Niche Populations in the BM}

To identify BM niche populations such as endothelial, stromal or perivascular cells femurs were digested in a HBSS1x solution containing Liberase $(1 \mathrm{U} / \mathrm{ml}$, Roche) and DNase I $\left(10 \mathrm{mU} / \mathrm{ml}\right.$, Sigma) for 40 minutes at $37^{\circ} \mathrm{C}$. After digestion, single-cell suspensions were obtained by gentle pipetting and mechanical dissociation of the remaining pieces through $70 \mu \mathrm{m}$-cell strainers (BD Falcon). Single-cell suspensions were washed with PEB and lysed with RBC1x for 5 minutes at RT. Cells were then washed, and a fraction was stained with the following antibodies for 30 minutes at $4^{\circ} \mathrm{C}$. For identifying niche components cells were stained with anti-CD45, anti-TER-119, anti-CD31 and anti-Sca-1. Stroma was identified by CD45-TER-119-CD31- and endothelial cells by CD45-TER-119-CD31+Sca-1+ profile.

\section{- Cell Cycle Assay using Ki67 in the BM}

To analyse cell cycle on hematopoietic progenitors, BM suspensions were surface stained as mentioned above (Hematopoietic Stem and Progenitor Cells) and subsequently fixed and permeabilized using the Foxp3/Transcription Factor Staining Buffer Set (Thermo Fisher) according to manufacturer's instructions. The cells were then stained for 30 minutes at $4^{\circ} \mathrm{C}$ with an eFluor 660-conjugated Ki67 antibody and DAPI.

\section{- Mature Leukocytes in Peripheral Blood}


For assessment of blood leukocytes, $0.3 \mathrm{ml}$ of blood were stained with anti-CD3e (1:100), anti-B220 (1:300), anti-Ly6G (1:200) and anti-CD11b (1:200) for $15 \mathrm{~min}$ at $4^{\circ} \mathrm{C}$. After, red blood cells were lysed with RBC Lysis Buffer for 10 minutes at RT and washed twice with PEB buffer. Cells were finally resuspended in $200 \mu$ PEB with True Count beads.

\section{- Estimation of Cell Numbers}

True count beads (BD) were prepared at a concentration of 10,000 beads per $\mathrm{ml}$ in PEB buffer. 200-300 $\mu$ l of this PEB buffer containing beads was added to single cell suspensions stained for flow cytometry as indicated above. A fixed number of beads were acquired in each experiment and the absolute number of cells were calculated as follows:

Cells/beads $\times 10,000$ beads/ $\mathrm{ml}$ PEB buffer $\times$ 0,3 ml PEB beads/fraction of tissue digested or stained.

Table 2. Antibody list for flow cytometry analysis

\begin{tabular}{|c|c|c|}
\hline Antibody & Clone & Company \\
\hline CD3ع- PerCP-Cy5.5 & $145-2 C 11$ & BioLegend \\
\hline CD3e- FITC & $145-2 C 11$ & BioLegend \\
\hline CD11b-BV510 & $\mathrm{M} 1 / 70$ & BioLegend \\
\hline CD31 (PECAM-1)-PE-Cy7 & 390 & eBioscience \\
\hline CD31 (PECAM-1)-APC & MEC13.3 & BioLegend \\
\hline CD34 efluor $\AA 660$ & RAM34 & Thermo Fisher \\
\hline CD36-APC & HM36 & BioLegend \\
\hline CD41-APC & eBioMWReg30 & eBioscience \\
\hline CD45-V450 & $3 F-11(R \cup O)$ & $\mathrm{BD}$ \\
\hline CD45R-APC-Cy7 & $A 3-6 B 2$ & $\mathrm{BD}$ \\
\hline CD48-APC-Cy7 & HM48-1 & BioLegend \\
\hline CD51-Biotin & RMV-7 & BioLegend \\
\hline CD54 (ICAM1)-APC & YN1/1.7.4 & BioLegend \\
\hline CD61-APC & 2C9.G2 & BioLegend \\
\hline CD62E (E-selectin)-Biotin & 10E9.6 & $\mathrm{BD}$ \\
\hline CD106 (VCAM1)-APC & 429 & BioLegend \\
\hline CD117 (c-kit)-PerCP-Cy5.5 & 2B8 & BioLegend \\
\hline CD117 (c-kit)-PE-Cy7 & 2B8 & $\mathrm{BD}$ \\
\hline CD150 (SLAM)-Alexa Fluor 488 & TC15-12F/2.2 & BioLegend \\
\hline
\end{tabular}




\begin{tabular}{|l|c|c|}
\hline CD150 (SLAM)-BV510 & TC15-12F/2.2 & BioLegend \\
\hline CD150 (SLAM)-Purified & TC15-12F/2.2 & BioLegend \\
\hline CCR2-PE & 475301 & R\&D systems \\
\hline CXCR2-PerCP-Cy5.5 & SA044G4 & BioLegend \\
\hline CXCR2-APC & SA044G4 & BioLegend \\
\hline CXCR4-PE & 2B11 & BD \\
\hline Ly-6A/E (Sca-1)-FITC & D7 & eBioscience \\
\hline Ly-6A/E (Sca-1)-PE-Cy7 & D7 & BD \\
\hline Ly6C-APC & HK1.4 & BioLegend \\
\hline Ly6G-Dylight 450 & 1A8 & BioXcell (conjugated in- house) \\
\hline Lineage cocktail & Several clones & BD \\
\hline Ki-67-e660 & SolA15 & Thermo Fisher \\
\hline RFP & Polyclonal & Rockland \\
\hline PDGFRa APC & APA5 & Thermo Fisher \\
\hline TER-119-Pacific Blue & Clone TER-119 & BioLegend \\
\hline
\end{tabular}

Table 3. Streptavidin list for flow cytometry analysis

\begin{tabular}{|l|c|c|}
\hline \multicolumn{1}{|c|}{ Antibody } & Clone & Company \\
\hline Streptavidin-DyLight 405 & $016-470-084$ & Thermo Fisher \\
\hline Streptavidin-488 & 454057 & Thermo Fisher \\
\hline Streptavidin APC-eFluor780 & $47-4317-82$ & eBioscience \\
\hline Streptavidin PE & & \\
\hline
\end{tabular}

\section{Molecular and Bioinformatics Analysis}

\section{RNA Isolation, Reverse Transcription and Real-Time PCR}

Total RNA was extracted from sorted cells with the RNA Extraction RNeasy Plus Mini-kit (QIAGEN) and first-strand cDNA was synthesized using a High Capacity cDNA Reverse Transcription Kit (Applied Biosystems; Carlsbad, CA) according to the manufacturer's protocol. Real-time quantitative PCR was carried out in an Applied Biosystems 7900HT Fast Real-Time PCR thermocycler using SYBR Green (4367659, Thermo Fisher Scientific). Expression was normalized to the expression of the $36 \mathrm{~b} 4$ housekeeping gene and all values were multiplied by the same arbitrary number for graphical purposes. Primer sequences are listed below:

\begin{tabular}{|c|c|c|}
\hline Gene & Primer orientation & Sequence \\
\hline \multirow{2}{*}{ 36B4 } & Forward & ACTGGTCTAGGACCCGAGAAG \\
\cline { 2 - 3 } & Reverse & TCCCACCTTGTCTCCAGTCT \\
\hline
\end{tabular}




\begin{tabular}{|c|c|c|}
\hline \multirow{2}{*}{ Cxcl1 } & Forward & GTCAGTGCCTGCAGACCATG \\
\cline { 2 - 3 } & Reverse & GGCTATGACTTCGGTTTGGG \\
\hline \multirow{2}{*}{ Cxcl12 } & Forward & TGCATCAGTGACGGTAAACCA \\
\cline { 2 - 3 } & Reverse & TTCTTCAGCCGTGCAACAATC \\
\hline \multirow{2}{*}{ Kitl } & Forward & CCCTGAAGACTCGGGCCTA \\
\cline { 2 - 3 } & Reverse & CAATTACAAGCGAAATGAGAGCC \\
\hline
\end{tabular}

Clonal analysis of circulating HSC

\section{Vector production}

SIN.LV.PGK.GFP.PRE vector stock was prepared as previously described (241). Briefly, concentrated LV stocks pseudotyped with the VSV.G envelope were produced by transient co-transfection of 4 plasmids in 293T cells and tittered as described (241).

\section{Transduction of hematopoietic progenitors}

Eight-week-old C57BL6/J mice were killed by $\mathrm{CO}_{2}$ inhalation, and $\mathrm{BM}$ was harvested by flushing femurs and tibiae with PBS-2\% FBS (FBS; Invitrogen). Lin NEG cells were purified using Lineage Cell Depletion Kit (Miltenyi Biotec) according to the manufacturer's instructions. Cells were then cultured in serum-free StemSpan medium (StemCell Technologies) containing penicillin, streptomycin, glutamine and a combination of mouse cytokines $(20 \mathrm{ng} / \mathrm{ml} \mathrm{IL-3,100} \mathrm{ng/ml} \mathrm{SCF,} 100 \mathrm{ng} / \mathrm{ml}$ Flt-3L, $50 \mathrm{ng} / \mathrm{ml}$ TPO all from Peprotech), at a concentration of $10^{6} \mathrm{cell} / \mathrm{s} / \mathrm{ml}$. Lin ${ }^{\text {NEG }}$ cells were pre-stimulated for 2-3 hours and then infected with the SINLV.PGK.GFP (MOI 100, $10^{8} \mathrm{TU} / \mathrm{ml}$ ). 12 hours after infection cells were washed and $5 \times 10^{5} \mathrm{BM}$ derived Lin $^{N E G}$ cells were injected into lethally irradiated recipient animals. A sample of cells was kept for 14 days in culture to assess GFP expression by FACS analysis and for genomic DNA extraction procedures.

For transplantation, 8-week-old wild-type female C57BL6/J mice were lethally irradiated $(1000 \mathrm{rad})$ and injected in the tail vein with $5 \times 10^{5}$ cells/mouse. FACS analysis was performed using lineage-specific antibodies (BD Biosciences Pharmingen) against CD11b, CD19, CD3 on cells obtained from blood collected at 6 weeks (and other time points) after transplant and analysed with FCS Express 3 software (De Novo Software). After 6 weeks of transplantation, transplanted 
mice containing barcoded library were surgically joined in parabiosis with sublethally irradiated mice (600 rad, to partially open BM niche) and left in parabiosis for 4 weeks. After 4 weeks, mice were surgically separated, and the BM and spleen of the "donor" mice was taken for clonal analysis. The "recipient" mouse was bled every 4 weeks, FACS sorted for the myeloid and lymphoid-GFP+ ${ }^{+}$population $\left(\mathrm{CD} 11 \mathrm{~b}^{+} \mathrm{GFP}^{+}\right.$and $\left.\mathrm{CD} 19 \mathrm{GFP}^{+}\right)$coming from the "donor" mice and send for clonal analysis. At the end of the experiment (16 weeks after parabiosis separation), femur, tibia, sternum, humerus and spleen from "recipient" mice was taken for analysis.

\section{Vector copy number (VCN) analysis}

Genomic DNA was extracted from cultured cells and tissues (BM, spleen) using the QIAGEN tissue DNA kit (QIAGEN). ddPCR analysis was performed with probes complementary to mouse genomic RPP30 (BIORAD, CAT10031255) and common $\boldsymbol{\psi}$-signal region of LV. VCN was determined as the ratio between the relative amounts of $L V$ versus total DNA evaluated by RPP30.

\section{Retrieval of integration sites (IS) from cell DNA}

For the retrieval of vector IS from genomic DNA, we adopted a sonication-based linker-mediated (LM) PCR method previously described (242, 243). Briefly, genomic DNA was sheared using a Covaris E220 Ultrasonicator (Covaris Inc., Woburn (MA)), generating fragments with an average size of $1000 \mathrm{bp}$. The fragmented DNA was then split in 3 technical replicates and subjected to end repair and $3^{\prime}$ adenylation using the NEBNext ${ }^{\circledR}$ Ultra ${ }^{\mathrm{TM}}$ DNA Library Prep Kit for Illumina ${ }^{\circledR}$ (New England Biolabs, Ipswich, MA.), and then ligated (DNA Technologies ligation kit, Skokie, IL.) to the two linker cassettes (LC) containing: sequence barcode, used for sample identification, and all the sequences required for Illumina paired end sequencing, tracked within our laboratory information management (244). Ligation products were then subjected to two rounds of exponential PCR and finally, the amplification products were sequenced using the Illumina Myseq/HiSeq platform (Illumina, San Diego, CA.).

\section{Analysis of Integration Sites}


Sequencing reads were processed by a dedicated bioinformatics pipeline (VISPA2) as previously described (244). Briefly, paired sequence reads were filtered for quality standards, barcodes identified for sample de-multiplexing of the sequence reads, the cellular genomic sequence mapped on the mouse (Mouse Genome_mm9) and the nearest RefSeq gene assigned to each unambiguously mapped integration site. For the quantification of the abundance of each clone we adopted an estimation method previously described (245) and implemented in the R package "SonicLength". This method estimates the abundance of each IS by counting the number of different DNA fragments containing the same vector/cell genome junctions and flanked by a genomic segment variable in size depending on the shear site position that is unique for each different cell genome present in the starting cell population. Therefore, the number of different shear sites assigned to an IS is proportional to the initial number of contributing cells, allowing to estimate the clonal abundance in the starting sample and avoiding the biases introduced by PCR amplification. As described, Sonic Length allows to correct for shear site saturation event that can impact on the quantification of highly abundant clones.

The entropy Shannon Diversity Index (H-index) has been used as a mathematical measure of the diversity of the transduced population (195). The complexity for each lineage and samples of our IS dataset was measured taking into account both the total number of different IS and their relative contribution (number of genomes per IS), thus considering the richness and evenness of the studied population.

Chao and Petersen-Schnabel models were used to estimate population size (194, 246). The cited models are based on capture-recapture methods, that are able to estimate the overall population size by exploiting repeated sampling (over time and/or in different places) of marked elements (animals or cells) and accounting for the number of shared elements among samplings. In gene therapy, population size estimators, such as these have been widely used to estimate the number of active repopulating HSC as lower bound estimation of the population size (193, 247).

Bulk RNA sequencing and analysis 


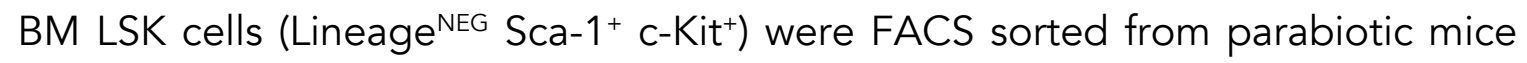
with typical purities $>95 \%$. For RNA extraction from sorted LSK cells the Pico Pure RNA Isolation Kit was used (KIT0214, Thermo Fisher). 0.2 - 0.4 ng of total RNA were used to amplify the cDNA using the SMART-Seq v4 Ultra Low Input RNA Kit (Clontech-Takara). $1 \mathrm{ng}$ of amplified cDNA was used to generate barcoded libraries using the Nextera XT DNA library preparation kit (Illumina). cDNA was fragmented and adapters added in a single reaction followed by an amplification and clean up. The size of the libraries was checked using the Agilent 2100 Bioanalyzer High Sensitivity DNA chip and their concentration was determined

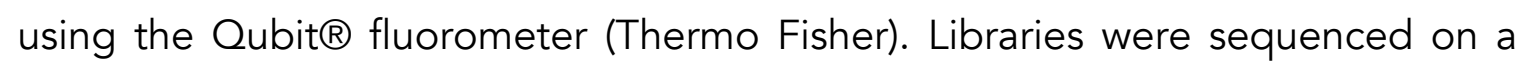
HiSeq2500 (Illumina) to generate 60 bases single end reads. The RNA sequencing experiments were performed at the Genomics Unit at CNIC. Read quality was determined with the application FastQC v0.11.5 (248). For data analysis, sequencing adaptor contaminations were removed from reads using Cutadapt v1.7.1 (249) and the resulting reads were mapped on the transcriptome (GRCm38 Ensembl gene-build 84) and quantified using RSEM v1.2.3 (250). Lowly expressed genes were removed, the rest were considered for statistical analysis with Limma. We considered lowly expressed genes those with no expression in 3 or more samples. Estimated counts from RSEM were normalized and differential expression tested using R package limma v3.32.2 (251). Differentially expressed genes were clustered by k-means. Genes with an adjusted p-value $<0.05$ were considered significant. The data is publicly available in GEO with accession number GSE148172.

\section{Sc-RNAseq data analysis}

The analysed single-cell dataset was released by Baryawno, et al., (190) and is publicly available in GEO with accession number GSE128423. The data counts were processed with the Seurat package v3.1.2 (252) to perform clustering and the differential expression analyses as described in the Seurat Standard Workflow. All code for single cell RNAseq was run on R v3.6.2. Genes with an adjusted pvalue $<0.05$ were considered significant.

\section{Immgen dataset}

Gene expression in BM populations was downloaded from Immgen (http://www.immgen.org/) using their dataset retrieval utility. The populations 
requested were "SC_LTSL_BM", "SC_STSL_BM", "SC_MPP34F_BM", "SC_CMP_BM_DR", "SC_MEP_BM" and "SC_GMP_BM".

\section{Protein Analysis}

We induced inflammation to facilitate detection of CXCL1. Mice were injected once i.p. with $5 \mathrm{mg} / \mathrm{ml}$ of endotoxin (LPS, Sigma) and plasma and bone marrow extracellular fluid (BMEF) were extracted 24 hours later. For plasma extraction, blood was collected in EDTA-coated tubes (Starstedt) and centrifuged $1000 \mathrm{~g}$ for 10 minutes and the supernatant was frozen until ELISA was performed. For BMEF extraction, femora were flushed with $1 \mathrm{ml}$ PBS, centrifuged at $1500 \mathrm{rpm} 5$ minutes at $4^{\circ} \mathrm{C}$, and supernatants frozen. CXCL1 was measured using commercially available ELISA reagents (R\&D Systems; Minneapolis; MN) following manufacturer's instructions. Samples were diluted 1:2 in PBS. For quantification of G-CSF levels in plasma we followed a similar ELISA procedure (R\&D Systems; Minneapolis; MN). Samples were diluted 1:4 in PBS.

\section{Imaging techniques}

\section{Histology}

For immunochemistry on paraffin sections, tissues were dehydrated, and paraffin wax embedded. $4 \mu \mathrm{m}$ sections were deparaffinised and brought to TBS buffer. Standard Haematoxylin and Eosin staining was carried out on the sections using the Leica automated stainer ST5020.

\section{Immunofluorescence staining of BM frozen sections}

For frozen sections of long bones, femur and tibia were fixed in $4 \%$ paraformaldehyde (PFA) overnight at $4^{\circ} \mathrm{C}$. Then, bones were decalcified in a PBS solution with EDTA $0.25 \mathrm{M}$ at $4^{\circ} \mathrm{C}$ for 15 days and thereafter incubated sequentially in 10\%, 20\% and 30\% sucrose/PBS at RT for 1 hour each, embedded and flash frozen in OCT (Tissue-Tek) using dry ice. Frozen sections were prepared $30 \mu \mathrm{m}$ thick with a Cryostat (CM1850, Leica), using Kawamoto's tape transfer method. For immunofluorescence staining, sections were rinsed with PBS and incubated with blocking buffer-containing PBS with 0.25\% Triton X100 (Sigma), $1 \%$ Normal Goat Serum (NGS) (Sigma) and 5\% BSA (Sigma) for $1 \mathrm{~h}$ at room 
temperature. Bones were then stained with antibodies against endomucin (Clone V.7C7, Santa Cruz Biotechnology, 1:200). Primary antibody staining was followed by 3 washes with PBS and 1h incubation at RT with Alexa Fluor 647-conjugated goat anti-rat secondary antibody (Molecular Probes, 1:500) or Alexa Fluor 488conjugated goat anti-rat (Molecular Probes, 1:500), and DAPI (Life Technologies, 1:1000). Slides were mounted with Mowiol 4-88 (MW 31000, Sigma). Images were captured at the Advanced Microscopy unit at CNIC.

\section{Whole-mount imaging of BM}

To measure HSC distance to $\mathrm{Cxcl} 1^{\text {TdTom }}$ cells, we performed whole mount immunostaining and tissue clearing of sternum or long bones, femur and tibia. Mice were euthanized with $\mathrm{CO}_{2}$ and the femurs excised and fixed at $4^{\circ} \mathrm{C}$ overnight in PBS with 4\% PFA. Then, the bones were decalcified as indicated above. Afterwards, the bones were permeabilized in methanol gradients in PBS for 30 $\min$ (PBS > MetOH 50\% > MetOH 80\% > MetOH 100\%). Then, the bones were bleached with Dent's bleach (15\% $\mathrm{H}_{2} \mathrm{O}_{2}, 16.7 \%$ DMSO in MetOH) for $1 \mathrm{~h}$ at RT, and then were rehydrated through descending methanol gradients in PBS (MetOH 80\% > MetOH 50\% > PBS). Then the bones were incubated with blocking buffer containing PBS with $0.3 \%$ Triton X100, 0.2\% BSA, 5\% DMSO, $0.1 \%$ azide and $25 \%$ FBS overnight at $4{ }^{\circ} \mathrm{C}$ with shaking. Afterwards, the bones were stained with anti-RFP, for the endogenous tomato (1:200) and endomucin (1:200) for 2 days at $4{ }^{\circ} \mathrm{C}$ with shaking. After washing for $24 \mathrm{~h}$ in washing buffer (PBS with $0.2 \%$ Triton $\mathrm{X} 100$ and $3 \% \mathrm{NaCl}$ ), tissues were stained with secondary antibodies Alexa Fluor 488-conjugated goat anti-rabbit (Molecular Probes, 1:500) and Alexa Fluor 647-conjugated goat anti-rat (Molecular Probes, 1:500) for 2 days. Then bones were washed for $24 \mathrm{~h}$ in washing buffer, and further stained with an anti-lineage panel cocktail (TER-119, RB6-8C5, RA3-6B2, M1/70, 145-2C11 at 1:50 dilution) and anti-CD41 (1:100) for 2 days at $4^{\circ} \mathrm{C}$ with shaking. After washing for $24 \mathrm{~h}$ in washing buffer the tissues were stained with CD150-PE antibody (1:50) and Streptavidin-405 (1:400) for 2 days at $4^{\circ} \mathrm{C}$ with shaking, and later washed in washing buffer for $24 \mathrm{~h}$. Finally, the bones were dehydrated in $\mathrm{MetOH}$ gradients in $\mathrm{dH}_{2} \mathrm{O}$ using glass containers for $30 \mathrm{~min}$ in each step (MetOH 50\% > MetOH 70\% $>\mathrm{MetOH} 90 \%>3 \times \mathrm{MetOH}$ 100\%), cleared for 30 min in $\mathrm{MetOH}$ with $50 \%$ BABB and afterwards in 100\% BABB (benzyl alcohol, benzyl benzoate 1:2) and imaged in a Leica SP8 X confocal microscopy system coupled to a DMI6000 inverted microscope at the Advanced Microscopy unit at CNIC. 


\section{Intravital Microscopy of Calvaria Bone Marrow}

$2 \times 10^{6}$ Lin $^{N E G}$ c-Kit+ cells were FACS-sorted from WT mice and labelled with the Cell Tracker Deep Red Dye kit (CTDR; Thermo Fisher) following the manufacturer's instructions. Labelled cells were intravenously injected into the BM, which was performed as described (253). Mice were anesthetized and hair in the skullcap was removed using an electric razor. The scalp was incised in the midline to expose the frontoparietal skull, and the conjunctive tissue covering the skull was carefully removed. The mouse's cranium was kept in place using a custom-made stereotactic holder. The mouse thus prepared was positioned under the intravital microscope described above and multipoint 4-dimensional captures every 3 minutes were performed for 2 hours. Blood vessels were visualized using green fluorescent Dextran (Molecular Probes) $(0.1 \mathrm{mg} / \mathrm{mice})$. Distances between HSPC or random points and CXCL1-producing cells were measured using ImageJ ( $\mathrm{NIH}$, Bethesda, MD).

\section{Image Analysis}

\section{Quantification of perivascular cells by immunofluorescence staining}

For the quantification of perivascular cells in immunofluorescence images perivascular cells were firstly defined as cells that were in direct contact with a vessel. These cells were quantified manually using Fiji is just Image $J(N I H)$ and the percentage was calculated among total cells labelled by the reporter fluorescence protein in each mouse line (Prx1, LepR, NG2, Cdh5, CXCL12 and CXCL1 reporter lines). Blood vessels were visualized using endomucin antibody and DAPI was used to stain the nucleus.

\section{Quantification of HSC distances in whole mount}

HSC were defined as $\mathrm{Lin}^{-} \mathrm{CD} 41^{-} \mathrm{CD} 150^{+}$cells in the whole mounts. Random points were projected only on marrow regions, carefully avoiding bone regions, using the $\mathrm{Lin}^{+}$channel as template for random spots generated on the mean intensity range, in comparable numbers to detected HSCs in the same field of view (FOV), using the spots tool of Imaris. Distances from HSCs or random points to $\mathrm{CXCL}^{+}$cells were quantified using the distance transformation channel of 
$\mathrm{CXCL}^{+}$cell-defined surfaces in 3-dimensions, using Imaris (Bitplane AG, Switzerland).

\section{Statistical Analysis}

Data from experiments in this study are shown as the mean values \pm standard error of the mean (SEM) or box and whiskers showing the median and percentiles. For comparisons between two groups, paired or unpaired 2-tailed Student t test was applied. For comparison with more than two groups, we used one-way or two-way analysis of variance (ANOVA) with Bonferroni multigroup post-test or Sidak multigroup post-test for measurement of distances. For the limiting dilution LT-reconstitution assays, we used Poisson's statistics performed with ELDA software (http://bioinf.wehi.edu.au/software/elda/). All statistical analyses were performed with GraphPad Prism software v7 and v8 (GraphPad software, California, USA) and R software (package: Vegan and R-capture for Markrecapture models and Sonic Length for quantification of IS abundance). P values $<0.05$ were considered statistically significant. ${ }^{\star} p<0.05 ;{ }^{\star \star} p<0.01 ;{ }^{\star \star \star} p<0.001$ and non-significant differences (ns) are indicated accordingly. 
$-138-$ 


\section{References}

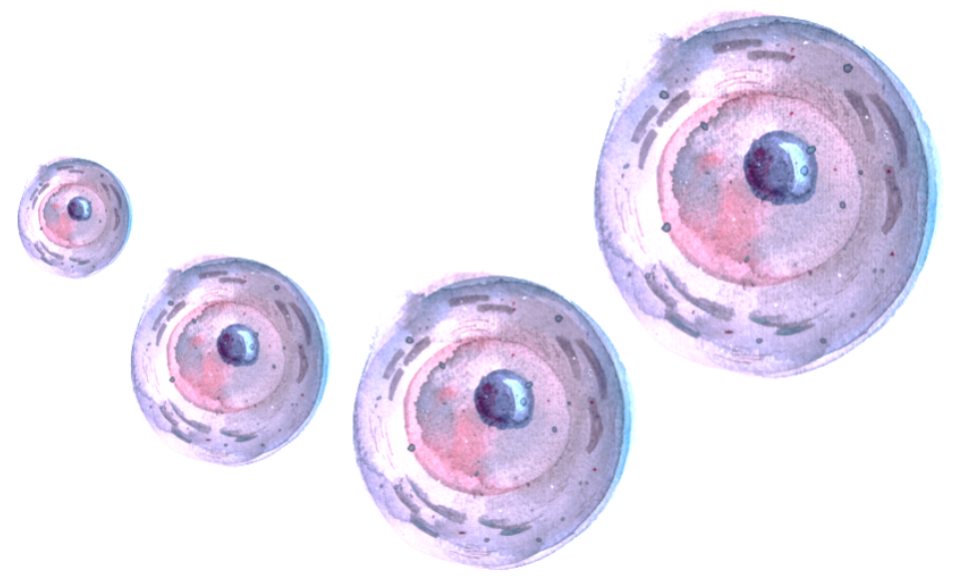


- 140 - 


\section{References}

1. S. Pinho, P. S. Frenette, Haematopoietic stem cell activity and interactions with the niche. Nat. Rev. Mol. Cell Biol. (2019), , doi:10.1038/s41580-019-0103-9.

2. D. E. Wright, A. J. Wagers, A. Pathak Gulati, F. L. Johnson, I. L. Weissman, Physiological migration of hematopoietic stem and progenitor cells. Science (80-. ). (2001), doi:10.1126/science.1064081.

3. S. Méndez-Ferrer, D. Lucas, M. Battista, P. S. Frenette, Haematopoietic stem cell release is regulated by circadian oscillations. Nature (2008), doi:10.1038/nature06685.

4. H. K. A. Mikkola, S. H. Orkin, The journey of developing hematopoietic stem cells. Development (2006), , doi:10.1242/dev.02568.

5. X. Gao, C. Xu, N. Asada, P. S. Frenette, The hematopoietic stem cell niche: From embryo to adult. Dev. (2018), , doi:10.1242/dev.139691.

6. Frenette, Hematopoietic stem cell trafficking. StemBook (2008), doi:10.3824/stembook.1.8.1.

7. A. Wilson, A. Trumpp, Bone-marrow haematopoietic-stem-cell niches. Nat. Rev. Immunol. (2006), , doi:10.1038/nri1779.

8. S. H. Orkin, L. I. Zon, Hematopoiesis: An Evolving Paradigm for Stem Cell Biology. Cell (2008), , doi:10.1016/j.cell.2008.01.025.

9. J. Palis, M. C. Yoder, Yolk-sac hematopoiesis: The first blood cells of mouse and man. Exp. Hematol. (2001), , doi:10.1016/S0301-472X(01)00669-5.

10. K. E. McGrath, J. M. Frame, K. H. Fegan, J. R. Bowen, S. J. Conway, S. C. Catherman, P. D. Kingsley, A. D. Koniski, J. Palis, Distinct Sources of Hematopoietic Progenitors Emerge before HSCs and Provide Functional Blood Cells in the Mammalian Embryo. Cell Rep. (2015), doi:10.1016/j.celrep.2015.05.036.

11. C. Böiers, J. Carrelha, M. Lutteropp, S. Luc, J. C. A. Green, E. Azzoni, P. S. Woll, A. J. Mead, A. Hultquist, G. Swiers, E. G. Perdiguero, I. C. Macaulay, L. Melchiori, T. C. Luis, S. Kharazi, T. Bouriez-Jones, Q. Deng, A. Pontén, D. Atkinson, C. T. Jensen, E. Sitnicka, F. Geissmann, I. Godin, R. Sandberg, M. F. T. R. de Bruijn, S. E. W. Jacobsen, Lymphomyeloid contribution of an immunerestricted progenitor emerging prior to definitive hematopoietic stem cells. Cell Stem Cell (2013), doi:10.1016/j.stem.2013.08.012.

12. M. F. T. R. De Bruijn, N. A. Speck, M. C. E. Peeters, E. Dzierzak, Definitive hematopoietic stem cells first develop within the major arterial regions of the mouse embryo. EMBO J. (2000), doi:10.1093/emboj/19.11.2465.

13. A. Medvinsky, E. Dzierzak, Definitive hematopoiesis is autonomously initiated by the AGM region. Cell (1996), doi:10.1016/S0092-8674(00)80165-8.

14. A. M. Müller, A. Medvinsky, J. Strouboulis, F. Grosveld, E. Dzierzakt, Development of hematopoietic stem cell activity in the mouse embryo. Immunity (1994), doi:10.1016/1074-7613(94)90081-7.

15. I. L. Weissman, Stem cells: Units of development, units of regeneration, and units in evolution. Cell (2000), , doi:10.1016/S0092-8674(00)81692-X. 
16. L. I. Zon, Intrinsic and extrinsic control of haematopoietic stem-cell self-renewal. Nature (2008), , doi:10.1038/nature07038.

17. M. J. Kiel, Ö. H. Yilmaz, T. Iwashita, O. H. Yilmaz, C. Terhorst, S. J. Morrison, SLAM family receptors distinguish hematopoietic stem and progenitor cells and reveal endothelial niches for stem cells. Cell (2005), doi:10.1016/j.cell.2005.05.026.

18. J. E. Till, E. A. McCulloch, A Direct Measurement of the Radiation Sensitivity of Normal Mouse Bone Marrow Cells. Radiat. Res. (1961), doi:10.2307/3570892.

19. A. M. Wu, L. Siminovitch, J. E. Till, E. A. McCulloch, Evidence for a relationship between mouse hemopoietic stem cells and cells forming colonies in culture. Proc. Natl. Acad. Sci. U. S. A. (1968), doi:10.1073/pnas.59.4.1209.

20. E. A. Mcculloch, L. Siminovitch, J. E. Till, Spleen-colony formation in anemic mice of genotype WWv. Science (80-. ). (1964), doi:10.1126/science.144.3620.844.

21. J. Domen, I. L. Weissman, Self-renewal, differentiation or death: Regulation and manipulation of hematopoietic stem cell fate. Mol. Med. Today (1999), , doi:10.1016/S1357-4310(99)01464-1.

22. G. J. Spangrude, S. Heimfeld, I. L. Weissman, Purification and characterization of mouse hematopoietic stem cells. Science (80-. ). (1988), doi:10.1126/science.2898810.

23. M. Osawa, K. I. Hanada, H. Hamada, H. Nakauchi, Long-term lymphohematopoietic reconstitution by a single CD34- low/negative hematopoietic stem cell. Science (80-. ). (1996), doi:10.1126/science.273.5272.242.

24. A. Wilson, G. M. Oser, M. Jaworski, W. E. Blanco-Bose, E. Laurenti, C. Adolphe, M. A. Essers, H. R. Macdonald, A. Trumpp, in Annals of the New York Academy of Sciences (2007).

25. C. Christodoulou, J. A. Spencer, S. C. A. Yeh, R. Turcotte, K. D. Kokkaliaris, R. Panero, A. Ramos, G. Guo, N. Seyedhassantehrani, T. V. Esipova, S. A. Vinogradov, S. Rudzinskas, Y. Zhang, A. S. Perkins, S. H. Orkin, R. A. Calogero, T. Schroeder, C. P. Lin, F. D. Camargo, Live-animal imaging of native haematopoietic stem and progenitor cells. Nature (2020), doi:10.1038/s41586020-1971-z.

26. A. Wilson, E. Laurenti, G. Oser, R. C. van der Wath, W. Blanco-Bose, M. Jaworski, S. Offner, C. F. Dunant, L. Eshkind, E. Bockamp, P. Lió, H. R. MacDonald, A. Trumpp, Hematopoietic Stem Cells Reversibly Switch from Dormancy to Self-Renewal during Homeostasis and Repair. Cell (2008), doi:10.1016/j.cell.2008.10.048.

27. M. B. Bowie, K. D. McKnight, D. G. Kent, L. McCaffrey, P. A. Hoodless, C. J. Eaves, Hematopoietic stem cells proliferate until after birth and show a reversible phase-specific engraftment defect. J. Clin. Invest. (2006), doi:10.1172/JCI28310.

28. M. B. Bowie, D. G. Kent, B. Dykstra, K. D. McKnight, L. McCaffrey, P. A. Hoodless, C. J. Eaves, Identification of a new intrinsically timed developmental checkpoint that reprograms key hematopoietic stem cell properties. Proc. Natl. Acad. Sci. U. S. A. (2007), doi:10.1073/pnas.0700460104. 
29. F. Arai, A. Hirao, M. Ohmura, H. Sato, S. Matsuoka, K. Takubo, K. Ito, G. Y. Koh, T. Suda, Tie2/angiopoietin-1 signaling regulates hematopoietic stem cell quiescence in the bone marrow niche. Cell (2004), doi:10.1016/j.cell.2004.07.004.

30. N. Cabezas-Wallscheid, D. Klimmeck, J. Hansson, D. B. Lipka, A. Reyes, Q. Wang, D. Weichenhan, A. Lier, L. Von Paleske, S. Renders, P. Wünsche, P. Zeisberger, D. Brocks, L. Gu, C. Herrmann, S. Haas, M. A. G. Essers, B. Brors, R. Eils, W. Huber, M. D. Milsom, C. Plass, J. Krijgsveld, A. Trumpp, Identification of regulatory networks in HSCs and their immediate progeny via integrated proteome, transcriptome, and DNA methylome analysis. Cell Stem Cell (2014), doi:10.1016/j.stem.2014.07.005.

31. E. M. Pietras, D. Reynaud, Y. A. Kang, D. Carlin, F. J. Calero-Nieto, A. D. Leavitt, J. A. Stuart, B. Göttgens, E. Passegué, Functionally Distinct Subsets of Lineage-Biased Multipotent Progenitors Control Blood Production in Normal and Regenerative Conditions. Cell Stem Cell (2015), doi:10.1016/j.stem.2015.05.003.

32. A. Sanjuan-Pla, I. C. Macaulay, C. T. Jensen, P. S. Woll, T. C. Luis, A. Mead, S. Moore, C. Carella, S. Matsuoka, T. B. Jones, O. Chowdhury, L. Stenson, M. Lutteropp, J. C. A. Green, R. Facchini, H. Boukarabila, A. Grover, A. Gambardella, S. Thongjuea, J. Carrelha, P. Tarrant, D. Atkinson, S. A. Clark, C. Nerlov, S. E. W. Jacobsen, Platelet-biased stem cells reside at the apex of the haematopoietic stem-cell hierarchy. Nature (2013), doi:10.1038/nature12495.

33. R. Yamamoto, Y. Morita, J. Ooehara, S. Hamanaka, M. Onodera, K. L. Rudolph, H. Ema, H. Nakauchi, Clonal analysis unveils self-renewing lineage-restricted progenitors generated directly from hematopoietic stem cells. Cell (2013), doi:10.1016/j.cell.2013.08.007.

34. A. Rodriguez-Fraticelli, S. Wolock, C. Weinreb, J. Sun, A. Klein, F. Camargo, Clonal analysis of lineage fate in unperturbed hematopoiesis. Exp. Hematol. (2017), doi:10.1016/j.exphem.2017.06.075.

35. K. B. Schoedel, M. N. F. Morcos, T. Zerjatke, I. Roeder, T. Grinenko, D. Voehringer, J. R. Göthert, C. Waskow, A. Roers, A. Gerbaulet, The bulk of the hematopoietic stem cell population is dispensable for murine steady-state and stress hematopoiesis. Blood (2016), doi:10.1182/blood-2016-03-706010.

36. K. Busch, K. Klapproth, M. Barile, M. Flossdorf, T. Holland-Letz, S. M. Schlenner, M. Reth, T. Höfer, H. R. Rodewald, Fundamental properties of unperturbed haematopoiesis from stem cells in vivo. Nature (2015), doi:10.1038/nature14242.

37. F. D. Camargo, Clonal analysis of native hematopoiesis. Exp. Hematol. (2015), doi:10.1016/j.exphem.2015.06.046.

38. S. Massberg, P. Schaerli, I. Knezevic-Maramica, M. Köllnberger, N. Tubo, E. A. Moseman, I. V. Huff, T. Junt, A. J. Wagers, I. B. Mazo, U. H. von Andrian, Immunosurveillance by Hematopoietic Progenitor Cells Trafficking through Blood, Lymph, and Peripheral Tissues. Cell (2007), doi:10.1016/j.cell.2007.09.047.

39. J. W. GOODMAN, G. S. HODGSON, Evidence for stem cells in the peripheral blood of mice. Blood (1962), doi:10.1182/blood.V19.6.702.702. 
40. T. Heidt, H. B. Sager, G. Courties, P. Dutta, Y. Iwamoto, A. Zaltsman, C. Von Zur Muhlen, C. Bode, G. L. Fricchione, J. Denninger, C. P. Lin, C. Vinegoni, P. Libby, F. K. Swirski, R. Weissleder, M. Nahrendorf, Chronic variable stress activates hematopoietic stem cells. Nat. Med. (2014), doi:10.1038/nm.3589.

41. C. Shi, T. Jia, S. Mendez-Ferrer, T. M. Hohl, N. V. Serbina, L. Lipuma, I. Leiner, M. O. Li, P. S. Frenette, E. G. Pamer, Bone Marrow Mesenchymal Stem and Progenitor Cells Induce Monocyte Emigration in Response to Circulating Tolllike Receptor Ligands. Immunity (2011), doi:10.1016/j.immuni.2011.02.016.

42. I. B. Mazo, E. J. Quackenbush, J. B. Lowe, U. H. Von Andrian, Total body irradiation causes profound changes in endothelial traffic molecules for hematopoietic progenitor cell recruitment to bone marrow. Blood (2002), doi:10.1182/blood.V99.11.4182.

43. A. Peled, I. Petit, O. Kollet, M. Magid, T. Ponomaryov, T. Byk, A. Nagler, H. Ben-Hur, A. Many, L. Shultz, O. Lider, R. Alon, D. Zipori, T. Lapidot, Dependence of human stem cell engraftment and repopulation of NOD/SCID mice on CXCR4. Science (80-. ). (1999), doi:10.1126/science.283.5403.845.

44. T. Nagasawa, H. Kikutani, T. Kishimoto, Molecular cloning and structure of a pre-B-cell growth-stimulating factor. Proc. Natl. Acad. Sci. U. S. A. (1994), doi:10.1073/pnas.91.6.2305.

45. A. Peled, V. Grabovsky, L. Habler, J. Sandbank, F. Arenzana-Seisdedos, I. Petit, H. Ben-Hur, T. Lapidot, R. Alon, The chemokine SDF-1 stimulates integrinmediated arrest of CD34+ cells on vascular endothelium under shear flow. J. Clin. Invest. (1999), doi:10.1172/JCI7615.

46. D. E. Wright, E. P. Bowman, A. J. Wagers, E. C. Butcher, I. L. Weissman, Hematopoietic stem cells are uniquely selective in their migratory response to chemokines. J. Exp. Med. (2002), doi:10.1084/jem.20011284.

47. T. Lapidot, A. Dar, O. Kollet, How do stem cells find their way home? Blood (2005), , doi:10.1182/blood-2005-04-1417.

48. Y. S. Tzeng, H. Li, Y. L. Kang, W. G. Chen, W. Cheng, D. M. Lai, Loss of Cxcl12/Sdf-1 in adult mice decreases the quiescent state of hematopoietic stem/progenitor cells and alters the pattern of hematopoietic regeneration after myelosuppression. Blood (2011), doi:10.1182/blood-2010-01-266833.

49. S. Méndez-Ferrer, T. V. Michurina, F. Ferraro, A. R. Mazloom, B. D. MacArthur, S. A. Lira, D. T. Scadden, A. Ma'ayan, G. N. Enikolopov, P. S. Frenette, Mesenchymal and haematopoietic stem cells form a unique bone marrow niche. Nature (2010), doi:10.1038/nature09262.

50. I. Petit, T. Ponomaryov, D. Zipori, L. Tsvee, G-CSF induces stem cell mobilization by decreasing bone marrow SDF-1 and up-regulating CXCR4. Nat. Immunol. (2002), doi:10.1038/ni813.

51. T. Nagasawa, S. Hirota, K. Tachibana, N. Takakura, S. I. Nishikawa, Y. Kitamura, N. Yoshida, H. Kikutani, T. Kishimoto, Defects of B-cell lymphopoiesis and bone-marrow myelopoiesis in mice lacking the CXC chemokine PBSF/SDF-1. Nature (1996), doi:10.1038/382635a0.

52. Y. R. Zou, A. H. Kottman, M. Kuroda, I. Taniuchi, D. R. Littman, Function of the chemokine receptor CXCR4 in heaematopolesis and in cerebellar development. Nature (1998), doi:10.1038/31269. 
53. Q. Ma, D. Jones, P. R. Borghesani, R. A. Segal, T. Nagasawa, T. Kismimoto, R. T. Bronson, T. A. Springer, Impaired B-lymphopoiesis, myelopoiesis, and derailed cerebellar neuron migration in CXCR4- and SDF-1-deficient mice. Proc. Natl. Acad. Sci. U. S. A. (1998), doi:10.1073/pnas.95.16.9448.

54. C. Hart, D. Drewel, G. Mueller, J. Grassinger, M. Zaiss, L. A. Kunz-Schughart, R. Andreesen, A. Reichle, E. Holler, B. Hennemann, Expression and Function of Homing-Essential Molecules and Enhanced In Vivo Homing Ability of Human Peripheral Blood-Derived Hematopoietic Progenitor Cells after Stimulation with Stem Cell Factor. Stem Cells (2004), doi:10.1634/stemcells.22-4-580.

55. J. L. Abkowitz, A. E. Robinson, S. Kale, M. W. Long, J. Chen, Mobilization of hematopoietic stem cells during homeostasis and after cytokine exposure. Blood (2003), doi:10.1182/blood-2003-01-0318.

56. J. L. Granick, S. I. Simon, D. L. Borjesson, Hematopoietic Stem and Progenitor Cells as Effectors in Innate Immunity. Bone Marrow Res. (2012), doi:10.1155/2012/165107.

57. M. H. Kim, J. L. Granick, C. Kwok, N. J. Walker, D. L. Borjesson, F. R. E. Curry, L. S. Miller, S. I. Simon, Neutrophil survival and c-kit+-progenitor proliferation in Staphylococcus aureus-infected skin wounds promote resolution. Blood (2011), doi:10.1182/blood-2010-07-296970.

58. F. Herisson, V. Frodermann, G. Courties, D. Rohde, Y. Sun, K. Vandoorne, G. R. Wojtkiewicz, G. S. Masson, C. Vinegoni, J. Kim, D. E. Kim, R. Weissleder, F. K. Swirski, M. A. Moskowitz, M. Nahrendorf, Direct vascular channels connect skull bone marrow and the brain surface enabling myeloid cell migration. Nat.

Neurosci. (2018), doi:10.1038/s41593-018-0213-2.

59. T. Papayannopoulou, Current mechanistic scenarios in hematopoietic stem/progenitor cell mobilization. Blood (2004), , doi:10.1182/blood-2003-051595.

60. T. Lapidot, I. Petit, Current understanding of stem cell mobilization: The roles of chemokines, proteolytic enzymes, adhesion molecules, cytokines, and stromal cells. Exp. Hematol. (2002), , doi:10.1016/S0301-472X(02)00883-4.

61. L. M. Pelus, S. Fukuda, Peripheral blood stem cell mobilization: The CXCR2 ligand $G R O \beta$ rapidly mobilizes hematopoietic stem cells with enhanced engraftment properties. Exp. Hematol. (2006), , doi:10.1016/j.exphem.2006.04.004.

62. I. G. Winkler, A. R. Pettit, L. J. Raggatt, R. N. Jacobsen, C. E. Forristal, V. Barbier, B. Nowlan, A. Cisterne, L. J. Bendall, N. A. Sims, J. P. Lévesque, Hematopoietic stem cell mobilizing agents G-CSF, cyclophosphamide or AMD3100 have distinct mechanisms of action on bone marrow HSC niches and bone formation. Leukemia (2012), doi:10.1038/leu.2012.17.

63. H. E. Broxmeyer, C. M. Orschell, D. W. Clapp, G. Hangoc, S. Cooper, P. A. Plett, W. C. Liles, X. Li, B. Graham-Evans, T. B. Campbell, G. Calandra, G. Bridger, D. C. Dale, E. F. Srour, Rapid mobilization of murine and human hematopoietic stem and progenitor cells with AMD3100, a CXCR4 antagonist. J. Exp. Med. (2005), doi:10.1084/jem.20041385.

64. W. C. Liles, H. E. Broxmeyer, E. Rodger, B. Wood, K. Hübel, S. Cooper, G. Hangoc, G. J. Bridger, G. W. Henson, G. Calandra, D. C. Dale, Mobilization of 
hematopoietic progenitor cells in healthy volunteers by AMD3100, a CXCR4 antagonist. Blood (2003), doi:10.1182/blood-2003-02-0663.

65. T. Itkin, S. Gur-Cohen, J. A. Spencer, A. Schajnovitz, S. K. Ramasamy, A. P. Kusumbe, G. Ledergor, Y. Jung, I. Milo, M. G. Poulos, A. Kalinkovich, A. Ludin, O. Kollet, G. Shakhar, J. M. Butler, S. Rafii, R. H. Adams, D. T. Scadden, C. P. Lin, T. Lapidot, Distinct bone marrow blood vessels differentially regulate haematopoiesis. Nature (2016), doi:10.1038/nature17624.

66. K. Vandoorne, D. Rohde, H. Y. Kim, G. Courties, G. Wojtkiewicz, L. Honold, F. F. Hoyer, V. Frodermann, R. Nayar, F. Herisson, Y. Jung, P. A. Désogère, C. Vinegoni, P. Caravan, R. Weissleder, D. E. Sosnovik, C. P. Lin, F. K. Swirski, M. Nahrendorf, Imaging the vascular bone marrow niche during inflammatory stress. Circ. Res. (2018), doi:10.1161/CIRCRESAHA.118.313302.

67. J. P. Lévesque, J. Hendy, Y. Takamatsu, P. J. Simmons, L. J. Bendall, Disruption of the CXCR4/CXCL12 chemotactic interaction during hematopoietic stem cell mobilization induced by gcsf or cyclophosphamide. J. Clin. Invest. (2003), doi:10.1172/JCl15994.

68. J. P. Lévesque, J. Hendy, I. G. Winkler, Y. Takamatsu, P. J. Simmons, Granulocyte colony-stimulating factor induces the release in the bone marrow of proteases that cleave c-KIT receptor (CD117) from the surface of hematopoietic progenitor cells. Exp. Hematol. (2003), doi:10.1016/S0301472X(02)01028-7.

69. B. Heissig, K. Hattori, S. Dias, M. Friedrich, B. Ferris, N. R. Hackett, R. G. Crystal, P. Besmer, D. Lyden, M. A. S. Moore, Z. Werb, S. Rafii, Recruitment of stem and progenitor cells from the bone marrow niche requires MMP-9 mediated release of Kit-ligand. Cell (2002), doi:10.1016/S0092-8674(02)007547.

70. J. P. Lévesque, Y. Takamatsu, S. K. Nilsson, D. N. Haylock, P. J. Simmons, Vascular cell adhesion molecule-1 (CD106) is cleaved by neutrophil proteases in the bone marrow following hematopoietic progenitor cell mobilization by granulocyte colony-stimulating factor. Blood (2001), doi:10.1182/blood.V98.5.1289.

71. A. Spiegel, S. Shivtiel, A. Kalinkovich, A. Ludin, N. Netzer, P. Goichberg, Y. Azaria, I. Resnick, I. Hardan, H. Ben-Hur, A. Nagler, M. Rubinstein, T. Lapidot, Catecholaminergic neurotransmitters regulate migration and repopulation of immature human CD34+ cells through Wnt signaling. Nat. Immunol. (2007), doi:10.1038/ni1509.

72. A. Spiegel, A. Kalinkovich, S. Shivtiel, O. Kollet, T. Lapidot, Stem Cell Regulation via Dynamic Interactions of the Nervous and Immune Systems with the Microenvironment. Cell Stem Cell (2008), , doi:10.1016/j.stem.2008.10.006.

73. Y. Katayama, M. Battista, W. M. Kao, A. Hidalgo, A. J. Peired, S. A. Thomas, P. $S$. Frenette, Signals from the sympathetic nervous system regulate hematopoietic stem cell egress from bone marrow. Cell (2006), doi:10.1016/j.cell.2005.10.041.

74. P. Dutta, H. B. Sager, K. R. Stengel, K. Naxerova, G. Courties, B. Saez, L. Silberstein, T. Heidt, M. Sebas, Y. Sun, G. Wojtkiewicz, P. F. Feruglio, K. King, J. N. Baker, A. M. Van Der Laan, A. Borodovsky, K. Fitzgerald, M. Hulsmans, F. 
Hoyer, Y. Iwamoto, C. Vinegoni, D. Brown, M. Di Carli, P. Libby, S. W. Hiebert, D. T. Scadden, F. K. Swirski, R. Weissleder, M. Nahrendorf, Myocardial infarction activates CCR2+ hematopoietic stem and progenitor cells. Cell Stem Cell (2015), doi:10.1016/j.stem.2015.04.008.

75. C. Pittendrigh, V. Bruce, Photoperiodism and Related Phenomena in Plants and Animals. Dly. Rhythm. as coupled Oscil. Syst. their Relat. to thermoPhotoperiod. (1959).

76. R. S. Edgar, E. W. Green, Y. Zhao, G. Van Ooijen, M. Olmedo, X. Qin, Y. Xu, M. Pan, U. K. Valekunja, K. A. Feeney, E. S. Maywood, M. H. Hastings, N. S. Baliga, M. Merrow, A. J. Millar, C. H. Johnson, C. P. Kyriacou, J. S. O’Neill, A. B. Reddy, Peroxiredoxins are conserved markers of circadian rhythms. Nature (2012), doi:10.1038/nature11088.

77. S. Panda, M. P. Antoch, B. H. Miller, A. I. Su, A. B. Schook, M. Straume, P. G. Schultz, S. A. Kay, J. S. Takahashi, J. B. Hogenesch, Coordinated transcription of key pathways in the mouse by the circadian clock. Cell (2002), doi:10.1016/S0092-8674(02)00722-5.

78. D. Druzd, A. De Juan, C. Scheiermann, Circadian rhythms in leukocyte trafficking. Semin. Immunopathol. (2014), , doi:10.1007/s00281-013-0414-4.

79. G. J. M. Maestroni, M. Cosentino, F. Marino, M. Togni, A. Conti, S. Lecchini, G. Frigo, Neural and endogenous catecholamines in the bone marrow. Circadian association of norepinephrine with hematopoiesis? Exp. Hematol. (1998).

80. D. Lucas, M. Battista, P. A. Shi, L. Isola, P. S. Frenette, Mobilized Hematopoietic Stem Cell Yield Depends on Species-Specific Circadian Timing. Cell Stem Cell (2008), , doi:10.1016/j.stem.2008.09.004.

81. C. Scheiermann, Y. Kunisaki, D. Lucas, A. Chow, J. E. Jang, D. Zhang, D. Hashimoto, M. Merad, P. S. Frenette, Adrenergic nerves govern circadian leukocyte recruitment to tissues. Immunity (2012), doi:10.1016/j.immuni.2012.05.021.

82. K. Golan, A. Kumari, O. Kollet, E. Khatib-Massalha, M. D. Subramaniam, Z. S. Ferreira, F. Avemaria, S. Rzeszotek, A. García-García, S. Xie, E. Flores-Figueroa, S. Gur-Cohen, T. Itkin, A. Ludin-Tal, H. Massalha, B. Bernshtein, A. K. Ciechanowicz, A. Brandis, T. Mehlman, S. Bhattacharya, M. Bertagna, H. Cheng, E. Petrovich-Kopitman, T. Janus, N. Kaushansky, T. Cheng, I. Sagi, M. Z. Ratajczak, S. Méndez-Ferrer, J. E. Dick, R. P. Markus, T. Lapidot, Daily Onset of Light and Darkness Differentially Controls Hematopoietic Stem Cell Differentiation and Maintenance. Cell Stem Cell (2018), doi:10.1016/j.stem.2018.08.002.

83. E. Bowers, A. Slaughter, P. S. Frenette, R. Kuick, O. M. Pello, D. Lucas, Granulocyte-derived TNFa promotes vascular and hematopoietic regeneration in the bone marrow. Nat. Med. (2018), doi:10.1038/nm.4448.

84. C. Scheiermann, P. S. Frenette, A. Hidalgo, Regulation of leucocyte homeostasis in the circulation. Cardiovasc. Res. (2015), , doi:10.1093/cvr/cvv099.

85. N. A-Gonzalez, J. A. Quintana, S. García-Silva, M. Mazariegos, A. G. de la Aleja, J. A. Nicolás-ávila, W. Walter, J. M. Adrover, G. Crainiciuc, V. K. Kuchroo, C. V. Rothlin, H. Peinado, A. Castrillo, M. Ricote, A. Hidalgo, Phagocytosis imprints 
heterogeneity in tissue-resident macrophages. J. Exp. Med. (2017), doi:10.1084/jem.20161375.

86. M. Casanova-Acebes, C. Pitaval, L. A. Weiss, C. Nombela-Arrieta, R. Chèvre, N. A-González, Y. Kunisaki, D. Zhang, N. Van Rooijen, L. E. Silberstein, C. Weber, T. Nagasawa, P. S. Frenette, A. Castrillo, A. Hidalgo, Rhythmic modulation of the hematopoietic niche through neutrophil clearance. Cell. 153 (2013), doi:10.1016/j.cell.2013.04.040.

87. R. Schofield, The relationship between the spleen colony-forming cell and the haemopoietic stem cell. A hypothesis. Blood Cells (1978).

88. S. J. Morrison, D. T. Scadden, The bone marrow niche for haematopoietic stem cells. Nature (2014), , doi:10.1038/nature12984.

89. F. G. Kapp, J. R. Perlin, E. J. Hagedorn, J. M. Gansner, D. E. Schwarz, L. A. O'Connell, N. S. Johnson, C. Amemiya, D. E. Fisher, U. Wölfle, E. Trompouki, C. M. Niemeyer, W. Driever, L. I. Zon, Protection from UV light is an evolutionarily conserved feature of the haematopoietic niche. Nature (2018), doi:10.1038/s41586-018-0213-0.

90. L. Ding, T. L. Saunders, G. Enikolopov, S. J. Morrison, Endothelial and perivascular cells maintain haematopoietic stem cells. Nature (2012), doi:10.1038/nature10783.

91. A. Greenbaum, Y. M. S. Hsu, R. B. Day, L. G. Schuettpelz, M. J. Christopher, J. N. Borgerding, T. Nagasawa, D. C. Link, CXCL12 in early mesenchymal progenitors is required for haematopoietic stem-cell maintenance. Nature (2013), doi:10.1038/nature11926.

92. L. Ding, S. J. Morrison, Haematopoietic stem cells and early lymphoid progenitors occupy distinct bone marrow niches. Nature (2013), doi:10.1038/nature11885.

93. A. Cordeiro Gomes, T. Hara, V. Y. Lim, D. Herndler-Brandstetter, E. Nevius, T. Sugiyama, S. Tani-ichi, S. Schlenner, E. Richie, H. R. Rodewald, R. A. Flavell, T. Nagasawa, K. Ikuta, J. P. Pereira, Hematopoietic Stem Cell Niches Produce Lineage-Instructive Signals to Control Multipotent Progenitor Differentiation. Immunity (2016), doi:10.1016/j.immuni.2016.11.004.

94. C. Nombela-Arrieta, G. Pivarnik, B. Winkel, K. J. Canty, B. Harley, J. E. Mahoney, S. Y. Park, J. Lu, A. Protopopov, L. E. Silberstein, Quantitative imaging of haematopoietic stem and progenitor cell localization and hypoxic status in the bone marrow microenvironment. Nat. Cell Biol. (2013), doi:10.1038/ncb2730.

95. M. Acar, K. S. Kocherlakota, M. M. Murphy, J. G. Peyer, H. Oguro, C. N. Inra, C. Jaiyeola, Z. Zhao, K. Luby-Phelps, S. J. Morrison, Deep imaging of bone marrow shows non-dividing stem cells are mainly perisinusoidal. Nature (2015), doi:10.1038/nature15250.

96. Y. Kunisaki, I. Bruns, C. Scheiermann, J. Ahmed, S. Pinho, D. Zhang, T. Mizoguchi, Q. Wei, D. Lucas, K. Ito, J. C. Mar, A. Bergman, P. S. Frenette, Arteriolar niches maintain haematopoietic stem cell quiescence. Nature (2013), doi:10.1038/nature12612.

97. A. Grüneboom, I. Hawwari, D. Weidner, S. Culemann, S. Müller, S. Henneberg, A. Brenzel, S. Merz, L. Bornemann, K. Zec, M. Wuelling, L. Kling, M. Hasenberg, 
S. Voortmann, S. Lang, W. Baum, A. Ohs, O. Kraff, H. H. Quick, M. Jäger, S. Landgraeber, M. Dudda, R. Danuser, J. V. Stein, M. Rohde, K. Gelse, A. I. Garbe, A. Adamczyk, A. M. Westendorf, D. Hoffmann, S. Christiansen, D. R. Engel, A. Vortkamp, G. Krönke, M. Herrmann, T. Kamradt, G. Schett, A. Hasenberg, M. Gunzer, A network of trans-cortical capillaries as mainstay for blood circulation in long bones. Nat. Metab. (2019), doi:10.1038/s42255-0180016-5.

98. D. N. Haylock, B. Williams, H. M. Johnston, M. C. P. Liu, K. E. Rutherford, G. A. Whitty, P. J. Simmons, I. Bertoncello, S. K. Nilsson, Hemopoietic Stem Cells with Higher Hemopoietic Potential Reside at the Bone Marrow Endosteum. Stem Cells (2007), doi:10.1634/stemcells.2006-0528.

99. J. Grassinger, D. N. Haylock, B. Williams, G. H. Olsen, S. K. Nilsson, Phenotypically identical hemopoietic stem cells isolated from different regions of bone marrow have different biologic potential. Blood (2010), doi:10.1182/blood-2009-12-260703.

100. C. E. Bozzini, M. E. Barrio Rendo, F. C. Devoto, C. E. Epper, Studies on medullary and extramedullary erythropoiesis in the adult mouse. Am. J. Physiol. (1970), doi:10.1152/ajplegacy.1970.219.3.724.

101. C. Kim, Homeostatic and pathogenic extramedullary hematopoiesis. J. Blood Med. (2010), doi:10.2147/jbm.s7224.

102. E. Lefrançais, G. Ortiz-Muñoz, A. Caudrillier, B. Mallavia, F. Liu, D. M. Sayah, E. E. Thornton, M. B. Headley, T. David, S. R. Coughlin, M. F. Krummel, A. D. Leavitt, E. Passegué, M. R. Looney, The lung is a site of platelet biogenesis and a reservoir for haematopoietic progenitors. Nature (2017), doi:10.1038/nature21706.

103. Y. Morita, A. Iseki, S. Okamura, S. Suzuki, H. Nakauchi, H. Ema, Functional characterization of hematopoietic stem cells in the spleen. Exp. Hematol. (2011), doi:10.1016/j.exphem.2010.12.008.

104. L. M. Calvi, G. B. Adams, K. W. Weibrecht, J. M. Weber, D. P. Olson, M. C. Knight, R. P. Martin, E. Schipani, P. Divieti, F. R. Bringhurst, L. A. Milner, H. M. Kronenberg, D. T. Scadden, Osteoblastic cells regulate the haematopoietic stem cell niche. Nature (2003), doi:10.1038/nature02040.

105. J. Zhang, C. Niu, L. Ye, H. Huang, X. He, W. G. Tong, J. Ross, J. Haug, T. Johnson, J. Q. Feng, S. Harris, L. M. Wiedemann, Y. Mishina, L. Li, Identification of the haematopoietic stem cell niche and control of the niche size. Nature (2003), doi:10.1038/nature02041.

106. R. S. Taichman, M. J. Reilly, S. G. Emerson, Human osteoblasts support human hematopoietic progenitor cells in in vitro bone marrow cultures. Blood (1996), doi:10.1182/blood.v87.2.518.bloodjournal872518.

107. T. M. Dexter, T. D. Allen, L. G. Lajtha, Conditions controlling the proliferation of haemopoietic stem cells in vitro. J. Cell. Physiol. (1977), doi:10.1002/jcp.1040910303.

108. S. K. Nilsson, H. M. Johnston, G. A. Whitty, B. Williams, R. J. Webb, D. T. Denhardt, I. Bertoncello, L. J. Bendall, P. J. Simmons, D. N. Haylock, Osteopontin, a key component of the hematopoietic stem cell niche and regulator of primitive hematopoietic progenitor cells. Blood (2005), 
doi:10.1182/blood-2004-11-4422.

109. S. Stier, Y. Ko, R. Forkert, C. Lutz, T. Neuhaus, E. Grünewald, T. Cheng, D. Dombkowski, L. M. Calvi, S. R. Rittling, D. T. Scadden, Osteopontin is a hematopoietic stem cell niche component that negatively regulates stem cell pool size. J. Exp. Med. (2005), doi:10.1084/jem.20041992.

110. H. Qian, N. Buza-Vidas, C. D. Hyland, C. T. Jensen, J. Antonchuk, R. Månsson, L. A. Thoren, M. Ekblom, W. S. Alexander, S. E. W. Jacobsen, Critical Role of Thrombopoietin in Maintaining Adult Quiescent Hematopoietic Stem Cells. Cell Stem Cell (2007), doi:10.1016/j.stem.2007.10.008.

111. H. Yoshihara, F. Arai, K. Hosokawa, T. Hagiwara, K. Takubo, Y. Nakamura, Y. Gomei, H. Iwasaki, S. Matsuoka, K. Miyamoto, H. Miyazaki, T. Takahashi, T. Suda, Thrombopoietin/MPL Signaling Regulates Hematopoietic Stem Cell Quiescence and Interaction with the Osteoblastic Niche. Cell Stem Cell (2007), doi:10.1016/j.stem.2007.10.020.

112. B. O. Zhou, L. Ding, S. J. Morrison, Hematopoietic stem and progenitor cells regulate the regeneration of their niche by secreting Angiopoietin-1. Elife (2015), doi:10.7554/eLife.05521.

113. J. Zhu, R. Garrett, Y. Jung, Y. Zhang, N. Kim, J. Wang, G. J. Joe, E. Hexner, Y. Choi, R. S. Taichman, S. G. Emerson, Osteoblasts support B-lymphocyte commitment and differentiation from hematopoietic stem cells. Blood (2007), doi:10.1182/blood-2006-08-041384.

114. T. Sugiyama, H. Kohara, M. Noda, T. Nagasawa, Maintenance of the Hematopoietic Stem Cell Pool by CXCL12-CXCR4 Chemokine Signaling in Bone Marrow Stromal Cell Niches. Immunity (2006), doi:10.1016/j.immuni.2006.10.016.

115. K. Tokoyoda, T. Egawa, T. Sugiyama, B. II Choi, T. Nagasawa, Cellular niches controlling $\mathrm{B}$ lymphocyte behavior within bone marrow during development. Immunity (2004), doi:10.1016/j.immuni.2004.05.001.

116. T. Nagasawa, New niches for B cells. Nat. Immunol. (2008), , doi:10.1038/ni0408-345.

117. Y. Omatsu, T. Sugiyama, H. Kohara, G. Kondoh, N. Fujii, K. Kohno, T. Nagasawa, The Essential Functions of Adipo-osteogenic Progenitors as the Hematopoietic Stem and Progenitor Cell Niche. Immunity (2010), doi:10.1016/j.immuni.2010.08.017.

118. M. Logan, J. F. Martin, A. Nagy, C. Lobe, E. N. Olson, C. J. Tabin, Expression of Cre Recombinase in the developing mouse limb bud driven by a Prxl enhancer. Genesis (2002), doi:10.1002/gene.10092.

119. B. O. Zhou, R. Yue, M. M. Murphy, J. G. Peyer, S. J. Morrison, Leptin-receptorexpressing mesenchymal stromal cells represent the main source of bone formed by adult bone marrow. Cell Stem Cell (2014), doi:10.1016/j.stem.2014.06.008.

120. J. L. Mignone, V. Kukekov, A. S. Chiang, D. Steindler, G. Enikolopov, Neural Stem and Progenitor Cells in Nestin-GFP Transgenic Mice. J. Comp. Neurol. (2004), doi:10.1002/cne.10964.

121. H. A. Himburg, C. M. Termini, L. Schlussel, J. Kan, M. Li, L. Zhao, T. Fang, J. P. Sasine, V. Y. Chang, J. P. Chute, Distinct Bone Marrow Sources of Pleiotrophin 
Control Hematopoietic Stem Cell Maintenance and Regeneration. Cell Stem Cell (2018), doi:10.1016/j.stem.2018.07.003.

122. J. M. Butler, D. J. Nolan, E. L. Vertes, B. Varnum-Finney, H. Kobayashi, A. T. Hooper, M. Seandel, K. Shido, I. A. White, M. Kobayashi, L. Witte, C. May, C. Shawber, Y. Kimura, J. Kitajewski, Z. Rosenwaks, I. D. Bernstein, S. Rafii, Endothelial Cells Are Essential for the Self-Renewal and Repopulation of NotchDependent Hematopoietic Stem Cells. Cell Stem Cell (2010), doi:10.1016/j.stem.2010.02.001.

123. H. A. Himburg, G. G. Muramoto, P. Daher, S. K. Meadows, J. L. Russell, P. Doan, J. T. Chi, A. B. Salter, W. E. Lento, T. Reya, N. J. Chao, J. P. Chute, Pleiotrophin regulates the expansion and regeneration of hematopoietic stem cells. Nat. Med. (2010), doi:10.1038/nm.2119.

124. I. G. Winkler, V. Barbier, B. Nowlan, R. N. Jacobsen, C. E. Forristal, J. T. Patton, J. L. Magnani, J. P. Lévesque, Vascular niche E-selectin regulates hematopoietic stem cell dormancy, self renewal and chemoresistance. Nat. Med. (2012), doi:10.1038/nm.2969.

125. H. Kobayashi, J. M. Butler, R. O’Donnell, M. Kobayashi, B. Sen Ding, B. Bonner, V. K. Chiu, D. J. Nolan, K. Shido, L. Benjamin, S. Rafii, Angiocrine factors from Akt-activated endothelial cells balance self-renewal and differentiation of haematopoietic stem cells. Nat. Cell Biol. (2010), doi:10.1038/ncb2108.

126. M. G. Poulos, P. Guo, N. M. Kofler, S. Pinho, M. C. Gutkin, A. Tikhonova, I. Aifantis, P. S. Frenette, J. Kitajewski, S. Rafii, J. M. Butler, Endothelial Jagged-1 Is necessary for homeostatic and regenerative hematopoiesis. Cell Rep. (2013), doi:10.1016/j.celrep.2013.07.048.

127. P. Guo, M. G. Poulos, B. Palikuqi, C. R. Badwe, R. Lis, B. Kunar, B. Sen Ding, S. Y. Rabbany, K. Shido, J. M. Butler, S. Rafii, Endothelial jagged-2 sustains hematopoietic stem and progenitor reconstitution after myelosuppression. J. Clin. Invest. (2017), doi:10.1172/JC192309.

128. C. Xu, X. Gao, Q. Wei, F. Nakahara, S. E. Zimmerman, J. Mar, P. S. Frenette, Stem cell factor is selectively secreted by arterial endothelial cells in bone marrow. Nat. Commun. (2018), doi:10.1038/s41467-018-04726-3.

129. A. T. Hooper, J. M. Butler, D. J. Nolan, A. Kranz, K. lida, M. Kobayashi, H. G. Kopp, K. Shido, I. Petit, K. Yanger, D. James, L. Witte, Z. Zhu, Y. Wu, B. Pytowski, Z. Rosenwaks, V. Mittal, T. N. Sato, S. Rafii, Engraftment and Reconstitution of Hematopoiesis Is Dependent on VEGFR2-Mediated Regeneration of Sinusoidal Endothelial Cells. Cell Stem Cell (2009), doi:10.1016/j.stem.2009.01.006.

130. M. Leiva, J. A. Quintana, J. M. Ligos, A. Hidalgo, Haematopoietic ESL-1 enables stem cell proliferation in the bone marrow by limiting TGF $\beta$ availability. Nat. Commun. (2016), doi:10.1038/ncomms10222.

131. P. L. Doan, J. L. Russell, H. A. Himburg, K. Helms, J. R. Harris, J. Lucas, K. C. Holshausen, S. K. Meadows, P. Daher, L. B. Jeffords, N. J. Chao, D. G. Kirsch, J. P. Chute, Tie21 bone marrow endothelial cells regulate hematopoietic stem cell regeneration following radiation injury. Stem Cells (2013), doi:10.1002/stem.1275.

132. M. Zhao, J. M. Perry, H. Marshall, A. Venkatraman, P. Qian, X. C. He, J. 
Ahamed, L. Li, Megakaryocytes maintain homeostatic quiescence and promote post-injury regeneration of hematopoietic stem cells. Nat. Med. (2014), doi:10.1038/nm.3706.

133. I. Bruns, D. Lucas, S. Pinho, J. Ahmed, M. P. Lambert, Y. Kunisaki, C. Scheiermann, L. Schiff, M. Poncz, A. Bergman, P. S. Frenette, Megakaryocytes regulate hematopoietic stem cell quiescence through CXCL4 secretion. Nat. Med. (2014), doi:10.1038/nm.3707.

134. P. L. Doan, J. P. Chute, The vascular niche: Home for normal and malignant hematopoietic stem cells. Leukemia (2012), , doi:10.1038/leu.2011.236.

135. S. Méndez-Ferrer, M. Battista, P. S. Frenette, in Annals of the New York Academy of Sciences (2010).

136. S. B. Vasamsetti, J. Florentin, E. Coppin, L. C. A. Stiekema, K. H. Zheng, M. U. Nisar, J. Sembrat, D. J. Levinthal, M. Rojas, E. S. G. Stroes, K. Kim, P. Dutta, Sympathetic Neuronal Activation Triggers Myeloid Progenitor Proliferation and Differentiation. Immunity (2018), doi:10.1016/j.immuni.2018.05.004.

137. S. Yamazaki, H. Ema, G. Karlsson, T. Yamaguchi, H. Miyoshi, S. Shioda, M. M. Taketo, S. Karlsson, A. Iwama, H. Nakauchi, Nonmyelinating schwann cells maintain hematopoietic stem cell hibernation in the bone marrow niche. Cell (2011), doi:10.1016/j.cell.2011.09.053.

138. O. Naveiras, V. Nardi, P. L. Wenzel, P. V. Hauschka, F. Fahey, G. Q. Daley, Bone-marrow adipocytes as negative regulators of the haematopoietic microenvironment. Nature (2009), doi:10.1038/nature08099.

139. R. J. Zhu, M. Q. Wu, Z. J. Li, Y. Zhang, K. Y. Liu, Hematopoietic recovery following chemotherapy is improved by BADGE-induced inhibition of adipogenesis. Int. J. Hematol. (2013), doi:10.1007/s12185-012-1233-4.

140. T. H. Ambrosi, A. Scialdone, A. Graja, S. Gohlke, A. M. Jank, C. Bocian, L. Woelk, H. Fan, D. W. Logan, A. Schürmann, L. R. Saraiva, T. J. Schulz, Adipocyte Accumulation in the Bone Marrow during Obesity and Aging Impairs Stem Cell-Based Hematopoietic and Bone Regeneration. Cell Stem Cell (2017), doi:10.1016/j.stem.2017.02.009.

141. A. Chow, D. Lucas, A. Hidalgo, S. Méndez-Ferrer, D. Hashimoto, C.

Scheiermann, M. Battista, M. Leboeuf, C. Prophete, N. Van Rooijen, M. Tanaka, M. Merad, P. S. Frenette, Bone marrow CD169+ macrophages promote the retention of hematopoietic stem and progenitor cells in the mesenchymal stem cell niche. J. Exp. Med. (2011), doi:10.1084/jem.20101688.

142. I. G. Winkler, N. A. Sims, A. R. Pettit, V. Barbier, B. Nowlan, F. Helwani, I. J. Poulton, N. Van Rooijen, K. A. Alexander, L. J. Raggatt, J. P. Lévesque, Bone marrow macrophages maintain hematopoietic stem cell (HSC) niches and their depletion mobilizes HSCs. Blood (2010), doi:10.1182/blood-2009-11-253534.

143. S. Kaur, L. J. Raggatt, S. M. Millard, A. C. Wu, L. Batoon, R. N. Jacobsen, I. G. Winkler, K. P. MacDonald, A. C. Perkins, D. A. Hume, J. P. Levesque, A. R. Pettit, Self-repopulating recipient bone marrow resident macrophages promote long-term hematopoietic stem cell engraftment. Blood (2018), doi:10.1182/blood-2018-01-829663.

144. A. Ludin, T. Itkin, S. Gur-Cohen, A. Mildner, E. Shezen, K. Golan, O. Kollet, A. Kalinkovich, Z. Porat, G. D'Uva, A. Schajnovitz, E. Voronov, D. A. Brenner, R. N. 
Apte, S. Jung, T. Lapidot, Monocytes-macrophages that express a-smooth muscle actin preserve primitive hematopoietic cells in the bone marrow. Nat. Immunol. (2012), doi:10.1038/ni.2408.

145. Y. Kawano, C. Fukui, M. Shinohara, K. Wakahashi, S. Ishii, T. Suzuki, M. Sato, N. Asada, H. Kawano, K. Minagawa, A. Sada, T. Furuyashiki, S. Uematsu, S. Akira, T. Uede, S. Narumiya, T. Matsui, Y. Katayama, G-CSF-induced sympathetic tone provokes fever and primes antimobilizing functions of neutrophils via PGE2. Blood (2017), doi:10.1182/blood-2016-07-725754.

146. X. Chen, H. Deng, M. J. Churchill, L. L. Luchsinger, X. Du, T. H. Chu, R. A. Friedman, M. Middelhoff, H. Ding, Y. H. Tailor, A. L. E. Wang, H. Liu, Z. Niu, H. Wang, Z. Jiang, S. Renders, S. H. Ho, S. V. Shah, P. Tishchenko, W. Chang, T. C. Swayne, L. Munteanu, A. Califano, R. Takahashi, K. K. Nagar, B. W. Renz, D. L. Worthley, C. B. Westphalen, Y. Hayakawa, S. Asfaha, F. Borot, C. S. Lin, H. W. Snoeck, S. Mukherjee, T. C. Wang, Bone Marrow Myeloid Cells Regulate Myeloid-Biased Hematopoietic Stem Cells via a Histamine-Dependent Feedback Loop. Cell Stem Cell (2017), doi:10.1016/j.stem.2017.11.003.

147. A. Nakamura-Ishizu, K. Takubo, M. Fujioka, T. Suda, Megakaryocytes are essential for HSC quiescence through the production of thrombopoietin. Biochem. Biophys. Res. Commun. (2014), doi:10.1016/j.bbrc.2014.10.095.

148. A. Nakamura-Ishizu, K. Takubo, H. Kobayashi, K. Suzuki-Inoue, T. Suda, CLEC-2 in megakaryocytes is critical for maintenance of hematopoietic stem cells in the bone marrow. J. Exp. Med. (2015), doi:10.1084/jem.20150057.

149. S. Pinho, T. Marchand, E. Yang, Q. Wei, C. Nerlov, P. S. Frenette, LineageBiased Hematopoietic Stem Cells Are Regulated by Distinct Niches. Dev. Cell (2018), doi:10.1016/j.devcel.2018.01.016.

150. T. S. Olson, A. Caselli, S. Otsuru, T. J. Hofmann, R. Williams, P. Paolucci, M. Dominici, E. M. Horwitz, Megakaryocytes promote murine osteoblastic HSC niche expansion and stem cell engraftment after radioablative conditioning. Blood (2013), doi:10.1182/blood-2012-10-463414.

151. M. Dominici, V. Rasini, R. Bussolari, X. Chen, T. J. Hofmann, C. Spano, D. Bernabei, E. Veronesi, F. Bertoni, P. Paolucci, P. F. Conte, E. M. Horwitz, Restoration and reversible expansion of the osteoblastic hematopoietic stem cell niche after marrow radioablation. Blood (2009), doi:10.1182/blood-200810-183459.

152. L. Zou, B. Barnett, H. Safah, V. F. LaRussa, M. Evdemon-Hogan, P. Mottram, S. Wei, O. David, T. J. Curiel, W. Zou, Bone marrow is a reservoir for CD4+CD25+ regulatory $T$ cells that traffic through CXCL12/CXCR4 signals. Cancer Res. (2004), doi:10.1158/0008-5472.CAN-04-1987.

153. J. Fujisaki, J. Wu, A. L. Carlson, L. Silberstein, P. Putheti, R. Larocca, W. Gao, T. I. Saito, C. Lo Celso, H. Tsuyuzaki, T. Sato, D. Côté, M. Sykes, T. B. Strom, D. T. Scadden, C. P. Lin, In vivo imaging of T reg cells providing immune privilege to the haematopoietic stem-cell niche. Nature (2011), doi:10.1038/nature10160.

154. Y. Hirata, K. Furuhashi, H. Ishii, H. W. Li, S. Pinho, L. Ding, S. C. Robson, P. S. Frenette, J. Fujisaki, CD150 high Bone Marrow Tregs Maintain Hematopoietic Stem Cell Quiescence and Immune Privilege via Adenosine. Cell Stem Cell (2018), doi:10.1016/j.stem.2018.01.017. 
155. J. Zhang, Q. Wu, C. B. Johnson, A. Olsson, A. Slaughter, M. May, B. Weinhaus, A. D'Alessandro, J. D. Engel, J. X. Jiang, J. M. Koffron, L. F. Huang, N. Salomonis, H. L. Grimes, D. Lucas, Three-dimensional mapping identifies distinct vascular niches for myelopoiesis. bioRxiv (2020), doi:10.1101/2020.04.02.014548.

156. A. Hérault, M. Binnewies, S. Leong, F. J. Calero-Nieto, S. Y. Zhang, Y. A. Kang, X. Wang, E. M. Pietras, S. H. Chu, K. Barry-Holson, S. Armstrong, B. Göttgens, E. Passegué, Myeloid progenitor cluster formation drives emergency and leukaemic myelopoiesis. Nature (2017), doi:10.1038/nature21693.

157. C. Lo Celso, H. E. Fleming, J. W. Wu, C. X. Zhao, S. Miake-Lye, J. Fujisaki, D. Côté, D. W. Rowe, C. P. Lin, D. T. Scadden, Live-animal tracking of individual haematopoietic stem/progenitor cells in their niche. Nature (2009), doi:10.1038/nature07434.

158. Y. Xie, T. Yin, W. Wiegraebe, X. C. He, D. Miller, D. Stark, K. Perko, R. Alexander, J. Schwartz, J. C. Grindley, J. Park, J. S. Haug, J. P. Wunderlich, H. Li, S. Zhang, T. Johnson, R. A. Feldman, L. Li, Detection of functional haematopoietic stem cell niche using real-time imaging. Nature (2009), doi:10.1038/nature07639.

159. S. K. Nilsson, H. M. Johnston, J. A. Coverdale, Spatial localization of transplanted hemopoietic stem cells: Inferences for the localization of stem cell niches. Blood (2001), doi:10.1182/blood.V97.8.2293.

160. M. Falchi, L. Varricchio, F. Martelli, F. Masiello, G. Federici, M. Zingariello, G. Girelli, C. Whitsett, E. F. Petricoin, S. K. Moestrup, A. Zeuner, A. R. Migliaccio, Dexamethasone targeted directly to macrophages induces macrophage niches that promote erythroid expansion. Haematologica (2015), doi:10.3324/haematol.2014.114405.

161. G. De Haan, S. S. Lazare, Aging of hematopoietic stem cells. Blood (2018), , doi:10.1182/blood-2017-06-746412.

162. S. J. Morrison, A. M. Wandycz, K. Akashi, A. Globerson, I. L. Weissman, The aging of hematopoietic stem cells. Nat. Med. (1996), doi:10.1038/nm09961011.

163. B. Dykstra, S. Olthof, J. Schreuder, M. Ritsema, G. De Haan, Clonal analysis reveals multiple functional defects of aged murine hematopoietic stem cells. J. Exp. Med. (2011), doi:10.1084/jem.20111490.

164. I. Beerman, D. Bhattacharya, S. Zandi, M. Sigvardsson, I. L. Weissman, D. Brydere, D. J. Rossia, Functionally distinct hematopoietic stem cells modulate hematopoietic lineage potential during aging by a mechanism of clonal expansion. Proc. Natl. Acad. Sci. U. S. A. (2010), doi:10.1073/pnas.1000834107.

165. Y. Liang, G. Van Zant, S. J. Szilvassy, Effects of aging on the homing and engraftment of murine hematopoietic stem and progenitor cells. Blood (2005), doi:10.1182/blood-2004-11-4282.

166. Z. Xing, M. A. Ryan, D. Daria, K. J. Nattamai, G. Van Zant, L. Wang, Y. Zheng, $\mathrm{H}$. Geiger, Increased hematopoietic stem cell mobilization in aged mice. Blood (2006), doi:10.1182/blood-2005-12-010272.

167. C. Gekas, T. Graf, CD41 expression marks myeloid-biased adult hematopoietic stem cells and increases with age. Blood (2013), doi:10.1182/blood-2012-09- 
457929.

168. B. J. Frisch, C. M. Hoffman, S. E. Latchney, M. W. LaMere, J. Myers, J. Ashton, A. J. Li, J. Saunders, J. Palis, A. S. Perkins, A. McCabe, J. N. P. Smith, K. E. McGrath, F. Rivera-Escalera, A. McDavid, J. L. Liesveld, V. A. Korshunov, M. R. Elliott, K. C. MacNamara, M. W. Becker, L. M. Calvi, Aged marrow macrophages expand platelet-biased hematopoietic stem cells via interleukin1B. JCl Insight (2019), doi:10.1172/jci.insight.124213.

169. H. Geiger, G. De Haan, M. Carolina Florian, The ageing haematopoietic stem cell compartment. Nat. Rev. Immunol. (2013), , doi:10.1038/nri3433.

170. D. J. Rossi, D. Bryder, J. Seita, A. Nussenzweig, J. Hoeijmakers, I. L. Weissman, Deficiencies in DNA damage repair limit the function of haematopoietic stem cells with age. Nature (2007), doi:10.1038/nature05862.

171. M. C. Florian, K. Dörr, A. Niebel, D. Daria, H. Schrezenmeier, M. Rojewski, M. D. Filippi, A. Hasenberg, M. Gunzer, K. Scharffetter-Kochanek, Y. Zheng, H. Geiger, Cdc42 activity regulates hematopoietic stem cell aging and rejuvenation. Cell Stem Cell (2012), doi:10.1016/j.stem.2012.04.007.

172. A. V. Ergen, N. C. Boles, M. A. Goodell, Rantes/Ccl5 influences hematopoietic stem cell subtypes and causes myeloid skewing. Blood (2012), doi:10.1182/blood-2011-11-391730.

173. N. Guidi, M. Sacma, L. Ständker, K. Soller, G. Marka, K. Eiwen, J. M. Weiss, F. Kirchhoff, T. Weil, J. A. Cancelas, M. C. Florian, H. Geiger, Osteopontin attenuates aging-associated phenotypes of hematopoietic stem cells. EMBO J. (2017), doi:10.15252/embj.201694969.

174. A. Köhler, V. Schmithorst, M. D. Filippi, M. A. Ryan, D. Daria, M. Gunzer, H. Geiger, Altered cellular dynamics and endosteal location of aged early hematopoietic progenitor cells revealed by time-lapse intravital imaging in long bones. Blood (2009), doi:10.1182/blood-2008-12-195644.

175. M. Saçma, J. Pospiech, R. Bogeska, W. de Back, J. P. Mallm, V. Sakk, K. Soller, G. Marka, A. Vollmer, R. Karns, N. Cabezas-Wallscheid, A. Trumpp, S. MéndezFerrer, M. D. Milsom, M. A. Mulaw, H. Geiger, M. C. Florian, Haematopoietic stem cells in perisinusoidal niches are protected from ageing. Nat. Cell Biol. (2019), doi:10.1038/s41556-019-0418-y.

176. M. Maryanovich, A. H. Zahalka, H. Pierce, S. Pinho, F. Nakahara, N. Asada, Q. Wei, X. Wang, P. Ciero, J. Xu, A. Leftin, P. S. Frenette, Adrenergic nerve degeneration in bone marrow drives aging of the hematopoietic stem cell niche. Nat. Med. (2018), doi:10.1038/s41591-018-0030-x.

177. M. G. Poulos, P. Ramalingam, M. C. Gutkin, P. Llanos, K. Gilleran, S. Y. Rabbany, J. M. Butler, Endothelial transplantation rejuvenates aged hematopoietic stem cell function. J. Clin. Invest. (2017), doi:10.1172/JC193940.

178. Y. H. Ho, R. del Toro, J. Rivera-Torres, J. Rak, C. Korn, A. García-García, D. Macías, C. González-Gómez, A. del Monte, M. Wittner, A. K. Waller, H. R. Foster, C. López-Otín, R. S. Johnson, C. Nerlov, C. Ghevaert, W. Vainchenker, F. Louache, V. Andrés, S. Méndez-Ferrer, Remodeling of Bone Marrow Hematopoietic Stem Cell Niches Promotes Myeloid Cell Expansion during Premature or Physiological Aging. Cell Stem Cell (2019), doi:10.1016/j.stem.2019.06.007. 
179. K. Schepers, E. M. Pietras, D. Reynaud, J. Flach, M. Binnewies, T. Garg, A. J. Wagers, E. C. Hsiao, E. Passegué, Myeloproliferative neoplasia remodels the endosteal bone marrow niche into a self-reinforcing leukemic niche. Cell Stem Cell (2013), doi:10.1016/j.stem.2013.06.009.

180. C. R. Walkley, J. M. Shea, N. A. Sims, L. E. Purton, S. H. Orkin, Rb Regulates Interactions between Hematopoietic Stem Cells and Their Bone Marrow Microenvironment. Cell (2007), doi:10.1016/j.cell.2007.03.055.

181. V. L. Battula, P. M. Le, J. C. Sun, K. Nguyen, B. Yuan, X. Zhou, S. Sonnylal, T. McQueen, V. Ruvolo, K. A. Michel, X. Ling, R. Jacamo, E. Shpall, Z. Wang, A. Rao, G. Al-Atrash, M. Konopleva, R. E. Davis, M. A. Harrington, C. W. Cahill, C. Bueso-Ramos, M. Andreeff, AML-induced osteogenic differentiation in mesenchymal stromal cells supports leukemia growth. JCl insight (2017), doi:10.1172/jci.insight.90036.

182. A. L. Boyd, J. C. Reid, K. R. Salci, L. Aslostovar, Y. D. Benoit, Z. Shapovalova, M. Nakanishi, D. P. Porras, M. Almakadi, C. J. V. Campbell, M. F. Jackson, C. A. Ross, R. Foley, B. Leber, D. S. Allan, M. Sabloff, A. Xenocostas, T. J. Collins, M. Bhatia, Acute myeloid leukaemia disrupts endogenous myelo-erythropoiesis by compromising the adipocyte bone marrow niche. Nat. Cell Biol. (2017), doi:10.1038/ncb3625.

183. J. W. Hussong, G. M. Rodgers, P. J. Shami, Evidence of increased angiogenesis in patients with acute myeloid leukemia. Blood (2000), doi:10.1182/blood.v95.1.309.001k17_309_313.

184. D. Passaro, A. Di Tullio, A. Abarrategi, K. Rouault-Pierre, K. Foster, L. ArizaMcNaughton, B. Montaner, P. Chakravarty, L. Bhaw, G. Diana, F. Lassailly, J. Gribben, D. Bonnet, Increased Vascular Permeability in the Bone Marrow Microenvironment Contributes to Disease Progression and Drug Response in Acute Myeloid Leukemia. Cancer Cell (2017), doi:10.1016/j.ccell.2017.08.001.

185. E. J. C. Rombouts, B. Pavic, B. Löwenberg, R. E. Ploemacher, Relation between CXCR-4 expression, Flt3 mutations, and unfavorable prognosis of adult acute myeloid leukemia. Blood (2004), doi:10.1182/blood-2004-02-0566.

186. A. C. Spoo, M. Lübbert, W. G. Wierda, J. A. Burger, CXCR4 is a prognostic marker in acute myelogenous leukemia. Blood (2007), doi:10.1182/blood-200605-024844.

187. B. Nervi, P. Ramirez, M. P. Rettig, G. L. Uy, M. S. Holt, J. K. Ritchey, J. L. Prior, D. Piwnica-Worms, G. Bridger, T. J. Ley, J. F. DiPersio, Chemosensitization of acute myeloid leukemia (AML) following mobilization by the CXCR4 antagonist AMD3100. Blood (2009), doi:10.1182/blood-2008-06-162123.

188. T. Matsunaga, N. Takemoto, T. Sato, R. Takimoto, I. Tanaka, A. Fujimi, T. Akiyama, H. Kuroda, Y. Kawano, M. Kobune, J. Kato, Y. Hirayama, S. Sakamaki, K. Kohda, K. Miyake, Y. Niitsu, Interaction between leukemic-cell VLA-4 and stromal fibronectin is a decisive factor for minimal residual disease of acute myelogenous leukemia. Nat. Med. (2003), doi:10.1038/nm909.

189. D. S. Krause, K. Lazarides, U. H. Von Andrian, R. A. Van Etten, Requirement for CD44 in homing and engraftment of BCR-ABL-expressing leukemic stem cells. Nat. Med. (2006), doi:10.1038/nm1489.

190. N. Baryawno, D. Przybylski, M. S. Kowalczyk, Y. Kfoury, N. Severe, K. 
Gustafsson, K. D. Kokkaliaris, F. Mercier, M. Tabaka, M. Hofree, D. Dionne, A. Papazian, D. Lee, O. Ashenberg, A. Subramanian, E. D. Vaishnav, O.

Rozenblatt-Rosen, A. Regev, D. T. Scadden, A Cellular Taxonomy of the Bone Marrow Stroma in Homeostasis and Leukemia. Cell (2019), doi:10.1016/j.cell.2019.04.040.

191. N. Severe, N. M. Karabacak, K. Gustafsson, N. Baryawno, G. Courties, Y. Kfoury, K. D. Kokkaliaris, C. Rhee, D. Lee, E. W. Scadden, J. E. Garcia-Robledo, T. Brouse, M. Nahrendorf, M. Toner, D. T. Scadden, Stress-Induced Changes in Bone Marrow Stromal Cell Populations Revealed through Single-Cell Protein Expression Mapping. Cell Stem Cell (2019), doi:10.1016/j.stem.2019.06.003.

192. I. Borges, I. Sena, P. Azevedo, J. Andreotti, V. Almeida, A. Paiva, G. Santos, D. Guerra, P. Prazeres, L. L. Mesquita, L. S. de B. Silva, C. Leonel, A. Mintz, A. Birbrair, Lung as a Niche for Hematopoietic Progenitors. Stem Cell Rev. Reports (2017), doi:10.1007/s12015-017-9747-z.

193. A. Biffi, E. Montini, L. Lorioli, M. Cesani, F. Fumagalli, T. Plati, C. Baldoli, S. Martino, A. Calabria, S. Canale, F. Benedicenti, G. Vallanti, L. Biasco, S. Leo, N. Kabbara, G. Zanetti, W. B. Rizzo, N. A. L. Mehta, M. P. Cicalese, M. Casiraghi, J. J. Boelens, U. Del Carro, D. J. Dow, M. Schmidt, A. Assanelli, V. Neduva, C. Di Serio, E. Stupka, J. Gardner, C. Von Kalle, C. Bordignon, F. Ciceri, A. Rovelli, M. G. Roncarolo, A. Aiuti, M. Sessa, L. Naldini, Lentiviral hematopoietic stem cell gene therapy benefits metachromatic leukodystrophy. Science (80-. ). (2013), doi:10.1126/science.1233158.

194. A. Chao, P. K. Tsay, S. H. Lin, W. Y. Shau, D. Y. Chao, The applications of capture-recapture models to epidemiological data. Stat. Med. (2001), doi:10.1002/sim.996.

195. B. Haegeman, J. Hamelin, J. Moriarty, P. Neal, J. Dushoff, J. S. Weitz, Robust estimation of microbial diversity in theory and in practice. ISME J. (2013), doi:10.1038/ismej.2013.10.

196. K. J. Eash, A. M. Greenbaum, P. K. Gopalan, D. C. Link, CXCR2 and CXCR4 antagonistically regulate neutrophil trafficking from murine bone marrow. J. Clin. Invest. (2010), doi:10.1172/JCl41649.

197. K. J. Eash, A. M. Greenbaum, P. Gopalan, G. A. Diaz, D. C. Link, CXCR2 Signals Act in Concert with CXCR4 to Regulate Neutrophil Release From the Bone Marrow. Blood (2009), doi:10.1182/blood.v114.22.235.235.

198. G. Cacalano, J. Lee, K. Kikly, A. M. Ryan, S. Pitts-Meek, B. Hultgren, W. I. Wood, M. W. Moore, Neutrophil and B cell expansion in mice that lack the murine IL-8 receptor homolog. Science (80-. ). (1994), doi:10.1126/science.8036519.

199. M. A. Stark, Y. Huo, T. L. Burcin, M. A. Morris, T. S. Olson, K. Ley, Phagocytosis of apoptotic neutrophils regulates granulopoiesis via IL-23 and IL-17. Immunity (2005), doi:10.1016/j.immuni.2005.01.011.

200. G. Mangialardi, A. Cordaro, P. Madeddu, The bone marrow pericyte: An orchestrator of vascular niche. Regen. Med. (2016), , doi:10.2217/rme-20160121.

201. K. Stark, K. Pekayvaz, S. Massberg, Role of pericytes in vascular immunosurveillance. Front. Biosci. - Landmark (2018), doi:10.2741/4615. 
202. C. J. Cummings, T. R. Martin, C. W. Frevert, J. M. Quan, V. A. Wong, S. M. Mongovin, T. R. Hagen, K. P. Steinberg, R. B. Goodman, Expression and function of the chemokine receptors CXCR1 and CXCR2 in sepsis. J. Immunol. (1999).

203. S. Nourshargh, R. Alon, Leukocyte Migration into Inflamed Tissues. Immunity (2014), , doi:10.1016/j.immuni.2014.10.008.

204. T. Girbl, T. Lenn, L. Perez, L. Rolas, A. Barkaway, A. Thiriot, C. del Fresno, E. Lynam, E. Hub, M. Thelen, G. Graham, R. Alon, D. Sancho, U. H. von Andrian, M. B. Voisin, A. Rot, S. Nourshargh, Distinct Compartmentalization of the Chemokines CXCL1 and CXCL2 and the Atypical Receptor ACKR1 Determine Discrete Stages of Neutrophil Diapedesis. Immunity (2018), doi:10.1016/j.immuni.2018.09.018.

205. A. Gomariz, P. M. Helbling, S. Isringhausen, U. Suessbier, A. Becker, A. Boss, T. Nagasawa, G. Paul, O. Goksel, G. Székely, S. Stoma, S. F. Nørrelykke, M. G. Manz, C. Nombela-Arrieta, Quantitative spatial analysis of haematopoiesisregulating stromal cells in the bone marrow microenvironment by $3 \mathrm{D}$ microscopy. Nat. Commun. (2018), doi:10.1038/s41467-018-04770-z.

206. S. Pinho, J. Lacombe, M. Hanoun, T. Mizoguchi, I. Bruns, Y. Kunisaki, P. S. Frenette, PDGFRa and CD51 mark human Nestin+ sphere-forming mesenchymal stem cells capable of hematopoietic progenitor cell expansion. J. Exp. Med. (2013), doi:10.1084/jem.20122252.

207. P. S. Frenette, S. Pinho, D. Lucas, C. Scheiermann, Mesenchymal stem cell: Keystone of the hematopoietic stem cell niche and a stepping-stone for regenerative medicine. Annu. Rev. Immunol. (2013), , doi:10.1146/annurevimmunol-032712-095919.

208. L. E. Purton, D. T. Scadden, Limiting Factors in Murine Hematopoietic Stem Cell Assays. Cell Stem Cell (2007), , doi:10.1016/j.stem.2007.08.016.

209. D. J. Rossi, D. Bryder, J. M. Zahn, H. Ahlenius, R. Sonu, A. J. Wagers, I. L. Weissman, Cell intrinsic alterations underlie hematopoietic stem cell aging. Proc. Natl. Acad. Sci. U. S. A. (2005), doi:10.1073/pnas.0503280102.

210. M. J. Kim, M. H. Kim, S. A. Kim, J. S. Chang, Age-related deterioration of hematopoietic stem cells. Int. J. Stem Cells (2008), , doi:10.15283/ijsc.2008.1.1.55.

211. F. E. Mercier, C. Ragu, D. T. Scadden, The bone marrow at the crossroads of blood and immunity. Nat. Rev. Immunol. (2012), , doi:10.1038/nri3132.

212. W. C. Wu, H. W. Sun, H. T. Chen, J. Liang, X. J. Yu, C. Wu, Z. Wang, L. Zheng, Circulating hematopoietic stem and progenitor cells are myeloid-biased in cancer patients. Proc. Natl. Acad. Sci. U. S. A. (2014), doi:10.1073/pnas.1320753111.

213. D. I. Gabrilovich, S. Nagaraj, Myeloid-derived suppressor cells as regulators of the immune system. Nat. Rev. Immunol. (2009), , doi:10.1038/nri2506.

214. M. Shimoto, T. Sugiyama, T. Nagasawa, Numerous niches for hematopoietic stem cells remain empty during homeostasis. Blood (2017), doi:10.1182/blood2016-09-740563.

215. T. Bondar, R. Medzhitov, p53-Mediated Hematopoietic Stem and Progenitor Cell Competition. Cell Stem Cell (2010), doi:10.1016/j.stem.2010.03.002. 
216. C. Waskow, S. Paul, C. Haller, M. Gassmann, H. R. Rodewald, Viable c-KitW/W mutants reveal pivotal role for c-Kit in the maintenance of lymphopoiesis. Immunity (2002), doi:10.1016/S1074-7613(02)00386-2.

217. L. A. Thorén, K. Liuba, D. Bryder, J. M. Nygren, C. T. Jensen, H. Qian, J. Antonchuk, S.-E. W. Jacobsen, Kit Regulates Maintenance of Quiescent Hematopoietic Stem Cells. J. Immunol. (2008), doi:10.4049/jimmunol.180.4.2045.

218. J. L. Granick, P. C. Falahee, D. Dahmubed, D. L. Borjesson, L. S. Miller, S. I. Simon, Staphylococcus aureus recognition by hematopoietic stem and progenitor cells via TLR2/MyD88/PGE2 stimulates granulopoiesis in wounds. Blood (2013), doi:10.1182/blood-2012-11-466268.

219. J. Hoggatt, P. Singh, T. A. Tate, B. K. Chou, S. R. Datari, S. Fukuda, L. Liu, P. V. Kharchenko, A. Schajnovitz, N. Baryawno, F. E. Mercier, J. Boyer, J. Gardner, D. M. Morrow, D. T. Scadden, L. M. Pelus, Rapid Mobilization Reveals a Highly Engraftable Hematopoietic Stem Cell. Cell (2018), doi:10.1016/j.cell.2017.11.003.

220. J. Sun, A. Ramos, B. Chapman, J. B. Johnnidis, L. Le, Y. J. Ho, A. Klein, O. Hofmann, F. D. Camargo, Clonal dynamics of native haematopoiesis. Nature (2014), doi:10.1038/nature13824.

221. A. Sinclair, L. Park, M. Shah, M. Drotar, S. Calaminus, L. E. M. Hopcroft, R. Kinstrie, A. V. Guitart, K. Dunn, S. A. Abraham, O. Sansom, A. M. Michie, L. Machesky, K. R. Kranc, G. J. Graham, F. Pellicano, T. L. Holyoake, CXCR2 and CXCL4 regulate survival and self-renewal of hematopoietic stem/progenitor cells. Blood (2016), doi:10.1182/blood-2015-08-661785.

222. T. Jaffer, D. Ma, The emerging role of chemokine receptor CXCR2 in cancer progression. Transl. Cancer Res. (2016), , doi:10.21037/tcr.2016.10.06.

223. S. Jaiswal, P. Natarajan, A. J. Silver, C. J. Gibson, A. G. Bick, E. Shvartz, M. McConkey, N. Gupta, S. Gabriel, D. Ardissino, U. Baber, R. Mehran, V. Fuster, J. Danesh, P. Frossard, D. Saleheen, O. Melander, G. K. Sukhova, D. Neuberg, P. Libby, S. Kathiresan, B. L. Ebert, Clonal Hematopoiesis and risk of atherosclerotic cardiovascular disease. N. Engl. J. Med. (2017), doi:10.1056/NEJMoa1701719.

224. S. Jaiswal, P. Natarajan, A. J. Silver, C. J. Gibson, A. G. Bick, E. Shvartz, M. McConkey, N. Gupta, S. Gabriel, D. Ardissino, U. Baber, R. Mehran, V. Fuster, J. Danesh, P. Frossard, D. Saleheen, O. Melander, G. K. Sukhova, D. Neuberg, P. Libby, S. Kathiresan, B. L. Ebert, Clonal Hematopoiesis and Risk of Atherosclerotic Cardiovascular Disease. N. Engl. J. Med. (2017), doi:10.1056/nejmoa1701719.

225. P. Libby, B. L. Ebert, CHIP (Clonal Hematopoiesis of Indeterminate Potential) potent and newly recognized contributor to cardiovascular risk. Circulation (2018), doi:10.1161/CIRCULATIONAHA.118.034392.

226. R. L. Bowman, L. Busque, R. L. Levine, Clonal Hematopoiesis and Evolution to Hematopoietic Malignancies. Cell Stem Cell (2018), , doi:10.1016/j.stem.2018.01.011.

227. S. Jaiswal, P. Fontanillas, J. Flannick, A. Manning, P. V. Grauman, B. G. Mar, R. C. Lindsley, C. H. Mermel, N. Burtt, A. Chavez, J. M. Higgins, V. Moltchanov, F. 
C. Kuo, M. J. Kluk, B. Henderson, L. Kinnunen, H. A. Koistinen, C. Ladenvall, G. Getz, A. Correa, B. F. Banahan, S. Gabriel, S. Kathiresan, H. M. Stringham, M. I. McCarthy, M. Boehnke, J. Tuomilehto, C. Haiman, L. Groop, G. Atzmon, J. G. Wilson, D. Neuberg, D. Altshuler, B. L. Ebert, Age-related clonal hematopoiesis associated with adverse outcomes. N. Engl. J. Med. (2014), doi:10.1056/NEJMoa1408617.

228. J. J. Fuster, S. MacLauchlan, M. A. Zuriaga, M. N. Polackal, A. C. Ostriker, R. Chakraborty, C. L. Wu, S. Sano, S. Muralidharan, C. Rius, J. Vuong, S. Jacob, V. Muralidhar, A. A. B. Robertson, M. A. Cooper, V. Andrés, K. K. Hirschi, K. A. Martin, K. Walsh, Clonal hematopoiesis associated with TET2 deficiency accelerates atherosclerosis development in mice. Science (80-. ). (2017), doi:10.1126/science.aag1381.

229. C. K. Ferrone, M. Blydt-Hansen, M. J. Rauh, Age-associated TET2 mutations: Common drivers of myeloid dysfunction, cancer and cardiovascular disease. Int. J. Mol. Sci. (2020), , doi:10.3390/ijms21020626.

230. A. Stadtmann, A. Zarbock, CXCR2: From bench to bedside. Front. Immunol. (2012), doi:10.3389/fimmu.2012.00263.

231. Y. Shi, L. Du, L. Lin, Y. Wang, Tumour-associated mesenchymal stem/stromal cells: Emerging therapeutic targets. Nat. Rev. Drug Discov. (2016), , doi:10.1038/nrd.2016.193.

232. A. Uccelli, L. Moretta, V. Pistoia, Mesenchymal stem cells in health and disease. Nat. Rev. Immunol. (2008), , doi:10.1038/nri2395.

233. K. Taniguchi, M. Karin, NF-B, inflammation, immunity and cancer: Coming of age. Nat. Rev. Immunol. (2018), , doi:10.1038/nri.2017.142.

234. M. Hanoun, M. Maryanovich, A. Arnal-Estapé, P. S. Frenette, Neural regulation of hematopoiesis, inflammation, and cancer. Neuron (2015), , doi:10.1016/j.neuron.2015.01.026.

235. I. Sörensen, R. H. Adams, A. Gossler, DLL1-mediated Notch activation regulates endothelial identity in mouse fetal arteries. Blood (2009), doi:10.1182/blood2008-08-174508.

236. M. J. Schloss, M. Horckmans, K. Nitz, J. Duchene, M. Drechsler, K. Bidzhekov, C. Scheiermann, C. Weber, O. Soehnlein, S. Steffens, The time-of-day of myocardial infarction onset affects healing through oscillations in cardiac neutrophil recruitment. EMBO Mol. Med. (2016), doi:10.15252/emmm.201506083.

237. Y. Kunisaki, A. Nishikimi, Y. Tanaka, R. Takii, M. Noda, A. Inayoshi, K. I. Watanabe, F. Sanematsu, T. Sasazuki, T. Sasaki, Y. Fukui, DOCK2 is a Rac activator that regulates motility and polarity during neutrophil chemotaxis. J. Cell Biol. (2006), doi:10.1083/jcb.200602142.

238. K. Vintersten, C. Monetti, M. Gertsenstein, P. Zhang, L. Laszlo, S. Biechele, A. Nagy, Mouse in red: Red fluorescent protein expression in mouse ES cells, embryos, and adult animals. Genesis (2004), doi:10.1002/gene.20095.

239. E. Passegué, E. F. Wagner, I. L. Weissman, JunB deficiency leads to a myeloproliferative disorder arising from hematopoietic stem cells. Cell (2004), doi:10.1016/j.cell.2004.10.010.

240. L. Madisen, T. A. Zwingman, S. M. Sunkin, S. W. Oh, H. A. Zariwala, H. Gu, L. L. 
Ng, R. D. Palmiter, M. J. Hawrylycz, A. R. Jones, E. S. Lein, H. Zeng, A robust and high-throughput Cre reporting and characterization system for the whole mouse brain. Nat. Neurosci. (2010), doi:10.1038/nn.2467.

241. E. Montini, D. Cesana, M. Schmidt, F. Sanvito, C. C. Bartholomae, M. Ranzani, F. Benedicenti, L. S. Sergi, A. Ambrosi, M. Ponzoni, C. Doglioni, C. Di Serio, C. Von Kalle, L. Naldini, The genotoxic potential of retroviral vectors is strongly modulated by vector design and integration site selection in a mouse model of HSC gene therapy. J. Clin. Invest. (2009), doi:10.1172/JCI37630.

242. S. Firouzi, Y. López, Y. Suzuki, K. Nakai, S. Sugano, T. Yamochi, T. Watanabe, Development and validation of a new high-throughput method to investigate the clonality of HTLV-1-infected cells based on provirus integration sites. Genome Med. (2014), doi:10.1186/gm568.

243. N. A. Gillet, N. Malani, A. Melamed, N. Gormley, R. Carter, D. Bentley, C. Berry, F. D. Bushman, G. P. Taylor, C. R. M. Bangham, The host genomic environment of the provirus determines the abundance of HTLV-1-infected T-cell clones. Blood (2011), doi:10.1182/blood-2010-10-312926.

244. G. Spinozzi, A. Calabria, S. Brasca, S. Beretta, I. Merelli, L. Milanesi, E. Montini, VISPA2: A scalable pipeline for high-throughput identification and annotation of vector integration sites. BMC Bioinformatics (2017), doi:10.1186/s12859-0171937-9.

245. C. C. Berry, N. A. Gillet, A. Melamed, N. Gormley, C. R. M. Bangham, F. D. Bushman, Estimating abundances of retroviral insertion sites from DNA fragment length data. Bioinformatics (2012), doi:10.1093/bioinformatics/bts004.

246. A. Chao, Estimating the Population Size for Capture-Recapture Data with Unequal Catchability. Biometrics (1987), doi:10.2307/2531532.

247. A. Aiuti, L. Biasco, S. Scaramuzza, F. Ferrua, M. P. Cicalese, C. Baricordi, F. Dionisio, A. Calabria, S. Giannelli, M. C. Castiello, M. Bosticardo, C. Evangelio, A. Assanelli, M. Casiraghi, S. Di Nunzio, L. Callegaro, C. Benati, P. Rizzardi, D. Pellin, C. Di Serio, M. Schmidt, C. Von Kalle, J. Gardner, N. Mehta, V. Neduva, D. J. Dow, A. Galy, R. Miniero, A. Finocchi, A. Metin, P. P. Banerjee, J. S. Orange, S. Galimberti, M. G. Valsecchi, A. Biffi, E. Montini, A. Villa, F. Ciceri, M. G. Roncarolo, L. Naldini, Lentiviral hematopoietic stem cell gene therapy in patients with wiskott-aldrich syndrome. Science (80-. ). (2013), doi:10.1126/science.1233151.

248. S. Andrews, FASTQC A Quality Control tool for High Throughput Sequence Data. Babraham Inst. (2015).

249. M. Martin, Cutadapt removes adapter sequences from high-throughput sequencing reads. EMBnet.journal (2011), doi:10.14806/ej.17.1.200.

250. B. Li, C. N. Dewey, RSEM: Accurate transcript quantification from RNA-Seq data with or without a reference genome. BMC Bioinformatics (2011), doi:10.1186/1471-2105-12-323.

251. M. E. Ritchie, B. Phipson, D. Wu, Y. Hu, C. W. Law, W. Shi, G. K. Smyth, Limma powers differential expression analyses for RNA-sequencing and microarray studies. Nucleic Acids Res. (2015), doi:10.1093/nar/gkv007.

252. A. Butler, P. Hoffman, P. Smibert, E. Papalexi, R. Satija, Integrating single-cell transcriptomic data across different conditions, technologies, and species. Nat. 
Biotechnol. (2018), doi:10.1038/nbt.4096.

253. A. Hidalgo, L. A. Weiss, P. S. Frenette, Functional selectin ligands mediating human CD34+ cell interactions with bone marrow endothelium are enhanced postnatally. J. Clin. Invest. (2002), doi:10.1172/JCI0214047. 
Annexes

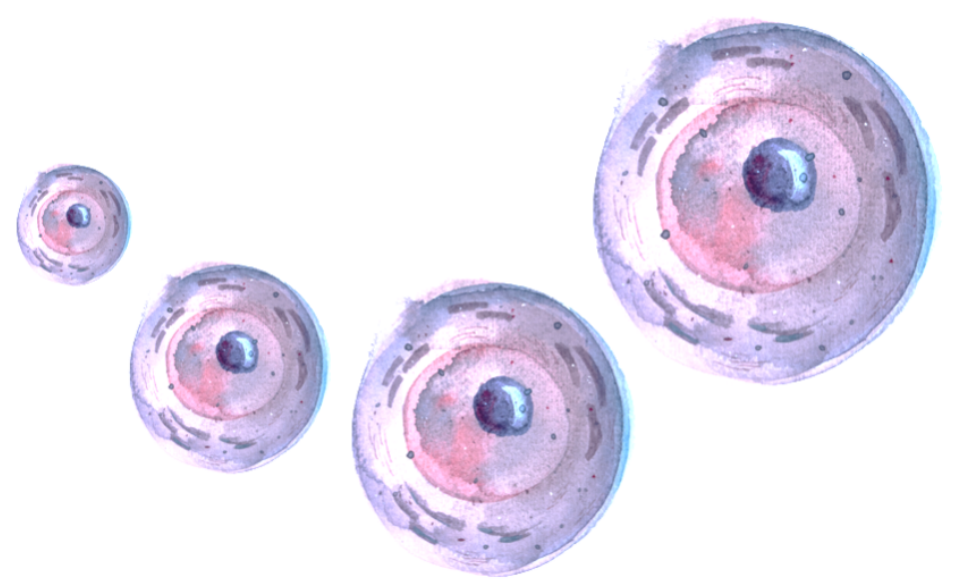

- 163 - 
- 164 - 


\section{Annexes}

\section{Abbreviations}

\begin{tabular}{|c|c|}
\hline ADR & Adrenergic Receptor \\
\hline AGM & Aorta-gonad-mesonephros \\
\hline AML & Acute Myeloid Leukemia \\
\hline ANGPT-1 & Angiopoietin-1 \\
\hline ANOVA & Analysis of Variance \\
\hline BM & Bone Marrow \\
\hline BMEF & Bone Marrow Extracellular Fluid \\
\hline BMNC & Bone Marrow Nuclear Cell \\
\hline BRDU & 5-bromo-2-deoxyuridine \\
\hline BSA & Bovine Serum Albumin \\
\hline CAR & CXCL12-abundant reticular cells \\
\hline$C D$ & Cluster of Differentiation \\
\hline CDH5 & Cadherin 5 \\
\hline CDNA & Complementary DNA \\
\hline CFU & Colony Forming Unit \\
\hline CFU-C & Colony Forming Unit Cell \\
\hline CFU-F & Colony Forming Unit Fibroblast \\
\hline CFU-S & Colony Forming Unit Spleen \\
\hline $\mathrm{CH}$ & Clonal Hematopoiesis \\
\hline $\mathrm{CHSC}$ & Circulating hematopoietic stem cell \\
\hline CLP & Common Lymphoid Progenitor \\
\hline CMP & Common Myeloid Progenitor \\
\hline CXCL & CXC chemokine ligand \\
\hline CXCR & CXC chemokine receptor \\
\hline DAPI & 4',6-diamidino-2-phenylindole \\
\hline DMSO & Dimethyl sulfoxide \\
\hline DNA & Deoxyribonucleic Acid \\
\hline DSRED & Discosoma-derived Red fluorescent protein \\
\hline DT & Diphtheria Toxin \\
\hline (E) & Embryonic day \\
\hline EC & Endothelial cell \\
\hline EDTA & Ethylenediaminetetraacetic acid \\
\hline EHT & Endothelial-to-hematopoietic transition \\
\hline ELISA & Enzyme-linked immunosorbent assay \\
\hline EMPS & Erythroid-Myeloid Progenitors \\
\hline FACS & Fluorescence Activated Cell Sorting \\
\hline
\end{tabular}




\begin{tabular}{|c|c|}
\hline FBS & Fetal Bovine Serum \\
\hline FCS & Forward Scatter \\
\hline FGF & Fibroblast Growth Factor \\
\hline G-CSF & Granulocyte Colony-Stimulating Factor \\
\hline GFAP & Glial Fibrillary Acidic Protein \\
\hline GFP & Green Fluorescence Protein \\
\hline GMP & Granulocyte Myeloid Progenitor \\
\hline HBSS & Hank's Balanced Salt Solution \\
\hline HDC & Histidine decarboxylase \\
\hline HEPES & 4-(2-Hydroxyethyl)-1-Piperazineethanesulfonic acid \\
\hline HSC & Hematopoietic stem cell \\
\hline HSPC & Hematopoietic stem and progenitor cell \\
\hline ICAM1 & Intercellular Adhesion Molecule 1 \\
\hline IL & Interleukin \\
\hline I.P. & Intraperitoneal \\
\hline IS & Integration site \\
\hline I.V. & Intravenous \\
\hline KO & Knockout \\
\hline LEPR & Leptin Receptor \\
\hline LPS & Lipopolysaccharide \\
\hline LRC & Label Retaining Cell \\
\hline LSC & Leukemic Stem Cell \\
\hline LSK & Lineage $^{\mathrm{NEG}} \mathrm{Sca}-1^{+} \mathrm{c}-\mathrm{Kit}^{+}$cells \\
\hline LT-HSC & Long Term Hematopoietic Stem Cell \\
\hline LTR & Long Term Repopulating \\
\hline LXR & Liver X Receptor \\
\hline LY6C & Lymphocyte antigen $6 \mathrm{C}$ \\
\hline LY6G & Lymphocyte antigen 6G \\
\hline MEP & Myeloid Erythroid Progenitor \\
\hline Ml & Myocardial Infarction \\
\hline MK & Megakaryocyte \\
\hline MMP & Matrix Metallopeptidase \\
\hline MP & Myeloid Progenitor \\
\hline MPL & Myeloproliferative Leukemia Protein \\
\hline MPP & Multipotent Progenitor \\
\hline MSC & Mesenchymal Stem Cell \\
\hline NA & Noradrenaline \\
\hline ND & Not Detected \\
\hline NES & Nestin \\
\hline NG2 & Neuron-glial antigen 2 \\
\hline NO & Nitric Oxide \\
\hline OCT & Optimal Cutting Temperature Compound \\
\hline
\end{tabular}




\begin{tabular}{l|l} 
OPN & Osteopontin \\
PBS & Phosphate-Buffered Saline \\
PDPN & Podoplanin \\
PFA & Paraformaldehyde \\
PGE 2 & Prostaglandin E2 \\
PRX1 & Paired-related homeobox gene-1 \\
QPCR & Quantitative PCR \\
RBC & Red blood cell \\
ROS & Reactive Oxygen Species \\
RPMI & Roswell Park Memorial Institute cell culture medium \\
RT & Room temperature \\
SCF & Stem cell factor \\
SDF-1 & Stromal cell-derived factor 1 (CXCL12) \\
SMA & Smooth Muscle Actin \\
SNS & Sympathetic nervous system \\
SSC & Side scatter \\
ST-HSC & Short term hematopoietic stem cell \\
THPO & Thrombopoietin \\
TIE2 & Tyrosine-protein kinase receptor 2 \\
TNF- $\alpha$ & Tumor Necrosis Factor $\alpha$ \\
TREGS & Regulatory T Cells \\
UV & Ultraviolet Light \\
VCAM1 & Vascular cell adhesion molecule 1 \\
VCN & Vector copy number \\
VLA & Very late antigen \\
WWF & Von Willebrand Factor \\
WT & Wild type \\
ZT & Zeitgeber time \\
&
\end{tabular}


$-168-$ 


\section{Publications}

Most of the data presented in this thesis is part of a manuscript currently under review in Nature.

Itziar Cossío, Daniela Cesana, José M. Adrover, Andrea Rubio-Ponce, Marianna Di Scala, Linnea A. Weiss, María Casanova-Acebes, Pierangela Gallina, Dariusz Przybylski, Juan A. Quintana, Christian Weber, David T. Scadden, Elena Almarza, Eugenio Montini and Andrés Hidalgo. Dissemination of blood stem cells through CXCR2 preserves hematopoietic fitness. Under review in Nature.

During this doctoral thesis the doctoral student has also participated in other publications:

1. De Koninck Magali, Lapi E, Badía-Careaga C, Cossío I, Giménez-Llorente D, Rodríguez-Corsino M, Andrada E, Hidalgo A, Manzanares M, X Real F, Losada A. Essential Roles of Cohesin STAG2 in Mouse Embryonic Development and Adult Tissue Homeostasis. Cell Reports (2020).

2. Sainz de Aja J, Menchero S, Rollan I, Barral A, Tiana M, Jawaid W, Cossío I, Alvarez A, Carreño-Tarragona G, Badia-Careaga C, Nichols J, Göttgens B, Isern J, Manzanares M. The pluripotency factor NANOG controls primitive haematopoiesis and directly regulates Tal1. EMBO J. 1;38(7): e99122 (2019).

3. Adrover JM, Del Fresno C, Crainiciuc G, Cuartero MI, Casanova-Acebes M, Weiss LA, Huerga-Encabo H, Silvestre-Roig C, Rossaint J, Cossío I, LechugaVieco AV, García-Prieto J, Gómez-Parrizas M, Quintana JA, Ballesteros I, Martin-Salamanca S, Aroca-Crevillen A, Chong SZ, Evrard M, Balabanian K, López J, Bidzhekov K, Bachelerie F, Abad-Santos F, Muñoz-Calleja C, Zarbock A, Soehnlein O, Weber C, Ng LG, Lopez-Rodriguez C, Sancho D, Moro MA, Ibáñez B, Hidalgo A. A Neutrophil Timer Coordinates Immune Defense and Vascular Protection. Immunity, 19;50(2):390-402.e10 (2019).

4. Cossío I, Lucas D, Hidalgo A. Neutrophils as regulators of the hematopoietic niche. Blood, 133(20):2140-2148 (2019). 


\section{Cell Reports}

\section{Essential Roles of Cohesin STAG2 in Mouse Embryonic Development and Adult Tissue Homeostasis}

\section{Graphical Abstract}

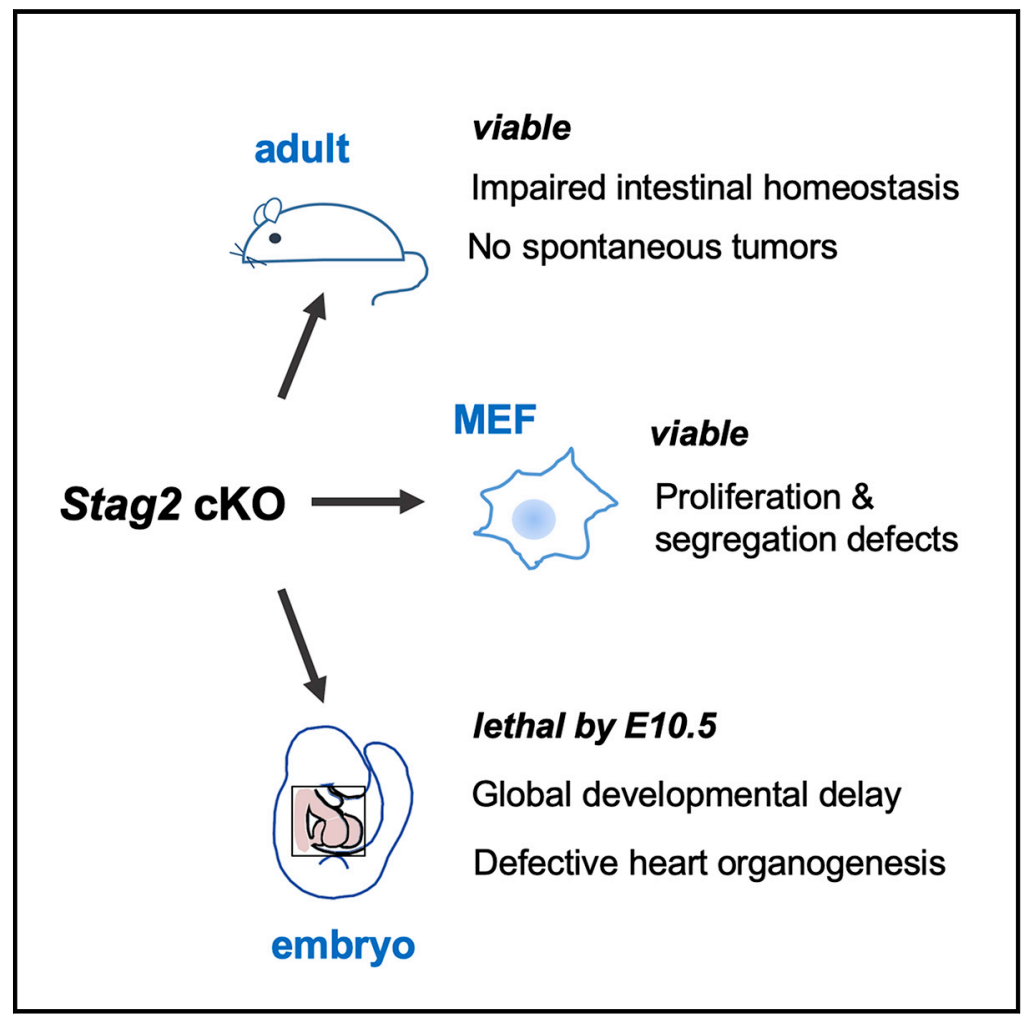

\section{Highlights}

- MEFs lacking STAG2 show reduced proliferation and mild cohesion defects

- Stag2 loss in adult mice reduces fitness but does not increase tumor incidence

- Stag2 KO embryos die by E10.5 with global developmental delay and malformed hearts

\section{Authors}

Magali De Koninck, Eleonora Lapi, Claudio Badía-Careaga, ..., Miguel Manzanares, Francisco X. Real, Ana Losada

\section{Correspondence}

alosada@cnio.es

\section{In Brief}

Cells carry STAG1- and STAG2-cohesin complexes whose functional specificity remains unclear. De Koninck et al. show that Stag2 deletion in mice results in embryonic lethality by mid-gestation. In contrast, STAG2 is not strictly required for viability in cells or adult tissues, and its loss is not sufficient to elicit tumorigenesis. 


\title{
Essential Roles of Cohesin STAG2 in Mouse Embryonic Development and Adult Tissue Homeostasis
}

\author{
Magali De Koninck, ${ }^{1,7}$ Eleonora Lapi, ${ }^{2,3,7}$ Claudio Badía-Careaga, ${ }^{4,7}$ Itziar Cossío, ${ }^{4}$ Daniel Giménez-Llorente, ${ }^{1}$ \\ Miriam Rodríguez-Corsino, ${ }^{1}$ Elena Andrada, ${ }^{2}$ Andrés Hidalgo, ${ }^{4}$ Miguel Manzanares, ${ }^{4,5}$ Francisco X. Real, ${ }^{2,3,6}$ \\ and Ana Losada ${ }^{1,8, *}$ \\ ${ }^{1}$ Chromosome Dynamics Group, Molecular Oncology Programme, Spanish National Cancer Research Centre (CNIO), 28029 Madrid, Spain \\ 2Epithelial Carcinogenesis Group, Molecular Oncology Programme, Spanish National Cancer Research Centre (CNIO), 28029 Madrid, Spain \\ ${ }^{3}$ CIBERONC, Madrid, Spain \\ ${ }^{4}$ Centro Nacional de Investigaciones Cardiovasculares (CNIC), 28029 Madrid, Spain \\ ${ }^{5}$ Centro de Biología Molecular "Severo Ochoa" (CBMSO), CSIC-UAM, 28049 Madrid, Spain \\ ${ }^{6}$ Departament de Ciències Experimentals i de la Salut, Universitat Pompeu Fabra, 08003 Barcelona, Spain \\ 7These authors contributed equally \\ 8Lead Contact \\ *Correspondence: alosada@cnio.es \\ https://doi.org/10.1016/j.celrep.2020.108014
}

\section{SUMMARY}

Cohesin mediates sister chromatid cohesion and 3D genome folding. Two versions of the complex carrying STAG1 or STAG2 coexist in somatic vertebrate cells. STAG2 is commonly mutated in cancer, and germline mutations have been identified in cohesinopathy patients. To better understand the underlying pathogenic mechanisms, we report the consequences of Stag2 ablation in mice. STAG2 is largely dispensable in adults, and its tissue-wide inactivation does not lead to tumors but reduces fitness and affects both hematopoiesis and intestinal homeostasis. STAG2 is also dispensable for murine embryonic fibroblasts in vitro. In contrast, Stag2-null embryos die by mid-gestation and show global developmental delay and defective heart morphogenesis, most prominently in structures derived from secondary heart field progenitors. Both decreased proliferation and altered transcription of tissue-specific genes contribute to these defects. Our results provide compelling evidence on cell- and tissue-specific roles of different cohesin complexes and how their dysfunction contributes to disease.

\section{INTRODUCTION}

Cohesin is a four-subunit complex that holds the sister chromatids together to ensure faithful DNA repair by homologous recombination and proper chromosome segregation during cell division (Morales and Losada, 2018; Nasmyth and Haering, 2009). It is present in all cells, and its cohesive function is essential for proliferation. In addition, cohesin contributes to the spatial organization of the genome and to the activation and repression of tissue-specific transcriptional programs together with architectural proteins such as CTCF and transcriptional regulators like Mediator (Dowen et al., 2014; Faure et al., 2012; Kagey et al., 2010; Merkenschlager and Nora, 2016). In the cohesin complexes present in vertebrate somatic cells, the Structural Maintenance of Chromosomes (SMC) heterodimer of SMC1A and SMC3 associates with the kleisin subunit RAD21 and with one of the two versions of the Stromal Antigen (SA/STAG) subunit, namely, STAG1 or STAG2 (Losada et al., 2000). The two variants are present in all tissues and cell types, but their functional specificity is not well established (Cuadrado and Losada, 2020).
We previously showed that genetic ablation of Stag1 in mice is embryonic lethal, which indicates that the two complexes are not redundant, at least during embryonic development (Remeseiro et al., 2012a). Lethality starts after embryonic day 11.5 (E11.5), but a small fraction of embryos survive to E18.5 and present severe developmental delay and general hypoplasia (Remeseiro et al., 2012b).

In Stag1-null mouse embryonic fibroblasts (MEFs), telomere cohesion is impaired, preventing efficient replication of telomeres and causing chromosome segregation defects in mitosis (Remeseiro et al., 2012a). Centromere and arm cohesion are not clearly affected, which suggests that cohesin-STAG1 is specifically required for telomere cohesion, whereas cohesin-STAG2 contributes to cohesion in other chromosomal regions. Results in human cells are in line with these findings, although the extent of cohesion defects reported in the absence of STAG2 is variable (Canudas and Smith, 2009; Kim et al., 2016; van der Lelij et al., 2017; Mullenders et al., 2015). Specific contributions of cohesin-STAG2 to DNA replication and repair have also been reported (Kong et al., 2014; Meisenberg et al., 2019; Mondal 
et al., 2019). In any case, a single variant is sufficient, and necessary, to maintain cell viability in culture (van der Lelij et al., 2017; Liu et al., 2018; Remeseiro et al., 2012a).

Cohesin variants also contribute distinctly to genome organization and gene regulation. In Stag1-null MEFs, cohesin distribution and their transcriptome are altered (Remeseiro et al., 2012b). In the pancreas of heterozygous Stag1 mice, the architecture of the Reg locus and the transcription of some of its genes are also changed compared with those in the pancreas of wild-type (WT) littermates, suggesting that STAG2 is not sufficient to compensate for the reduced levels of STAG1 (Cuadrado et al., 2015). In human mammary epithelial cells, downregulation of STAG1 or STAG2 results in distinct changes in gene expression and chromatin contacts (Kojic et al., 2018). Cohesin-STAG1 and cohesin-STAG2 colocalize with CTCF and play a major role in the preservation of topologically associating domain (TAD) borders. In contrast, cohesin-STAG2 is also present at enhancers lacking CTCF that are bound by other transcriptional regulators (Cuadrado et al., 2019; Faure et al., 2012; Kojic et al., 2018; Sasca et al., 2019) Importantly, cohesin-STAG1 cannot occupy these non-CTCF cohesin sites even when STAG2 is absent (Kojic et al., 2018). Specific distribution of the two cohesin variants has also been reported in hematopoietic stem cells (HSCs), in which the loss of STAG2 decreases the transcription of lineage-specification genes and promotes stem cell renewal (Viny et al., 2019). In mouse embryonic stem cells, cohesin-STAG2 promotes compaction of Polycomb domains and the establishment of long-range interaction networks between distant Polycomb-bound promoters that are important for gene repression (Cuadrado et al., 2019).

Germline mutations in genes encoding cohesin and its regulatory factors are at the origin of a group of human syndromes collectively known as cohesinopathies. Cornelia de Lange syndrome (CdLS) is the most common of them, and up to $60 \%$ of the patients carry heterozygous mutations in NIPBL, a protein involved in loading cohesin on chromatin (Liu et al., 2009; Sarogni et al., 2020). Clinical features often include growth retardation, intellectual disability, facial dysmorphism, and congenital heart defects. Recently, germline mutations in STAG1 and STAG2 have been identified in patients with features partially overlapping those of CdLS and other cohesinopathies (Lehalle et al., 2017; Mullegama et al., 2019; Soardi et al., 2017; Yuan et al., 2019). Somatic mutations in cohesin genes, particularly in STAG2, have also been identified in several tumor types (De Koninck and Losada, 2016). STAG2 has been recognized as one of the 12 genes significantly mutated in 4 or more cancer types (Lawrence et al., 2014). Among them, STAG2 loss is most frequent in urothelial bladder cancer (Balbás-Martinez et al., 2013; Taylor et al., 2014). The evidence emerging from the study of diseases associated with both germline and somatic cohesin mutations strongly suggests that gene deregulation, rather than defects in chromosome segregation, underlies the pathogenic mechanism (Balbás-Martinez et al., 2013; Liu et al., 2009; Mullenders et al., 2015).

Given the growing importance of STAG2 in human disease, we generated a Stag2 conditional knockout (cKO) mouse strain to identify specific functions of STAG2 at the cellular and organismal levels.

\section{RESULTS}

\section{Mild Cohesion Defects and Slower Proliferation in STAG2-Deficient MEFs}

First, we generated a cKO allele of the Stag2 gene, which is located on the $X$ chromosome (Figure S1). Next, Stag2 cKO MEFs were isolated from E12.5 embryos resulting from mating Stag2 $2^{\text {lox/lox }}$ females with males carrying $h U B C-C r e E R T 2$ for ubiquitous, tamoxifen-induced activation of the Cre recombinase. Upon addition of 4-hydroxy-tamoxifen (4-OHT) to the culture medium, STAG2 protein levels in treated MEFs (KO) dropped below $5 \%$ of the amount present in untreated MEFs (WT), and compensatory upregulation of STAG1 could be observed (Figure 1A). The doubling time of STAG2-deficient cells was higher than the WT (Figure 1B), but flow cytometry analysis did not reveal differences in the cell cycle profiles of WT and KO MEFs (Figure 1C). A statistically significant increase in the percentage of caspase-3-positive cells was observed in the $\mathrm{KO}$ MEF cultures (14\% versus 3\% in WT MEFs; Figure 1D), suggesting a contribution of cell death to the higher doubling time. We next examined sister chromatid cohesion and chromosome segregation. For these experiments, Stag2 was deleted under serum-starved conditions, and cells going through the first mitosis after release from the G0 arrest were collected. Very few cases of severe cohesion defects (i.e., complete sister chromatid unpairing) were detected in metaphase spreads from WT or KO MEFs $(1.3 \%$ and $3 \%$ of chromosomes per metaphase examined, respectively; Figure 1E, bottom left). However, we did observe a larger fraction of chromosomes with mild cohesion defects (i.e., increased distance between sister centromeres; $26 \%$ in KO versus $11 \%$ in WT MEFs; Figure 1E, bottom right). We also found a ca. 2-fold increase in the percentage of anaphase cells with lagging chromosomes and/or chromosome bridges among KO MEFs compared with that of WT MEFs $(29 \%$ versus $17 \%$ ), although the difference did not reach statistical significance (Figure 1F). Finally, we observed that the proportion of metaphases with an abnormal chromosome number increased the longer MEFs were grown in the absence of STAG2 (Figure $1 \mathrm{G}$ ). Overall, these defects are milder than those identified in Stag1-null MEFs (Remeseiro et al., 2012a) or in C2C12 myoblasts or HeLa cells after STAG2 downregulation by small interfering RNA (siRNA) (Canudas and Smith, 2009; Remeseiro et al., 2012a). We conclude that primary cultured cells almost completely lacking STAG2 can proliferate, although at slower rates. They maintain sufficient cohesion to divide successfully but mis-segregate chromosomes more frequently that WT cells.

\section{STAG2 Inactivation in Adult Mice Does Not Lead to} Spontaneous Tumors

To determine whether STAG2 is essential in adulthood, male and female 4-week-old Stag2 cKO mice carrying or not carrying the $h U B C-C r e E R T 2$ transgene (hereafter referred to as KO and WT, respectively) were fed with a tamoxifen-containing diet (TMX). We did not observe an acute loss of viability in the KO mice, but long-term follow up revealed that their survival was significantly shorter than that of WT mice (Figure 2A). At 12 weeks, loss of the STAG2 protein was confirmed in a large fraction of cells $(>80 \%)$ in all tissues from $\mathrm{KO}$ mice analyzed by 
A

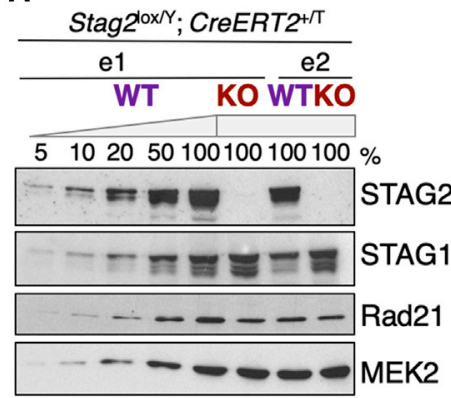

B

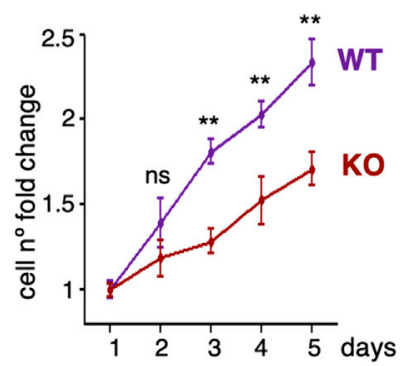

C
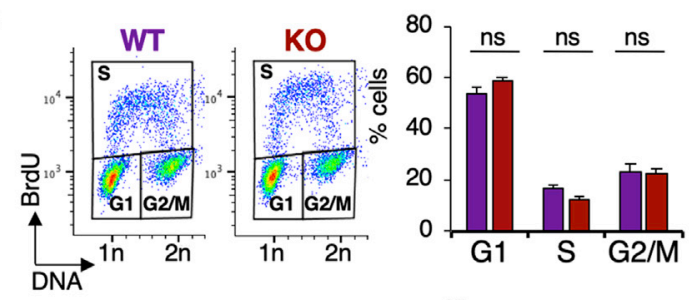

D
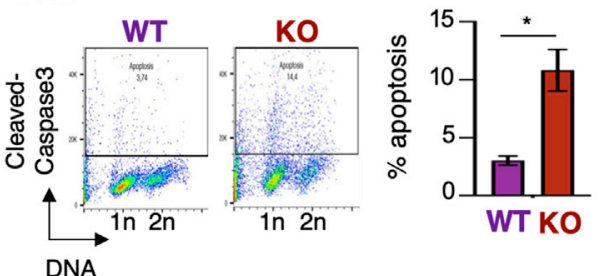

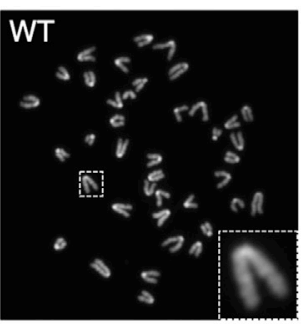

proper cohesion

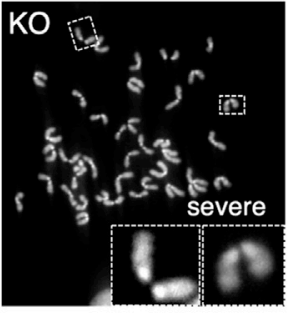

defective cohesion
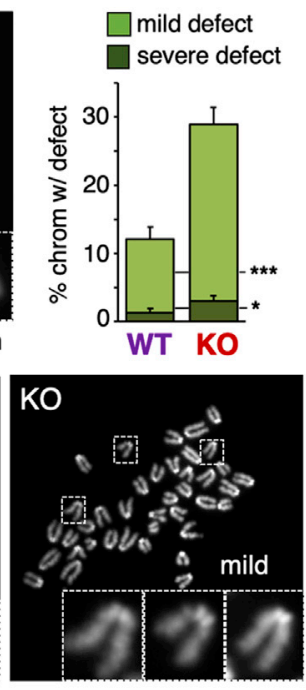

F

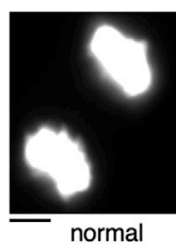

G

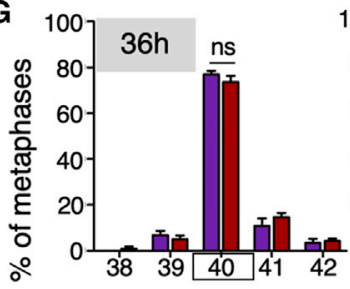

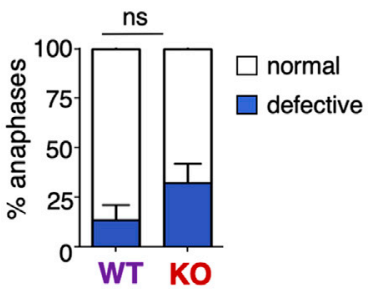

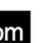
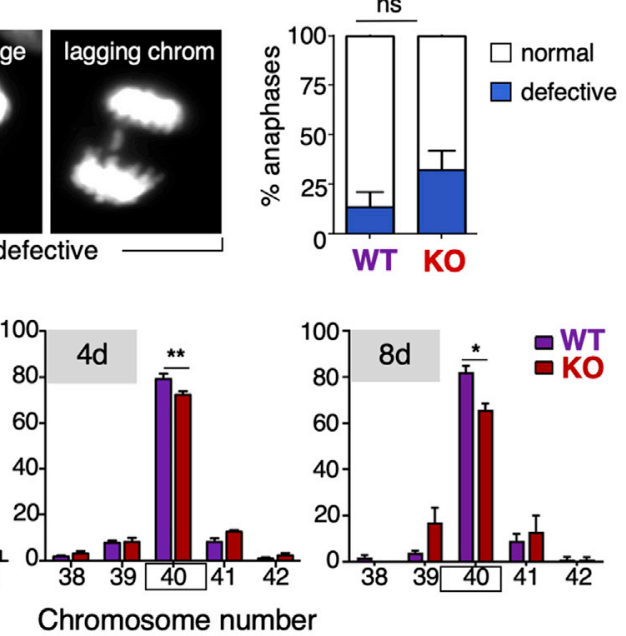

Figure 1. STAG2-Deficient MEFs Display Slower Proliferation and Increased Rates of Chromosome Missegregation

(A) Immunoblot analyses of whole-cell extracts of Stag2-cKO MEFs from two embryos (e1 and e2) untreated or treated for 4 days with 4-OHT (WT and KO hereafter). Decreasing amounts (shown as \% of maximal) of WT MEF extract were loaded to estimate STAG2 depletion levels. MEK2 is used as a loading control. (B) Growth curves of WT and KO MEFs representing the average fold increase in cell number relative to the number of cells seeded on day 1 . Data are from MEFs from 2 embryos, each analyzed in triplicates (mean \pm SEM).

(C) Representative BrdU incorporation profiles by fluorescence-activated cell sorting (FACS) in WT and KO MEFs, and bar graph showing values for $\mathrm{n}=4$ (mean \pm SEM).

(D) Representative FACS profiles of cleaved caspase 3 staining in WT and KO MEFs, and bar graph showing the fraction of apoptotic cells in $n=3$ (mean \pm SEM). (E) Representative metaphase spreads from Stag2-WT and -KO MEFs, and quantification (mean \pm SEM) of chromosomes showing centromeric cohesion defects (severe or mild) At least 100 metaphases from MEFs from 3 different embryos were inspected. Scale bar, $10 \mu \mathrm{m}$.

(F) Images of normal and defective anaphase cells found among WT and KO MEFs (left), and their quantification (right, mean \pm SEM). At least 100 anaphases from MEFs from 3 different embryos were inspected. Scale bar, $5 \mu \mathrm{m}$.

(G) Quantification of chromosome number frequency in metaphase spreads of WT and KO MEFs (mean \pm SEM). For the first time point, MEFs were serum starved for 3 days in $\pm 4-\mathrm{OHT}$ and released for $36 \mathrm{~h}$ to reach the first mitosis; " $4 \mathrm{~d}$ " and " $8 \mathrm{~d}$ " indicate number of days asynchronously growing MEFs were kept \pm 4 -OHT cells before analysis. At least 100 metaphases from MEFs from 3 different embryos were inspected. Mann-Whitney test; ${ }^{\star * *} p<0.001,{ }^{\star *} p<0.01,{ }^{\star} p<0.05 ; n s, p \geq$ 0.05 .

immunohistochemistry (Figure 2B, left panel; Figure 2C, first point in the graph). However, over time, the fraction of STAG2negative cells dropped dramatically in the more proliferative tissues (e.g., intestine and spleen) and to a lesser extent in tissues with moderate (i.e., lymph node) or low proliferation rates (i.e., liver, pancreas or brain; Figure 2C; compare the labeling of STAG2 in the lymph node and the pancreas of a 35-week-old $\mathrm{KO}$ mouse in Figure $2 \mathrm{~B}$ ). These results suggest that recombination-mediated deletion of the Stag2 gene is incomplete and disadvantageous to cells; as a consequence, WT unrecombined cells rapidly outcompete mutant cells in highly proliferative tissues. Similar results have been found in other genetic mouse models (Hay et al., 2005; Ireland et al., 2004). Given the proposed role of STAG2 as tumor suppressor, we searched for evidence of malignancy in the $\mathrm{KO}$ mice. There were no preneoplastic or neoplastic lesions in the full necropsies of KO mice $(n=13)$. Likewise, a macroscopic assessment failed to reveal neoplasms in a large cohort of mice $(n=63)$ of up to 70 weeks age, indicating 
A

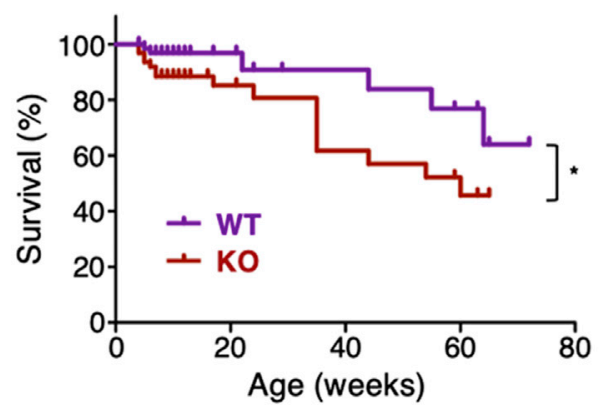

C

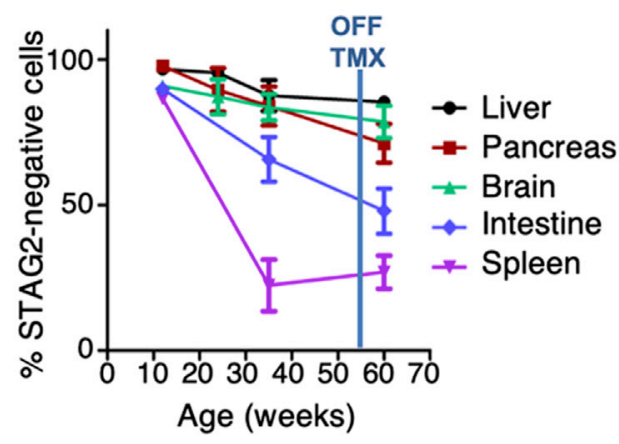

B

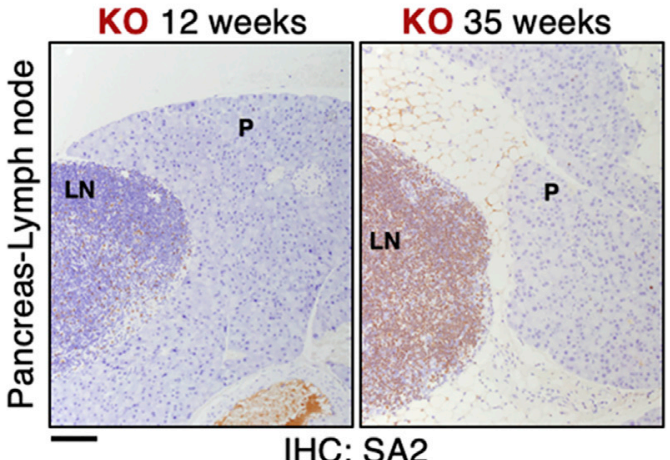

IHC: SA2

D
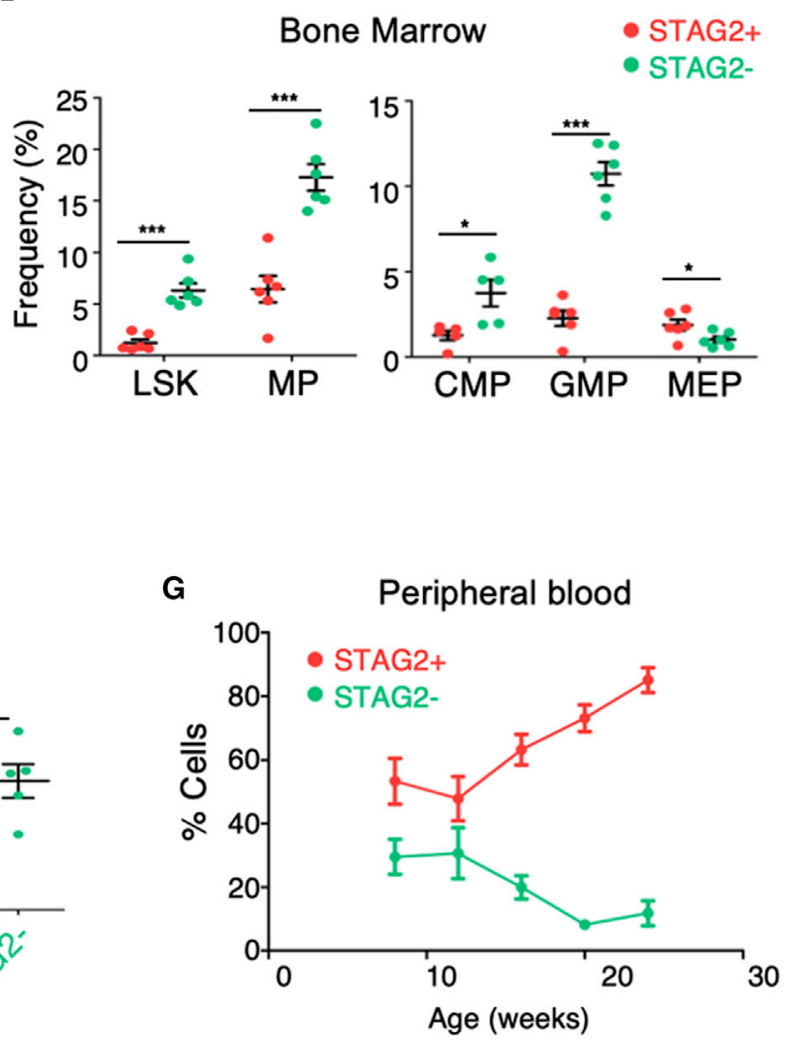

Figure 2. Effects of STAG2 Ablation in Adult Mice

(A) Kaplan-Meier survival curves of Stag2-KO and -WT mice. Four-week-old Stag2-cKO male and female mice carrying the Cre-ERT2 allele (KO; $\mathrm{n}=63)$, or not (WT; $n=66)$, were continuously fed on a TMX-containing diet and monitored thrice weekly. No sex differences in survival were observed. Gehan-BreslowWilcoxon test; ${ }^{*} p<0.05$

(B) Representative images of STAG2 expression in sections of pancreas (P) and associated lymph node (LN) of KO mice at 12 (left) and 35 (right) weeks of age, assessed by immunohistochemistry (IHC). Scale bar, $100 \mu \mathrm{m}$.

(C) Percentage of recombined STAG2-negative cells in various organs over time was assessed by IHC. Representative microphotographs were quantified with ImageJ software (3 micrographs per mouse; $\mathrm{n}=3$ to 6 mice depending on the time point). Error bars indicate SEM.

(D) Flow cytometry analysis of bone marrow HSPCs in 12-week-old KO mice $(n=6)$. Left, LSK (Lin- c-Kit+ Sca1+); MP (Lin- c-Kit+). Right, CMP (Lin- c-Kit+ CD34+ CD1632-); GMP (Lin- c-Kit+ CD34+ CD1632+); MEP (Lin- C-Kit+ CD34- CD1632-). Error bars indicate SEM. Unpaired t test; ${ }^{*} p<0.05$; ${ }^{* * *} p<0.001$. (E) Flow cytometry analysis of bone marrow Ter119+ cells in 12-week-old KO mice $(n=6)$. Error bars indicate SEM. Unpaired $t$ test; ${ }^{* *} p<0.01$.

(F) Colony-forming unit assay using FACS-sorted GFP and Tomato total bone marrow cells from 12-week-old KO mice $(n=5)$. Error bars indicate SEM. Unpaired $t$ test; ${ }^{* *} \mathrm{p}<0.01$.

(G) Flow cytometry analysis of GFP+ (STAG2-) and Tomato+ (STAG2+) leukocytes in peripheral blood of KO mice over time $(n=5)$. Error bars indicate SEM. 
that Stag2 inactivation on its own does not increase spontaneous tumor incidence in adult mice.

\section{STAG2 Supports Normal Hematopoiesis in Adult Mice} Compared with controls, KO mice treated with TMX for 8 weeks (starting at 4 weeks of age) displayed mild reductions in peripheral blood leukocyte, erythrocyte, and platelet counts, pointing to anomalies in hematopoiesis (Figures S2A and S2B). We took advantage of the dual-fluorescent Cre reporter (Rosa26_ACTBtdTomato_EGFP) carried by the $\mathrm{KO}$ animals to study the relative abundance of the WT and KO hematopoietic populations: unrecombined WT cells display cell-membrane-localized tdTomato fluorescence, whereas Cre-recombinase-expressing cells and their progeny display membrane-localized EGFP fluorescence. Analyses of leukocytes from peripheral blood and spleen of $\mathrm{KO}$ animals carrying the Stag2 cKO allele, the $h U B C-C r e E R T 2$ transgene, and the aforementioned Cre reporter revealed an enrichment in myeloid cells (monocytes and neutrophils) and a significant reduction in T lymphocytes among STAG2-deficient (GFP+) cells compared with that of unrecombined (Tomato+) cells (Figure $\mathrm{S} 2 \mathrm{C}$ ). To address whether this phenotype was due to a defect in hematopoiesis, we analyzed stem cell populations in the bone marrow. A clear expansion of the LSK (Lin- Sca1+ CKit+) population and myeloid progenitor (MP) compartment was associated with STAG2 loss (Figure 2D, left). Further analysis of MPs showed an increased frequency in common MPs (CMPs) and granulocyte-monocyte progenitors (GMPs) and a decrease in megakaryocyte-erythrocyte progenitors (MEPs) among STAG2-deficient (GFP+) cells, in agreement with the findings in peripheral blood (Figure 2D, right). Reductions in MEPs paralleled a decrease in bone marrow Ter119+ erythroid cells in KO mice (Figures 2E and S2D). Functional analyses revealed a higher colony-forming capacity of STAG2-deficient hematopoietic cells than that of WT (Figure 2F), which is concordant with the increase in LSK cells. The loss in lymphoid potential might explain the reduced chimerism of mutant cells over time in peripheral blood (Figure 2G). These results support the notion that STAG2 loss leads to increased self-renewal and to impaired differentiation of HSCs. We observe an increase in granulocyte and macrophage populations at the expense of the erythroid cells (myeloid skewing) and an overall competitive disadvantage when WT HSCs are present. These data are consistent with previous reports on the contribution of cohesin to normal hematopoiesis and the occurrence of cohesin mutations in myeloid malignancies (Mullenders et al., 2015; Thol et al., 2014; Viny et al., 2019). In contrast with the reduced proliferation of Stag2 KO MEFs, the enhanced self-renewal of Stag2 KO HSCs provides important evidence for supporting a STAG2 tumor suppressor role as well as possible tissue-specific effects of Stag2 inactivation.

\section{STAG2 Is Required for Intestinal Homeostasis}

Shortly after the initiation of a TMX diet at 4 weeks, the survival curve of $\mathrm{KO}$ mice diverted from that of WT mice (Figure $2 \mathrm{~A}$ ), the former also showing reduced body weight (Figure 3A). Histological analyses of 8-week-old animals failed to reveal major alterations in tissues of $\mathrm{KO}$ mice with the exception of the intestine, in which patches of epithelial erosion and necrosis were observed. Moderate or severe lesions were present in $60 \%$ of mutant mice, whereas WT mice showed much milder and less abundant lesions (Figure 3B). We analyzed proliferation and apoptosis in the small bowel: intestinal crypts from $\mathrm{KO}$ mice showed a significant reduction of bromodeoxyuridine-positive (BrdU+) cells (Figure 3C), suggesting reduced regeneration capacity. In addition, and in agreement with the findings in cultured MEFs, we found a significant increase in apoptosis measured as active caspase-3 labeling (Figure 3D). To acquire further insight into the requirement of STAG2 for intestinal cell renewal, we generated primary organoid cultures from the small intestine of TMX-treated KO mice carrying the fluorescent reporter (Figure 3E, left). STAG2-null GFP+ cells yielded fewer and smaller organoids than STAG2 proficient Tomato+ cells (Figure 3E, right). From these findings, we conclude that STAG2 is also required for intestinal homeostasis.

\section{Stag2-Null Embryos Display Developmental Delay by E9.5 and Die Soon Afterward}

To assess the effect of Stag2 inactivation in embryogenesis, Sta$g 2^{\text {lox/lox }}$ females were crossed with males carrying the CAG-Cre transgene, which codes for a Cre recombinase that is expressed ubiquitously from the zygote stage. Because Stag2 is an X-linked gene, male embryos resulting from this cross would be either WT or null (KO) for Stag2, whereas females would be WT or heterozygous (see genotyping strategy in Figure S1D). There were no Stag2-null males among the offspring, whereas heterozygous females were born at sub-Mendelian ratio (Figure 4A, last column). To determine at what point during embryonic development STAG2 becomes essential, we extracted and genotyped litters at different times after conception. We found live KO male embryos at the expected Mendelian ratios at E8.5 and E9.5, but not later (Figure 4A). In all studies presented hereafter, we used exclusively male embryos, either WT or KO. Immunohistochemical analyses of embryo sections with STAG2-specific antibodies confirmed tissue-wide absence of the protein (Figure 4B). Importantly, E9.5 KO embryos were visibly smaller than their WT littermates with variable penetrance of the phenotype (mild and severe examples shown in Figure 4C). To establish whether the growth delay is accompanied by developmental delay, we counted somites along the dorsal axis of embryos extracted at E8.5, E9.5, and E10.5 (see detail in Figure 4C). Somite number provides a reliable readout of developmental stage between E8 and E10.5. Although all embryos presented similar somite counts by E8.5, a significantly reduced number of somites was observed in mutant embryos by E9.5. By E10.5, the difference in somite counts between WT and $\mathrm{KO}$ embryos was equivalent to a 1-day lag (Figure 4D). Thus, a loss of STAG2 causes a generalized developmental delay, noticeable by E9.5 with variable penetrance, and results in death by E10.5.

Aberrant Heart Morphogenesis in Stag2-Null Embryos To identify developmental defects that could explain embryonic lethality, we analyzed the histology of E9.5 KO embryos with both mild and severe growth phenotypes ( $\mathrm{KO}$ mild and $\mathrm{KO}$ severe, respectively) and compared them with two different types of WT controls: littermates (age-matched, WT1) and embryos from different litters but with the same number of somites 
A

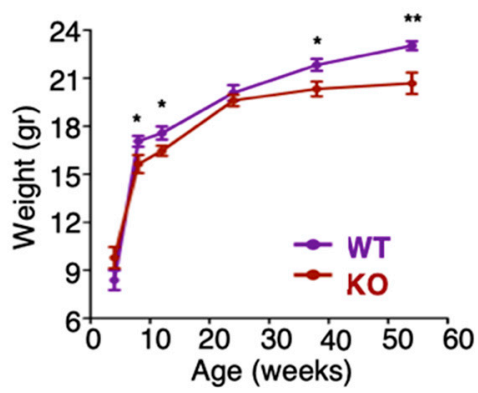

B

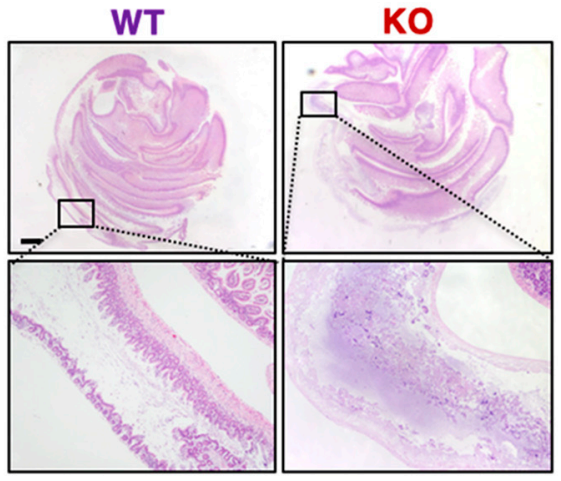

- Severe

ㅁ Moderate

$\square$ Mild

№ne

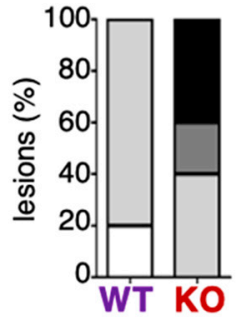

C

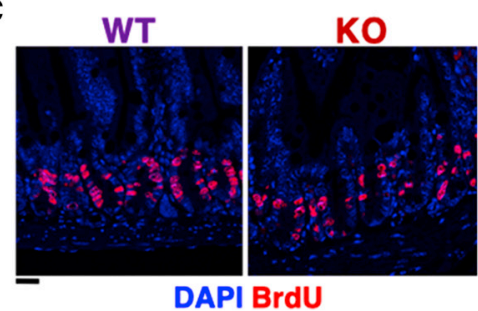

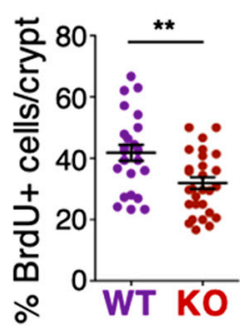

D

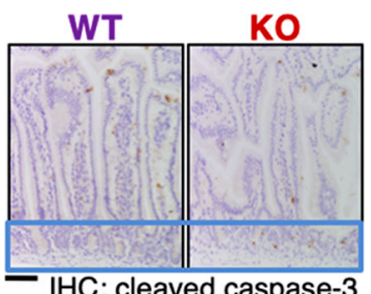

IHC: cleaved caspase-3

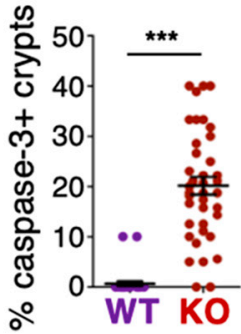

E

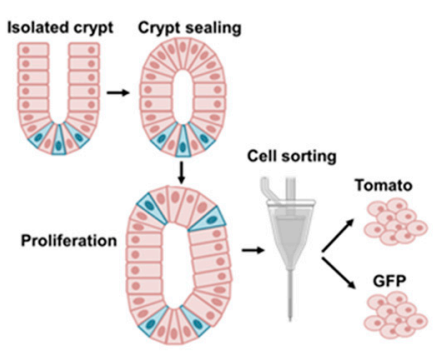

STAG2+

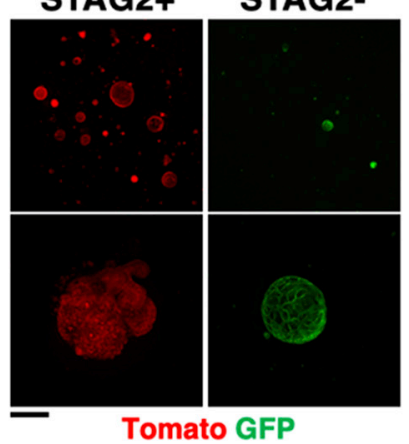

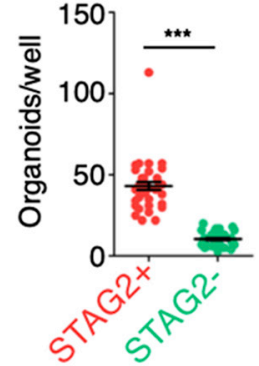

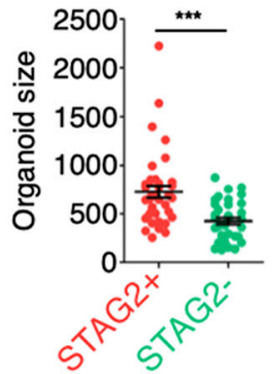

Figure 3. Requirement of STAG2 for Intestinal Cell Renewal

(A) Weight of KO and WT mice over time ( $n=18, W T ; n=18, K O)$. Error bars indicate SEM. One-sided Mann-Whitney U test; ${ }^{*} p<0.05$; ${ }^{* *} p<0.01$.

(B) Representative images of H\&E-stained small intestine sections of 8-week-old WT and KO mice (left) and semiquantitative assessment of severity of lesions (right). Scale bar, $1 \mathrm{~mm}$. ( $n=5, W T ; n=5, K O)$.

(C) Immunofluorescence analysis of BrdU (red) in sections of 8-week-old WT and KO intestine. Nuclei are counterstained with 4',6-diamidino-2-phenylindole (DAPI; blue). Scale bar, $25 \mu \mathrm{m}$. The percentage of BrdU+ cells per crypt is shown in the graph on the right $(n=24, W T ; n=29, K O)$. Error bars indicate SEM. Twotailed Mann-Whitney $U$ test; ${ }^{* *} \mathrm{p}<0.01$.

(D) Immunohistochemical analysis of cleaved caspase-3 in sections of 8 week-old WT and KO intestine. Nuclei are counterstained with hematoxylin. Crypt region is indicated by a dashed box. Scale bar, $50 \mu \mathrm{m}$. The percentage of crypts per section showing cleaved caspase- 3 staining is plotted on the right $(n=30, W T ; n=$ 39, KO). Error bars indicate SEM. Two-tailed Mann-Whitney U test; ${ }^{* \star} \mathrm{p}<0.001$.

(E) Experimental design for intestinal organoid generation (left). Confocal microscopy images of Tomato+ (STAG2+) and GFP+ (STAG2-) organoids (middle). Scale bar, $100 \mu \mathrm{m}$. Quantification of the number and size of organoids (in pixels) obtained from cells sorted from primary intestinal organoids (5,000 cells/well) (right). Error bars indicate SEM. Paired t test; ${ }^{\star \star \star} \mathrm{p}<0.001$.

(stage-matched, WT2). Although KO severe embryos showed aberrant morphology of several structures, most tissues and organs from KO mild embryos did not present obvious malformations but were clearly more similar to stage-matched than to age-matched controls (see neural tube [NT] in Figure 5; see other structures in Figure S3). A remarkable exception to this general trend was a selective defect in the developing heart. At E9.5, the murine heart presents a multichambered conformation as a 
A

\begin{tabular}{|c|c|c|c|c|c|c|c|c|}
\hline \multirow{2}{*}{ genotype } & E8.5 & E9.5 & \multicolumn{2}{|c|}{ E10.5 } & \multicolumn{2}{|c|}{ E12.5 } & \multicolumn{2}{|c|}{ born } \\
\hline & $\begin{array}{ll}\% & n\end{array}$ & $\%$ & $\%$ & & $\%$ & & $\%$ & $\mathrm{n}$ \\
\hline WT & 3012 & $25 \quad 29$ & 28 & 15 & 34 & 25 & 35 & 32 \\
\hline HET & $12.5 \quad 5$ & 22.526 & 24 & 13 & 33 & 24 & 16 & 15 \\
\hline WT & 32.513 & 22.526 & 20 & 11 & 27 & 20 & 49 & 45 \\
\hline alive & $20 \quad 10$ & 35 & 2 & 1 & 0 & 0 & 0 & 0 \\
\hline dead & 0 & 0 & 26 & 14 & 6 & 4 & 0 & 0 \\
\hline
\end{tabular}

B

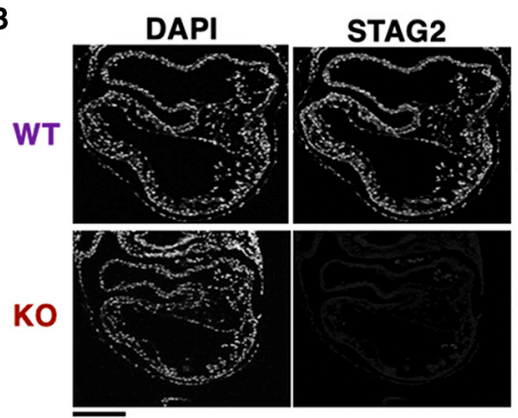

D

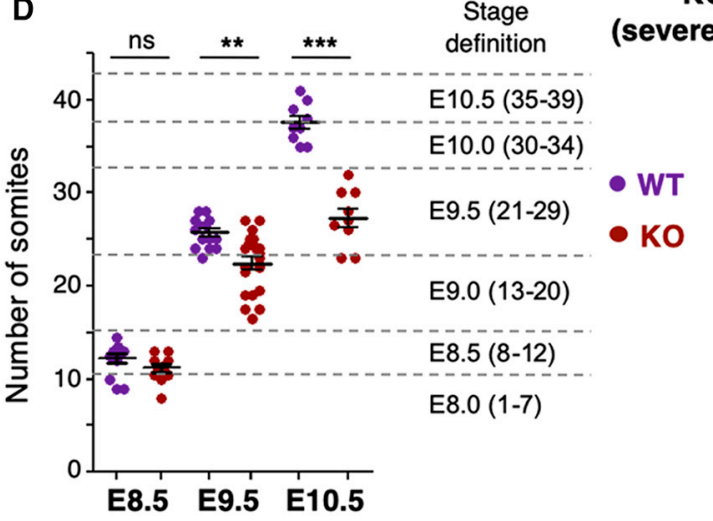

Figure 4. STAG2 Becomes Essential by Midgestation

(A) Viability of STAG2-deficient embryos at different stages of development. We genotyped 6, 14, 7, and 13 litters at E8.5, E9.5, E10.5, and E12.5, respectively, as well as 11 litters at weaning ("born"). Phenotypes and genotypes for Stag2 are female WT $($ lox $/+)$, female HET $(\Delta /+)$, male WT (lox/Y), male $\mathrm{KO}(\Delta / \mathrm{Y})$.

(B) Immunofluorescence staining of STAG2 in transverse heart sections of WT and KO male embryos at E9.5. Nuclei are counterstained with DAPI. Scale bar, $200 \mu \mathrm{m}$.

(C) Representative images of WT and KO male embryos (mild and severe phenotypes) at E9.5 and E10.5. Scale bar, $1 \mathrm{~mm}$. A detail of the somites apparent along the dorsal axis of the embryo is shown at the bottom.

(D) Somite counts of WT and KO male embryos: 6 litters at E8.5 ( $n=13$, WT; $n=10, K O), 9$ litters at E9.5 ( $n=19, W T ; n=25, K O)$, and 4 litters at E10.5 ( $n=10, W T ; n=10, K O)$. Two-tailed Student's t test, ${ }^{\star \star *} p<0.001,{ }^{\star \star} p<0.01 ; n s, p \geq 0.05$. The number of somites expected at each stage of development is indicated on the right.

LV was only smaller than age-matched controls (WT1) due to general developmental delay, but it was not different from stage-matched controls (WT2) (Figure 5D). The IFT of $\mathrm{KO}$ mild embryos appeared normal, but the OFT showed an aberrant rightward turning at the junction with the ventricular myocardium (white arrowhead in WT1 and KO mild under OFT in Figure $5 \mathrm{~B}$ ). Moreover, the length of the OFT was significantly reduced in $\mathrm{KO}$ mild embryos compared with that of both controls (Figure 5E). The defects described above were exacerbated in $\mathrm{KO}$ severe embryos, which displayed distended atria and ventricles with no visible indication of a future septum between right and left chambers (black arrowheads in WT1 and KO severe,

result of linear heart tube extension and looping (see scheme in Figure 5A). Two prospective ventricles and two prospective atria can be distinguished, although there is still no septation between them. The inflow tract (IFT) in the posterior pole of the heart tube allows blood to enter, and the outflow tract (OFT) is an extension of the ventricle that allows blood to flow out and will become the aorta and pulmonary trunk in an adult heart (Kelly et al. 2014). Histological analyses of serial heart sections (Figures $5 \mathrm{~A}$ and $5 \mathrm{~B})$ revealed a smaller right ventricle (RV) in KO mild embryos than that in both controls, whereas no clear differences were found in the left ventricle (LV; compare images for WT1, KO mild, and WT2 under HCs [heart chambers] in Figure 5B). Quantification of the ventricular area confirmed this observation; although the RV was smaller in KO mild embryos than in both age- (WT1) and stage-matched (WT2) controls (Figure 5C), the under $\mathrm{HC}$, in Figure 5B), and abnormal RV development (asterisk in Figure 5B). In these mutants, both the OFT and the IFT were distended. Unlike at E9.5, when penetrance was variable, all $\mathrm{KO}$ embryos displayed severe cardiac anomalies by E10.5, as well as extensive necrosis and apoptosis (Figure 5F). Thus, defective heart function may account for the embryonic lethality of Stag2 KO embryos.

\section{Decreased Proliferation in Stag2-Mutant Embryos}

To shed light into the cellular mechanisms leading to the defects described above, we first confirmed that both STAG1 and STAG2 are expressed in the heart of E9.5 WT embryos by using immunofluorescence (Figure S4). These findings are consistent with reported data from single-cell RNA sequencing (RNA-seq) of E8.25 murine embryos, which shows similar patterns of 
A
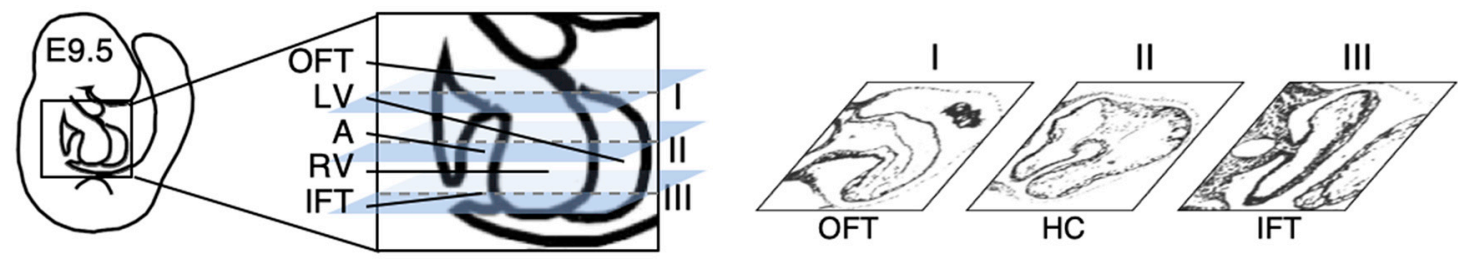

B

E9.5

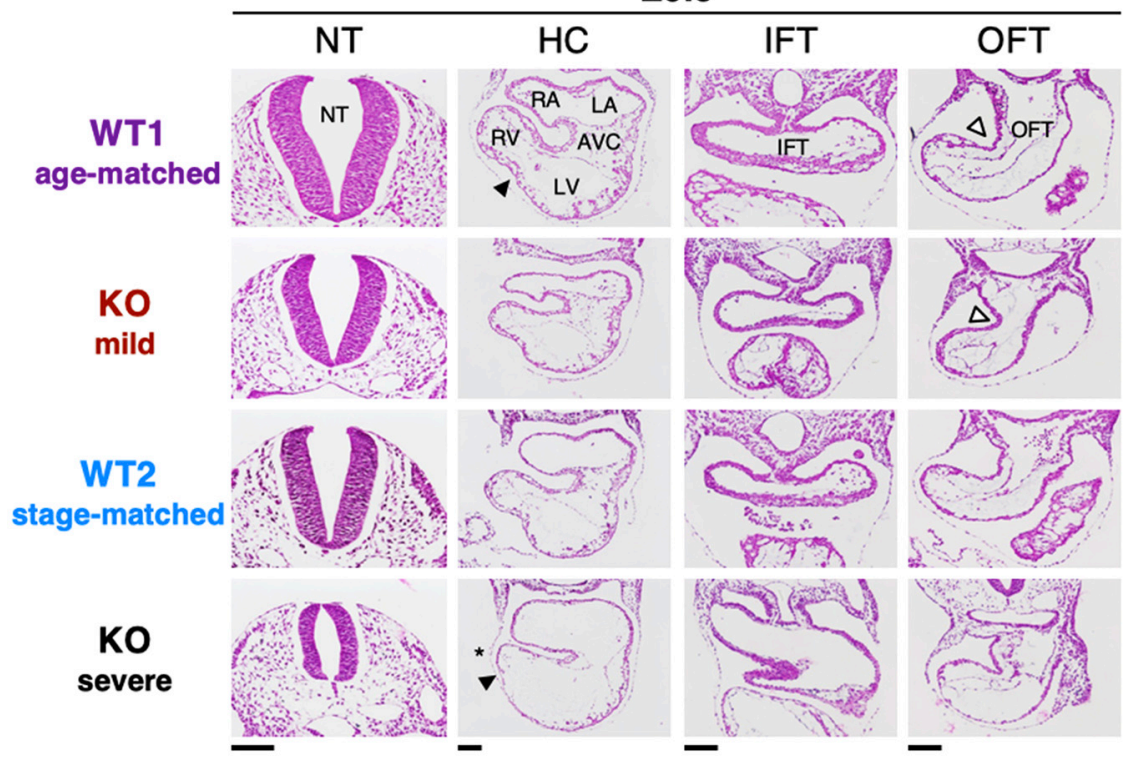

$\mathbf{F}$

E10.5

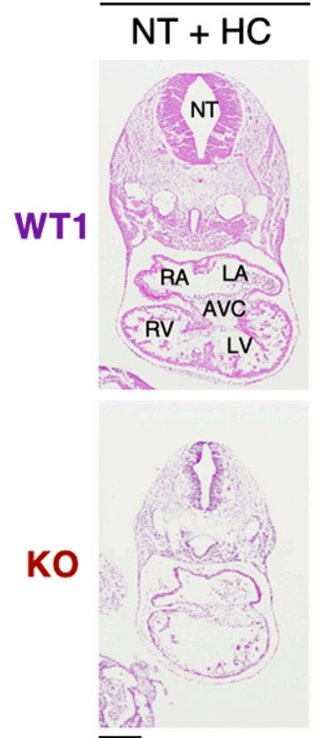

C

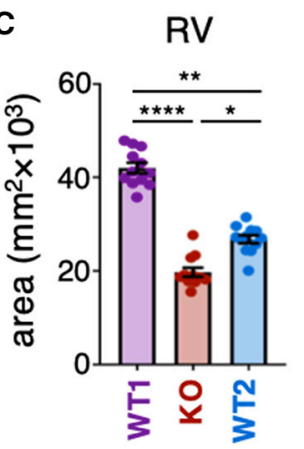

D

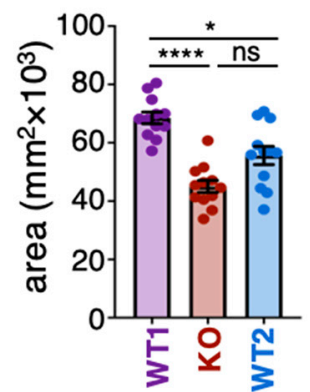

E

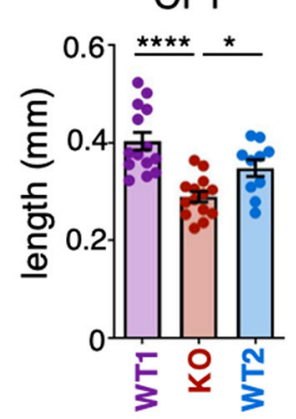

Figure 5. Heart Defects in Stag2-Mutant Embryos

(A) Scheme showing the different regions of an E9.5 embryonic heart and the approximate position and orientation of the transverse sections used for analysis in (B).

(B) H\&E-stained sections of KO (mild and severe), WT1 (age-matched control), and WT2 (stage-matched control) E9.5 male embryo neural tube (NT), heart chambers (HCs), inflow tract (IFT), and outflow tract (OFT). RA, right atrium; LA, left atrium; AVC, atrioventricular canal; RV, right ventricle; LV, left ventricle. Black arrowheads indicate the position of the prospective septum between right and left chambers. White arrowheads point at the OFT curve. Asterisk highlights the small size of the RV in the KO severe embryo. Scale bars (valid for entire column), $100 \mu \mathrm{m}$.

(C) RV size measured in H\&E-stained sections (12 sections from 4 embryos per genotype) from E9.5 WT1 (age-matched control), KO (mild), and WT2 (stagematched control) male embryos. Mean \pm SEM are shown. Kruskal-Wallis test and Dunn's multiple comparison post-test; ${ }^{\star \star \star} p<0.001,{ }^{\star \star} p<0.01$, ${ }^{*} p<0.05$; $n s, p$ $\geq 0.05$. 
expression for both genes (Ibarra-Soria et al., 2018). We next analyzed proliferation and apoptosis in E9.5 WT1, WT2, and $\mathrm{KO}$ mild embryos to uncover primary defects. Heart sections, as well as sections containing the NT for comparison, were labeled with anti-phosphohistone H3 $(\mathrm{H} 3 \mathrm{P})$ to detect proliferating cells and with Terminal deoxynucleotidyl transferase deoxyuridine triphosphate (dUTP) nick end labeling (TUNEL) to mark apoptotic cells. To better identify the different heart compartments, co-staining of Islet 1 (ISL1) was used. ISL1 is a transcription factor expressed in anterior and posterior secondary heart field (ASHF and PSHF, respectively) progenitors that is progressively turned off in their descendants as they migrate into, and populate, the heart tube through its anterior and posterior ends (OFT and IFT, respectively; see scheme in Figure S5A; Cai et al., 2003). The fraction of H3P-positive cells in the HCs (atria and ventricles) was significantly lower in the mutants than in their littermate age-matched WT1 controls but was similar to WT2 stage-matched controls ( $\mathrm{HC}$ in Figures 6A, 6B, and S5B). These differences were reproduced in ASHF and OFT, as well as in the NT, whereas they were less prominent in PSHF and IFT (Figures 6A and 6B; Figure S5B). There was also increased apoptosis in mutant NT and HCs compared with that of both controls, although the number of TUNEL-positive cells was very low in all cases and inter-individual variability was high (Figure 6C). These results suggest that the global developmental delay observed in Stag2 mutants at E9.5 is mainly due to a decrease in the proliferative capacity of mutant cells.

\section{Specific Defects in Secondary Heart Field Progenitors in} Stag2-Mutant Embryos

Although decreased proliferation might account for the global growth delay observed in the heart (and other organs) in mutant embryos, it failed to explain why morphological defects were more evident in certain heart structures, i.e., the OFT and RV. Interestingly, these structures derive from second heart field (SHF) progenitors, whereas the LV derives from the first heart field (FHF) progenitors (Kelly et al., 2014). More specifically, ISL1+ progenitors present in the ASHF migrate into the heart tube contributing to the OFT and RV (Figure S5A). We reasoned that the reduced size of RV and OFT length observed in mutant embryos compared with stage-matched WT2 controls (Figures $5 \mathrm{C}$ and $5 \mathrm{E}$ ) could result from altered migration of ASHF progenitors (ISL1+) into the OFT. To test this possibility, we quantified total cell numbers in heart sections as well as in the NT. We found that in the NT, HC, and OFT, cellularity of $\mathrm{KO}$ mild embryos was lower than that in WT1 and more similar to WT2 embryos (Figure 6D), consistent with their smaller size (Figure 5) and reduced proliferation rates (Figure 6B). In contrast, cell numbers in the ASHF were similar in KO and WT1 littermates (Figure 6D), despite mutants showing a much reduced proliferation rate (Figure $6 \mathrm{~B}$ ). Moreover, although the fraction of ISL1+ progenitors in ASHF was similar in all embryos, it decreased in the OFT of KO mild embryos compared with that of both controls (Figure 6E). Thus, impaired migration of ASHF progenitors into the heart tube could explain the morphological defects in RV and OFT observed in mutant hearts.

\section{Altered Transcription of Cardiac Development \\ Regulators in Stag2-Mutant Embryos}

To address whether gene regulation by cohesin could contribute to the phenotypes described above, we compared the heart transcriptomes of E9.5 Stag2-KO and -WT embryos by RNAseq. To exclude variation related to developmental stage, we selected littermate embryos of both genotypes with a similar number of somites. To identify tissue-specific changes, we extracted RNA from the heart and from the NT lying adjacent to the heart. There were 1,881 differentially expressed genes (DEGs; false discovery rate [FDR] < 0.05) between WT samples of the two tissues, which we used to define a cardiac-enriched and a neural-enriched gene set (1,116 and 765 genes, respectively). Gene Ontology analysis confirmed the functional specificity of these gene sets ("cardiac" and "neural" genes, for simplicity; Figure 7A; Table S1). STAG2 loss had a greater impact on the heart transcriptome, as shown in the heatmap (Figure 7A). Accordingly, pairwise comparisons between WT and KO samples for each tissue identified 846 DEGs in the heart but only 5 in the NT (FDR < 0.05; Figure 7B; Table S2). Among the DEGs in the heart, there were 222 and 112 genes from the cardiac and neural gene sets, respectively, indicating that tissue-specific genes were preferentially affected by STAG2 loss (Figure 7C; Table S1). Moreover, among heart DEGs, most cardiac genes were downregulated, whereas the neural genes were upregulated (Figure 7D). These findings agree with the proposed role of cohesin-STAG2 in tissue-specific transcription, promoting the activation of genes specifying a tissue (i.e., cardiac genes in heart) and repression of alternative gene programs (e.g., neural genes in heart) (Kojic et al., 2018). A closer look at the list of DEGs in the heart revealed several cardiomyocyte markers and well-established SHF regulators among the downregulated genes (Figure $7 \mathrm{~B}$, right). For instance, Fgf8 and Hand2 contribute to the survival of ASHF progenitors, whereas Wnt5a activity is critical for their deployment into the OFT (Park et al., 2006; Sinha et al., 2015; Tsuchihashi et al., 2011), consistent with the defects described in the previous section. These data suggest that the loss of STAG2 alters the expression of genes encoding important regulators of heart remodeling by SHF progenitors. This finding, together with decreased proliferation, likely contributes to the observed defects in heart morphogenesis.

\section{DISCUSSION}

A major challenge in cohesin biology is to understand the specific functions of STAG1 and STAG2 (Cuadrado et al., 2019; Kojic et al., 2018; Wutz et al., 2020). To address this question, we

(D) LV size, as in (C).

(E) The outer curve of the OFT was measured in 10-12 sections from 4 embryos of each genotype stained as shown in Figure 6. Mean \pm SEM are shown. KruskalWallis test and Dunn's multiple comparison post-test; ${ }^{* \star *} \mathrm{p}<0.001$, ${ }^{*} \mathrm{p}<0.05$; $\mathrm{ns}, \mathrm{p} \geq 0.05$.

(F) H\&E-stained transverse sections encompassing the NT and the heart of a WT and a KO male embryo at E10.5. Heart regions indicated as in (A). Scale bar, $250 \mu \mathrm{m}$. 

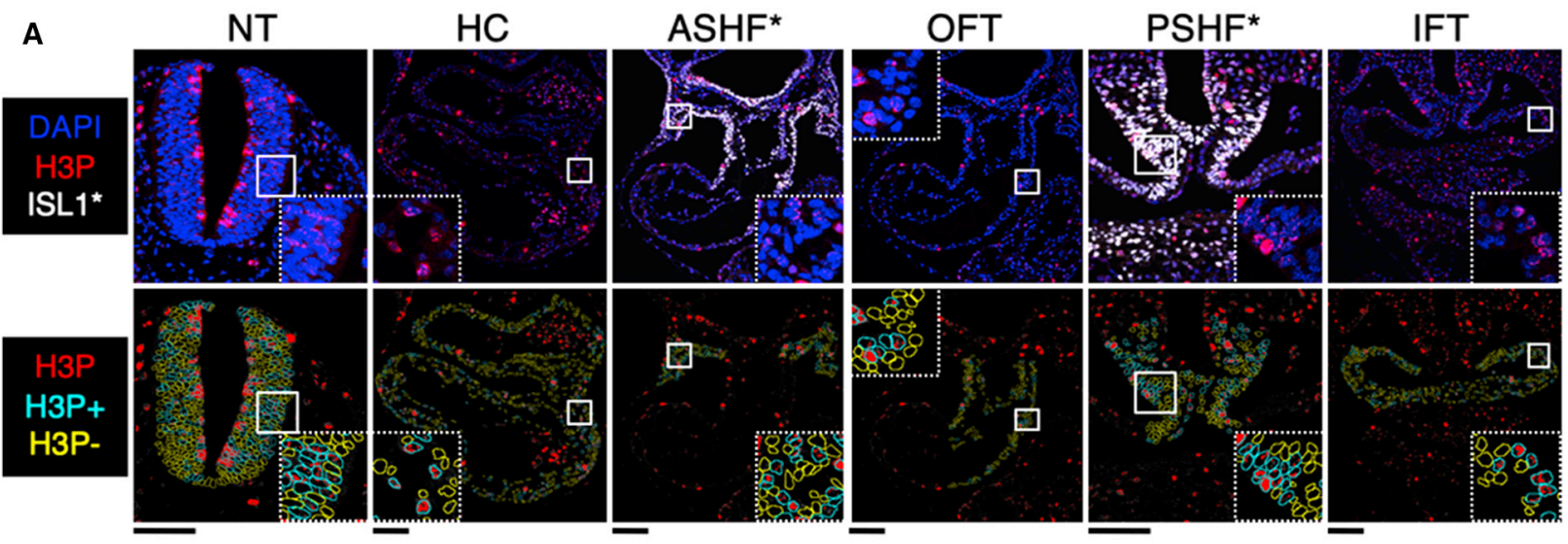

B
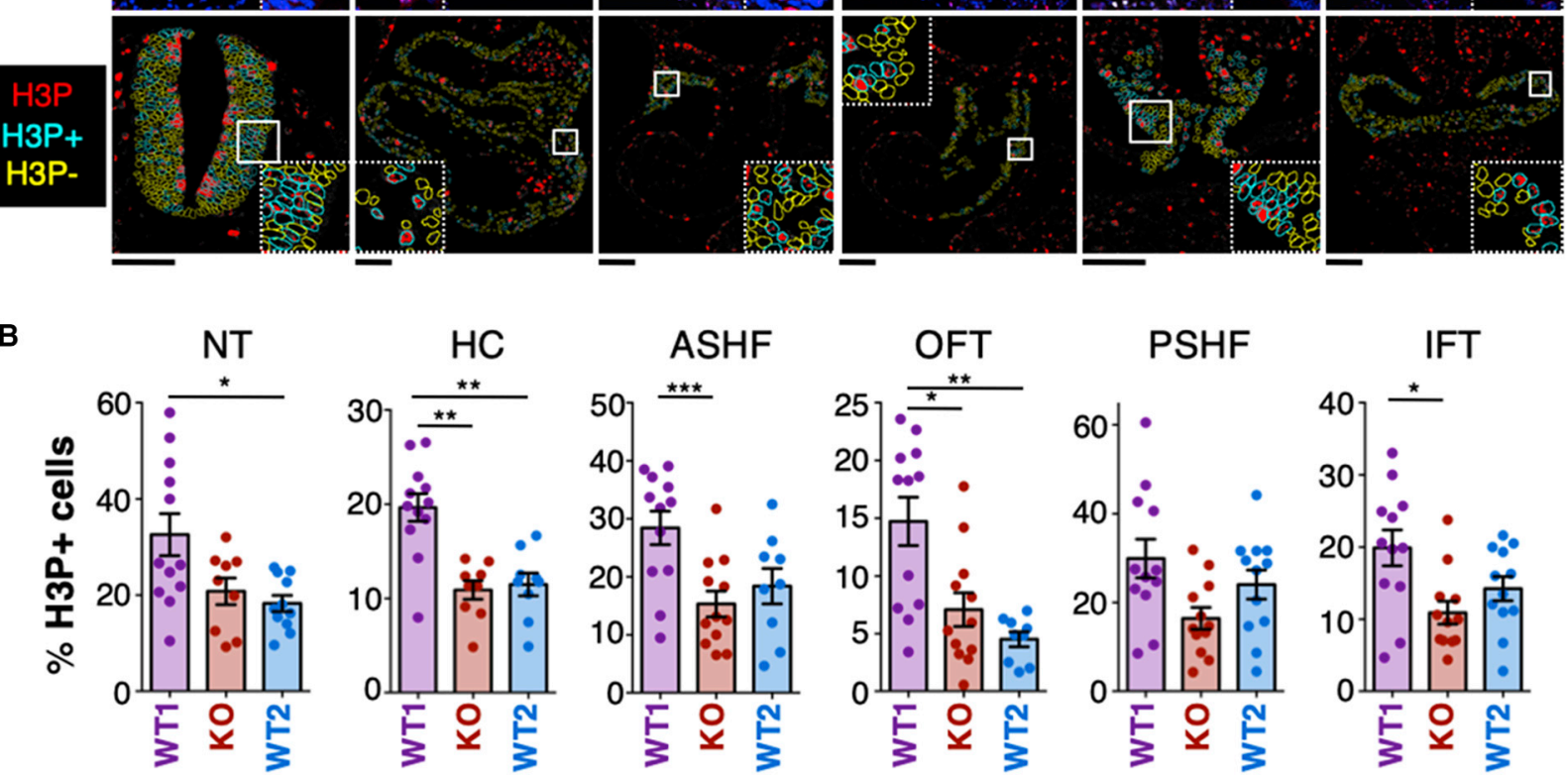

C
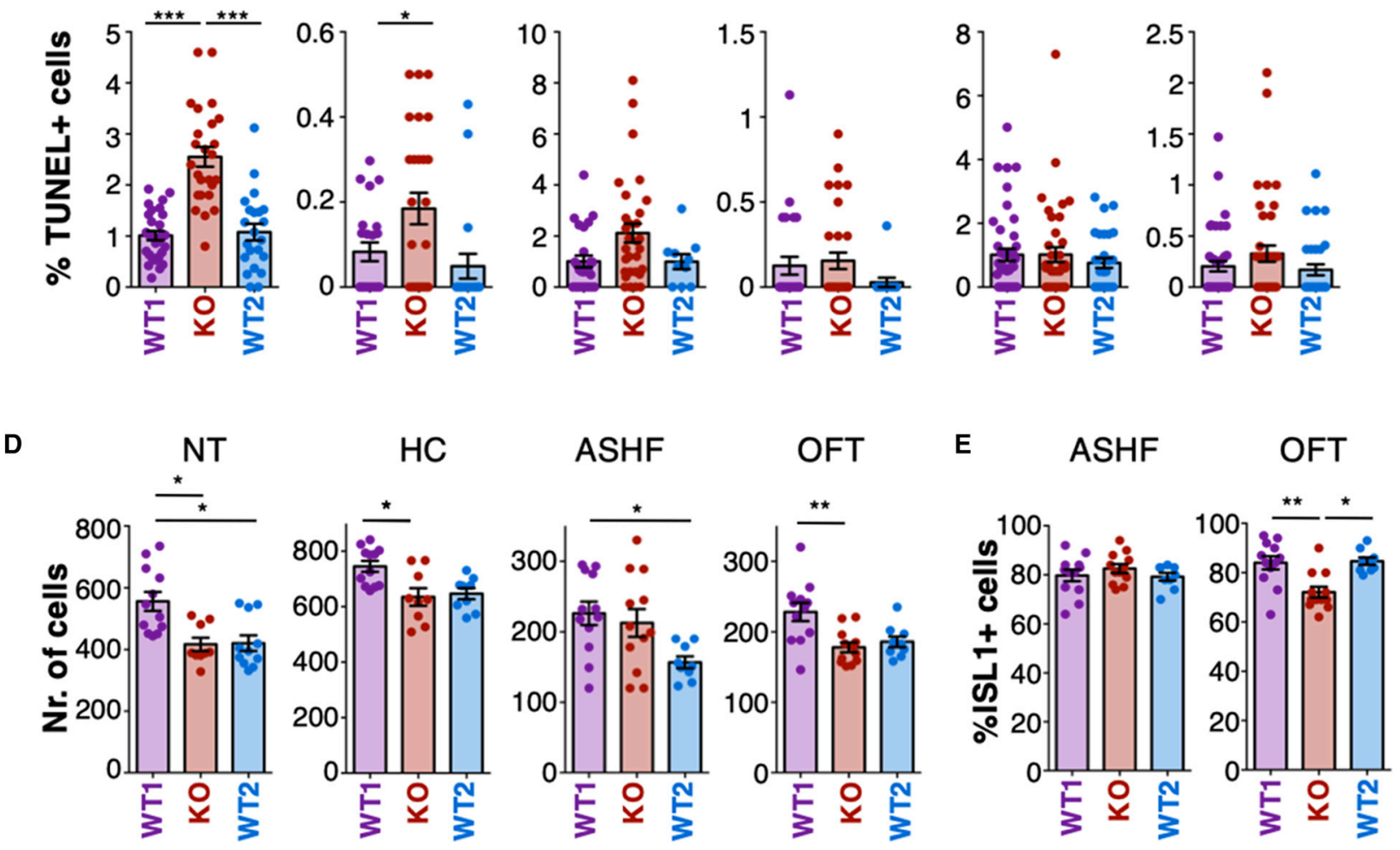
A

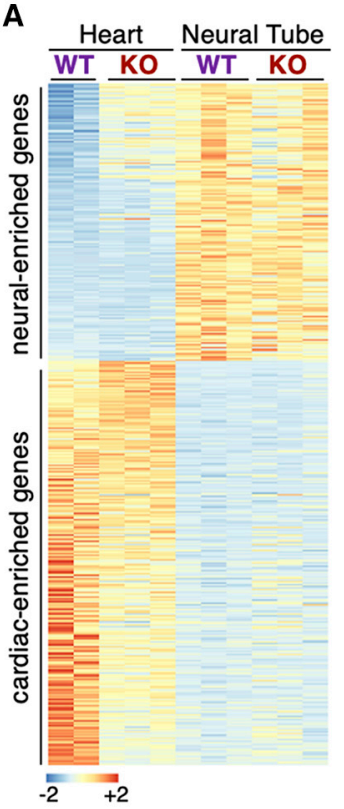

B

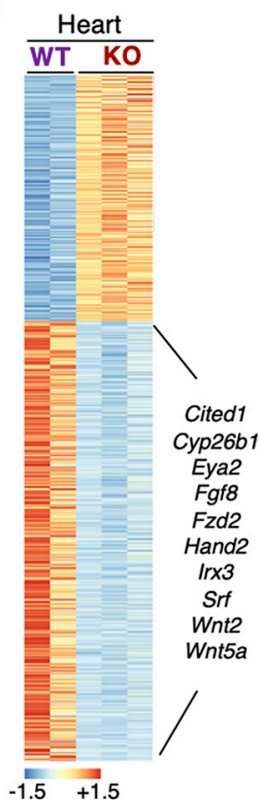

C

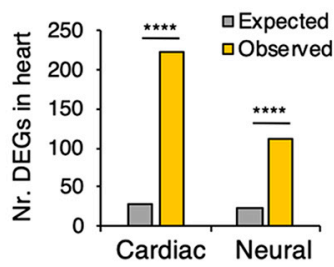

D

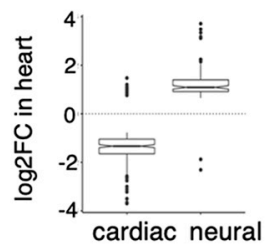

Figure 7. Transcriptional Deregulation in Stag2-Null Embryos

(A) Heatmap showing relative expression of 1,116 cardiac- and 765 neural-enriched genes in all samples. Gene sets were defined by differential expression between WT heart and WT NT samples.

(B) Heatmap of 846 DEGs in WT and KO heart samples. Among the downregulated genes, we highlight some with established roles in cardiomyocyte differentiation and SHF progenitors.

(C) Expected versus observed number of cardiac and neural genes found among heart DEGs. Total number of expressed genes was 21,653. Fisher's exact test; ${ }^{* * * *}<0.0001(\mathrm{p}<2 \mathrm{E}-12)$.

(D) Boxplot of expression changes in the cardiac and neural genes identified as DEGs in the heart. See also Table S1.

result from a decreased proliferation capacity of the mutant cells, as suggested by our findings using organoids, and/or from altered regulation of the balance between self-renewal and differentiation in the intestine, as shown in HSCs. In fact, a recent previously characterized a Stag1-KO mouse (Remeseiro et al., 2012a, 2012b), and we have now generated a Stag2-KO mouse. Recently, a study describing the consequences of Stag2 ablation in the hematopoietic system of adult mice demonstrated a specific role for STAG2 in balancing self-renewal and differentiation in hematopoietic precursors (Viny et al., 2019). Here, we describe instead the consequences of ubiquitous STAG2 elimination in embryos and adult mice.

Whole-body deletion of Stag2 in young mice does not result in acute loss of viability, which suggests that STAG1 can largely compensate for the lack of STAG2 postnatally. Efficiency of Cre-mediated recombination of the Stag2-cKO allele was high in adult tissues 8 weeks after Cre induction, but the fraction of recombined cells decreased notably over the subsequent weeks in proliferative tissues despite continuous TMX administration. This observation indicates a clear proliferative disadvantage of STAG2-deficient cells and does not allow us to rule out that a more severe phenotype might be disclosed upon achieving a more complete or sustained depletion of STAG2. Consistent with results obtained upon Stag2 deletion in HSCs by using Mx1-Cre, ubiquitous deletion results in increased self-renewal and defective lineage commitment in this compartment (Viny et al., 2019). Our histopathological analyses also detected defects in gastrointestinal tract homeostasis. This phenotype could study shows that cohesin promotes repression of differentiation genes in Drosophila intestinal stem cells to maintain stemness and intestinal homeostasis (Khaminets et al., 2020). However, TMX toxicity in the gut has been reported and was focused specifically in the stomach (Keeley et al., 2019). Thus, toxicity resulting from continued TMX administration - a condition used here to avoid expansion of unrecombined Stag2 WT cells-may also contribute to the intestinal phenotype. A more detailed analysis of the effects of STAG2 loss in the intestine, by using a variety of TMX administration protocols and a time course, is warranted.

In contrast to the redundancy and functional compensation of the STAG proteins in adult mice, embryos require both proteins to complete development. Constitutive inactivation of Stag1 in the germline is embryonic lethal and causes severe development delay, with incomplete penetrance, but no obvious organ malformation (Remeseiro et al., 2012a, 2012b). In contrast, inactivation of Stag2 in the germline leads to earlier lethality, starting at E9.5. This phenotype is associated with a general developmental delay and a dramatic effect on heart development with no Stag2-null embryos surviving beyond E10.5. Histopathological characterization of $\mathrm{KO}$ mild embryos revealed specific defects in heart structures derived from the SHF, the RV, and OFT. More detailed analyses detected an accumulation of progenitor

Figure 6. Reduced Cell Proliferation and Impaired Migration of ASHFs in the Developing Heart of Stag2-Null Embryos

(A) Representative transverse sections of H3P staining of E9.5 WT1 embryos. Top row: original immunofluorescence signal of DAPI (blue), H3P (red), and ISL1 (white, only shown in regions marked with *). Both morphological criteria and ISL1 staining were used to demarcate ASHF and PSHF regions. Bottom row: H3P signal converted to a binary image, with representation of nuclei selection. Similar images for KO and WT2 embryos are shown in Figure S5. Scale bars, $100 \mu \mathrm{m}$. (B) Quantification of H3P-positive cells as readouts for proliferation in E9.5 male embryos. A total of 9-12 non-consecutive sections from 3-4 embryos were analyzed per genotype and region.

(C) Quantification of TUNEL-positive cells as readouts for apoptosis in the same embryos. At least 10 sections from 3-4 embryos were analyzed per genotype and region.

(D and E) Quantification of the total number of cells per section (D) and ISL1-positive cells (E) in the indicated regions. Same sections as in (B) were analyzed. For (B)-(D), mean \pm SEM are shown. Kruskal-Wallis test and Dunn's multiple comparison post-test; ${ }^{* \star *} p<0.001,{ }^{* *} p<0.01,{ }^{*} p<0.05 ; n s, p \geq 0.05$. 
(ISL1+) cells in the ASHF of mutant embryos as well as their reduced presence in the OFT. Impaired migration of ASHF progenitors into the heart tube could account for these observations. Alternative explanations would be premature differentiation or increased apoptosis of ISL1+ progenitors in the OFT of the mutant embryos, but they would not account for the increase in cell numbers in the ASHF. Interestingly, defects in the migration of progenitors has also been proposed as the cause of heart defects in zebrafish and murine embryos after reducing the levels of cohesin or its loader NIPBL (Muto et al., 2011; Santos et al., 2016; Schuster et al., 2015). The transcriptomic changes identified in the heart of $\mathrm{KO}$ embryos could contribute to this defect, although the underlying cellular and molecular mechanisms need to be identified. Increasing evidence supports the notion that the presence of cohesin-STAG2 at enhancer elements independently of CTCF promotes cell-type-specific transcription, a function that is not compensated by cohesin-STAG1 (Cuadrado et al., 2019; Kojic et al., 2018; Viny et al., 2019). Consistent with this idea, we observed altered tissue-specific transcription patterns in $\mathrm{KO}$ embryonic hearts, with a lower expression of cardiac genes and de-repression of genes from other lineages. Thus, we propose that defects in both proliferation and lineage specification contribute to the heart abnormalities observed in the STAG2-deficient embryos. We cannot ascertain whether these heart defects are the primary cause of embryonic death. However, the heart is one of the first organs to start differentiating in the embryo and the first one to become functional (Bruneau, 2013). Impaired heart function would make embryos unable to sustain further development, thus masking defects in other organs arising later.

Cohesinopathy cases with STAG2 mutations have been reported recently. Male patients display mild phenotypes, lack heart defects, and carry missense variants. In contrast, ventricular septal defects and other heart anomalies have been described in female patients carrying loss-of-function or missense variants (Lehalle et al., 2017; Mullegama et al., 2017, 2019; Soardi et al., 2017; Yuan et al., 2019). Because STAG2 is an X-linked gene, the embryonic lethality of Stag2-null male murine embryos reported here explains why inactivating germline mutations will most likely not be tolerated in males, whereas heterozygous females may survive through the selection of cells in which the WT allele is not silenced by the $X$ inactivation process. Heterozygous female embryos resulting from mating Stag $2^{\text {lox/lox }}$ females with CAG-Cre males arrive normally to mid-gestation (E12.5), but they are born at sub-Mendelian ratios, indicating problems in the last stages of embryonic development (Figure 4A). Although the heterozygous females that are born appear as healthy as their WT littermates, more specific studies will be required to address the potential resemblance with the phenotypes of female patients carrying heterozygous mutations in STAG2.

In summary, here, we show the distinct functional properties of STAG2 at the cellular and organismal levels in mice, compared with those of STAG1. Cells lacking cohesin-STAG2 are viable both in vitro (tissue culture) and in vivo (in embryos and adult tissues), confirming that cohesin-STAG1 is sufficient to fulfill essential cohesin functions (van der Lelij et al., 2017; Liu et al., 2018). However, their decreased proliferation and altered tran- scriptomes lead to embryonic lethality, a result that provides further compelling evidence for cell- and tissue-specific roles of the two cohesin complexes and how their dysfunction may contribute to disease. We speculate that genomic changes derived from decreased accuracy of chromosome segregation and/or DNA repair as well as transcriptional alterations affecting cell identity and stem cell physiology may underlie the behavior of STAG2 mutant tumors. Although inactivation of Stag2 in adult mice did not increase tumor incidence in our study, similar to other major tumor suppressor genes such as Cdkn2a or $R b$ (Krimpenfort et al., 2001; Sharpless et al., 2001; Vooijs and Berns, 1999), these mice will be useful to model the cooperation of STAG2 mutations with other genetic alterations for promoting tumorigenesis in a wide variety of cell types.

\section{STAR $\star$ METHODS}

Detailed methods are provided in the online version of this paper and include the following:

- KEY RESOURCES TABLE

- RESOURCE AVAILABILITY

Lead Contact

○ Materials Availability

Data and Code Availability

- EXPERIMENTAL MODEL AND SUBJECT DETAILS

O Generation of a conditional knockout allele for Stag2

O MEFs

- METHOD DETAILS

O MEF characterization

O Histopathology and immunohistochemical (IHC) analyses of adult tissue sections

○ Hematological analyses

O Hematopoietic cell isolation and Flow Cytometry

O In vitro colony-forming unit assays

O Establishment of intestinal organoids

O Hematoxylin and immunofluorescence staining of mouse embryo sections

O RNA-sequencing

- QUANTIFICATION AND STATISTICAL ANALYSIS

\section{SUPPLEMENTAL INFORMATION}

Supplemental Information can be found online at https://doi.org/10.1016/j. celrep.2020.108014.

\section{ACKNOWLEDGMENTS}

We acknowledge the excellent technical support of the CNIO Mouse Genome Editing unit led by Sagrario Ortega and the CNIC Microscopy unit, in particular Verónica Labrador, as well as the help of Natalia del Pozo, Ana Cuadrado, Dácil Alonso, Alba de Martino, Eduardo Caleiras, and Cristian Perna. This work has been supported by the State Research Agency (AEI), Spanish Ministry of Science and Innovation, with cofunding of the European Regional Development Funds (grants BFU2013-48481-R and BFU2016-79841-R to A.L., SAF2015-70553-R to F.X.R., BFU2017-84914-P and BFU2015-72319-EXP to M.M., and BES-2014-069166 fellowship to M.D.K.), and a grant to F.X.R. and a Postdoctoral Contract to E.L. from the Fundación Científica de la Asociación Española Contra el Cáncer. Both CNIO and CNIC are supported by Instituto de Salud Carlos III (ISCIII) and Severo Ochoa Centers of Excellence 
(SEV-2015-0510 and SEV-2015-0505). The CNIC is also supported by the Pro CNIC Foundation.

\section{AUTHOR CONTRIBUTIONS}

E.L. and M.R.-C. generated the Stag2 cKO mouse; M.D.K. characterized MEFs; E.L., E.A., and I.C. carried out the studies in adult mice; M.D.K. and C.B.-C. performed the embryo studies; D.G.-L. analyzed RNA-seq data; A.H., M.M., F.X.R., and A.L. supervised the study and contributed to experimental design and data interpretation.

\section{DECLARATION OF INTERESTS}

The authors declare no competing interests

Received: March 30, 2020

Revised: June 15, 2020

Accepted: July 17, 2020

Published: August 11, 2020

\section{REFERENCES}

Balbás-Martínez, C., Sagrera, A., Carrillo-de-Santa-Pau, E., Earl, J., Márquez, M., Vazquez, M., Lapi, E., Castro-Giner, F., Beltran, S., Bayés, M., et al. (2013). Recurrent inactivation of STAG2 in bladder cancer is not associated with aneuploidy. Nat. Genet. 45, 1464-1469.

Belteki, G., Haigh, J., Kabacs, N., Haigh, K., Sison, K., Costantini, F., Whitsett, J., Quaggin, S.E., and Nagy, A. (2005). Conditional and inducible transgene expression in mice through the combinatorial use of Cre-mediated recombination and tetracycline induction. Nucleic Acids Res. 33, e51.

Bruneau, B.G. (2013). Signaling and transcriptional networks in heart development and regeneration. Cold Spring Harb. Perspect. Biol. 5, a008292.

Cai, C.-L., Liang, X., Shi, Y., Chu, P.-H., Pfaff, S.L., Chen, J., and Evans, S. (2003). Isl1 identifies a cardiac progenitor population that proliferates prior to differentiation and contributes a majority of cells to the heart. Dev. Cell 5, 877-889.

Canudas, S., and Smith, S. (2009). Differential regulation of telomere and centromere cohesion by the Scc3 homologues SA1 and SA2, respectively, in human cells. J. Cell Biol. 187, 165-173.

Carretero, M., Ruiz-Torres, M., Rodríguez-Corsino, M., Barthelemy, I., and Losada, A. (2013). Pds5B is required for cohesion establishment and Aurora B accumulation at centromeres. EMBO J. 32, 2938-2949.

Cuadrado, A., and Losada, A. (2020). Specialized functions of cohesins STAG1 and STAG2 in 3D genome architecture. Curr. Opin. Genet. Dev. 61, 9-16.

Cuadrado, A., Remeseiro, S., Graña, O., Pisano, D.G., and Losada, A. (2015). The contribution of cohesin-SA1 to gene expression and chromatin architecture in two murine tissues. Nucleic Acids Res. 43, 3056-3067.

Cuadrado, A., Giménez-Llorente, D., Kojic, A., Rodríguez-Corsino, M., Cuartero, Y., Martín-Serrano, G., Gómez-López, G., Marti-Renom, M.A., and Losada, A. (2019). Specific Contributions of Cohesin-SA1 and Cohesin-SA2 to TADs and Polycomb Domains in Embryonic Stem Cells. Cell Rep. 27, 35003510.e4.

De Koninck, M., and Losada, A. (2016). Cohesin mutations in cancer. Cold Spring Harb. Perspect. Med. 6, a026476.

Dowen, J.M., Fan, Z.P., Hnisz, D., Ren, G., Abraham, B.J., Zhang, L.N., Weintraub, A.S., Schujiers, J., Lee, T.I., Zhao, K., and Young, R.A. (2014). Control of cell identity genes occurs in insulated neighborhoods in mammalian chromosomes. Cell 159, 374-387.

Faure, A.J., Schmidt, D., Watt, S., Schwalie, P.C., Wilson, M.D., Xu, H., Ramsay, R.G., Odom, D.T., and Flicek, P. (2012). Cohesin regulates tissue-specific expression by stabilizing highly occupied cis-regulatory modules. Genome Res. 22, 2163-2175.

Hay, T., Patrick, T., Winton, D., Sansom, O.J., and Clarke, A.R. (2005). Brca2 deficiency in the murine small intestine sensitizes to p53-dependent apoptosis and leads to the spontaneous deletion of stem cells. Oncogene 24, 38423846.

Ibarra-Soria, X., Jawaid, W., Pijuan-Sala, B., Ladopoulos, V., Scialdone, A., Jörg, D.J., Tyser, R.C.V., Calero-Nieto, F.J., Mulas, C., Nichols, J., et al. (2018). Defining murine organogenesis at single-cell resolution reveals a role for the leukotriene pathway in regulating blood progenitor formation. Nat. Cell Biol. 20, 127-134.

Ireland, H., Kemp, R., Houghton, C., Howard, L., Clarke, A.R., Sansom, O.J., and Winton, D.J. (2004). Inducible Cre-mediated control of gene expression in the murine gastrointestinal tract: effect of loss of $\beta$-catenin. Gastroenterology 126, 1236-1246.

Kagey, M.H., Newman, J.J., Bilodeau, S., Zhan, Y., Orlando, D.A., van Berkum, N.L., Ebmeier, C.C., Goossens, J., Rahl, P.B., Levine, S.S., et al. (2010). Mediator and cohesin connect gene expression and chromatin architecture. Nature 467, 430-435.

Keeley, T.M., Horita, N., and Samuelson, L.C. (2019). Tamoxifen-Induced Gastric Injury: Effects of Dose and Method of Administration. Cell. Mol. Gastroenterol. Hepatol. 8, 365-367.

Kelly, R.G., Buckingham, M.E., and Moorman, A.F. (2014). Heart fields and cardiac morphogenesis. Cold Spring Harb. Perspect. Med. 4, a015750.

Khaminets, A., Ronnen-Oron, T., Baldauf, M., Meier, E., and Jasper, H. (2020). Cohesin controls intestinal stem cell identity by maintaining association of Escargot with target promoters. eLife 9, e48160.

Kim, J.S., He, X., Orr, B., Wutz, G., Hill, V., Peters, J.M., Compton, D.A., and Waldman, T. (2016). Intact Cohesion, Anaphase, and Chromosome Segregation in Human Cells Harboring Tumor-Derived Mutations in STAG2. PLoS Genet. 12, e1005865.

Kojic, A., Cuadrado, A., De Koninck, M., Giménez-Llorente, D., RodríguezCorsino, M., Gómez-López, G., Le Dily, F., Marti-Renom, M.A., and Losada, A. (2018). Distinct roles of cohesin-SA1 and cohesin-SA2 in 3D chromosome organization. Nat. Struct. Mol. Biol. 25, 496-504.

Kong, X., Ball, A.R., Jr., Pham, H.X., Zeng, W., Chen, H.Y., Schmiesing, J.A., Kim, J.S., Berns, M., and Yokomori, K. (2014). Distinct functions of human cohesin-SA1 and cohesin-SA2 in double-strand break repair. Mol. Cell. Biol. 34, 685-698.

Krimpenfort, P., Quon, K.C., Mooi, W.J., Loonstra, A., and Berns, A. (2001) Loss of p16Ink4a confers susceptibility to metastatic melanoma in mice. $\mathrm{Na}$ ture 413, 83-86.

Lambert, J.F., Benoit, B.O., Colvin, G.A., Carlson, J., Delville, Y., and Quesenberry, P.J. (2000). Quick sex determination of mouse fetuses. J. Neurosci. Methods 95, 127-132.

Lawrence, M.S., Stojanov, P., Mermel, C.H., Robinson, J.T., Garraway, L.A., Golub, T.R., Meyerson, M., Gabriel, S.B., Lander, E.S., and Getz, G. (2014). Discovery and saturation analysis of cancer genes across 21 tumour types. Nature 505, 495-501.

Lehalle, D., Mosca-Boidron, A.-L., Begtrup, A., Boute-Benejean, O., Charles, P., Cho, M.T., Clarkson, A., Devinsky, O., Duffourd, Y., Duplomb-Jego, L., et al. (2017). STAG1 mutations cause a novel cohesinopathy characterised by unspecific syndromic intellectual disability. J. Med. Genet. 54, 479-488.

Liu, J., Zhang, Z., Bando, M., Itoh, T., Deardorff, M.A., Clark, D., Kaur, M., Tandy, S., Kondoh, T., Rappaport, E., et al. (2009). Transcriptional dysregulation in NIPBL and cohesin mutant human cells. PLoS Biol. 7, e1000119.

Liu, Y., Xu, H., Van der Jeught, K., Li, Y., Liu, S., Zhang, L., Fang, Y., Zhang, X., Radovich, M., Schneider, B.P., et al. (2018). Somatic mutation of the cohesin complex subunit confers therapeutic vulnerabilities in cancer. J. Clin. Invest. 128, 2951-2965.

Losada, A., Yokochi, T., Kobayashi, R., and Hirano, T. (2000). Identification and characterization of SA/Scc3p subunits in the Xenopus and human cohesin complexes. J. Cell Biol. 150, 405-416.

Love, M.I., Huber, W., and Anders, S. (2014). Moderated estimation of fold change and dispersion for RNA-seq data with DESeq2. Genome Biol. 15, 550. Meisenberg, C., Pinder, S.I., Hopkins, S.R., Wooller, S.K., Benstead-Hume, G., Pearl, F.M.G., Jeggo, P.A., and Downs, J.A. (2019). Repression of 
Transcription at DNA Breaks Requires Cohesin throughout Interphase and Prevents Genome Instability. Mol. Cell 73, 212-223.e7.

Merkenschlager, M., and Nora, E.P. (2016). CTCF and Cohesin in Genome Folding and Transcriptional Gene Regulation. Annu. Rev. Genomics Hum. Genet. 17, 17-43.

Mondal, G., Stevers, M., Goode, B., Ashworth, A., and Solomon, D.A. (2019). A requirement for STAG2 in replication fork progression creates a targetable synthetic lethality in cohesin-mutant cancers. Nat. Commun. 10, 1686.

Morales, C., and Losada, A. (2018). Establishing and dissolving cohesion during the vertebrate cell cycle. Curr. Opin. Cell Biol. 52, 51-57.

Mullegama, S.V., Klein, S.D., Mulatinho, M.V., Senaratne, T.N., Singh, K., UCLA Clinical Genomics Center; Nguyen, D.C., Gallant, N.M., Strom, S.P., Ghahremani, S., Rao, N.P., and Martinez-Agosto, J.A. (2017). De novo lossof-function variants in STAG2 are associated with developmental delay, microcephaly, and congenital anomalies. Am. J. Med. Genet. A. 173, 1319-1327. Mullegama, S.V., Klein, S.D., Signer, R.H., UCLA Clinical Genomics Center; Vilain, E., and Martinez-Agosto, J.A. (2019). Mutations in STAG2 cause an Xlinked cohesinopathy associated with undergrowth, developmental delay, and dysmorphia: Expanding the phenotype in males. Mol. Genet. Genomic Med. 7, e00501.

Mullenders, J., Aranda-Orgilles, B., Lhoumaud, P., Keller, M., Pae, J., Wang, K., Kayembe, C., Rocha, P.P., Raviram, R., Gong, Y., et al. (2015). Cohesin loss alters adult hematopoietic stem cell homeostasis, leading to myeloproliferative neoplasms. J. Exp. Med. 212, 1833-1850.

Muto, A., Calof, A.L., Lander, A.D., and Schilling, T.F. (2011). Multifactorial origins of heart and gut defects in nipbl-deficient zebrafish, a model of Cornelia de Lange Syndrome. PLoS Biol. 9, e1001181.

Nasmyth, K., and Haering, C.H. (2009). Cohesin: its roles and mechanisms. Annu. Rev. Genet. 43, 525-558.

Park, E.J., Ogden, L.A., Talbot, A., Evans, S., Cai, C.-L., Black, B.L., Frank, D.U., and Moon, A.M. (2006). Required, tissue-specific roles for Fgf8 in outflow tract formation and remodeling. Development 133, 2419-2433.

Remeseiro, S., Cuadrado, A., Carretero, M., Martínez, P., Drosopoulos, W.C., Cañamero, M., Schildkraut, C.L., Blasco, M.A., and Losada, A. (2012a). Cohesin-SA1 deficiency drives aneuploidy and tumourigenesis in mice due to impaired replication of telomeres. EMBO J. 31, 2076-2089.

Remeseiro, S., Cuadrado, A., Gómez-López, G., Pisano, D.G., and Losada, A. (2012b). A unique role of cohesin-SA1 in gene regulation and development. EMBO J. 31, 2090-2102.

Ruzankina, Y., Pinzon-Guzman, C., Asare, A., Ong, T., Pontano, L., Cotsarelis, G., Zediak, V.P., Velez, M., Bhandoola, A., and Brown, E.J. (2007). Deletion of the developmentally essential gene ATR in adult mice leads to age-related phenotypes and stem cell loss. Cell Stem Cell 1, 113-126.

Santos, R., Kawauchi, S., Jacobs, R.E., Lopez-Burks, M.E., Choi, H., Wikenheiser, J., Hallgrimsson, B., Jamniczky, H.A., Fraser, S.E., Lander, A.D., and Calof, A.L. (2016). Conditional Creation and Rescue of Nipbl-Deficiency in Mice Reveals Multiple Determinants of Risk for Congenital Heart Defects. PLoS Biol. 14, e2000197.

Sarogni, P., Pallotta, M.M., and Musio, A. (2020). Cornelia de Lange syndrome: from molecular diagnosis to therapeutic approach. J. Med. Genet. 57, 289-295.
Sasca, D., Yun, H., Giotopoulos, G., Szybinski, J., Evan, T., Wilson, N.K., Gerstung, M., Gallipoli, P., Green, A.R., Hills, R., et al. (2019). Cohesin-dependent regulation of gene expression during differentiation is lost in cohesin-mutated myeloid malignancies. Blood 134, 2195-2208.

Schuster, K., Leeke, B., Meier, M., Wang, Y., Newman, T., Burgess, S., and Horsfield, J.A. (2015). A neural crest origin for cohesinopathy heart defects. Hum. Mol. Genet. 24, 7005-7016.

Sharpless, N.E., Bardeesy, N., Lee, K.H., Carrasco, D., Castrillon, D.H., Aguirre, A.J., Wu, E.A., Horner, J.W., and DePinho, R.A. (2001). Loss of p16Ink4a with retention of p19Arf predisposes mice to tumorigenesis. Nature 413, 86-91.

Sinha, T., Li, D., Théveniau-Ruissy, M., Hutson, M.R., Kelly, R.G., and Wang, J. (2015). Loss of Wnt5a disrupts second heart field cell deployment and may contribute to OFT malformations in DiGeorge syndrome. Hum. Mol. Genet. 24, 1704-1716.

Soardi, F.C., Machado-Silva, A., Linhares, N.D., Zheng, G., Qu, Q., Pena, H.B. Martins, T.M.M., Vieira, H.G.S., Pereira, N.B., Melo-Minardi, R.C., et al. (2017). Familial STAG2 germline mutation defines a new human cohesinopathy. NPJ Genom. Med. 2, 7.

Taylor, C.F., Platt, F.M., Hurst, C.D., Thygesen, H.H., and Knowles, M.A. (2014). Frequent inactivating mutations of STAG2 in bladder cancer are associated with low tumour grade and stage and inversely related to chromosomal copy number changes. Hum. Mol. Genet. 23, 1964-1974.

Thol, F., Bollin, R., Gehlhaar, M., Walter, C., Dugas, M., Suchanek, K.J., Kirchner, A., Huang, L., Chaturvedi, A., Wichmann, M., et al. (2014). Mutations in the cohesin complex in acute myeloid leukemia: clinical and prognostic implications. Blood 123, 914-920.

Tsuchihashi, T., Maeda, J., Shin, C.H., Ivey, K.N., Black, B.L., Olson, E.N., Yamagishi, H., and Srivastava, D. (2011). Hand2 function in second heart field progenitors is essential for cardiogenesis. Dev. Biol. 351, 62-69.

van der Lelij, P., Lieb, S., Jude, J., Wutz, G., Santos, C.P., Falkenberg, K., Schlattl, A., Ban, J., Schwentner, R., Hoffmann, T., et al. (2017). Synthetic lethality between the cohesin subunits STAG1 and STAG2 in diverse cancer contexts. eLife 6, e26980.

Viny, A.D., Bowman, R.L., Liu, Y., Lavallée, V.-P., Eisman, S.E., Xiao, W., Durham, B.H., Navitski, A., Park, J., Braunstein, S., et al. (2019). Cohesin Members Stag1 and Stag2 Display Distinct Roles in Chromatin Accessibility and Topological Control of HSC Self-Renewal and Differentiation. Cell Stem Cell 25, 682-696.e8.

Vooijs, M., and Berns, A. (1999). Developmental defects and tumor predisposition in Rb mutant mice. Oncogene 18, 5293-5303.

Wutz, G., Ladurner, R., St Hilaire, B.G., Stocsits, R.R., Nagasaka, K., Pignard, B., Sanborn, A., Tang, W., Várnai, C., Ivanov, M.P., et al. (2020). ESCO1 and CTCF enable formation of long chromatin loops by protecting cohesin ${ }^{\text {STAG1 }}$ from WAPL. eLife 9, e52091.

Yuan, B., Neira, J., Pehlivan, D., Santiago-Sim, T., Song, X., Rosenfeld, J., Posey, J.E., Patel, V., Jin, W., Adam, M.P., et al. (2019). Clinical exome sequencing reveals locus heterogeneity and phenotypic variability of cohesinopathies. Genet. Med. 21, 663-675. 


\section{STAR $\star M E T H O D S$}

\section{KEY RESOURCES TABLE}

\begin{tabular}{|c|c|c|}
\hline REAGENT or RESOURCE & SOURCE & IDENTIFIER \\
\hline \multicolumn{3}{|l|}{ Antibodies } \\
\hline MEK2 mouse monoclonal & $\mathrm{BD}$ & Cat\# M24520 \\
\hline Rad21 rabbit polyclonal & Carretero et al., 2013 & $N / A$ \\
\hline STAG1 rat monoclonal & Kojic et al., 2018 & $\mathrm{~N} / \mathrm{A}$ \\
\hline STAG1 rabbit polyclonal & Remeseiro et al., 2012a & $\mathrm{N} / \mathrm{A}$ \\
\hline STAG2 mouse monoclonal & SCBT & Cat\# SC-81852; RRID:AB_2199948 \\
\hline BrdU-FITC & $\mathrm{BD}$ & Cat\# 556028; RRID:AB_396304 \\
\hline Lineage cocktail & $\mathrm{BD}$ & Cat\# 558451 \\
\hline Ly-6A/E (Sca-1)-PE-Cy7 & $\mathrm{BD}$ & Cat\# 558162; RRID:AB_647253 \\
\hline CD117 (c-kit)-PerCP-Cy5.5 & BioLegend & Cat\# 105824; RRID:AB_2131597 \\
\hline CD48-APC-Cy7 & BioLegend & Cat\# 103432; RRID:AB_2561463 \\
\hline CD150 (SLAM)-BV510 & BioLegend & Cat\# 115929; RRID:AB_2562189 \\
\hline CD34-e Fluor 660 & eBioscience & Cat\# 50034182; RRID:AB_10596826 \\
\hline CD16/32- BV605 & $\mathrm{BD}$ & Cat\# 563006; RRID:AB_2737947 \\
\hline CD11b (Mac1)-PE-Cy7 & BioLegend & Cat\# 101216; RRID:AB_312799 \\
\hline Ly6G-Dylight 450; conjugated in house & BioXcell & N/A \\
\hline CD3ع- PerCP-Cy5.5 & BioLegend & Cat\# 100328; RRID:AB_893318 \\
\hline CD45R (B220)-APC-Cy7 & $\mathrm{BD}$ & Cat\# 552094; RRID:AB_394335 \\
\hline Streptavidin DyLight 405 & Jackson Immunoresearch & Cat\# 016-470-084; RRID:AB_2337248 \\
\hline Ter119-Pacific Blue & BioLegend & Cat\# 116231; RRID:AB_2149212 \\
\hline $\mathrm{BrdU}(\mathrm{MoBu}-1)$ & Santa Cruz & Cat\# 51514; RRID:AB_626519 \\
\hline Cleaved-caspase3 (ASP175) & Cell Signaling & Cat\# 9661; RRID:AB_2341188) \\
\hline $\mathrm{H} 3 \mathrm{P}$ rabbit polyclonal & Millipore & Cat\# 06-570; RRID:AB_310177 \\
\hline ISL1 mouse monoclonal & DSHB Hybridoma Bank & Cat\# 39.4D5; RRID:AB_2314683 \\
\hline \multicolumn{3}{|l|}{ Chemicals, Peptides, and Recombinant Proteins } \\
\hline Prolong Gold Antifade Reagent & Life Technologies & Cat\# P36930 \\
\hline Matrigel & Corning & Cat\# 356231 \\
\hline Cell recovery solution & Corning & Cat\# 354253 \\
\hline Dispase II solution & GIBCO & Cat\# 17105041 \\
\hline Formalin & Sigma & Cat\# HT501128-4L \\
\hline Biotin-16-dUTP & Roche & Cat\# 11093070910 \\
\hline TRI reagent & Sigma & Cat\# T9424 \\
\hline \multicolumn{3}{|l|}{ Critical Commercial Assays } \\
\hline Cytofix/Cytoperm kit & $\mathrm{BD}$ & Cat\# 554722 \\
\hline FITC Active Caspase-3 Apoptosis kit & $\mathrm{BD}$ & Cat\# 550480 \\
\hline Terminal Transferase recombinant kit & Roche & Cat\# 03333574001 \\
\hline \multicolumn{3}{|l|}{ Deposited Data } \\
\hline RNA-seq datasets in mouse embryos & This paper & GEO: GSE152298 \\
\hline \multicolumn{3}{|l|}{ Experimental Models: Organisms/Strains } \\
\hline Stag2 conditional knockout mice & This paper & $\mathrm{N} / \mathrm{A}$ \\
\hline Tg.hUBC-CreERT2 mice & Ruzankina et al., 2007 & $N / A$ \\
\hline Tg.CAG-Cre mice & Belteki et al., 2005 & $N / A$ \\
\hline \multicolumn{3}{|l|}{ Oligonucleotides } \\
\hline Primer Stag2 f1: TGGTGCTTGGGATCAGATTT & This paper & N/A \\
\hline Primer Stag2 r1: TCCСTCATCAAAGTCGAAAA & This paper & $N / A$ \\
\hline
\end{tabular}




\begin{tabular}{|c|c|c|}
\hline \multicolumn{3}{|l|}{ Continued } \\
\hline REAGENT or RESOURCE & SOURCE & IDENTIFIER \\
\hline Primer Stag2 r2: AACAGCCTGAGCAAAGAATCC & This paper & N/A \\
\hline Primer Sry fwd: TGGGACTGGTGACAATTGTC & Lambert et al., 2000 & N/A \\
\hline Primer Sry rev: GAGTACAGGTGTGCAGCTCT & Lambert et al., 2000 & N/A \\
\hline \multicolumn{3}{|l|}{ Recombinant DNA } \\
\hline Stag2 targeting vector & EUCOMM & PG00032_A_D11-3 \\
\hline \multicolumn{3}{|l|}{ Software and Algorithms } \\
\hline GraphPad Prism (statistical analysis) & GraphPad Software Inc & $\begin{array}{l}\text { https://www.graphpad.com/scientific- } \\
\text { software/prism/ }\end{array}$ \\
\hline Flow Jo v10.0.8 (flow cytometry analysis) & Flow Jo LLC & https://www.flowjo.com/solutions/flowjo \\
\hline LAS AF v3.8 (imaging) & Leica & $\begin{array}{l}\text { https://www.leica-microsystems.com/products/ } \\
\text { microscope-software/p/leica-application-suite/ }\end{array}$ \\
\hline Definiens Developer XD v2.5 (imaging) & Definiens Inc - AstraZeneca & N/A \\
\hline NIS Elements D3.2 and 4.30 (imaging) & Nikon & $\begin{array}{l}\text { https://www.microscope.healthcare.nikon.com/ } \\
\text { products/software/nis-elements }\end{array}$ \\
\hline FIJI v1.52b & & https://imagej.net/Fiji \\
\hline Lexogen Quantseq pipeline (RNA-seq analysis) & BlueBee & $\begin{array}{l}\text { https://www.lexogen.com/store/quantseq- } \\
\text { data-analysis-bluebee-platform/ }\end{array}$ \\
\hline DESeq2 (RNA-seq analysis) & Love et al., 2014 & $\begin{array}{l}\text { http://www.bioconductor.org/packages/release/ } \\
\text { bioc/html/DESeq2.html }\end{array}$ \\
\hline
\end{tabular}

\section{RESOURCE AVAILABILITY}

\section{Lead Contact}

Further information and requests for resources and reagents should be directed to and will be fulfilled by the Lead Contact, Ana Losada (alosada@cnio.es).

\section{Materials Availability}

Resources generated in this study are available upon request. Requests of Stag2 cKO mice should be addressed to A. Losada (alosada@cnio.es).

Data and Code Availability

RNA-sequencing datasets have been deposited in the Gene Expression Omnibus (GEO) under the accession number GSE152298

\section{EXPERIMENTAL MODEL AND SUBJECT DETAILS}

Generation of a conditional knockout allele for Stag2

The targeting vector PG00032_A_D11-3 (EUCOMM) containing loxP sites flanking exon 7 of the Stag2 gene and a SA-lacZ-Neo cassette flanked by FRT sites integrated in intron 6 was electroporated into G4 mouse embryonic stem cells (Figure S1A). Clones were selected in G418 and screened by Southern blotting for homologous recombination (Figure S1B). Positive clones were infected with adeno-FLP to remove the selection cassette and create the conditional allele and microinjected into C57BL/6BrdCrHsd-Tyr morulae. Germline transmitting chimeras were screened by PCR (primers f1: $5^{\prime}$-TGGTGCTTGGGATCAGATTT-3' and r1: $5^{\prime}-$ TCCCTCATCAAAGTCGAAAA-3') and selected to generate the colonies (Figure S1C). Mice carrying the Stag2 cKO allele were crossed with mice carrying a tamoxifen inducible Cre-ERT2 allele (Tg.hUBC-CreERT2) for MEF isolation and adult mice viability assays or with a constitutively active Cre (Tg.CAG-Cre) to assess embryonic development and lethality (Figure S1D). All crosses were maintained in a predominantly C57BL/6 background. To induce recombination in adult animals, 4 week-old mice were fed for variable periods of time with a TMX-containing diet, as specified. For the experiments in adult animals, both male and female mice were analyzed. For experiments in embryos, only male embryos were used, as explained in main text. All animal procedures were approved by local and regional ethics committees (Institutional Animal Care and Use Committee and Ethics Committee for Research and Animal Welfare, Instituto de Salud Carlos III) and performed according to the European Union guidelines. 
MEFs

MEFs were isolated from E12.5 embryos resulting from mating Stag $2^{\text {lox/lox }}$ females with $T g . h U B C-C r e E R T 2^{+/ T}$ males and genotyped for Stag2 (with a mixture of primers f1, $r 1$ and r2: 5'- AACAGCCTGAGCAAAGAATCC-3'), CreERT2 (fwd: 5'- TGAAGCTCCGGTTाT GAACT-3'; rev: 5'- GGTTCTTGCGAACCTCATCAC-3') and the Sry Y chromosome marker (fwd: 5'-TGGGACTGGTGACAATTGTC-3': rev: 5'- GAGTACAGGTGTGCA GCTCT-3'). MEFs were cultured in DMEM supplemented with $20 \%$ FBS and $1 \%$ penicillin-streptomycin and grown at $37^{\circ} \mathrm{C}$ under $90 \%$ humidity and $5 \% \mathrm{CO}_{2}$.

\section{METHOD DETAILS}

\section{MEF characterization}

For each experiment, MEFs derived from 2-4 different embryos were analyzed. To ablate STAG2 expression, conditional Stag $2^{\text {lox } / Y} ;$ CreERT2 $2^{+/ T}$ MEFs were cultured in the presence of $1 \mu \mathrm{M}$ 4-hydroxy tamoxifen (4-OHT) for 3-4 days and the efficiency of depletion was assessed by immunoblotting. The same cells cultured without 4-OHT served as control.

To assess proliferation, MEFs pretreated for 4 days with 4-OHT were seeded at low confluence in multiwell plates (3 wells per time point). In the following days, cells were collected and counted in a Neubauer hemocytometer.

For cell cycle analysis, MEFs grown for 4 days in medium with or without 4-OHT were collected after a 30 min pulse with $30 \mu \mathrm{M}$ BrdU, fixed and incubated with a FITC-conjugated anti-BrdU antibody and DNA was stained with $50 \mu \mathrm{g} / \mathrm{ml}$ propidium iodide. To study apoptosis, MEFs grown for 4 days in the presence of 4-OHT were collected (both adhered and floating cells) and fixed and permeabilized using the BD Cytofix/Cytoperm kit (BD 554722). Cells stained with DAPI and the FITC Active Caspase-3 Apoptosis kit (BD 550480) according to manufacturer instructions. Flow cytometry was performed in a FACS Canto II cytometer and profiles were analyzed using FlowJo 10.0 .8 software.

For analysis of mitotic defects, MEFs were serum-starved (0.1\% FBS) for 3 days in the presence or absence of $4-\mathrm{OHT}$, switched to medium supplemented with $20 \%$ FBS, and collected after $36 \mathrm{~h}$. For chromosome spreads, $0.1 \mu \mathrm{g} / \mathrm{ml}$ colcemid was added to the medium 3-4 h before harvesting. Cells were swollen in $0.03 \mathrm{M}$ sodium citrate, fixed in methanol:acetic acid 3:1 and dropped onto slides. For anaphase analysis, cells were seeded onto coverslips at the time of release from G0 arrest. In both cases, cells were stained with $1 \mu \mathrm{g} / \mathrm{ml} \mathrm{DAPI}$, mounted with Vectashield and imaged using a Leica DM6000 microscope with LAS AF software.

Histopathology and immunohistochemical (IHC) analyses of adult tissue sections

Tissues from adult mice were analyzed following standard histopathology procedures. Mice were sacrificed by cervical dislocation and a complete necropsy was performed; the following tissues were analyzed histologically: bladder, pancreas and associated lymph nodes, spleen, kidney, liver, lung, heart, brain, gastrointestinal tract, thymus, thyroid and parathyroid. WT littermates were sacrificed and used as controls. Organs were fixed in $4 \%$ neutral buffered $(\mathrm{pH} 6.9)$ formaldehyde for $24 \mathrm{~h}$ and embedded in paraffin. After standard H-E staining, sections were analyzed in a Leica DM5000B microscope by a trained veterinary pathologist and histological findings were recorded. After scanning of histological slides (AxioScan 4.1, Zeiss), representative microphotographs were taken.

IHC was performed on 2.5- $\mu \mathrm{m}$ sections of formalin-fixed paraffin-embedded tissues, unless otherwise indicated. After deparaffinization and rehydration, antigen retrieval was performed by boiling in citrate buffer $\mathrm{pH} 6$ for 10 min and endogenous peroxidase was inactivated with $3 \% \mathrm{H}_{2} \mathrm{O}_{2}$-methanol for 30 min at room temperature (RT). Sections were blocked with $2 \%$ BSA in PBS and incubated with anti-STAG2 and anti-cleaved caspase-3 (Asp175). After washing, the Envision secondary reagent (DAKO) was added for 40 min at RT and sections were washed three times with PBS. 3,3'-Diaminobenzidine tetrahydrochloride (DAB) was used as a chromogen. Sections were lightly counterstained with hematoxylin, dehydrated and mounted. A non-related lgG was used as a negative control. STAG2 expression in sections of liver, pancreas, brain, spleen and intestine of KO mice was assessed by IHC at 12, 24, 35 and 60 weeks of age. Representative microphotographs were taken and quantified with ImageJ software. For IF, after deparaffinization, rehydration and antigen retrieval, sections were incubated with 3\% BSA $0.1 \%$ Triton in PBS for 45 min at room temperature and incubated with primary anti-BrdU overnight at $4^{\circ} \mathrm{C}$. After washing with $0.1 \%$ Triton/PBS, an Alexa Fluor 555-labeled goat anti-rabbit Ig secondary antibody was added for $45 \mathrm{~min}$, sections were washed, and nuclei were counterstained with DAPI. After washing with PBS, sections were mounted with Prolong Gold Antifade Reagent (Life Technologies, P36930). Images were acquired using a confocal microscope (Leica, SP5).

\section{Hematological analyses}

Peripheral blood was extracted from the mouse cheek and collected in EDTA-coated tubes. Standard complete blood counts were performed using an automated analyzer (Abacus Junior Vet, CVM Diagnóstico Veterinario S.L, Navarra, Spain) according to the manufacturers' instructions. For the GFP/Tomato blood competition experiment, KO mice received a TMX diet from weaning and blood was collected at 8, 12, 16, 20, 24 weeks of age. After RBC depletion using hypotonic lysis buffer, GFP or Tomato positive cells were quantified on a LSRII Fortessa (BD and analyzed with FlowJo v10 software (Tree Star, Ashland, OR). 
Hematopoietic cell isolation and Flow Cytometry

Bone marrow was isolated from the tibia and femur; peripheral blood was obtained by cardiac puncture; spleens were disaggregated and homogenized through a 70- $\mu$ m strainer; all these samples were incubated for 10 min in RBC lysis buffer and resuspended in PBS. For the analysis of LSK and MPs, cells were incubated with the biotinylated lineage antibody cocktail (CD3e, B220, CD11b, Gr1 and Ter119), together with streptavidin conjugated to DyLight 405, anti-Sca-1 (D7) -PE-Cy7 (BD), and anti- c-Kit (2B8) -PerCP-Cy5.5 for $30 \mathrm{~min}$. For the analysis of myeloid progenitors, cells were incubated with the previous antibodies plus anti-CD34 -eF660 (RAM34) and anti-Fc $\gamma$ RII/III - BV605 (2.4G2). For the analysis of bone marrow Ter119+ cells, cells were incubated with anti-Ter119-Pacific Blue for $15 \mathrm{~min}$. For the analysis of peripheral blood leukocytes (PBL) and splenocytes, cells were stained with anti-Ly6G -DyLight 405 (1A8, in-house conjugated), anti-B220 labeled with APCCy7 (RA3-6B2), and anti-Cd11b -PECy7 (Mac1) and anti-CD3e -PerCPCy5.5 (145-2C11) for 15 min. Samples were collected on a LSRII Fortessa (BD) and analyzed with FlowJo v10 software (Tree Star, Ashland, OR).

In vitro colony-forming unit assays

To evaluate self-renewal capacity, 20,000 Tomato or GFP ${ }^{+}$bone marrow cells from Stag2 KO mice were FACS-sorted, seeded in cytokine-supplemented methylcellulose tubes, and plated in duplicates in $35 \mathrm{~mm}$ culture dishes (NUNC A/S; Roskilde, Denmark). Cultures were incubated at $37^{\circ} \mathrm{C}$ in a $5 \% \mathrm{CO}_{2}$ atmosphere. The number of CFU-Cs was scored on day 7 using an inverted microscope. Cells were sorted with a FACSAria Instrument (BD).

\section{Establishment of intestinal organoids}

Small intestines of 8 week-old mice were opened longitudinally, washed with cold PBS supplemented with antibiotics and gently scraped to remove villi. The tissue was chopped into around $5 \mathrm{~mm}$ pieces, further washed with cold PBS and antibiotic and incubated in $8 \mathrm{mM}$ EDTA with PBS for $5 \mathrm{~min}$ at RT and then for $30 \mathrm{~min}$ on ice. Tissue fragments were vigorously shaken with cold PBS. The supernatant was enriched for crypts. This procedure was repeated twice and the supernatant joined. This fraction was passed through a $70-\mu \mathrm{m}$ cell strainer (BD Bioscience) to remove residual villous material. Isolated crypts were centrifuged at $800 \mathrm{rpm}$ for 3 min to separate crypts from single cells. The final fraction consisted of essentially pure crypts and was embedded in in growth factor-reduced and phenol red-free Matrigel (Corning, 356231). Matrigel-crypts suspensions (20 $\mu \mathrm{L}$ drops) were plated onto 6-well plates, allowed to settle in a humidified incubator at $37^{\circ} \mathrm{C} / 5 \% \mathrm{CO}_{2}$, and overlaid with $2 \mathrm{~mL}$ of culture medium (Advanced DMEM/ F12 (Invitrogen)) containing growth factors (50ng/ml EGF (Peprotech), 500 ng/ml R-spondin (Sigma) and $100 \mathrm{ng} / \mathrm{ml}$ Noggin (Peprotech)). Isolated crypts were allowed to close in culture medium for 2-4 days. For sorting experiments, Matrigel was removed with Cell Recovery Solution (Corning, 354253) on ice and the cell suspension was washed with PBS, then with washing medium, and centrifuged at $1200 \mathrm{rpm}$ for $5 \mathrm{~min}$ at $4^{\circ} \mathrm{C}$. Then, sealed crypts were digested with Dispase II solution (10 mg/mL) (GIBCO, 17105041) for $15-$ $20 \mathrm{~min}$ in a rotating wheel at room temperature. The digestion was stopped with $2 \mathrm{mM}$ EDTA and single cells were obtained by mechanical disruption with a syringe with a $21 \mathrm{G}$ needle. Dissociated cells were passed through cell strainer and single, viable (DAPI ${ }^{-}$, GFP $^{+}$or Tomato ${ }^{+}$cells were sorted by flow cytometry using an FACS Ariall (BD Biosciences). Sorted cells were collected in crypt culture medium and embedded in Matrigel in 96-well plates (5000 cells/well) for the clonal growth experiments. Organoids were allowed to grow for 7 days and images were acquired with a CCD-microscope. Three microphotographs in the $Z$ axis were taken in order to collect the majority of the organoids. Then, a Z stack was done using ImageJ software. Quantification was performed with tailored routines programmed in Definiens XD v2.5 software.

\section{Hematoxylin and immunofluorescence staining of mouse embryo sections}

Whole-mount embryos were dissected in PBS at RT and imaged using a Leica MZ10F microscope and LAS 3.8 software. DNA from yolk sacs was used to genotype for Stag2 and Sry. Embryos were fixed in 10\% formalin solution at pH 7 (Sigma HT501128-4L) overnight at $4{ }^{\circ} \mathrm{C}$, dehydrated in an ethanol series and stored in ethanol $70 \%$ at $4{ }^{\circ} \mathrm{C}$ until further processing. Embryos were embedded in paraffin and sectioned transversely at 5- $\mu \mathrm{m}$. H-E staining was performed by standard procedures [WT1 $(n=3), K O$ mild $(n=4)$, WT2 $(n=3)$ and KO severe $(n=2)$ at E9.5; WT $(n=3)$ and KO $(n=3)$ at E10.5]. Sections were imaged with a Nikon Eclipse 90i microscope and NIS Elements D 3.2 imaging software.

Co-immunostaining for H3P-ISL1-TUNEL was performed using 4 embryos per genotype (WT1, KO mild and WT2). For TUNEL, the Terminal Transferase recombinant kit (Roche 03333574 001) and biotin-16-dUTP (Roche 11093070 910) were used. Sections were imaged with a Nikon A1R confocal microscope and NIS Elements 4.30 software. H3P signal was quantified with a custom-made ImageJ macro, taking into account both late G2 and M-phase signals. Statistical significance was determined by Kruskal-Wallis test and Dunn's Multiple Comparison post-test using GraphPad Prism 5.03.

\section{RNA-sequencing}

Whole mount KO and WT embryos at E9.5 (21-23 pairs of somites) were placed in cold PBS. The whole heart along with surrounding SHF regions and a section of heart-proximal neural tube were dissected, snap-frozen and stored at $-80^{\circ} \mathrm{C}$. Per genotype and region, 3 replicates were prepared pooling material from 3 embryos that were processed with TRI reagent (Sigma T9424) and homogenized with syringe and needle (25-30G). Chloroform and phase lock tubes (QuantaBio 2302830) were used for phase separation and a subsequent precipitation with ethanol was performed at $-20^{\circ} \mathrm{C}$. RNA samples were analyzed using a Bioanalyzer 2100 (Agilent) and the 


\section{Cell Reports}

Article

RNA 6000 Pico kit. Libraries were prepared using the QuantSeq 3' mRNA-seq Library Prep Kit FWD (Lexogen) and sequenced on an Illumina HiSeq 2500 platform. For alignment and gene counting, we applied the Lexogen QuantSeq 2.2.3 pipeline provided by BlueBee, designed for use with the libraries described above. We decided to remove one of the WT heart replicates due to initial inferior RNA integrity and a failure to cluster with the rest of the WT heart samples. The differential expression analyses have been performed with DeSeq2, excluding genes with no reads in any of the samples. Results were filtered by $p$ value $<0.05$ and FDR $<0.05$. In the heatmaps, color intensities correspond to the relative expression levels for each gene among conditions, normalized using the mean and standard deviation.

\section{QUANTIFICATION AND STATISTICAL ANALYSIS}

Information about sample size and statistical test applied for each experiment can be found in the figure legends and in the Method Details section. Difference between groups was defined as significant when $p<0.05$. 


\title{
The pluripotency factor NANOG controls primitive hematopoiesis and directly regulates Tal1
}

\author{
Julio Sainz de Aja ${ }^{1}$, Sergio Menchero ${ }^{1}$, Isabel Rollan ${ }^{1}$, Antonio Barral ${ }^{1}$, Maria Tiana ${ }^{1}$, Wajid Jawaid ${ }^{2,3}$, \\ Itziar Cossio ${ }^{1}$, Alba Alvarez ${ }^{1}$, Gonzalo Carreño-Tarragona ${ }^{1,4}$, Claudio Badia-Careaga ${ }^{1}$, \\ Jennifer Nichols ${ }^{2,5}$, Berthold Göttgens ${ }^{2,3}$ iD, Joan Isern ${ }^{1,6}$ iD \& Miguel Manzanares ${ }^{1,{ }^{*}}$ (D)
}

\begin{abstract}
Progenitors of the first hematopoietic cells in the mouse arise in the early embryo from Brachyury-positive multipotent cells in the posterior-proximal region of the epiblast, but the mechanisms that specify primitive blood cells are still largely unknown. Pluripotency factors maintain uncommitted cells of the blastocyst and embryonic stem cells in the pluripotent state. However, little is known about the role played by these factors during later development, despite being expressed in the postimplantation epiblast. Using a dual transgene system for controlled expression at postimplantation stages, we found that Nanog blocks primitive hematopoiesis in the gastrulating embryo, resulting in a loss of red blood cells and downregulation of erythropoietic genes. Accordingly, Nanogdeficient embryonic stem cells are prone to erythropoietic differentiation. Moreover, Nanog expression in adults prevents the maturation of erythroid cells. By analysis of previous data for NANOG binding during stem cell differentiation and CRISPR/Cas9 genome editing, we found that Tal1 is a direct NANOC target. Our results show that Nanog regulates primitive hematopoiesis by directly repressing critical erythroid lineage specifiers.
\end{abstract}

Keywords erythropoiesis; gastrulation; Nanog; primitive hematopoiesis; Tal1 Subject Categories Development \& Differentiation; Transcription DOI 10.15252/embj.201899122 | Received 30 January 2018| Revised 24 January 2019 | Accepted 25 January 2019| Published online 27 February 2019

The EMBO Journal (2019) 38: e99122

\section{Introduction}

Blood cells first appear during mouse development in the extraembryonic yolk sac at embryonic day (E) 7.5. These are primarily erythroid cells, needed to provide oxygen for the exponential embryo growth at these stages (Baron et al, 2012). This initial primitive hematopoiesis is not generated by hematopoietic stem cells, which first appear later in development (around E10.5) and provide the basis for definitive hematopoiesis (Jagannathan-Bogdan \& Zon, 2013).

The precursors of the first erythroid cells are already present at the initial stages of gastrulation, in the nascent mesoderm at the posterior end of the embryo (Lawson et al, 1991; Huber et al, 2004); moreover, detailed fate mapping suggests that these cells are specified in the epiblast before gastrulation (Kinder et al, 1999; Padron-Barthe et al, 2014). Hematopoietic precursors are specified after the determination of the early mesoderm from the epiblast, which is driven by the sequential action of the transcription factors encoded by Brachyury and Mesp1 and ends in the expression of FLK1 (encoded by $K d r$ ), which marks most mesodermal cells at gastrulation (Pfister et al, 2007; Chan et al, 2013; Scialdone et al, 2016). Subsequently, primitive hematopoiesis progenitors start expressing a battery of lineage-specific transcription factor genes such as Tal1, Gata1, and Klf1 as they migrate to the extraembryonic region and generate the blood islands of the yolk sac (Dore \& Crispino, 2011; Baron et al, 2012).

Despite the knowledge acquired in recent years on the regulation of gastrulation and lineage determination of blood cells, we still do not fully understand how hematopoietic precursors are specified from within the pool of common mesodermal cells present in the posterior-proximal region of the gastrulating embryo. In other words, it remains unclear how the first differentiated cell type to appear in the postimplantation embryo (the primitive hematopoietic cells) is specified from a multipotent population of mesodermal progenitors, and how lineage-specific genes are turned on in this rapid transition. In this study, we provide evidence for an involvement in this process of the homeobox transcription factor gene Nanog.

NANOG is a constituent of the core set of transcription factors, together with OCT4 and SOX2, involved in establishing and maintaining embryonic pluripotency, both in the blastocyst and in embryonic stem (ES) cells in culture (Chambers \& Tomlinson, 2009). Loss of Nanog in the early blastocyst results in embryonic

\footnotetext{
1 Centro Nacional de Investigaciones Cardiovasculares Carlos III (CNIC), Madrid, Spain

2 Wellcome-Medical Research Council Cambridge Stem Cell Institute, Cambridge, UK

3 Department of Haematology, Cambridge Institute for Medical Research, University of Cambridge, Cambridge, UK

4 Department of Haematology, Hospital 12 de Octubre, Madrid, Spain

5 Department of Physiology, Development and Neuroscience, University of Cambridge, Cambridge, UK

6 Department of Experimental \& Health Sciences, University Pompeu Fabra (UPF), Barcelona, Spain

*Corresponding author. Tel: +34914531200; E-mail: mmanzanares@cnic.es
} 
death at implantation (Mitsui et al, 2003); however, Nanog-deficient ES cells are still able to maintain pluripotency, although they are prone to differentiate (Chambers et al, 2007). In the preimplantation embryo, Nanog is expressed throughout the epiblast. During implantation, Nanog is turned off, only to be re-expressed at E6.0 in the posterior part of the epiblast, where the primitive streak will form and gastrulation takes place shortly after (Hart et al, 2004; Osorno et al, 2012). Later, expression is restricted to primordial germ cells, with Nanog playing a crucial role in their development (Chambers et al, 2007; Yamaguchi et al, 2009; Zhang et al, 2018). Aside from its function in the germline, there is little or no previous evidence for Nanog playing any other role in the postimplantation epiblast or in the gastrulating embryo.

Here, we show that sustained expression of Nanog beyond gastrulation blocks differentiation of red blood cells during primitive hematopoiesis. This phenotype can be recapitulated in the adult, where Nanog leads to an increase in the number of megakaryocyteerythroid precursors (MEPs), possibly by blocking their differentiation. Hematopoietic differentiation of Nanog-deficient ES cells is enhanced, further supporting the hypothesis that Nanog blocks the erythroid lineage in the epiblast of the gastrulating embryo. Furthermore, by re-analyzing single-cell RNA-seq data from gastrulating embryos (Scialdone et al, 2016) and NANOG ChIP-seq data in ES and epiblast-like cells (Murakami et al, 2016), together with CRISPR/ Cas9-mediated genome editing, we found that NANOG directly represses the expression of the erythroid specifier Tall. Together, these findings suggest that Nanog controls the early specification of hematopoietic cells from mesodermal precursors during gastrulation.

\section{Results}

\section{Nanog blocks erythropoiesis in developing mouse embryos}

Nanog loss of function is lethal at preimplantation stages (Mitsui et al, 2003), therefore preventing analysis of the putative role of Nanog later in development, when it is re-expressed at the posterior part of the gastrulating embryo (Hart et al, 2004). To overcome this obstacle, we used an inducible TetON transgenic model $\left(\right.$ Nanog $\left.^{\mathrm{tg}}\right)$ in which Nanog expression is induced by the administration of doxycycline (dox) (Piazzolla et al, 2014). We induced Nanog from E6.5 in order to prolong its expression beyond E7.5, when it is normally turned off (Hart et al, 2004), and examined the embryos at E9.5. Visual examination of freshly dissected dox-treated embryos showed some growth retardation and craniofacial defects, but the most notable effect was a lack of blood (Fig 1A). To confirm this observation, we carried out whole-mount in situ hybridization for Hbb-bhl, which encodes the beta-like embryonic hemoglobin (Wilkinson et al, 1987) and for Redrum, an erythroid-specific long non-coding RNA (Alvarez-Dominguez et al, 2014; Paralkar et al, 2014). In untreated (control) Nanog ${ }^{t g}$ embryos at E9.5, Hbb-bh1 labels primitive red blood cells that are distributed throughout the yolk sac. Expression of Nanog up to this stage resulted in near complete blockade of $H b b$-bh1 expression (Fig 1A). Redrum is expressed in the developing aorta-gonad-mesonephros (AGM) region, surely from erythroid cells circulating along the aorta, and in the tail bud. Nanog induction led to loss of Redrum expression in the AGM region, but interestingly not in the tail bud that is not a site of embryonic erythropoiesis (Fig 1A). We also checked if the apparent lack of blood was accompanied by vascular defects. Immunostaining for Endomucin, expressed in embryonic endothelial cells, revealed no substantial differences at E9.5 between dox-treated and untreated Nanog ${ }^{\text {tg }}$ embryos, as is observed in the correct patterning of intersomitic vessels (Fig 1B). Furthermore, CD31 staining showed that yolk sac vasculature was equally unaffected in dox-treated embryos (Fig EV1A). We also examined heart morphology at these stages, to address if other mesodermal derivatives showed developmental defects. Hearts of freshly dissected E9.5 dox-treated embryos beat normally, and both overall morphology and histological sections showed no defects (Fig EV1B). Prolonged Nanog expression in the embryo thus causes a deficit in primitive red blood cells that is accompanied by lack of expression of erythroid-specific genes, but does not affect early vascular or cardiac development.

To characterize the effect of Nanog induction on hematopoiesis, we analyzed progenitors and red blood cells by flow cytometry of dispersed individual yolk sacs from E9.5 embryos using c-Kit (a marker of early uncommitted progenitors), CD41 (erythroid progenitors; Mitjavila-Garcia et al, 2002), CD71, and Ter119 (Borges et al, 2012). Dox-treated Nanog ${ }^{t g}$ embryos showed a dramatic reduction in erythroblast cells $\left(\mathrm{CD} 71^{+}\right.$Ter119 ${ }^{+}$; Fig 1C and D), which supports the above results. However, the total number of hematopoietic progenitor populations $\left(\mathrm{cKit}^{+} \mathrm{CD} 41^{+}\right.$and $\mathrm{CD} 41^{+}$, respectively) remained unchanged (Fig $1 \mathrm{E}$ and $\mathrm{F}$ ). We examined the morphology of erythroblasts from circulating blood of E9.5 dox-treated and untreated embryos by Giemsa staining (Fraser et al, 2007) and found that the few remaining primitive erythroid cells showed a normal morphology (Fig EV1C). Taken together, these results suggest that Nanog causes a blockade in hematopoietic progenitors, preventing their differentiation toward erythroblast cells.

\section{Nanog downregulates the expression of key erythroid determination genes}

We next investigated how prolonged Nanog expression to E9.5 influences hematopoietic gene expression. For this, we isolated progenitor and mature populations by flow cytometry as described above (Fig 1C and D), and conducted RT-qPCR to examine the expression of core lineage determinants of hematopoietic fate: Tal1, Runx1, Gata1, and Klf1 (Palis et al, 1999; Yokomizo et al, 2008; Kuvardina et al, 2015). Gain of Nanog expression in erythroblasts (CD71 ${ }^{+}$ Ter $119^{+}$population) resulted in significant downregulation of Tal1 and increase of Runx1 (Fig 1G). However, despite consistent gain of Nanog expression, we did not observe differences of gene expression in earlier progenitors (Fig EV1D).

To examine whether similar changes occur at earlier stages, we induced Nanog expression from E5.5 to E7.5, a time window spanning initiation of primitive hematopoiesis. Whole-mount in situ hybridization showed decreased expression of Gata1 and Klf1 in the extraembryonic region, corresponding to the blood island domain (Fig EV1E). RT-qPCR of individual dox-treated or control E7.5 Nanog ${ }^{\text {tg }}$ embryos showed decreased expression of the core erythropoietic genes Tal1, Gata1, and Klf1 but no change in Runx1 (Fig EV1F). A possible explanation for our observations would be that Nanog expression causes a general blockade of mesodermal specification, with the downregulation of early hematopoiesis genes being merely a secondary effect of this. We therefore tested the 
expression of lineage determinants expressed at gastrulation (Brachyury and Eomes) and the early mesodermal gene Kdr (Shalaby et al, 1995; Palis et al, 1999). Exogenous Nanog induced the expression of both Brachyury and Eomes, in line with published data (Teo et al, 2011), but did not alter Kdr expression (Fig EV1F). Together, these results suggest that Nanog blocks erythroid fate and is able to specifically downregulate the early expression of erythropoietic genes during the initial determination of primitive hematopoiesis.

\section{Nanog-induced hematopoietic defects are cell intrinsic}

The results presented so far suggest that Nanog blocks specifically erythroid progenitors during primitive hematopoiesis. To test if this is the case, we aimed to rescue the observed genotype by generating chimeric embryos by injection of wild-type ES cells constitutively expressing GFP (Diaz-Diaz et al, 2017) into Nanog ${ }^{\text {tg }}$ blastocysts. The resulting embryos were treated in utero with dox at E6.5 and examined for GFP fluorescence at E10.5. Those showing no overall contribution (no $\mathrm{GFP}^{+}$cells) were used as controls, whereas embryos containing $\mathrm{GFP}^{+}$cells were considered chimeras (Fig 2A and B). Erythroid cells were evaluated in individual embryos by flow cytometry analysis of CD71 and Ter119, as described earlier (Fig 1E and F).

Chimeras with high contribution of wild-type ES cells had circulating blood in both the embryo and the yolk sac, despite dox treatment, contrasting with embryos with no contribution (Fig 2B). Chimeras showed a recovery of erythroid cells, with high contribution from $\mathrm{GFP}^{+}$wild-type ES-derived cells (Fig 2C). Quantification of erythroid populations in chimeras showed an increased content of $\mathrm{CD} 71^{+}$Ter $119^{+}$cells (Fig 2D); this increase did not occur when the experiment was repeated without dox treatment (Fig 2E). The number of $\mathrm{GFP}^{-}$cells (derived from Nanog expressing cells) in doxtreated chimeras did not differ from that in controls (with no contribution of $\mathrm{GFP}^{+}$cells), demonstrating that the recovery of the erythroid populations in chimeras was entirely due to the wild-type ES cells (Fig 2F). These results indicate that the effect of Nanog on erythroid progenitors is primarily cell autonomous, and not secondary to Nanog-induced changes in other cell types.

\section{Loss of Nanog enhances hematopoietic differentiation of ES cells}

To investigate the effect of the absence of Nanog on the erythroid lineage, we tested the potential of ES cells with homozygous Nanog loss of function (Chambers et al, 2007) to differentiate into blood cells in culture (Irion et al, 2010). $\mathrm{Nanog}^{-/-}$and wild-type control ES cells of the parental strain (E14Tg2a) were used to generate embryoid bodies (EB). EBs were allowed to differentiate for up to 7 days in hematopoietic differentiation media. After disaggregation and culture, different colony-forming units (CFU) were scored between days 5 and 7 (D5-D7; Fig 3A). Despite a trend for a decrease in the number of common myeloid progenitors (CFUGEMM), Nanog ${ }^{-1-}$ EBs generated significantly more primitive erythroid colonies (Ery-P) than controls, as well as a significantly higher number of mature erythroid colonies (BFU-E; burst forming unit erythroid) in the presence of cytokines driving a broader hematopoietic differentiation. Interestingly, there was no betweengenotype difference in granulocyte-monocyte (CFU-GM) progenitors, but monocyte (CFU-M) or granulocyte (CFU-G) progenitors were produced more abundantly from wild type than from Nanog $^{-1-}$ EBs (Fig 3A). This last observation is possibly due to a decrease in common myeloid progenitors together with a significant increase of erythroid progenitors in the mutants. $\mathrm{Nanog}^{-/-}$ES cells thus have an increased potential for specific differentiation to red blood cells.

To investigate how the absence of Nanog affects the gene networks involved in erythroid specification, we monitored control and $\mathrm{Nanog}^{-1-}$ ES-derived EBs for the expression of selected markers over 10 days of differentiation. Brachyury expression was examined as a marker of initial mesoderm specification, a necessary first step for the establishment of hematopoietic lineages in EBs (Fehling et al, 2003). Brachyury expression markedly increased at day 3 in wild-type cells, as previously described (Robertson et al, 2000), but in $\mathrm{Nanog}^{-1-}$ EBs this expression peak was delayed until day 5 (Fig 3B). Nanog is thus likely required for the correct temporal activation of Brachyury. We next checked the expression of genes encoding the erythroid-specific factors Tal1, Gata1, and Klf1 and the embryonic hemoglobin gene $H b b$-bh1. In wild-type EBs, erythroid gene expression peaks around day 5, 2 days after Brachyury activation. In $\mathrm{Nanog}^{-/-}$EBs, erythroid gene expression peaked a day later, at day 6. However, this is only 1 day after the onset of Brachyury expression, contrasting the 2-day delay in wild-type EBs (Fig 3B). Given the requirement of Brachyury expression for hematopoietic differentiation (Fehling et al, 2003), we aligned the expression dynamics of wild-type and $\mathrm{Nanog}^{-1-}$ cells to the day of Brachyury induction (Fig EV2A). To validate this approach, we

Figure 1. Effect of Nanog on erythropoietic development.

A Dox-induced prolongation of Nanog expression in Nanog ${ }^{\text {tg }}$ embryos up to E9.5 results in lack of blood (left) and downregulation of erythropoietic gene expression. The center and right panels show whole-mount in situ hybridization for Hbb-bhI (in embryos with intact yolk sacs) and for the long non-coding RNA Redrum. Asterisks mark the aorta-gonad-mesonephros (AGM) region and arrows the tail bud. Embryos of the same genotype but not treated with dox were used as controls (-dox). Scale bars, $500 \mu \mathrm{m}$.

B Endomucin staining of vessels in control (-dox) or treated (+dox) E9.5 Nanog ${ }^{\text {tg }}$ embryos. On the right, higher magnifications of the boxed areas. Scale bar, 500 $\mu$ m.

C Representative FACS plot of the distribution of the CD71 and Ter119 populations in dissected yolk sacs from untreated and dox-treated E9.5 Nanog ${ }^{\text {tg }}$ embryos.

D Quantification of the CD71 ${ }^{+}$Ter $119^{+}$population in controls (-dox, black dots; $n=8$ ) and Nanog expressing (+dox, red dots; $n=7$ ) E9.5 yolk sacs. Each replicate contained a pool of 5 (-dox) or 8 (+dox) E9.5 Nanog ${ }^{\text {tg }}$ embryos. ${ }^{* *} p<0.0005$; Student's $t$-test. Horizontal line represents mean values and error bars standard deviation (SD).

E Representative FACS plots showing the distribution of CKit and CD41 populations in yolk sacs from untreated controls (-dox) and Nanog expressing (+dox) E9.5 Nanog ${ }^{\text {tg }}$ embryos.

F Quantification of different progenitor populations in yolk sacs from control (-dox, black dots; $n=8$ ) and Nanog expressing (+dox, red dots; $n=7$ ) E9.5 embryos. Each replicate contained a pool of 5 (-dox) or 8 (+dox) E9.5 Nanog ${ }^{\text {tg }}$ embryos. Horizontal line represents mean values and error bars SD.

G Differences in the expression levels of Nanog and selected hematopoietic genes in the CD71 $1^{+}$Ter $119^{+}$population of control $(-$dox; $n=7)$ and $N a n o g$ expressing (+dox; $n=4)$ E9.5 embryos. ${ }^{* *} P<0.005,{ }^{* *} p<0.0005$; Student's $t$-test. Horizontal line represents mean values and error bars SD. 
A
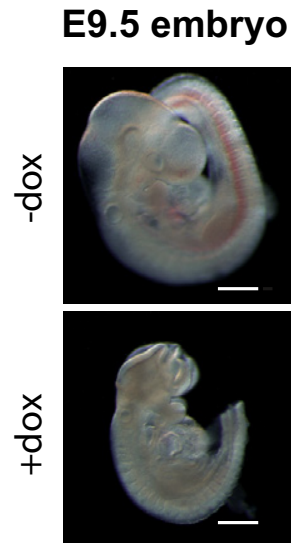

$H b b-b h 1$
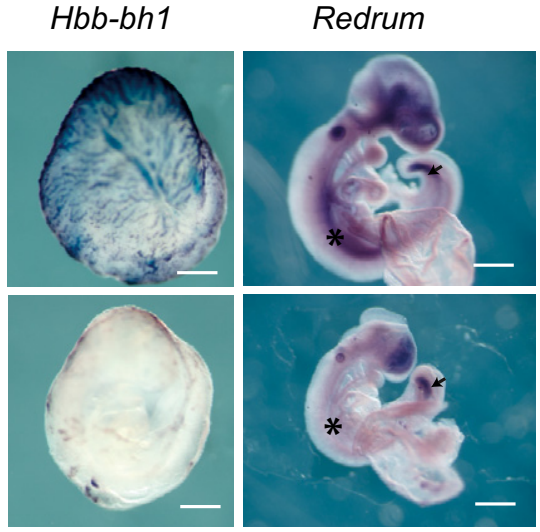

B

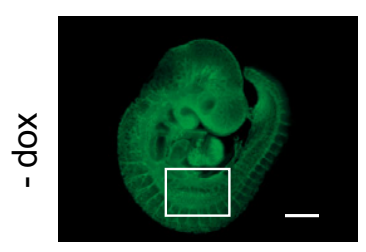

Endomucin

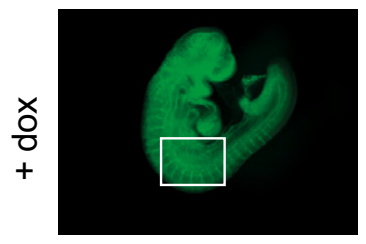

C E9.5 yolk sacs
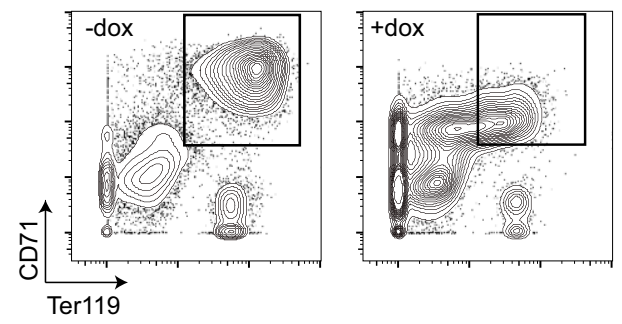

E

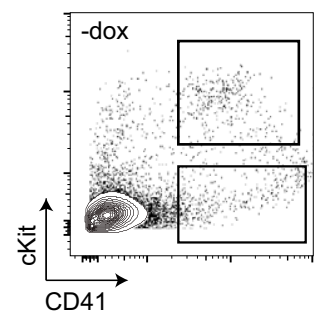

D

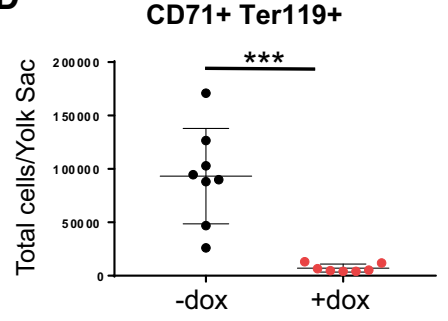

\section{G E9.5 circulating cells}
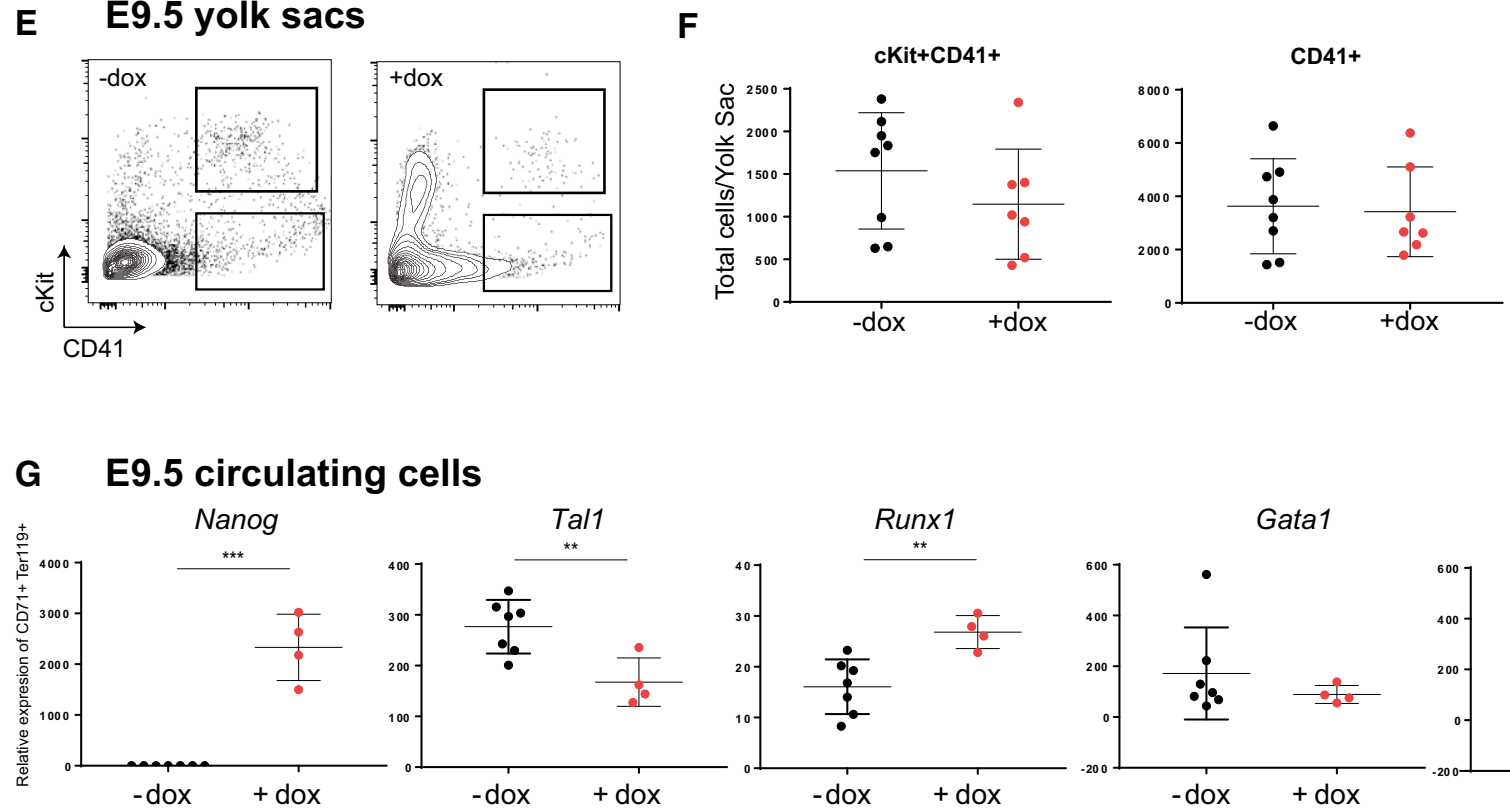

Figure 1.

examined the expression of $K d r$, a pan-mesodermal gene that acts downstream of Brachyury; relative to the timing of Brachyury induction, dynamics of $K d r$ expression coincided in wild-type and Nanog $^{-1-}$ EBs. In contrast, erythroid gene activation occurred earlier in $\mathrm{Nanog}^{-1-}$ EBs than in wild-type controls (Fig EV2B). Thus, although mesoderm induction is delayed in $\mathrm{Nanog}^{-1-} \mathrm{EBs}$, once it occurs the $\mathrm{Nanog}^{-/-}$mesodermal cells show an elevated potential for erythroid differentiation.

To further study the effect of loss of Nanog, we deleted a floxed allele from a heterozygous ES cell line (Nanog ${ }^{\text {flox/-}}$; Zhang et al, 2018) by transfecting Cre recombinase and differentiating sorted $\mathrm{GFP}^{+}$cells (that is activated upon Cre recombination) from ES to 
A

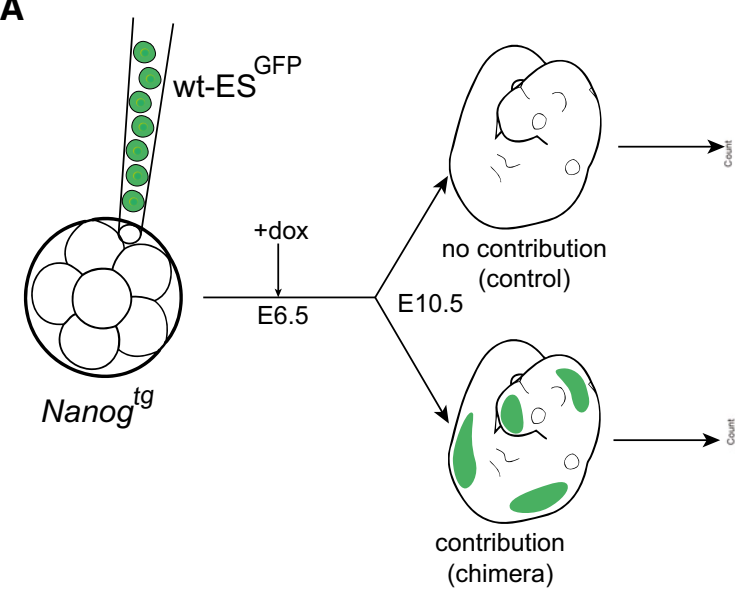

B E10.5 embryo

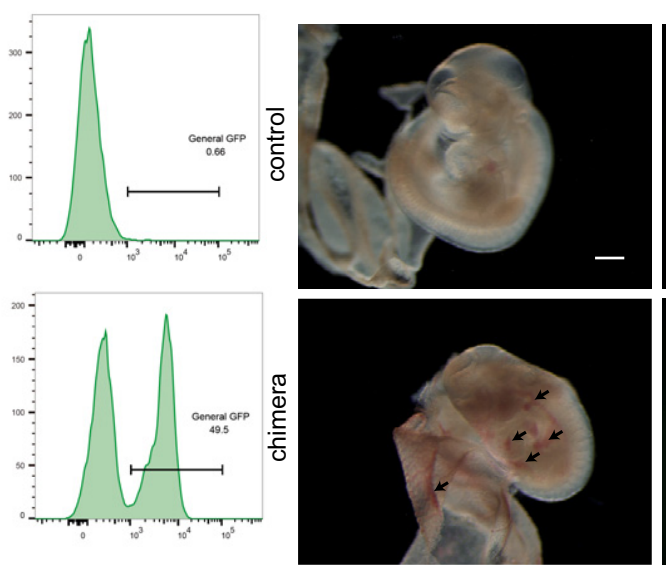

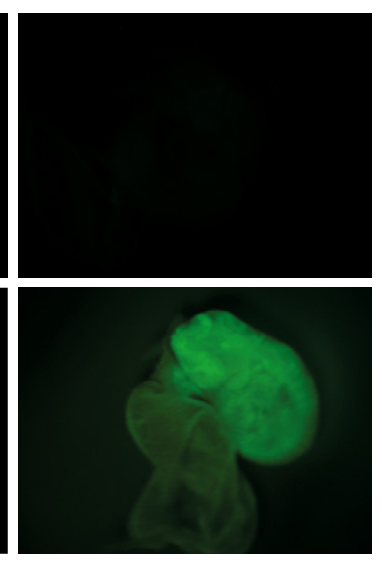

\section{E10.5 embryo}
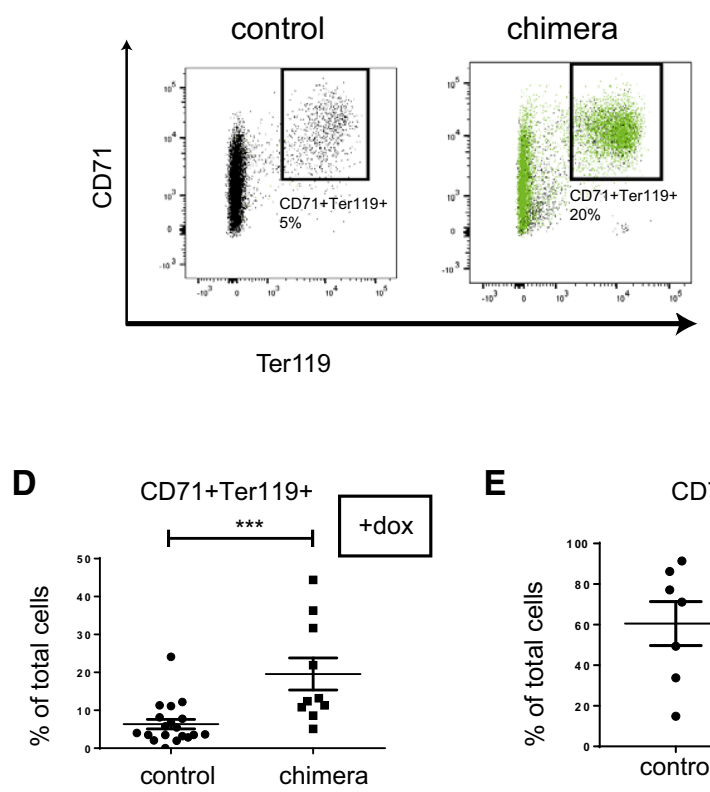

E

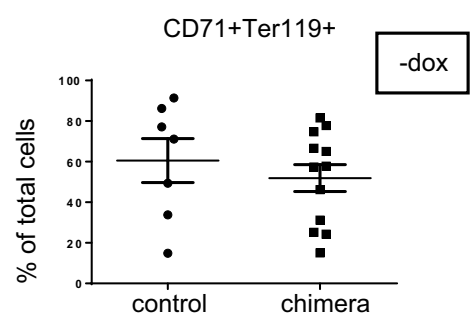

$\mathbf{F}$

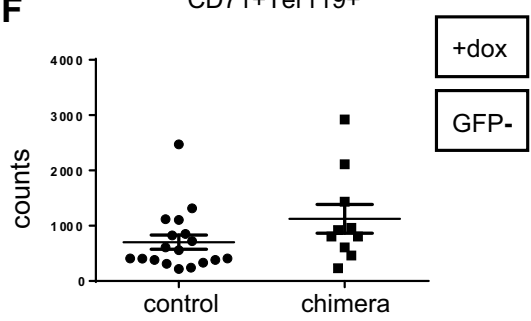

Figure 2. Wild-type ES cells rescue erythroid maturation in chimeric embryos.

A Experimental design for chimera generation, and contribution of GFP cells to chimeric embryo (right hand side panels).

B Freshly dissected dox-treated Nanog ${ }^{\text {tg }}$ E10.5 embryos without (control) and with (chimera) contribution of wt-ES ${ }^{\text {GFP }}$ cells (left, brightfield; right, GFP). Arrows mark the presence of blood in chimeric embryos that is absent from controls. Scale bar, $500 \mu \mathrm{m}$.

C Representative FACS plots showing of red blood cell maturation as determined by CD71/Ter119 staining in single dox-treated E10.5 control (left) and chimeric (right) embryos.

D-F Quantification of the CD71 ${ }^{+}$Ter119 ${ }^{+}$population in single dox-treated E10.5 embryos (D; control, $n=18$; chimera, $n=10$ ), untreated embryos (E; control, $n=7$; chimera, $n=12$ ), and in GFP ${ }^{-}$cells (not derived from wild-type ES cells) from dox-treated embryos (F; control, $n=18 ;$ chimera, $\left.n=10\right)$. *** $P<0.0005 ;$ Student's $t$ test. Horizontal line represents mean values and error bars SD.

epiblast-like cells (Hayashi et al, 2011; Murakami et al, 2016). This process recapitulates in culture the transition from pluripotent cells of the blastocyst to primed cells of the epiblast (Buecker et al, 2014), a time window during development when Nanog is expressed. Mutant cells (Nanog del/-) upregulate Brachyury following the same dynamics as control heterozygote Nanog ${ }^{\text {flox/- }}$ cells. However, they show precocious activation of erythroid gene expression (Fig EV2C), in line with our previous observations.

\section{Blockade of adult erythrocyte maturation by Nanog}

Nanog has mostly been analyzed in early developmental stages and in pluripotent stem cells. However, some reports have described its expression and roles in adult tissues and cells (Tanaka et al, 2007; Kohler et al, 2011; Piazzolla et al, 2014). In light of our findings during embryonic hematopoiesis, we explored the effects of Nanog during erythroid differentiation in the adult. 
A
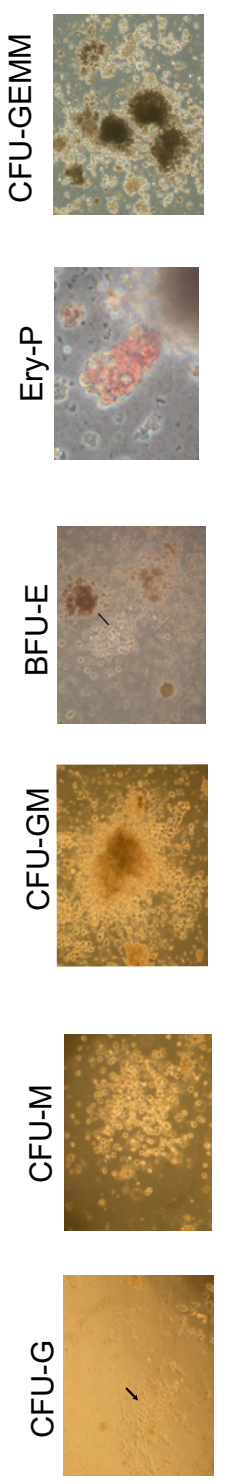

ES cell-derived embryoid bodies

D5

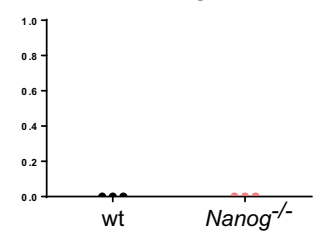

D6
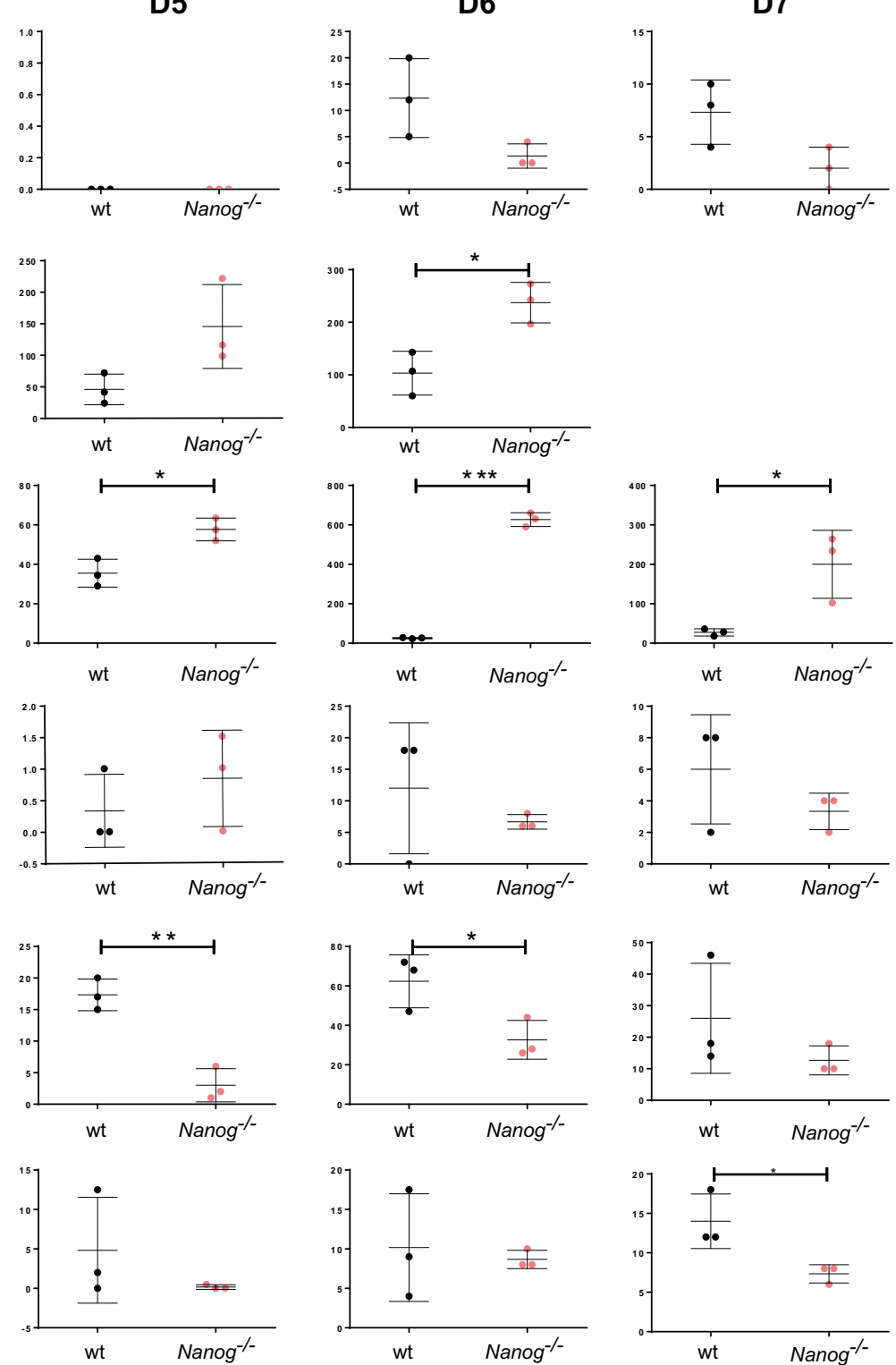
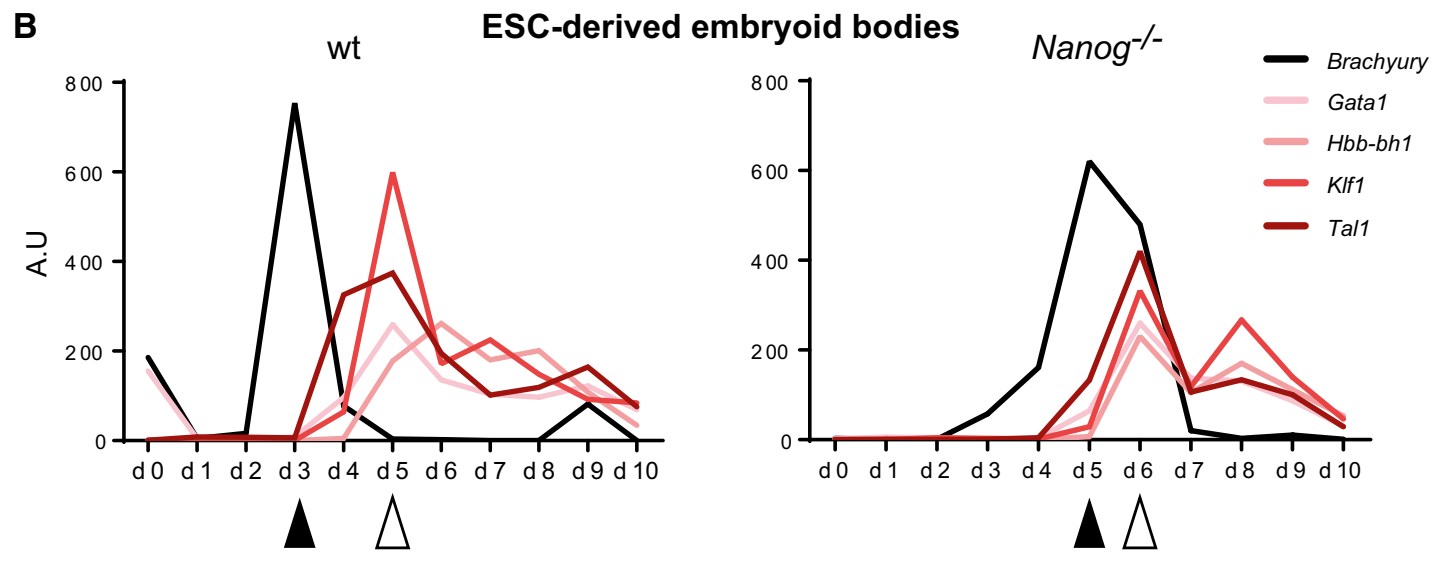

Figure 3. 
Figure 3. Nanog-knockout ES cells show increased potential to generate erythroid precursors.

A Quantification of colony-forming units generated by wild-type (wt) and knockout (Nanog ${ }^{-1}$ ) ES cells after culture of EBs for 5 (D5), 6 (D6), or 7 (D7) days and plating disaggregated cells in different hemogenic-promoting conditions. Panels on the left show representative images of mouse hematopoietic colonies obtained after 12 days of culture in specific media. CFU-GEMM, progenitors giving rise to granulocytes, erythrocytes, monocytes, and megakaryocytes; BFU-E, burst forming unitserythroid; Ery-P, colony-forming primitive erythroid; CFU-GM, granulocyte-monocyte precursors; CFU-M, monocyte precursors; CFU-G, granulocyte precursors. No CFU-GEMM are detected at D5 and no BFU-E at D7. For both wt and knockout cells, $n=3$ each with three technical replicates. ${ }^{*} P<0.05,{ }^{\star \star} P<0.005$, ${ }^{* \star *} P<0.00005$; Student's $t$-test. Horizontal line represents mean values and error bars SD.

B RT-qPCR determination of the relative expression of Brachyury and selected hematopoietic genes in control (wt, right) and knockout (Nanog ${ }^{-1-}$, left) ES cells $(n=3)$ during 10 days of EB differentiation in hematopoietic cytokine-enriched medium. Black arrowheads indicate the peak of Brachyury expression and white arrowheads the time of maximum hematopoietic gene expression.

Nanog expression was systemically induced in adult Nanog ${ }^{\text {tg }}$ mice by 5-day treatment with dox in drinking water, and the mice were then sacrificed and bone marrow extracted (dox ${ }^{+}$; Fig 4A). As controls, we used untreated mice of the same genotype (dox-). Analysis of erythrocyte maturation with CD71 and Ter119 (Socolovsky et al, 2001; Zhang et al, 2003) revealed an increase in immature populations (basophilic and polychromatic erythroblasts; S2 and S3, respectively) together with a decrease in the number of more differentiated erythroblasts (orthochromatic erythroblasts, S4; Fig $4 \mathrm{~B}$ and $\mathrm{C}$ ). This result suggested a block in the differentiation of erythrocyte precursors, so we next quantified bone marrow progenitors by flow cytometry using lineage cocktail, c-kit, Sca-1, CD34, and CD16/32 (Fig 4D; Challen et al, 2009).

Induced Nanog expression triggered a decrease in absolute cell numbers of hematopoietic stem cells (lineage-Sca ${ }^{+} \mathrm{cKit}^{+}$; LSK) and common myeloid progenitors (CMP), but no changes in granulocyte-macrophage progenitors. Interestingly, this was accompanied by a significant increase in megakaryocyte-erythroid progenitors (MEP; Fig 4E). Analysis of the expression of key erythroid genes by RT-qPCR in sorted MEPs revealed a significant reduction of Tal1 in dox-treated mice (Fig 4F). Together, these results indicate that Nanog can block the maturation of red blood cells during adult hematopoiesis together with the downregulation of key erythroid factors. This leads to defective differentiation of these populations and therefore to an accumulation of their precursors.

We further characterized this phenotype by RNA-seq on the MEPs from dox-treated and untreated adult Nanog ${ }^{t g}$ mice. Genes downregulated in MEPs from dox-treated animals were enriched in functional terms related to bone marrow cell populations, and more specifically MEPs (Fig EV3A). This confirms that Nanog is repressing the transcriptional program for erythroid progenitors (Fig EV3B). On the other hand, genes that are upregulated upon Nanog induction are highly enriched in the mast cell program (Fig EV3A and B; Dataset EV1). Most interestingly, deletion of Tal1 during adult hematopoiesis results in production of mast cells from MEPs, while under normal conditions these cells derive from granulocyte-monocyte progenitors (Salmon et al, 2007). This is accompanied by an upregulation of Gata2 (Salmon et al, 2007), a critical specifier of mast cells (Ohmori et al, 2015), that we also see increased upon Nanog expression in MEPs (Fig EV3B; Dataset EV1). Furthermore, the expression of Cebpa, a factor that represses mast cell lineage (Iwasaki et al, 2006), is downregulated in the Nanogexpressing MEP population (Fig EV3B; Dataset EV1). However, we believe that the positive regulation of the mast cell program is not a physiological role of Nanog, because this cell type does not appear during gastrulation (as erythroid progenitors do) but at later stages in the yolk sac and the AGM (Gentek et al, 2018) where Nanog is not expressed. Thus, we consider that upregulation of the mast cell program is a secondary consequence of the downregulation of erythroid lineage factors, such as Tal1, in Nanog expressing MEPs.

To extend these observations, we next carried out bone marrow transplantation of $\operatorname{Nanog}^{t g}$ mice to wild-type irradiated recipients (Fig 4G). After 3 months of engraftment and recovery, more than $95 \%$ of peripheral blood cells were derived from donor mice $(n=7$; Fig $4 \mathrm{H}$ ). We treated the mice for 4 months with dox to induce Nanog expression only in hematopoietic cells, and found that at that point the host cells had been partially able to recolonize the bone marrow and contribute to peripheral blood cells (ranging from 20 to $80 \%$; Fig $4 \mathrm{H}$ ). We then purified bone marrow from the transplanted mice and analyzed chimerism in different progenitor populations. While LSK, CMPs of GMPs show variable degrees of contribution of wild-type cells and Nanog expressing cells, MEPs are almost exclusively derived from the host (Fig 4I). These results indicate that the expression of Nanog in MEPs causes them to be outcompeted by wild-type cells during bone marrow reconstitution, possibly due to their decreased ability to differentiate and generate mature erythroid cells.

\section{A distal NANOG-binding element represses Tal1 expression in the embryo}

Nanog-mediated downregulation of erythroid specification genes in both the embryo and the adult strongly suggests that some of these genes are likely direct transcriptional targets of NANOG. If so, we would expect to find mutually exclusive expression of Nanog and these genes at the time of initial hematopoietic specification in the gastrulating embryo. We therefore analyzed single-cell expression data from E7.0 nascent mesoderm (Scialdone et al, 2016), when Nanog is still expressed in the posterior-proximal region of the embryo (Hart et al, 2004), and examined the number of cells expressing both Nanog and markers of mesoderm (Brachyury, Cdx2) and hematopoiesis (Tal1, Runx1, Gata1, Klf1; Fig 5A). For all of these genes, we found the expected proportion of co-expressing cells with Nanog with the exception of Tall (Fig 5A and B). We confirmed that Nanog can downregulate Tall at early stages by culturing Nanog ${ }^{\text {tg }}$ embryos with or without dox from E6.5 to E6.75 ex utero, which did not alter normal development (Fig 5C). Tal1 failed to be upregulated in dox-treated embryos, whereas other hematopoietic genes such as Klf1 were unaffected (Fig 5D). We further confirmed that Nanog downregulates Tall by whole-mount in situ hybridization of E7.5 embryos treated with dox in utero (Fig 5E).

This evidence strongly suggests that Tal1 is a direct transcriptional target of NANOG during early gastrulation at the onset of 
hematopoietic determination. To investigate this possibility, we analyzed published ChIP-seq data for NANOG binding in ES and EpiLCs, which correspond to the E6.0 epiblast in the mouse embryo (Murakami et al, 2016). This study describes a broad resetting of
NANOG-occupied genomic regions in the transition from ES cells to EpiLCs, resembling the developmental progress from the naïve inner cell mass of the blastocyst to the primed epiblast at gastrulation (Hayashi et al, 2011; Morgani et al, 2017). We examined a number
A

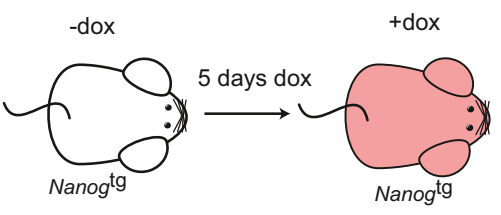

D Adult bone marrow

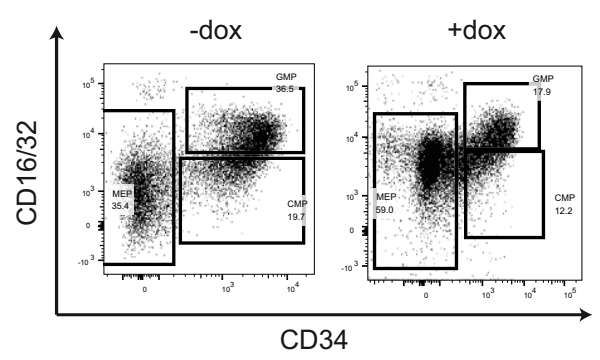

B Adult bone marrow

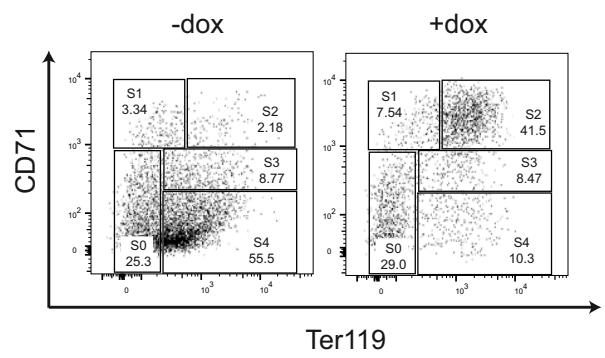

C

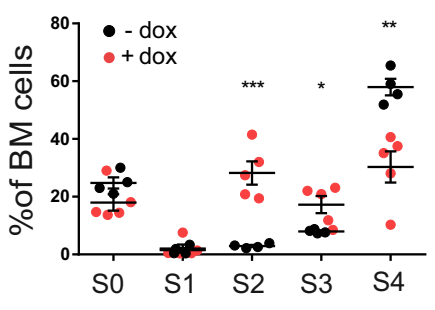

$\mathbf{E}$

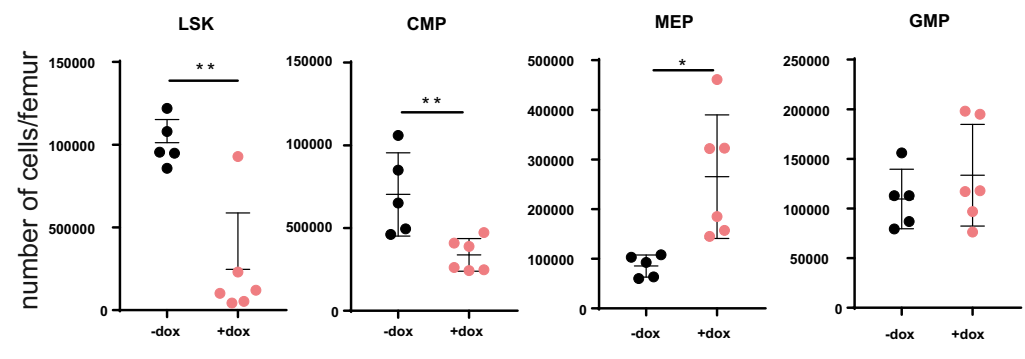

\section{F Adult MEPs}
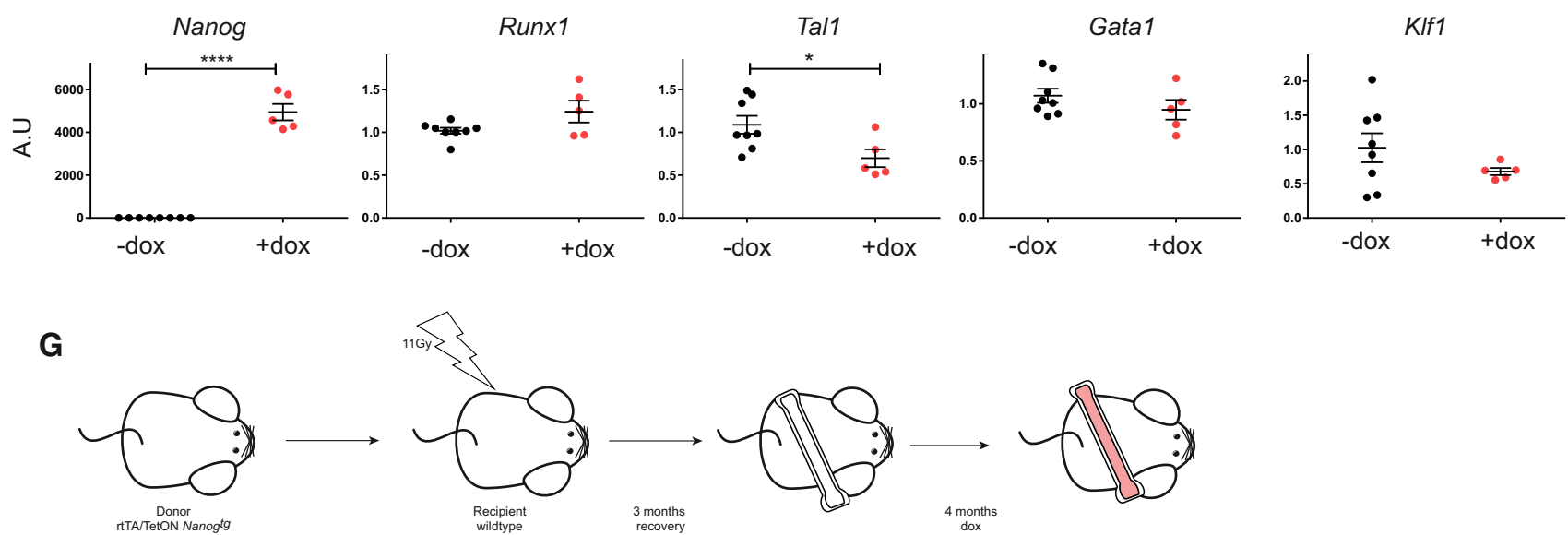

H

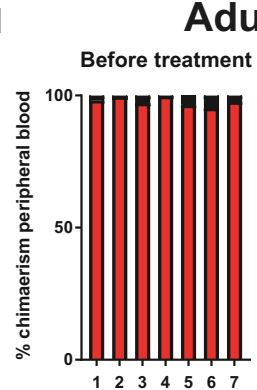

I

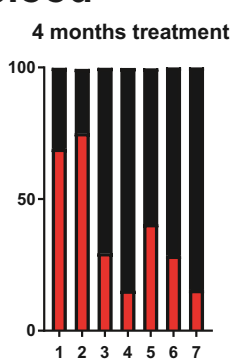

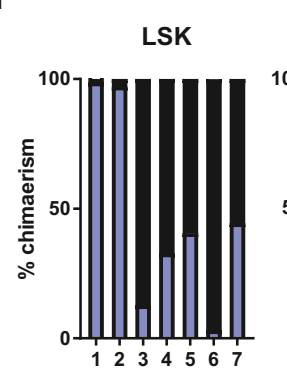

Adult bone marrow

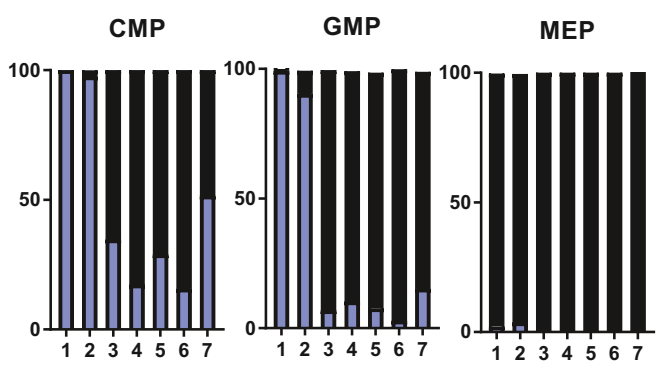

Figure 4. 
Figure 4. Induced Nanog expression blocks erythroid maturation in adult mice.

A Experimental design for the treatment of adult Nanog $^{\text {tg }}$ mice.

B Representative FACS plots showing the distribution of different populations distinguished by CD71/Ter119 staining in whole bone marrow from untreated (-dox) or treated (+dox) adult mice. S0 (double negative cell), S1 (proerythroblast), S2 (basophilic erythroblast), S3 (polychromatic erythroblast), and S4 (orthochromatic erythroblast) are different stages of blood maturation.

C Quantification of the S1-S4 erythroid populations (-dox, $n=4 ;+$ dox, $n=5$ ). ${ }^{*} p<0.05,{ }^{* *} p<0.005,{ }^{* * *} p<0.0005$; Student's $t$-test. Horizontal line represents mean values and error bars SD.

D Representative FACS plots showing the distribution of CD16/32 and CD34 hematopoietic precursors sorted from the $\mathrm{CKit}^{+} \mathrm{SCa1}^{-} \mathrm{LIN}^{-}$bone marrow of untreated (-dox) or treated (+dox) adult Nanog ${ }^{\text {tg }}$ mice.

E Quantification of precursor populations based on CD16/32 and CD34 sorting, as total number of cells per individual femur $(-$ dox, $n=5 ;+$ dox, $n=6)$. ${ }^{*} P<0.05$, ${ }^{* *} P<0.005$; Student's $t$-test. Horizontal line represents mean values and error bars SD.

F RT-qPCR quantification of the relative expression of hematopoietic genes in megakaryocyte-erythroid progenitors (MEP; - dox, $n=8 ;+$ dox, $n=5$ ). ${ }^{*} P<0.05$, ${ }^{* * * \star} P<0.00005$; Student's $t$-test. Horizontal line represents mean values and error bars SD.

C Experimental design for the transplant of Nanog ${ }^{\text {tg }}$ bone marrow to wild-type recipients and treatment of chimeric mice.

$\mathrm{H}$ Contribution of Nanog $^{\text {tg }}$ transplanted bone marrow cells to peripheral blood before (left) and after (right) dox treatment. Percentage of host-derived cells (CD45.1 ${ }^{+}$) are shown in black, and of donor derived cells (CD45.1/CD45.2 double + ) in red. Individual mice are indicated on the $x$-axis $(n=7)$.

I Contribution of Nanog $^{\text {tg }}$ transplanted cells to LSK, CMP, GMP, and MEP populations purified from bone marrow. Percentage of host-derived cells (CD45.1 ${ }^{+}$) are show in black, and of donor derived cells (CD45.1/CD45.2 double + ) in blue. Individual mice are indicated on the $x$-axis $(n=7)$.

of genomic loci, detecting binding at the Nanog locus itself in both ES cells and EpiLCs (Fig EV4A) and in Cdx2 only in ES cells (Fig EV4B). Neither cell type showed evidence of NANOG bound regions surrounding Runx1 (Fig EV4C) or Klf1 (Fig EV4D). Interestingly, EpiLCs, but not ES cells, showed NANOG binding 22 kilobases upstream of Tall, in an intron of the neighboring Stil gene (Fig EV4E). We also detected NANOG binding downstream of Gatal (Fig EV4F); however, these regions could be functionally related to the neighboring Eras and Hdac6 genes, which are associated with pluripotency and early stem cell differentiation (Takahashi et al, 2003; Chen et al, 2013).

Analysis of the Tal1/Stil NANOG bound region in EpiLCs (Fig 5F) revealed bona fide consensus binding sites (Fig EV5A). To investigate the function of this region, we deleted it by CRISPR/ Cas9-mediated genome editing (Ran et al, 2013) by microinjection in one-cell stage embryos and examined the transcriptional consequences in early development. Gene expression was analyzed by RT-qPCR in individual edited E6.5 embryos. As controls, we used embryos of the same batch showing no evidence of deletion of the Tal1/Stil NANOG bound region (Fig EV5B). Tal1 expression was significantly increased in targeted embryos, whereas other genes such as Klf1, Gfilb, or Runx1 were unaffected (Fig 5G). Deletion of this genomic region did not alter Stil expression, despite the location of the site within this gene (Fig 5G). These assays provide strong evidence that this specific genomic region acts as a cis-regulatory element in the Nanog-mediated repression of Tall in the early mouse embryo.

In order to further confirm these observations and address the effect of the deletion on Tall expression and its dependence on Nanog, we analyzed the transition from ES to EpiLC in culture as above. For this, we generated lines deleted for the distal Tall element by genome editing as previously described in vivo, but in ES cells derived from the $\mathrm{Nanog}^{\mathrm{tg}}$ mouse (Figs $5 \mathrm{H}$ and EV5C). We observe that non-treated Nanog ${ }^{\text {tg }}$ ES cells (non-deleted control) show a significant increase in Tal1 expression when they transit to EpiLCs (Fig 5H), what would be the equivalent of the initial expression of Tal1 in the embryo. However, if dox is added to the medium, this increase of Tal1 between ES and EpiLC is no longer significant. Thus, in this experimental setting, increased expression of Nanog is able to block at least partially the early induction of Tal1, in line with our in vivo results. Nevertheless, when we repeat his experiment but with two independent ES cell lines where the NANOGbound distal element (dTal1) has been deleted (Nanog ${ }^{\text {tg }}$; TTal1 $^{\text {del\#1 }}$ and $\mathrm{Nanog}^{\text {tg }} ; \mathrm{dTal1}^{\mathrm{del}{ }^{\sharp 2}}$ ), EpiLC become unresponsive to Nanog upon dox treatment and still upregulate Tal1 as cells not treated with dox. These results show that the distal element we have characterized is necessary for correct initiation of Tal1 expression, and that it mediates the response of Tal1 to Nanog.

\section{Discussion}

Red blood cell precursors are the first cell type to be specified from nascent mesoderm during mouse gastrulation (Kinder et al, 1999; Baron et al, 2012). While the genes and networks that determine primitive hematopoietic cells are well understood (Isern et al, 2011; Kingsley et al, 2013), much less is known about how precursors are specified during the early stages of primitive streak formation (Padron-Barthe et al, 2014). Here, we show that the pluripotency factor NANOG regulates the transition from multipotent mesodermal progenitors to red blood cell precursors in these early steps, at least partially through the direct regulation of the lineage specifier Tal1.

Despite the well-characterized role of pluripotency factors in embryonic stem cells and the preimplantation embryo (Chambers \& Tomlinson, 2009), their function at later developmental stages has received much less attention, even if they are expressed up to gastrulation in mice (Yeom et al, 1996; Hart et al, 2004; Osorno et al, 2012) and primates (Nakamura et al, 2016). Oct4 is involved in proliferation of the primitive streak (DeVeale et al, 2013), in correct trunk elongation of the trunk (Aires et al, 2016), and some evidence points to it having a role in mesoderm and subsequent hematopoietic specification (Kong et al, 2009). However, no clear function is known for Nanog after implantation apart from the regulation of germline development (Chambers et al, 2007). Single-cell RNA-seq expression data from gastrulating embryos (Scialdone et al, 2016) show that Nanog is expressed in a subset of mesodermal precursors. This situation is reminiscent of the heterogeneities in Nanog expression in the preimplantation embryo, which drives lineage segregation of the epiblast and the primitive endoderm 
A

E7.0 embryo

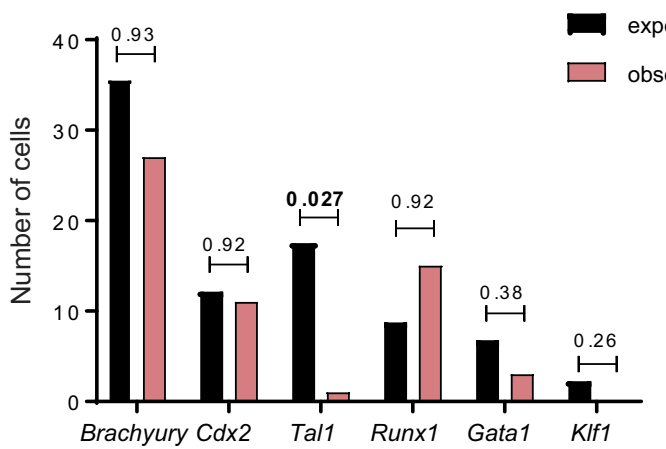

C

E6.5 embryo
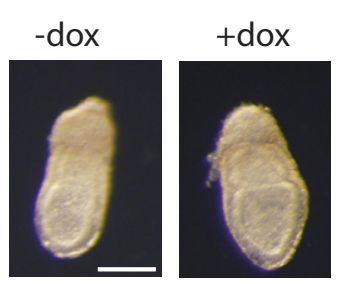

ex utero

E

\section{E7.5 embryo}

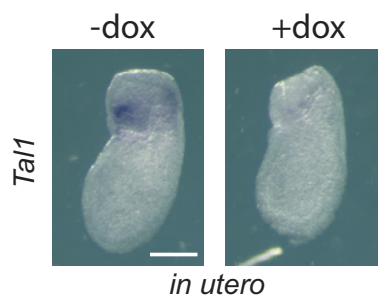

D
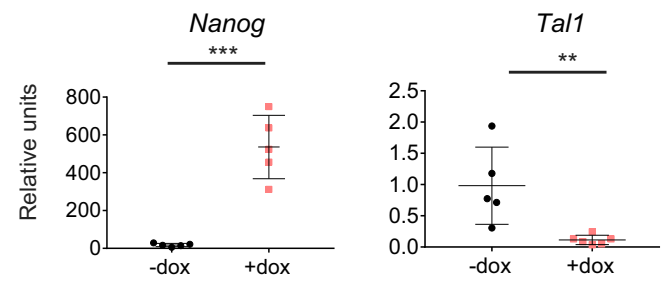

KIf1

$\mathbf{F}$

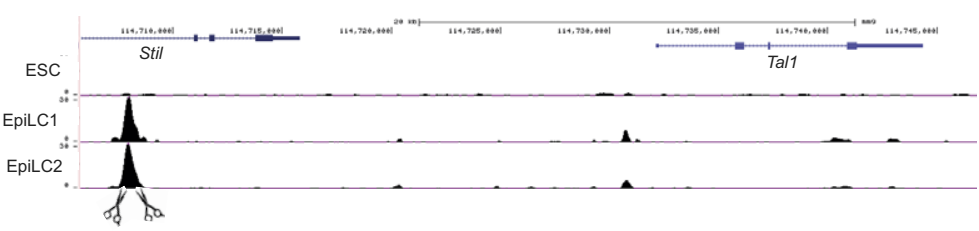

G E6.5 embryo
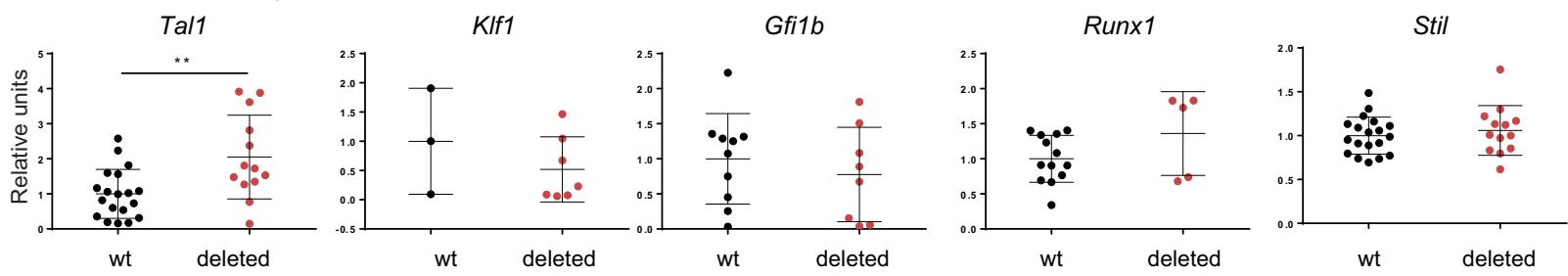

H ES to EpiL cell transition
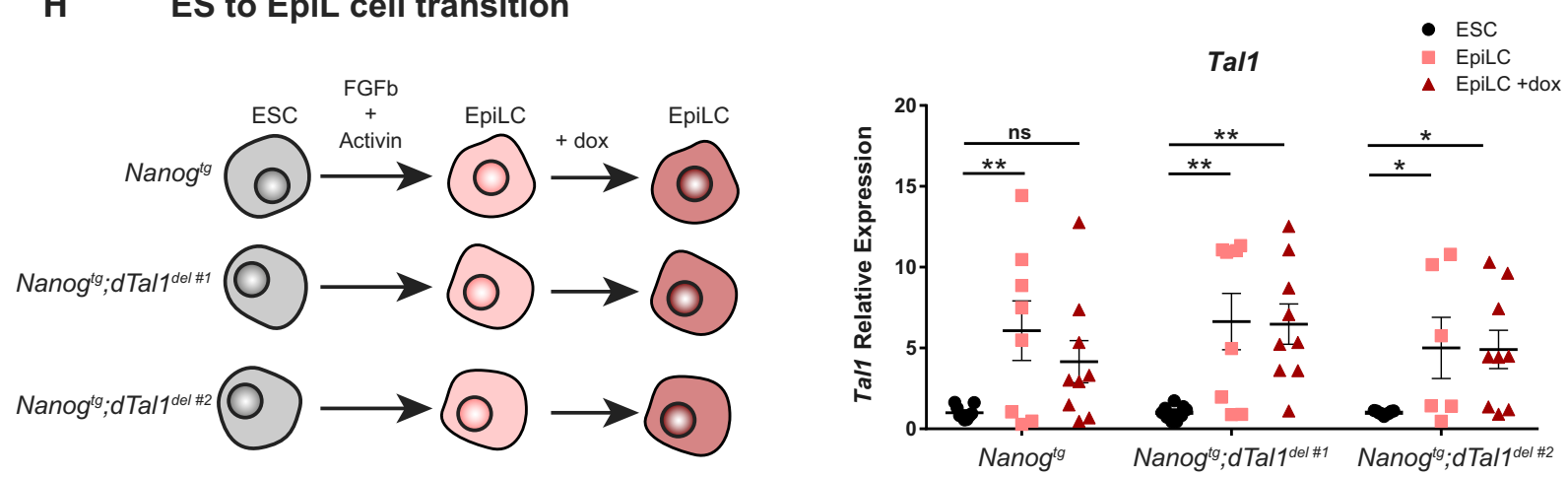

Figure 5. 
Figure 5. Direct transcriptional regulation of Tal1 expression by Nanog.

A Expected and observed number of mesodermal $\left(\mathrm{Flk1}^{+}\right)$cells of the $\mathrm{E} 7.0$ mouse embryo expressing Nanog and selected mesodermal or hematopoietic gene expression, based on single-cell RNA-seq data (Scialdone et al, 2016). Statistical significance was calculated with a hypergeometric test.

B PCA showing the distribution of Flk1 ${ }^{+}$E7.0 mesoderm cells expressing Nanog (green) or Tal1 (red). The single cell expressing both genes is shown in yellow and indicated by an arrow.

C E6.5 Nanog ${ }^{\text {tg }}$ embryos after $8 \mathrm{~h}$ ex utero culture in the presence (+dox) or absence (-dox) of doxycycline. Scale bar, $100 \mu \mathrm{m}$.

D RT-qPCR quantification of the relative expression of Nanog, Tal1, and $K I f 1$ in individual untreated embryos (-dox) or treated embryos $(+$ dox $)(n=5)$. ** $P<0.005$, ${ }^{* * *} P<0.0005$; Student's $t$-test. Horizontal line represents mean values and error bars SD.

E Whole-mount in situ hybridization of Tal1 in E7.5 untreated (-dox) or in utero treated (+dox) Nanog ${ }^{\text {tg }}$ embryos. Scale bar, $100 \mu \mathrm{m}$.

F UCSC browser view of the Tal1/Still region (mm9; chr4:114,705,753-114,756,741), indicating the presence of the NANOG binding peak, determined by ChIP-seq, in EpiLCs (2 replicates are shown) but not in ES cells (Murakami et al, 2016); the binding peak was deleted by CRISPR/Cas9 genome editing (scissors).

G RT-qPCR determination of relative expression in wild-type and CRISPR-deleted embryos $(n=5)$ of Tal1 (wt, $n=19$; deleted, $n=13)$, KIfI (wt, $n=3$; deleted, $n=6$ ), Gfilb (wt, $n=10$; deleted, $n=8$ ), RunxI (wt, $n=13$; deleted, $n=5$ ), and Stil (wt, $n=19$; deleted, $n=13$ ). ${ }^{*} p<0.005$, Student's $t$-test. Horizontal line represents mean values and error bars SD.

H Experimental design for ES to EpiL cell differentiation of Nanog ${ }^{\text {tg }}$ cells and two independent clones (Nanog ${ }^{\text {tg }} ;$ TTal $^{\text {del\#1 }}$ and Nanog ${ }^{\text {tg }} ; d T a l 1^{\text {del\#z }}$ ) where the binding site for NANOG distal to Tal1 has been deleted (left). On the right, relative expression of Tal1 determined by RT-qPCR for each ES cell line (ESC; $n=9$ for all three lines) and EpiL cells without (EpiLC; Nanog ${ }^{\text {tg }}$ and Nanog $^{\text {tg }} ;$ dTal1 $^{\text {del\#1 }}, n=8 ;$ Nanog $^{\text {tg }} ;$ dTalI $^{\text {del\#2 }}, n=6$ ) or with dox treatment (EpiLC + dox; $n=9$ for all three lines). The genotype of the cell lines is indicated below. Values were normalized to Nanog ${ }^{\text {tg } E S C .}{ }^{*} P<0.05,{ }^{* \star} P<0.01$, ns $=$ not significant; ANOVA with Fisher post-test. Horizontal line represents mean values and error bars standard error of the mean (SEM).

(Xenopoulos et al, 2015). Our results suggest that a similar situation may occur during specification of the first mesodermal lineages. Nanog expression in Brachyury-positive cells would maintain them in a pan-mesodermal multipotent state, whereas its downregulation would allow the expression of early hematopoietic lineage specifiers, driving their differentiation to primitive red blood cells. This process, however, occurs during a limited time window during the initial phases of gastrulation, as Nanog is quickly downregulated by E8.0-8.5 (Hart et al, 2004; Scialdone et al, 2016). By this stage, mesodermal progenitors have ingressed through the primitive streak and are no longer able to activate the early hematopoietic program, a process that also involves restricted spatial signaling through the Wnt and Bmp pathways (Cheng et al, 2008; Myers \& Krieg, 2013; Mimoto et al, 2015). Therefore, this Nanog-mediated switch would act to control the rapid specification of blood precursors, the first lineage determination event in gastrulation, and required to supply the embryo with oxygen to support its subsequent exponential growth.

We also show that Nanog directly represses the master hematopoietic regulator Tal1 (Porcher et al, 2017) through an upstream regulatory element located in an intron of the neighboring Stil gene. Interestingly, this site is occupied by NANOG only during the differentiation of ES cells to EpiLCs (Murakami et al, 2016). This change in binding site usage during this transition again suggests that Nanog has specific roles in the postimplantation pregastrulating epiblast (the in vivo equivalent of EpiLCs; Hayashi et al, 2011) that are distinct from those operating during the pluripotent state. Tal1 is certainly a prime candidate for mediating at least partially the effects of Nanog on erythropoiesis, as we found that it is consistently repressed at different embryonic stages and in adult erythroid progenitors. However, surely other genes involved in early erythroid development, such as Gata1, could be also direct Nanog targets during this process. Further studies will unravel the full network regulated by Nanog at these stages.

In the adult, Nanog expression leads to defective erythroid-cell maturation, as also occurs in the embryo, and to an accumulation of MEPs showing downregulation of Tal1. This can be explained by a defect in the differentiation of these progenitors, and the phenotype we observe is reminiscent of the adult-specific Tal1 knockout (Hall et al, 2005). It is therefore tempting to speculate that the regulatory circuit acting in the early embryo can be reenacted in the adult solely by induction of Nanog.

Hematopoietic differentiation of $\mathrm{Nanog}^{-1-}$ ES cells (Chambers et al, 2007) confirms the proposed role for Nanog in erythroid development. Although $\mathrm{Nanog}^{-/-}$cells show an initial delay in the activation of early pan-mesodermal markers such as Brachyury, once this occurs, they show a faster and more coherent expression of erythroid genes. Directed differentiation reveals that the lack of Nanog promotes the red blood cell potential of these cells, which show a marked increase in both primitive and more mature erythroid colony formation. Our results show that Nanog acts as a barrier to red blood cell development. Controlled downregulation of Nanog during the initial phases of differentiation may present a novel approach to boosting the generation of red blood cells from pluripotent stem cells, a major clinical need (Kaufman, 2009).

\section{Materials and Methods}

\section{Animal model}

We obtained the Nanog/rtTA mouse line (R26-M2rtTA;Colla1-tetONanog) (Piazzolla et al, 2014) from Manuel Serrano (CNIO, Madrid) and Konrad Hochedlinger (Harvard Stem Cell Institute). This is a double transgenic line that carries the M2-rtTA gene inserted at the Rosa26 locus and a cassette containing Nanog cDNA under the control of a doxycycline-responsive promoter (tetO) inserted downstream of the Colla1 locus. Mice were genotyped by PCR of tail-tip DNA as previously described (Hochedlinger et al 2005; Piazzolla et al, 2014). Mice were housed and maintained in the animal facility at the Centro Nacional de Investigaciones Cardiovasculares (Madrid, Spain) in accordance with national and European Legislation. Procedures were approved by the CNIC Animal Welfare Ethics Committee and by the Area of Animal Protection of the Regional Government of Madrid (ref. PROEX 196/14).

Double-homozygote transgenic males were mated with CD1 females, which were then treated with doxycycline (dox) to induce the Nanog cassette by replacing normal drinking water with a $7.5 \%$ sucrose solution containing dox $(1 \mathrm{mg} / \mathrm{ml})$, with replacement with fresh solution after 2 days. For transgene induction in embryos to 
be harvested at E7.5, a single $100 \mu$ intraperitoneal injection of $25 \mu \mathrm{g} / \mu \mathrm{l}$ doxycycline was administered to pregnant females at E5.5, followed by dox administration in drinking water as above.

\section{RT-qPCR assays}

RNA was isolated from ESCs or sorted E9.5 cells using the RNeasy Mini Kit (Qiagen) and then reverse transcribed using the High Capacity cDNA Reverse Transcription Kit (Applied Biosystems). RNA from individual E6.5-7.5 embryos or sorted bone marrow populations was isolated using the Arcturus PicoPure RNA Isolation Kit (Applied Biosystems) and reverse transcribed using the Quantitect Kit (Qiagen).

cDNA was used for quantitative PCR (qPCR) with Power SYBR ${ }^{\circledR}$ Green (Applied Biosystems) in a 7900HT Fast Real-Time PCR System (Applied Biosystems). Expression of each gene was normalized to the expression of the housekeeping genes Actin and Ywhaz. Primers used are listed in Dataset EV2.

\section{Flow cytometry}

E9.5 and E10.5 whole embryos or dissected yolk sacs were disaggregated with $0.25 \%$ collagenase type I (Stemcell Technologies) at $37^{\circ} \mathrm{C}$ for $30 \mathrm{~min}$, and the cells were washed with PBS containing $2 \%$ FBS (Gibco) and filtered through a $70-\mu \mathrm{m}$ mesh. The single-cell suspension was then incubated for $30 \mathrm{~min}$ at $4^{\circ} \mathrm{C}$ with the following antibodies: anti-CD71-FITC (BD Biosciences), anti-Ter119-APC (BD Biosciences), anti-cKit-PEcy7 (BD Biosciences), and anti-CD41-PE (BD Biosciences). Samples were analyzed with the BD LSRFortessa flow cytometer.

Bone marrow of adult mice was obtained from femurs and tibias crushed in a mortar and filtered through a $70-\mu \mathrm{m}$ mesh to obtain single-cell suspensions. For hematopoietic cell maturation assays, a small fraction of the bone marrow was separated and the rest was depleted of red blood cells by lysis in FACSLysing solution (BD Biosciences). Antibodies used for blood maturation assay were antiCD71-FITC (BD Biosciences) and anti-Ter119-APC (BD Biosciences). Antibodies for BM precursor sorting were Biotinylated lineage cocktail (BD Biosciences), anti-CD34(RAM34)-FITC (BD Biosciences), anti-cKit-PEcy7 (BD Biosciences), anti-CD16/32-BV605 (BD Biosciences), and anti-Sca1-PerCP-Cy5.5 (BD Biosciences).

\section{Cytospin cell preparation}

For peripheral blood cytospin preparations, E9.5 embryos were dissected in warm PBS with $2 \%$ FBS and EDTA $0.5 \mathrm{mM}$, puncturing the yolk sac and the heart to let blood disperse into the media. All the preparation was passed through a $70-\mu \mathrm{m}$ filter, centrifuged for $5 \mathrm{~min}$ at $135 \mathrm{~g}$, and resuspended in a final volume of $200 \mu \mathrm{l} \mathrm{PBS}$. Cells were collected on a glass slide on a Thermo ScientificCytospin 4 Cytocentrifuge for $10 \mathrm{~min}$ at $200 \mathrm{rpm}$ and stained with MayGrünwald-Giemsa. Slides were scanned on a NanoZoomer-2.0RS C110730 scanner (Hamamatsu).

\section{Cell culture}

ESCs were maintained in serum-free conditions with Knock out serum replacement (Thermo Fisher), LIF (produced in-house), and 2i (CHIR-99021, Selleckchem; and PD0325901, Axon). BT12 and E14Tg2a ESCs were kindly provided by Ian Chambers and Austin Smith (Chambers et al, 2007). ESC was differentiated toward hematopoiesis according to published protocols (Sroczynska et al, 2009; Irion et al, 2010; Lesinski et al, 2012).

For embryoid body formation, 5000 ESCs were plated in StemPro34 medium supplemented with nutrient supplement (Gibco) and $2 \mathrm{mM}$ l-glutamine (l-Gln), penicillin/streptomycin (Gibco), $50 \mu \mathrm{g} / \mathrm{ml}$ ascorbic acid, $200 \mu \mathrm{g} / \mathrm{ml}$ iron-saturated transferrin, $4 \mathrm{ng} /$ $\mathrm{ml}$ recombinant human BMP4, and $4 \times 10^{-4}$ monothioglycerol. After 2.5 days, to the cultures were added $5 \mathrm{ng} / \mathrm{ml}$ recombinant human fibroblast growth factor 2 (rhFGF2; basic fibroblast growth factor $[\mathrm{bFGF}]), 5 \mathrm{ng} / \mathrm{ml}$ recombinant human activin $\mathrm{A}, 5 \mathrm{ng} / \mathrm{ml}$ recombinant human VEGF (rhVEGF), $20 \mathrm{ng} / \mathrm{ml}$ recombinant murine thrombopoietin (TPO), and $100 \mathrm{ng} / \mathrm{ml}$ recombinant murine stem cell factor (rmSCF). Cytokines were obtained from R\&D Systems Inc. or Peprotech. EBs were dissociated at day 6 by treatment with $0.05 \%$ trypsin-EDTA at $37^{\circ} \mathrm{C}$ for $2-5 \mathrm{~min}$.

Dissociated EBs at day 5 and 6 were plated in Methocult SF M3436 methylcellulose medium for quantification of primitive erythroid progenitor cells (BFU-E). Dissociated EBs at days 5, 6, and 7 were plated in Methocult GF M3434 methylcellulose medium for quantification of erythroid progenitor cells (CFU-E), granulocytemacrophage progenitor cells (CFU-GM, CFU-G, CFU-M), and multipotential granulocyte, erythroid, macrophage, and megakaryocyte progenitor cells (CFU-GEMM). Cells were plated in triplicate on ultra-low attachment surface plates (Corning) at 50,000 cells per plate. Plates were incubated in high humidity chambers for 12 days at $37^{\circ} \mathrm{C}$ and $5 \% \mathrm{CO}_{2}$. Whole plates were counted. For qPCR, EBs were directly lysed in extraction buffer and frozen at $-80^{\circ} \mathrm{C}$.

Nanog-floxed ES cells (Nanog ${ }^{\text {flox/- }}$; Zhang et al, 2018) were transfected with a Cre-expressing plasmid to induce recombination using Lipofectamine 2000 (Invitrogen). After 48 h, GFP-positive cells (Nanog ${ }^{\text {del/-}}$ ) and GFP-negative cells used as control (Nanog ${ }^{\text {flox/- }}$ ) were sorted using a FACS Aria Cell Sorter. Differentiation toward EpiLCs was induced by plating $5 \times 10^{4}$ ES cells on a well of a 24-well plate coated with human plasma fibronectin $(10 \mu \mathrm{g} / \mathrm{ml}$, Sigma $)$ in N2B27 medium supplemented with $20 \mathrm{ng} / \mathrm{ml}$ Activin A (Preprotech),12 ng/ml bFGF (Preprotech), and 1\% Knock out serum replacement (Thermo Fisher) for 3 days.

Embryonic stem cells from Nanog ${ }^{\text {tg }}$ mice were derived following standard procedures (Nagy et al, 2003). Differentiation to EpiLCs was performed in $\operatorname{Nanog}^{\text {tg }}$ ES cells and in two different clones of Nanog $^{\text {tg }}$ ES cells where the binding site upstream of Tal1 was deleted (Nanog ${ }^{\text {tg }}$; dTal1 $^{\text {del } \# 1}$ and ${ }^{\# 2}$ ). Differentiation was induced by plating $3 \times 10^{4}$ ES cells on a well of a 24 -well plate and using the same conditions above-mentioned. After 3 days of differentiation, doxycycline $(2 \mathrm{ng} / \mathrm{ml})$ was added to the medium of the corresponding wells to induce Nanog expression. One day later, EpiLCs with or without doxycycline treatment were lysed for RNA isolation.

\section{In situ hybridization and immunohistochemsitry}

Embryos were collected in cold PBS, transferred to 4\% PFA, and fixed overnight at $4^{\circ} \mathrm{C}$. After washing, embryos were dehydrated through increasing concentrations of PBS-diluted methanol $(25,50$, 75 , and $2 \times 100 \%$ ). In situ hybridization in whole-mount embryos was performed as described (Ariza-McNaughton \& Krumlauf, 2002; 
Acloque et al, 2008). Signal was developed with anti-digoxigeninAP (Roche) and BM-Purple (Roche). Images were acquired with a Leica MZ-12 dissecting microscope. Probes for in situ were obtained by PCR of cDNA with the primers listed in Dataset EV2.

For immunohistochemistry in whole mount, embryos were fixed overnight at $4^{\circ} \mathrm{C}$ in $4 \%$ paraformaldehyde, followed by overnight incubation at $4^{\circ} \mathrm{C}$ in primary antibody diluted 1:100 (rat monoclonal anti-endomucin, Santa Cruz sc-65495; or rat monoclonal anti-CD31, Santa Cruz sc-18916), washed and incubated overnight at $4^{\circ} \mathrm{C}$ with 1:500 Alexa Fluor 488 goat anti-rat (Termo Fisher Scientific, A11006) for Endomucin or HRP goat anti-rat (Termo Fisher Scientific, 31470) for CD31. For histology, embryos fixed as above were dehydrated through an ethanol series, cleared with xylene, embedded in paraffin, sectioned at $5 \mu \mathrm{m}$, and stained with hematoxylin and eosin.

\section{RNA-seq}

RNA was isolated from three replicates each of approximately 20,000 MEPs purified by sorting from adult untreated and doxtreated Nanog ${ }^{t g}$ mice. Sequencing was performed by the CNIC Genomics Unit using the GAIIx sequencer. Adapters were removed with Cutadapt v1.14 and sequences were mapped and quantified using RSEM v1.2.20 to the transcriptome set from Mouse Genome Reference NCBIM37 and Ensembl Gene Build version 67. Differentially expressed genes between the two groups were normalized and identified using the limma bioconductor package. Only $P$-values $<0.05$ adjusted through Benjamini-Hochberg procedure were considered as significant. Hierarchical clustering was performed on Z-scored values of the selected genes to generate an overview of the expression profile. Functional enrichment analysis was conducted using Enrichr (Kuleshov et al, 2016).

\section{CRISPR/Cas9 genome editing}

sgRNAs were designed using the CRISPR Design Tool from the Zhang Lab at MIT (http://crispr.mit.edu/). Sequences of guide RNAs are indicated in Fig EV5A. The two guide RNAs at $25 \mathrm{ng} / \mu \mathrm{l}$ were incubated with the Cas9 protein (PNA bio) at $30 \mathrm{ng} / \mu \mathrm{l}$ and microinjected into the pronuclei of (CBAxC57)F1 zygotes $(1,490)$; 1,075 surviving embryos were transferred to $\mathrm{CD} 1$ pseudopregnant females. 105 embryos were recovered at E6.5, and after discarding delayed or malformed embryos, 72 were lysed in $100 \mu$ l extraction buffer from the Arcturus PicoPure RNA Isolation Kit (Applied Biosystems). Aliquots of $10 \mu \mathrm{l}$ were used for DNA extraction for PCR genotyping, and the remaining $90 \mu \mathrm{l}$ was used for RNA extraction for RT-qPCR. Embryos for which we did not obtain a clear genotype were discarded, as well as those for which RT-qPCR of housekeeping genes did not reach a minimal threshold.

Embryonic stem cells from Nanog ${ }^{t g}$ mice were electroporated with Cas9 protein and sgRNAs as above. Individual clones were picked, genotyped as above, karyotyped, and expanded for further use.

\section{Statistical analysis}

Statistical analysis was performed with the use of two-tailed Student's unpaired $t$-test analysis (when the statistical significance of differences between two groups was assessed) or one-way
ANOVAs with subsequent Fisher post-test (when the statistical significance of differences between more than two groups was assessed). Prism software version 7.0 (Graphpad Inc.) was used. For the analysis of the expected proportion of co-expressing cells with Nanog, we used a hypergeometric test in R.

\section{Data availability}

Sequencing data have been deposited at GEO under accession number GSE119467 (https://www.ncbi.nlm.nih.gov/geo/query/ acc.cgi?acc $=$ GSE119467) .

Expanded View for this article is available online.

\section{Acknowledgements}

We thank Manuel Serrano and Konrad Hochedlinger for the Nanog ${ }^{\text {tg }}$ mouse line; Miguel Torres and Covadonga Díaz for the ES-GFP cell line; Austin Smith, Ian Chambers, and Harry G. Leitch for Nanog $^{-1-}$ ES cell lines; Luis Miguel Criado and the CNIC Transgenesis Unit for chimera generation; Elena LopezJimenez, Giovanna Giovinazzo, and the CNIC Pluripotent Cell Technology Unit for derivation of Nanog ${ }^{\text {tg }}$ ES cells; Simon Mendez-Ferrer and Abel SánchezAguilera for support and discussions; Cristina Gutierrez-Vazquez, Teresa Rayon, Hector Sanchez-Iranzo, and Andrés Hidalgo for comments on the manuscript; Simon Bartlett for English editing; and members of Manzanares laboratory for continued support. This work was supported by the Spanish government (grant BFU2014-54608-P and BFU2017-84914-P to MM; grants RYC-201109209 and BFU-2012-35892 to JI). The Gottgens and Nichols laboratories are supported by core funding from the Wellcome Trust and MRC to the Wellcome and MRC Cambridge Stem Cell Institute. The CNIC is supported by the Spanish Ministry of Science, Innovation and Universities (MINECO) and the Pro CNIC Foundation, and is a Severo Ochoa Center of Excellence (SEV-2015-0505).

\section{Author contribution}

JSA and MM designed the research with input from JI, BG, and JN. JSA performed the majority of the experiments, with the help of SM for embryo work and tissue culture; IR for genome editing; $A B$ and MT for ES cell work; WJ and AA for data analysis; IC, AB, and CC-T for analysis of adults; and CB-C for in situs. JSA and MM wrote the manuscript with input from all authors.

\section{Conflict of interest}

The authors declare that they have no conflict of interest.

\section{References}

Acloque H, Wilkinson DG, Nieto MA (2008) In situ hybridization analysis of chick embryos in whole-mount and tissue sections. Methods Cell Biol 87: $169-185$

Aires R, Jurberg AD, Leal F, Novoa A, Cohn MJ, Mallo M (2016) Oct4 is a key regulator of vertebrate trunk length diversity. Deu Cell 38: 262-274

Alvarez-Dominguez JR, Hu W, Yuan B, Shi J, Park SS, Gromatzky AA, van Oudenaarden A, Lodish HF (2014) Global discovery of erythroid long noncoding RNAs reveals novel regulators of red cell maturation. Blood 123: $570-581$

Ariza-McNaughton L, Krumlauf R (2002) Non-radioactive in situ hybridization: simplified procedures for use in whole-mounts of mouse and chick embryos. Int Reu Neurobiol 47: 239-250 
Baron MH, Isern J, Fraser ST (2012) The embryonic origins of erythropoiesis in mammals. Blood 119: 4828-4837

Borges L, lacovino M, Mayerhofer T, Koyano-Nakagawa N, Baik J, Garry DJ, Kyba M, Letarte M, Perlingeiro RC (2012) A critical role for endoglin in the emergence of blood during embryonic development. Blood 119: $5417-5428$

Buecker C, Srinivasan R, Wu Z, Calo E, Acampora D, Faial T, Simeone A, Tan M, Swigut T, Wysocka J (2014) Reorganization of enhancer patterns in transition from naive to primed pluripotency. Cell Stem Cell 14: 838-853

Challen GA, Boles N, Lin KK, Goodell MA (2009) Mouse hematopoietic stem cell identification and analysis. Cytometry A 75: 14-24

Chambers I, Silva J, Colby D, Nichols J, Nijmeijer B, Robertson M, Vrana J, Jones K, Grotewold L, Smith A (2007) Nanog safeguards pluripotency and mediates germline development. Nature 450: 1230-1234

Chambers I, Tomlinson SR (2009) The transcriptional foundation of pluripotency. Development 136: 2311-2322

Chan SS, Shi X, Toyama A, Arpke RW, Dandapat A, lacovino M, Kang J, Le G, Hagen HR, Garry DJ, Kyba M (2013) Mesp1 patterns mesoderm into cardiac, hematopoietic, or skeletal myogenic progenitors in a contextdependent manner. Cell Stem Cell 12: 587-601

Chen PB, Hung JH, Hickman TL, Coles AH, Carey JF, Weng Z, Chu F, Fazzio TC (2013) Hdac6 regulates Tip60-p400 function in stem cells. Elife 2: e01557

Cheng X, Huber TL, Chen VC, Gadue P, Keller GM (2008) Numb mediates the interaction between Wnt and Notch to modulate primitive erythropoietic specification from the hemangioblast. Development 135: 3447-3458

DeVeale B, Brokhman I, Mohseni P, Babak T, Yoon C, Lin A, Onishi K, Tomilin A, Pevny L, Zandstra PW, Nagy A, van der Kooy D (2013) Oct4 is required $\sim$ E7.5 for proliferation in the primitive streak. PLoS Genet 9: e1003957

Diaz-Diaz C, Fernandez de Manuel L, Jimenez-Carretero D, Montoya MC, Claveria C, Torres M (2017) Pluripotency surveillance by Myc-driven competitive elimination of differentiating cells. Deu Cell 42:585-599.e4

Dore LC, Crispino JD (2011) Transcription factor networks in erythroid cell and megakaryocyte development. Blood 118: 231-239

Fehling HJ, Lacaud G, Kubo A, Kennedy M, Robertson S, Keller G, Kouskoff V (2003) Tracking mesoderm induction and its specification to the hemangioblast during embryonic stem cell differentiation. Development 130: $4217-4227$

Fraser ST, Isern J, Baron MH (2007) Maturation and enucleation of primitive erythroblasts during mouse embryogenesis is accompanied by changes in cell-surface antigen expression. Blood 109: 343-352

Gentek R, Ghigo C, Hoeffel G, Bulle MJ, Msallam R, Gautier G, Launay P, Chen J, Ginhoux F, Bajenoff M (2018) Hemogenic endothelial fate mapping reveals dual developmental origin of mast cells. Immunity 48 : $1160-1171.05$

Hall MA, Slater NJ, Begley CG, Salmon JM, Van Stekelenburg LJ, McCormack MP, Jane SM, Curtis DJ (2005) Functional but abnormal adult erythropoiesis in the absence of the stem cell leukemia gene. Mol Cell Biol 25: $6355-6362$

Hart AH, Hartley L, Ibrahim M, Robb L (2004) Identification, cloning and expression analysis of the pluripotency promoting Nanog genes in mouse and human. Deu Dyn 230: 187-198

Hayashi K, Ohta H, Kurimoto K, Aramaki S, Saitou M (2011) Reconstitution of the mouse germ cell specification pathway in culture by pluripotent stem cells. Cell 146: 519-532

Huber TL, Kouskoff V, Fehling HJ, Palis J, Keller G (2004) Haemangioblast commitment is initiated in the primitive streak of the mouse embryo. Nature 432: 625-630
Irion S, Clarke RL, Luche H, Kim I, Morrison SJ, Fehling HJ, Keller GM (2010) Temporal specification of blood progenitors from mouse embryonic stem cells and induced pluripotent stem cells. Development 137: 2829-2839

Isern J, He Z, Fraser ST, Nowotschin S, Ferrer-Vaquer A, Moore R, Hadjantonakis AK, Schulz V, Tuck D, Gallagher PG, Baron MH (2011) Single-lineage transcriptome analysis reveals key regulatory pathways in primitive erythroid progenitors in the mouse embryo. Blood 117: 4924-4934

Iwasaki H, Mizuno S, Arinobu Y, Ozawa H, Mori Y, Shigematsu H, Takatsu K, Tenen DG, Akashi $\mathrm{K}$ (2006) The order of expression of transcription factors directs hierarchical specification of hematopoietic lineages. Genes Deu 20: 3010-3021

Jagannathan-Bogdan M, Zon LI (2013) Hematopoiesis. Development 140: $2463-2467$

Kaufman DS (2009) Toward clinical therapies using hematopoietic cells derived from human pluripotent stem cells. Blood 114: 3513-3523

Kinder SJ, Tsang TE, Quinlan CA, Hadjantonakis AK, Nagy A, Tam PP (1999) The orderly allocation of mesodermal cells to the extraembryonic structures and the anteroposterior axis during gastrulation of the mouse embryo. Development 126: $4691-4701$

Kingsley PD, Greenfest-Allen E, Frame JM, Bushnell TP, Malik J, McGrath KE, Stoeckert CJ, Palis J (2013) Ontogeny of erythroid gene expression. Blood 121: e5-e13

Kohler EE, Cowan CE, Chatterjee I, Malik AB, Wary KK (2011) NANOG induction of fetal liver kinase-1 (FLK1) transcription regulates endothelial cell proliferation and angiogenesis. Blood 117: 1761-1769

Kong KY, Williamson EA, Rogers JH, Tran T, Hromas R, Dahl R (2009) Expression of $\mathrm{SCl}$ in mesoderm rescues hematopoiesis in the absence of Oct-4. Blood 114: 60-63

Kuleshov MV, Jones MR, Rouillard AD, Fernandez NF, Duan Q, Wang Z, Koplev S, Jenkins SL, Jagodnik KM, Lachmann A, McDermott MG, Monteiro CD, Gundersen CW, Ma'ayan A (2016) Enrichr: a comprehensive gene set enrichment analysis web server 2016 update. Nucleic Acids Res 44: W90-7

Kuvardina ON, Herglotz J, Kolodziej S, Kohrs N, Herkt S, Wojcik B, Oellerich T, Corso J, Behrens K, Kumar A, Hussong H, Urlaub H, Koch J, Serve H, Bonig H, Stocking C, Rieger MA, Lausen J (2015) RUNX1 represses the erythroid gene expression program during megakaryocytic differentiation. Blood 125 $3570-3579$

Lawson KA, Meneses JJ, Pedersen RA (1991) Clonal analysis of epiblast fate during germ layer formation in the mouse embryo. Development 113 : 891-911

Lesinski DA, Heinz N, Pilat-Carotta S, Rudolph C, Jacobs R, Schlegelberger B, Klump H, Schiedlmeier B (2012) Serum- and stromal cell-free hypoxic generation of embryonic stem cell-derived hematopoietic cells in vitro, capable of multilineage repopulation of immunocompetent mice. Stem Cells Transl Med 1: 581-591

Mimoto MS, Kwon S, Green YS, Goldman D, Christian JL (2015) GATA2 regulates Wnt signaling to promote primitive red blood cell fate. Deu Biol 407: 1-11

Mitjavila-Garcia MT, Cailleret M, Godin I, Nogueira MM, Cohen-Solal K, Schiavon V, Lecluse Y, Le Pesteur F, Lagrue AH, Vainchenker W (2002) Expression of CD41 on hematopoietic progenitors derived from embryonic hematopoietic cells. Development 129: 2003-2013

Mitsui K, Tokuzawa Y, Itoh H, Segawa K, Murakami M, Takahashi K, Maruyama M, Maeda M, Yamanaka S (2003) The homeoprotein Nanog is required for maintenance of pluripotency in mouse epiblast and ES cells. Cell 113: $631-642$ 
Morgani S, Nichols J, Hadjantonakis AK (2017) The many faces of Pluripotency: in vitro adaptations of a continuum of in vivo states. BMC Deu Biol 17: 7

Murakami K, Gunesdogan U, Zylicz JJ, Tang WWC, Sengupta R, Kobayashi T, Kim S, Butler R, Dietmann S, Surani MA (2016) NANOG alone induces germ cells in primed epiblast in vitro by activation of enhancers. Nature 529: $403-407$

Myers CT, Krieg PA (2013) BMP-mediated specification of the erythroid lineage suppresses endothelial development in blood island precursors. Blood 122: 3929 - 3939

Nagy A, Gertsensten M, Vintersten K, Behringer R (2003) Manipulating the mouse embryo: a laboratory manual. Cold Spring Harbor, NY: Cold Spring Harbor Laboratory Press

Nakamura T, Okamoto I, Sasaki K, Yabuta Y, Iwatani C, Tsuchiya H, Seita Y, Nakamura S, Yamamoto T, Saitou M (2016) A developmental coordinate of pluripotency among mice, monkeys and humans. Nature 537: 57-62

Ohmori S, Moriguchi T, Noguchi Y, Ikeda M, Kobayashi K, Tomaru N, Ishijima Y, Ohneda O, Yamamoto M, Ohneda K (2015) GATA2 is critical for the maintenance of cellular identity in differentiated mast cells derived from mouse bone marrow. Blood 125: 3306-3315

Osorno R, Tsakiridis A, Wong F, Cambray N, Economou C, Wilkie R, Blin G, Scotting PJ, Chambers I, Wilson V (2012) The developmental dismantling of pluripotency is reversed by ectopic Oct4 expression. Development 139: $2288-2298$

Padron-Barthe L, Temino S, Villa del Campo C, Carramolino L, Isern J, Torres M (2014) Clonal analysis identifies hemogenic endothelium as the source of the blood-endothelial common lineage in the mouse embryo. Blood 124: $2523-2532$

Palis J, Robertson S, Kennedy M, Wall C, Keller G (1999) Development of erythroid and myeloid progenitors in the yolk sac and embryo proper of the mouse. Development 126: 5073-5084

Paralkar VR, Mishra T, Luan J, Yao Y, Kossenkov AV, Anderson SM, Dunagin M, Pimkin M, Gore M, Sun D, Konuthula N, Raj A, An X, Mohandas N, Bodine DM, Hardison RC, Weiss MJ (2014) Lineage and species-specific long noncoding RNAs during erythro-megakaryocytic development. Blood 123: $1927-1937$

Pfister S, Steiner KA, Tam PP (2007) Gene expression pattern and progression of embryogenesis in the immediate post-implantation period of mouse development. Gene Expr Patterns 7: 558-573

Piazzolla D, Palla AR, Pantoja C, Canamero M, de Castro IP, Ortega S, GomezLopez G, Dominguez O, Megias D, Roncador G, Luque-Garcia JL, Fernandez-Tresguerres B, Fernandez AF, Fraga MF, Rodriguez-Justo M, Manzanares M, Sanchez-Carbayo M, Garcia-Pedrero JM, Rodrigo JP, Malumbres $M$ et al (2014) Lineage-restricted function of the pluripotency factor NANOG in stratified epithelia. Nat Commun 5: 4226

Porcher C, Chagraoui H, Kristiansen MS (2017) SCL/TAL1: a multifaceted regulator from blood development to disease. Blood 129: 2051-2060

Ran FA, Hsu PD, Wright J, Agarwala V, Scott DA, Zhang F (2013) Genome engineering using the CRISPR-Cas9 system. Nat Protoc 8: 2281-2308

Robertson SM, Kennedy M, Shannon JM, Keller G (2000) A transitional stage in the commitment of mesoderm to hematopoiesis requiring the transcription factor SCL/tal-1. Development 127: 2447-2459
Salmon JM, Slater NJ, Hall MA, McCormack MP, Nutt SL, Jane SM, Curtis DJ (2007) Aberrant mast-cell differentiation in mice lacking the stem-cell leukemia gene. Blood 110: 3573-3581

Scialdone A, Tanaka Y, Jawaid W, Moignard V, Wilson NK, Macaulay IC, Marioni JC, Gottgens B (2016) Resolving early mesoderm diversification through single-cell expression profiling. Nature 535: 289-293

Shalaby F, Rossant J, Yamaguchi TP, Gertsenstein M, Wu XF, Breitman ML, Schuh AC (1995) Failure of blood-island formation and vasculogenesis in Flk-1-deficient mice. Nature 376: 62-66

Socolovsky M, Nam H, Fleming MD, Haase VH, Brugnara C, Lodish HF (2001) Ineffective erythropoiesis in Stat5a(-/-)5b(-/-) mice due to decreased survival of early erythroblasts. Blood 98: $3261-3273$

Sroczynska P, Lancrin C, Pearson S, Kouskoff V, Lacaud G (2009) In vitro differentiation of mouse embryonic stem cells as a model of early hematopoietic development. Methods Mol Biol 538: 317-334

Takahashi K, Mitsui K, Yamanaka S (2003) Role of ERas in promoting tumour-like properties in mouse embryonic stem cells. Nature 423 : $541-545$

Tanaka Y, Era T, Nishikawa S, Kawamata S (2007) Forced expression of Nanog in hematopoietic stem cells results in a gammadeltaT-cell disorder. Blood 110: 107-115

Teo AK, Arnold SJ, Trotter MW, Brown S, Ang LT, Chng Z, Robertson EJ, Dunn NR, Vallier $L$ (2011) Pluripotency factors regulate definitive endoderm specification through eomesodermin. Genes Deu 25: $238-250$

Wilkinson DG, Bailes JA, Champion JE, McMahon AP (1987) A molecular analysis of mouse development from 8 to 10 days post coitum detects changes only in embryonic globin expression. Development 99: 493-500

Xenopoulos P, Kang M, Puliafito A, Di Talia S, Hadjantonakis AK (2015) Heterogeneities in Nanog expression drive stable commitment to pluripotency in the mouse blastocyst. Cell Rep 10: 1508-1520

Yamaguchi S, Kurimoto K, Yabuta Y, Sasaki H, Nakatsuji N, Saitou M, Tada $\mathrm{T}$ (2009) Conditional knockdown of Nanog induces apoptotic cell death in mouse migrating primordial germ cells. Development 136 : $4011-4020$

Yeom YI, Fuhrmann G, Ovitt CE, Brehm A, Ohbo K, Gross M, Hubner K, Scholer HR (1996) Germline regulatory element of Oct-4 specific for the totipotent cycle of embryonal cells. Development 122: $881-894$

Yokomizo T, Hasegawa K, Ishitobi H, Osato M, Ema M, Ito Y, Yamamoto M, Takahashi S (2008) Runx1 is involved in primitive erythropoiesis in the mouse. Blood 111: 4075 - 4080

Zhang J, Socolovsky M, Gross AW, Lodish HF (2003) Role of Ras signaling in erythroid differentiation of mouse fetal liver cells: functional analysis by a flow cytometry-based novel culture system. Blood 102: 3938 - 3946

Zhang M, Leitch HG, Tang WWC, Festuccia N, Hall-Ponsele E, Nichols J, Surani MA, Smith A, Chambers I (2018) Esrrb complementation rescues development of Nanog-null germ cells. Cell Rep 22: 332-339

License: This is an open access article under the terms of the Creative Commons Attribution 4.0 License, which permits use, distribution and reproduction in any medium, provided the original work is properly cited. 


\section{Immunity}

\section{A Neutrophil Timer Coordinates Immune Defense and Vascular Protection}

\section{Graphical Abstract}

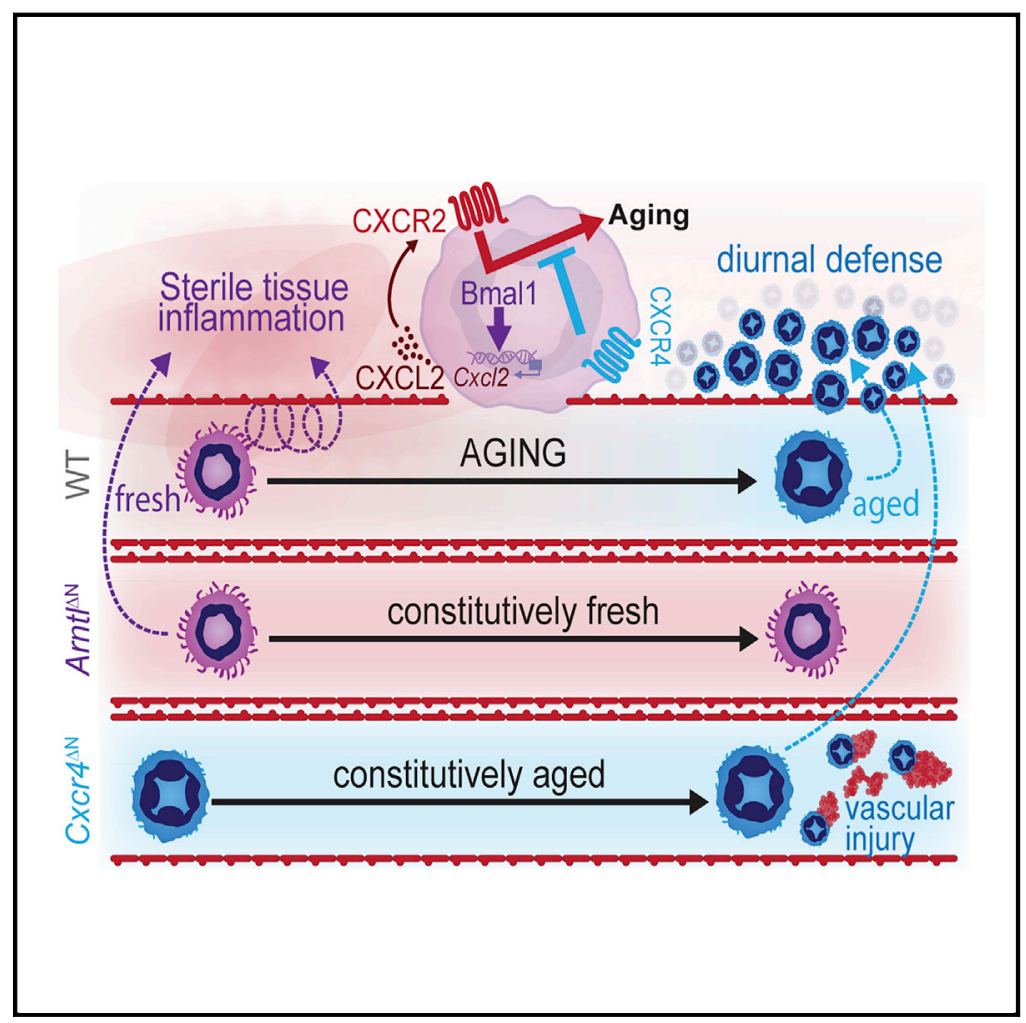

Highlights

- Neutrophil aging is an intrinsically driven, bona fide circadian process

- Bmal1 and CXCR2 induce neutrophil aging, whereas CXCR4 antagonizes it

- Diurnal aging critically dictates how and when neutrophils migrate into tissues

- Aging favors neutrophil clearance, thereby protecting the cardiovascular system

\section{Authors}

José M. Adrover, Carlos del Fresno, Georgiana Crainiciuc, ...,

María A. Moro, Borja Ibáñez, Andrés Hidalgo

\section{Correspondence}

ahidalgo@cnic.es

\section{In Brief}

Neutrophils display circadian oscillations in numbers and phenotype in the circulation. Adrover and colleagues now identify the molecular regulators of neutrophil aging and show that genetic disruption of this process has major consequences in immune cell trafficking, anti-microbial defense, and vascular health. 


\title{
A Neutrophil Timer Coordinates Immune Defense and Vascular Protection
}

\author{
José M. Adrover, ${ }^{1}$ Carlos del Fresno, ${ }^{2}$ Georgiana Crainiciuc, ${ }^{1}$ Maria Isabel Cuartero,,${ }^{3,4}$ María Casanova-Acebes, ${ }^{1,16}$ \\ Linnea A. Weiss, ${ }^{1,17}$ Hector Huerga-Encabo, ${ }^{5}$ Carlos Silvestre-Roig, ${ }^{6,7}$ Jan Rossaint, ${ }^{8}$ Itziar Cossío, ${ }^{1}$ \\ Ana V. Lechuga-Vieco, ${ }^{2}$ Jaime García-Prieto, ${ }^{2}$ Mónica Gómez-Parrizas, ${ }^{2}$ Juan A. Quintana, ${ }^{1}$ Ivan Ballesteros, ${ }^{1}$ \\ Sandra Martin-Salamanca, ${ }^{1}$ Alejandra Aroca-Crevillen, ${ }^{1}$ Shu Zhen Chong, ${ }^{9}$ Maximilien Evrard, ${ }^{9}$ Karl Balabanian, ${ }^{10}$ \\ Jorge López, ${ }^{11}$ Kiril Bidzhekov, ${ }^{6}$ Françoise Bachelerie, ${ }^{10}$ Francisco Abad-Santos, ${ }^{12}$ Cecilia Muñoz-Calleja, ${ }^{11}$ \\ Alexander Zarbock, ${ }^{8}$ Oliver Soehnlein, ${ }^{6,7}$ Christian Weber, ${ }^{6,13}$ Lai Guan Ng, ${ }^{9}$ Cristina Lopez-Rodriguez, ${ }^{5}$ David Sancho, ${ }^{2}$ \\ María A. Moro, ${ }^{3,4}$ Borja Ibáñez, 2,14,15 and Andrés Hidalgo ${ }^{1,6,18, *}$ \\ ${ }^{1}$ Area of Developmental and Cell Biology, Centro Nacional de Investigaciones Cardiovasculares Carlos III (CNIC), Madrid, Spain \\ ${ }^{2}$ Area of Myocardial Pathophysiology, Centro Nacional de Investigaciones Cardiovasculares Carlos III (CNIC), Madrid, Spain \\ 3Unidad de Investigación Neurovascular, Department of Pharmacology, Faculty of Medicine, Universidad Complutense \\ ${ }^{4}$ Instituto de Investigación Hospital 12 de Octubre (i+12), Madrid, Spain \\ ${ }^{5}$ Immunology Unit, Department of Experimental and Health Sciences, Pompeu Fabra University, Barcelona \\ ${ }^{6}$ Institute for Cardiovascular Prevention (IPEK), Ludwig-Maximillians-Universität München \\ ${ }^{7}$ German Centre for Cardiovascular Research (DZHK), partner site Munich Heart Alliance, Munich, Germany \\ ${ }^{8}$ Department of Anesthesiology, Intensive Care, and Pain Medicine, University of Münster, Germany \\ 9Singapore Immunology Network (SlgN), Agency for Science, Technology and Research (A*STAR), Biopolis, Singapore \\ ${ }^{10}$ Inserm Unité Mixte de Recherche (UMR) S996, Université Paris-Sud, Laboratory of Excellence in Research on Medication and Innovative \\ Therapeutics, Clamart, France \\ ${ }^{11}$ Department of Immunology, Instituto de Investigación Sanitaria Princesa, Hospital Universitario de La Princesa, Madrid, Spain \\ ${ }^{12}$ Department of Clinical Pharmacology, Instituto Teófilo Hernando, Hospital Universitario de La Princesa, Instituto de Investigación Sanitaria \\ Princesa, Madrid, Spain \\ ${ }^{13}$ Department of Biochemistry, Cardiovascular Research Institute Maastricht (CARIM), Maastricht University, Maastricht, the Netherlands \\ ${ }^{14} \mathrm{CIBER}$ de Enfermedades Cardiovasculares (CIBERCV), Madrid, Spain \\ ${ }^{15}$ Department of Cardiology, Instituto de Investigación Sanitaria (IIS)-Fundación Jiménez Díaz, Madrid, Spain \\ ${ }^{16}$ Present address: Tisch Cancer Institute, Mount Sinai School of Medicine, New York City, New York, USA \\ 17Present address: Centro Nacional de Biotecnología, Madrid, Spain \\ ${ }^{18}$ Lead Contact \\ *Correspondence: ahidalgo@cnic.es \\ https://doi.org/10.1016/j.immuni.2019.01.002
}

\section{SUMMARY}

Neutrophils eliminate pathogens efficiently but can inflict severe damage to the host if they over-activate within blood vessels. It is unclear how immunity solves the dilemma of mounting an efficient anti-microbial defense while preserving vascular health. Here, we identify a neutrophil-intrinsic program that enabled both. The gene Bmal1 regulated expression of the chemokine CXCL2 to induce chemokine receptor CXCR2-dependent diurnal changes in the transcriptional and migratory properties of circulating neutrophils. These diurnal alterations, referred to as neutrophil aging, were antagonized by CXCR4 (C-X-C chemokine receptor type 4) and regulated the outer topology of neutrophils to favor homeostatic egress from blood vessels at night, resulting in boosted anti-microbial activity in tissues. Mice engineered for constitutive neutrophil aging became resistant to infection, but the persistence of intravascular aged neutrophils predisposed them to thrombo-inflammation and death. Thus, diurnal compartmentalization of neu- trophils, driven by an internal timer, coordinates immune defense and vascular protection.

\section{INTRODUCTION}

The capacity of neutrophils not only to kill pathogens but also to inflict severe damage to tissues suggests the existence of a protective mechanism that balances these opposing functions. Both anti-microbial immunity and vascular inflammation are known to follow circadian patterns (Man et al., 2016; Muller et al., 1989; Scheiermann et al., 2013), suggesting that such mechanisms may be temporally regulated. The nature of this mechanism, however, remains enigmatic.

While studying neutrophils in the steady state, we previously identified a natural phenotypic shift of circulating neutrophils that followed a strict diurnal regime (Casanova-Acebes et al., 2013). Neutrophils released from the bone marrow display high CD62L that is progressively reduced during the day, while surface CXCR4 (C-X-C chemokine receptor type 4) increases prior to their natural egress from blood, a process referred to as clearance (Casanova-Acebes et al., 2013). This process of neutrophil aging has been proposed to be regulated by the gut microbiota and to favor a pro-inflammatory phenotype that predisposes to vascular inflammation (Zhang et al., 2015). 
Contrasting with this model of extrinsically driven neutrophil aging, studies have shown that intrinsic programs controlled by the molecular clock also regulate immune cell properties (Druzd et al., 2017; Nguyen et al., 2013; Silver et al., 2012). Because the mechanisms regulating aging and its physiological consequences remain uncertain, we explored whether neutrophils are endowed with an intrinsic program that controls diurnal aging, tunes their anti-microbial functions, and limits vascular inflammation.

In transcriptome analyses of circulating neutrophils performed at different times, we found regulation of clock-related genes and the CXCR2 signaling pathway. Bmal1 (brain and muscle aryl hydrocarbon receptor nuclear translocator [ARNT]-like 1; encoded by Arnt/) regulated expression of CXCL2 (chemokine [C-X-C motif] ligand 2), a CXCR2 ligand that controlled neutrophil aging in a cell-autonomous manner. Deletion of Arntl or Cxcr2 from neutrophils prevented phenotypic aging, whereas deletion of $\mathrm{Cxcr} 4$, a negative regulator of CXCR2 signaling, resulted in unrestrained aging. Neutrophil aging disrupted cytoskeletal integrity to specifically prevent rolling and accumulation in inflamed areas without affecting homeostatic migration into naive tissues at night. In turn, this temporal regulation of trafficking regulated diurnal responses to infections, while at the same time removing neutrophils from the bloodstream, thereby protecting vessels from inflammatory injury. This process may underlie the circadian susceptibility of mammals to cardiovascular disease.

\section{RESULTS}

\section{A Neutrophil-Intrinsic Timer Drives Diurnal Aging}

In a small cohort of healthy volunteers, we found diurnal changes in neutrophil markers similar to those associated with neutrophil aging in mice (Casanova-Acebes et al., 2013), suggesting conservation of this phenomenon across species (Figure S1A). In healthy mice, the number of aged neutrophils in blood follows diurnal patterns with a peak at around zeitgeber time 5 (ZT5, i.e., $5 \mathrm{~h}$ after lights on), while non-aged or "fresh" neutrophils predominate at ZT13 (Casanova-Acebes et al., 2013). These diurnal patterns persisted in constant darkness and could be entrained by light shift (Figures S1B and S1C), indicating that neutrophil aging is a bona fide circadian process. To identify genetic programs that were temporally regulated in neutrophils, we compared the transcriptomes of circulating neutrophils purified from wild-type (WT) mice at these two times. We identified changes in over 1,300 genes related to pathways of inflammation, migration, and apoptosis (Figures 1A, 1B, and S1D; Table S1), which suggested modulation of these processes during the day. Given the diurnal pattern of aging, we inspected genes of the molecular clock because they are known to regulate immune rhythms (Man et al., 2016; Scheiermann et al., 2013). Expression of clock-related genes, including Arntl (encoding Bmal1) and Clock, increased at ZT5, while others, like Per2, were decreased at this time (Figures 1B, S1D, and S1E). Transcriptional analyses at multiple times of day revealed circadian oscillations for all these genes in circulating neutrophils (Figure 1C). They also demonstrated reduced expression of Sell (encoding CD62L) at ZT5 but no changes for Cxcr4 (Figure 1B). We also noticed reduced expression of $C x c r 2$, whose expression displayed diurnal patterns (Figure 1D), at ZT5 (Figure 1B). Further, CXCR2 agonists induced phenotypic changes in neutrophils that resembled those seen during natural aging, namely reductions in CXCR2 and CD62L on the cell surface (Figures 1E and S1F).

Guided by the temporal expression patterns of these genes, we predicted that Bmal1 and CXCR2 might be required for diurnal neutrophil aging. Although CXCR4 did not present transcriptional oscillations (Figure 1D), its presence on the cell surface changed diurnally (Figure 1E) (Casanova-Acebes et al., 2013), and this receptor is known to antagonize CXCR2 signaling (Martin et al., 2003), suggesting that CXCR4 might also contribute to aging. Analyses of blood from wild-type animals revealed ligands for both receptors in plasma, with oscillating amounts of CXCL12, and constitutively low amounts of CXCL2 (Figure 1F).

To formally test the possibility that these genes regulated neutrophil aging, we generated mice with neutrophil-specific deficiency in Arntl, Cxcr2, or Cxcr4 (herein referred to as $A r n t I^{\Delta \mathrm{N}}$, $C x c r 2^{\Delta N}$, and $C x c r 4^{\Delta N}$ ) by using the $h M R P 8^{c r e}$ driver line, which resulted in robust depletion of the receptors from the surface of neutrophils (Figures S2A and S2B). Immunoblot analysis of the Bmal1 protein confirmed efficient depletion in $\left.A r n t\right|^{\Delta \mathrm{N}}$ neutrophils and revealed natural reductions of this protein at ZT13 in wild-type neutrophils and unchanged amounts in $C x c r 2^{\Delta \mathrm{N}}$ and $\mathrm{CxCr}^{\Delta \mathrm{N}}$ neutrophils (Figure S2C). We then assessed surface $\mathrm{CD62L}$, a marker reduced during aging (Casanova-Acebes et al., 2013; Uhl et al., 2016; Zhang et al., 2015) (Figure 1E). To ensure that potential alterations were cell-intrinsic, we generated bone marrow transplant chimeras of wild-type and of each of the mutant donors. We found elevated CD62L in circulating neutrophils from $\left.A r n t\right|^{\Delta N}$ and $C x c r 2^{\Delta N}$ donors, suggesting disrupted aging in these mutants (Figure $2 \mathrm{~A}$ ). In contrast, $\mathrm{CxCr} 4^{\Delta \mathrm{N}}$ neutrophils were low for CD62L, which suggested constitutive aging (Figure 2A). If these alterations were caused by disruption of diurnal aging, we predicted that CD62L would not change over time. Indeed, in vivo metabolic pulse and chase of neutrophils with bromodeoxyuridine (BrdU) demonstrated that the temporal changes in CD62L seen in wild-type neutrophils were abrogated in $\mathrm{Arnt}^{\Delta \mathrm{N}}$ and $\mathrm{CxCr}^{\Delta \mathrm{N}}$ mutants (Figure 2B). To further confirm that these genes regulated the natural dynamics of aging, we measured CD62L through full diurnal cycles in all mutant mice. Although surface CD62L exhibited diurnal oscillations in wildtype neutrophils, all three mutants presented disrupted patterns, and $A r n t^{\Delta \mathrm{N}}$ mutants showed a complete loss of rhythmicity (Figure 2C). Accordingly, surface CXCR4 also lost diurnal oscillations in $\mathrm{Arnt}^{\Delta \mathrm{N}}$ neutrophils (data not shown).

To dissect the antagonistic role of CXCR4 in aging, we pretreated wild-type neutrophils with CXCL12, the main ligand for CXCR4, before exposing them to a CXCR2 agonist. Given the constitutive aging of $\mathrm{CxCr}^{4 \mathrm{~N}}$ neutrophils, we hypothesized that stimulation through CXCR4 might prevent CXCR2-dependent responses. Indeed, CXCL12 blunted both the reductions in CD62L and the chemotaxis elicited through CXCR2 (Figure S2D). In addition, neutrophils expressing Cxcr4 ${ }^{\text {WHIM }}$, a hyper-signaling variant of CXCR4 (Balabanian et al., 2012), displayed constitutive elevations in CD62L (Figure S2E). Combined, these data indicated that Bmal1 and CXCR2 promote diurnal aging and that CXCR4 prevents it. 

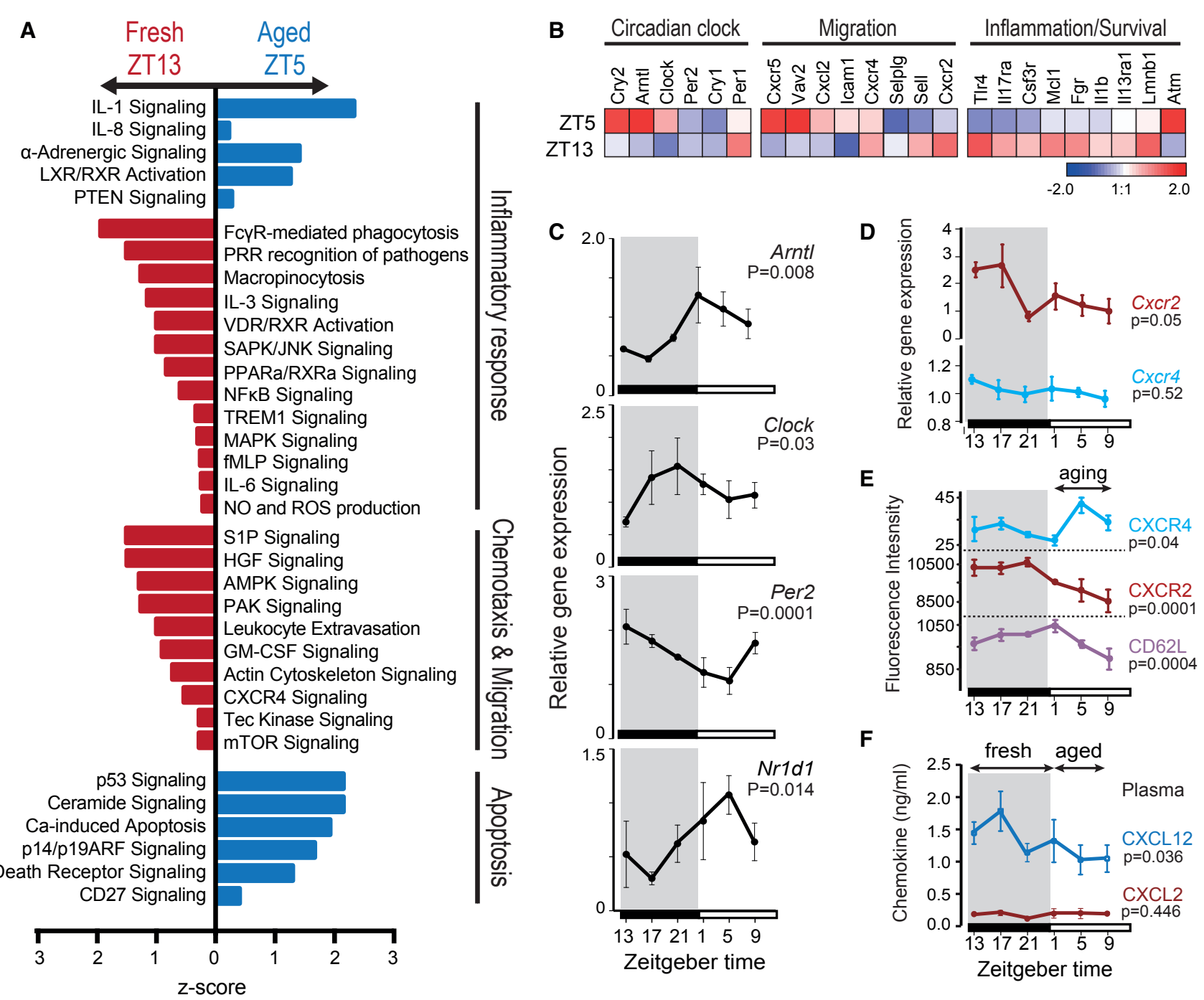

Figure 1. Cell-Intrinsic Rhythms in Circulating Neutrophils, Related to Figure S1

(A) Molecular pathways differentially regulated in circulating wild-type neutrophils at ZT5 versus ZT13. Comparisons are presented as $Z$ score values.

(B) Heatmap of selected genes at ZT5 versus ZT13, including genes of the circadian clock and genes encoding proteins related to migration and inflammation. The color scale indicates fold changes of expression for each gene.

(C) Diurnal expression of the indicated clock genes in neutrophils isolated from the circulation of wild-type mice at the indicated zeitgeber times. Shaded areas represent night; $\mathrm{n}=2-6$ mice per time point.

(D) Diurnal expression of $C x c r 2$ and $C x c r 4$ in circulating wild-type neutrophils at the indicated times; $n=2-6$ mice per time point. The diurnal curves are repeated (dashed lines) to better appreciate the pattern.

(E) Surface CXCR4, CXCR2, and CD62L measured at different diurnal times by flow cytometry; $n=5$ mice. Highlighted is the time of aging (ZT1-ZT9), when CD62L and CXCR2 go down and CXCR4 goes up. The diurnal curves are repeated (dashed lines) to better appreciate the pattern.

(F) Diurnal changes of CXCL2 and CXCL12 in the plasma of wild-type mice; $n=5-10$ mice per time point. The diurnal curves are repeated (dashed lines) to better appreciate the pattern.

All values are presented as mean \pm SEM. $p$ values were determined by amplitude versus zero t test analyses (see Quantification and Statistical Analysis) to test for circadian behavior (C-F).

\section{Aging-Driven Transcriptional Programs}

Having identified Bmal1, CXCR2, and CXCR4 as intrinsic regulators of diurnal aging, we used the neutrophil-specific mutant mice as models to examine how programmed diurnal aging impacted neutrophil physiology. We first performed transcriptomic analyses of blood neutrophils extracted from $A r n t l^{\Delta \mathrm{N}}$, $\mathrm{Cxcr}^{\Delta \mathrm{N}}$, and $\mathrm{Cxcr} 4^{\Delta \mathrm{N}}$ mice at ZT5 and compared them with the profiles of wild-type neutrophils at ZT5 and ZT13. Principal component analyses of the five groups revealed that neutrophils that displayed a CD62 $\mathrm{L}^{\mathrm{HI}}$ fresh phenotype (including wild-type at ZT13, Arntl $^{\Delta \mathrm{N}}$, and $C x c r 2^{\Delta \mathrm{N}}$ ) clustered together, whereas those that shared an aged phenotype (wild-type at ZT5 and $C x c r 4^{\Delta N}$ ) separated from the fresh cluster (Figure 2D), and many genes were differentially regulated among the fresh and aged groups (Figure S2F). Consistent with a role in diurnal aging, when we contrasted the transcriptomes of wild-type and mutant mice at 
A

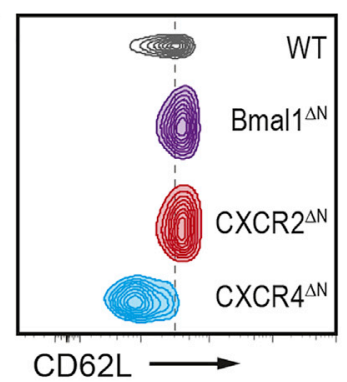

C

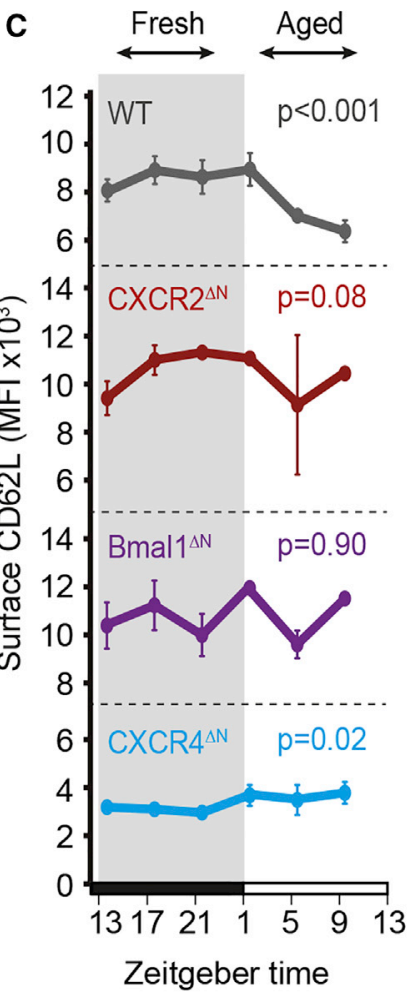

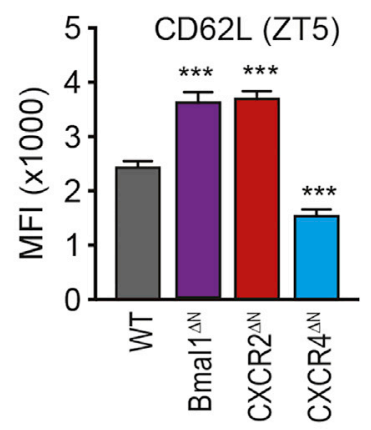

D

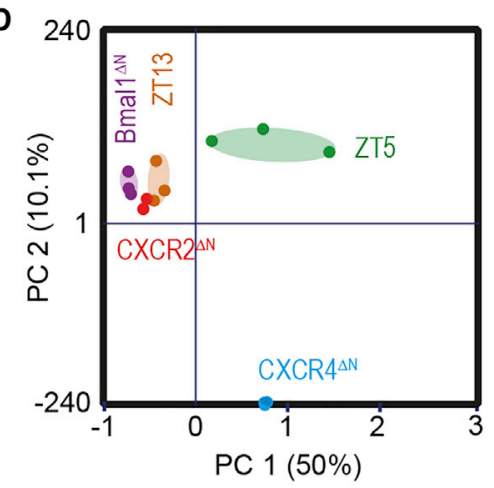

E

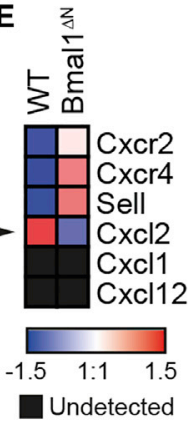

PC $1(50 \%)$
B

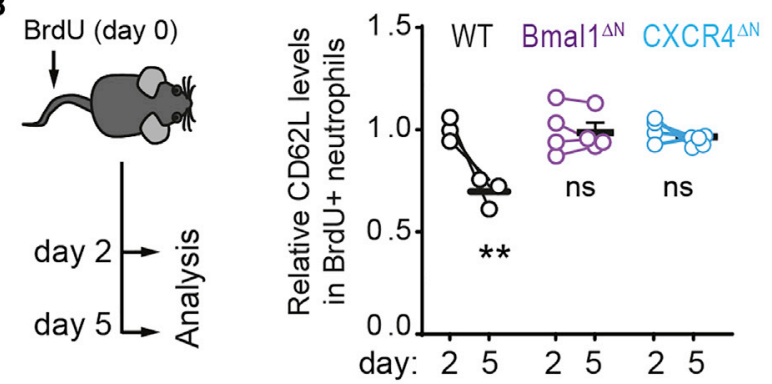

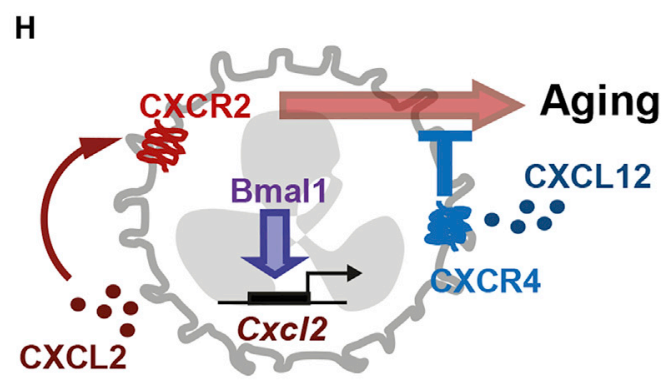

ChIP
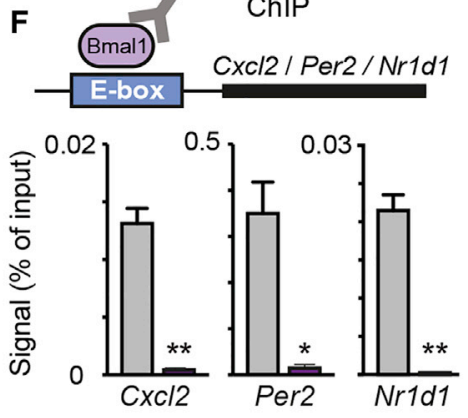

$\square$ WT $\square$ Bmal1 $1^{\Delta \mathrm{N}}$
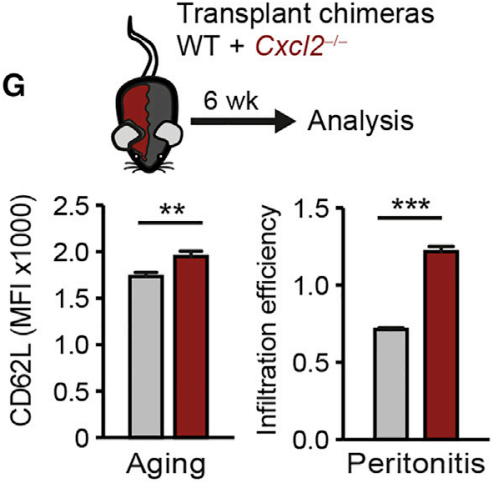

$\square$ Wild-type $\square \mathrm{Cxcl2}-1$

Figure 2. Bma1, CXCR2, and CXCR4 Form a Diurnal Timer in Neutrophils, Related to Figure S2

(A) Surface expression of CD62L in wild-type and mutant neutrophils at ZT5. Cytometric data are from transplant chimeras of wild-type with each mutant. Bars at right show median fluorescence intensity; $\mathrm{n}=14-30$ mice per group.

(B) In vivo BrdU labeling followed by analysis of CD62L in BrdU+ cells 2 and 5 days after injection. Note that as labeled neutrophils enter the bloodstream, they lose CD62L over time in WT mice, but not in $A r n t /^{\Delta \mathrm{N}}$ and $C x c r 4^{\Delta \mathrm{N}}$ mutants. Data are normalized to day 2 in each group; $\mathrm{n}=3-5$ mice per group.

(C) Diurnal surface CD62L in circulating neutrophils in wild-type and mutant mice, as determined by flow cytometry. The times when neutrophils are phenotypically fresh or aged are indicated on top; $n=3-10$ mice per time point. $p$ values were determined by amplitude versus zero $t$ test analyses.

(D) Principal component analyses of differentially expressed genes in circulating neutrophils from wild-type neutrophils at ZT5 and ZT13, and $A r n t t^{\Delta \mathrm{N}}, C x c r 2^{\Delta \mathrm{N}}$, and $\mathrm{CxCr} 4^{\Delta \mathrm{N}}$ mutants at ZT5.

(E-G) Bmal1-regulated expression of CXCL2 controls aging. (E) Heatmap showing differential expression of aging-related genes in wild-type and Arnt ${ }^{\Delta \mathrm{N}}$ neutrophils. Expression of $\mathrm{Cxcl} 1$ and $\mathrm{Cxcl} 12$ was undetectable. Data are from triplicate samples of each group obtained at ZT5. (F) Experimental design and ChIP analyses of Bmal1 binding to E-box-containing promoter regions of $\mathrm{CxCl}$, Per2, and $\mathrm{Nr} 1 \mathrm{~d} 1$ in wild-type and Arnt $/^{\Delta \mathrm{N}}$ neutrophils. (G) Experimental setup and phenotype of $\mathrm{CxCl}^{-/-}$neutrophils in transplantation chimeras. $\mathrm{CxCl}{ }^{-1-}$ neutrophils display elevated $\mathrm{CD} 62 \mathrm{~L}$ expression and enhanced migration to zymosantreated peritoneum, both of which are consistent with disrupted aging.

(H) Model of neutrophil aging: CXCR2 signaling drives aging, whereas CXCR4 antagonizes these signals and prevents it. Bmal1 regulates CxCl2 expression to promote autocrine aging.

Except where indicated, all values are mean $\pm \mathrm{SEM}$. ${ }^{\star \star} \mathrm{p}<0.01 ;{ }^{* \star *} \mathrm{p}<0.001$ as determined by one-way ANOVA (A), upaired $\mathrm{t}$ test $(\mathrm{B}$ and $\mathrm{F})$, or paired $\mathrm{t}$ test (D and $G)$. 
both ZT5 and ZT13, we found that the diurnal changes in gene expression of wild-type neutrophils were absent or blunted in Arntl $^{\Delta \mathrm{N}}$ and $\mathrm{Cxcr}_{4}{ }^{\Delta \mathrm{N}}$ neutrophils (Figure S2G). These findings aligned with the phenotypic data (Figures $2 \mathrm{~A}$ and $2 \mathrm{C}$ ) and define diurnal aging as a global transcriptional program of circulating neutrophils that occurs naturally during the day and that could be recapitulated in the mutant mice.

We next focused on genetic programs that consistently changed when independently interrogating the effect of time (ZT5 versus ZT13) and genotype (Arnt ${ }^{\Delta \mathrm{N}}$ versus $\left.\mathrm{Cxcr} 4^{\Delta \mathrm{N}}\right)$. We noticed prominent regulation of the IL-8 (interleukin 8) signaling pathway (a ligand for human CXCR2; Figure $\mathrm{S} 2 \mathrm{H}$ ), which was in line with our previous results and suggested engagement of CXCR2 during aging. Analyses of our sequencing data revealed that among aging-related genes, only expression of $\mathrm{CxCl} 2$, a CXCR2 ligand expressed by neutrophils (Li et al., 2016), was reduced in $\mathrm{Arnt}^{\Delta \mathrm{N}}$ relative to wild-type neutrophils (Figure 2E), suggesting that this chemokine could provide a link between Bmal1 and CXCR2 during aging. Indeed, chromatin immunoprecipitation (ChIP) assays with wild-type neutrophils revealed that Bmal1 bound predicted E-box elements in the promoter regions not only of known target clock genes (Per2 and Nr1d1), but also of the $\mathrm{Cxcl} 2$ gene (Figure 2F). Further analysis of bone marrow chimeras from wild-type and $\mathrm{CxCl} 2^{-1-}$ donors confirmed that this chemokine was required for neutrophil aging in a cellautonomous manner (Figure 2G). Consistently, in vivo blockade of CXCL2, but not of another CXCR2 ligand (CXCL1), blunted the aging phenotype of wild-type neutrophils without affecting Cxcr2 ${ }^{\Delta N}$ mutants (Figure S2l). These findings explained the defective aging seen in $\left.A r n t\right|^{\Delta \mathrm{N}}$ neutrophils (Figure 2A) and revealed that Bmal1-driven production of CXCL2 controlled neutrophil aging through autocrine CXCR2 signaling.

To independently assess the cell-intrinsic nature of aging, we tracked the kinetics of fresh neutrophils transferred into recipient mice at ZT5 (the time of maximal aging). Although host mice became enriched in fresh neutrophils over time, the transferred neutrophils became progressively aged (Figure S2J), further supporting that neutrophil aging is intrinsically driven.

Combined, these findings supported a model whereby diurnal neutrophil aging is driven by Bmal1 through regulation of $\mathrm{CxCl} 2$ expression. This chemokine in turn signals through CXCR2 to induce phenotypic aging, whereas CXCR4 antagonizes these signals and prevents aging (Figure $2 \mathrm{H}$ ).

\section{Aging-Regulated Migration of Neutrophils}

The transcriptomic analyses additionally identified pathways that changed significantly (-log ( $p$ value) $>1.3$ ), including cytokine signaling, activation of nuclear receptors, toll-like receptor signaling, leukocyte extravasation, and actin cytoskeleton signaling (Figures $1 \mathrm{~A}$ and $\mathrm{S} 2 \mathrm{H}$ ). Because many of these pathways ultimately regulate the migration of neutrophils into tissues to exert immune functions, we investigated the in vivo trafficking patterns associated with neutrophil aging.

We considered two migratory modalities that are relevant in neutrophil physiology: migration into healthy tissues (or clearance, which follows diurnal cycles) (Casanova-Acebes et al., 2018; Scheiermann et al., 2012), and migration into inflamed tissues. We took advantage of our neutrophil-specific mouse models to exclude cell-extrinsic factors influenced by time, such as diurnal changes in adhesion molecules reported on endothelial cells (Scheiermann et al., 2012). In addition, because CXCR2 plays prominent roles in multiple homeostatic and inflammatory scenarios that may not be related to aging, we restricted our subsequent analyses to Bmal1 and CXCR4 mutants as models for fresh and aged neutrophils, respectively.

We first generated parabiotic pairs of wild-type and mutant mice to compare the migration efficiency of fresh $\left.(A r n t)^{\Delta N}\right)$ and aged $\left(C x c r 4^{\Delta N}\right)$ neutrophils relative to wild-type neutrophils in the same physiological context (Figure 3A). We found that homeostatic clearance of $\left.A r n t\right|^{\Delta \mathrm{N}}$ neutrophils into multiple tissues of wild-type partners was strongly impaired, whereas it was unaffected for Cxcr4 $4^{\Delta N}$ neutrophils (Figure 3B), indicating that neutrophil aging was required for clearance into tissues.

We next examined the migration of the aging mutant neutrophils into inflamed tissues using zymosan-induced peritonitis in the parabiotic pairs. To our surprise, we found the opposite response: enhanced migration of $\left.A r n t\right|^{\Delta \mathrm{N}}$ fresh neutrophils and reduced infiltration by $\mathrm{CxCr} 4^{\Delta \mathrm{N}}$ aged neutrophils (Figure $3 \mathrm{C}$ ). Using an independent model of constitutive aging (mice lacking endothelial selectins, Selp; Sele ${ }^{-1-}$ mice; Casanova-Acebes et al., 2013), we confirmed that aged neutrophils displayed intact clearance at steady state (Figure S3A) but impaired migration to inflamed tissues (Figure 3B). In contrast, impaired aging of neutrophils expressing the hyper-signaling Cxcr4 ${ }^{\text {WHIM }}$ mutation resulted in enhanced migration to inflamed tissues (Figure S2E). Whole-mount imaging of inflamed cremasteric muscles from transplantation chimeras confirmed the differential capacity of fresh and aged neutrophils to infiltrate inflamed tissues relative to wild-type cells (Figures 3D and $3 \mathrm{E}$ ), and this became even more prominent when comparing the migration of constitutively aged and fresh neutrophils within the same mouse (Figure 3F). Importantly, these findings aligned with enhanced inflammatory recruitment of wild-type neutrophils when they were phenotypically fresh (ZT13), and this diurnal preference was lost in $A r n t^{\Delta \mathrm{N}}$ and $\mathrm{CxCr}^{\Delta \mathrm{N}}$ mutant mice (Figure S3B). These data reveal that aging instructs a diurnal switch in the migratory preference of neutrophils, from inflammatory to homeostatic.

\section{Surface Topology and Rolling Efficiency Are Regulated during Diurnal Aging}

To search for the mechanisms underlying the distinct migratory patterns of fresh and aged neutrophils, we examined the different steps of the recruitment cascade (rolling, adhesion, and extravasation) in the cremasteric microcirculation with intravital microscopy (Figure 4A). We found elevated rolling, adhesion, and extravasation efficiencies of $A r n t l^{\Delta \mathrm{N}}$ neutrophils and significant reductions for Cxcr4 ${ }^{\Delta N}$ neutrophils (Figure 4B). The defects in the recruitment cascade of $C x c r 4^{\Delta \mathrm{N}}$ aged neutrophils were independently reproduced in Selp; Sele ${ }^{-I-}$-derived aged neutrophils (Figure S4A; Video S1). In contrast to rolling, the crawling dynamics of neutrophils on the vessel wall and within tissues (Figures S4B and S4C), as well as the migration to various chemoattractants (Figure S4D), were unaffected by aging. Furthermore, analyses in auto-perfused flow chambers coated with P-selectin alone or together with ICAM-1 (intercellular adhesion molecule 1) and CXCL1 and connected to the circulation of wild-type mice (Figure S4E) revealed elevated rolling efficiencies 

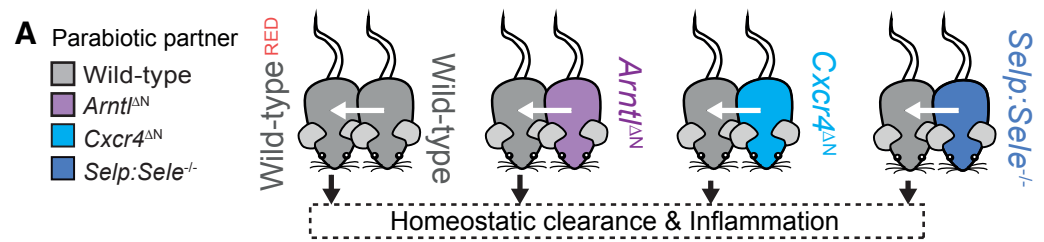

B

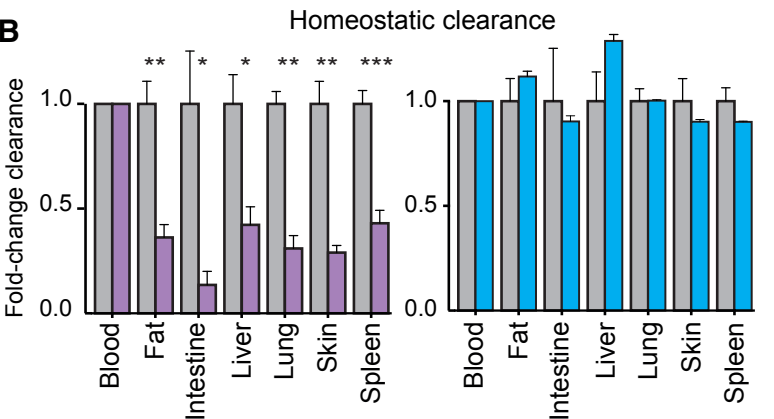

Figure 3. Diurnal Aging Impairs Inflammatory Recruitment but Favors Homeostatic Clearance, Related to Figure S3

(A) Experimental setup to test recruitment in parabiotic pairs. Mutant neutrophils that enter tissues of their wild-type ${ }^{\mathrm{RED}}$ partners (expressing DsRed) allowed estimation of their migratory capacity relative to wild-type cells.

(B) Quantification of $A r n t t^{\Delta \mathrm{N}}$ (left) and $C x c r 4^{\Delta \mathrm{N}}$ (right) neutrophils cleared in multiple tissues of wild-type partners at ZT5; values are adjusted to ratios in blood and normalized to wild-type neutrophils cleared in wild-type partners; $\mathrm{n}=3-8$ mice.

(C) Infiltration efficiency of control and mutant neutrophils into the peritoneum of wild-type parabiotic partners at $Z T 5 ; n=3-5$ mice.

( $D$ and $E$ ) Whole-mount staining of TNF- $\alpha$-treated cremaster muscles from transplant chimeras of wildtype and $A r n t l^{\Delta \mathrm{N}}$ donors (D) or wild-type and Cxcr $4^{\Delta \mathrm{N}}$ donors $(E)$, showing extravasated neutrophils and vessels. Extravasated neutrophils are quantified in Figure 4B (extravasation).

(F) Whole-mount staining as in (D), comparing constitutively fresh and aged neutrophils in Bmal1 ${ }^{\Delta \mathrm{N}}$ mice (fresh, red) set in parabiosis with Selp; Sele ${ }^{-/-;}$GFP (aged, green) partners. The relative infiltration of each partner is quantified in the right bar graph. Scale bars for (D)-(F), $70 \mu \mathrm{m}$. Insets scale bars, $10 \mu \mathrm{m}$.

All bars show mean \pm SEM. ${ }^{*} \mathrm{p}<0.05 ;{ }^{* *} \mathrm{p}<0.01$; ${ }^{* * *} \mathrm{p}<0.001$ as determined by unpaired $(\mathrm{B})$ or paired ( $\mathrm{C}$ and $\mathrm{F}$ ) $\mathrm{t}$ test analysis.

and subsequent adhesion for neutrophils at ZT13 relative to ZT5 (Figure S4F), indicating that diurnal changes in rolling and adhesion were cell intrinsic. Combined, these data suggested that diurnal aging impaired inflammatory recruitment by specifically targeting rolling, a rate-limiting step during leukocyte recruitment (Ley et al., 2007).

Because rolling is largely mediated by endothelial selectins, the data implied that aging targeted selectin ligands on neutrophils. However, binding analyses using soluble P-and E-selectin antibody chimeras revealed only modest changes in selectin binding, regardless of time of day or genetic background (Figure S4G), suggesting that biosynthesis of selectin ligands was unlikely to cause the loss of rolling during aging. Effective engagement of selectins under flow additionally demands correct topology at the neutrophil's surface to optimize ligand presentation at the tip of microvilli, a type of membrane protrusion that relies on a network of cortical actin (Finger et al., 1996; Simon et al., 2007; von Andrian et al., 1995). Analyses of actin distribution with immunofluorescence staining and of surface topology with scanning electron microscopy revealed dramatic reductions in cortical $\beta$-actin in aged neutrophils, which coincided with a reduced number of microvilli both in wild-type mice at ZT5 (Figures 4C and 4D) and in genetically induced aged mice (Cxcr4 ${ }^{\Delta \mathrm{N}}$; Figures S4H and S4I). These data revealed that disruption of the neutrophil's cortical architecture during aging impairs migration to inflamed tissues.

\section{Homeostatic Migration Does Not Require Rolling}

These observations, however, failed to explain why rollingdefective aged neutrophils maintained an intact capacity to enter non-inflamed tissues under homeostasis (Figure 3B). To address this issue, we analyzed homeostatic and inflammatory recruitment in control mice and in mice with impaired rolling due to the lack of endothelial selectins (Selp; Sele ${ }^{-1-}$ mice; Frenette et al., 1996). Although adhesion to inflamed vessels required selectin-mediated rolling as expected, we found that spontaneous adhesion during homeostatic recruitment to the skin occurred even in the absence of rolling (Figure 4E). Video analyses of the dermal microvasculature at times of clearance (ZT9) confirmed that neutrophils arrested suddenly, without the need of a preceding rolling step (Figure 4F; Video S2). Thus, neutrophil aging maintains homeostatic clearance but prevents inflammatory recruitment by disabling selectin-mediated rolling (Figure 4G).

To determine how the differential migratory properties of fresh and aged neutrophils affected tissue injury, we induced ischemic inflammation of the brain by occlusion of the middle cerebral artery. Because the brain is devoid of neutrophils at steady state (Figure S4J), this model allows measuring of the contribution of infiltrating neutrophils to tissue damage (Cuartero et al., 2013; Sreeramkumar et al., 2014). In line with our prediction, brain injury was only exacerbated in $A r n t{ }^{\Delta \mathrm{N}}$ mice enriched in fresh neutrophils (Figure S4K), suggesting that preferential migration of fresh neutrophils during inflammation contributes to tissue injury.

\section{Diurnal Aging Boosts Anti-microbial Defense}

The observations so far raised the possibility that regulation of neutrophil migration during the day might be a primary role of diurnal aging; it could drive compartmentalization of aged 
A

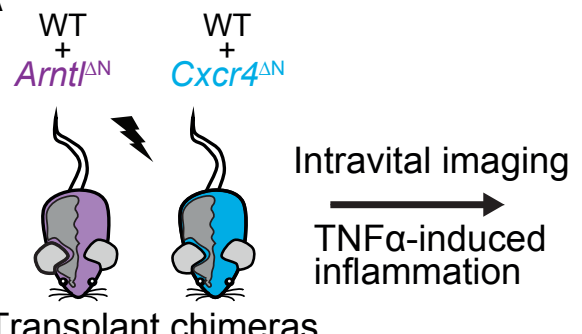

Transplant chimeras

C
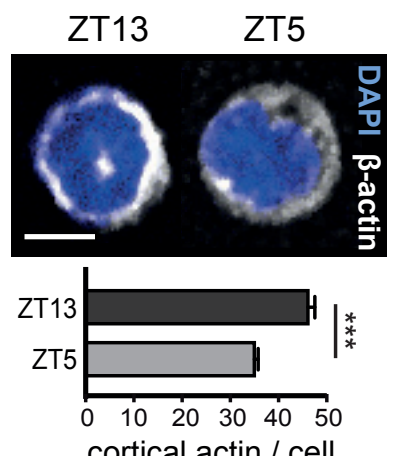

F Wild-type

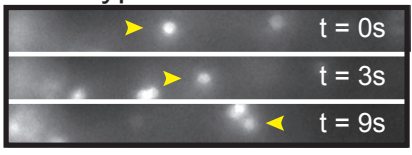

Selp:Sele-r-

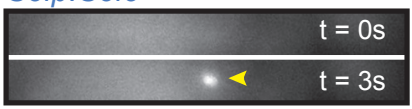

Flow $\rightarrow$
B Rolling

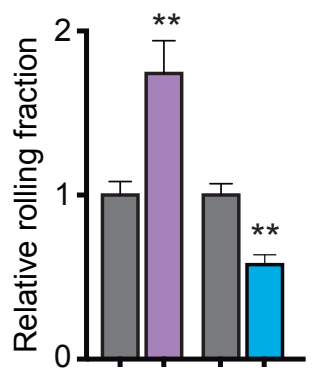

D ZT13
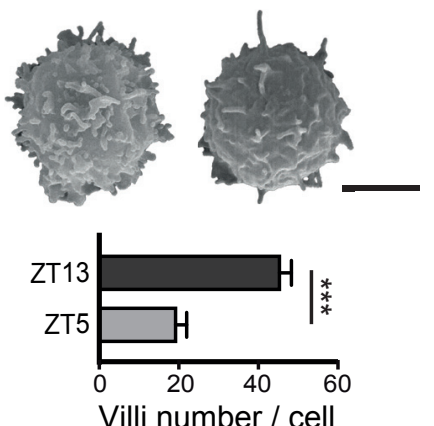

Villi number / cell

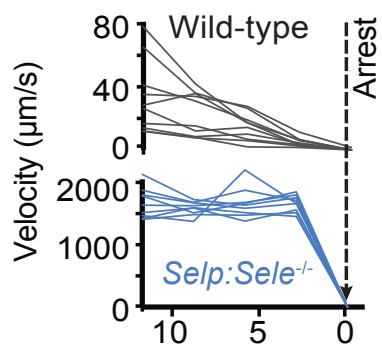

Time before arrest (s)

G
Adhesion

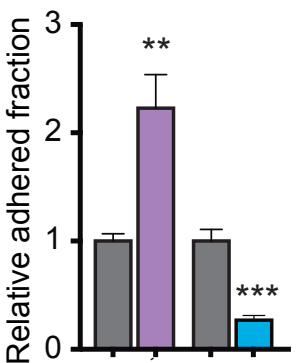

E

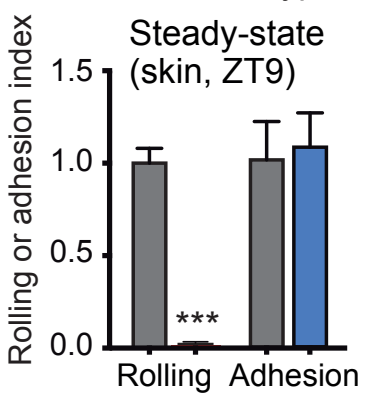

Extravasation

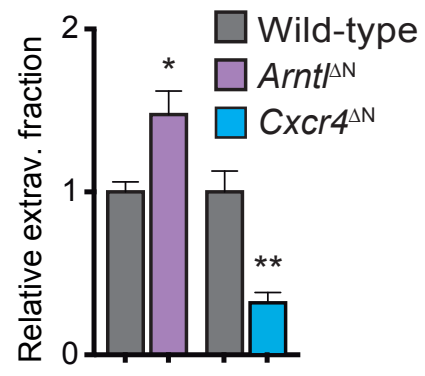

Steady-state

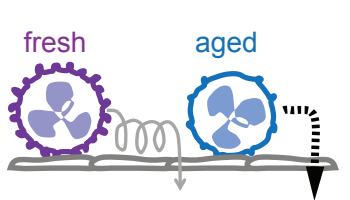

Inflammation

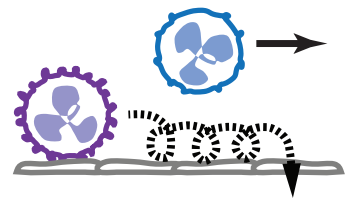

Figure 4. Microvilli Collapse and Impaired Rolling Are Hallmarks of Aged Neutrophils, Related to Figure S4

(A) Strategy for competitive recruitment of neutrophils in bone marrow chimeras, at ZT5.

(B) Relative frequencies of rolling, adherent, and extravasated fresh $\left(A r n t l^{\Delta N}\right)$ and aged $\left(C x c r 4^{\Delta N}\right)$ neutrophils, normalized to wild-type controls in chimeric mice; $\mathrm{n}=30-61$ venules from 5-6 mice.

(C) $\beta$-actin staining in wild-type neutrophils at ZT5 and ZT13, and frequency of neutrophils with cortical distribution of actin; $n=324-330$ cells per group.

(D) Scanning electron micrographs of wild-type neutrophils at $Z T 5$ and $Z T 13$, and number of microvilli on their surface. Scale bar, $5 \mu \mathrm{m} ; n=23-29$ cells per group.

(E) Rolling and adhesion of neutrophils on cremasteric venules after treatment with TNF- $\alpha$ (inflammation), or on naive dermal microvessels at ZT9-13 (steady state) in wild-type or Selp;Sele ${ }^{-1-}$ mice. $n=50-55$ venules from 4-5 mice (steady state) and 25-27 venules from 3-5 mice (inflamed cremaster). ND, none detected. (F) Kinetics of neutrophils ( $\left(\mathrm{H} 6 \mathrm{G}^{+}\right.$, yellow arrows) prior to firm arrest on dermal microvessels at steady state. Left, representative sequential intravital frames with neutrophils arresting in the last sequence (reverse arrows). Right, flow or roll dynamics of neutrophils before firm arrest; $n=10$ cells shown per group.

(G) Model for the preferential recruitment of fresh and aged neutrophils into inflamed or naive tissues, respectively.

Bars show mean \pm SEM. ${ }^{\star} \mathrm{p}<0.05 ;{ }^{* \star} \mathrm{p}<0.01$; ${ }^{* \star \star} \mathrm{p}<0.001$ as determined by paired $(\mathrm{B})$ or unpaired $\mathrm{t}$ test analysis $(\mathrm{C}$ and $\mathrm{D})$ or non-parametric MannWhitney test (E).

neutrophils into tissues at night in anticipation of pathogens potentially breaking into tissues, while at the same time reducing their numbers within vessels to minimize injury when the chance of immune activation is higher.

To test this possibility, we analyzed the diurnal dynamics of aged neutrophils in the circulation of wild-type and mutant mice throughout the day. The analyses revealed striking differences: in wild-type mice, aged neutrophils peaked at ZT5 and disappeared at ZT13, whereas in $A r n t{ }^{\Delta \mathrm{N}}$ mice, they displayed non-oscillating low numbers and $C x c r 4^{\Delta N}$ animals presented constitutive elevations in aged neutrophils in blood (Figure 5A). Notably, the absolute number of neutrophils maintained normal oscillations in the blood of $A r n t l^{\Delta \mathrm{N}}$ mutants (Figure S5A), indicating that neutrophil numbers and aging are regulated through different mechanisms. We therefore used these mouse models to determine how aging-driven trafficking regulated immune defense and vascular health.

We first infected mice with Candida albicans, using a protocol that allows systemic spread, targets the kidneys, and is controlled by neutrophils (Del Fresno et al., 2018; Lionakis 
A

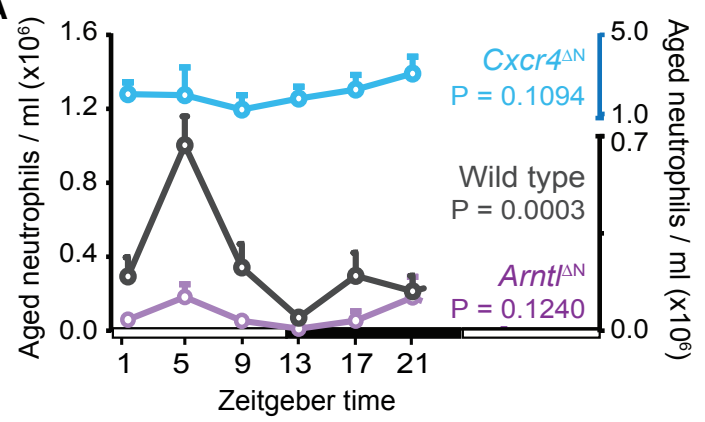

B

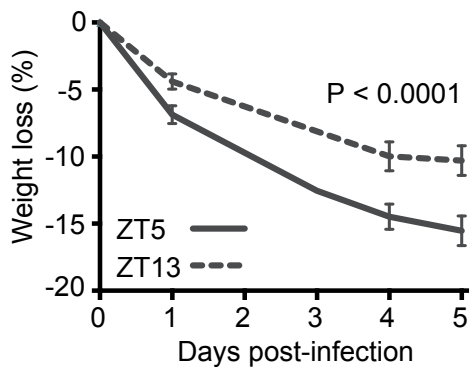

C

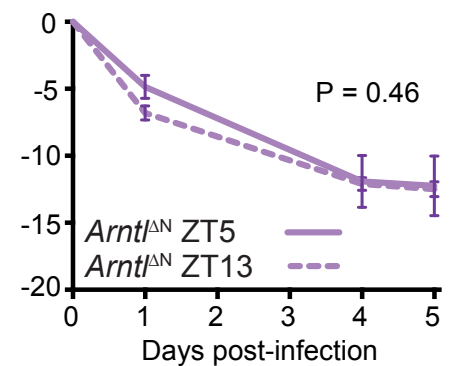

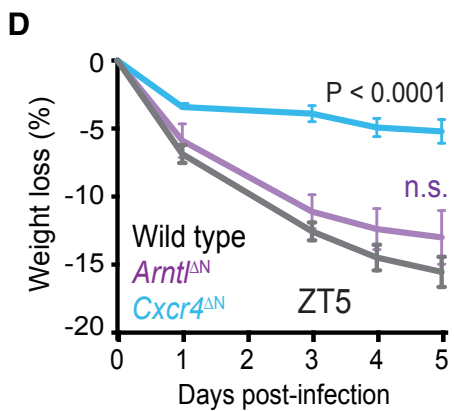
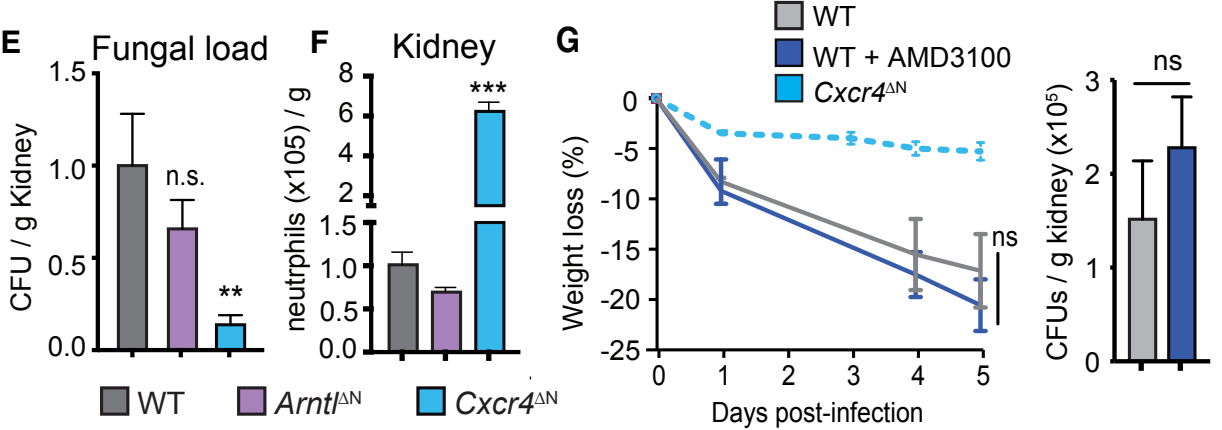

Figure 5. Neutrophil Aging Confers Diurnal Protection against Infection, Related to Figure S5

(A) Diurnal numbers of $\mathrm{CD}_{2} \mathrm{~L}^{\mathrm{lo}}$ aged neutrophils in the blood of wild-type, Arnt/ ${ }^{\Delta \mathrm{N}}$, and $\mathrm{Cxcr} 4^{\Delta \mathrm{N}}$ mice; $\mathrm{n}=3-6 \mathrm{mice}$. See also Figure S5A.

(B and C) Weight loss kinetics of wild-type (B) and Arnt/ ${ }^{\Delta N}$ mice (C) infected with C. albicans at ZT5 or ZT13; $n=5-21$ mice.

(D) Weight-loss curves of wild-type, Arnt/ ${ }^{\Delta \mathrm{N}}$, and $C x c r 4^{\Delta \mathrm{N}}$ mice infected at ZT5; $\mathrm{n}=12-14$ mice.

(E) Fungal burden at day 5 in the kidneys from the mice in (D), normalized to WT.

(F) Number of neutrophils in the kidneys of non-infected mice; $n=4$ mice per group.

(G) Kinetics of weight loss in control or AMD-treated wild-type mice after systemic C. albicans infection at ZT5. The dashed line shows weight loss in Cxcr4 ${ }^{\Delta \mathrm{N}}$ mice as in Figure 4D for reference. Bars at right show fungal burden in kidneys at day 5 post-infection; $n=10$ mice.

Data are shown as mean \pm SEM. ${ }^{\star \star} p<0.01$; ${ }^{* \star *} p<0.001$; n.s., not significant, as determined by amplitude versus zero $t$ test (A), two-way ANOVA (B-D and G), one-way ANOVA with Dunnett's multigroup correction (E and F), and unpaired t test analysis (G).

et al., 2011). Wild-type mice displayed diurnal patterns of response to infection, with increased resistance at ZT13 as defined by reduced weight loss, fungal load in kidneys, and improved survival (Figures 5B, S5B, and S5C). Importantly, the initial time of infection was critical for the long-term immune response because the effect could be seen several days after infection. Resistance to Candida at ZT13 coincided with more neutrophils in naive kidneys and fewer in blood (Figures S5D and S5E), suggesting that their presence in tissues at the time of infection conferred protection. Remarkably, the diurnal variation in susceptibility to Candida infection was abolished in $\mathrm{Arnt}^{\mathrm{\Delta N}}$ mice (Figure $5 \mathrm{C}$ ), indicating that neutrophil aging was needed to anticipate the infection. These observations predicted that mice with constitutively aged neutrophils clearing into tissues might perform better against infection. Indeed, $C x c r 4^{\Delta N}$ mice had more neutrophils in naive kidneys and manifested remarkable resistance to infection and reduced fungal spread (Figures 5D and 5F). However, because $\mathrm{CxCr} 4^{\Delta \mathrm{N}}$ mice displayed neutrophilia (Figure S5A), this observation could be alternatively explained by elevated numbers rather than by the aging status of neutrophils. To discriminate between these possibilities, we treated wild-type mice with a single injection of the CXCR4 antagonist AMD3100, a treatment that causes acute neutrophilia (Devi et al., 2013) but did not induce aging (Figures S5F and S5G). Despite neutrophil counts that were even higher than those in $C x c r 4^{\Delta N}$ mice,
AMD3100-treated mice were as susceptible to Candida infection as untreated wild-type mice (Figure 5G), indicating that the aging status, rather than the number of neutrophils, conferred protection against Candida. In addition, the capacity of fresh (ZT13 or $\mathrm{Arnt}^{\Delta \mathrm{N}}$ ) and aged (ZT5 and Cxcr4 ${ }^{\Delta \mathrm{N}}$ ) neutrophils to phagocytose Candida conidia to produce reactive oxygen species (Figures S5H-S5J) and to secrete cytokines (Figure S5K) were similar to wild-type cells, which supported the contention that neutrophil migration, rather than other cellular processes, was the relevant process regulated by aging. We obtained evidence of similar diurnal variations in the response to bacterial sepsis, which was also lost in $\mathrm{Arnt}^{\Delta \mathrm{N}}$ mice (Figure S5L), further revealing a general influence of neutrophil aging in responses to infection. Thus, aging-driven clearance of neutrophils into tissues orchestrates anti-microbial defense.

\section{Constitutive Neutrophil Aging Predisposes to Vascular Inflammation}

To define whether diurnal neutrophil aging additionally conferred protection to vessels, we used a model of acute myocardial infarction (AMI) induced by ischemia reperfusion of the left anterior descending coronary artery, in which inflammation originates intravascularly without prior neutrophil extravasation (VintenJohansen, 2004). Similar to infections, the extent of cardiac damage displayed diurnal variations, with larger infarct sizes at 
A

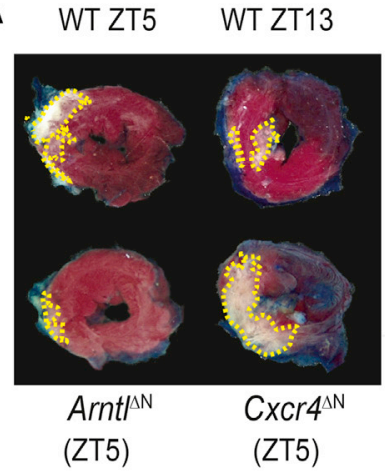

B C

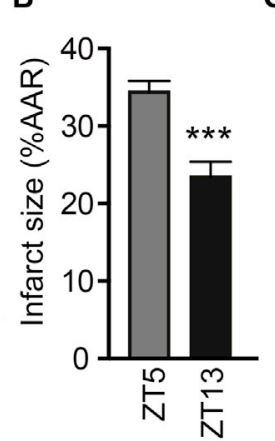

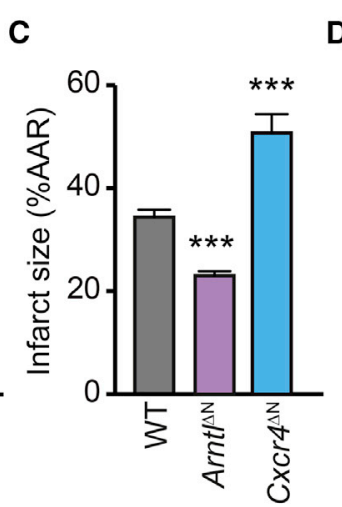

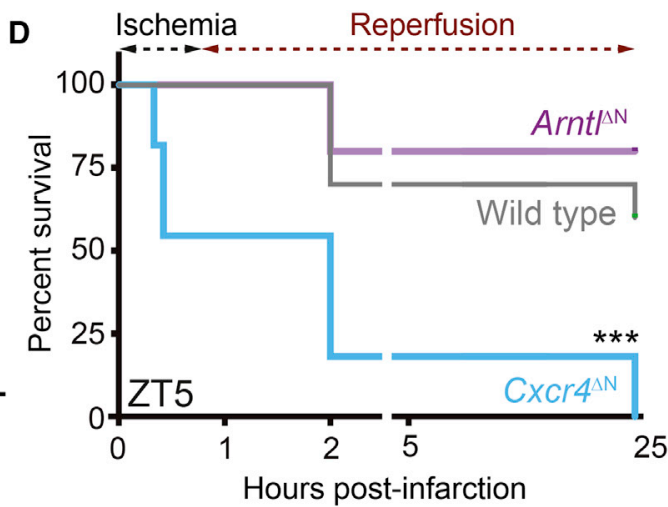

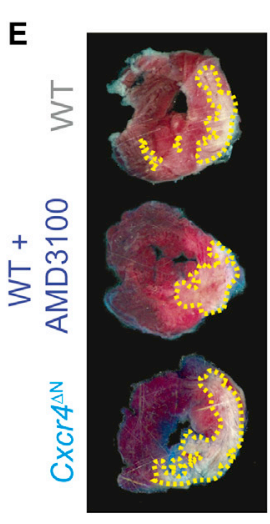
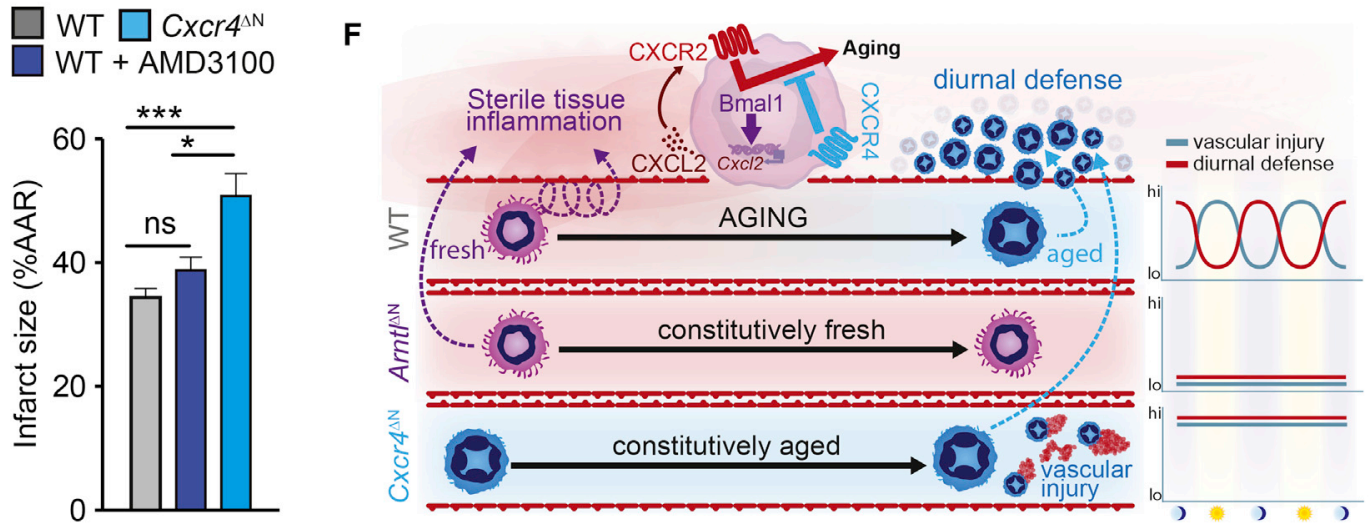

Figure 6. Aged Neutrophils Aggravate Myocardial Infarction, Related to Figure S6

(A) Representative images of hearts from wild-type mice subjected to ischemia reperfusion at ZT5 or ZT13, or the indicated mutant mice at ZT5. Dotted yellow lines highlight areas of dead myocardium; $n=4-8$ mice per group from 3 experiments.

(B) Infarct sizes in wild-type mice at different diurnal times, after correction for areas at risk (AAR; see related Figure S5); $\mathrm{n}=5-8$ mice.

(C) Infarct sizes in wild-type, $\left.A r n t\right|^{\Delta \mathrm{N}}$, and $C x c r 4^{\Delta \mathrm{N}}$ mice at $\mathrm{ZT5}$ (see related Figure S5); $\mathrm{n}=4-8$ mice.

(D) Survival curves of wild-type, $A r n t t^{\Delta \mathrm{N}}$, and $C x c r 4^{\Delta \mathrm{N}}$ mice subjected to myocardial infarction at ZT5; $\mathrm{n}=9-11$ mice.

(E) Representative images of hearts from untreated or AMD3100-treated wild-type mice and Cxcr4 $4^{\Delta \mathrm{N}}$ mice. Surgeries were performed at ZT5 and dead myocardium is highlighted as in (A). Bars at right show quantification of infarcted areas in the same groups; $n=4-5$ mice from one experiment.

Bars show mean \pm SEM. ${ }^{\star} p<0.05 ;{ }^{\star \star \star} p<0.001$; n.s., not significant, as determined by one-way ANOVA with Dunnett's multigroup correction (C and $\left.E\right)$, unpaired $\mathrm{t}$ test analysis (B), and log-rank test (D).

(F) Molecular regulators and consequences of disrupted neutrophil aging. Defective aging (Arnt/ $\left.{ }^{\Delta N}\right)$ impairs the evening boost in anti-microbial defense but protects from vascular injury; instead constitutive aging $\left(\mathrm{Cxcr}^{\Delta \mathrm{N}}\right)$ enhances the response to infections but exacerbates thrombo-inflammation.

ZT5 (Figures 6A, 6B, and S6A). Accordingly, infarct sizes after only $1 \mathrm{~h}$ of reperfusion were remarkably larger in $\mathrm{CxCr} 4^{\Delta \mathrm{N}}$ mice and smaller in $A r n t I^{\Delta \mathrm{N}}$ mice (Figure $6 \mathrm{C}$ ), and this correlated with early death of all $C x c r 4^{\Delta N}$ mice (Figure 6D). This dramatic response was not caused by increased numbers of circulating neutrophils in $\mathrm{CxCr}^{\Delta \mathrm{N}}$ mice because treatment of wild-type mice with AMD3100 did not aggravate myocardial injury (Figure $6 \mathrm{E}$ ). Thus, the presence of aged neutrophils in the circulation is detrimental for tissues after vascular ischemia and reperfusion, whereas their diurnal clearance is protective.

We examined potential mechanisms by which aged neutrophils might exacerbate vascular injury in $C x c r 4^{\Delta N}$ mice. Using a model of ischemia reperfusion in the cremaster muscle that allows high-resolution live imaging of affected vessels, we found disseminated thrombi in microvessels of $\mathrm{CxCr}^{\Delta \mathrm{N}}$ mice (Figure S6C; Video S3). Depletion of neutrophils in $\mathrm{Cxcr} 4^{\Delta \mathrm{N}}$ mice prevented thrombi formation and improved survival after infarction (Figures S6B and S6C), indicating that both responses were mediated by neutrophils. Although neutrophil extracellular traps (NETs) can promote thrombosis (Fuchs et al., 2010), they were not responsible for the response of $C x c r 4^{\Delta N}$ mice because two different NET inhibitors failed to prevent thrombus formation in reperfused venules (Figure S6D). In addition, endothelial proliferation and apoptosis, as well as vascular permeability, were not affected at baseline across multiple tissues, including hearts, of $\mathrm{Arnt}^{\Delta \mathrm{N}}$ and Cxcr4 ${ }^{\Delta \mathrm{N}}$ mice (Figures S6E-S6G), indicating that aging did not directly compromise basal vascular health prior to the ischemic insult.

Overall, these observations suggest that neutrophil aging is critically driven by an internal program, as we failed to find contributions from other factors, including reactive oxygen species (ROS; data not shown) or the intestinal microbiota (Figures $\mathrm{S} 6 \mathrm{H}-\mathrm{S} 6 \mathrm{~K})$, both of which had been previously associated with neutrophil senescence or aging, respectively (Harbort et al., 2015; Zhang et al., 2015). In turn, aging controls diurnal compartmentalization of neutrophils into tissues and out of the 
circulation, thereby balancing immune protection and vascular protection (Figure 6F; Video S4).

\section{DISCUSSION}

Mammalian immunity is not constant in quantity (e.g., number of recruited or mobilized leukocytes) or quality throughout the day, as it adapts to varying diurnal challenges from the environment, including the chance of exposure to infectious pathogens (Man et al., 2016). Likewise, damage to the cardiovascular system, in both humans and model organisms, follows circadian patterns (Muller et al., 1989; Scheiermann et al., 2013). Because neutrophils are major mediators of anti-microbial defense and vascular inflammation, we predicted that the diurnal variations in both processes could be mechanistically explained by the existence of a neutrophil-intrinsic program (or "timer") that regulated their activity through the day. In this study, we identified and characterized this program and revealed that it underlies the circadian susceptibility of mice to infection and vascular inflammation.

We have found that the diurnal program of neutrophils is coordinated by the circadian-related protein Bmal1 in coordination with two chemokine receptors: CXCR2, which drives aging, and CXCR4, which antagonizes it. Multiple functional assays allowed us to confirm that time-of-day differences in wild-type cells could be faithfully recapitulated by the respective mutants: Arntl $^{\Delta \mathrm{N}}$ cells resembled night (fresh) neutrophils, whereas Cxcr $4^{\Delta \mathrm{N}}$ mutants behaved similar to daytime (aged) neutrophils. Before release into the bloodstream, maturing neutrophils are retained within the marrow in an environment with high CXCR4 signaling (Eash et al., 2009, 2010), which raises the intriguing possibility that this diurnal timer is inhibited until neutrophils are released into blood. Once in blood, functional analyses of mice in which we disabled each component of this timer revealed that preferential invasion of inflamed or naive tissues is compartmentalized in time. Under steady-state conditions, neutrophils released from the marrow gradually lost their ability to enter inflammatory sites and prepared for clearance into tissues. This migratory switch was intrinsically regulated, but it likely coordinated with extrinsic programs because disruption of rhythms in vascular cells can also affect the diurnal entry of leukocytes in tissues (Scheiermann et al., 2012) and because CXCL12, which is not produced by neutrophils, negatively regulated diurnal aging through CXCR4.

We found that one potential benefit of diurnal infiltration into naive tissues was to optimize immune defense, as demonstrated by the loss of diurnal oscillations in the response against fungal or bacterial infections when Arntl was deleted from neutrophils. Removal of Cxcr4, the negative regulator of the neutrophil timer, instead caused unrestrained aging and enhanced antimicrobial responses, while at the same time precipitating severe thrombo-inflammatory reactions following ischemia reperfusion. In contrast, Arntl $^{\Delta \mathrm{N}}$ mutants displayed attenuated damage during myocardial infarction, altogether indicating that an intact neutrophil clock was important to balance anti-microbial defense and cardiovascular inflammation.

Among the various transcriptional pathways activated during aging, we identified those related to leukocyte extravasation and actin cytoskeleton signaling, an observation that allowed us to identify disruption of cortical actin polymerization as a key molecular event linking diurnal aging with alterations in the migratory properties of neutrophils. Although the mechanisms underlying these cytoskeletal changes remain to be elucidated, this observation is consistent with early studies showing disrupted actin polymerization on human CD62 $\mathrm{L}^{\mathrm{lo}}$ neutrophils (TanjiMatsuba et al., 1998). Diurnal loss of microvilli was particularly relevant because these structures allow presentation of glycoconjugate ligands to endothelial selectins under flow (von Andrian et al., 1995), thus explaining the dramatic loss of rolling and migration of aged neutrophils to inflamed areas. At the same time, loss of microvilli might conceivably enhance the exposure of $\beta 2$ integrins present on the cell body (Erlandsen et al., 1993) and favor rolling-independent arrest as seen in the naive dermal microvasculature. The fact that a similar behavior of constitutive adhesion in non-inflamed vessels is displayed by patrolling monocytes (Auffray et al., 2007) suggests that this mechanism could be a common property of myeloid leukocytes endowed with homeostatic surveillance roles.

Overall, our findings are consistent with a model in which the oscillatory nature of the aging program enables alert states of neutrophils that are useful to anticipate infections but must be shut down when the risk of infection is low to prevent damage to the vasculature. We note that Bmal1-driven aging of neutrophils may not necessarily adjust to behavioral rhythms because we found that neutrophil aging peaked in the morning in both humans and mice, which are species with opposed activity periods. We therefore propose that a major purpose of aging is to ensure temporal separation of neutrophil-mediated responses within vessels from those in tissues, thereby optimizing defense without compromising vascular health.

The diurnal aging pattern of neutrophils aligns with studies showing temporally gated responses for other leukocyte subsets, including monocytes, macrophages, or $\mathrm{T}$ helper-17 (Th17) cells (Nguyen et al., 2013; Silver et al., 2012; Yu et al., 2013), which may be useful to temporally concentrate immune response against specific pathogens in different tissues (Tognini et al., 2017). Different from these other leukocytes, however, the existence of a circadian program in neutrophils was not intuitive because their lifetime in the circulation is generally accepted to be less than one day (Summers et al., 2010), which implies that there cannot be true circadian oscillations of gene expression within a given neutrophil. Further, at present we do not know whether aging is regulated by the transcriptional properties of Bmal1 or by the core circadian clock. For these reasons, we envision this system to function like a cellular timer (rather than a true circadian clock) that resets with every new wave of neutrophils released from the bone marrow. In other words, for short-lived cells such as neutrophils, the clock appears to regulate oscillations on a population scale by acting as a timer at the cellular level.

Given the high prevalence of infections and cardiovascular disease, a final question is whether the identification of a diurnal program in neutrophils could offer therapeutic alternatives for these life-threatening complications. In principle, targeting CXCR2 or CXCR4 with specific agonists might allow pharmacological and transient manipulation of the timer. This "chronoprogramming" of neutrophils could allow the generation of phenotypes that promote defense or protect the vasculature, as needed. We expect that manipulation of the timer will not 
have detrimental consequences because animals with impaired or enhanced neutrophil aging do not present gross anomalies or spontaneous susceptibility to disease at baseline, at least under specific-pathogen-free conditions (data not shown). Thus, for humans at risk of cardiovascular events, it might be advisable to block aging, whereas immunocompromised patients susceptible to infections might benefit from drugs that promote it. We are currently exploring strategies that exploit the unique temporal properties of neutrophils.

\section{STAR $\star$ METHODS}

Detailed methods are provided in the online version of this paper and include the following:

- KEY RESOURCES TABLE

- CONTACT FOR REAGENT AND RESOURCE SHARING

- EXPERIMENTAL MODEL AND SUBJECT DETAILS

$$
\text { O Mice }
$$

O Human Studies

- METHOD DETAILS
O Analysis of Human Samples
O Parabiosis

O Cytometry and Cell Sorting

Whole-Mount Staining of Excised Cremaster Muscles

O RNA Isolation, Reverse Transcription, and rtPCR

C Chromatin Immunoprecipitation (ChIP)

Intravital Imaging of the Mouse Skin

Intravital Microscopy of the Cremaster Muscle

O Intravital Imaging of Ischemia and Reperfusion Injury

Analysis of Neutrophil Clearance in the Steady State

Q Quantification of Neutrophil Numbers in Tissues

O Chemokine Quantification in Plasma

O RNA-Sequencing

O Western Blotting

O Generation of Transplant Chimeras

O Circadian Analysis of Aging Markers and RNA Extraction

$\bigcirc$ Analysis of Neutrophil Aging in Light-Dark and DarkDark Light Regimes

O Entrainment of Neutrophil Aging by Inverted Light Regime

O Infection with Candida albicans

○ Heat-Killed Candida albicans (HKC) Phagocytosis Assay

O Mouse Model of Acute Myocardial Infarction

Brain Ischemia

O Scanning Electron Microscopy

O CXCR2 and CXCR4 Cross-Inhibition Assays

O Neutrophil Depletion

O Zymosan-Induced Peritonitis

O Soluble Selectin Binding Assays

○ Auto-Perfused Flow Chamber Assay

O Cortical Beta-Actin Quantification

- ROS Quantification

O Chemotaxis Assay

- Multiplex Cytokine Assay

- AMD3100-Induced Neutrophilia

O Neutrophil Transfer Experiments
○ BrdU Labelling

O In Vivo CXCL1 and CXCL2 Blockade

$\bigcirc$ Cecal Ligation and Puncture (CLP)-Induced Sepsis

- Evans Blue Vascular Permeability Assay

- Analysis of Endothelial Proliferation and Apoptosis

- Analysis of Neutrophil Aging in Microbiota-Depleted and Germ-free Mice

- QUANTIFICATION AND STATISTICAL ANALYSIS

O RNA-Sequencing Data Analysis

O Statistical Analysis

O Amplitude versus Zero Test

- DATA AND SOFTWARE AVAILABILITY

\section{SUPPLEMENTAL INFORMATION}

Supplemental Information includes six figures, three tables, and four videos and can be found with this article online at https://doi.org/10.1016/j.immuni. 2019.01.002.

\section{ACKNOWLEDGMENTS}

We thank all members of the Hidalgo Lab for discussion and insightful comments; J.M. Ligos, R. Nieto, and M. Vitón for help with sorting and cytometric analyses; I. Ortega and E. Santos for animal husbandry; D. Rico, M.J. Gómez, C. Torroja, and F. Sanchez-Cabo for insightful comments and help with transcriptomic analyses; V. Labrador, E. Arza, A.M. Santos, and the Microscopy Unit of the CNIC for help with microscopy; S. Aznar-Benitah, U. Albrecht, Q.-J. Meng, B. Staels, and H. Duez for the generous gift of mice; J.A. Enriquez and J. Ávila for scientific insights; and J.M. García and A. Diez de la Cortina for art. This study was supported by Intramural grants from $A^{*} S T A R$ to L.G.N., BES-2013-065550 to J.M.A., BES-2010-032828 to M.C.-A, and JCl-201214147 to L.A.W (all from Ministerio de Economía, Industria y Competitividad; MEIC). Additional MEIC grants were SAF2014-61993-EXP to C.L.-R.; SAF2015-68632-R to M.A.M. and SAF-2013-42920R and SAF201679040Rto D.S. D.S. also received 635122-PROCROP H2020 from the European Commission and ERC CoG 725091 from the European Research Council (ERC). ERC AdG 692511 PROVASC from the ERC and SFB1123-A1 from the Deutsche Forschungsgemeinschaft were given to C.W.; MHA VD1.2/ $81 \mathrm{Z1600212}$ from the German Center for Cardiovascular Research (DZHK) was given to C.W. and O.S.; SFB1123-A6 was given to O.S.; SFB914-B08 was given to O.S. and C.W.; and INST 211/604-2, ZA 428/12-1, and ZA 428/ 13-1 were given to A.Z. This study was also supported by PI12/00494 from Fondo de Investigaciones Sanitarias (FIS) to C.M.; PI13/01979, Cardiovascular Network grant RD 12/0042/0054, and CIBERCV to B.I.; SAF2015-65607-R, SAF2013-49662-EXP, and PCIN-2014-103 from MEIC; and co-funding by Fondo Europeo de Desarrollo Regional (FEDER) to A.H. The CNIC is supported by the MEIC and the Pro CNIC Foundation and is a Severo Ochoa Center of Excellence (MEIC award SEV-2015-0505).

\section{AUTHOR CONTRIBUTIONS}

J.M.A., C.d.F., M.I.C., M.C.-A., L.A.W., H.H.-E., C.S.-R., J.R., J.A.Q., G.C. J.G.-P., M.G.-P., S.M.-S., M.E., and J.L. performed experiments; C.W., K.B., and F.B. contributed essential reagents; A.Z., O.S., C.L.-R., M.A.M., B.I., D.S., L.N., J.M.A., and A.H. designed and supervised experiments; F.A. and C.M. coordinated the study on humans; A.H. designed and supervised the study. A.H. and J.M.A. wrote the manuscript, which was edited by all authors.

\section{DECLARATION OF INTERESTS}

The authors declare no competing interests.

Received: August 24, 2018

Revised: November 23, 2018

Accepted: January 2, 2019

Published: January 29, 2019; corrected online: November 6, 2019 


\section{REFERENCES}

Auffray, C., Fogg, D., Garfa, M., Elain, G., Join-Lambert, O., Kayal, S., Sarnacki, S., Cumano, A., Lauvau, G., and Geissmann, F. (2007). Monitoring of blood vessels and tissues by a population of monocytes with patrolling behavior. Science 317, 666-670.

Balabanian, K., Brotin, E., Biajoux, V., Bouchet-Delbos, L., Lainey, E., Fenneteau, O., Bonnet, D., Fiette, L., Emilie, D., and Bachelerie, F. (2012). Proper desensitization of CXCR4 is required for lymphocyte development and peripheral compartmentalization in mice. Blood 119, 5722-5730.

Casanova-Acebes, M., Pitaval, C., Weiss, L.A., Nombela-Arrieta, C., Chèvre, R., A-González, N., Kunisaki, Y., Zhang, D., van Rooijen, N., Silberstein, L.E., et al. (2013). Rhythmic modulation of the hematopoietic niche through neutrophil clearance. Cell 153, 1025-1035.

Casanova-Acebes, M., Nicolás-Ávila, J.A., Li, J.L., García-Silva, S., Balachander, A., Rubio-Ponce, A., Weiss, L.A., Adrover, J.M., Burrows, K., A-González, N., et al. (2018). Neutrophils instruct homeostatic and pathological states in naive tissues. J. Exp. Med. 215, 2778-2795.

Cuartero, M.I., Ballesteros, I., Moraga, A., Nombela, F., Vivancos, J., Hamilton, J.A., Corbí, A.L., Lizasoain, I., and Moro, M.A. (2013). N2 neutrophils, novel players in brain inflammation after stroke: modulation by the PPAR $\gamma$ agonist rosiglitazone. Stroke 44, 3498-3508.

Del Fresno, C., Saz-Leal, P., Enamorado, M., Wculek, S.K., Martínez-Cano, S., Blanco-Menéndez, N., Schulz, O., Gallizioli, M., Miró-Mur, F., et al. (2018). DNGR-1 in dendritic cells limits tissue damage by dampening neutrophil recruitment. Science 362, 351-356.

Devi, S., Wang, Y., Chew, W.K., Lima, R., A-González, N., Mattar, C.N., Chong, S.Z., Schlitzer, A., Bakocevic, N., Chew, S., et al. (2013). Neutrophil mobilization via plerixafor-mediated CXCR4 inhibition arises from lung demargination and blockade of neutrophil homing to the bone marrow. J. Exp. Med. 210, 2321-2336.

Druzd, D., Matveeva, O., Ince, L., Harrison, U., He, W., Schmal, C., Herzel, H., Tsang, A.H., Kawakami, N., Leliavski, A., et al. (2017). Lymphocyte Circadian Clocks Control Lymph Node Trafficking and Adaptive Immune Responses. Immunity 46, 120-132.

Eash, K.J., Means, J.M., White, D.W., and Link, D.C. (2009). CXCR4 is a key regulator of neutrophil release from the bone marrow under basal and stress granulopoiesis conditions. Blood 113, 4711-4719.

Eash, K.J., Greenbaum, A.M., Gopalan, P.K., and Link, D.C. (2010). CXCR2 and CXCR4 antagonistically regulate neutrophil trafficking from murine bone marrow. J. Clin. Invest. 120, 2423-2431.

Erlandsen, S.L., Hasslen, S.R., and Nelson, R.D. (1993). Detection and spatial distribution of the beta 2 integrin (Mac-1) and L-selectin (LECAM-1) adherence receptors on human neutrophils by high-resolution field emission SEM. J. Histochem. Cytochem. 41, 327-333.

Faust, N., Varas, F., Kelly, L.M., Heck, S., and Graf, T. (2000). Insertion of enhanced green fluorescent protein into the lysozyme gene creates mice with green fluorescent granulocytes and macrophages. Blood 96, 719-726.

Finger, E.B., Bruehl, R.E., Bainton, D.F., and Springer, T.A. (1996). A differential role for cell shape in neutrophil tethering and rolling on endothelial selectins under flow. J. Immunol. 157, 5085-5096.

Frenette, P.S., Mayadas, T.N., Rayburn, H., Hynes, R.O., and Wagner, D.D. (1996). Susceptibility to infection and altered hematopoiesis in mice deficient in both P-and E-selectins. Cell 84, 563-574.

Fuchs, T.A., Brill, A., Duerschmied, D., Schatzberg, D., Monestier, M., Myers, D.D., Jr., Wrobleski, S.K., Wakefield, T.W., Hartwig, J.H., and Wagner, D.D. (2010). Extracellular DNA traps promote thrombosis. Proc. Natl. Acad. Sci. USA 107, 15880-15885.

García-Prieto, J., Villena-Gutiérrez, R., Gómez, M., Bernardo, E., Pun-García, A., García-Lunar, I., Crainiciuc, G., Fernández-Jiménez, R., Sreeramkumar, V., Bourio-Martínez, R., et al. (2017). Neutrophil stunning by metoprolol reduces infarct size. Nat. Commun. 8, 14780.

Harbort, C.J., Soeiro-Pereira, P.V., von Bernuth, H., Kaindl, A.M., CostaCarvalho, B.T., Condino-Neto, A., Reichenbach, J., Roesler, J., Zychlinsky,
A., and Amulic, B. (2015). Neutrophil oxidative burst activates ATM to regulate cytokine production and apoptosis. Blood 126, 2842-2851.

Hasenberg, A., Hasenberg, M., Männ, L., Neumann, F., Borkenstein, L., Stecher, M., Kraus, A., Engel, D.R., Klingberg, A., Seddigh, P., et al. (2015). Catchup: a mouse model for imaging-based tracking and modulation of neutrophil granulocytes. Nat. Methods 12, 445-452.

Hidalgo, A., Peired, A.J., Wild, M., Vestweber, D., and Frenette, P.S. (2007). Complete identification of $\mathrm{E}$-selectin ligands on neutrophils reveals distinct functions of PSGL-1, ESL-1, and CD44. Immunity 26, 477-489.

Hidalgo, A., Chang, J., Jang, J.E., Peired, A.J., Chiang, E.Y., and Frenette, P.S. (2009). Heterotypic interactions enabled by polarized neutrophil microdomains mediate thromboinflammatory injury. Nat. Med. 15, 384-391.

Janich, P., Pascual, G., Merlos-Suárez, A., Batlle, E., Ripperger, J., Albrecht U., Cheng, H.Y., Obrietan, K., Di Croce, L., and Benitah, S.A. (2011). The circadian molecular clock creates epidermal stem cell heterogeneity. Nature 480 , 209-214.

Ley, K., Laudanna, C., Cybulsky, M.I., and Nourshargh, S. (2007). Getting to the site of inflammation: the leukocyte adhesion cascade updated. Nat. Rev. Immunol. 7, 678-689.

Li, B., and Dewey, C.N. (2011). RSEM: accurate transcript quantification from RNA-Seq data with or without a reference genome. BMC Bioinformatics 12, 323.

Li, J.L., Lim, C.H., Tay, F.W., Goh, C.C., Devi, S., Malleret, B., Lee, B., Bakocevic, N., Chong, S.Z., Evrard, M., et al. (2016). Neutrophils SelfRegulate Immune Complex-Mediated Cutaneous Inflammation through CXCL2. J. Invest. Dermatol. 136, 416-424.

Lionakis, M.S., Lim, J.K., Lee, C.C., and Murphy, P.M. (2011). Organ-specific innate immune responses in a mouse model of invasive candidiasis. J. Innate Immun. 3, 180-199.

Man, K., Loudon, A., and Chawla, A. (2016). Immunity around the clock. Science 354, 999-1003.

Martin, C., Burdon, P.C., Bridger, G., Gutierrez-Ramos, J.C., Williams, T.J., and Rankin, S.M. (2003). Chemokines acting via CXCR2 and CXCR4 control the release of neutrophils from the bone marrow and their return following senescence. Immunity 19, 583-593.

Muller, J.E., Tofler, G.H., and Stone, P.H. (1989). Circadian variation and triggers of onset of acute cardiovascular disease. Circulation 79, 733-743.

Nguyen, K.D., Fentress, S.J., Qiu, Y., Yun, K., Cox, J.S., and Chawla, A. (2013). Circadian gene Bmal1 regulates diurnal oscillations of Ly6C(hi) inflammatory monocytes. Science 341, 1483-1488.

Nie, Y., Waite, J., Brewer, F., Sunshine, M.J., Littman, D.R., and Zou, Y.R. (2004). The role of CXCR4 in maintaining peripheral B cell compartments and humoral immunity. J. Exp. Med. 200, 1145-1156.

Passegué, E., Wagner, E.F., and Weissman, I.L. (2004). JunB deficiency leads to a myeloproliferative disorder arising from hematopoietic stem cells. Cell $119,431-443$.

Radu, M., and Chernoff, J. (2013). An in vivo assay to test blood vessel permeability. J. Vis. Exp. 73, e50062.

Rittirsch, D., Huber-Lang, M.S., Flierl, M.A., and Ward, P.A. (2009). Immunodesign of experimental sepsis by cecal ligation and puncture. Nat. Protoc. 4, 31-36.

Robinson, M.D., McCarthy, D.J., and Smyth, G.K. (2010). edgeR: a Bioconductor package for differential expression analysis of digital gene expression data. Bioinformatics 26, 139-140.

Schageman, J., Zeringer, E., Li, M., Barta, T., Lea, K., Gu, J., Magdaleno, S., Setterquist, R., and Vlassov, A.V. (2013). The complete exosome workflow soIution: from isolation to characterization of RNA cargo. BioMed Res. Int. 2013, 253957.

Scheiermann, C., Kunisaki, Y., Lucas, D., Chow, A., Jang, J.E., Zhang, D., Hashimoto, D., Merad, M., and Frenette, P.S. (2012). Adrenergic nerves govern circadian leukocyte recruitment to tissues. Immunity 37, 290-301.

Scheiermann, C., Kunisaki, Y., and Frenette, P.S. (2013). Circadian control of the immune system. Nat. Rev. Immunol. 13, 190-198. 
Schindelin, J., Rueden, C.T., Hiner, M.C., and Eliceiri, K.W. (2015). The ImageJ ecosystem: An open platform for biomedical image analysis. Mol. Reprod. Dev. 82, 518-529.

Schloss, M.J., Horckmans, M., Nitz, K., Duchene, J., Drechsler, M., Bidzhekov, K., Scheiermann, C., Weber, C., Soehnlein, O., and Steffens, S. (2016). The time-of-day of myocardial infarction onset affects healing through oscillations in cardiac neutrophil recruitment. EMBO Mol. Med. 8, 937-948.

Silver, A.C., Arjona, A., Walker, W.E., and Fikrig, E. (2012). The circadian clock controls toll-like receptor 9 -mediated innate and adaptive immunity. Immunity $36,251-261$.

Simon, S.I., Nyunt, T., Florine-Casteel, K., Ritchie, K., Ting-Beall, H.P., Evans, E., and Needham, D. (2007). Dynamics of neutrophil membrane compliance and microstructure probed with a micropipet-based piconewton force transducer. Ann. Biomed. Eng. 35, 595-604.

Sreeramkumar, V., Adrover, J.M., Ballesteros, I., Cuartero, M.I., Rossaint, J., Bilbao, I., Nácher, M., Pitaval, C., Radovanovic, I., Fukui, Y., et al. (2014). Neutrophils scan for activated platelets to initiate inflammation. Science 346, 1234-1238.

Sturn, A., Quackenbush, J., and Trajanoski, Z. (2002). Genesis: cluster analysis of microarray data. Bioinformatics 18, 207-208.

Summers, C., Rankin, S.M., Condliffe, A.M., Singh, N., Peters, A.M., and Chilvers, E.R. (2010). Neutrophil kinetics in health and disease. Trends Immunol. 31, 318-324.

Tanji-Matsuba, K., van Eeden, S.F., Saito, Y., Okazawa, M., Klut, M.E., Hayashi, S., and Hogg, J.C. (1998). Functional changes in aging polymorphonuclear leukocytes. Circulation 97, 91-98.
Tognini, P., Thaiss, C.A., Elinav, E., and Sassone-Corsi, P. (2017). Circadian Coordination of Antimicrobial Responses. Cell Host Microbe 22, 185-192.

Uhl, B., Vadlau, Y., Zuchtriegel, G., Nekolla, K., Sharaf, K., Gaertner, F., Massberg, S., Krombach, F., and Reichel, C.A. (2016). Aged neutrophils contribute to the first line of defense in the acute inflammatory response. Blood 128, 2327-2337.

Vinten-Johansen, J. (2004). Involvement of neutrophils in the pathogenesis of lethal myocardial reperfusion injury. Cardiovasc. Res. 61, 481-497.

Vintersten, K., Monetti, C., Gertsenstein, M., Zhang, P., Laszlo, L., Biechele, S., and Nagy, A. (2004). Mouse in red: red fluorescent protein expression in mouse ES cells, embryos, and adult animals. Genesis 40, 241-246.

von Andrian, U.H., Hasslen, S.R., Nelson, R.D., Erlandsen, S.L., and Butcher, E.C. (1995). A central role for microvillous receptor presentation in leukocyte adhesion under flow. Cell 82, 989-999.

Yu, X., Rollins, D., Ruhn, K.A., Stubblefield, J.J., Green, C.B., Kashiwada, M., Rothman, P.B., Takahashi, J.S., and Hooper, L.V. (2013). TH17 cell differentiation is regulated by the circadian clock. Science 342, 727-730.

Zarbock, A., Lowell, C.A., and Ley, K. (2007). Spleen tyrosine kinase Syk is necessary for E-selectin-induced alpha(L)beta(2) integrin-mediated rolling on intercellular adhesion molecule-1. Immunity 26, 773-783.

Zhang, D., Chen, G., Manwani, D., Mortha, A., Xu, C., Faith, J.J., Burk, R.D. Kunisaki, Y., Jang, J.E., Scheiermann, C., et al. (2015). Neutrophil ageing is regulated by the microbiome. Nature $525,528-532$. 


\section{STAR $\star$ METHODS}

\section{KEY RESOURCES TABLE}

\begin{tabular}{|c|c|c|}
\hline REAGENT or RESOURCE & SOURCE & IDENTIFIER \\
\hline \multicolumn{3}{|l|}{ Antibodies } \\
\hline CXCR4-APC (Human) & eBioscience & Clone 12G5; RRID: AB_1944349 \\
\hline CD11b-FITC (Human) & eBioscience & Clone ICRF44 \\
\hline CD16-Pacific Blue (Human) & $\mathrm{BD}$ & Clone 3G8 \\
\hline CD62L-PE (Human) & $\mathrm{BD}$ & Clone DREG56 \\
\hline CD11C-APC (Human) & $\mathrm{BD}$ & Clone B-ly6 \\
\hline bio & eBioscience & Clone $1 \mathrm{~A} 8$ \\
\hline Ly6G-Dylight 450 & BioXcell (conjugated in-house) & Clone 1A8; RRID: AB_1107721 \\
\hline Ly6G-Dylight 650 & BioXcell (conjugated in-house) & Clone 1A8; RRID: AB_1107721 \\
\hline Ly6G-FITC & eBioscience & Clone 1A8; RRID: AB_2572532 \\
\hline CD45-PerCP-Сy5.5 & Biolegend & Clone 30-F11; RRID: AB_893344 \\
\hline CD11b-PE & Tonbo Biosciences & Clone M1/70; RRID: AB_2621746 \\
\hline CD11b-FITC & $\mathrm{BD}$ & Clone M1/70; RRID: AB_394774 \\
\hline CXCR2-PerCP-Cy5.5 & Biolegend & Clone SA044G4; RRID: AB_2565695 \\
\hline CXCR4-APC & eBioscience & Clone 2B11; RRID: AB_10670877 \\
\hline CD41-PE & eBioscience & Clone MWReg30 RRID: AB_2538354 \\
\hline Ly6C-FITC & Biolegend & Clone HK1.4 \\
\hline CD62L-APC & eBioscience & Clone MEL-14; RRID: AB_469410 \\
\hline CD62L-FITC & eBioscience & Clone MEL-14; RRID: AB_465109 \\
\hline Anti-CXCL1 & $R \& D$ & MAB453; RRID: AB_2087696 \\
\hline Anti-CXCL2 & $R \& D$ & MAB452; RRID: AB_2230058 \\
\hline Anti-CXCL12 & $R \& D$ & MAB350; RRID: AB_2088149 \\
\hline Anti-Ly6G (depleting antibody) & BioXCell & BE0075-1; RRID: AB_1107721 \\
\hline \multicolumn{3}{|c|}{ Experimental Models: Organisms/Strains } \\
\hline WT & Charles River & C57BL/6 \\
\hline Mrp8 ${ }^{\mathrm{CRE}}$ & Passegué et al., 2004 & B6.Cg-Tg(S100A8-cre,-EGFP) 1llw \\
\hline$C x c r 2^{f l / f 1}$ & Schloss et al., 2016 & C57BL/6-Cxcr2 $2^{\operatorname{tm} 1 R m r a}$ \\
\hline$C x c r 2^{\Delta N}$ & This paper & $\mathrm{N} / \mathrm{A}$ \\
\hline Cxcr4 $4^{f 1 / f l}$ & Nie et al., 2004 & B6.129P2-Cxcr4 $4^{\mathrm{tm} 2 Y z o}$ \\
\hline$C x c r 2^{\Delta N}$ & This paper & $\mathrm{N} / \mathrm{A}$ \\
\hline Cxcr2 $2^{\text {WHIM }}$ & Balabanian et al., 2012 & $\mathrm{Cxcr} 4^{/ 1013}$ \\
\hline Arntf $f^{1 / f 1}$ & Janich et al., 2011 & B6.129S4(Cg)-Arnt| ${ }^{\text {tm1Weit }}$ \\
\hline$\left.A r n t\right|^{\Delta N}$ & This paper & $\mathrm{N} / \mathrm{A}$ \\
\hline Selp; Sele ${ }^{-1-}$ & Frenette et al., 1996 & B6.129S2-Sele ${ }^{t m 1 H y n}$ Selp ${ }^{t m 1 H y n}$ \\
\hline DsRED & Vintersten et al., 2004 & B6.Cg-Tg(CAG-DsRedMST)1Nagy \\
\hline Lyz2 ${ }^{\text {GFP }}$ & Faust et al., 2000 & B6.129P-Lyz2 ${ }^{\text {tm1(EGFP)1.1Graf/Mmmh }}$ \\
\hline Candida albicans & Del Fresno et al., 2018 & SC5314 \\
\hline \multicolumn{3}{|c|}{ Chemicals, Peptides, and Recombinant Proteins } \\
\hline CXCL12 (recombinant) & $R \& D$ & $460-S D$ \\
\hline CXCL1 (recombinant) & $R \& D$ & $453-\mathrm{KC}$ \\
\hline CXCL2 (recombinant) & $R \& D$ & 452-M2 \\
\hline TNF- $\alpha$ & $R \& D$ & 410-MT-050 \\
\hline AMD3100 & Tocris & Cat\# 3299 \\
\hline
\end{tabular}




\begin{tabular}{lll}
\hline Continued & & \\
\hline REAGENT or RESOURCE & SOURCE & IDENTIFIER \\
\hline Oligonucleotides & & N/A \\
\hline Primers for qPCR, see Table S3 & This paper & \\
\hline Critical Commercial Assays & & DY275 \\
\hline CXCL1 quantification kit & R\&D & DY276-05 \\
CXCL2 quantification kit & R\&D & DSA00 \\
CXCL12 quantification kit & R\&D & PPX-10-MXTZ766 \\
Mouse ProcartaPlex ${ }^{\text {TM }}$ & Thermo Scientific & \\
\hline Software and Algorithms & & Schindelin et al., 2015 \\
\hline ImageJ & NIH & RRID: SCR_007370 \\
Imaris & Bitplane & RRID: SCR_015775 \\
Genesis & TÜ Graz & RRID: SCR_002798 \\
Prism & Graphpad & RRID: SCR_008520 \\
\hline Flowjo vX & Treestar & \\
\hline
\end{tabular}

\section{CONTACT FOR REAGENT AND RESOURCE SHARING}

Reagents used in this study are readily available from the noted commercial suppliers in the method itself. Further information and requests for other resources and reagents should be directed to and will be fulfilled by the Lead Contact, Andrés Hidalgo (ahidalgo@ cnic.es).

\section{EXPERIMENTAL MODEL AND SUBJECT DETAILS}

\section{Mice}

All experiments were performed in 7- to 18-week-old male C57BL/6 mice kept in a specific pathogen-free facility at Centro Nacional de Investigaciones Cardiovasculares (CNIC) under a $12 \mathrm{~h}$ light and $12 \mathrm{~h}$ dark schedule (lights on at $7 \mathrm{am}$, off at $7 \mathrm{pm}$ ), with water and chow available ad libitum. To generate mice with neutrophil-specific deficiency, we crossed Arntl/flfl (kindly provided by S. Benitah) (Janich et al., 2011), Cxcr4 ${ }^{\text {fl/fl }}$ (Nie et al., 2004) or Cxcr2 fl/f (Schloss et al., 2016) with hMRP8CRE mice (Passegué et al., 2004), Cxcr4 ${ }^{\text {WHIM }}$ mice with a hyper-signalling form of CXCR4 have been described (Balabanian et al., 2012) and were used as donors to generate BM chimeras. Cxc/2 ${ }^{-1-}$ mice were obtained from Jackson. In some control experiments we crossed Cxcr ${ }^{f l / f}$ mice with the Ly6G ${ }^{\mathrm{CRE}}$ mice (Hasenberg et al., 2015). Mice deficient in $\mathrm{P}$ an E-selectins (Selp; Sele ${ }^{-/-}$) have been previously described (Frenette et al., 1996). To obtain reporter mice for intravital microscopy (IVM) studies, we crossed Selp; Sele ${ }^{-/-}$mice with transgenic mice expressing DsRed under the control of the $\beta$-actin promoter (DsRed ${ }^{\text {Tg; }}$ Vintersten et al., 2004) or with the Lyz2 ${ }^{\text {GFP }}$ reporter mouse (Selp; Sele ${ }^{-1-;}$ GFP mice)(Faust et al., 2000). Both were also used in wild-type reporters for some intravital imaging experiments. In control experiments, we confirmed that wild-type mice used as controls yielded a similar phenotype compared with $h M R P 8^{\text {cre }}$ alone, Arntfl/fl and Cxcr/ ${ }^{f f l}$ mice (data not shown). No specific randomization method was followed in this study. All experimental procedures were approved by the Animal Care and Ethics Committee of CNIC and the regional authorities.

\section{Human Studies}

The study comprised blood from 12 healthy volunteers withdrawn at 12am, 4pm, 8pm, 12pm, 4am, and 8am. The study complied with current Spanish legislation on clinical research in humans and was approved by the Ethics Committee for Clinical Research of Hospital Universitario de la Princesa. All volunteers gave written informed consent to participate in the study.

\section{METHOD DETAILS}

\section{Analysis of Human Samples}

Total blood obtained from 12 healthy volunteers at 12am, 4pm, 8pm, 12pm, 4am and 8am and erythrocytes lysed in hypotonic buffer. Cells were incubated in 100 $\mu$ PBS buffer containing 2 mM EDTA and 1\% BSA (PEB buffer) with the following antibodies: anti-CXCR4-allophycocianin (APC; clone 12G5), anti-CD11b-FITC (clone ICRF44; both from eBiosciences), anti-CD16-pacific blue (clone 3G8), anti-CD62L-phycoerythrin (PE; clone DREG56), anti-CD11c-APC and 7AAD (all from BD Biosciences). Cells were washed and analysed in a Canto flow cytometer at the Hospital de la Princesa, Madrid. 


\section{Parabiosis}

We followed previously published procedures (Casanova-Acebes et al., 2013). Briefly, anesthetized mice were shaved and matched incisions were made from the olecranon to the knee joint, then olecranon and knee were attached by a single suture from one mouse to the other, using 5-0 polypropylene, and the dorsal and ventral skins were stitched by continuous suture. Analyses were done 4 to 6 weeks after surgery.

\section{Cytometry and Cell Sorting}

Cytometric analyses were performed using a Sony SP6800 Spectral Analyzer (Sony Biotechnology, Japan) or a LSRII Fortessa. For human sample cytometry we used a Canto flow cytometer (BD BioSciences). All cell sorting experiments were performed using an FACS Aria cell sorter (BD Biosciences), except the circadian sorting of blood neutrophils for qPCR analysis, which was performed using a Sony SH800S Cell Sorter. In all cases we obtained purities $>95 \%$. All analyses, except for human samples, were done at the Cellomics Unit of the CNIC. All antibodies and streptavidin conjugates used in this study are listed in Table S2.

\section{Whole-Mount Staining of Excised Cremaster Muscles}

Excised cremaster muscles were fixed in $4 \%$ paraformaldehyde at $4^{\circ} \mathrm{C}$ overnight. Fixed samples were washed 3 times in PBS containing $0.5 \%$ Triton-x 100 (PBST) and blocked for $2 \mathrm{~h}$ in PBST 25\% FBS at room temperature with shaking. Staining of neutrophils was performed using a biotinylated anti-Mrp14 antibody (clone 2B10 kindly provided by Dr. N. Hogg, Cancer Research UK, London) and blood vessels with an anti-CD31 antibody (BD Biosciences) in $10 \% \mathrm{FBS}-\mathrm{PBST}$ overnight at $4{ }^{\circ} \mathrm{C}$ with shaking. Cremaster muscles were then washed and incubated with secondary antibody (goat anti-rabbit-Alexa 405 or -Alexa 647; Life Technologies) and Alexa-488 conjugated Streptavidin in 10\%FBS-PBST for $4 \mathrm{~h}$ at room temperature. Samples were then washed and mounted in Mowiol 4-88 (Mw 31,000; Sigma). Imaging of whole-mount intestines was performed using a Leica SP8 X confocal microscopy system coupled to a DMI6000 inverted microscope, with 10x (HC PL Fluotar 10x/0.3 Dry) or 63x (HC PL Apo CS2 63x/1.4 OIL) magnification objectives. For in-depth quantification, large Z-stack and panoramic-stitched images were taken with a Nikon A1R confocal system coupled to a Nikon Eclipse-Ti inverted microscope with the following lines: Diode 402nm Argon Laser 457, 476, 488, 514nm Diode 561nm HeNe Laser 642nm using a Plan Apo 10x/0,45 dry objective and the software NIS Elements AR 4.30.02 (Build 1053 LO, 64 bits, Nikon Instruments, Tokyo, Japan) for acquisition of confocal 3D tile-scan images of the whole cremaster muscle, which were afterwards analysed using Imaris (Bitplane, Zurich, Switzerland). All imaging was performed at the Microscopy \& Dynamic Imaging Unit of CNIC.

RNA Isolation, Reverse Transcription, and rtPCR

Total RNA was prepared with the RNA Extraction RNeasy Plus Mini-kit (QIAGEN) and RNA was reverse-transcribed with the High-Capacity cDNA Reverse Transcription kit (Applied Biosystems; Carlsbad, CA) according to the manufacturer's protocol. Real-time quantitative PCR (SYBR-green, Applied Biosystems) assays were performed with an Applied Biosystems 7900HT Fast Real-Time PCR System sequencer detector. Expression was normalized to the expression of the 36b4 housekeeping gene. Primer sequences are listed in the Table S3.

\section{Chromatin Immunoprecipitation (ChIP)}

Neutrophils were sorted from the bone marrow as indicated previously and fixed in $0.75 \%$ formaldehyde for 10 min at room temperature. Formaldehyde was then quenched with glycine (final concentration $0.26 \mathrm{M}$ ) for $5 \mathrm{~min}$. After washing twice with cold PBS, cells were pelleted and frozen at $-80^{\circ} \mathrm{C}$. Each sample was lysed in $0.25 \mathrm{ml}$ of lysis buffer $(1 \% \mathrm{SDS}, 10 \mathrm{Mm}$ EDTA, $50 \mathrm{mM}$ Tris-HCl pH 8 , $1 \mathrm{mM}$ PMSF, $5 \mu \mathrm{g} / \mathrm{ml}$ leupeptin-aprotinin, $1 \mu \mathrm{g} / \mathrm{ml}$ pepstatin A, $10 \mathrm{mM} \mathrm{NaF}, 10 \mathrm{mM}$ sodium orthovanadate, and $10 \mathrm{mM} \beta$-glycerophosphate) for $30 \mathrm{~min}$ with rotation at room temperature. Lysates were sonicated using the Diagenode Bioruptor sonication system (Diagenode, Bioruptor UCD-200TM-EX). Each sample was sonicated for two rounds of six cycles (30s ON and 30s OFF) at the high power setting to obtain DNA fragments between 500 and $1000 \mathrm{bp}$. After sonication, samples were centrifuged to remove insoluble debris, supernatants were collected and $5 \%$ of each sample was separated to use as a measure of chromatin input for normalization. The rest of the sample was diluted 1/10 in ChIP dilution buffer (1\% TritonX-100, $20 \mathrm{mM} \mathrm{Tris-HCl,} \mathrm{pH} 8,2 \mathrm{mM}$ EDTA, $150 \mathrm{mM} \mathrm{NaCl}, 1 \mathrm{mM}$ PMSF, $5 \mu \mathrm{g} / \mathrm{ml}$ leupeptin, $5 \mu \mathrm{g} / \mathrm{ml}$ aprotinin, $1 \mu \mathrm{g} / \mathrm{ml}$ pepstatin A, $10 \mathrm{mM} \mathrm{NaF}, 10 \mathrm{mM}$ sodium orthovanadate, and $10 \mathrm{mM} \beta$-glycerophosphate) for immunoprecipitation. Samples were precleared with protein A Sepharose beads (GE Healthcare, 17-0780-01) that were previously pre-adsorbed with fish sperm DNA (Roche, 11467140 001) and bovine serum albumin (New England Biolabs, Ref. B9001S) for 1 hour at $4^{\circ} \mathrm{C}$. Anti-Bmal1 antibody (ChIP Grade [ab3350]) was added to the lysates after removing the preclearing beads and incubated overnight at $4^{\circ} \mathrm{C}$. Pre-adsorbed protein $\mathrm{A}$ Sepharose beads were then added, incubated for 1 hour at $4^{\circ} \mathrm{C}$, and then washed three times with ChIP washing buffer $(0.1 \%$ SDS, $1 \%$ TX-100, $20 \mathrm{mM} \mathrm{Tris-HCl,} \mathrm{pH} 8,2 \mathrm{mM}$ EDTA, and $150 \mathrm{mM} \mathrm{NaCl}$ ) and once with final washing buffer (0.1\% SDS, 1\% TX-100, $20 \mathrm{mM}$ Tris-HCl pH 8, 2 mM EDTA, and 500 mM $\mathrm{NaCl}$ ). To elute DNA, beads were gently shaken with $200 \mu$ l elution buffer (1\% SDS and $100 \mathrm{mM} \mathrm{NaHCO}$ ) for 45 min at room temperature. To reverse the crosslinking, samples were incubated overnight at $65^{\circ} \mathrm{C}$. Then samples were incubated with $3 \mu \mathrm{RNase}$ (Roche, 11119915001$) 30 \mathrm{~min}$ at $37^{\circ} \mathrm{C}$ prior to the addition of $8 \mu$ of Proteinase K (Roche, 3115828001 ) for 1 hour at $50^{\circ} \mathrm{C}$ and DNA was purified by ethanol precipitation. Immunoprecipitated chromatins and their respective inputs before immunoprecipitation were analyzed by RT-qPCR using the primers listed in Table S3. The primers for the Cxcl2, Per2 and Nr1d1 promoter regions were designed in the vicinity of E-box sequences. 
Intravital Imaging of the Mouse Skin

For intravital microscopy of the dermal microcirculation, the dorsal side of the ear of anesthetized mice was mounted on a custombuilt support, and we acquired images from several venules in 2 minute-long time-lapse videos at $3 \mathrm{~s}$ intervals. We used the VIVO system built by $3 i$ (Intelligent Imaging Innovations, Dever, CO) upon an Axio Examiner Z.1 workstation (Zeiss, Oberkochen, Germany) and mounted on a 3D motorized stage (Sutter Instrument, Novato, CA). The system was equipped with a CoolLED pE widefield fluorescence LED light source (CoolLED Ltd. UK) and a quad pass filter cube with Semrock Di01-R405/488/561/635 dichroic and FF01-446/523/600/677 emitter. A plan-apochromat 40x W NA1.0 objective (Zeiss) was used and images were collected with a CoolSnap HQ2 camera (Photometrics, Tucson, AZ). The system was run on a Dell Precision T7500 computer system (Dell Inc., Round Rock, TX) using the SlideBook software (Intelligent Imaging Innovations). Acquisitions were made at ZT5, ZT9 or ZT13 and neutrophils were stained with an AF647-conjugated anti-Ly6G antibody (clone 1A8, BioXcell), while blood vessels were visualized using red fluorescent Dextran (Molecular Probes). In some groups, anti-P and E-selectin antibodies or Rat lgG control antibody (25 $\mu \mathrm{g} / \mathrm{mouse}$ ) were injected $2 \mathrm{~h}$ before imaging. Quantification was done using the Image $(\mathrm{NIH}$, Bethesda, MD). Cells were considered adhered if they remained stationary on the venule for over 30 seconds. Vessel diameters were measured using the Image J software (Schindelin et al., 2015) to normalize the number of adherent cells.

\section{Intravital Microscopy of the Cremaster Muscle}

Intravital microscopy of the cremaster muscle after TNF- $\alpha$ stimulation (R\&D Systems, $0.5 \mu \mathrm{g}$ intrascrotal injection) was performed as previously reported (Hidalgo et al., 2009) using the VIVO system indicated above. For confocal IVM, we used laser stacks for 488,561 and $640 \mathrm{~nm}$ beams coupled with a confocal scanner (Yokogawa CSUX-A1; Yokogawa, Japan) and images were acquired with $0.5 \mu \mathrm{m}$ Z-intervals. The SlideBook software was used for acquisition and analysis. Ten to twenty venules segments per mouse were analysed 150 to 210 min after TNF- $\alpha$ treatment in multiple fluorescence channels (Cy3/561 for PE, FITC/488 for FITC and Cy5/640 for APC) and bright-field images with $1 \times 1$ or $2 \times 2$ binning with 3 second interval for 2 min on each field of view. For double staining with PE- and FITC-conjugated antibodies, acquisition was facilitated in single (FITC) and quadrant (PE) filters in order to avoid bleed-through of fluorescent signals between channels. For the visualization of leukocytes, fluorescently labelled anti-Ly6G-APC, anti-Ly6C-FITC and anti-CD62L-APC antibodies were injected intravenously at $1 \mu \mathrm{g} / \mathrm{mouse}$.

For analysis of rolling and adhered cells to the inflamed endothelium we used the SlideBook software. Counts of rolling or adhered cells in 2-minute captures (captured at 3 second intervals) were normalized using the width of the vessel to allow comparison among all vessels. For adhesion or rolling efficiency indices, these data were compared with the frequency of free-flowing WT and experimental cells in the blood for each mouse, which was obtained from cytometric analysis of blood neutrophils for each parabiont or BM chimeric mouse. Kinetic parameters for crawling neutrophils were calculated using ImageJ, with the help of the Manual Tracking plugin (Fabrice Cordelières, Institut Curie, France) and the Chemotaxis and Migration Tool (Gerhard Trapp and Elias Horn, ibidi GmbH, Germany). Analyses of extravasated neutrophil were performed on large tile-scans of whole-mounted cremaster muscles with Imaris (Bitplane AG, Switzerland). We performed blind automatic counting of extravasated neutrophils by masking out the vessels using CD31 ${ }^{+}$fluorescence (on the BMT WT:Arnt/ ${ }^{\Delta \mathrm{N}}$, WT: Cxcr4 ${ }^{\Delta \mathrm{N}}$ chimeras and Selp; Sele ${ }^{-1-}:$ Arntl ${ }^{\Delta \mathrm{N}}$ parabionts) or manually delimiting the vessels using brightfield or laminin fluorescence (on Selp; Sele ${ }^{-1-}$ with WT parabionts). Kinetic parameters for extravasated neutrophils were obtained in the DsRed ${ }^{\mathrm{Tg}}$ with Selp; Sele ${ }^{-1-}-$ Lyz2 $^{\mathrm{GFP}}$ parabionts using automatic tracking of cells with Imaris.

Intravital Imaging of Ischemia and Reperfusion Injury

Mouse cremaster were prepared as indicated without TNF- $\alpha$ stimulation. Upon exteriorization of the muscle we intravenously injected fluorescent antibodies to label cellular populations. Ly6G-APC (clone 1A8; BioXcell) for neutrophils, CD41-PE (eBioscience) for platelets, Ly6C-FITC (Biolegend) and CD62L-APC (BD Bioscience). Ischemia was achieved by occlusion of the incoming and outgoing vessels by clamping the tissue connecting the muscle and the animal's body with a 15mm Micro Serrefine clamp (Fine Science Tools, Heidelberg, Germany) for 45 minutes. Reperfusion was achieved by removal of the clamp. In some experiments neutrophils were depleted prior ischemia by injecting an anti-Ly6G-antibody (see below). Recordings were made with 3 second interval for 2 minutes for each field of view. Imaging was performed before and during ischemia, and during reperfusion. Some mice were treated intravenously with $300 \mu \mathrm{g}$ of $\mathrm{Cl}$-amidine (Cayman Chemical Company, Ann Arbor) 1h before imaging, or with $500 \mu \mathrm{g}$ DNAse I (Roche, Basel, Switzerland) immediately before imaging.

\section{Analysis of Neutrophil Clearance in the Steady State}

11- to 18-week-old DsRed ${ }^{T g}$ mice were analysed after 1 month in parabiosis with non-fluorescent $C x c r 2^{\Delta \mathrm{N}}, C x c r 4^{\Delta \mathrm{N}}, A r n t l^{\Delta \mathrm{N}}$ and Selp; Sele ${ }^{-1-}$ mice or wild-type controls. The blood of each WT mice was analysed and used to obtain the ratio of neutrophils derived from each partner. Mice were sacrificed with $\mathrm{CO}_{2}$ and carefully perfused with $30 \mathrm{ml}$ of PBS to remove all blood. Tissues (white adipose tissue or WAT, large intestine, liver, lung, skin and spleen) were extracted and kept in cold PBS (except liver, kept at room temperature in HBSS) and processed immediately. Skin, large intestine (colon), lung and WAT were digested in HBSS with liberase $\left(1 \mathrm{U} / \mathrm{ml}\right.$, Roche) and DNAse I $\left(1 \mathrm{mU} / \mathrm{ml}\right.$, Sigma) for $30 \mathrm{~min}$ at $37^{\circ} \mathrm{C}$. Bone marrow and spleen were mechanically dissociated to prepare single-cell suspensions by flushing and straining, respectively. Enrichment of leukocytes in liver was performed by centrifugation using a 36\% Percoll (GE Healthcare, diluted in HBSS) gradient. Colons were pre-incubated with HBSS containing $5 \mathrm{mM}$ EDTA for $45 \mathrm{~min}$ at $37^{\circ} \mathrm{C}$ before digestion to remove epithelial cells. Blood counts were analysed in an automated 
hemocytometer (Abacus Junior, Diatron; Holliston, USA) and blood red blood cells (RBC) lysed in a hypotonic buffer. Single-cell suspensions from tissues were incubated with fluorescently-conjugated antibodies against CD45, CD11b and Ly6G (BioXcell) and analysed in a Sony SP6800 Spectral Analyzer. DsRed+ Ly6G ${ }^{\text {hi }}$ cells and DsRed ${ }^{N E G}$ Ly6G $^{H I}$ cells discriminated host- (DsRedTg) from partner-derived neutrophils. To normalize values between the different parabiotic pairs we corrected the ratios of host versus partner-derived neutrophils in each tissue with the ratios present in blood of each parabiotic pair. Deviations from the original ratio in blood was used to estimate the efficiency of migration of partner-derived neutrophils in each organ. Finally, infiltration efficiencies were normalized to the values obtained for the DsRed ${ }^{\top \mathrm{g}}: \mathrm{WT}$ pairs, which was set as the control group.

\section{Quantification of Neutrophil Numbers in Tissues}

In some experiments, we measured absolute numbers of neutrophils present in tissues (see Neutrophil clearance assays). Truecount beads (Truecount absolute counting tubes, BD) were prepared at a concentration of 10,000 beads per ml of PBS buffer. $300 \mu$ l of the bead suspension were added to single cell suspensions stained for flow cytometry, and then neutrophil number values were calculated based on the number of beads per tube and corrected by the weight or volume of tissue analysed.

\section{Chemokine Quantification in Plasma}

CXCL12, CXCL1 and CXCL2 amounts were measured in plasma samples taken every 4h from WT mice using commercially available ELISA reagents, following the manufacturer's protocol (R\&D Systems; Minneapolis; MN).

\section{RNA-Sequencing}

Blood neutrophils were FACS sorted using by Ly6G and DAPI labelling, with typical purities > 95\%. cDNA amplification from neutrophil RNA (1275pg) and generation of index-tagged sequencing libraries were carried out using the Ovation ${ }^{\circ}$ Single Cell RNA-Seq System (NuGEN $\mathbb{R}$ Technologies, San Carlos CA). Libraries were quantified using a Quant-iTTM dsDNA HS assay with the Q-bit fluorometer (Life Technologies, Carlsbad, California). Average library size and size distribution were determined using a High Sensitivity DNA assay in an Agilent 2100 Bioanalyzer (Agilent Technologies, Santa Clara CA). Libraries were normalized to 10nM using Tris-Cl 10mM, pH8.5 with 0.1\% Tween 20. Libraries were applied to an Illumina flow cell for cluster generation (True Seq SR Cluster Kit V2 cBot) and $61 \mathrm{nt}$ long, single-end reads were generated on a Genome Analyzer Ilx, using the TruSeq SBS Kit v5 (Illumina) and following the standard RNA sequencing protocol. Reads were further processed using the CASAVA package (Illumina) to demultiplex reads according to adapter indexes and to produce fastq files. Read quality was determined with the application FastQC (Schageman et al., 2013). The RNA sequencing experiments were performed at the Genomics Unit at CNIC.

\section{Western Blotting}

Blood neutrophils were sorted based on Ly6G (clone 1A8) expression. Purified cells were lysed in RIPA buffer containing 50 mM Tris- $\mathrm{HCl}, \mathrm{pH} 8 ; 150 \mathrm{mM} \mathrm{NaCl} ; 1 \%$ Triton X-100; 0.5\% sodium deoxycholate; $0.1 \%$ SDS; $1 \mathrm{mM}$ PMSF (Sigma) and a protease inhibitor cocktail (Sigma). Proteins from $2 \times 10^{5}$ lysed cells were separated by $10 \%$ SDS-PAGE and transferred onto PVDF membrane. Membranes were incubated overnight with antibodies against Bmal1 (Bethyl labs) and $\beta$-Actin (Abcam) at 1:1000 dilutions, and then thoroughly washed and incubated with HRP-conjugated anti-rabbit or anti-mouse antibody (1:1000; GE Healthcare Life Sciences). Blots were visualized using the chemiluminescent Luminata Forte Western HRP Substrate (Millipore).

\section{Generation of Transplant Chimeras}

To address the aging status of mutants in the same physiological context as wild-type cells, we generated mixed bone marrow chimeras, which has the added advantage of allowing simultaneous staining and analysis of willd-type and mutant cells. Donor BM cells were harvested from DsRed ${ }^{\top \mathrm{g}}$ or experimental models by flushing the femur with PBS. Recipient wild-type C57BL/6 mice were lethally irradiated (two 6Gy doses, $3 \mathrm{~h}$ apart) before receiving 1 million bone marrow nucleated cells by intravenous injection. For mixed chimeras, equal numbers of experimental and DsRed ${ }^{T g} \mathrm{BM}$ cells were mixed before intravenous injection. Engraftment of recipient animals was assessed 8-10 weeks after transplantation by analysis of the percentage of mutant and DsRed ${ }^{\mathrm{Tg}}$ leukocytes in blood by flow cytometry.

\section{Circadian Analysis of Aging Markers and RNA Extraction}

Circadian blood samples were extracted every $4 \mathrm{~h}$ during $24 \mathrm{~h}$ from wild-type or experimental mice, starting at ZT1 (Zeitgeber time, 1 hour after the onset of light, 7:00 at the CNIC's animal facility). For circadian surface marker analysis, RBC were lysed in hypotonic lysis buffer $\left(0.15 \mathrm{M} \mathrm{KH}_{4} \mathrm{Cl}, 0.01 \mathrm{M} \mathrm{KHCO3}\right.$ and $0.01 \mathrm{M}$ EDTA in water) and incubated 15 minutes with $0.25 \mu \mathrm{g}$ anti-Ly6G (1A8 clone, BioXcell), -CXCR2 (Biolegend) and -CXCR4 (eBioscience) antibodies, washed and analysed in a Sony SP6800 Spectral Analyser. Analysis was performed using Flowjo vX (Tree Star Inc, Ashland, OR). For circadian RNA assays, blood taken at above circadian time points was lysed for RBCs and sorted using a Sony SH800S Cell Sorter (Sony Biotechnology, Japan) based on viable Ly6G ${ }^{+}$ cells. RNA was extracted as indicated above.

\section{Analysis of Neutrophil Aging in Light-Dark and Dark-Dark Light Regimes}

To check the circadian nature of neutrophil aging we compared twice a day (ZT5 and ZT13) the number of CD62 $\mathrm{L}^{\mathrm{LO}}$ aged neutrophils for 4 consecutive days in WT mice subjected to $12 \mathrm{~h}: 12 \mathrm{~h}$ light: dark cycles, and in mice subjected to constant darkness in a light 
cabinet (LFC 3-16 with EF700ET programmer from E. Becker \& Co) starting 24h after the onset of the light schedule. We analysed blood neutrophils at the indicated time points by flow cytometry as indicated above.

\section{Entrainment of Neutrophil Aging by Inverted Light Regime}

We maintained wild-type mice in 12h: 12h light-dark or dark-light (inverted light cycle) regimes using light-cabinets (Light/ Dark Chamber LT400 from Parkbio). After 3 weeks of this inverted regime we analysed peripheral blood neutrophils every 6 hours for 24 hours by flow cytometry, as indicated above.

\section{Infection with Candida albicans}

Mice were intravenously infected with $1.5 \times 10^{5} \mathrm{C}$. albicans conidia (SC5314 strain) and monitored daily for weight loss and general health following our institutional guidance. Infections were performed either at ZT5 or ZT13. Kidney fungal burden was determined at day 6 post-infection by plating organ homogenates in serial dilutions on YPD plates (Sigma); colony-forming units (CFUs) were counted after growth for $48 \mathrm{hr}$ at $30^{\circ} \mathrm{C}$. Flow cytometry analysis of renal leukocyte infiltrates was performed on cell suspensions obtained from kidney homogenates obtained by digestion with $0.025 \mathrm{mg} / \mathrm{ml}$ of Liberase TL (Roche) for 10 min at $37^{\circ} \mathrm{C}$ and filtered through $40 \mu \mathrm{m}$ cell strainers (BD PharMingen). Phenotypic analyses of renal leukocyte infiltration were performed by flow cytometry.

\section{Heat-Killed Candida albicans (HKC) Phagocytosis Assay}

Candida albicans conidia were heat-killed by boiling for 30 minutes. To quantify the phagocytic capacity, BM-sorted neutrophils were stained with $5 \mu \mathrm{M}$ CFSE and exposed to labelled HKC labelled with $2.5 \mu \mathrm{M}$ Cell Violet-labelled (both from Molecular probes) at a $30: 1$ ratio, for $15 \mathrm{~min}$ at $37^{\circ}$. After washing, cells were recovered on ice with PBS containing $5 \mathrm{mM}$ EDTA. To remove bound but not internalized HKC, cells were incubated in Trypsin-EDTA ( $0.25 \%$; Life Technologies) for 15 minutes at $37^{\circ}$ prior to analysis by flow cytometry. Cells were fixed in $4 \%$ paraformaldehyde and neutrophils engulfing HKC were identified as double-positive cells for Cell Violet and CFSE. Cytospin preparations were also done for microscopic inspection of phagocytosis.

\section{Mouse Model of Acute Myocardial Infarction}

Male 8- to 12-week-old mice were subjected to 45 min occlusion of the left anterior descending (LAD) coronary artery followed by $1 \mathrm{~h}$ reperfusion (for infarct size). For survival experiments, the LAD was reperfused for up to $24 \mathrm{~h}$ and mice monitored hourly for the first $6 \mathrm{~h}$, and at 16, 20 and $24 \mathrm{~h}$. The I/R procedure was performed as previously described (García-Prieto et al., 2017). In some experiments mice were depleted of neutrophils as indicated below. Briefly, fully anesthetized animals were intubated and temperature controlled throughout the experiment at $36.5^{\circ} \mathrm{C}$ to prevent hypothermic cardioprotection. Thoracotomy was then performed and the LAD was ligated with a nylon 8/0 monofilament suture for $45 \mathrm{~min}$. The electrocardiogram was monitored (MP36R, Biopac Systems Inc.) to confirm total coronary artery occlusion (ST-segment elevation) throughout the $45 \mathrm{~min}$ ischemia. At the end of the ischemia, the chest was closed and animals were kept with $100 \% \mathrm{O}_{2}$ and analgesized with buprenorphine (subcutaneous injection, $0.1 \mathrm{mg} / \mathrm{kg}$ ). For quantification of infarct size, mice were re-anesthetized and re-intubated, and the LAD coronary artery was re-occluded by ligating the suture in the same position as the original infarction. Animals were then sacrificed and $1 \mathrm{~mL}$ of $1 \%$ Evans Blue dye (Sigma) was infused IV to delineate the Area at Risk (AAR: myocardium lacking blood flow, i.e., negative for blue dye staining). The left ventricle (LV) was isolated, cut into transverse slices (5-7 1-mm thick slices per LV), and both sides were imaged. To delineate the infarcted (necrotic) myocardium, slices were incubated in triphenyltetrazolium chloride (TTC, Sigma) at $37^{\circ} \mathrm{C}$ for 15 min. The slices were then re-photographed, weighed, and regions negative for Evans Blue staining (AAR) and for TTC (infarcted myocardium) were quantified using ImageJ ( $\mathrm{NIH}$, Bethesda, MD). Percentage values for AAR and infarcted myocardium were corrected to mg independently for each slice. Absolute AAR and infarct size were determined as the mg:mg ratio of AAR:LV and infarcted myocardium:AAR, respectively. Outcome assessment was performed blind to condition (mouse type, zeitgeber time or treatment).

\section{Brain Ischemia}

To induce brain ischemia (without reperfusion injury) we followed previously described protocols (Sreeramkumar et al., 2014). Mice were anesthetized with isoflurane $1.5 \%-2 \%$ in a mixture of $80 \%$ air and $20 \%$ oxygen, and body temperature was maintained with a heating pad during the surgical procedure and anaesthesia recovery. Mice were subjected to permanent focal cerebral ischemia (middle cerebral artery occlusion - pMCAO) through the distal occlusion of middle cerebral artery by ligature of the trunk just before its bifurcation between the frontal and parietal branches with a 9-0 suture, in combination with the occlusion of the ipsilateral common carotid artery. Following surgery, individual animals were returned to their cages with free access to water and food. All the groups were performed and quantified in a randomized fashion by investigators blinded to groups. Physiological parameters were not significantly different among the different groups studied. Infarct size was determined by magnetic resonance imaging 48 hours after MCAO using a BIOSPEC BMT 47/40 (Bruker, Ettlingen, Germany). Infarct volume was calculated using the ImageJ software (NIH, USA) from the T2-weighted images. With the observer masked to the experimental conditions, the areas of the infarcted tissue (InfArea), the whole ipsilesional hemisphere (IpsArea) and the whole contralesional hemisphere (ContrArea) were delineated for each slice. Then, infarct volume, expressed as percentage of the hemisphere that is infarcted (\% $\mathrm{IH})$ was calculated using the formula: $\% \mathrm{IH}=\mathrm{InfVol} /$ ContrVol$^{\star} 100$ where InfVol (Infarcted Tissue Volume) $=\Sigma$ InfArea $_{i} /$ Swellinglndex $_{\mathrm{i}}$, ContrVol (Contralesional

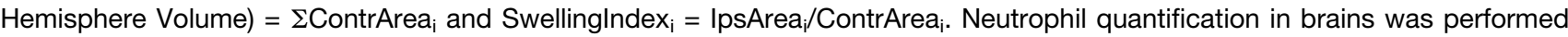
$48 \mathrm{~h}$ after surgery. The ipsilateral cortex was dissected, placed in ice-cold PBS and dissociated into a single cell suspension by 
mechanical dissociation. Cell suspensions were filtered on $70-\mu \mathrm{m}$ nylon mesh strainers and centrifuged at $300 \mathrm{~g}$ for $10 \mathrm{~min}$ at room temperature. Pellets were resuspended in $8 \mathrm{~mL}$ of $35 \%$ Percoll and overlaid on the top of $5 \mathrm{ml} \mathrm{HBSS}$. The gradient was centrifuged at $800 \mathrm{~g}$ for 40 minutes at $4^{\circ} \mathrm{C}$ and cell pellets resuspended for staining with anti-CD11b-FITC and anti-Ly-6G-PE antibodies (BD Bioscence). Stained cells were analysed in a FACSCalibur flow cytometer with CellQuest software (BD Pharmingen, San Jose, CA) and data were analysed using FlowJo software (Tree Star Inc, Ashland, OR).

\section{Scanning Electron Microscopy}

Blood from WT, Arnt/ ${ }^{\Delta \mathrm{N}}$ and Cxcr4 ${ }^{\Delta \mathrm{N}}$ was harvested, RBC lysed and leukocytes stained with Ly6G (1A8, BioXcell) before sorting in a FACS Aria sorter (BD Biosciences). Sorted cells were immediately centrifuged and fixed using $4 \%$ PFA plus $2.5 \%$ glutaraldehyde in PBS for $2 \mathrm{~h}$ at $4{ }^{\circ} \mathrm{C}$. Cells were then dehydrated by serial 5 min incubations in increasing concentrations of ethanol, (30\%>50\%>70\% $>80 \%>90 \%>100 \%$ ). Samples were dried in an automated critical point dryer (Leica EM CDP 300) and then coated in a rotarypumped coating system (Quorum Technologies Q150RS). Imaging was performed at 10kV with a field emission microscope (JEOL 6335F). Critical point drying, coating and imaging of the samples was performed at ICTS National Centre of Electron Microscopy (UCM, Madrid, Spain).

\section{CXCR2 and CXCR4 Cross-Inhibition Assays}

Wild-type mice were bled, RBC lysed and leukocytes resuspended in RPMI 1640 (Invitrogen). Some cells were pretreated with CXCL12 (50 ng/ml, R\&D Systems) for 5 minutes at $37^{\circ} \mathrm{C}$ while others were left untreated. Cells were allowed to migrate towards CXCL1 (50ng/ml, R\&D Systems) or CXCL2 (50ng/ml, R\&D Systems) through $6.5 \mathrm{~mm}$ transwells with $5 \mu \mathrm{m}$ pore polycarbonate membrane insert (Corning, NY, USA), for $1 \mathrm{~h}$ at $37^{\circ} \mathrm{C}$. Transmigrated cells were collected and stained with anti-Ly6G and antiCD62L antibodies for cytometric analysis. Migration to only media was also used as a control. Quantification was performed using Truecount beads, as indicated above.

\section{Neutrophil Depletion}

In some experiments mice were depleted of neutrophils prior to ischemia/reperfusion. Mice were injected 100 $\mu \mathrm{g}$ of anti-Ly6G antibody (1A8 clone; BioXCell; West Lebanon, $\mathrm{NH}$ ) intraperitoneally for 2 consecutive days resulting in $>93 \%$ reduction in blood neutrophil counts. Lymphocyte and monocyte counts were not affected by this treatment (Casanova-Acebes et al., 2013).

\section{Zymosan-Induced Peritonitis}

Transplantation chimeras or the wild-type partner in parabiotic pairs were treated with zymosan (1mg, intraperitoneal injection, Sigma). After $2 \mathrm{~h}$ we took blood samples and obtained the peritoneal lavage for cytometric analyses and cell count. We compared the ratios of neutrophils from each donor in the peritoneum and blood to estimate the migration efficiencies of mutant cells (ratio in peritoneum / ratio in blood). We also compared the migration efficiency of neutrophils in wild-type mice at ZT5 and ZT13. In this case we measured the absolute number of neutrophils in the peritoneal lavage using counting beads (Truecount absolute counting tubes, $\mathrm{BD}$ ) and normalized migration relative to the absolute number of neutrophils in blood.

For experiments analyzing the dynamics of neutrophil aging during inflammation, we treated wild-type mice with zymosan (1mg, i.p.) and after $24 \mathrm{~h}$ we analyzed blood neutrophils twice per day (ZT5 and ZT13) for 4 consecutive days. We estimated the absolute number of aged $\left(\mathrm{CD}_{2} \mathrm{~L}^{\mathrm{LO}}\right)$ neutrophils by flow cytometry as indicated above.

\section{Soluble Selectin Binding Assays}

Transplantation chimeras were bled and RBC lysed, then cells washed in RPMI 1640 containing 5\% FBS and stained them using E- or $\mathrm{P}$-selectin/human IgM chimeras as reported (Hidalgo et al., 2007). Cells were further incubated for 15 min with a FITC-conjugated anti-human IgM (Jackson Immunoresearch) and anti-Ly6G-APC (BioXcell) antibodies. Control samples contained 5mM EDTA. Cells were analysed in a Sony SP6800 Spectral Analyzer.

\section{Auto-Perfused Flow Chamber Assay}

In order to investigate the number of rolling and adherent cells, we used a microflow chamber system (Zarbock et al., 2007). 20 $\mathrm{x}$ $200\lceil\mathrm{~m}$ rectangular glass capillaries were filled with P-selectin $(50\lceil\mathrm{~g} / \mathrm{ml})$ alone or in combination with ICAM-1 (15 $\mu \mathrm{g} / \mathrm{ml})$ and/or CXCL1 $(25\lceil\mathrm{~g} / \mathrm{ml})$ f for $2 \mathrm{hr}$ and blocked for $2 \mathrm{hr}$ with $1 \%$ casein (Pierce Chemicals, Dallas, TX). One side of the chamber was connected to a PE 10 tubing and inserted into the carotid artery. The other side of the chamber was connected to a PE 50 tubing and used to control the wall shear stress, which was calculated as described (Zarbock et al., 2007). Microscopy was conducted with a Zeiss Axioskop (Carl Zeiss, Inc., Thornwood, NY) with a saline immersion objective (SW 20, N.A. 0.5). Recordings were taken using an SW40/0.75 objective and a digital camera (Sensicam QE). Capturing on P-selectin was analyzed after 2 minutes and chemokine-induced arrest was analyzed after 6 minutes of perfusion.

\section{Cortical Beta-Actin Quantification}

Sorted neutrophils were cytospun onto Superfrost Plus microscope slides (Thermo Scientific, Waltham, USA) with a Shandon Cytospin 4 (Thermo Scientific) for 5 minutes at 500 RPM in medium acceleration. Then cells were fixed with $4 \%$ PFA in PBS for 10 minutes and blocked with $5 \%$ goat serum, $5 \%$ BSA in saline in a humid chamber for 30 minutes. Finally, cells were stained with a rabbit 
anti-mouse beta-actin antibody (ab8227, Abcam) and with a secondary goat anti-rabbit antibody conjugated with AF568. Then slides were mounted with Mowiol and captured with a Leica SP8 X confocal microscopy system. Analysis of captured images was performed using Imaris (Bitplane).

\section{ROS Quantification}

Red blood cell-lysed blood was plated in RPMI in 96-well polystyrene microplates (Corning Falcon, New York, USA) and stimulated with 50nM of phorbol 12-myristate 13-acetate (PMA) for 1h. Cells were then stained with 5mM Dihydroethidium (DHE, Thermo Fisher, Waltham, USA) for 20 minutes and stained for cytometric analysis.

\section{Chemotaxis Assay}

Whole blood was harvested and red blood cells were lysed. Cells were plated in $6.5 \mathrm{~mm}$ polycarbonate transwells with $5 \mu \mathrm{m}$ pores (Corning, Corning, USA) in RPMI medium. In the bottom well, a single chemokine was added to allow chemotactic migration: 25mg/ml CXCL12 (R\&D), 20ng/ml CXCL1 (R\&D), 5ng/ml LTB4 (Tocris), 100mM fMLP (Sigma) or 10ng/ml CCL2 (R\&D). Transwells were incubated $2 \mathrm{~h}$ at $37^{\circ} \mathrm{C}$ and transmigrated cells were harvested from the bottom well and stained for cytometric analysis. The number of transmigrated cells was assessed by the presence of a known number of Truecount beads (BD Biosciences).

\section{Multiplex Cytokine Assay}

Mice were i.p. injected with Zymosan as indicated above. After 2 hours peritoneal lavages were collected and neutrophils stained for FACS sorting. One million sorted neutrophils were incubated for 3h in RPMI containing 0.5\% BSA and 100,000 heat-killed C. albicans conidia. Supernatants were collected and frozen at $-80^{\circ} \mathrm{C}$ until cytokine quantification was performed. CXCL1, IL-10, IL-1 $\beta$, IL-12, TNF- $\alpha$, G-CSF, IL-23, CXCL2, IL-6 and CCL2 were measured in neutrophil supernatants using the Mouse ProcartaPlex ${ }^{\mathrm{TM}}$ Multiplex Immunoassay (PPX-10-MXTZ766), following the manufacturer's protocol (Thermo Scientific, Waltham, USA). For TGF- $\beta 1$ detection, samples were activated with $\mathrm{HCl}$ and measured using commercial TGF beta-1 Mouse ProcartaPlex ${ }^{\mathrm{TM}}$ Simplex Kit (EPX01A-20608901). Data acquisition was performed on a MagPix instrument (Luminex Inc, Houston, TX) using xPONENT v4.2 software (Luminex) and analyzed with ProcartaPlex Analyst software (v1.0; Thermo Scientific).

\section{AMD3100-Induced Neutrophilia}

$2.5 \mathrm{mg} / \mathrm{kg}$ of AMD3100 (Tocris) was injected intraperitoneally into wild-type mice $1 \mathrm{~h}$ before analysis. Then blood neutrophils were analyzed at ZT5 in an automated hemocytometer (Abacus Junior) and stained for cytometric analysis as previously described. At this time (1h after injection of AMD3100) we subjected control or AMD3100-treated mice to AMI and Candida albicans infection, as previously described.

\section{Neutrophil Transfer Experiments}

To increase the yield of fresh neutrophils we used AMD3100-treated mice as neutrophil donors (see Figure S5G). To minimize ex vivo manipulation of neutrophils we transferred $200 \mu \mathrm{l}$ of freshly extracted blood from donor mice by i.v. injection into host wild-type mice at ZT5. Aging markers in host and donor cells were then analyzed 5 minutes and 5 hours after inoculation in the peripheral blood of host mice by flow cytometry as indicated above.

\section{BrdU Labelling}

For metabolic labelling with 5-Bromodeoxyuridine, mice were intraperitoneally injected with a single dose of 2.5mg BrdU (BD Biosciences). Blood samples were collected at indicated times and stained for Ly6G, CD62L, CXCR2 and CXCR4, followed by fixation and intracellular labelling of BrdU using an APC-conjugated anti-BrdU antibody as per manufacturer's instructions (BD Biosciences).

\section{In Vivo CXCL1 and CXCL2 Blockade}

For in vivo blockade of CXCL1 and CXCL2, mice were injected intraperitoneally twice with $50 \mu \mathrm{g}$ of isotype or monoclonal antibody against CXCL1 (MAB453, R\&D) or CXCL2 (MAB452, R\&D) the night before the analysis (-17h) and the same day (-5h). Blood from treated mice was harvested and analysed by flow cytometry as previously described. In a set of mice, intravital imaging was performed to analyse neutrophil behaviour in the microvasculature of the cremaster muscle by intravital microscopy as previously described.

\section{Cecal Ligation and Puncture (CLP)-Induced Sepsis}

CLP was performed as previously described (Rittirsch et al., 2009). Briefly, the peritoneal cavity of ketamine/xylazine-anesthetised mice was exposed with a small incision and the cecum was exteriorized. $80 \%$ of the cecum distal to the ileo-cecal valve was ligated using non-absorbable 7-0 suture. A 23-gauge needle was then used to puncture both walls of the distal end of the cecum, and a small drop of faeces was extruded through the perforation. The ligated and punctured cecum was relocated inside the peritoneal cavity and both peritoneum and skin were closed. Mice were then treated with s.c. injection of Buprenorphine. Control mice (sham) were included with the same procedure but without ligation or puncture. 


\section{Evans Blue Vascular Permeability Assay}

To address whether the constitutive presence of fresh or aged neutrophils in the mutant mice affected the vascular integrity, we performed vascular permeability assays as previously described (Radu and Chernoff, 2013). In brief, a 0.5\% solution of Evans blue in sterile PBS was prepared and $200 \mu \mathrm{l}$ of the solution was i.v. injected into WT or mutant mice. 5 minutes after the transfer mice were sacrificed and tissues extracted and weighted. Then, tissues were submerged in $1 \mathrm{ml}$ formamide and incubated at $50^{\circ} \mathrm{C}$ for $24 \mathrm{~h}$. Tissues were removed and the tubes centrifuged for 5 minutes at $645 \mathrm{~g}$. Finally, supernatants were measured for absorbance at $610 \mathrm{~nm}$ using an xMark Microplate Spectrophotometer (BioRad) plate reader. The vascular permeability test was performed in untreated and LPS-treated $(10 \mathrm{mg} / \mathrm{kg})$ mice.

\section{Analysis of Endothelial Proliferation and Apoptosis}

We processed tissues from WT or mutant mice as previously described (see section Analysis of neutrophil clearance in the steadystate) to obtain single cell suspensions. One half of the suspension was used to measure apoptosis and the rest to assess proliferation. For proliferation we measured Ki67 by intra-nuclear staining in endothelial cells. Cells were stained with anti-CD45 conjugated with PerCP/Cy5.5 (BioLegend) and anti-CD31-APC (eBioScience) and then fixed and permeabilized using the Fix/Perm and Perm Buffers (eBiosciences) according to manufacturer's instructions. Cells were then stained for 20 minutes at $4^{\circ} \mathrm{C}$ with an antimouse and rat Ki67 antibody labelled with eFluor660 (Thermo Fisher) and analysed by flow cytometry.

For quantification of apoptotic endothelial cells we measured Annexin V binding and DAPI labelling. Cell suspensions were incubated with 1:200 of anti-CD45 and anti-CD31, washed twice in cold PBS and resuspended in Annexin V Binding Buffer (10 mM Hepes, $\mathrm{pH}$ adjusted to 7.4 with $\mathrm{NaOH}, 140 \mathrm{mM} \mathrm{NaCl}, 2.5 \mathrm{mM} \mathrm{CaCl}_{2}$ ) at a concentration of 1 million cells per ml. $100 \mu$ l of this cell suspension ( $1 \times 10^{5}$ cells) was stained with PE-conjugated Annexin V (Invitrogen) for 15 minutes at room temperature in the dark. Finally, $400 \mu$ of binding buffer containing DAPI was added to each tube and analyzed by flow cytometry within one hour.

\section{Analysis of Neutrophil Aging in Microbiota-Depleted and Germ-free Mice}

For microbiota depletion studies we followed a previously published protocol (Zhang et al., 2015). Briefly, mice were fed with a cocktail of antibiotics (ABX) including ampicillin (1 g/l), neomycin (1 g/l), metronidazol $(1 \mathrm{~g} / \mathrm{l})$ and vancomycin $(1 \mathrm{~g} / \mathrm{l})$ in drinking water for 4 weeks prior to analysis by flow cytometry. For germ-free mice we compared by flow cytometry SPF-housed with germ free (GF) mice for markers of aging state (see method of Circadian analysis of aging markers and RNA extraction).

\section{QUANTIFICATION AND STATISTICAL ANALYSIS}

Specific quantification protocols are detailed in each method above.

\section{RNA-Sequencing Data Analysis}

For data analysis, sequencing adaptor contaminations were removed from reads using Cutadapt and the resulting reads were mapped on the transcriptome (GRCm38 Ensembl gene-build 70) and quantified using RSEM v1.17 (Li and Dewey, 2011). Only genes with at least one count per million in at least 2 samples were considered for statistical analysis. Data were then normalized and differential expression was tested using the Bioconductor package EdgeR (Robinson et al., 2010). Raw and Benjamini-Hochberg adjusted $p$ values were calculated for each of the comparisons of interest. Non-adjusted $p$ values were used to identify overrepresented pathways using Ingenuity Pathway Analysis (IPA, Quiagen, https://www.ingenuity.com/). These results were quantitatively validated by qPCR analyses for a collection of relevant genes, and functionally validated using in vivo assays as detailed in the manuscript. K-means clustering, PCA analysis and heatmap representations were produced using the Genesis software (Sturn et al., 2002).

\section{Statistical Analysis}

Unless otherwise indicated, data are represented as mean values \pm standard error of the mean (SEM). Paired or unpaired $t$ test was used when 2 groups were compared, and comparison of more than two datasets was done using one-way analysis of variance (ANOVA) with Turkey's post-test. Where applicable, normality was estimated using D'Agostino \& Pearson or Shapiro-Wilk normality test. Log-rank analysis was used for Kaplan-Meier survival curves. Sample exclusion was not performed unless evident signs of disease were found in a mouse, in which case statistically significant outliers were identified using Grubb's test (ESD method). Comparisons of two-time curves were performed using two-way ANOVA. All statistical analyses were performed using Prism v6 (GraphPad Software, California, USA). A p value below 0.05 was considered statistically significant; non-significant differences (ns) are indicated accordingly.

\section{Amplitude versus Zero Test}

For determination of diurnal patterns, we performed COSINOR fitting of circadian curves, using the curve-fitting module of Graphpad Prism with the equation $Y=$ Baseline + Amplitude $x \cos$ (Frecuency $X+$ Phaseshift), where Baseline = average of Ymax and Ymin; Amplitude $=0.5 \times(Y \max -\mathrm{Ymin})$, Frecuency $=0.2618\left(2^{*} \pi / 24\right)$ and Phaseshift= value of $\mathrm{X}$ at Ymax. To determine whether a diurnal curve displayed an oscillating pattern we used the COSINOR-calculated amplitudes and compared them with a hypothetical zeroamplitude curve (i.e., with no circadian behaviour) assuming that both curves have identical standard deviations. We finally compared 
the two curves' amplitudes using unpaired t test analyses. This analysis gave a better estimation of circadian patterns considering all time points rather than comparing only two times. Through the text this is termed "amplitude versus zero test."

\section{DATA AND SOFTWARE AVAILABILITY}

The accession number for the raw data for the RNA sequencing analyses is GEO: GSE102310. Any other pieces of data are available on request. The data are presented in the main manuscript and in the supplementary materials. RNA-seq data are deposited in the Genome Expression Omnibus under accession number GSE86619. 


\section{Review Series}

\section{HUMAN NEUTROPHILS}

\section{Neutrophils as regulators of the hematopoietic niche}

Itziar Cossío, ${ }^{1, \star}$ Daniel Lucas, ${ }^{2, *}$ and Andrés Hidalgo $0^{1,3, *}$

${ }^{1}$ Area of Cell and Developmental Biology, Fundación Centro Nacional de Investigaciones Cardiovasculares (CNIC) Carlos III, Madrid, Spain; ${ }^{2}$ Division of Experimental Hematology and Cancer Biology, Cincinnati Children's Hospital Medical Center, Cincinnati, OH; and ${ }^{3}$ Institute for Cardiovascular Prevention (IPEK), Ludwig-Maximillians-Universität, Munich, Germany

The niche that supports hematopoietic stem and progenitor cells (HSPCs) in the bone marrow is a highly dynamic structure. It maintains core properties of HSPCs in the steady state, and modulates their proliferation and differentiation in response to changing physiological demands or pathological insults. The dynamic and environment-sensing properties of the niche are shared by the innate immune system. Thus, it is not surprising that innate immune cells, including macrophages and neutrophils, are now recognized as important regulators of the hematopoietic niche and, ultimately, of the stem cells from which they derive. This review synthesizes emerging concepts on niche regulation by immune cells, with a particular emphasis on neutrophils. We argue that the unique developmental, circadian, and migratory properties of neutrophils underlie their critical contributions as regulators of the hematopoietic niche. (Blood. 2019;133(20):2140-2148)

\section{Introduction}

Neutrophils are innate, polymorphonuclear leukocytes that act as the first line of host defense against invading pathogens. Central to their function is their ability to be recruited to sites of infection, to recognize and phagocytose microbes, and to kill pathogens through a combination of cytotoxic mechanisms (reviewed in Mayadas et $\mathrm{al}^{1}$ ). These include the production of reactive oxygen species (ROS), the release of antimicrobial peptides, and the extrusion of their nuclear contents to form neutrophil extracellular traps. Beyond their prominent immune roles, recent years have seen a remarkable emergence of unexpected nonimmune functions of neutrophils in homeostasis as well as in diseases with an important inflammatory component, including systemic lupus and cancer. ${ }^{2}$

A wealth of recent studies have begun to dissect the function of immune cells, including neutrophils, in the bone marrow. These studies most prominently highlight the diversity of properties of a cell type that not long ago was regarded as purely cytotoxic and proinflammatory. Here, we review fundamental aspects of neutrophil and bone marrow niche biology, and discuss the functional interplay between neutrophils and other immune cells within these niches that help to preserve hematopoietic stem and progenitor cells (HSPCs). We finally consider temporal regulation of the hematopoietic niche driven in part by the unique circadian properties of neutrophils, as this highlights novel layers of interaction between immunity and hematopoiesis.

\section{Developing neutrophils and neutrophils in development}

Neutrophils are short-lived cells, as they are generally believed to circulate for only 6 to 12 hours in mice and humans. ${ }^{3,4}$ Their short lifespan in circulation demands constant production and release from the bone marrow, with an estimated production rate in humans of $\sim 10^{10}$ cells per day. ${ }^{5}$ Given their indispensable antimicrobial roles but potential toxic activity in tissues, both excessive and deficient production of neutrophils can have major detrimental consequences for the organism. Indeed, neutrophil homeostasis is tightly regulated through a balance between granulopoiesis, storage, and egress from the bone marrow, intravascular margination, clearance, constitutive death by apoptosis, ${ }^{6}$ and elimination through phagocytosis in specific organs. ${ }^{5,7}$

Neutrophils are formed within the bone marrow through a series of progressively differentiated precursors in a process termed granulopoiesis. The most immature long-term or short-term stem cells give rise to multipotent progenitors, common myeloid progenitors, and granulocyte-macrophage progenitors (GMPs). Only recently, GMPs have been shown to produce neutrophil-committed proliferative precursors ( $\mathrm{NeP}$ and pre$\mathrm{Neu}$ ) that differentiate into nonproliferative immature neutrophils, and give rise to the mature neutrophils that are released into the bloodstream ${ }^{8,9}$ (Figure 1).

The ultimate elimination of neutrophils is as important as their production, and these 2 processes must be tightly coordinated to maintain a constant supply and steady number of neutrophils in blood. ${ }^{10}$ This is important because overproduction of neutrophils can aggravate cytotoxic damage in healthy tissues as seen in many inflammatory diseases, whereas neutropenia inevitably results in recurrent infections and, paradoxically, chronic inflammatory states. ${ }^{11} \mathrm{~A}$ key mechanism regulating neutrophil homeostasis was reported in a seminal study by Ley and colleagues, and involves the interleukin 23 (IL-23)/IL-17/granulocyte colony-stimulating factor (G-CSF) feedback circuit. ${ }^{12}$ Senescent 


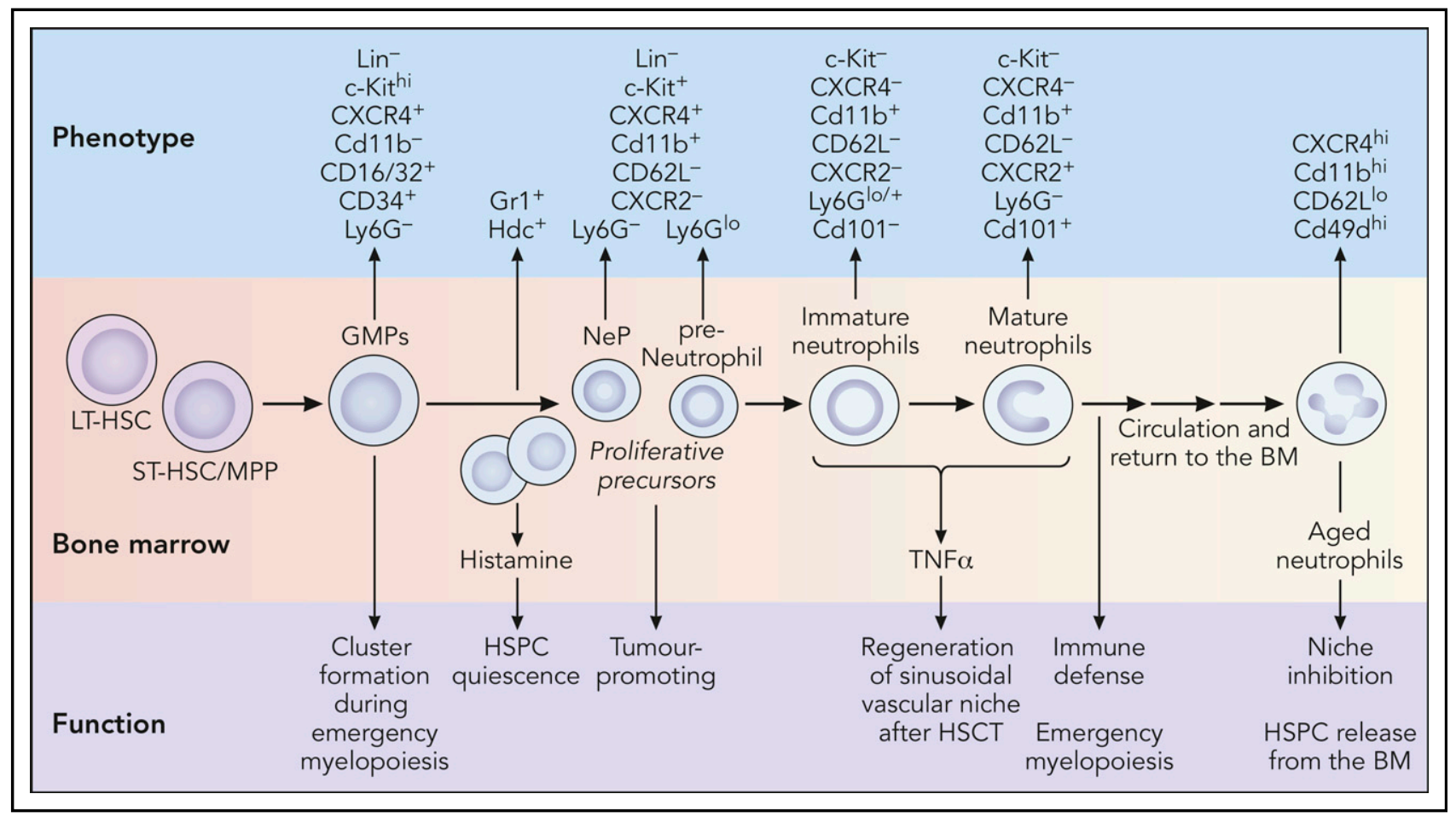

Figure 1. Functional and phenotypic diversity of neutrophils in the bone marrow. Neutrophils are produced inside of the bone marrow (BM) through progressive maturation of hematopoietic progenitors (long-term hematopoietic stem cells [LT-HSCs] to GMPs). Proliferative precursors (NeP and preNeu) differentiate into immature neutrophils and finally into mature neutrophils that are released into blood. A fraction of aged neutrophils return into the marrow after several hours in the circulation. Top and bottom panels indicate specific phenotypes and functions, respectively, of neutrophils at each stage of their life cycle. HSC, hematopoietic stem cell; HSCT, hematopoietic stem cell transplantation; HSPC, hematopoietic stem and progenitor cell; MPP, multipotent progenitor; ST, short-term; TNF, tumor necrosis factor. Professional illustration by Patrick Lane, ScEYEnce Studios.

neutrophils that migrate to peripheral tissues are phagocytosed by tissue-resident phagocytes, including macrophages and dendritic cells, ${ }^{12}$ in a process that relies, at least partially, on the liver $\mathrm{X}$ receptors (LXRs). ${ }^{13}$ Activation of LXRs in engulfing phagocytes inhibits transcription of 1123 , a cytokine that boosts granulopoiesis by promoting the production of IL-17, which in turn induces the production by stromal cells of G-CSF, the main granulopoietic factor. ${ }^{13}$ This homeostatic loop becomes evident in mice deficient in adhesion molecules, in which neutrophils have impeded egress from blood into tissues and consequent reduced uptake by tissue phagocytes, leading to unleashed production of IL-23 and IL-17, and therefore supraphysiological levels of G-CSF that drive the overproduction and release of neutrophils into blood. ${ }^{12}$ This study was important not only for identifying a mechanism for homeostatic regulation of neutrophil numbers, but also for providing the first link between neutrophils and functional regulation of hematopoiesis. The receptor CXCR2 is not only needed for the normal release of neutrophils from the bone marrow into blood, but also for their migration into tissues. Deficiency in $\mathrm{Cxcr} 2$ or its ligand CXCL5 produced by intestinal cells also results in dysregulation of the IL17/G-CSF axis and microbiota composition, resulting in elevated medullary granulopoiesis and neutrophilia. ${ }^{12,14}$ Interestingly, studies in antibiotic-treated mice demonstrated a reciprocal regulation, whereby the microbiota are an important innate stimulus for IL-17-producing cells in the intestine and G-CSF production, thereby participating in neutrophil production and immune competence of the organism. ${ }^{15}$

The cross talk between mature immune cells and hematopoietic stem cells (HSCs) is already evident from embryonic life, a stage at which specific populations of primitive immune cells have an essential role in determining HSC fate. For instance, yolk sacderived macrophages that migrate to the fetal liver around embryonic day 10.5 contribute substantially to the first wave of hematopoiesis. ${ }^{16}$ The fetal liver serves as the main hematopoietic organ during embryonic development until HSCs move to the bone marrow, which becomes the primary site of hematopoiesis from the perinatal period onward. It is striking that yolk sac-derived macrophages persist in functionally distinct tissues in adulthood such as in brain (microglia), epidermis (Langerhans cells), and lung (alveolar macrophages), ${ }^{16}$ among many other tissues, implying that early dissemination of immune cells is important for prenatal and adult life in hematopoietic and nonhematopoietic organs. Also during embryonic life, a subset of primitive neutrophils that lies in the dorsal aorta of the zebrafish embryo was shown to play an important role in determining HSC fate. ${ }^{17}$ These cells were shown to be the main source of tumor necrosis factor $\alpha$ (TNF $\alpha)$, a cytokine needed for the emergence and specification of HSCs in the embryo, thereby providing an example of early immune-driven determination of HSC fate in development. ${ }^{17}$

\section{The hematopoietic bone marrow niche}

HSPCs proliferate and differentiate in a highly regulated manner, thus giving rise to all immune subsets in the bone marrow, or after migrating into extramedullary hematopoietic or lymphoid organs. Regulation of hematopoiesis requires a highly dynamic and tightly regulated orchestration of stem cellintrinsic programs. ${ }^{18}$ Notably, the realization that HSPCs lost 
repopulating ability when placed outside of the marrow led to the formulation of the "niche" concept, which proposed a specific stem cell-supportive environment inside of the medullary space. ${ }^{19,20}$ This supportive niche is composed of a plethora of cellular components, which regulate HSPC activity by supplying growth regulators and retention factors. The specific location of HSCs in the vast medullary space has been controversial (reviewed in Wei and Frenette ${ }^{21}$ ); many studies pointed to localization close to the endosteal region, ${ }^{22-25}$ whereas others studies suggested that HSCs localized randomly in the bone marrow or were perisinusoidal. ${ }^{26}$ It has become increasingly clear that the vast majority of HSCs in the marrow localize adjacent to blood vessels, therefore proximal to perivascular cells. Endothelial cells are key sources of CXCL12 and the cytokine stem cell factor (SCF) that maintain HSPCs. ${ }^{27-30}$ In addition to the endothelium, rare populations of perivascular cells are also key sources of CXCL12 and SCF. Aided by the use of multiparametric imaging with different markers and lineagespecific reporter genes, the complex heterogeneity that exists among stromal cells is now being clarified. Based on the brightness and morphology of Nestin-green fluorescent protein-positive $\left(\mathrm{GFP}^{+}\right)$cells, 2 subsets of mesenchymal progenitor cells were identified. ${ }^{24}$ Nes-GFP-bright cells are scarce and associate with arterioles, whereas the GFP-dim cells are more abundant, reticular shaped, and associate with sinusoids. Interestingly, quiescent HSCs preferentially localize near arteriolar cells. Nes-GFP-bright cells express the pericyte markers $\mathrm{NG}^{+}$and $\alpha$-smooth muscle actin and produce abundant CXCL12 needed for HSC localization and quiescence. ${ }^{31}$ In contrast, Nes-GFP-dim cells, which can be also identified by expression of the leptin receptor $\left(\operatorname{LepR}^{+}\right)$, are an important source of SCF that help maintain constant numbers of HSCs in the bone marrow. ${ }^{31}$

Besides cells of mesenchymal origin, early findings provided strong evidence that the nervous system also regulates the hematopoietic niche and HSPC properties. ${ }^{32-34}$ Sympathetic nerves that align with the medullary vasculature regulate the expression of stromal CXCL12 and thereby the traffic of HSPCs in and out of the bone marrow under homeostasis or stress conditions. ${ }^{34-36}$ Specifically, release of the neurotransmitter noradrenaline by sympathetic nerves signals stromal cells through the $\beta 3$-adrenergic receptor, leading to rapid downregulation of $\mathrm{Cxcl} 12$ expression. Interestingly, studies showed that noradrenaline secretion follows a circadian pattern controlled by the core genes of the molecular clock, which elegantly explained the diurnal release of HSPCs into blood. ${ }^{34}$ Glial fibrillary acidic protein $\left(\mathrm{GFAP}^{+}\right)$nonmyelinating Schwann cells that ensheath sympathetic nerves are also functional regulators of HSC proliferation by providing active transforming growth factor $\beta 1$ (TGF $\beta 1) .{ }^{37}$

\section{Niche regulation by \\ hematopoietic descendants}

In addition to stromal niche components and sympathetic nerves, a growing list of hematopoietic cells that descend from HSPCs have been shown to influence HSC homeostasis and fate, including macrophages, megakaryocytes (MKs), regulatory T cells (Tregs), and neutrophils. Bone marrow-resident macrophages were the first among this progeny shown to favor retention of HSPCs by reinforcing the function of $\mathrm{Nestin}^{+}$cells and osteoblasts, ${ }^{38-40}$ an effect that opposes the niche-inhibiting and mobilizing effects of the sympathetic nervous system. Experiments in which $\mathrm{CD}_{169^{+}}$macrophages were acutely depleted demonstrated that their elimination was sufficient to induce HSPC egress into the bloodstream. ${ }^{38}$ Interestingly, macrophages regulate HSPCs also under stress; in a transplantation setting, radiation eliminates the majority of leukocytes but spares a population of resident macrophages that repopulate the spleen and marrow via autonomous cell division. ${ }^{41}$ These $\mathrm{CD} 169^{+}$radiation-resistant macrophages are needed for optimal donor-derived HSC reconstitution. ${ }^{41}$

MKs, the precursors of platelets, are in close contact with sinusoidal vessels in the marrow, where they extend cytoplasmic protrusions into the vessel lumen to release newly produced platelets. A subset of HSCs localizes near MKs in the sinusoids, and this spatial relationship was shown by several studies to correlate with regulation of HSC pool size. ${ }^{42,43}$ Specifically, HSPCs expanded dramatically after depletion of MK in Cxcl4Cre;iDTR mice. These effects could be pinned down to the production of key regulators of HSPC proliferation by MKs, including CXCL4 and TGF $\beta 1$, both of which promote HSC quiescence. Consequently, deletion of these factors from MKs resulted in increased HSC numbers in the steady state. In contrast to these results, a separate study showed that depletion of MKs resulted in reduction of HSC numbers despite a similar loss of quiescence, an effect that was accounted for by the production of thrombopoietin. ${ }^{44}$ Besides homeostasis, MKs can promote HSPC recovery after ablation with irradiation by secretion of fibroblast growth factor $1^{43}$ or indirectly through osteoblast expansion. ${ }^{45}$

The bone marrow is a major reservoir of a population of $\mathrm{CD} 4^{+} \mathrm{CD} 25^{+} \mathrm{T}$ lymphocytes with immune-modulatory functions or Tregs. ${ }^{46} \mathrm{~A}$ subset of Tregs that expresses high levels of the stem marker CD150 was found in the endosteal region of the bone marrow, proximal to HSPCs. ${ }^{47,48}$ CD150high Tregs control HSPC quiescence and engraftment through the production of adenosine generated via the CD39 ectoenzyme, ${ }^{48}$ and it has been proposed that Tregs confer immune privilege to the HSPC niche. ${ }^{47}$

In summary, ample evidence now shows that the hematopoietic niche is regulated, secured, and nurtured by the very descendants of HSPCs residing therein, perhaps providing a regulatory loop that feeds on output cells and benefits from the exquisite sensing properties of mature immune cells. Given the precedents described earlier in this section, it is not surprising that other hematopoietic cell lineages can actively regulate the bone marrow niche. In the next section, we focus our discussion on neutrophils, the most abundant among HSPC descendants, whose extreme sensitivity to stress, tissue damage, and even temporal cues may provide additional layers of regulation of the bone marrow niche.

\section{Regulation of HSPC quiescence and proliferation by neutrophils}

Besides perivascular cells and MKs, myeloid cells have been shown to maintain HSPC quiescence through a negative 
feedback histaminergic circuit. Indeed, a myeloid population expressing the histidine decarboxylase produces histamine (Figure 1). This biogenic amine inhibits active cycling of a myeloid-biased histidine decarboxylase-high HSC population through the histamine receptor 2 , and promotes its self-renewal. ${ }^{49}$ This pathway elicited by granulocytes and possibly other myeloid subsets was important for HSPC maintenance because ablation of histamine-producing cells caused myeloid-biased HSCs and progenitors to exit dormancy and induced loss of serial transplantation capacity. ${ }^{49}$

Along the same line, neutrophils stimulate emergency myelopoiesis via production of ROS, which oxidizes the phosphatase and tensin homolog phosphatase to directly activate HSPC proliferation upon acute infection or inflammation. ${ }^{50}$ We expect that, as we continue to extend our knowledge on neutrophil biology in the bone marrow, new mechanisms by which these cells directly regulate HSPC fate will emerge. At present, however, the most prominent known roles of neutrophils on HSPCs are mediated through regulation of their niche, as discussed next.

\section{Role of neutrophils in regeneration of the} bone marrow niche

HSC transplantation (HSCT) remains the only curative treatment of most malignant and nonmalignant hematopoietic diseases. In this procedure, the diseased host hematopoietic cells are wiped out by high-dose chemotherapy or radiotherapy. Healthy HSPCs and more mature hematopoietic cells are then transferred into the recipient's circulation where they home to the bone marrow to engraft and regenerate a new hematopoietic system. Unfortunately, the treatments used to eliminate the host hematopoietic cells invariably cause an almost complete destruction of the vascular HSPC niche in the bone marrow. Specifically, they ablate the sinusoidal vasculature and associated perivascular cells, while leaving arteries and arterioles mostly intact. ${ }^{51-53} \mathrm{Al}-$ though the transplanted HSPCs can engraft for short periods near endosteal arterioles and $\mathrm{MKs}_{1}{ }^{43,45}$ long-term restoration of normal hematopoiesis demands reestablishment of a healthy sinusoidal network, ${ }^{51-53}$ as initially demonstrated by Rafii and colleagues. ${ }^{52}$ Indeed, deletion of vascular-borne vascular endothelial growth factor receptor 2 does not affect baseline hematopoiesis, but strongly impairs regeneration of the vasculature and the hematopoietic compartment after injury. ${ }^{52}$ In addition to the aforementioned functions of supporting nutrients and providing a niche for HSPCs, the sinusoidal network also produces many molecules like Notch ligands and pleiotrophin that promote HSPC engraftment specifically after injury. ${ }^{51,54-57}$ Thus, regeneration of the sinusoidal network is the rate-limiting step in restoring healthy hematopoiesis after HSCT, and a longstanding question has been which environmental cues instruct vascular regeneration of the damaged niche.

We recently discovered that bone marrow $\mathrm{Gr} 1^{+} \mathrm{CD} 115^{-}$neutrophils drive sinusoidal regeneration after transplantation. ${ }^{58} \mathrm{We}$ noticed that, in mice transplanted with total bone marrow mononuclear cells, regeneration of the host vascular niche correlated directly with the number of donor hematopoietic cells transplanted, and adoptive transfer experiments demonstrated that only bone marrow neutrophils were capable of driving sinusoidal regeneration. In agreement, depletion of mature neutrophils from the initial graft or genetic ablation of donorderived neutrophils delayed regeneration of the vasculature. These experiments indicated that neutrophils are both necessary and sufficient to drive vascular regeneration after HSCT. Imaging experiments showed that bone marrow neutrophils are selectively recruited to the injured sinusoids, where they secrete $\mathrm{TNF} \alpha$, a cytokine that promoted endothelial cell survival and regeneration of the sinusoids ${ }^{58}$ (Figure 2). After transplantation, donor HSPCs initiate a proregenerative program that greatly increases their proliferation and their capacity to generate neutrophils and other myeloid cells. ${ }^{59}$ These findings suggested that newly generated neutrophils can promote regeneration of the sinusoidal network, which in turn facilitates hematopoietic progenitor engraftment. ${ }^{58}$ This positive feedback loop continues until the sinusoidal network is restored and the bone marrow returns to homeostasis. Surprisingly, the signals and mechanisms that sense regeneration of the sinusoidal niche, halt further vessel growth, and induce HSPC return to quiescence are almost completely unknown, although it is likely that TGF $\beta$ signaling plays a major role in this process. ${ }^{53,60}$ Identification of these mechanisms may lead to the development of better therapies to promote faster myeloid cell recovery, with restoration of innate immunity and reduced infections after HSCT.

Although the role of neutrophils in bone marrow regeneration was previously unclear, it was well established that they contributed to tissue regeneration (reviewed in Wang ${ }^{61}$ ). Neutrophils are recruited to injured tissues via damage-associated molecular patterns, ${ }^{62}$ where they can exert both positive and negative effects in the regeneration program. This is dependent on cellular context and in the amount and type of neutrophils recruited to each tissue. ${ }^{61,63}$ In the context of vascular development and repair, it is now clear that different neutrophil subsets cross talk with endothelial cells to regulate their function.

As described earlier in "Developing neutrophils and neutrophils in development," embryonic neutrophils induce generation of definitive HSCs by signaling via TNF $\alpha$ to the hemogenic endothelium. ${ }^{17}$ The Phillipson group identified a vascular endothelial growth factor receptor 1-positive neutrophil subset in the circulation (representing $\sim 5 \%$ of blood neutrophils) that is selectively recruited to hypoxic tissues, where they induce vessel growth via matrix metallopeptidase 9 release. ${ }^{64-66}$ Intriguingly, however, blood-borne neutrophils are unable to induce vascular regeneration in the marrow despite expressing high amounts of $\mathrm{TNF} \alpha$, a limitation that may reflect their inability to home to injured sinusoids after adoptive transfer. ${ }^{58}$ An emerging concept is that neutrophils are a heterogeneous population both in tissues and in peripheral blood, and that they can adopt unique physiological functions. ${ }^{67,68}$ In the particular case of medullary regeneration, we highlight that there are at least 2 subsets of angiogenic neutrophils: 1 in the bone marrow that acts on niche-associated sinusoids and 1 in the periphery that acts on peripheral vessels. A recent study by the $\mathrm{Ng}$ group also showed that classically defined bone marrow $\mathrm{Gr} 1^{+} \mathrm{CD} 115^{-}$neutrophils are in fact a heterogeneous population that comprises a proliferating neutrophil progenitor as well as immature and mature neutrophils with transcriptional signatures distinct from those of circulating neutrophils. ${ }^{8}$ It will be interesting to dissect the behavior of each of these 


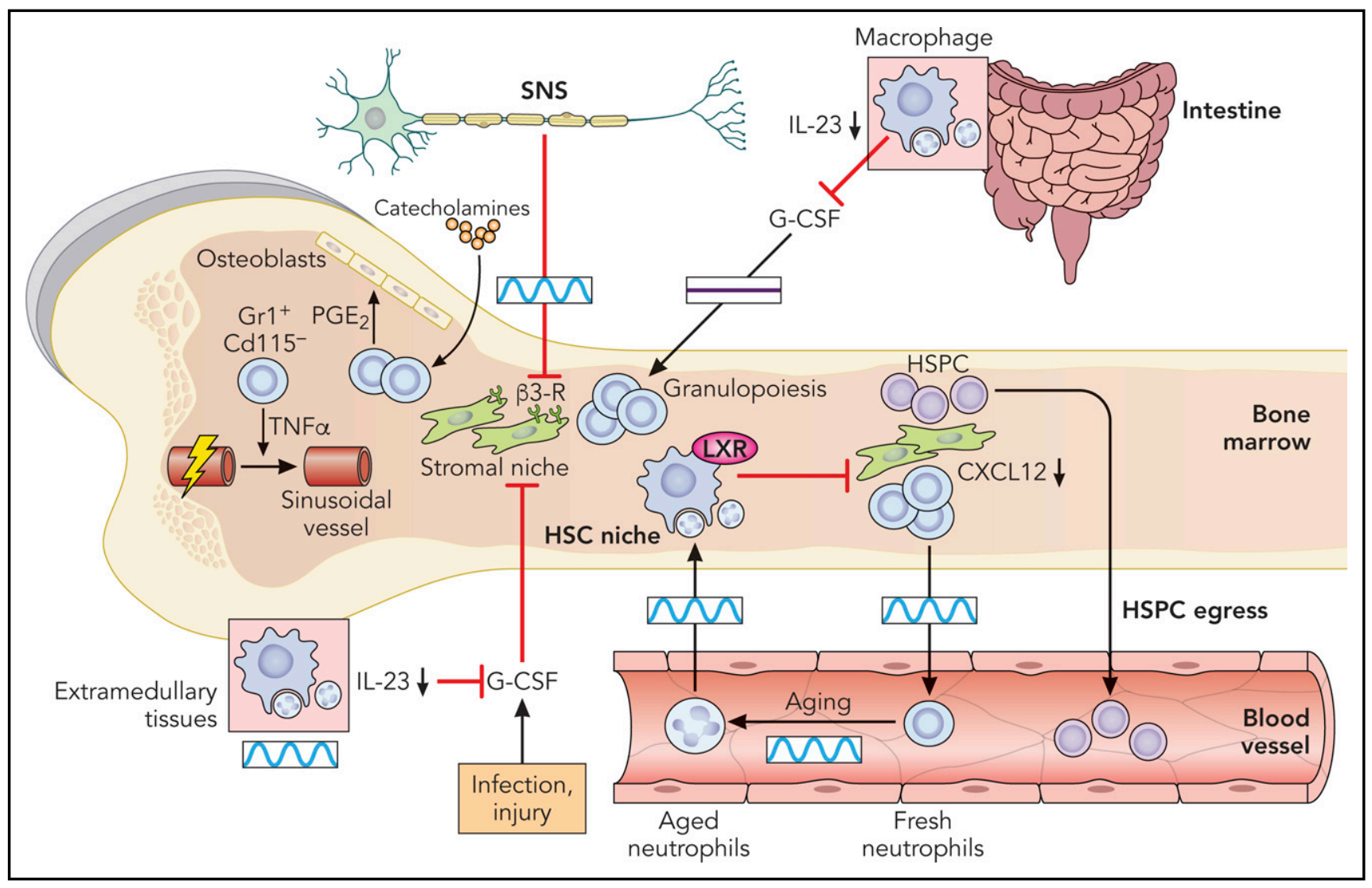

Figure 2. Regulation of the hematopoietic bone marrow niche. The sympathetic nervous system (SNS) exerts control on the HSC niche by the circadian release of catecholamine, which targets $\beta 3$-adrenergic receptors on stroma cells. The same signals can act through neutrophils to produce prostaglandin $\mathrm{E}_{2}$ ( $\mathrm{PG} \mathrm{E}_{2}$ ) and stimulate the osteoblastic niche. The stromal niche is also circadianally regulated by aged neutrophils that return to the bone marrow after only several hours in the circulation. Aged neutrophils that infiltrate the bone marrow are engulfed by macrophages and activation of the LXRs lead to inhibition of the hematopoietic niche. Excessive G-CSF production associated with several inflammatory processes or impaired neutrophil clearance in extramedullary tissues is also a potent inhibitor of the HSPC niche. All of these regulatory mechanisms ultimately inhibit production of CXCL12, thereby promoting HSPC egress into blood. This has been shown in the intestine, where neutrophil infiltration in the mucosa and engulfment of neutrophils by tissue-resident macrophages inhibits the IL-23/IL-17/G-CSF axis and remotely supports niche activity in a circadian-independent manner. Boxes indicate the presence or absence of circadian oscillations in each tissue. Professional illustration by Patrick Lane, ScEYEnce Studios.

medullary neutrophil subsets after HSCT and their contribution to vascular niche regeneration.

Bone marrow neutrophils are recruited specifically to injured sinusoids. This direct interaction is clearly important for the sinusoids as areas of the bone marrow that have no neutrophils showed no sinusoidal regeneration. ${ }^{58}$ In the steady state, neutrophil trafficking is regulated, almost exclusively, via CXCR2 and CXCR4. ${ }^{69}$ However, pharmacological blockade of both pathways does not affect neutrophil recruitment to injured vessels, ${ }^{58}$ thereby indicating the existence of an unidentified mechanism in the sinusoids, induced by damage to the vasculature that specifically recruits neutrophils to injured bone marrow vessels.

In addition to aiding regeneration of the vascular niche, neutrophils have been reported to support niche activity by enhancing the capacity of preosteoblastic cells to produce osteopontin, an important retention factor for HSPCs in the marrow. ${ }^{70}$ Interestingly, adrenergic stimulation of neutrophils through the $\beta 3$ receptor induced production of prostaglandin E2, a well-known support factor for hematopoiesis, ${ }^{71}$ which in turn induced osteoblastic activity through the EP4 receptor. ${ }^{70}$ Thus, neutrophils appear to counteract, to some extent, the inhibitory effects that catecholamines exert on the niche, thereby preventing excessive HSPC mobilization (Figure 2). The identification of neutrophils as intermediary and regulators of the mobilization process provides important mechanistic links between the various pathways that regulate hematopoietic niches.

\section{Circadian regulation of the hematopoietic niche}

In almost all life forms on Earth, the planet's rotation has led to the evolution of daily circadian cycles of 24 hours. In mammals, peripheral clocks are normally synchronized with the environment by entrainment from daily exposure to light-dark cycles. The central circadian pacemaker located in the suprachiasmatic nuclei receives photic information conducted from the retina. The synchrony between autonomous circadian clocks found in all major organs and tissues is maintained by a complex network, involving neuronal signaling, secretion of hormones, and metabolic cues (reviewed in Scheiermann et $\mathrm{al}^{72}$ ). As discussed in "The hematopoietic bone marrow niche," the bone marrow is extensively innervated by autonomic nerve fibers, including sympathetic nerves, which play important physiological roles in the bone marrow. Sympathetic nerves have been shown to be 
responsible for cytokine-elicited mobilization of HSPCs outside of the bone marrow into blood, ${ }^{32}$ although active signaling in monocytic cells has also been demonstrated. ${ }^{39}$ G-CSF, a cytokine broadly used in the clinic to mobilize HSPCs into circulation for transplantation therapies, promotes the release of noradrenaline by autonomic neurons located in the periphery. Released adrenaline mediates the suppression of osteoblasts located in the endosteal marrow, thereby reducing the synthesis of CXCL12 and causing HSPC mobilization. ${ }^{32}$ Additionally, sympathetic nerves regulate perivascular Nestin-GFP ${ }^{+}$stem cells by acting on $\beta 3$ adrenergic receptors. ${ }^{35}$ This neuralmesenchymal axis is responsible for the circadian expression of CXCL12 by bone marrow stromal cells, which causes the homeostatic release of HSPCs into circulation. ${ }^{34}$ In mice, the lowest levels of CXCL12 protein in the medullary space coincide with HSPC egress around zeitgeber time 5 (ZT5, or 5 hours after the onset of light), and the highest CXCL12 levels occur at ZT13 and correlate with the lowest numbers of circulating HSPCs. ${ }^{34}$ In contrast to mice, humans display inverted circadian oscillations with maximum levels of progenitors in blood in the evening. ${ }^{73}$

Given the bidirectional flux between blood and marrow, it is not surprising that adrenergic nerves also control the expression of endothelial-adhesion molecules in the medullary vasculature, as these adhesion molecules are necessary for HSPC homing back to the marrow. ${ }^{74}$ It is also likely that a cross talk exists between the levels of the chemokine CXCL12 and those of endothelialadhesion molecules; for instance, in mice, higher levels of CXCL12 at night correlate with a higher retention of HSCs in the bone marrow. The genuine circadian nature of this process was illustrated by jet-lag experiments showing that repeated shifts in light cycle were sufficient to ablate circadian HSPC recruitment into tissues. ${ }^{74}$

Interestingly, much like HSPCs, mature leukocytes infiltrate the bone marrow in a circadian manner, ${ }^{74}$ with peak homing to the marrow and other organs at ZT13 in mice. The circadian migration of mature leukocytes (and HSPCs) may be beneficial to provide a readily available set of tissue-resident leukocytes that mediate immune defense during the animal's active phase, when the individual's probability of injury or encountering pathogens is highest. The circadian fluxes of mature leukocytes that return to the marrow also suggest potential regulation of bone marrow niches by cells that have "sampled" the extramedullary environment. For example, it is likely that various myeloid cell subsets regulate circadian oscillations in HSPC activity through $\mathrm{TNF} \alpha$. Indeed, this cytokine has been shown to regulate circadian migration, proliferation, and differentiation through modulation of ROS and melatonin signaling in HSPCs, and its medullary levels are controlled in part by neutrophils and monocytes. ${ }^{58,75}$

\section{Neutrophil aging and temporal control of the hematopoietic niche}

Neutrophils are the most abundant myeloid population in the bone marrow. Because of the short lifespan, vast amounts of neutrophils must be released into the blood every day to maintain homeostatic numbers. ${ }^{10}$ This implies that, even under homeostatic conditions, large numbers must also be eliminated every day, yet possible functions for these naturally cleared neutrophils were enigmatic. ${ }^{76,77}$

Only recently, we and others discovered that circulating neutrophils undergo circadian fluctuations that affect not only neutrophil numbers, but also their phenotype. This spontaneous change over time is referred to as neutrophil aging. ${ }^{78}$ As neutrophils age in the circulation, their repertoire of surface receptors change: they upregulate markers like CXCR4 and verylate-activation antigen 4 , both of which are important for the retention in and homing to the marrow, ${ }^{76,77,79}$ and downregulate others including CD62L (L-selectin) and CXCR277,80 (Figure 1). This CXCR4 ${ }^{\text {hi }}$ CD62L lo population of aged neutrophils follows marked circadian oscillations throughout the day and is completely cleared out from circulation by night (ZT13), when the active behavioral phase of the mice begins. ${ }^{77}$ Interestingly, recent studies in mice have proposed that aged neutrophils gain immune competence by enhancing $\beta 2$ integrin-dependent adhesion, as well as their capacity to phagocytose and to form DNA-based neutrophil extracellular traps. ${ }^{81,82}$ In addition, microbiota-derived metabolites have been proposed to drive neutrophil aging through Toll-like receptor signaling, ${ }^{82}$ although our own data suggest that cell-intrinsic circadian programs can also drive aging. ${ }^{80}$ Thus, although the evolutionary drive and physiological role of this diurnal aging process of neutrophils remains to be fully elucidated, it is tempting to speculate that diurnal "priming" of neutrophils is needed for these cells to fully mature and to fulfill additional functions after their lifetime in blood, once they have cleared into tissues.

Aged neutrophils that clear from the circulation into tissues are believed to be ultimately engulfed and eliminated by tissue macrophages. ${ }^{78,83}$ The bone marrow is one of the tissues in which aged neutrophils are cleared in larger numbers. Clearance in this organ not only serves to control neutrophil numbers but also, importantly, generates homeostatic signals that modulate the bone marrow niche. ${ }^{77}$ When aged neutrophils infiltrate the mouse bone marrow between ZT5 and ZT13, they are engulfed by tissue-resident macrophages. This efferocytic process generates LXR-dependent, but otherwise undefined, signals that downregulate the number of niche cells and, consequently, the amount of CXCL12 in the marrow, thereby promoting HSPC egress into blood (Figure 2). The numbers of CXCL12-producing reticular cells and osteoblasts in the bone marrow consistently increase when neutrophils are experimentally depleted, indicating that neutrophils modulate the size of the niche stroma. More importantly, interruption of this natural niche-inhibitory pathway by depletion of circulating neutrophils or macrophages completely blunted the diurnal oscillations of HSPCs in blood, indicating that circadian clearance of aged neutrophils drives rhythms in the hematopoietic niche. ${ }^{77}$ These findings in mice reveal a coalescence of hematopoietic, neural, and immune inputs in the bone marrow to provide multilayered regulation of hematopoiesis. Importantly, alterations of this axis appear to powerfully influence disease, as shown in the context of cardiovascular disease or cancer. ${ }^{84-86}$

Contrary to common belief, homeostatic clearance of aged neutrophils is not unique to the bone marrow, spleen, or liver: it also takes place in many extramedullary tissues such as the lung, skin, or muscle in which they can perform tissue-specific roles. $2,78,87$ Surprisingly, infiltration of neutrophils in the 
intestinal mucosa enhances bone marrow niche activity remotely, by preventing $1 / 23$ transcription in intestinal macrophages. Similar to the aforementioned "neutrostat" model, ${ }^{12}$ inhibition of this cytokine results in reduced systemic levels of G-CSF and preserved niche function, thereby preventing excessive mobilization of HSPCs into blood. However, unlike the rhythmic inhibitory roles of marrow-infiltrating neutrophils, ${ }^{77}$ infiltration in the intestine does not follow circadian patterns and, consequently, niche regulation from the intestine does not influence the diurnal oscillations of circulating $\mathrm{HSPCs}^{87}$ (Figure 2). These recent findings expand the regulatory mechanisms of hematopoietic niches to include distant anatomical sites, and are consistent with the reported effects of microbiota-derived signals emanating from the gut in regulating stem cell and niche activity. ${ }^{88-90}$

\section{Concluding remarks}

It is becoming increasingly clear that dysregulation of hematopoiesis is an important underlying driver of disease. Therefore, understanding the multiple regulatory mechanisms of this highly dynamic process becomes a question of major biomedical relevance. Disturbance of neural regulation of the niche occurs during organismal aging ${ }^{91}$ and is also prominent in the context of ischemic disease, ${ }^{92,93}$ whereas dysregulated cytokine pathways appear to be more common in cancer. ${ }^{84}$ We have discussed here emerging evidence that neutrophils provide additional layers of regulation in hematopoiesis. The realization that neutrophils influence multiple aspects of niche physiology, from maintenance of the mesenchymal niche to HSPC quiescence, demands urgent evaluation of their contribution to inflammatory disease and hematological malignancies. Neutrophils are also instrumental in regenerating the injured vascular niche and may provide strategies to accelerate regeneration of patients undergoing HSCT. More generally, we propose that the unique temporal properties of neutrophils, their basal presence in multiple tissues, and exquisite capacity to sense danger make these cells ideal intermediaries for niche regulation and repair not only in the bone marrow, but also in other tissues that demand rapid responses to environmental challenges.

\section{Acknowledgments}

This work was supported in part by SAF2015-65607-R and Fondo Europeo de Desarrollo Regional (FEDER) (A.H.); Ministerio de Ciencia, Innovacion y Universidades (MCIU) for fellowship BES-2014-068915 (I.C.); and R01 HL136529-01 from the National Institutes of Health, National Heart, Lung, and Blood Institute (D.L.). The Centro Nacional de Investigaciones Cardiovasculares (CNIC) was supported by the $\mathrm{MCIU}$ and the Pro CNIC Foundation, and is a Severo Ochoa Center of Excellence (MCIU award SEV-2015-0505).

\section{Authorship}

Contribution: I.C., D.L., and A.H. wrote this article.

Conflict-of-interest disclosure: The authors declare no competing financial interests.

ORCID profile: A.H., 0000-0001-5513-555X.

Correspondence: Andrés Hidalgo, Area of Cell and Developmental Biology, Fundación Centro Nacional de Investigaciones Cardiovasculares Carlos III, Madrid 28029, Spain; e-mail: ahidalgo@cnic.es.

\section{Footnotes}

Submitted 26 October 2018; accepted 3 December 2018. Prepublished online as Blood First Edition paper, 21 March 2019; DOI 10.1182/blood2018-10-844571.

*I.C., D.L., and A.H. contributed equally to the writing of this review article.

\section{REFERENCES}

1. Mayadas TN, Cullere X, Lowell CA. The multifaceted functions of neutrophils. Annu Rev Pathol. 2014;9(1):181-218.

2. Nicolás-Ávila JA, Adrover JM, Hidalgo A. Neutrophils in homeostasis, immunity, and cancer. Immunity. 2017;46(1):15-28.

3. Lahoz-Beneytez J, Elemans M, Zhang Y, et al. Human neutrophil kinetics: modeling of stable isotope labeling data supports short blood neutrophil half-lives. Blood. 2016;127(26): 3431-3438.

4. Summers $C$, Rankin SM, Condliffe AM, Singh N, Peters AM, Chilvers ER. Neutrophil kinetics in health and disease. Trends Immunol. 2010 31(8):318-324.

5. von Vietinghoff S, Ley K. IL-17A controls IL$17 \mathrm{~F}$ production and maintains blood neutrophil counts in mice. J Immunol. 2009;183(2): 865-873.

6. Luo HR, Loison F. Constitutive neutrophil apoptosis: mechanisms and regulation. Am J Hematol. 2008;83(4):288-295.

7. Bratton DL, Henson PM. Neutrophil clearance: when the party is over, clean-up begins. Trends Immunol. 2011;32(8):350-357.
8. Evrard M, Kwok IWH, Chong SZ, et al. Developmental analysis of bone marrow neutrophils reveals populations specialized in expansion, trafficking, and effector functions. Immunity. 2018;48(2):364-379.

9. Zhu YP, Padgett L, Dinh HQ, et al. Identification of an early unipotent neutrophil progenitor with pro-tumoral activity in mouse and human bone marrow. Cell Rep. 2018; 24(9):2329-2341

10. Scheiermann C, Frenette PS, Hidalgo A Regulation of leucocyte homeostasis in the circulation. Cardiovasc Res. 2015;107(3): 340-351.

11. Kruger $P$, Saffarzadeh $M$, Weber $A N$, et al. Neutrophils: between host defence, immune modulation, and tissue injury. PLoS Pathog. 2015;11(3):e1004651.

12. Stark MA, Huo Y, Burcin TL, Morris MA, Olson TS, Ley K. Phagocytosis of apoptotic neutrophils regulates granulopoiesis via IL-23 and IL-17. Immunity. 2005;22(3):285-294

13. Hong C, Kidani Y, A-Gonzalez N, et al. Coordinate regulation of neutrophil homeostasis by liver $X$ receptors in mice. J Clin Invest. 2012;122(1):337-347.

14. Mei J, Liu Y, Dai N, et al. Cxcr2 and Cxcl5 regulate the IL-17/G-CSF axis and neutrophil homeostasis in mice. J Clin Invest. 2012 122(3):974-986.

15. Deshmukh HS, Liu Y, Menkiti OR, et al. The microbiota regulates neutrophil homeostasis and host resistance to Escherichia coli K1 sepsis in neonatal mice. Nat Med. 2014;20(5): 524-530.

16. Gomez Perdiguero E, Klapproth K, Schulz C et al. Tissue-resident macrophages originate from yolk-sac-derived erythro-myeloid progenitors. Nature. 2015;518(7540): 547-551.

17. Espín-Palazón R, Stachura DL, Campbell CA et al. Proinflammatory signaling regulates hematopoietic stem cell emergence. Cell. 2014;159(5):1070-1085.

18. Gao X, Xu C, Asada N, Frenette PS. The hematopoietic stem cell niche: from embryo to adult. Development. 2018;145(2):139691.

19. Morrison SJ, Scadden DT. The bone marrow niche for haematopoietic stem cells. Nature. 2014;505(7483):327-334.

20. Schofield R. The relationship between the spleen colony-forming cell and the haemopoietic stem cell. Blood Cells. 1978;4(1-2): 7-25. 
21. Wei Q, Frenette PS. Niches for hematopoietic stem cells and their progeny. Immunity. 2018; 48(4):632-648

22. Adams GB, Chabner KT, Alley IR, et al. Stem cell engraftment at the endosteal niche is specified by the calcium-sensing receptor. Nature. 2006;439(7076):599-603.

23. Guezguez B, Campbell CJ, Boyd AL, et al. Regional localization within the bone marrow influences the functional capacity of human HSCs. Cell Stem Cell. 2013;13(2):175-189.

24. Kunisaki Y, Bruns I, Scheiermann C, et al. Arteriolar niches maintain haematopoietic stem cell quiescence. Nature. 2013; 502(7473):637-643

25. Nombela-Arrieta C, Pivarnik G, Winkel B, et al. Quantitative imaging of haematopoietic stem and progenitor cell localization and hypoxic status in the bone marrow microenvironment [published correction appears in Nat Cell Biol. 2013;15(8):1016]. Nat Cell Biol. 2013;15(5): $533-543$

26. Acar M, Kocherlakota KS, Murphy MM, et al. Deep imaging of bone marrow shows nondividing stem cells are mainly perisinusoidal. Nature. 2015;526(7571):126-130.

27. Ding L, Saunders TL, Enikolopov G, Morrison SJ. Endothelial and perivascular cells maintain haematopoietic stem cells. Nature. 2012; 481(7382):457-462

28. Ding L, Morrison SJ. Haematopoietic stem cells and early lymphoid progenitors occupy distinct bone marrow niches [published correction appears in Nature. 2014:514(7521): 262]. Nature. 2013;495(7440):231-235

29. Greenbaum A, Hsu YM, Day RB, et al. CXCL12 in early mesenchymal progenitors is required for haematopoietic stem-cell maintenance. Nature. 2013;495(7440):227-230.

30. Xu C, Gao X, Wei Q, et al. Stem cell factor is selectively secreted by arterial endothelial cells in bone marrow. Nat Commun. 2018;9(1): 2449.

31. Asada N, Kunisaki Y, Pierce $H$, et al. Differential cytokine contributions of perivascular haematopoietic stem cell niches. Nat Cell Biol. 2017:19(3):214-223.

32. Katayama Y, Battista M, Kao WM, et al. Signals from the sympathetic nervous system regulate hematopoietic stem cell egress from bone marrow. Cell. 2006;124(2):407-421.

33. Méndez-Ferrer S, Battista M, Frenette PS. Cooperation of beta(2)- and beta(3)-adrenergic receptors in hematopoietic progenitor cell mobilization. Ann N Y Acad Sci. 2010;1192(1): 139-144.

34. Méndez-Ferrer S, Lucas D, Battista M, Frenette PS. Haematopoietic stem cell release is regulated by circadian oscillations. Nature. 2008;452(7186):442-447

35. Méndez-Ferrer $S$, Michurina TV, Ferraro $F$, et al. Mesenchymal and haematopoietic stem cells form a unique bone marrow niche. Nature. 2010;466(7308):829-834.

36. Vasamsetti SB, Florentin J, Coppin E, et al. Sympathetic neuronal activation triggers myeloid progenitor proliferation and differentiation. Immunity. 2018;49(1):93-106.
37. Yamazaki S, Ema H, Karlsson G, et al. Nonmyelinating Schwann cells maintain hematopoietic stem cell hibernation in the bone marrow niche. Cell. 2011;147(5):1146-1158.

38. Chow A, Lucas D, Hidalgo A, et al. Bone marrow $\mathrm{CD} 169+$ macrophages promote the retention of hematopoietic stem and progenitor cells in the mesenchymal stem cell niche. J Exp Med. 2011;208(2):261-271.

39. Christopher MJ, Rao M, Liu F, Woloszynek JR Link DC. Expression of the G-CSF receptor in monocytic cells is sufficient to mediate hematopoietic progenitor mobilization by G-CSF in mice. J Exp Med. 2011;208(2): 251-260.

40. Winkler IG, Sims NA, Pettit AR, et al. Bone marrow macrophages maintain hematopoietic stem cell (HSC) niches and their depletion mobilizes HSCs. Blood. 2010;116(23): 4815-4828.

41. Kaur S, Raggatt LJ, Millard SM, et al. Selfrepopulating recipient bone marrow residen macrophages promote long-term hematopoietic stem cell engraftment. Blood. 2018; 132(7):735-749.

42. Bruns I, Lucas D, Pinho S, et al. Megakaryocytes regulate hematopoietic stem cell quiescence through CXCL4 secretion. Nat Med. 2014:20(11):1315-1320.

43. Zhao M, Perry JM, Marshall H, et al. Megakaryocytes maintain homeostatic quiescence and promote post-injury regeneration of hematopoietic stem cells. Nat Med. 2014;20(11):1321-1326.

44. Nakamura-Ishizu A, Takubo K, Fujioka M, Suda T. Megakaryocytes are essential for HSC quiescence through the production of thrombopoietin. Biochem Biophys Res Com mun. 2014:454(2):353-357.

45. Olson TS, Caselli A, Otsuru S, et al. Megakaryocytes promote murine osteoblastic HSC niche expansion and stem cell engraftment after radioablative conditioning. Blood. 2013;121(26):5238-5249.

46. Zou L, Barnett B, Safah H, et al. Bone marrow is a reservoir for CD4+CD25+ regulatory $T$ cells that traffic through CXCL12/CXCR4 signals. Cancer Res. 2004;64(22):8451-8455.

47. Fujisaki J, Wu J, Carlson AL, et al. In vivo imaging of Treg cells providing immune privilege to the haematopoietic stem-cell niche. Nature. 2011;474(7350):216-219.

48. Hirata $Y$, Furuhashi $\mathrm{K}$, Ishii $\mathrm{H}$, et al. CD150(high) bone marrow Tregs maintain hematopoietic stem cell quiescence and immune privilege via adenosine. Cell Stem Cell. 2018;22(3):445-453.

49. Chen X, Deng H, Churchill MJ, et al. Bone marrow myeloid cells regulate myeloid-biased hematopoietic stem cells via a histaminedependent feedback loop. Cell Stem Cell. 2017;21(6):747-760.

50. Kwak HJ, Liu P, Bajrami B, et al. Myeloid cellderived reactive oxygen species externally regulate the proliferation of myeloid progenitors in emergency granulopoiesis. Immunity. 2015;42(1):159-171.

51. Doan PL, Russell JL, Himburg HA, et al. Tie2(+) bone marrow endothelial cells regulate hematopoietic stem cell regeneration following radiation injury. Stem Cells. 2013;31(2):

327-337

52. Hooper AT, Butler JM, Nolan DJ, et al. Engraftment and reconstitution of hematopoiesis is dependent on VEGFR2-mediated regeneration of sinusoidal endothelial cells. Cell Stem Cell. 2009;4(3):263-274.

53. Leiva M, Quintana JA, Ligos JM, Hidalgo A. Haematopoietic ESL-1 enables stem cell proliferation in the bone marrow by limiting TGF $\beta$ availability. Nat Commun. 2016;7(1): 10222.

54. Butler JM, Nolan DJ, Vertes EL, et al. Endothelial cells are essential for the selfrenewal and repopulation of Notch dependent hematopoietic stem cells. Cell Stem Cell. 2010;6(3):251-264.

55. Himburg HA, Muramoto GG, Daher P, et al. Pleiotrophin regulates the expansion and regeneration of hematopoietic stem cells. Nat Med. 2010;16(4):475-482.

56. Himburg HA, Yan $X$, Doan $P L$, et al. Pleiotrophin mediates hematopoietic regeneration via activation of RAS. J Clin Invest. 2014;124(11):4753-4758.

57. Poulos MG, Guo $P$, Kofler NM, et al. Endothelial Jagged-1 is necessary for homeostatic and regenerative hematopoiesis. Cell Reports. 2013;4(5):1022-1034.

58. Bowers E, Slaughter A, Frenette PS, Kuick R, Pello OM, Lucas D. Granulocyte-derived TNF $\alpha$ promotes vascular and hematopoietic regeneration in the bone marrow. Nat Med. 2018;24(1):95-102.

59. Pietras EM, Reynaud D, Kang YA, et al. Functionally distinct subsets of lineage-biased multipotent progenitors control blood production in normal and regenerative conditions. Cell Stem Cell. 2015;17(1):35-46.

60. Brenet F, Kermani P, Spektor R, Rafii S, Scandura JM. TGF $\beta$ restores hematopoietic homeostasis after myelosuppressive chemotherapy. J Exp Med. 2013;210(3):623-639.

61. Wang J. Neutrophils in tissue injury and repair Cell Tissue Res. 2018;371(3):531-539

62. Pittman K, Kubes P. Damage-associated molecular patterns control neutrophil recruitment. J Innate Immun. 2013;5(4):315-323

63. Kovtun A, Messerer DAC, ScharffetterKochanek K, Huber-Lang M, Ignatius A. Neutrophils in tissue trauma of the skin, bone, and lung: two sides of the same coin. $\mathrm{J}$ Immunol Res. 2018;2018:8173983.

64. Christoffersson G, Lomei J, O'Callaghan P, Kreuger J, Engblom S, Phillipson M. Vascular sprouts induce local attraction of proangiogenic neutrophils. J Leukoc Biol. 2017;102(3) 741-751.

65. Christoffersson G, Vågesjö E, Vandooren J, et al. VEGF-A recruits a proangiogenic MMP9-delivering neutrophil subset that induces angiogenesis in transplanted hypoxic tissue. Blood. 2012;120(23):4653-4662.

66. Massena S, Christoffersson G, Vågesjö E, et al. Identification and characterization of VEGF-Aresponsive neutrophils expressing CD49d, VEGFR1, and CXCR4 in mice and humans. Blood. 2015;126(17):2016-2026. 
67. Alvarenga DM, Mattos MS, Araújo AM, Antunes MM, Menezes GB. Neutrophil biology within hepatic environment. Cell Tissue Res. 2018;371(3):589-598.

68. Silvestre-Roig C, Hidalgo A, Soehnlein O Neutrophil heterogeneity: implications for homeostasis and pathogenesis. Blood. 2016; 127(18):2173-2181.

69. Eash KJ, Greenbaum AM, Gopalan PK, Link DC. CXCR2 and CXCR4 antagonistically regulate neutrophil trafficking from murine bone marrow. J Clin Invest. 2010;120(7):2423-2431.

70. Kawano Y, Fukui C, Shinohara M, et al. G-CSFinduced sympathetic tone provokes fever and primes antimobilizing functions of neutrophils via PGE2. Blood. 2017;129(5):587-597.

71. Frisch BJ, Porter RL, Gigliotti BJ, et al. In vivo prostaglandin E2 treatment alters the bone marrow microenvironment and preferentially expands short-term hematopoietic stem cells. Blood. 2009;114(19):4054-4063

72. Scheiermann C, Gibbs J, Ince L, Loudon A Clocking in to immunity. Nat Rev Immunol. 2018;18(7):423-437.

73. Lucas D, Battista M, Shi PA, Isola L, Frenette PS. Mobilized hematopoietic stem cell yield depends on species-specific circadian timing Cell Stem Cell. 2008;3(4):364-366.

74. Scheiermann C, Kunisaki Y, Lucas D, et al. Adrenergic nerves govern circadian leukocyte recruitment to tissues. Immunity. 2012;37(2): 290-301.

75. Golan K, Kumari A, Kollet O, et al. Daily onset of light and darkness differentially controls hematopoietic stem cell differentiation and maintenance. Cell Stem Cell. 2018;23(4): 572-585.
76. Martin C, Burdon PC, Bridger G, GutierrezRamos JC, Williams TJ, Rankin SM. Chemokines acting via CXCR2 and CXCR4 control the release of neutrophils from the bone marrow and their return following senescence. Immunity. 2003;19(4):583-593.

77. Casanova-Acebes M, Pitaval C, Weiss LA, et al. Rhythmic modulation of the hematopoietic niche through neutrophil clearance. Cell. 2013;153(5):1025-1035.

78. Adrover JM, Nicolás-Ávila JA, Hidalgo A. Aging: a temporal dimension for neutrophils. Trends Immunol. 2016;37(5):334-345.

79. Papayannopoulou T. Mechanisms of stem-/ progenitor-cell mobilization: the anti-VLA-4 paradigm. Semin Hematol. 2000;37(1 suppl 2):11-18

80. Adrover JM, Del Fresno C, Crainiciuc G, et al. A neutrophil timer coordinates immune defense and vascular protection. Immunity. 2019:50(2):390-402.

81. Uhl B, Vadlau Y, Zuchtriegel G, et al. Aged neutrophils contribute to the first line of defense in the acute inflammatory response. Blood. 2016;128(19):2327-2337

82. Zhang D, Chen G, Manwani D, et al. Neutrophil ageing is regulated by the microbiome. Nature. 2015;525(7570) 528-532.

83. A-Gonzalez N, Quintana JA, García-Silva S, et al. Phagocytosis imprints heterogeneity in tissue-resident macrophages. J Exp Med. 2017;214(5):1281-1296.

84. Coffelt SB, Wellenstein MD, de Visser KE. Neutrophils in cancer: neutral no more. Nat Rev Cancer. 2016;16(7):431-446.

85. Dutta $P$, Sager HB, Stengel KR, et al. Myocardial infarction activates CCR2(+) hematopoietic stem and progenitor cells. Cell Stem Cell. 2015;16(5):477-487.

86. Nahrendorf M, Swirski FK, Aikawa E, et al. The healing myocardium sequentially mobilizes two monocyte subsets with divergent and complementary functions. J Exp Med. 2007; 204(12):3037-3047.

87. Casanova-Acebes M, Nicolás-Ávila JA, Li JL, et al. Neutrophils instruct homeostatic and pathological states in naive tissues. J Exp Med. 2018;215(11):2778-2795.

88. Luo $Y$, Chen GL, Hannemann N, et al. Microbiota from obese mice regulate hematopoietic stem cell differentiation by altering the bone niche. Cell Metab. 2015;22(5): 886-894.

89. Maslowski KM, Vieira AT, Ng A, et al. Regulation of inflammatory responses by gut microbiota and chemoattractant receptor GPR43. Nature. 2009;461(7268):1282-1286.

90. Shi C, Jia T, Mendez-Ferrer S, et al. Bone marrow mesenchymal stem and progenitor cells induce monocyte emigration in response to circulating Toll-like receptor ligands. Immunity. 2011;34(4):590-601.

91. Maryanovich $\mathrm{M}$, Zahalka $\mathrm{AH}$, Pierce $\mathrm{H}$, et al. Adrenergic nerve degeneration in bone marrow drives aging of the hematopoietic stem cell niche. Nat Med. 2018;24(6):782-791.

92. Courties G, Herisson F, Sager HB, et al. Ischemic stroke activates hematopoietic bone marrow stem cells. Circ Res. 2015;116(3): 407-417.

93. Dutta P, Courties G, Wei Y, et al. Myocardia infarction accelerates atherosclerosis. Nature. 2012;487(7407):325-329. 